\title{
New strategies to improve multilateration systems in the air traffic control
}

IVÁN ANTONIO MANTILLA GAVIRIA 



\section{New Strategies to Improve Multilateration Systems in the Air Traffic Control}

Doctoral Thesis by

Ivan A. Mantilla Gaviria

Supervisors:

Juan V. Balbastre Tejedor

Gaspare Galati 

national and international level.

First Edition, 2013

\section{cc Iván Antonio Mantilla Gaviria}

(C) of the present edition:

Editorial Universitat Politècnica de València

www.editorial. upv.es

ISBN: 978-84-9048-089-2 (printed version)

Any unauthorized copying, distribution, marketing, editing, and in general any other exploitation, for whatever reason, of this piece of work or any part thereof, is strictly prohibited without the authors' expressed and written permission. 


\section{Finnancial Support}

The research developed in this thesis has been supported by a FPU scholarship (AP2008-03300) from the Spanish Ministry of Education. 
To My Mom, Cecilia (R.I.P.), this goal is also yours 



\section{Abstract}

This thesis is focused on the Mode S Multilateration (MLAT) systems for the Air Traffic Control (ATC) operations, specifically on their layout design and on the process of cooperative targets localization performed by them. It addresses the development of new design and localization strategies. The design strategies are based on the application of metaheuristic optimization techniques, whilst the localization ones are based on the application and combination of regularization methods along with some current localization algorithms.

The design strategies are composed of an effective, general procedure to emplace both the standard and enhanced MLAT receiving stations on a surveillance volume, and a set of design strategies to be used with such a proposed procedure. This procedure uses the metaheuristic of Genetic Algorithms (GA), and is intended to obtain useful design parameters that allow optimal system configurations that provide suitable performance levels. Furthermore, the procedure developed in this thesis is able to evaluate and improve previous system designs, as well as possible system enhancements. For this context, we overcome several issues like the lack of a general model that relates the system performance parameters with those ones that can be simulated on a computer, the setting of the design problem as a computer optimization one, the development and application of the numerical tools to analyze and evaluate the MLAT systems performance, the complexity evaluation of the resulting computer optimization problem, and the application and modification of the GA components for solving such a optimization problem. To validate the contributions in this context, some simulations are performed on a real airport scenario.

The localization strategies are composed of a set of developed regularized location estimators, a set of developed additional improvements, and the use of some current localization algorithms. These strategies can be used for both surface and wide areas surveillance, and solve some practical problems like the loss of position accuracy when using a small number of stations, or 
for small areas where the stations are close to each other (e.g., airport surface surveillance), and the mitigation of some errors due to multipath effect. Moreover, the proposed localization strategies are found to be highly statistically and numerically efficient, in contrast to current ones that are efficient in only one sense (i.e., statistically or numerically). For this context, we overcome several issues like the identification and evaluation of the numerical causes of the above mentioned problems, the analysis of the current localization algorithms, the adaptation of the regularization methods theory for solving the localization problem, the development of some numerical additional tools that allows the real time implementation of such proposed algorithms, and the combination of the current algorithms with the proposed ones. To validate the contributions in this context, some simulations with both simulated and real data scenarios are performed.

Keywords: Multilateration, Passive Localization, Mode S, Airport Surveillance, Combinatorial Optimization, Metaheuristic Techniques, Radar Architectures and Systems, Air Traffic Control, Regularization Methods, Inverse Problems. 


\section{Resumen}

Esta tesis está centrada en los sistemas de Multilateración (MLAT) Modo S para las operaciones de control de tráfico aéreo (ATC), específicamente en su diseño y en el proceso de localización de blancos cooperativos realizado por los mismos. Ésta aborda el desarrollo de nuevas estrategias de diseño y de localización. Las estrategias de diseño se basan en la aplicación de técnicas de optimización metaheurística, mientras que las de localización están basadas en la aplicación y combinación de métodos de regularización junto con algunos algoritmos actuales.

Las estrategias de diseño están compuestas por un procedimiento general, y efectivo, que permite la ubicación, en el volumen de vigilancia, de estaciones receptoras para sistemas MLAT estándar y mejorados, así como de un conjunto de estrategias que se utilizan con dicho procedimiento. Este procedimiento utiliza la metaheurística de los Algoritmos Genéticos (GA), y tiene como objetivo el cálculo de parámetros de diseño útiles, que permiten obtener configuraciones de sistema que proveen los niveles de rendimiento adecuados. Además, el procedimiento propuesto en esta tesis se puede utilizar también para la evaluación y mejora de otros diseños previos, así como para la mejora o ampliación de sistemas ya desplegados. En este contexto, se han superado ciertos problemas como la escasez de modelos generales que relacionen los parámetros de rendimiento del sistema, con aquellos que pueden ser simulados por ordenador, la configuración del problema de diseño como un problema de optimización por ordenador, el desarrollo y aplicación de herramientas numéricas para analizar y evaluar el rendimiento de los sistemas MLAT, la evaluación de la complejidad del problema de optimización resultante, y la aplicación y modificación de algunos componentes de los GA para la solución de dicho problema de optimización. Las contribuciones de esta parte se validan por medio de simulaciones de un escenario aeroportuario real.

Las estrategias de localización están compuestas por un conjunto de algoritmos regularizados de localización, desarrollados en esta tesis, por un conjunto de mejoras adicionales, y por ciertos algoritmos actuales de 
localización. Dichas estrategias pueden ser utilizadas tanto para vigilancia en superficie como para área amplia, y solucionan algunos problemas reales como la pérdida de precisión cuando se dispone de un número reducido de estaciones, o para áreas pequeñas donde todas las estaciones están muy cercanas unas de otras (por ejemplo, para vigilancia de superficie aeroportuaria), y la reducción de algunos errores debidos al efecto multicamino. Además, las estrategias de localización desarrolladas en esta tesis son altamente eficientes en sentido estadístico y numérico, en contraste con las actuales que lo son únicamente en un sentido (estadístico o numérico). En este contexto, se han superado ciertos problemas como la identificación y evaluación de las causas numéricas de los problemas anteriormente mencionados, el análisis de los algoritmos de localización actuales, la adaptación de la teoría de los métodos de regularización a la solución de los problemas de localización, el desarrollo de algunas herramientas numéricas adicionales que permitan la implementación, en tiempo real, de los algoritmos propuestos, y la combinación de los algoritmos actuales con los propuestos. Las contribuciones de esta parte se validan con simulaciones en escenarios con datos de medidas simuladas y en escenarios con datos de medidas reales.

Palabras claves: Multilateración, Localización Pasiva, Modo S, Vigilancia de Superficie, Optimización Combinatoria, Técnicas Metaheurísticas, Arquitecturas y Sistemas Radar, Control de Tráfico Aéreo, Métodos de Regularización, Problemas Inversos. 


\section{Resum}

Esta tesi està centrada en els sistemes de Multilateració (MLAT) Mode S per a les operacions de control de trànsit aeri (ATC), específicament en el seu disseny i en el procés de localització de blancs cooperatius realitzat pels mateixos. Esta aborda el desenvolupament de noves estratègies de disseny i de localització. Les estratègies de disseny es basen en l'aplicació de tècniques d'optimització metaheurística, mentre que les de localització estan basades en l'aplicació i combinació de mètodes de regularització junt amb alguns algoritmes actuals.

Les estratègies de disseny estan compostes per un procediment general, i efectiu, que permet la ubicació, en el volum de vigilància, d'estacions receptores per a sistemes MLAT estàndard i millorats, així com d'un conjunt d'estratègies que s'utilitzen amb el l'anomenat procediment. Este procediment utilitza la metaheurística dels Algoritmes Genètics (GA), i té com a objectiu el càlcul de paràmetres de disseny útils, que permeten obtindre configuracions de sistema que proveïxen els nivells de rendiment adequats. A més, el procediment proposat en esta tesi es pot utilitzar també per a l'avaluació i millora d'altres dissenys previs, així com per a la millora o ampliació de sistemes ja desplegats. En este context, s'han superat certs problemes com l'escassetat de models generals que relacionen els paràmetres de rendiment del sistema, amb aquells que poden ser simulats per ordinador, la configuració del problema de disseny com un problema d'optimització per ordinador, el desplegament i aplicació de ferramentes numèriques per a analitzar i avaluar el rendiment dels sistemes MLAT, l'avaluació de la complexitat del problema d'optimització resultant, i l'aplicació i modificació d'alguns components dels GA per a la solució del mencionat problema d'optimització. Les contribucions d'esta part es validen per mitjà de simulacions d'un escenari aeroportuari real.

Les estratègies de localització estan compostes per un conjunt d'algoritmes regularitzats de localització, desenvolupats en esta tesi, per un conjunt de millores addicionals, i per certs algoritmes actuals de localització. Les anomenades estratègies poden ser utilitzades tant per a 
vigilància en superfície com per a àrea àmplia, i solucionen alguns problemes reals com la pèrdua de precisió quan es disposa d'un número reduït d'estacions, o per a àrees xicotetes on totes les estacions estan molt pròximes les unes de les altres (per exemple, per a vigilància de superfície aeroportuària), i la reducció d'alguns errors deguts a este efecte multicamí. A més, les estratègies de localització desenvolupades en esta tesi són altament eficients en sentit estadístic i numèric, en contrast amb les actuals que ho són únicament en un sentit (estadístic o numèric). En este context, s'han superat certs problemes com la identificació i avaluació de les causes numèriques dels problemes anteriorment mencionats, l'anàlisi dels algoritmes de localització actuals, l'adaptació de la teoria dels mètodes de regularització a la solució dels problemes de localització, el desenvolupament d'algunes ferramentes numèriques addicionals que permeten la implementació, en temps real, dels algoritmes proposats, i la combinació dels algoritmes actuals amb els proposats. Les contribucions d'esta part es validen amb simulacions en escenaris amb dades de mesures simulades i en escenaris amb dades de mesures reals.

Paraules claus: Multilateració, Localització Passiva, Mode S, Vigilància de Superfície, Optimització Combinatòria, Tècniques Metaheurísticas, Arquitectures i Sistemes Radar, Control de Trànsit Aeri, Mètodes de Regularització, Problemes Inversos. 


\section{Acknowledgements}

The beginning, development and achievement of this thesis would not have been possible without the invaluable help of many people. First and foremost, I want to thank specially the infinite support of my mom, Cecilia, whom this work is dedicated, and who always gave me the best advices and encouragements to never give up and always reach my goals. Thanks a lot for ever.

Thanks to my God for everything received and the opportunities that arise in my life.

I offer my special gratitude to my supervisor, Dr. Juan V. Balbastre, who gave me the opportunity to come Spain, who has personally and technically supported me throughout these years with his knowledge and advice, and who has helped me to growth as a person and researcher, allowing me the space to conduct my own experiences.

I am very grateful with my external supervisor, Dr. Gaspare Galati, for his invaluable scientific support, very important to the successful achievement of this thesis, and also for hosting me in his research group, the Radar and Navigation Laboratory, at Tor Vergata University (Rome, Italy) during my two research periods there.

I am also very grateful with Dr. Mauro Leonardi from Tor Vergata University (Rome, Italy), and member of the Radar and Navigation Laboratory. I really appreciate his personal and scientific help during my time in Italy. Thanks for all the time we spent discussing about the usefulness of regularization methods for MLAT localization, and for your touristic information for traveling in Italy.

I would like to thank to Dr. Alejandro Díaz from Universidad Politécnica de Cartagena (Cartagena, Spain) for his teachings when investigated in optimization of microwave devices for my Master Thesis. I really appreciate his advices and all the time he spent working with me. The acquired knowledge in research and optimization methods has been very useful for me during these years. 
I would like to show my gratitude to all the colleagues and friends that I have known over these years in the research group GEA at the Universidad Politécnica de Valencia. I would like to mention Ruy, Tatitana, Silvia, Oscar, Oneira, Mauricio, Emilio, Catalina, and Camilo. Each of them helped me to have good moments in the day to day work. Likewise, I do not forget the administrative staff, Isidora and Mati, for her support in managing all the administrative aspects for conferences trips, scholarship payments, and university certifications.

Thanks to the Indra S.A. CNS/ATM department. Part of this thesis was motivated by the projects with this company about software tools for designing and evaluating MLAT systems. Specially thanks to Eng. Ramón Flores and Eng. Miguel Muñoz. All our discussions about the design and evaluation of MLAT systems have been of great help for developing the second part of this thesis.

Thanks for the company ERA A.S. for proviging the real recording data for testing the localization algorithms. These data have helped me to validate the usefulness of the third part of this thesis and to reach more suitable results.

A special word of thanks to Dr. Fernando Niño, who was the promoter of my trip to Spain. My doctoral studies and thesis are also part of his important efforts for promoting the international educational exchanges for Colombian students.

I wish also to thank the Spansh Ministry of Education for the financial support of this thesis under the FPU shoclarships program.

Many special thanks to my father, Ivan José, to my brother, Habib, and to my sister, Nohora. It is a pleasure for me to acknowledge the help given by them during these years. Thanks for their calls, emails, confidence and fondness.

A special thanks to my Spanish friend, Luis, for his personal advices and support during the last year of this work.

I would also like to thank to all of my friends that have helped me in one way or another. Thanks a lot.

Ivan A. Mantilla Gaviria

Valencia, Spain, 2013 


\section{Contents}

Abstract

Abbreviations and Acronyms..................................................................xxi

\section{Part I Introduction}

1 General Information About This Thesis ...............................................1

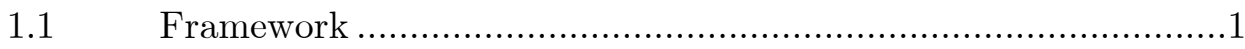

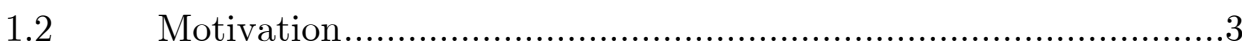

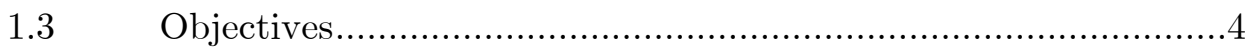

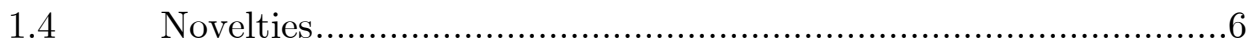

1.5 Organization of the Thesis ..................................................6

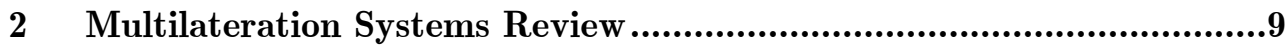

$2.1 \quad$ Operating Principles of Multilateration …..................................9

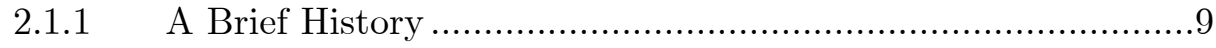

2.1.2 Definition and Theoretical Foundations ...............................13

2.1.3 Signals Used in MLAT Systems .......................................29

2.2 Multilateration Systems in the CNS/ATM Scheme ...................32

2.3 A General Scheme for Multilateration Systems .......................33 


\section{Part II Multilateration Systems Design and Deployment}

$3 \quad$ Overall Frame and Design Problem .................................................41

3.1 Multilateration System Design and its Particularities ..............41

3.1.1 Performance Parameters for MLAT Systems ....................... 43

3.1.2 Design System Parameters .....................................................45

3.1.3 Performance Parameters vs. Design Parameters ....................50

3.1.4 Particularities for the MLAT Stations Deployment ..............52

3.2 Solutions for MLAT Systems Design: State of the Art .............53

4 Optimization Theory: A Short Review ............................................59

$4.1 \quad$ Combinatorial Optimization Problems......................................59

4.1.1 Complexity of a CO Problem ................................................61

4.1.2 On the Solution of CO problems ..........................................62

4.2 Introduction to Metaheuristic Optimization Methods...............63

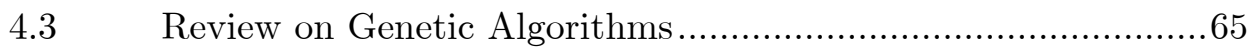

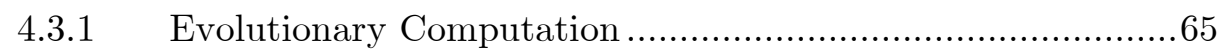

4.3.2 Genetic Algorithms.........................................................66

5 General Procedures for Optimizing the Multilateration Layout .........73

5.1 The Multilateration System Design as a Combinatorial Optimization Problem ......................................................................73

5.1.1 Mapping the MLAT System Design to a CO Problem..........75

5.2 Complexity Analysis of the MLAT Design Problem .................78

$5.3 \quad$ Description of the Overall Design Procedure ..............................84

5.3.1 General Framework ........................................................... 84

5.3.2 Iterative Numerical Procedure .............................................. 87

$5.4 \quad$ Proposed Design Strategies ................................................... 90

5.4.1 Standard MLAT System with a Fixed Number of Stations .. 91

5.4.2 Standard MLAT System with a Variable Number of Stations.

5.4.3 Enhanced MLAT System with a Fixed Number of Stations .94

5.5 Simulation and Results ..................................................... 95

5.5.1 MLAT System with a Fixed Number of TDOA Stations......96

5.5.2 MLAT System with a Variable Number of TDOA Stations .98

5.5.3 MLAT System with a Fixed Number of TDOA/AOA Stations 


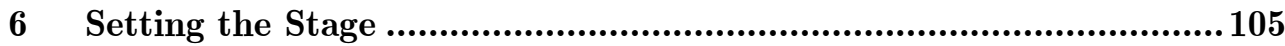

6.1 Localization Problem in Mode S Multilateration .................... 105

6.1.1 General Problem Description............................................... 106

6.2 An Inverse Problem Description ............................................... 109

6.2.1 Ill-Conditioned Problems ...................................................... 110

6.2.2 Solving Ill-Conditioned Problems .......................................... 111

6.3 Singular Value Decomposition (SVD) and Other Numerical

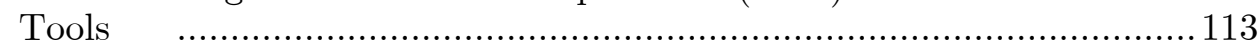

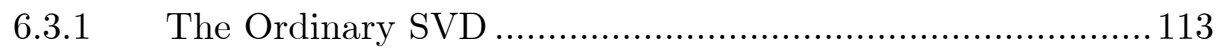

6.3.2 Null Space and Range of a Matrix .................................... 114

6.3.3 The Generalized SVD (GSVD) ....................................... 114

6.3.4 Classification of the Ill-Conditioned Problems...................... 115

6.3.5 Basic Parameters to Analyze Ill-Conditioned Problems ...... 116

6.4 Least Squares (LS) and the Pseudoinverse ............................ 117

7 Localization Algorithms for Mode S Multilateration..........................119

7.1 A Prelude to Localization Algorithms ........................................ 119

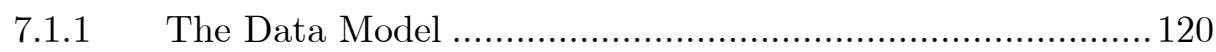

7.1.2 The Numerical Algorithm................................................. 122

7.1.3 The General Framework for Localization Algorithms ......... 122

7.1.4 Setting a General Notation................................................. 123

7.2 Open Form Algorithms ......................................................... 125

7.2.1 Taylor-Series Expansion Algorithm..................................... 125

7.3 Closed Form Algorithms .................................................... 129

7.3.1 Schmidt Algorithm (Plane Intersection)............................. 130

7.3.2 Smith and Abel Algorithm (Spherical Interpolation) .......... 132

7.3.3 Friedlander Algorithm (Spherical Interpolation) ................. 134

7.3.4 Schau and Robinson Algorithm (Spherical Intersection) .....137

7.3.5 Chan and Ho Algorithm (Spherical Interpolation with

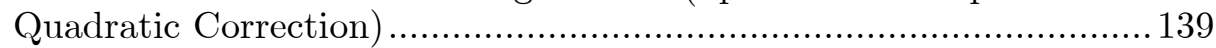

7.3.6 Bancroft Algorithm .......................................................... 144

7.3.7 Wikipedia Algorithm (Plane Intersection) .......................... 146

7.3.8 Summary of Localization Algorithms .................................. 147

7.4 Projected Algorithms .......................................................... 148

7.5 Analysis of Ill-Conditioned Problems in Mode S Multilateration. 
7.6 Simulation and Results: Comparison of Localization Algorithms 155

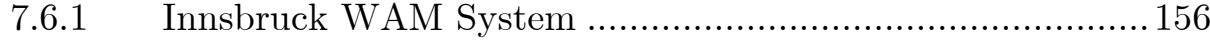

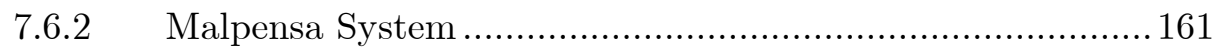

7.6.3 Linate System (Surface Movement).................................... 164

7.6.4 Numerical Comparison and General Conclusions for Simulated

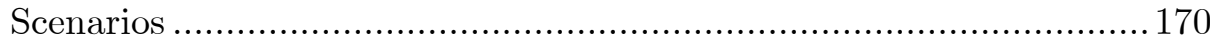

7.7 Experimental With Real Data ........................................... 175

8 Multilateration Localization by Using Regularization Algorithms .... 185

8.1 Solving Localization Problem by Tikhonov Regularization..... 187

8.1.1 Solving Regularized MLE by SVD ................................. 189

8.1.2 Effect of Tikhonov Regularization on the Localization

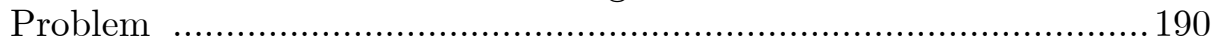

8.1.3 Regularization Error of Tikhonov Regularization................. 191

8.1.4 Estimation of the Regularization Parameter for Tikhonov.. 192

8.2 Solution by SVD Based Methods ......................................... 197

8.2.1 Solution by Truncated SVD (T-SVD) .............................. 198

8.2.2 Estimation of the Regularization Parameter for T-SVD .....200

8.2.3 Solution by T-SVD with a Sub-Set Selection (T-SVD SS) .. 201

8.3 Solution by Total Least Squares (TLS) Based Methods .........201

8.3.1 Solution by Truncated TLS (T-TLS) ...............................202

8.4 General Localization Strategy and Additional Improvements. 203

8.4.1 Additional Improvements ..............................................206

8.5 Simulation and Results: Analysis for Regularized Location

Estimators .......................................................................... 210

8.5.1 Accuracy Analysis for Innsbruck WAM System..................211

8.5.2 Accuracy Analysis for Malpensa WAM System ..................213

8.5.3 Accuracy Analysis for Linate (Surface) LAM System .........219

8.6 Simulation and Results: Analysis of the Starting Point Quality.. .

8.6.1 Feasible Options for LAM Operations.................................222

8.6.2 Feasible Options for WAM Operations .............................. 228

8.6.3 Accuracy Analysis for LAM Operations ............................. 229

8.6.4 Accuracy Analysis for WAM Operations............................2238

8.7 Experimental With Real Data: General Localization Strategy247

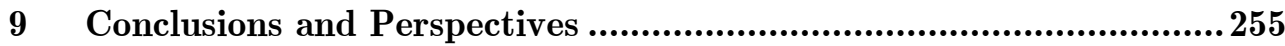

9.1 Conclusions for Design Strategies............................................. 255

9.2 Conclusions for Localization Strategies ................................ 257 
Contents $\quad$ xix

Future Work ....................................................... 261

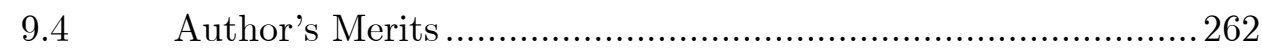

A Development of Combinatorial by Means of Stirling's Approximation..

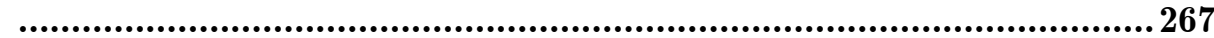

B Development of Averaging Kernels for Tikhonov Regularization .....269

C Simulated Scenarios .................................................................271

C.1 Scenarios with Real Geometry and Simulated Measurements. 271

C.1.1 Innsbruck System ............................................... 272

C.1.2 Malpensa System .................................................. 273

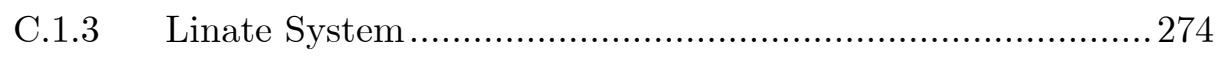

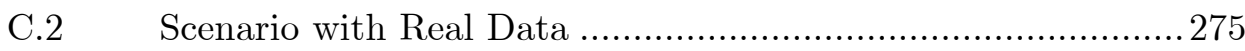

C.2.1 Tallinn LAM System (ERA A.S.) ..............................2275

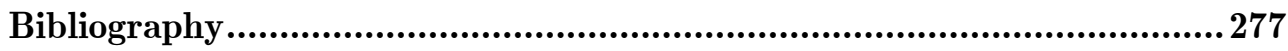





\section{List of Figures}

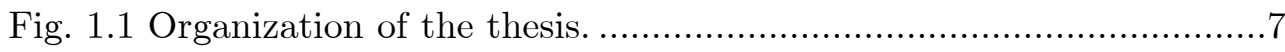

Fig. 2.1 The first hyperbolic positioning system: "Hyperbolic Audio

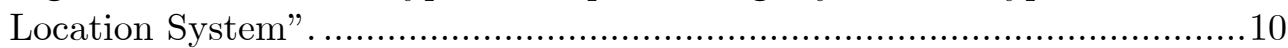

Fig. 2.2 Hyperbolic navigation system principle.....................................11

Fig. 2.3 General scheme for a MLAT system........................................ 15

Fig. 2.4 Section of a hyperboloid for a pair of receiving stations in MLAT

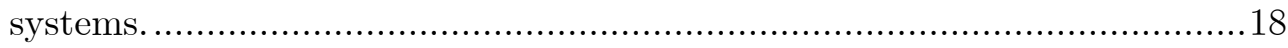

Fig. 2.5 Intersection of three hyperboloids. ........................................... 18

Fig. 2.6 Hyperbolas for four stations and a target located in an arbitrary position. The reference station is the station number one (the black one) and the target is represented by the magenta circle..................................19

Fig. 2.7 Error sources classification for MLAT systems.............................22

Fig. 2.8 Eccentricity and perpendicularity of the hyperbolas....................27

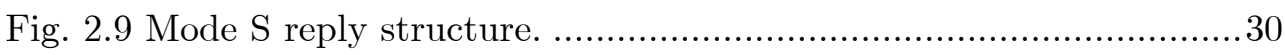

Fig. 2.10 A classification of the main surveillance systems. ........................33

Fig. 2.11 General logic architecture for an MLAT system.........................34

Fig. 2.12 General flowchart of MLAT functions. .......................................... 35

Fig. 3.1 Examples of LoS and NLOS scenarios. ......................................46

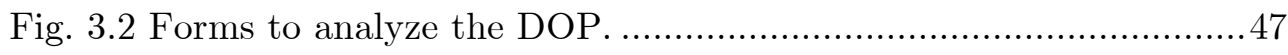

Fig. 3.3 Performance parameters vs. design parameters. ...........................50

Fig. 4.1 Trajectory-based methods vs. Population-based methods: Illustration of the search space exploration...............................................64

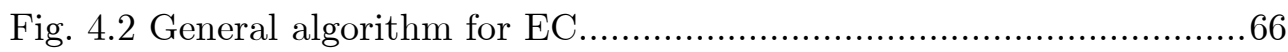

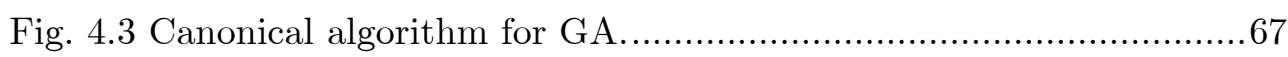

Fig. 4.4 Stochastic uniform selection operator. ........................................ 70

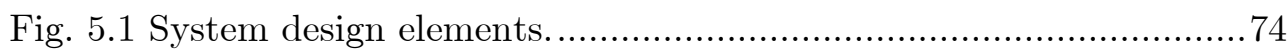


Fig. 5.2 Number of possible solutions in respect of the search space size and of the number of required stations. The vertical scales are logarithmic......81

Fig. 5.3 Joint analysis of the problem complexity ................................... 83

Fig. 5.4 General framework for designing MLAT systems. ........................85

Fig. 5.5 Flowchart for the iterative procedure (the framework core).........87

Fig. 5.6 Barcelona - El Prat airport layout with the possible sites to

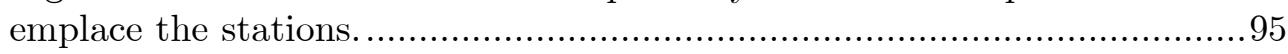

Fig. 5.7 Horizontal accuracy for the design with a fixed number of TDOA

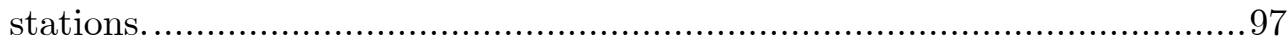

Fig. 5.8 GA convergence for the design with a fixed number of TDOA

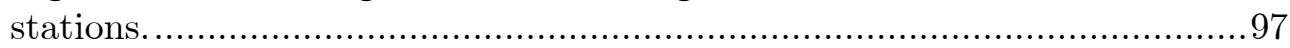

Fig. 5.9 SPoD for the design with a fixed number of TDOA stations.........98

Fig. 5.10 Horizontal accuracy for the design with a variable number of

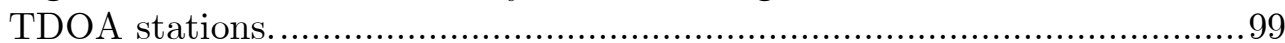

Fig. 5.11 GA convergence for the design with a variable number of TDOA

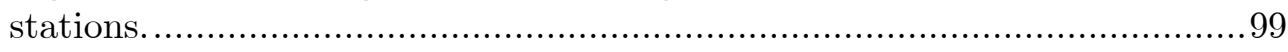

Fig. 5.12 SPoD for the design with a variable number of TDOA stations. 100

Fig. 5.13 Horizontal accuracy for the design with a fixed number of TDOA/AOA stations.................................................................... 101

Fig. 5.14 GA convergence for the design with a fixed number of

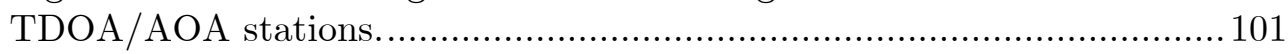

Fig. 5.15 SPoD for the design with a fixed number of TDOA/AOA stations. 102

Fig. 6.1 Localization problem in Mode S Multilateration......................... 106

Fig. 6.2 General scheme of a localization problem in MLAT................... 108

Fig. 6.3 Regularization strategy..................................................... 111

Fig. 7.1 General framework for localization algorithms.......................... 123

Fig. 7.2 General notation for localization algorithms............................... 124

Fig. 7.3 Geometrical interpretation of projected algorithms. ................... 149

Fig. 7.4 Ill-conditioned problem in MLAT systems: an example................151

Fig. 7.5 Condition number for the first seven Taylor iterations. Vertical axis represents the condition number and horizontal axis the Monte-Carlo trials.

152

Fig. 7.6 Characterization of the singular value spectrum for the MLAT localization problem (vertical axes in log scale). 154

Fig. 7.7 2D R.M.S error for Innsbruck system: comparison of localization algorithms. 
Fig. 7.8 Vertical R.M.S error for Innsbruck system: comparison of localization algorithms. 158

Fig. 7.9 2D bias for Innsbruck system: comparison of localization algorithms. 159

Fig. 7.10 Vertical bias for Innsbruck system: comparison of localization algorithms. 160

Fig. 7.11 2D R.M.S error for Innsbruck system: comparison of projected localization algorithms. 160

Fig. 7.12 2D bias for Innsbruck system: comparison of projected localization algorithms. 161

Fig. 7.13 2D R.M.S error for Malpensa system: comparison of localization algorithms. 162

Fig. 7.14 Vertical R.M.S for Malpensa system: comparison of localization algorithms. 163

Fig. 7.15 2D bias for Malpensa system: comparison of localization algorithms. 163

Fig. 7.16 Vertical bias for Malpensa system: comparison of localization algorithms. 163

Fig. 7.17 2D R.M.S error for Malpensa system: comparison of projected localization algorithms. 165

Fig. 7.18 2D bias for Malpensa system: comparison of projected localization algorithms. 165

Fig. 7.19 2D R.M.S error for Linate system: comparison of localization algorithms. 166

Fig. 7.20 2D bias for Linate system: comparison of localization algorithms. 166

Fig. 7.21 2D R.M.S error for Linate system: comparison of projected localization algorithms. 167

Fig. 7.22 2D bias for Linate system: comparison of projected localization algorithms. 167

Fig. 7.23 2D R.M.S error for Linate system in the presence of the failure of station 1: comparison of localization algorithms. 169

Fig. 7.24 2D bias for Linate system in the presence of the failure of station 1: comparison of localization algorithms. 169

Fig. 7.25 2D R.M.S error for Linate system in the presence of the failure of station 1: comparison of projected localization algorithms. 169

Fig. 7.26 2D bias for Linate system in the presence of the failure of station 1: comparison of projected localization algorithms. 170 
Fig. $7.27 \mathrm{X}(\mathrm{t})$ for statistical and algebraic approach based models: comparison of localization algorithms. 179

Fig. $7.28 \mathrm{X}(\mathrm{t})$ for numerical approach based models: comparison of localization algorithms. 179

Fig. $7.29 \mathrm{Y}(\mathrm{t})$ for statistical and algebraic approach based models: comparison of localization algorithms. 180

Fig. 7.30 $\mathrm{Y}(\mathrm{t})$ for numerical approach based models: comparison of localization algorithms. 180

Fig. 7.31 $\mathrm{X}(\mathrm{t})$ for statistical and algebraic approach based models: comparison of projected localization algorithms. 181

Fig. $7.32 \mathrm{X}(\mathrm{t})$ for numerical approach based models: comparison of projected localization algorithms 181

Fig. 7.33 $\mathrm{Y}(\mathrm{t})$ for statistical and algebraic approach based models: comparison of projected localization algorithms. 182

Fig. $7.34 \mathrm{Y}(\mathrm{t})$ for numerical approach based models: comparison of

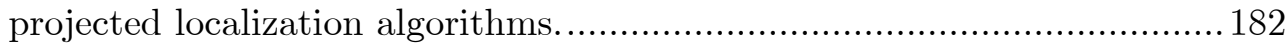

Fig. 8.1 Basic scheme for the general localization strategy..................... 205

Fig. 8.2 Solution selection by nearest estimated neighbour..................... 207

Fig. 8.3 Flowchart for the general strategy with the additional

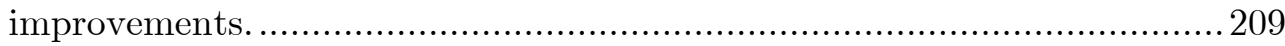

Fig. 8.4 2D R.M.S error for Innsbruck system. ....................................... 212

Fig. 8.5 Vertical R.M.S error for Innsbruck system............................... 212

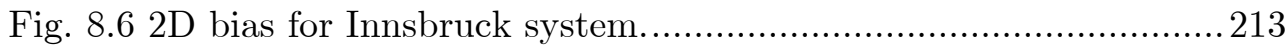

Fig. 8.7 Vertical bias for Innsbruck system............................................ 213

Fig. 8.8 2D R.M.S error for Malpensa system......................................... 214

Fig. 8.9 Vertical R.M.S error for Malpensa system..................................2 214

Fig. 8.10 2D bias for Malpensa system...............................................214

Fig. 8.11 2D R.M.S error for Malpensa system by using barometric altitude. 216

Fig. 8.12 Vertical R.M.S error for Malpensa system by using barometric altitude. 216

Fig. 8.13 2D bias for Malpensa system by using barometric altitude........217

Fig. 8.14 Vertical bias for Malpensa system by using barometric altitude. 217

Fig. 8.15 2D R.M.S error for Linate system with a fixed starting point...221

Fig. 8.16 2D bias for Linate system with a fixed starting point. .221

Fig. 8.17 2D R.M.S error for Linate system with starting point from projected version of Schau\&Robinson algorithm 222 
Fig. 8.18 2D bias for Linate system with starting point from projected version of Schau\&Robinson algorithm. 223

Fig. 8.19 2D R.M.S error for Linate system with a fixed starting point: failure of station 1 . 224

Fig. 8.20 2D bias for Linate system with a fixed starting point: failure of station 1 224

Fig. 8.21 2D R.M.S error for Linate system with starting point from projected version of Schau\&Robinson algorithm: failure of station 1.......225

Fig. 8.22 2D bias for Linate system with starting point from projected version of Schau\&Robinson algorithm: failure of station 1......................2225

Fig. 8.23 Feasible options for the starting point in LAM operations for the non-projected version of Taylor based algorithms. 228

Fig. 8.24 Feasible options for the starting point in LAM operations for the projected version of Taylor based algorithms. 228

Fig. 8.25 Feasible options for the starting point in WAM operations for the non-projected version of Taylor based algorithms................................... 228

Fig. 8.26 2D R.M.S error with starting point provided by $\mathrm{Schau}_{3 \mathrm{D}}$......... 230

Fig. 8.27 2D bias with starting point provided by $\mathrm{Schau}_{3 \mathrm{D}}$ …................. 230

Fig. 8.28 2D R.M.S error with starting point provided by $\mathrm{Schau}_{3 \mathrm{D}}$ : failure of station 1 .... 230

Fig. 8.29 2D bias with starting point provided by $\mathrm{Schau}_{3 \mathrm{D}}$ : failure of station

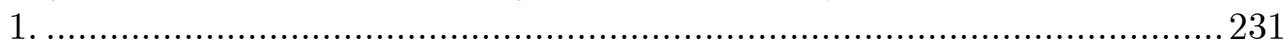

Fig. 8.30 2D R.M.S error with starting point provided by $\mathrm{Schau}_{3 \mathrm{D}}+$ fixed altitude. 232

Fig. 8.31 2D bias with starting point provided by $\mathrm{Schau}_{3 \mathrm{D}}+$ fixed altitude. 232

Fig. 8.32 2D R.M.S error with starting point provided by $\mathrm{Schau}_{3 \mathrm{D}}+$ fixed altitude: failure of station 1 . 232

Fig. 8.33 2D bias with starting point provided by $\mathrm{Schau}_{3 \mathrm{D}}+$ fixed altitude: failure of station 1 . 233

Fig. 8.34 2D R.M.S error with starting point set to a fixed coordinate. ...2234

Fig. 8.35 2D bias with starting point set to a fixed coordinate. 234

Fig. 8.36 2D R.M.S error with starting point set to a fixed coordinate: failure of station 1 . 234

Fig. 8.37 2D bias with starting point set to a fixed coordinate: failure of station 1 235

Fig. 8.38 2D R.M.S error with starting point provided by $\mathrm{Schau}_{2 \mathrm{D}}+$ fixed altitude. 236 
Fig. 8.39 2D bias with starting point provided by $\mathrm{Schau}_{2 \mathrm{D}}+$ fixed altitude. 236

Fig. 8.40 2D R.M.S error with starting point provided by $\mathrm{Schau}_{2 \mathrm{D}}+$ fixed altitude: failure of station 1 . 236

Fig. $8.412 \mathrm{D}$ bias with starting point provided by $\mathrm{Schau}_{2 \mathrm{D}}+$ fixed altitude: failure of station 1 . 237

Fig. 8.42 2D R.M.S error with starting point provided by $\mathrm{Schau}_{3 \mathrm{D}}$......... 239

Fig. 8.43 Vertical R.M.S error with starting point provided by $\operatorname{Schau}_{3 \mathrm{D}}$. 240

Fig. 8.44 2D bias with starting point provided by $\mathrm{Schau}_{3 \mathrm{D}}$.....................240

Fig. 8.45 2D R.M.S error with starting point provided by $\mathrm{Schau}_{3 \mathrm{D}}+$ fixed altitude. 241

Fig. 8.46 2D bias with starting point provided by $\mathrm{Schau}_{3 \mathrm{D}}+$ fixed altitude. 241

Fig. 8.47 2D R.M.S error with starting point set to a fixed coordinate. ...242

Fig. 8.48 2D bias with starting point set to a fixed coordinate. 242

Fig. 8.49 2D R.M.S error with starting point provided by $\mathrm{Schau}_{2 \mathrm{D}}+$ fixed altitude. 243

Fig. 8.50 2D bias with starting point provided by $\mathrm{Schau}_{2 \mathrm{D}}+$ fixed altitude. 243

Fig. 8.51 2D R.M.S error with starting point provided by $\mathrm{Schau}_{3 \mathrm{D}}+$

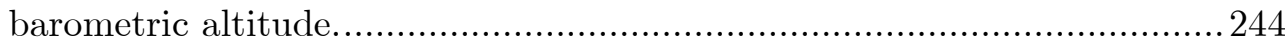

Fig. 8.52 Vertical R.M.S error with starting point provided by $\mathrm{Schau}_{3 \mathrm{D}}+$ barometric altitude. 244

Fig. 8.53 2D bias with starting point provided by $\mathrm{Schau}_{3 \mathrm{D}}+$ barometric altitude. .245

Fig. 8.54 X(t) for Tikhonov based RLE.................................................. 250

Fig. 8.55 Y(t) for Tikhonov based RLE.................................................... 251

Fig. $8.56 \mathrm{X}(\mathrm{t})$ for T-SVD based RLE....................................................... 252

Fig. 8.57 Y(t) for T-SVD based RLE....................................................... 253

Fig. C.1 Innsbruck system layout (top: vertical profile, bottom: horizontal profile).

Fig. C.2 Malpensa system layout for the first takeoff line: Malpensa 1 (top: horizontal profile, bottom: vertical profile). 273

Fig. C.3 Linate system layout and the simulated surface movement........274

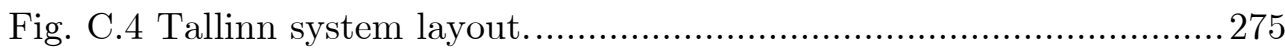

Fig. C.5 Line of sight profile for Tallinn system..................................... 276 


\section{List of Tables}

Table 2.1 Summary of MLAT measurement errors..................................24

Table 3.1 Qualitative scale for the PDOP. ................................................4 46

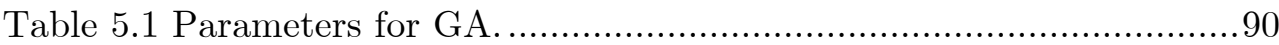

Table 7.1 Summary of localization algorithms. A: Alegbraic, S: Statistical,

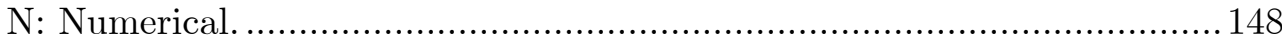

Table 7.2 Mean 2D R.M.S error and 2D bias values for localization algorithms. Values given in meters. ....................................................... 173

Table 7.3 Mean vertical R.M.S error and vertical bias values for localization algorithms. Values given in meters. ................................................. 173

Table 7.4 Mean 2D R.M.S error and 2D bias values for projected localization algorithms. Values given in meters. ........................................ 174

Table 7.5 Mean 2D R.M.S error and 2D bias values for the localization algorithms, at Linate scenario, in the presence of a failure of station 1. Values given in meters.

Table 7.6 Statistical parameters for the error distributions of localization algorithms. Values given in meters. 183

Table 7.7 Statistical parameters for the error distributions of projected localization algorithms. Values given in meters. .................................... 183

Table 8.1 Possible sources for the starting point. ...................................227

Table 8.2 Mean of the 2D bias over the simulated path of Linate system. Values given in meters. 237

Table 8.3 Mean of the 2D R.M.S error over the simulated path of Linate system. Values given in meters. 238

Table 8.4 Mean of the 2D bias over the simulated path of Linate system: failure of station 1 . Values given in meters. 238

Table 8.5 Mean of the 2D R.M.S error over the simulated path of Linate system: failure of station 1 . Values given in meters. 238 
Table 8.6 Mean of the 2D bias over the entire takeoff line of Malpensa system. Values given in meters.

Table 8.7 Mean of the 2D R.M.S error over the entire takeoff line of Malpensa system. Values given in meters.

247

Table 8.8 Statistical parameters for the error distributions of the Tikhonov based RLE. Values given in meters................................................. 254

Table 8.9 Statistical parameters for the error distributions of the T-SVD

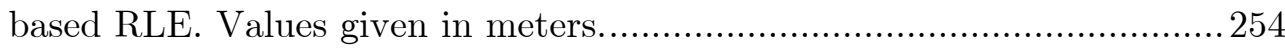

Table C.1 Stations coordinates for the Innsbruck WAM system..............272

Table C.2 Stations coordinates for the Malpensa WAM system...............273

Table C.3 Stations coordinates for the Linate LAM system....................2274 


\section{List of Symbols}

$\boldsymbol{X} \quad$ Generic notation for a matrix

$\boldsymbol{x} \quad$ Generic notation for a vector

$x \quad$ Generic notation for a scalar

$(\cdot)^{T} \quad$ Transpose

$(\cdot)^{-1}$ Inverse matrix

$(\cdot)^{\dagger} \quad$ Moore-Penrose pseudoinverse (or simply pseudoinverse matrix)

$(\cdot)$ ! Factorial number

$\|\cdot\|_{p} \quad$ p-norm operator

$\|\cdot\|_{F} \quad$ Frobenius norm

$\hat{*} \quad$ Estimated value

$\langle\because$,$\rangle \quad Lorenz inner product$

$E[\cdot] \quad$ Expected value of random variable

$\mathcal{N}(\cdot) \quad$ Null space operator

$\boldsymbol{N}(\cdot) \quad$ Covariance matrix for the random error vector

$\boldsymbol{U} \quad$ Left singular matrix

$\boldsymbol{\Sigma} \quad$ Matrix of singular values

$\boldsymbol{V} \quad$ Right singular matrix

$\boldsymbol{L} \quad$ Regularization matrix of Tikhonov

$\boldsymbol{A}_{\lambda}^{-1} \quad$ Regularized inverse matrix of Tikhonov

$\boldsymbol{\Xi} \quad$ Resolution matrix

ח Permutation matrix

$\boldsymbol{I}_{n} \quad n \times n$ identity matrix

$\boldsymbol{\theta}$ Target position vector

$\boldsymbol{\vartheta}_{i} \quad$ Position vector for the $i$ th receiving station

$\boldsymbol{m}$ Measurement vector

$\boldsymbol{n}$ Random error vector

$\boldsymbol{u}_{i} \quad$ ith left singular vector

$\boldsymbol{v}_{i} \quad i$ th right singular vector

$\xi_{i}^{T} \quad i$ th averaging kernel

$\rho(\cdot) \quad$ Residual error norm function

$\Omega(\cdot) \quad$ Smooting norm function 
$\Lambda(\cdot) \quad$ Likelihood function

$P(A \mid B) \quad$ Condition probability of $A$, given $B$

$\sigma_{T O A_{i}}^{2} \quad$ Variance for the $i$ th TOA measurement error

$N_{A} \quad$ Number of stations forming an array

$N_{S} \quad$ Number of stations

$n \quad$ Number of coordinates to be estimated

$r_{i} \quad$ Propagation distance from the target to the $i$ th station

$t_{e} \quad$ Emission time

$\sigma_{i} \quad i$ th singular value

$r_{\epsilon} \quad$ Numerical rank of a matrix

$R_{S} \quad$ Target range

$K \quad$ Number of Taylor iterations

$\lambda$ Regularization parameter of Tikhonov

$f_{i} \quad i$ th filter factor of Tikhonov

$k_{\epsilon} \quad$ Discrete regularization parameter for T-SVD 


\section{Abbreviations and Acronyms}

\begin{tabular}{ll} 
A.M.S.L & Above Mean Sea Level \\
ADS & Automatic Dependent Surveillance \\
ADS-B & Automatic Dependent Surveillance - Broadcast \\
ANSP & Air Navigation Services Provider \\
AOA & Angle of Arrival \\
ASDE & Airport Surface Detection Equipment \\
ATC & Air Traffic Control \\
ATCRBS & Air Traffic Control Radar Beacon System \\
ATM & Air Traffic Management \\
CNS & Communication Navigation and Surveillance \\
CO & Combinatorial Optimization \\
CPS & Central Processing Subsystem \\
CRLB & Cràmer-Rao Lower Bound \\
DF & Downlink Format \\
DGPS & Differential Global Positioning System \\
DOP & Dilution of Precision \\
DTM & Digital Terrain Model \\
DXF & Data Exchange Format \\
ECAC & European Civil Aviation Conference \\
EUROCAE & European Organisation for Civil Aviation Equipment \\
EUROCONTROL & European Organisation for the Safety of Air Navigation \\
FIM & Fisher Information Matrix \\
FRUIT & False Replies Unsynchronized in Time \\
GA & Genetic Algorithm \\
GCV & Generalized Cross Validation \\
GPS & Global Positioning System \\
GSVD & Generalized Singular Value Decomposition \\
HAOA & Horizontal Angle of Arrival \\
HDOP & Horizontal Dilution of Precision \\
IATA & International Air Transport Association \\
ICAO & International Civil Aviation Organization \\
& \\
\hline
\end{tabular}




\begin{tabular}{|c|c|}
\hline KF & Kalman Filter \\
\hline LAM & Local Area Multilateration \\
\hline LORAN & LOng RAnge Navigation \\
\hline LoS & Line of Sight \\
\hline LP & Linear Programming \\
\hline LS & Least Squares \\
\hline ML & Maximum Likelihood \\
\hline MLAT & Multilateration \\
\hline MLE & Maximum Likelihood Estimator \\
\hline MOPS & Minimum Operational Performance Specification \\
\hline MSSR & Monopulse Secondary Surveillance Radar \\
\hline $\mathrm{NaN}$ & Not a Number \\
\hline NLOS & Non Line of Sight \\
\hline NP & Nondeterministic Polynomial \\
\hline NP & Nonlinear Programming \\
\hline PDOP & Position Dillution of Precision \\
\hline PoD & Probability of Detection \\
\hline PoFD & Probability of False Detection \\
\hline PoFID & Probability of False Identification \\
\hline PoI & Probability of Identification \\
\hline PoL & Probability of Localization \\
\hline PSR & Primary Surveillance Radar \\
\hline QP & Quadratic Programming \\
\hline R.M.S & Root Mean Square \\
\hline RLE & Regularized Location Estimator \\
\hline RTD & Rount Trip Delay \\
\hline SM & Measurement Selection \\
\hline SNR & Signal to Noise Ration \\
\hline SPoD & System Probability of Detection \\
\hline SS & Solution Selection \\
\hline SSR & Secondary Surveillance Radar \\
\hline SVD & Singular Value Decomposition \\
\hline TCAS & Traffic alert and Collision Avoidance System \\
\hline TDOA & Time Difference of Arrival \\
\hline TLS & Total Least Squares \\
\hline TMA & Terminal Manoeuvring Area \\
\hline TOA & Time Of Arrival \\
\hline T-SVD & Truncated - Singular Value Decomposition \\
\hline T-SVD SS & $\begin{array}{l}\text { Truncated - Singular Value Decomposition Subset } \\
\text { Selection }\end{array}$ \\
\hline T-TLS & Truncated - Total Least Squares \\
\hline UAT & Universal Access Transceiver \\
\hline VAOA & Vertical Angle of Arrival \\
\hline VDOP & Vertical Dilution of Precision \\
\hline
\end{tabular}


VHF

WAM

Very High Frequency

Wide Area Multilateration 

Part I

Introduction 



\section{General Information About This Thesis}

\subsection{Framework}

Multilateration (MLAT) systems are a powerful means for the surveillance function of Air Traffic Control (ATC) operations. These systems are intended to extract, and display to air traffic controllers, the position and identification of aircrafts (taxiing, taking off/landing, in the approach or enroute phases of flight) or vehicles equipped with a Secondary Surveillance Radar (SSR) transponder [1-2]. In these systems, a number of ground receiving stations, with capabilities to measure some physical characteristics of signals emitted by transponders, such as Time of Arrival (TOA) -for the standard version-, Round Trip Delay (RTD) or Angle of Arrival (AOA) for the enhanced versions-, are placed in some strategic locations around the coverage area, and they are connected with a Central Processing Subsystem (CPS) to compute the target (i.e., aircraft or vehicle) position. In the standard, and most widely extended, configuration of MLAT systems, the Mode $\mathrm{S}$ transmissions and the unsolicited transponder emissions (i.e., the squitter) as well as the responses to interrogations elicited by the MLAT system, can be used. These signals are received by the ground stations and their TOAs are measured and sent to the CPS, where the transponder position is calculated. This calculation is based on the Time Difference of Arrival (TDOA) principle, where mathematical intersections of multiples hyperbolas (or hyperboloids), which have been created with relative time differences, are solved.

These systems include both hardware and software (as most systems do) components. The hardware is mainly composed by the ground stations, the CPS, other auxiliary electronic devices and communication data links, and the software one is composed by a set of procedures which perform some specific functions in both ground stations and CPS.

For the hardware implementation, a design must be carried out to choose a suitable number of stations and to set them up around the coverage area, in order to satisfy some specific requirements and 
restrictions. This is an ad-hoc process (particular for every scenario), which is usually achieved by trial and error and is subject to the particular experience of system dealers. Through this process, the most important aspect is the spatial distribution of the stations, relative to the coverage area, as the system accuracy strongly depends on that factor. Once the hardware implementation is finalized, the system is ready to perform the set of specific functions.

Basically, the elementary MLAT system functions are: the TOA measurements, the target identification, the target position estimation, the integrity analysis, the target tracking, and the system synchronization. As a matter of fact, each of such functions always introduces a disturbance (noise) term, whose orders of magnitudes have a big influence on the maximum achievable system accuracy. On the other hand, in order to reduce the position errors, a post MLAT function, called data fusion, and which is common to all surveillance systems (e.g., primary radar, secondary radar, MLAT and Automatic Dependant Surveillance - Broadcast -ADS-B) deployed in the coverage area, can be implemented to combine the position estimated by them and thus obtaining a final position, which generally could be more accurate and reliable than the individual ones.

The overall MLAT performance strongly depends on three aspects: the quality of the system layout design, on the kind of measurements and their accuracy, and the quality of the localization process, which can involve the target position estimation, which is the localization itself, and the tracking.

Regarding to the first aspect (system layout design), its main goal is to deploy the minimum number of stations, in order to obtain the requested system coverage and performance, meeting all the regulatory standards, and the constraints imposed by each particular scenario, with the minimum cost. In general, choosing the number of stations and their locations to cope with all the requirements is not an obvious task and the system designer has to make several attempts, by trial and error, before obtaining a satisfactory spatial distribution of the stations. Moreover, an important number of parameters, like Line of Sight (LoS), Probability of Detection (PoD), Position Dilution of Precision (PDOP), Signal-to-Noise Ratio (SNR), multipath effects, instrumental errors, the kind of measurements to be used (e.g., TOA/TDOA, RTD, AOA), etc., must be taken into account when designing these systems. As it can be expected, the system design process, performed by trial and error, is subject to several non controllable and subjective parameters which can lead to non optimal designs.

The localization is the function that determines the target position by using, as inputs, the measurements and the system geometry. The algorithms that calculate the position are currently classified into two families: closed form algorithms and open form algorithms. In both cases, a system of highly nonlinear equations must be solved to obtain the aircraft 
position (more exactly, the transponder one). On the other hand, the error at this stage mainly depends on the spatial distribution of the ground stations (system geometry), the SNR, multipath, measurements accuracy (noise presented in the TOA, TDOA, RTD or AOA), the system transient performance, an on the efficiency on the localization algorithm to construct and to solve the system of equations. There are several works where the total error at this stage is studied [3-7]. It can be seen that in normal conditions the total Root Mean Square (R.M.S) error is between 5-100 m, depending on the scenario characteristics. However, in those publications the authors comment about an important and critical phenomenon in the system of equations called ill-conditioning of the problem. This means that the system of equations does not meet the three Hadamard conditions [8], namely: the solution exists, the solution is unique and the solutions continuously depends on the problem data. The effect of this problem is that the system accuracy became very bad (e.g., errors greater than $500 \mathrm{~m}$ ) or simply the solution (aircraft position) does not exists. Likewise, this problem is present in all the state of the art of the localization algorithms.

The system synchronization is another critical function, which also can be an error source. However, this function is not directly related to the target localization process. Therefore, its study is not part of this thesis, wherein a perfect synchronization is assumed. Finally, the integrity function is another additional function that can be embedded or not in the system and it is neither studied in this thesis.

Few works have been found regarding to the two situations commented above, for this reason, we propose new strategies, for the system design and target localization, that allow improving the overall system performance for ATC operations.

\subsection{Motivation}

The multilateration systems are being widely deployed over a high number of airports around the world, for the aircraft surveillance and control in all flight phases, and in many cases they are replacing the classical secondary surveillance radar. The European Organisation for the Safety of Air Navigation (EUROCONTROL) has published in his report "The ATM Surveillance Strategy for ECAC" [9] that the MLAT systems will be one of the three pillars for the ATC infrastructures after 2020. The latter shows the present and future relevance that these systems have in the operations of all air spaces around the world. Likewise, the international scientific community has made clear that problems like the position uncertainty, for some coverage areas, or poor accuracies in other ones, are the main 
challenges, which can be solved in order to make these systems $100 \%$ reliable, allowing an optimum management of the growing world air traffic.

Regarding to the efficient MLAT systems design, no relevant works have been proposed, and currently this process must be performed by trial and error. On the other hand, for the target localization process, two interesting lines have been explored. One of them consists of developing an important number of localization strategies, which are intended to efficiently estimate the target position. However, besides these algorithms are also subject to the same problems commented before in $\S 1.1$, they are only efficient in one sense: statistically or numerically, but not in both. It is a current limitation of the state of the art which has motivated us to develop the present thesis. The second solution that has been proposed in the recent years is the data fusion, which basically consists of combining the information provided by more than one system and thus obtaining a final position more "accurate and reliable". This solution, although has been successfully applied, it does not solve the root of the problems, commented before in $\S 1.1$, because it is a post-process that, first, in many cases it can has inputs with a high level of errors and, it always requires more than one deployed system. Therefore, the researches proposed in this thesis are focused on improving these systems from their more elementary structures: before operations -system design- and before data fusion -localization-. This is achieved by means of new strategies, based on metaheuristic optimization techniques and regularization methods, some of which have already been successfully tested in other scientific fields, like the image processing or geophysics.

\section{$1.3 \quad$ Objectives}

The general objective of this thesis is to develop new strategies to design and operate the MLAT systems, used for ATC operations, in a more efficient way. The design strategies are based on the utilization of metaheuristic optimization techniques and they are intended to find the optimal spatial distribution of the system ground stations, taking into account the most relevant system operation parameters. The strategies to operate the systems are based on the development of new localization algorithms which allow solving the problems of position uncertainty and poor accuracy that the current systems can present. Moreover, these algorithms are intended to be more statistically and numerically efficient than the current state of the art. The new strategies can be applied to design, deploy and operate the MLAT systems for airport surface surveillance as well as for takeoff-landing, approach and enroute control. An important advance in the current knowledge of air traffic control is expected from the development of these strategies, because they solve 
several deficiencies that have been made clear, by the international scientific community, in the last years.

To reach the general idea of this thesis we propose the following particular objectives:

- To develop, adapt and apply some metaheuristic optimization techniques, in order to obtain new strategies that can be applied in the efficient and automatic design of the MLAT systems layout. With these strategies, it will be possible to obtain the optimal spatial distribution of the system ground stations, taking into account all the relevant parameters which influence the accuracy, for any scenario. These strategies will allow designing and deploying the MLAT systems in a more efficient way, leading to an important reduction of the time and economic costs with regard to the classical procedures based on a trial and error approach.

- To develop a general, novel framework to understand, evaluate and compare the localization algorithms. This new framework allows the classification of localization algorithms in a wider sense than the classical one of open and closed form algorithms.

- To simulate and evaluate the efficiency of the most representative localization algorithms, in order to identify the ones that provides the better statistical or numerical characteristics.

- To develop and adapt the mathematical theory of regularization methods to solve the target localization in MLAT systems. It allow us to overcome the ill-conditioning of the resulting system of equations (i.e., of an inverse problem), to reduce the errors due to measurements noise and those due to a potential reduction of the number of system ground stations and, possibly, to mitigate errors which can be originated by external factors like those produced by the multipath effect. With this theory it will be possible to implement more accurate, reliable and fault-tolerant systems.

- To develop a general localization strategy that combines the proposed localization algorithms, a set of proposed additional improvements, and the tracking algorithms. It allows the general improvement of the localization process by making use of all the available resources in the general ATC infrastructure.

- To simulate and analyze the full MLAT system performance, in order to validate the design, deployment and operations process. It allows to evaluate the proposed strategies as well as to implement the necessaries adjustments to obtain the best possible accuracy levels. To reach this objective, simulated and real data will be used. 


\subsection{Novelties}

As we have stated before, in this thesis new design and localization strategies, which allow improving the overall MLAT system performance, are proposed. In this sense, the particular novelties and contributions of this thesis can by summarized as follows:

- We have unified all the relevant aspects for MLAT system design into a unique source.

- We have set the MLAT system design as a Combinatorial Optimization $(\mathrm{CO})$ problem, demonstrating also that there is no a deterministic algorithm that solves this problem in a polynomial computer time.

- We have developed and general strategy to the efficient and automatic design of MLAT systems. This strategy makes use of all the real parameters that control the overall system accuracy, as well as, of any kind of restriction under any kind of scenario.

- We have developed a new general framework that classifies the localization algorithms, and define them as the pair composed by a data model and a numerical algorithm.

- We have developed some regularized location estimators that are more statistically and numerically efficient than the current ones in the state of the art.

- We have developed a general localization strategy, which can be used for any surveillance scenario, and which shows certain robustness under situations of measurements perturbed by random noises and by the corresponding ones produced by the multipath effect.

\subsection{Organization of the Thesis}

Since this thesis describes the research of two contexts for MLAT systems, we have structured it into three parts, nine chapters, and three annexes, as shown in Fig. 1.1.

The first part, Introduction, is composed of two chapters, 1 and 2, that are horizontal to the entire thesis. The Chapter $\mathbf{1}$ describes the general information of this thesis, whereas the Chapter $\mathbf{2}$ provides a short, but complete review for the theory of MLAT systems.

The second part, Multilateration Systems Design and Deployment, is composed of three chapters: 3, 4, and 5. The Chapter 3 provides a complete description of the design of MLAT systems, describing all the parameters that have been taken into account, and the state of the art for the different solutions already in the literature. The Chapter 4 


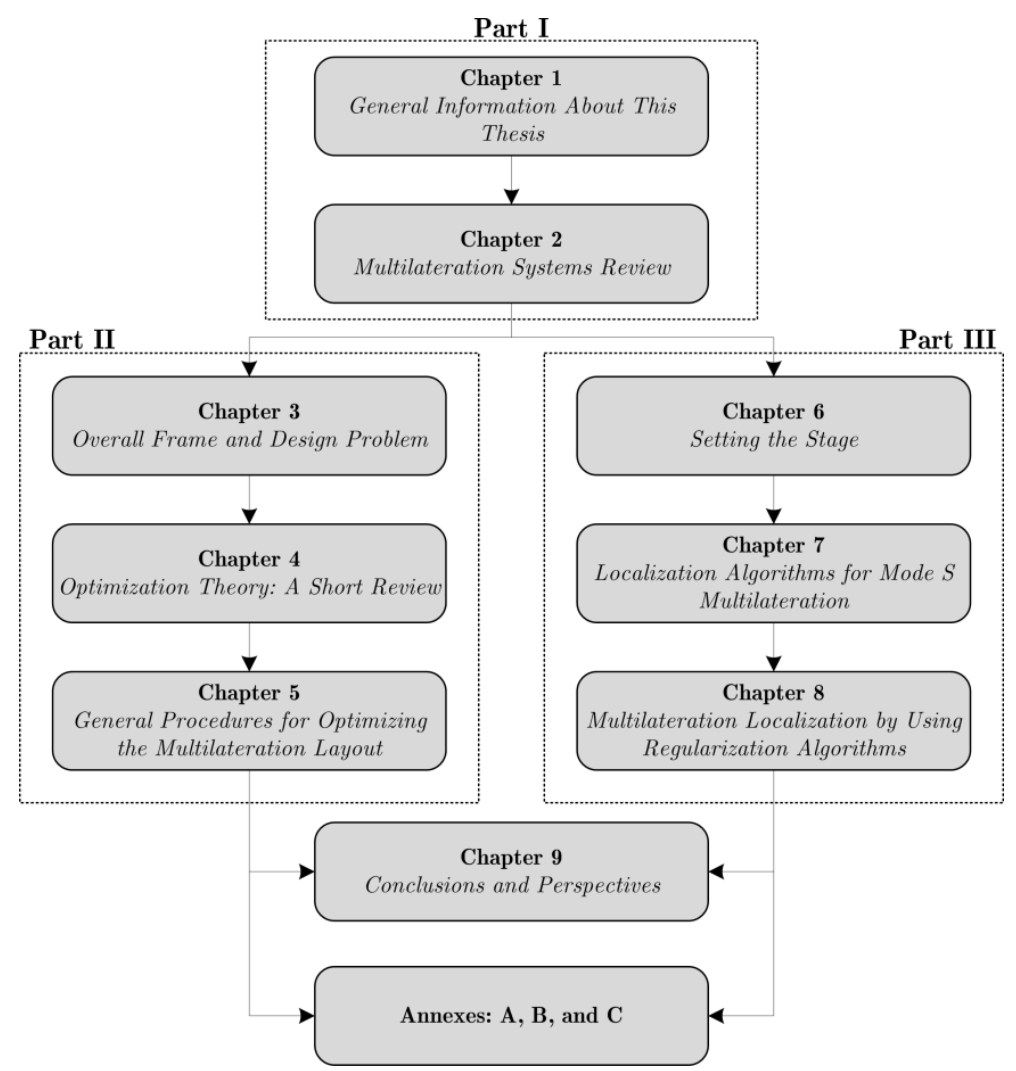

Fig. 1.1 Organization of the thesis.

provides a short revision of the optimization theory, describing the combinatorial optimization problems, and the optimization algorithms used and adapted for this thesis. Finally, the Chapter $\mathbf{5}$ provides the complexity analysis for the MLAT design problem as a combinatorial optimization one, and the proposed general procedures for optimizing the MLAT systems layout.

The third part, Multilateration Algorithms and Their Improvements, is composed of three chapters: 6,7 , and 8 . The Chapter 6 provides a short revision of the mathematics for analyzing and solving inverse problems. The Chapter $\mathbf{7}$ provides a complete analysis for the current localization algorithms for MLAT systems, the proposed general framework for them, and the corresponding accuracy analysis. In this chapter, the simulations are performed with both simulated and real data scenarios. Finally, the Chapter $\mathbf{8}$ provides the proposed regularized location estimators, and the proposed general localization strategy with a set of additional improvements. Also for this chapter, the simulations are performed with both simulated and real data scenarios. 
Finally, the Chapter 9 provides the general conclusions, perspectives, and contribution to the knowledge reached in this thesis. The Annex A provide a mathematical demonstration required for Chapter 5, the Annex B a mathematical demonstration required for Chapter 8, the Annex C describes the simulated and real data scenarios used for the simulations in Chapter 7 and Chapter 8 . 


\section{Multilateration Systems Review}

In this chapter, we provide a general review for the Multilateration (MLAT) systems context. We start by providing a description of the operating principles of these systems, covering from their history, the definition and theoretical foundations, and the different signals that are used for target localization with those systems. Then, we briefly describe the position of MLAT systems within the Air Traffic Control (ATC) infrastructures, and finalize the chapter by providing a general scheme for understanding the complete operation of MLAT as a surveillance system. The main purpose of this chapter is to place the reader into the scientific and technological field this thesis is dealing.

\subsection{Operating Principles of Multilateration}

In this section, we describe the operating principle of MLAT systems. Their history, from their first development, going through their evolution toward the navigation systems, until the current application as cooperative and independent surveillance systems, is described. Then, the definition and theoretical foundations are also provided. The basic mathematical principle, its geometrical interpretation, the kind of measurements that are used, the noises affecting them, and the influence of these noisy measurements into the target localization process are thoroughly described. Finally, this section is finalized by describing the different signals available for using along with the MLAT systems in the ATC infrastructures.

\subsubsection{A Brief History}

The multilateration systems, also commonly as a kind of hyperbolic positioning systems, arose during the First World War. From that time dates the first localization application based on the hyperbolic positioning principle, which was called "Hyperbolic Audio Location System" [10]. This application was based on the relative time of arrival measurements of sound 


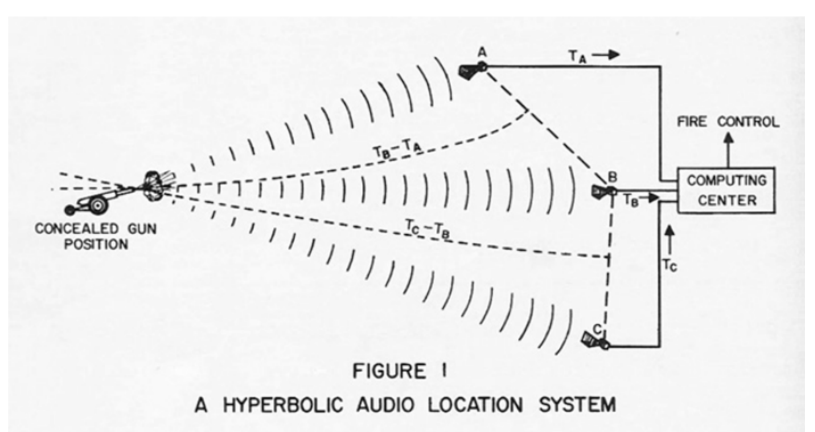

Fig. 2.1 The first hyperbolic positioning system: "Hyperbolic Audio Location System".

signals. Such a system was used for determining the hidden war cannons location in the battlefields. The original scheme of this system, taken from [10], is reproduced in Fig. 2.1.

In that system, the time of arrivals, of the sounds of explosions, to the receiving stations ( $A, B$ and $C$ in Fig. 2.1), were determined by using a chronograph installed in the "computing center". Then, these measurements were used to manually determine how far was the cannon from the station $B$ in respect of station $A$ and how far from the station $C$ in respect of station $B$. Finally, the user drew two hyperbolas $\left(T_{B}-T_{A}\right.$ and $T_{C}-T_{B}$ in Fig. 2.1), whose intersection was taken as the cannon location. The main limitations of this system were the short-range of the sound waves and the low accuracy of the time measurements device.

From the First World War, during the emergence of civil commercial aviation, came up the need of accurately know the location of the aircrafts in respect of the departure and arrival fields. In that time started the development of the aids for air navigation. Later, during the Second World War, radiofrequency generators capable of delivering signals of kilowatts of power and devices capable of measuring the time of arrival, with a precision of one millionth of a second, were developed. With these tools, the first hyperbolic navigation systems [11] were created within the class of radio navigation systems. These systems can be considered as an inverse ${ }^{1}$ evolution of that one shown in Fig. 2.1.

One of the first hyperbolic navigation systems was the $\mathrm{GEE}^{2}$, which was fully operative in 1942, and used by the British Royal Air Force during the

\footnotetext{
${ }^{1}$ Note the term "inverse" refers to the fact that in the first application in Fig. 2.1, the signal source location was variable, its position was calculated in a fixed central site and the corresponding receiving stations were also fixed. By the contrary, in the hyperbolic navigation systems, the signal sources are fixed (system stations) and the aircraft, whose location is evidently not fixed, is at the same time receiver and the site where the position is calculated.

${ }^{2}$ GEE is not an acronym, it is really the short for "Grid".
} 


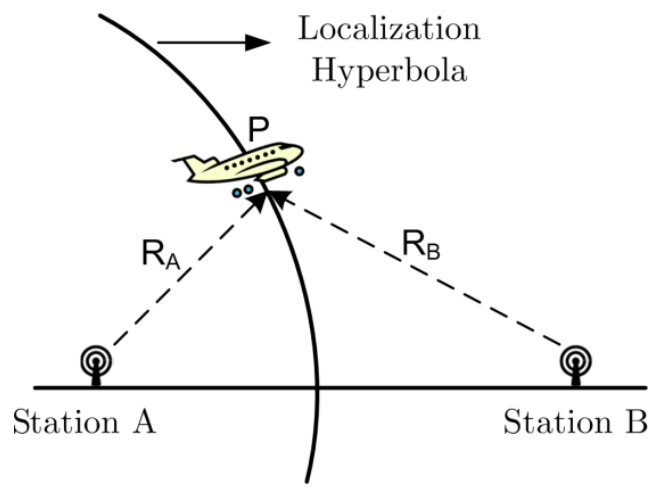

Fig. 2.2 Hyperbolic navigation system principle.

Second World War. Another one was the LORAN (LOng RAnge Navigation), which was fully operative in the spring of 1943. Finally, it is worth mentioning also other posterior/improved systems as DECCA, LORAN-B, LORAN-C and the OMEGA.

The hyperbolic navigation systems use a set of ground stations, deployed in sites with known locations and synchronized in time and phase. These stations transmit electromagnetic signals, which are received by the aircraft, where a physical parameter such as time of arrival or phase is measured. With the measurements and the adequate navigation algorithms, the aircraft position, in respect of a reference site, is calculated by the airborne navigation equipment. The principle governing this kind of navigation systems is shown in Fig. 2.2.

Fig. 2.2 shows a scenario with two transmitting stations, denoted for convenience as $A$ and $B$, and an aircraft located in a point $P$ in the space ${ }^{3}$. Both stations transmit electromagnetic signals at the same time ${ }^{4}$ (due to the synchronization scheme) and, because the distance from the aircraft to each station is, in general, different, it receives the signals at different time instants. The signal propagation delays, from each station to the aircraft, are directly related to the corresponding distance between each station and aircraft. If we denote these propagation times by $\Delta t_{\overline{A P}}$ and $\Delta t_{\overline{B P}}$ respectively, they can be defined as:

$$
\Delta t_{\overline{A P}}=\frac{\overline{A P}}{c}=\frac{R_{A}}{C}
$$

\footnotetext{
${ }^{3}$ For practical issues, we consider a two-dimensional example, although the same concepts can be straightforwardly extended to the three-dimensional case.

${ }^{4}$ For the sake of simplicity, simultaneous transmissions are considered. However, is more usual to find systems synchronized in such a way that stations transmit at different times.
} 


$$
\Delta t_{\overline{B P}}=\frac{\overline{B P}}{c}=\frac{R_{B}}{c}
$$

where $c$ is the speed of light in free space $\left(3 \times 10^{8} \mathrm{~m} / \mathrm{s}\right)$ and, $R_{A}$ and $R_{B}$ are the distances from the station $A$ and $B$ to the aircraft, respectively.

The mathematical principle of these systems lies in the fact that the difference between the distances from each station to the aircraft is equal to a constant that represents a hyperbola (or hyperboloid for 3D case) in the space, whose foci are located at the station positions. Such a hyperbola is known as "localization hyperbola" and it defines a line (or surface for 3D case) over which the aircraft should be located, as shown in Fig. 2.2. Mathematically, it is expressed by:

$$
\overline{A P}-\overline{B P}=c\left(\Delta t_{\overline{A P}}-\Delta t_{\overline{B P}}\right)=\gamma
$$

where, clearly, the constant $\gamma$ depends on the distances difference or, equivalently, on the differences between the corresponding propagation times. If a third station is used, a new localization hyperbola can be determined thus reducing the number of possible location points only to the intersections of these two hyperbolas. Theoretically, with three stations, a two-dimensional position can be calculated, whilst with four, the threedimensional case is solved.

The concepts described above, although something differently, have allowed the development of Multilateration (MLAT) systems for Air Traffic Control (ATC). As it will be shown later, one of the main differences between the MLAT systems and hyperbolic navigation systems is that for the first ones, due to the fact that they are essentially surveillance systems, the target position is calculated in a central station rather than in the target itself. It is clear to see that this concept is more similar to that one described by Fig. 2.1.

With the rise and growth of civil aviation, the highly precise control and surveillance of air space became mandatory. The latter drove the development of surveillance and control systems for civil aviation applications. Chronologically, the surveillance systems evolution can be cited as follows: Primary Surveillance Radar (PSR), Secondary Surveillance Radar (SSR), Monopulse Secondary Surveillance Radar (MSSR), Secondary Surveillance Radar - Selective Mode (SSR - Mode S) and followed by the Multilateration (MLAT) systems. Furthermore, the development of satellite navigation allowed the Automatic Dependent Surveillance (ADS), whose main application nowadays is the ADS Broadcast (ADS - B).

The reasons behind the development of MLAT systems arise from the necessity of automatically obtaining the target identification and to increase the accuracy and coverage levels for surface surveillance, because the 
classical systems, like Airport Surface Detection Equipment (ASDE) which is based on PSR, did not allow such features. Moreover, these systems suffered false target detections and performance degradation due to rain. On the other hand, the performance characteristics of the surveillance and control systems for wide areas (flight phases) did not provided enough accuracy for surface operations ${ }^{5}$.

The first test of a MLAT system, which had been published, was made in 1970 (previously to the Mode S development) by the Bendix Corporation [12] (USA). This system used interrogation directive beams to elicit replies to an Air Traffic Control Radar Beacon System (ATCRBS) transponder. The time of arrival was estimated and then sent to a central computer, which calculated the transponder position by using similar algorithms to those used in LORAN and Global Positioning System (GPS). The tests for this system were carried out only for surface targets in the Logan International Airport at Boston, Massachusetts, USA. The result of these tests showed the feasibility of implementing such system for air traffic control and surveillance. However, by that time, this system was considered highly expensive. Thereafter, with the development and the commissioning of Mode S, and the Traffic alert and Collision Avoidance System (TCAS), the need to know whether MLAT systems could be implemented on this kind of new signals arose. One of the tests to proof the latter was carried out also in the Logan International Airport by the Lincoln Laboratory in 1986 [13]. The new results indicated the viability of implementing these systems with these signals for air traffic control and surveillance operations. Finally, as it will be shown later, the overall performance of these systems has been so good that, nowadays, there exits the corresponding version for flight phases.

\subsubsection{Definition and Theoretical Foundations}

In this part, a formal and more modern definition of MLAT systems for air traffic control and surveillance, as well as the theoretical foundations of them, are provided. As it has been commented before, such a foundation is based on the mathematical intersection of multiples spatial hyperbolas (or hyperboloids). So, in this part, this foundation is described in depth and focused on MLAT systems.

Before continuing with the definition of a MLAT system, it is worth mentioning that the air traffic control and surveillance systems can be classified into two groups, according to the roll the target plays in its

${ }^{5}$ Due to the small spatial separations among surface targets and due to the high acceleration levels which are experimented by them, the accuracy levels required for surface surveillance and control are higher than those required for flight phases. 
positioning (see Fig. 2.10 later). These two groups are non-cooperative systems and cooperative systems. In the first group, the target position calculation does not depend on any event or function executed by the target itself. An example of this group is the PSR, where a rotating antenna transmits high power electromagnetic signals which propagate to the target, then, these signals are reflected by the target structure (e.g., aircraft fuselage), received again by the rotated antenna and, finally, by means of advanced correlation techniques the target position is obtained [14]. On the other hand, in the second group, the target actively participates in its positioning. In these systems the target position calculation directly depends on the reception of an electromagnetic signal emitted by the target. Examples of these systems are the SSR Mode S, MLAT systems and ADS B. Moreover, another important classification of the air traffic control and surveillance systems is related with the entity where the target position is calculated. In this sense, if the target position is calculated by the system itself, this is called independent system, whilst if the position is calculated by an external entity and the particular system only read the data, this is called dependent system.

\section{Definition}

A MLAT system is a cooperative/independent positioning system, which is basically composed by a set of receiving stations (generally jointly synchronized) and one or more interrogating stations deployed throughout a coverage area, and a computing center where a set of algorithms are executed to obtain the position of either surface or air targets. Such algorithms are based on the differences of some measured parameter, of a signal emitted by a target device commonly called transponder (for surface vehicles it is called "non-transponder" device because, although it plays the same role than a transponder, it has non-avionics requirements), in each of the receiving stations. In these systems, the signal emitted by target transponders can be generated due to an external request, i.e., an interrogation made by an interrogating station that can be either part of the system itself or external to it, or synchronously and spontaneously by the target itself.

Nowadays, MLAT systems have become an important part of the ATC infrastructures. In this field, MLAT systems are used to inform air traffic controllers of the presence, identification and position of aircrafts or vehicles within the corresponding coverage area. To do this, they measure some physical parameter of the signal emitted by the target transponders, which, for its standard form, is the Time of Arrival (TOA) of the signal to every receiving station. These measurements are sent to a Central Processing

Subsystem (CPS) where they are processed to detect the target presence, to obtain its identification and to estimate the transponder position (i.e., that 


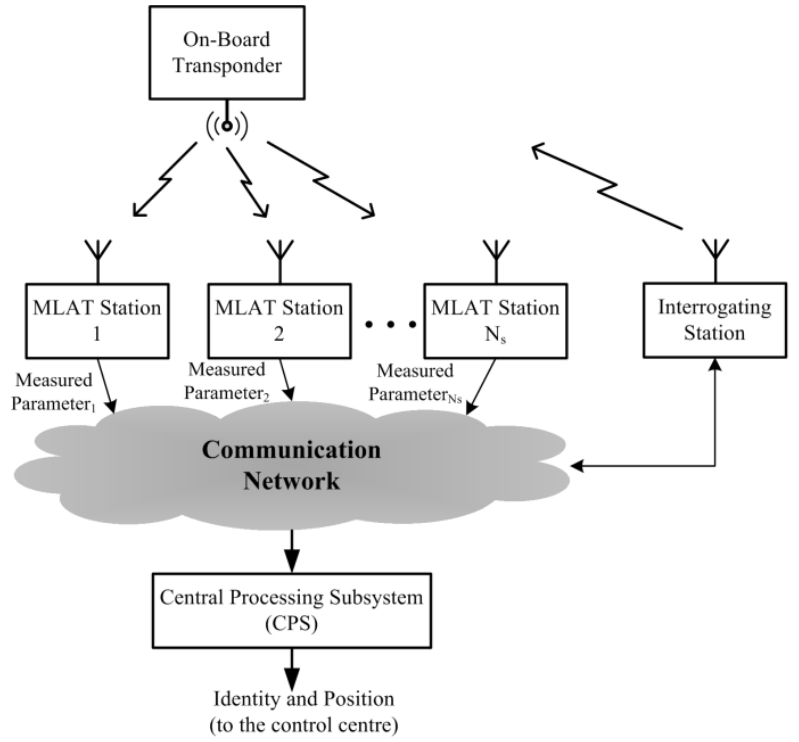

Fig. 2.3 General scheme for a MLAT system.

of the aircrafts or vehicles). Besides the TOA measurements, other kinds of measurements are possible, like: the Round-Trip Delay (RTD), which is basically the time between the transmission of an interrogation signal and the reception of its corresponding reply; the Angle of Arrival (AOA) which can be vertical (VAOA) or horizontal (HAOA), and the time integration, which basically consists in the use of several TOA measurements, coming from the same target, at consecutive instants. When MLAT systems implement these kinds of measurements, in addition to the TOA ones, they are referred as enhanced MLAT systems (henceforth the enhanced form).

MLAT systems can be deployed for almost any kind of air space. In this sense, when MLAT systems are used for airport and approach surveillance, they are called Local Area Multilateration (LAM) and, when they are used for wider areas (e.g., en-route areas), they are called Wide Area Multilateration (WAM).

A general scheme for a MLAT system is shown in Fig. 2.3. This figure shows an on-board transponder device (or the "non-transponder" device for surface vehicles) emitting a signal, which can be a spontaneous transmission or elicited by the interrogating station, that is received by a set of $N_{S}$ ground stations. These stations measure the signal TOA and send this data to the CPS by using a communication network, which can be either a wired (e.g., twisted pair, coaxial, optical fibre) or wireless (e.g., radio) one. In the 
CPS, the target identification and position are obtained and, finally, this information is sent to the air traffic controller display ${ }^{6}$.

In a general frame, it is considered that every station is capable of measuring the signal TOA; however, there exist other MLAT architectures in which the corresponding TOAs are measured in the CPS instead of at the receiving station. In this kind of architecture, the time delay between the receiving station antenna and the CPS must be precisely characterized and then added to the TOA values.

The target detection and identification are performed by means of the decoding and processing of the data contained in the signal emitted by the on-board transponder. Both decoding and processing are out of the scope of this thesis, so, we assume the systems we analyze perform these functions properly. On the other hand, the localization process, its accuracy and its reliability are the core of this thesis. For this reason, the theoretical foundations of MLAT systems discussed in the next subsection are focused on these particular topics.

\section{Theoretical Foundations}

As it has been commented before, a MLAT system is composed of a number of receiving stations, whose positions are accurately known, which measure, in a synchronized way, the time of reception/arrival to them, of a signal emitted by an on-board transponder device. These data are sent to the CPS, where they are used to obtain the information about the target situation (i.e., basically the target position) by means of the Time Difference of Arrival (TDOA) technique, as depicted in Fig. 2.3.

The TDOA technique calculates the difference between the signal TOAs to each station in respect of the TOA of one of them, which is designated as the reference station. Such a quantity, commonly denoted as TDOA, mathematically represents a spatial hyperboloid (or hyperbola) on which the target (specifically the transponder device) should be located. Thus, with one TDOA value (i.e., two stations) the target can be located on a hyperbolic surface characterized by the TDOA quantity and the corresponding stations position, which are the foci of such a hyperbola. The Fig. 2.2 also holds for this principle. Therefore, with more than one TDOA, all the possible target situations can be reduced only to those points belonging to the spatial intersection (or intersections) of the hyperbolic surfaces. Numerically, it means that, with a suitable number of stations, the target position can be calculated as the solution to the mathematical problem of estimating the intersection point (or points) of the hyperbolic surfaces represented by each available TDOA. This problem is stated as a

${ }^{6}$ As shown later, this information can be previously processed by a tracking and data fusion modules before it is sent to the air traffic controller display. 
highly non-linear system of hyperbolic equations, where, in general sense, the unknowns are the intersection points (i.e., the possible target positions). This concept is described below.

The TOA quantity measured by each station is a relative clock time (not an absolute propagation time), which can be expressed in any time unit, generally in seconds, and mathematically it can be described as follows:

$$
\begin{gathered}
\widehat{T O A}_{i}=t_{e}+\frac{r_{i}}{c}+n_{i} \\
\widehat{T O A}_{i}=t_{e}+\frac{1}{c} \sqrt{\left(x-x_{i}\right)^{2}+\left(y-y_{i}\right)^{2}+\left(z-z_{i}\right)^{2}}+n_{i}
\end{gathered}
$$

where the superscript "^^" denotes a measured quantity, $t_{e}$ is the signal emission time in the transponder, which is also an unknown clock time, $r_{i}$ is the exact distance (obviously unknown) from the target to the $i$ th station, $c$ is the speed of light in free-space and $n_{i}$ is a term that represents the TOA measurement error, whose magnitude depends on several parameters like the hardware instrumental errors, the quality of the received signal (commonly measured by the Signal-to-Noise Ratio -SNR-), the transmission and reception antenna gains, the synchronization scheme, analogue-todigital converter sampling errors, quantisation errors, multipath effect, etc. For the MLAT localization problem this term is treated in a statistical sense rather than with the punctual values. The exact transponder position is denoted as $(x, y, z)$ and the one of each station as $\left(x_{i}, y_{i}, z_{i}\right)$.

If we designate, without loss of generality, the reference station as the station number 1 , the TDOA between the $i$ th station and the reference one can be expressed as the following non-linear equation:

$$
\begin{gathered}
\widehat{T D O A}_{i, 1}=\widehat{T O A}_{i}-\widehat{T O A}_{1} \\
\widehat{T D O A} A_{i, 1}=\frac{1}{c} \sqrt{\left(x-x_{i}\right)^{2}+\left(y-y_{i}\right)^{2}+\left(z-z_{i}\right)^{2}} \\
-\frac{1}{c} \sqrt{\left(x-x_{1}\right)^{2}+\left(y-y_{1}\right)^{2}+\left(z-z_{1}\right)^{2}}+n_{i, 1}
\end{gathered}
$$

where $n_{i, 1}$ is the equivalent measurement error term for the TDOA quantity, which is also treated in a statistical sense.

In (2.4) it can be observed that the unknown term of signal emission time $t_{e}$ has been eliminated from the TOA model (2.3) to the TDOA one, thus avoiding the need to know or to estimate it. This is one of the advantages of using the TDOA technique. On the other hand, (2.4) represents a hyperboloid whose foci are in the stations position and on which, in the noiseless case, the target is located, as shown in Fig. 2.4. In this figure, for clarity, only a section of the hyperboloid is shown. From this figure is clear to see how with only a pair of receiving stations the target 


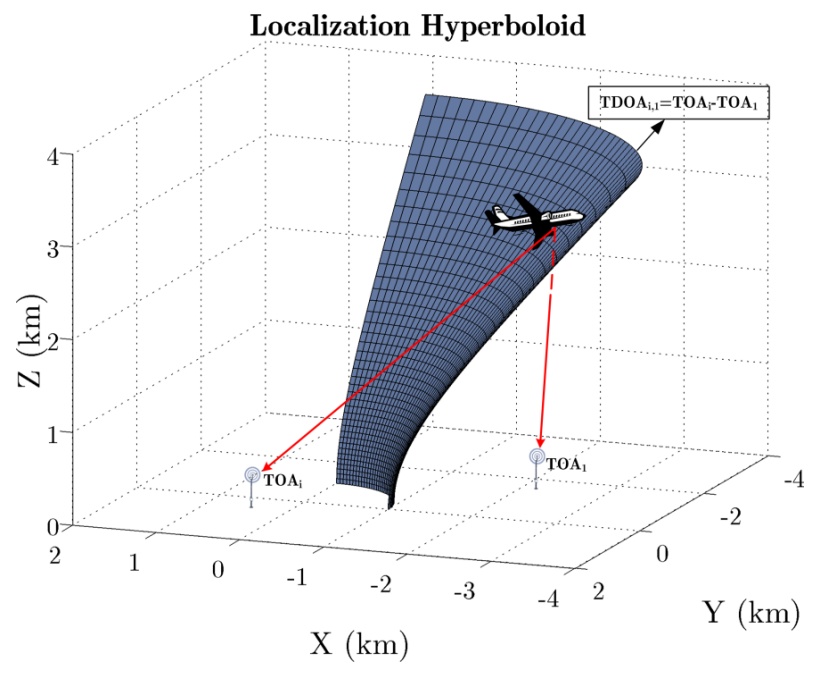

Fig. 2.4 Section of a hyperboloid for a pair of receiving stations in MLAT systems.

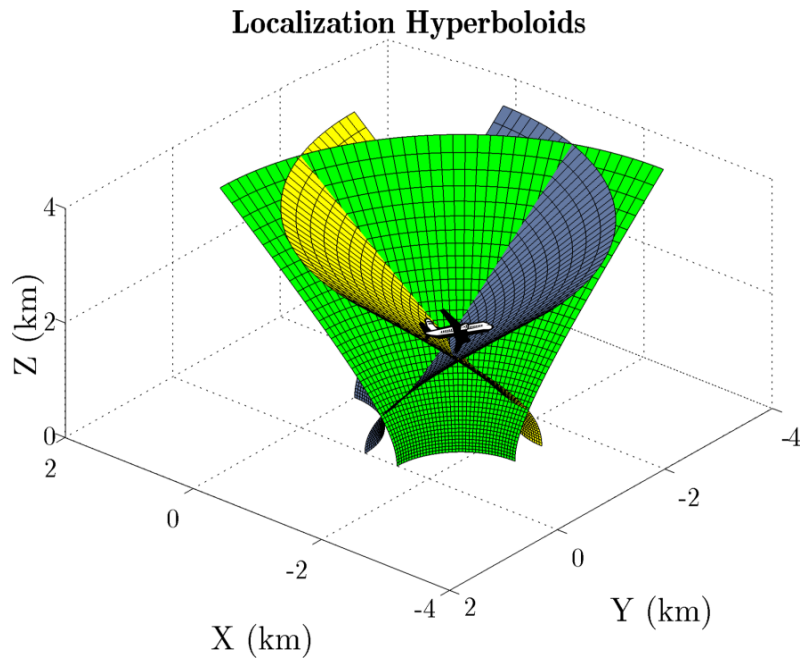

Fig. 2.5 Intersection of three hyperboloids.

can be located on a hyperbolic surface of the space. Because we are interested in a punctual (numerical) position value rather than in a surface or volume, it is necessary to obtain more than one hyperboloid in the form of (2.4), i.e., the use of more than two receiving stations is required. As it has been commented before, to obtain this numerical value, the most general is to set a system of hyperbolic equations in the form of (2.4) and solve it under some conditions. This system of equations is highly nonlinear on the target position and must be solved by any available and reliable analytical or numerical tool. More details about it are given later. 


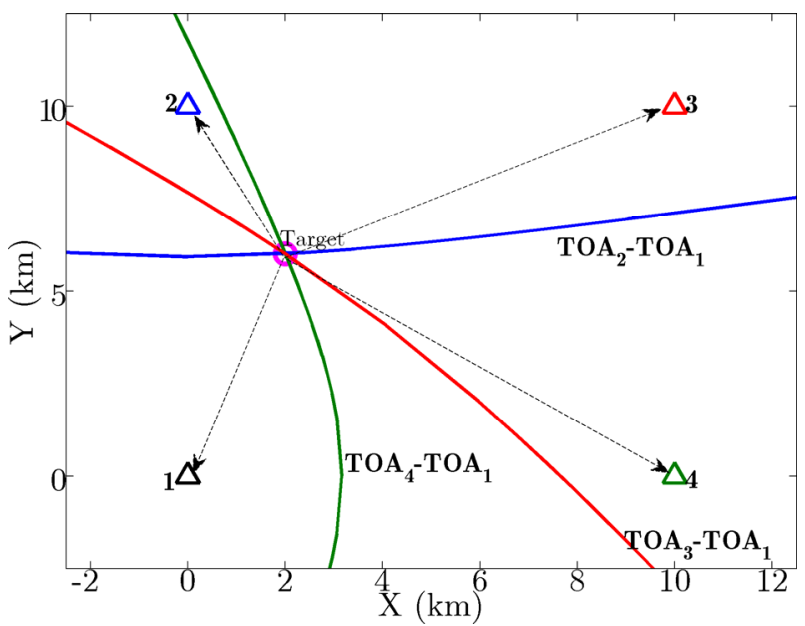

Fig. 2.6 Hyperbolas for four stations and a target located in an arbitrary position. The reference station is the station number one (the black one) and the target is represented by the magenta circle.

Mathematically, we require at least three stations (i.e., two hyperbolic equations) to calculate a position in a spatial reference frame with two dimensions (henceforth called 2D localization), typically $(x, y)$ and, at least four (i.e., three hyperbolic equations) for three dimensions (henceforth called 3D localization), i.e., the full Cartesian set $(x, y, z)$. Nevertheless, due to the measurement errors, which are contained in the term $n_{i, 1}$ in (2.4), the hyperboloids intersection is really an intersection dynamic area (or volume) rather than purely a point. However, by the moment we assume, for the sake of simplicity, the intersection is only a point, and in a posterior chapter we will emphasize on its actual characteristics. The latter is illustrated in Fig. 2.5.

For a clearer illustration, Fig. 2.6 shows an example for a 2D localization situation. Thus, the locus of points on which the target is located is a hyperbola instead of a hyperboloid. It can be observed, for example, for the $T D O A$ obtained from the station number 2 and the reference one that, with this value $\left(T O A_{2}-T O A_{1}\right)$, the target can only be located on the blue hyperbola. The same hold for the other TDOA lines. Finally, if at least two hyperbolas are mathematically combined, the possible target localizations can be reduced, normally, to a spatial point, as shown in Fig. 2.6. Furthermore, it is worth to comment that more than one intersection point (or area) can appear; it depends on the particular system geometry (i.e., the spatial distribution of the station) and the target position relative to this. If it happens, it is necessary to find any redundant information which allows discarding the remaining (false) intersections. This redundant information can come from adding more stations (i.e., more hyperbolic equations), by 
using other kind of measurements (e.g., RTD, AOA, etc.), by using a priori information collected by the system itself (e.g., predicted positions from tracking algorithms), or by using information about the target position coming from other, external, sources or systems.

As it has been stated before, when the transponder emitted signal is received by only three stations, in this case a two dimensional position $(x, y)$ can be calculated and this solution is referred as $2 D$ solution. Nevertheless, with the use of an external source that provides the target altitude, e.g., the barometric altitude contained in a Mode $\mathrm{C}$ reply [15], a three dimensional position can be obtained based on both $2 D$ solution and target altitude. However, it is worth saying that due to the difference between the barometric altitude and the target height over a geographical reference frame (commonly known as geometric altitude), this kind of solution can be less accurate than the one obtained when four stations (or more than four) receive the signal, in which case is referred as the $3 D$ solution. Finally, when more than four stations receive the transponder emitted signal, with the use of adequate mathematical algorithms, all of these measurements can be combined in such a way that the final solution, in principle, is more accurate than that one obtained only with four stations, as it will be shown later in this thesis.

We have seen that the MLAT systems collect a certain set of measurements, from a set or receiving stations, and then obtain a target position by solving a numerical problem (i.e., a system of hyperbolic equations). So, this process can be mathematically seen as a domain change in which the measurements, a domain, are converted into another domain, the spatial one. If we denote the target position as the column vector $\boldsymbol{\theta}=[x, y, z]^{T}$ and the set of measurements as $\widehat{\boldsymbol{m}}_{M L A T}=\left[\widehat{m}_{1}, \ldots, \widehat{m}_{i}, \ldots, \widehat{m}_{M}\right]^{T}$, where $\widehat{m}_{i}$ represents any kind of measurement available for MLAT systems (e.g., TOA, TDOA, RTD, AOA, etc.), and $M$ is the total number of measurements, the MLAT localization problem can be comprised into a mathematical operator form as follows:

$$
\widehat{\boldsymbol{\theta}}=\mathcal{M}\left\{\widehat{\boldsymbol{m}}_{M L A T}\right\}
$$

where we have used $\widehat{\boldsymbol{\theta}}$ to emphasize that the target position as obtained by a MLAT system is an estimated parameter, and $\mathcal{M}$ is an operator we have called $M L A T$ operator, which converts a $\mathbb{R}^{M}$ vector space element into a $\mathbb{R}^{3}$ vector space one, in the most general case. This operator is essentially a numerical inverse problem and the particular form depends on the kind of measurements comprised in vector $\widehat{\boldsymbol{m}}_{M L A T}$ and the particular method used to solve it. The MLAT operator has some characteristics; it is a highly nonlinear operator, although it can be linearly approximated, it amplifies the measurement errors contained in $\widehat{\boldsymbol{m}}_{M L A T}$, by a factor that depends on the 
system geometry, and introduces them into the target position $\widehat{\boldsymbol{\theta}}$. Therefore, it is considered as a non-exact operator. In this sense, the accuracy of the MLAT operator (i.e., that of the MLAT system, which henceforth is called system accuracy) mainly depends on three factors, namely, the system geometry, the measurement noise, and the robustness of the methodology used to form and to solve the numerical problem, the latter commonly known as localization algorithm. The system geometry and the measurement noise set the best system accuracy that can be achieved with the particular MLAT system, whereas the possibility to reach this best accuracy depends directly on the localization algorithm. In other words, the system geometry and the measurement noise set the best case and the localization algorithm set the efficiency of the MLAT operator to achieve it. Thus, it is clear to understand that the best system accuracy can be obtained by using the adequate system geometry, the measurements with the possible lowest noise, and the most efficient localization algorithm. The basics and the relation among these three aspects are briefly discussed below.

The measurements made by the MLAT system stations can be affected by several phenomena, which produce certain amount of error on them and which, at the same time, produce inaccuracies in the target localization. There exist several classifications for the sources generating these errors, for example, according with their origin, whether they come from an internal or external source to the system, according to the system component which is affected by them or depending whether all the receiving stations are equally affected or not. In the following we will briefly comment a classification of these error sources based on whether they are internal or external to the system. Moreover, although we will mainly focus the description for the TOA/TDOA measurements, most of the developments also apply for other measurements like RTD or AOA. In Fig. 2.7 the main errors, according to this classification, are summarized.

Fig. 2.7 shows the classification of the error sources into two sets. The first one comprises those errors that are externally generated to the system and the other one comprises those that are internally generated. The phenomena generating these errors (external or internal) can equally or unequally affect all the receiving stations. In this sense, every station is affected by a certain amount of error sources, whose effects can be represented by an error term $\left(n_{i}\right.$ in Fig. 2.7) contained in the corresponding measurement and whose value is, in a wide sense, time-dependent. Generally, this is a random term and, therefore, it must be statistically analyzed. The statistical characteristics of this error term are discussed later. Before it, we will provide a short conceptual description of the error sources. 


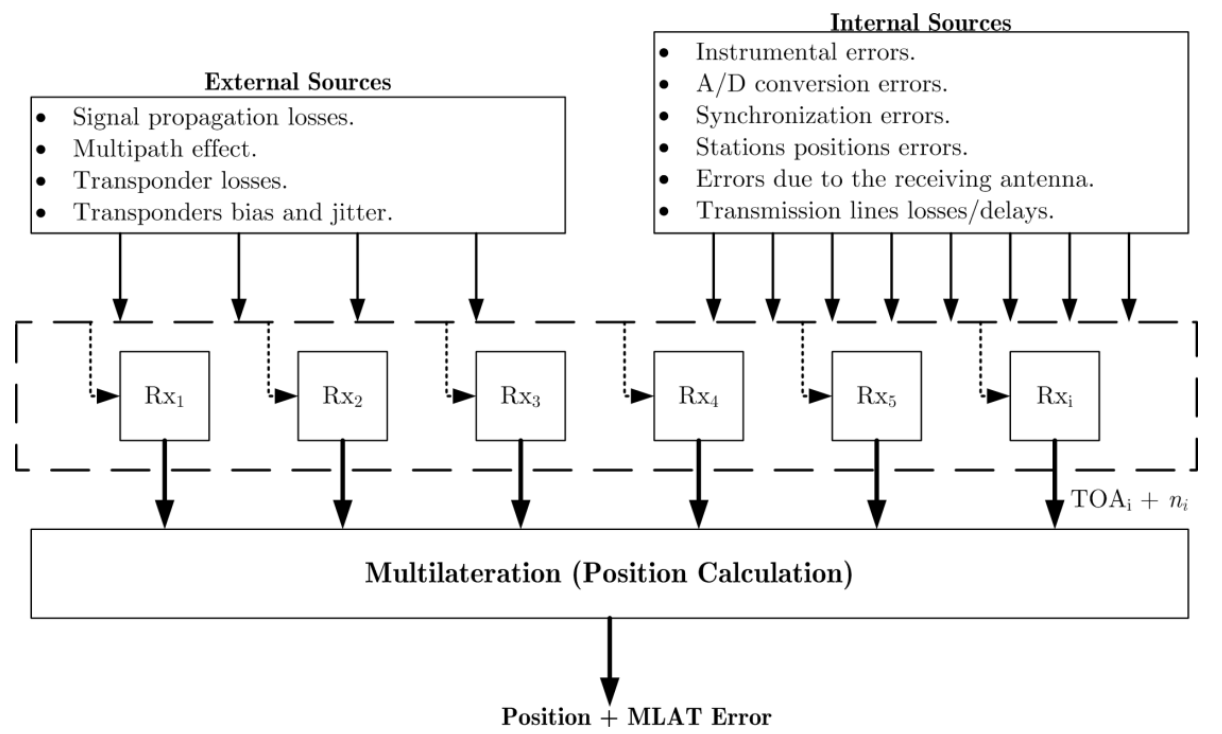

Fig. 2.7 Error sources classification for MLAT systems.

As far as the measurement errors originated for reasons external to the system operation itself, they cannot be adjusted in the system, although some advanced techniques can be implemented to compensate the inaccuracies produced by them. Among these errors are those due to the signal propagation losses, to the multipath effect, to any transponder losses and to any bias (time delay) or jitter on the transponder. The signal propagation losses are directly related with the Signal-to-Noise Ratio (SNR), and the greater the distance from the target to the receiving stations, the lower the SNR. This kind of error, as it is evident, differently affects each station. The multipath effect produces the station receives several copies of the same signal. It can be due to reflections from building or mountains, even from other targets. This error differently affects each station and can severely degrade the system accuracy. However, due to the fact that this error is highly terrain model dependant, and because it can be mitigated by simply changing the station position, among other simple strategies, it is normally not taken into account in a theoretical system analysis. The transponder losses are related to the signal propagation ones, because, as in any transmission/reception system, the quality of the received signal depends also on the quality of the transmitted one. In this sense, any transponder deficiency, low amplification or low transmitting antenna gain will reduce the SNR at the receiving station, thus increasing also the signal propagation losses. This effect, for the same target, equally affects all the receiving stations. Finally, the errors originated by the 
transponder bias and jitter are very important but for RTD measurements, not for TOA/TDOA, AOA or time integration.

On the other hand, the measurement errors generated by internal sources are errors which appear for reasons internal to the system operation, architecture itself. Therefore, the magnitude of these errors directly depends on the manufacture of devices and on the general system architecture, among others. Among this kind of errors are those due to manufacturing defects (instrumental error), to the Analog-to-Digital (A/D) conversion process, to the synchronization scheme, to inaccuracies on the stations position data, to the receiving antennas and to the transmission lines and/or microwave links losses and delays. The instrumental error is due to any manufacturing defect. This error is always present and equally affects all stations independently of the target position. The A/D conversion process errors appear due to the sample, quantisation and codification steps that must be performed on this process. This error is always present and the magnitude of this directly depends on the hardware quality. For the same manufacturer, this error equally affects all the receiving stations. The error due to the synchronization scheme appears if the stations are not equally aligned on time. It depends on the synchronization scheme used (they are commented later) and can severely affect the system accuracy. The error due to the receiving antennas is directly related to the errors due to signal propagation losses, because, depending on the receiving antenna used (mainly to his gain parameter) these errors can be mitigated or even augmented. This error equally affects the receiving stations in the sense that, maybe, all of them use the same antenna, but it can also differently affect them because several antenna parameters depend on the target position (like antenna gain which normally is not constant for all the directions). The error due to the transmission lines losses and delays appears because when signals propagate along them, they experiment electromagnetic losses and time delays. This kind of error differently affects each station. Finally, the errors due to possible inaccuracies on the station positions data are not relevant and are negligible when advanced topographic equipments are used.

All the measurement errors described above, as we have previously commented, can be comprised into an equivalent error term $n_{i}$, which possesses some statistical characteristics. In this sense, all the measurement errors described above can be statistically classified as random and systematic errors. As was demonstrated in [16], the errors of random nature can be assumed, for every receiving station, as jointly Gaussian distributed with zero mean and standard deviation $\sigma$, where the value of the latter depends on the MLAT architecture used and the sources affecting the particular station. On the other hand, the systematic errors are, in general sense, time invariant and they are always present with the same magnitude, 
for example, the time delays in the transmission lines from the receiving antenna to the CPS. Such errors can be fully monitored, accurately measured and corrected. For this reason, in all the mathematical analysis of MLAT systems is totally valid to neglect the effect of these errors and assume the MLAT measurement errors as only jointly Gaussian distributed.

Particularly, each measurement error due either to internal or external sources can be characterized by an individual Gaussian distribution which is also zero-mean and with a given standard deviation. In the following table the most relevant random errors are summarized along with their main characteristics.

Table 2.1 Summary of MLAT measurement errors.

\begin{tabular}{|c|c|}
\hline Error & Characteristics \\
\hline Instrumental & $\begin{array}{l}\text { The corresponding standard deviation must be provided } \\
\text { by the system manufacturer. }\end{array}$ \\
\hline A/D conversion & $\begin{array}{l}\text { The corresponding standard deviation must be provided } \\
\text { by the system manufacturer. }\end{array}$ \\
\hline Synchronization & $\begin{array}{l}\text { The standard deviation depends on the synchronization } \\
\text { scheme used and the corresponding standard deviation } \\
\text { value must be provided by the system manufacturer. } \\
\text { Depending on the synchronization scheme this standard } \\
\text { deviation can be equal or not for all stations. }\end{array}$ \\
\hline $\begin{array}{c}\text { Signal } \\
\text { propagation }\end{array}$ & $\begin{array}{l}\text { The standard deviation for this kind of errors depends } \\
\text { on the SNR at the reception point. Therefore, this error } \\
\text { is particular for every spatial point and for every } \\
\text { receiving station. Due to the fact that SNR depends, in } \\
\text { addition to the propagation losses, on the transmission } \\
\text { and reception hardware, this error can include also } \\
\text { other kind of errors which were separately described } \\
\text { before. These errors are those due to the transponder } \\
\text { losses and those due to the receiving station losses (e.g., } \\
\text { due to the antenna gain). }\end{array}$ \\
\hline $\begin{array}{l}\text { Transponder bias } \\
\text { and jitter }\end{array}$ & $\begin{array}{l}\text { This error is present and is relevant for RTD } \\
\text { measurements. The magnitude of the standard } \\
\text { deviation depends on the transponder device and it is } \\
\text { equal for all stations. }\end{array}$ \\
\hline
\end{tabular}

Finally, once known the standard deviations for every error source, affecting a particular station, the corresponding standard deviation for the Gaussian distribution which describes the total measurement error can be estimated as the root-sum-square of all contributions as follows: 


$$
\sigma_{T O A_{i}}^{2}=\sum_{j} \sigma_{\text {Source }}^{2}, i
$$

where $\sigma_{T O A_{i}}$ is the corresponding standard deviation for the $i$ th receiving station and $\sigma_{\text {source }_{j, i}}$ is the corresponding one of the $j$ th error source affecting the ith receiving station. Furthermore, it is important to emphasize that, although (2.6) has been described for TOA/TDOA measurements, exactly the same equation holds for other kind of measurements like RTD or AOA. In order to standardize some terminology, hereafter we will refer to the measurements errors effect as measurement accuracy, which for the TOA case is called TOA accuracy.

The measurement vector $\widehat{\boldsymbol{m}}_{T D O A}$ (cf. (2.5)), for the TDOA measurements case with a number of $N_{s}$ receiving stations, and for any target position $\boldsymbol{\theta}$, can be expressed as follows:

$$
\begin{aligned}
\widehat{\boldsymbol{m}}_{T D O A}(\boldsymbol{\theta})= & {\left[\begin{array}{c}
\widehat{T O A}_{2}(\boldsymbol{\theta})-\widehat{T O A}_{1}(\boldsymbol{\theta}) \\
\widehat{T O A}_{3}(\boldsymbol{\theta})-\widehat{T O A}_{1}(\boldsymbol{\theta}) \\
\vdots \\
\widehat{T O A}_{N_{S}}(\boldsymbol{\theta})-\widehat{T O A}_{1}(\boldsymbol{\theta})
\end{array}\right]+\boldsymbol{n}(\boldsymbol{\theta}) } \\
& =\left[\begin{array}{c}
\widehat{T D O A}_{2,1}(\boldsymbol{\theta}) \\
\widehat{T D O A}_{3,1}(\boldsymbol{\theta}) \\
\vdots \\
\widehat{T D O A_{N_{S}, 1}}
\end{array}\right]+\boldsymbol{n}(\boldsymbol{\theta})
\end{aligned}
$$

being $\boldsymbol{n}$ the corresponding TDOA measurement error vector, which is zero mean Gaussian distributed with covariance matrix defined by:

$$
\begin{aligned}
& \boldsymbol{N}(\boldsymbol{\theta}) \\
& =\left[\begin{array}{cccc}
\sigma_{T O A_{2}}^{2}(\boldsymbol{\theta})+\sigma_{T O A_{1}}^{2}(\boldsymbol{\theta}) & \sigma_{T O A_{1}}^{2}(\boldsymbol{\theta}) & \cdots & \sigma_{T O A_{1}}^{2}(\boldsymbol{\theta}) \\
\sigma_{T O A_{1}}^{2}(\boldsymbol{\theta}) & \sigma_{T O A_{3}}^{2}(\boldsymbol{\theta})+\sigma_{T O A_{1}}^{2}(\boldsymbol{\theta}) & \cdots & \sigma_{T O A_{1}}^{2}(\boldsymbol{\theta}) \\
\vdots & \vdots & \ddots & \vdots \\
\sigma_{T O A_{1}}^{2}(\boldsymbol{\theta}) & \sigma_{T O A_{1}}^{2}(\boldsymbol{\theta}) & \cdots & \sigma_{T O A_{N_{S}}}^{2}(\boldsymbol{\theta})+\sigma_{T O A_{1}}^{2}(\boldsymbol{\theta})
\end{array}\right]
\end{aligned}
$$

This covariance matrix can be considered as the noise covariance that is introduced into the MLAT operator in (2.5). Nevertheless, as we commented before, the MLAT operator accuracy does not depend only on this noise covariance but also on the spatial distribution of the stations (i.e., the system geometry) relative to the corresponding coverage area. Moreover, the influence of the measurement errors is magnified by the system geometry in the MLAT operator. In the following, the system geometry influence and how much it magnifies the measurement errors is described. 
The system geometry refers to the spatial arrangement of the receiving stations relative to the coverage area. Furthermore, the system geometry also depends on the kind of measurements which are used by the receiving stations, e.g., TOA/TDOA, RTD or AOA. It is because each of these measurements spatially represents a specific geometric figure; for instance, in the case of TOA/TDOA is a hyperboloid or hyperbola, and in the case of RTD is a sphere or circumference. Nevertheless, for the sake of simplicity, we will focus this description only on TOA/TDOA measurements, although the same theory fully holds for the other ones.

The system geometry influence on the system accuracy is directly related with the eccentricity of the hyperboloids or hyperbolas derived from each TDOA measurement and on their perpendicularity. This idea is described in Fig. 2.8. As we have commented, every TDOA measurement contains some amount of error (cf. (2.4) or (2.7)), which is characterized by a Gaussian distribution of zero-mean and the standard deviation given by (2.6). This fact makes TDOA measurements to be non-exact but lying on a strip centred at the theoretical curve. The strip width depends on the standard deviation magnitude of each measurement error distribution. The latter is illustrated in Fig. 2.8 for a system composed by four stations deployed in a square geometry and for two different points in the coverage area, part (a) and part (b) respectively. In this figure, for clarity only two hyperbolas are shown for every point, with the solid lines representing the exact measurement values and the dotted ones the boundaries set by the measurements noise errors. Under this situation, we can clearly see in Fig. 2.8 that the target can be located (with a given probability) in an area rather than at a point. This area is called uncertainty area and the smaller it is the higher the system accuracy is and vice versa. By comparing both parts of Fig. 2.8 it can be seen that the size of the uncertainty areas is different, although they are depicted by assuming the same amount of measurement errors. This size difference is due to the eccentricity of the hyperbolas and also on how much perpendicular are the sheets of them. For example, in part (a) of Fig. 2.8 we can see two hyperbolas with relative high eccentricity and nearly perpendicular. In this case, the uncertainty area has a smaller size than that one of the part (b) of Fig. 2.8, whose hyperbolas are clearly not orthogonal, and one of these $\left(T O A_{4}-T O A_{1}\right.$ : the green one) has a small eccentricity. Both eccentricity and the perpendicularity of the hyperbolas are mathematically comprised in a factor called Dilution of Precision (DOP), which is a unit-less deterministic quantity that represent the quality of the system geometry for calculating the target position in a particular point in the coverage area (or volume), i.e., how much the system geometry magnifies the measurement errors into the system accuracy. Such a quantity is a property of the system and it is particular to each configuration. The DOP is, in a general sense, space- 


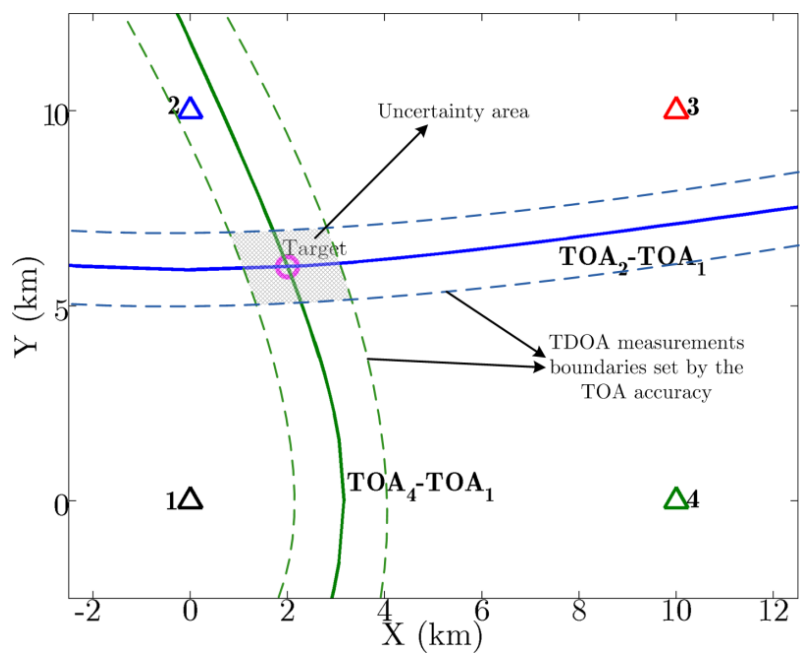

(a) A point inside the square geometry.

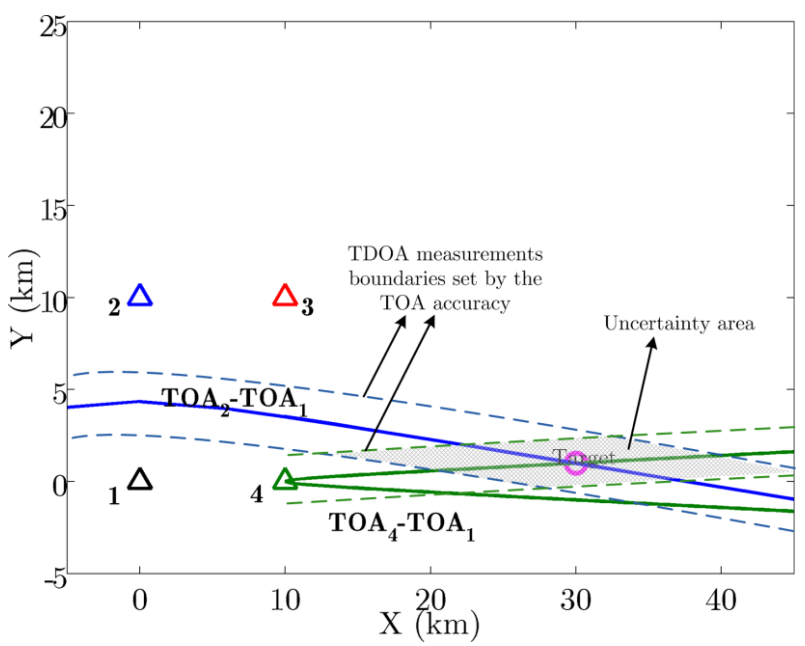

(b) A point outside the square geometry.

Fig. 2.8 Eccentricity and perpendicularity of the hyperbolas.

variant. Therefore, it is right to think that, with the same measurement accuracy and the same system geometry, the system accuracy for different points within the coverage area will be different.

The most general form of the DOP is the Position Dilution of Precision (PDOP), which is a factor (also unit-less) representing the magnification of the measurement errors in a three-dimensional space, usually in a rectangular frame $(x, y, z)$. The ideal value of PDOP is the unity and the greater this parameter is the higher is the noise magnification. There are 
other two forms of the DOP, derived from the PDOP, which are relevant in the theoretical system accuracy analysis. These are the Horizontal Dilution of Precision (HDOP) and the Vertical Dilution of Precision (VDOP). The HDOP represents the quality of the system geometry for calculating the $2 \mathrm{D}$ target position, commonly referred to a $(x, y)$ plane, whereas the VDOP represents the same but for the vertical component (i.e., the $z$ ) of the target position vector.

The PDOP does not depend only on the system geometry but it is also related with the number of stations. For example, in Fig. 2.8, if the same squared geometry is formed by more than four stations, the PDOP decreases (i.e., it is improved). So, in a wide sense, but not fully general, the greater the number of stations is the smaller the PDOP, and also the HDOP and VDOP.

Both measurements accuracy and the DOP provide the theoretical system accuracy, that is, the best accuracy that can be achieved with a particular MLAT system composed by a set of stations, drawing a specific geometry, where each of these measure some parameter(s) with a specific and statistically definable measurement accuracy. From a mathematical point of view, this is also the theoretical accuracy of the MLAT operator in (2.5). Equivalently to the case of the DOP, the theoretical system accuracy also can be divided into three forms. These are the 3D accuracy that is denoted by $\sigma_{x y z}$ or $\sigma_{3 D}$, the $2 \mathrm{D}$ (or horizontal) accuracy that is denoted as $\sigma_{x y}$ or $\sigma_{2 D}$, and the vertical accuracy that is denoted as $\sigma_{z}$. The measurement accuracy and DOP must be analyzed and adjusted in a system design process, in order to obtain an adequate configuration that provides the best possible theoretical system accuracy.

Due to the fact that the DOP is a deterministic quantity, it is right to say that the target position, as provided by the MLAT system, theoretically has the same statistical behaviour of the measurement noises, i.e., the target position in MLAT system can be modelled as a Gaussian distribution whose mean is the exact target position value and whose standard deviation is provided by the measurement noises and DOP.

Finally, the third element of the MLAT operator in (2.5) is the localization algorithm, which is the one that provides a numerical value for the target position. In the ideal case, the target position as provided by this algorithm should only have the inaccuracies due to the measurements noise and DOP. However, this is not always true and the algorithm also introduces errors into the system accuracy but in a different way than those due to the measurement accuracy or DOP. The errors introduced by the localization algorithm have their origin in the problem definition and numerical solution of it, and they can be treated in terms of efficiency, i.e., how much the localization algorithm reaches the theoretical accuracy as set by the measurements accuracy and DOP. There are a considerable number 
of localization algorithms, but a detailed description of this aspect is the aim of Chapter 7 of this thesis.

Summarizing this subsection, we can consider the MLAT localization problem as a mathematical operator, whose accuracy depends on three elements. The first two elements, the measurements and the system geometry, set the best accuracy levels (or lower bounds of accuracy) that can be reached by the system. On the other hand, the third one is the localization algorithm, which provides a target position value making use of the measurements and the system geometry, and whose final accuracy, which henceforth we will call operational system accuracy, can reach the theoretical one depending on its efficiency.

\subsubsection{Signals Used in MLAT Systems}

Until now we have described a brief history of multilateration, its definition and its theoretical foundations. In these subsections we have stated that a MLAT system measures the TOA, or other physical parameter, of signals emitted by the on-board transponder device, which can be installed either in an aircraft or in a surface vehicle. Thereby, the aim of this subsection is to describe those signals, and their main characteristics, including the corresponding message format.

\section{Secondary Surveillance Radar (SSR) Signals}

The SSR system is basically composed by a ground rotating antenna, which transmits interrogation signals at $1030 \mathrm{MHz}$ [17]. Such an interrogation signal is received by the aircrafts within the SSR coverage area, processed, and then, replied with a $1090 \mathrm{MHz}$ signal. This signal is called Mode $A / C$ reply because the kind of information it contains, which are the identification in Code $\mathrm{A}$ and the barometric altitude codified in a Code $\mathrm{C}$ (with a resolution of 100 feet). Due to the fact that all aircrafts are equipped with the corresponding transponder device, called Mode A/C transponder, this signal can be used by the MLAT receiving stations for measuring the TOA, and also for the CPS to automatically identify the target.

On the other hand, the use of Mode A/C signals has some limitations related with the quality of the signal. Regarding to the identification issues, the problem of code swaps can sometimes appears, because the codes A are not unique. Furthermore, the TOA (or other parameter) measurement accuracy mainly depends on the quality of the received signal, which can be negatively affected by some propagation effects (e.g., scattering, reflections, multipath effects, etc.). However, this last limitation is inherent to MLAT systems and does not represent a strong operative problem, due to the fact that it is taken into account during the design process. Another limitation 


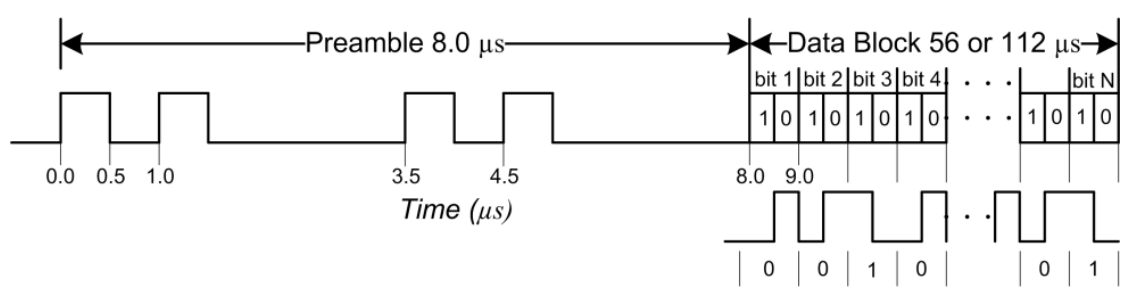

Fig. 2.9 Mode S reply structure.

of the SSR signals is the update rate, because it directly depends on the corresponding interrogation rate, which is commonly limited to reduce the effect of FRUIT $^{7}$. In high/medium traffic density scenarios it can be an important limitation for the MLAT system because the excess of interrogations/replies can generates capacity channel problems. Finally, the range of these signals is around $250 \mathrm{NM}$ [17], although it depends on the propagation degradations and the traffic density in the coverage area.

\section{Secondary Surveillance Radar (SSR) Mode S Signals}

The SSR Mode S system operation is similar to that of the SSR. The signal physical characteristics and limitations are the same than those of the SSR Mode A/C signals, i.e., the SSR Mode S signal is also a $1090 \mathrm{MHz}$ signal which is transmitted as a reply to a $1030 \mathrm{MHz}$ interrogation signal.

The main difference with the SSR Mode A/C signals is that the Mode S allows a selective interrogation, i.e., the Mode $\mathrm{S}$ only interrogates those targets of interest. These selective interrogations are possible because the aircrafts (or surface vehicles) transmit, within the reply message, a globallyunique identification called ICAO address [15]. Thus the SSR Mode S system interrogates only one particular aircraft. The ICAO address is composed by 24 bits and for sending this kind of signal the aircraft must be equipped with a Mode S transponder. The Mode S replies are made up from a train of pulses, modulated on the $1090 \mathrm{MHz}$ carrier, according to the ICAO recommendations [15], as shown in Fig. 2.9.

The signal preamble is used to detect the reply and to measure its TOA (usually by using the leading and trailing edges of the pulses) or, better, by maximum likelihood estimation [18]. Mathematically, each pulse can be defined as follows:

$$
g(t)=\operatorname{rect}\left(\frac{t}{T_{1}}\right) \otimes \operatorname{rect}\left(\frac{t}{T_{2}}\right)
$$

\footnotetext{
${ }^{7}$ FRUIT: False Replies Unsynchronized in Time.
} 
where $T_{1}(0.05-0.1 \mu s)$ is the edge duration, $T_{2}(0.5 \mu s)$ is the pulse duration and $\otimes$ is the convolution operator. From these pulses not only the TOA can be measured but also other parameters like the direction of arrival. The Data Block section in Fig. 2.9 is the reply part that contains the particular message, e.g., the ICAO address, barometric altitude or any other information.

The use of Mode S signals allows MLAT systems to unambiguously identify every target present in its coverage area. Furthermore, the resolution of the barometric altitude contained in one of its messages is 25 feet, which is higher than that of the SSR Mode A/C.

Regarding to the system update rate, which can be achieved with these signals, it is still low, but due to the selective interrogation capabilities it is better than that provided by the SSR Mode A/C signals. Finally, the range of these signals is also around $250 \mathrm{NM}$.

\section{Mode S Squitter}

The aircrafts that are equipped with a Mode $\mathrm{S}$ transponder device have also the capability of sending, periodically and without the need of any interrogation, a signal which contains a message with only the ICAO address. Such a signal is called Mode $S$ acquisition squitter (or simply Mode $\mathrm{S}$ squitter). The Mode $\mathrm{S}$ transponders with this option activated send this signal every second.

The Mode S squitter allows the implementation of a MLAT system without the need of an external system with interrogation capabilities or its own interrogation module. This operation mode is called passive multilateration and it is described later.

The Mode $\mathrm{S}$ squitter signal is physically the same than SSR Mode S signal, with the only difference of the update rate and the message content. Therefore, the limitations and range are the same for both.

\section{ADS - B Data Links}

The ADS - B technology transmits, by a data radio-link, data about the target state (e.g., position, velocity, intended trajectory, identification, etc.) that are obtained from its navigation systems, including the on-board GNSS devices. The main radio-links used by this technology are the 1090 $\mathrm{MHz}$ channel, whose corresponding signal is called Mode S $1090 \mathrm{MHz}$ Extended Squitter (Mode S ES), the VHF Digital Link Mode 4 (VHF Mode 4 ), and the Universal Access Transceiver (UAT). Nowadays, the most used and widely extended signal for MLAT systems is the Mode S ES; therefore, it is the only one described in this thesis.

The Mode $\boldsymbol{S} \boldsymbol{E S}$ is essentially the same signal than the Mode S squitter but with an extension (this is why it is called Extended) in the message 
content. The message extension can include (depending on the phase of flight) the data obtained from the on-board navigation systems, for example, the GPS position (Latitude, Longitude and geometric height), velocity, position uncertainty, etc.

The update rate for this message, when the corresponding option is activated, is $6 \mathrm{~Hz}$. Finally, physically the Mode S ES has the same characteristics and limitations than those of the SSR Mode S and the Mode S squitter.

\subsection{Multilateration Systems in the CNS/ATM Scheme}

The civil aviation operations are organized, technically and operationally speaking, under the scheme denominated as Communications, Navigation and Surveillance / Air Traffic Management (CNS / ATM). In this scheme, the term Communications refers to the entire infrastructure used for the information (data and voice) interchange either air-to-ground or ground-toground. The term Navigation refers to the ground and on-board (avionics) infrastructure that allow the aircrafts to know their situation at any time and thus navigate from one point to another one in the airspace. Surveillance refers to the specific ground infrastructure that allows to the air traffic controllers to know, at any time, the identification, position, course and intended route of all the aircrafts and surface vehicles present in the airspace under their responsibility. Finally, Air Traffic Management refers to the organization and management of the airspace, which allows to the aircrafts operators to fulfil with their flight plans, providing at the same time the maximum safety levels.

The MLAT systems are framed within the term Surveillance as a ground-based cooperative/independent system. Cooperative because they use signals that are emitted by an on-board transponder device, generally Mode S signals, and independent because they calculate the target position autonomously from other information sources. These systems can be seen as the direct replacement of the classical SSR Mode S, because they allow to provide much more services than the classical SSR based systems [9]. The Fig. 2.10 shows a brief summary of the most important surveillance systems. 


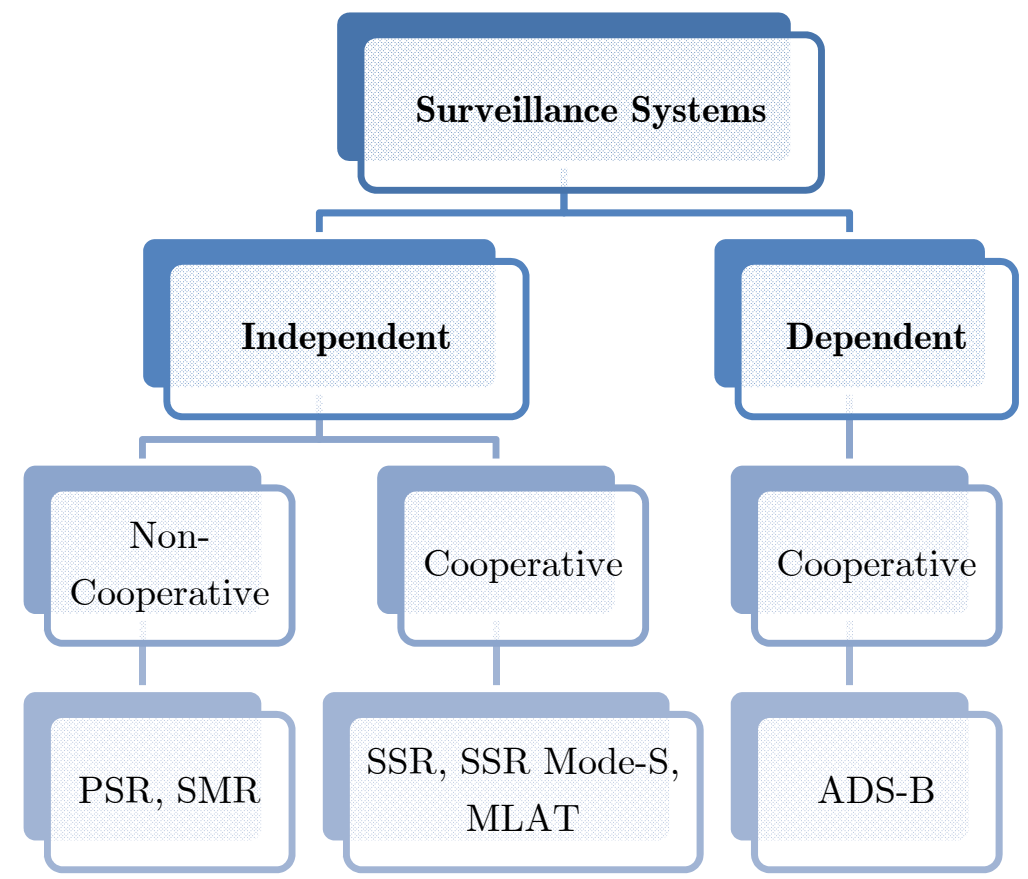

Fig. 2.10 A classification of the main surveillance systems.

\subsection{A General Scheme for Multilateration Systems}

In this subsection the different functions of MLAT systems are introduced. A general architecture is described and then each of its components, focusing on those relevant for this thesis.

\subsubsection{General Architecture for MLAT Systems}

A MLAT system is composed by a set of equipments performing a set of functions, which allow achieving the main purposes of these systems: the automatic identification and localization of a target emitting any of the available signals. The MLAT architecture depends on the equipments used to perform the system functions and their configurations. In Fig. 2.11 a general logical architecture of a MLAT system is shown. Hereafter, the different architectures are discussed based on this general scheme, describing also each of its functions and parts.

The Fig. 2.11 shows a MLAT system in his more general and full version, which is composed by set of receiving stations -Rx- (which in turn are composed of other devices), interrogation stations (Int.), a 


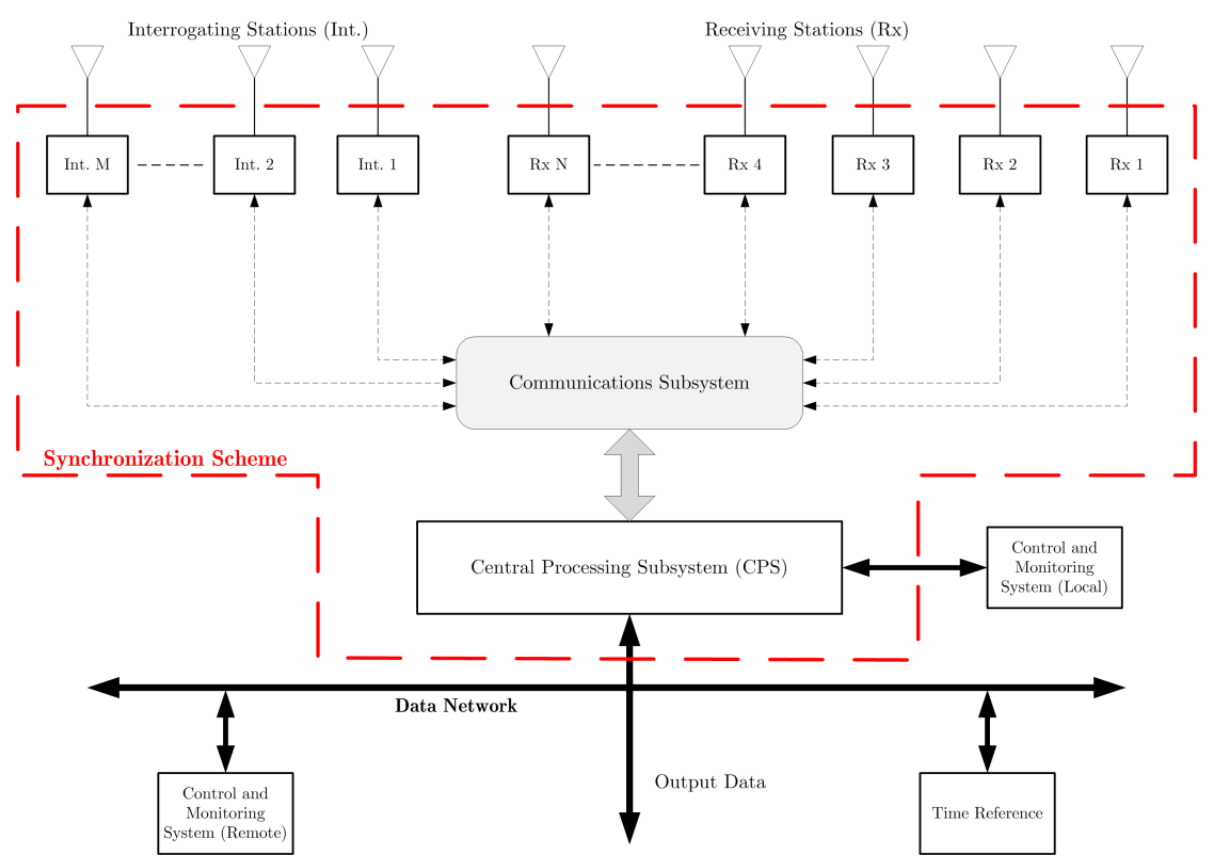

Fig. 2.11 General logic architecture for an MLAT system.

communication subsystem, a Central Processing Subsystem (CPS), a time reference system, and an external data network. Within this scheme other equipments that make part of the general ATC infrastructure can be mentioned but, for the sake of simplicity, they are omitted. On the other hand, all the elements mentioned above run some functions that allow the main purposes of MLAT systems. Within these functions are the signals detection, the target identification, the TOA/TDOA (or other physical parameters) measurements, the target position calculation, the stations (interrogating and receiving) and CPS synchronization, the data transmission/reception among the different system components, the control and monitoring, the data integrity evaluation, the calculated/obtained data format and the transmission of these to a control centre.

In general terms, one (or more than one) interrogating station sends an interrogation signal (normally at $1030 \mathrm{MHz}$ ); then, the receiving stations receive the corresponding reply signal sent by a transponder device, measure its TOA and send such data, along with the message contained in the signal, to the CPS by means of the communication subsystem. In the CPS, the target is identified, its position is calculated and the data contained in the signal message is analyzed. Then, the CPS sends to the control centre, by using the data network, all the calculated and read data in a specific format (usually in ASTERIX [19] format) for presentation on the air traffic controllers displays. Other data processing functions can be 


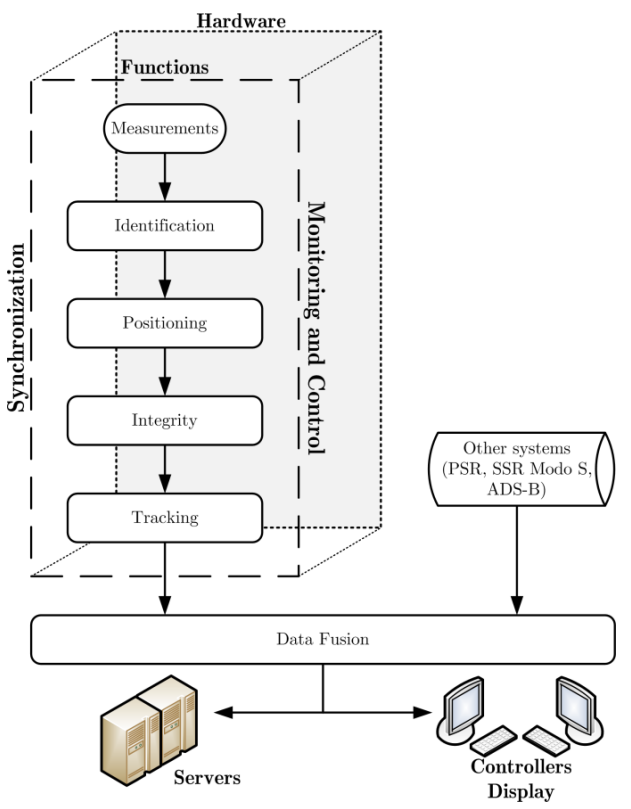

Fig. 2.12 General flowchart of MLAT functions.

used like the data integrity analysis and evaluation, as well as possible failures detection. It is important to emphasize that before sending the data from the CPS to the control centre, the position data are processed by tracking and data fusion modules, the latter in the case of existing another surveillance system (e.g., PSR, SSR, ADS-B, etc.). Additionally, in the CPS, a synchronization scheme is continuously running to fix all the stations (interrogating and receiving) on the same time reference frame for the TOA measurements. Finally, the time reference module provides to the system a unified and highly precise time reference. The control and monitoring system allows the system configuration (locally or remotely) as well as the monitoring of all its parts. All these functions are summarized in Fig. 2.12.

As we have already stated, each element of the MLAT system architecture depends on the type of scheme used to execute the functions described in Fig. 2.12. Of particular interest to this thesis are the functions of interrogation (which can be considered as a part of the measurement function), measurement, synchronization and positioning. Therefore, in the following paragraphs, the most important aspects about them are shortly described.

According to the interrogation function, the MLAT system can operate in active mode (also called active multilateration) or passive mode (also called passive multilateration). In the active mode, at least a dedicated and synchronized interrogation station (or several ones) is present 
in the system coverage area; thus, the system itself requests the reply signals from the transponders present in the coverage area, in addition to those that are spontaneously sent by them (e.g., squitters and extended squitters). On the other hand, in passive mode the system does not have any dedicated interrogation station and, therefore, it only uses the signals spontaneously sent by transponders and those that are elicited by external interrogating stations of other surveillance system present in the coverage area (e.g., SSR Mode S).

According to the measurement function (particularly for TOA/TDOA measurements) the MLAT system can use a direct measurement scheme or a cross-correlation scheme. In the direct measurement scheme, the MLAT system directly measures the signals TOAs either in each station or in the CPS and then obtains the TDOA quantities by using the expression described by (2.4). On the other hand, in the cross-correlation scheme the system directly measures (or estimates) the TDOA quantities in the CPS. It is worth saying that the TOA/TDOA measurement function is also called time-stamping.

According to the synchronization function the MLAT system can use a common clock scheme or a distributed clock scheme. In the common clock scheme the system uses only one ${ }^{8}$ clock allocated in the CPS to perform the TOA/TDOA measurements. On the other hand, in the distributed clock scheme a local clock is implemented for every receiving station and therefore, the TOA measurements are performed in each of these. So an additional tool must be used to time align all the local clocks. Among these additional tools there are mainly three options, namely, the reference transponder, standalone GNSS and common-view GNSS. The reference transponder option uses a reference transponder that sends synchronization signals to all the stations. The standalone GNSSS option implement a GNSS receiver and time aligns all the receiving stations clocks with some GNSS constellation, like GPS. Finally, the common-view GNSS also time aligns all the receiving stations clocks with a GNSS constellation, but by using the same satellite(s) for all the stations (from this comes the name common-view); so, additional processing must be implemented for this option.

According to the positioning function the MLAT system can obtains the target position in 3D or 2D, and for each of these it can use different strategies. In the case of 3D, the MLAT system can directly calculate the three coordinates or it can use additional/external information about the target height. In the case of $2 \mathrm{D}$, the system can use the two coordinates as extracted from the 3D localization or it can use projected models. For now, the important aspect is to know that there exist these possibilities to obtain

${ }^{8}$ Regardless of the redundant parts for reliability and availability issues. 
the target position, because the full description of all these is one of the objectives of the third part of this thesis. 

Part II

Multilateration Systems Design and Deployment 



\section{Overall Frame and Design Problem}

The second part of this thesis covers all related with development of new strategies to design and deploy MLAT systems. In Chapter 2, we have studied all the basic theory of these systems, their origin, the theoretical foundations, their geometrical interpretation, their different functions as a surveillance system, and the influence of the system geometry and the measurement noise on the target localization process. Now, in this chapter, we use that theory to describe the overall frame and design problem for MLAT systems. We describe the design problem from technical point of view and its particularities. In this part, we study all the necessary concepts to understand the key idea of designing MLAT systems. Finally, a complete review of the state of the art, for the current solutions for designing MLAT systems, is provided. The purpose of this chapter is to provide the reader all the necessary concepts to design MLAT systems, and to prepare it for understanding the proposed general design strategies, which are described in Chapter 5.

\subsection{Multilateration System Design and its Particularities}

In general, the MLAT system design starts with a set of requirements and restrictions which must be satisfied with a limited amount of resources (e.g., economics, logistics, time, etc.). Within the requirements, aspects such as the coverage area size (commonly known as surveillance volume), system accuracy, update rate, minimum probability of detection, minimum probability of identification or maximum probability of false alarm, among others, can be found. On the other hand, within the restrictions are those imposed by the regulatory bodies either internationals, like the International Civil Aviation Organization (ICAO), nationals, like the corresponding National Supervisory Authority (NSA), and even the Air Navigation Service Provider (ANSP). Other kinds of restrictions that can 
be found are those imposed by the nature of the scenario to be covered, for example, the presence of high density urban areas can limit the emplacement of some stations, the airport buildings (in the case of airport surveillance) can significantly affect the number of required stations (both receiving and interrogating). In the same sense, other restrictions are those imposed by the presence, in the surveillance volume of interest, of other surveillance systems like an SSR (either Mode A/C or Mode S), because it can make the difference between installing or not dedicated interrogating stations for the MLAT system.

Based on the stated above, it is clear that a MLAT system design consists in the maximization of the requirements compliance (e.g., of the theoretical system performance), by using the fewest amount of resources, and satisfying all the restrictions. To do this, basically a set of performance parameters must be analyzed and calibrated, at the same time the stations are suitably deployed, and the remaining infrastructure is selected.

As we have seen in Fig. 2.12, a MLAT system is composed of a set of hardware on which a certain set of functions are executed. Therefore, in a MLAT system each of these components, hardware and functions, should be calibrated and optimized to meet the main system design objective described before. The hardware design and optimization is out of the scope of this thesis. Regarding to the functions, some of these are associated to a specific hardware, e.g., a receiving station or the CPS, and other ones are related to the whole system. The functions associated only to a specific part of the system can be individually designed, calibrated and optimized. This is the case of the synchronization, measurement and identification functions, which can be run either on a receiving station or on the CPS, and the integrity and tracking functions that are always run on the CPS. On the other hand, the positioning (or localization) function, although it is executed on the CPS, depends on the whole system as we have seen in $\S 2.1 .2$; i.e., this function strongly depends on the system geometry, as well as on the measurements accuracy, which also depends on the synchronization scheme and on other factors, and the particular localization algorithms that are implemented on the CPS. So, this function cannot be individually designed, calibrated and optimized. On the contrary, it must be designed by taking into account the whole system. In this sense, the measurement function is adjusted in every station (or CPS) and the localization algorithms can be implemented individually in the CPS and easily modified after the system is operative. However, obtaining the suitable system geometry is not trivial, and the higher the number of stations or the complexity of the coverage area the more complex the design process can be. Moreover, measurements or synchronization functions, and the remaining ones, can be easily (and economically) modified but not the system geometry, which can imply strong expenses due to civil engineering 
changes or new communication links. For this reason, the design process in this thesis is oriented to the system performance optimization based on all those aspects related to the system geometry, i.e., the number of stations and their position in the surveillance volume of interest, but taking also into account the other related functions.

There exist a variety of parameters to evaluate the MLAT system performance once it is operative, which we call system performance parameters. Most of them strongly depend on the positioning function, which essentially is the core function of MLAT systems and, as it has been commented before, they are usually set as the system requirements to be satisfied by the final design. However, such a set of performance parameters, due to their nature, cannot be taken into account directly from the system design and, therefore, they have to be taken into account by means of another set of parameters, which we have defined as system design parameters. In other words, the system design parameters allows the optimization of the positioning function from a theoretical perspective which, at the same time, is one the functions that optimize (or maximize) the system performance.

In the following we briefly describe the most important performance parameters for MLAT systems and the corresponding design parameters along with the particularities to introduce them into a design process. Furthermore, a general scheme to illustrate the relation between performance and design parameters is shown. Additionally, some guidelines about the stations deployment are provided in the last part of this subsection.

\subsubsection{Performance Parameters for MLAT Systems}

The most relevant parameters to analyze and evaluate the MLAT system performance are [1-2]: the System Probability of Detection (SPoD), the Probability of Identification (PoI), system capacity, latency, start-up time, the Probability of False Detection (PoFD), the Probability of False Identification (PoFID) and the system availability. Each of these is described below.

\section{System Probability of Detection (SPoD)}

The SPoD is the probability of generating a valid position report, within the predefined accuracy requirements, every time period (the system updating period). The position must be only calculated by the MLAT system. It is, position data obtained from other systems like the ADS-B are not valid reports for the SPoD computation.

Another concept related with the SPoD is the probability of detection of one station, which is related to the time percentage that the station is 
available and working under the required standards. This kind of probability must be provided by the manufacturer and in this thesis it is denoted as $P o D_{\text {station }}$.

\section{Probability of Identification (PoI)}

The $P o I$ is the probability of generating a valid target identification report every time period.

\section{System Capacity}

The system capacity is defined as the number of targets that can be processed by the CPS without introducing additional time delays rather than those previously stipulated.

\section{Latency}

The latency is defined as the elapsed time since a Mode S signal is detected and the corresponding target report is generated.

\section{Start - Up Time}

The start-up time is defined as the elapsed time since the system is switched on and it is fully operative. The switch on time also includes the time restoration due to possible power losses.

\section{Probability of False Detection (PoFD)}

The PoFD is the probability of generating a report corresponding to a false target.

\section{Probability of False Identification (PoFID)}

The PoFID is the probability of providing an invalid identification of a valid target.

\section{Operational System Accuracy}

The operational system accuracy is the one that must be provided by the system once it is operative. As we have stated before, it is not always the same than the theoretical one, which is the accuracy allowed by the system geometry and the measurement noises, because during the design process the effects of the localization algorithms are not taken into account.

This accuracy is obtained as the mean square error between the positions obtained from a system measurement campaign and those provided by a reference system, like a GPS with differential capabilities (Differential GPS -DGPS-), another already accepted and certified surveillance system present in the surveillance volume or, even with trajectory reconstruction algorithms. 


\section{Availability}

The availability refers to the probability of the system being able to perform the corresponding function at the start time of any operation. In other terms, this is the parameter measuring how reliable is the system under any eventuality.

\subsubsection{Design System Parameters}

The most relevant parameters to design a MLAT system, based on the positioning function, are the DOP (PDOP, HDOP and VDOP can be used), the Line of Sight (LoS) coverage, the theoretical system accuracy, the measurements error balance and the redundancy. Each of these is described below.

\section{Line of Sight (LoS) Coverage}

The LoS coverage is a parameter particular to each station and is related with the propagation path followed by the signal emitted by the transponder from the target to the receiving station. In this sense, it is considered that a station has LoS to a particular spatial point if the emitted signal propagates from this point through the receiving station without being obstructed or perturbed by any natural or artificial obstacle, i.e., in a straight line. On the contrary, if the signal is received by the station being only propagated by any path different to the shortest path (the straight line) between transponder and the receiving station, then it is considered that such station does not have LoS to that point. The latter is known as a Non LoS situation, or simply NLOS. This concept is depicted in Fig. 3.1. In part (a) of this figure, a scenario of LoS situations is shown whereas in part (b) two possible situations of NLOS are shown. The fixed NLOS situation can be (and must be) taken into account in the design process, whilst the temporary one cannot be taken into account and the possible errors due to it must be corrected in the measurement or localization functions.

It is evident from the analysis of this parameter that, after defining the surveillance volume, the set of those sites where the stations provide the highest percentage of LoS over the entire volume must be identified.

\section{Dilution Of Precision (DOP)}

Although we have previously defined this parameter, in this part a description focused on the design process is provided. As we have commented, the DOP is a unit-less factor that provides information about the quality of the system geometry for calculating the target position in a spatial point. Furthermore, the corresponding parameters to analyze the quality of the system geometry for $3 \mathrm{D}, 2 \mathrm{D}$ and vertical localization are the 


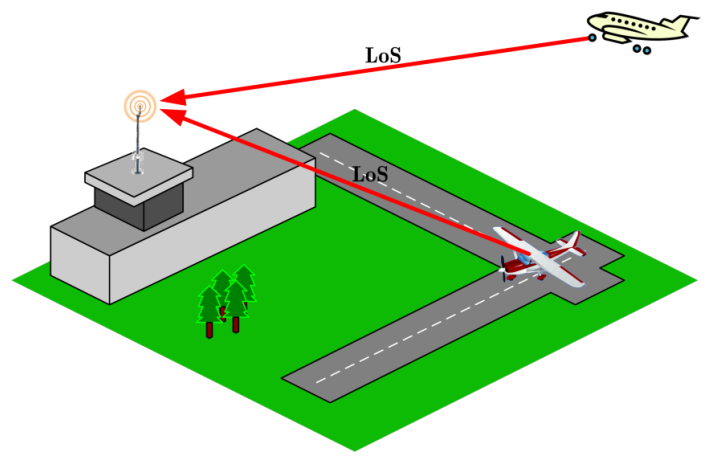

(a) Line of Sight scenario.

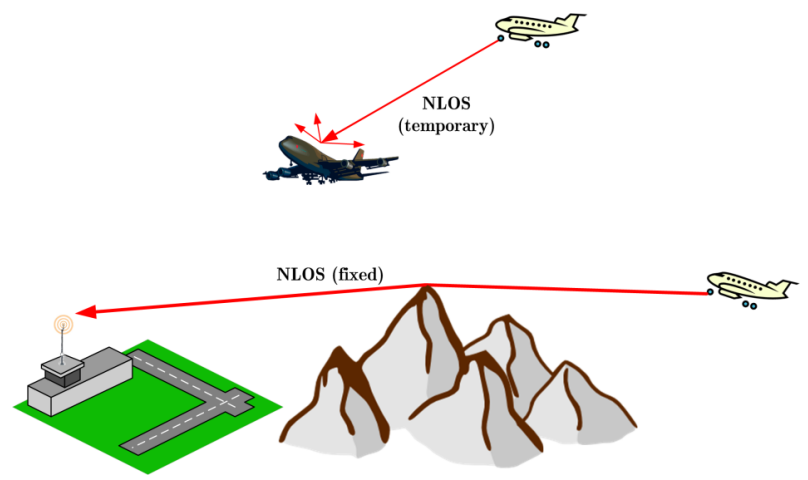

(b) No Line of Sight scenario.

Fig. 3.1 Examples of LoS and NLOS scenarios.

PDOP, HDOP and VDOP respectively. Because the DOP is unit-less, its interpretation is not trivial and, therefore, a special equivalence scale must be used. For this case, a qualitative scale that associates the DOP numerical values with a quality level of the system geometry (e.g., poor, medium, high) is commonly used. An example of such scale is shown in Table 3.1 for PDOP. However, the equivalences shown in this table are only indicative because it depends also on the scenario to be covered.

Table 3.1 Qualitative scale for the PDOP.

\begin{tabular}{|c|l|}
\hline \hline PDOP & Quality \\
\hline 1 & Ideal \\
\hline $1-2$ & High \\
\hline $2-5$ & Good \\
\hline $5-10$ & Moderate \\
\hline $10-20$ & Poor \\
\hline$>20$ & Very poor \\
\hline \hline
\end{tabular}




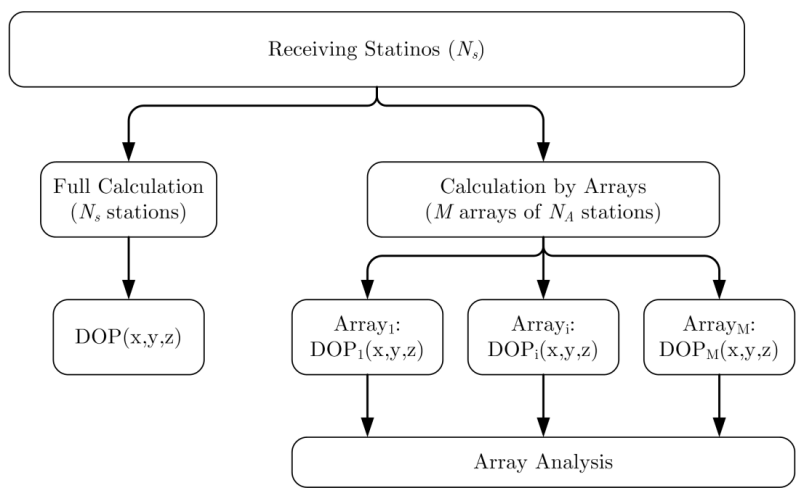

Fig. 3.2 Forms to analyze the DOP.

It is worth to remember that this parameter only depends on the geometric distribution of the stations; it is, on the stations position relative to each point of the surveillance volume. Therefore, for a fixed amount of measurement errors, the theoretical system accuracy (or equivalently the lower bounds of the system accuracy) only depends on the DOP (PDOP, HDOP or VDOP). Although one can think that only the theoretical system accuracy analysis is enough for a design process, the DOP plays an important role in the sense that, commonly, the stations cannot be deployed in those sites that, theoretically, yield the best geometric configurations.

The DOP must be jointly analyzed with the LoS because, for practical issues, it is better (and also more realistic) to calculate the DOP only at those points satisfying the LoS requirements.

The DOP analysis is independent of the measurements or synchronization architecture. It depends, for a particular spatial point, on the number of stations having LoS to such point and the particular geometry formed by them. Based on it, the DOP can be analyzed or calculated by using all the stations having LoS to each point, or by combining all of these in arrays of $N_{A}$ stations and obtaining one DOP value for each array. The first form is called full calculation and the second one calculation by arrays. The selected form to analyze the DOP depends on the design strategy and on the localization scheme used by the manufacturer. Furthermore, as the localization algorithms, for the DOP calculation at least three stations are required, in whose case it is only possible to obtain the HDOP; nor PDOP neither VDOP. To obtain these latter it is necessary the use of at least four stations. This is summarized in the Fig. 3.2.

When the DOP analysis is performed by means of the full calculation, for every point only one DOP value is obtained, whilst for the calculation 
by arrays, as many DOP values as arrays are obtained for every point. The number of arrays, each of them composed by $N_{A}$ stations, at any particular point $(x, y, z)$, is denoted by $M(x, y, z)$ and is given by the following expression:

$$
M(x, y, z) \equiv\left(\begin{array}{c}
N_{c}(x, y, z) \\
N_{A}
\end{array}\right)=\frac{N_{c}(x, y, z) !}{\left(N_{c}(x, y, z)-N_{A}\right) ! N_{A} !}
$$

where $N_{c}(x, y, z)$ is the number of stations having LoS to the analyzed point.

As shown in Fig. 3.2, when the calculation by arrays is used, an array analysis must be performed in order to identify whether or not there exists a number of arrays proving a DOP value smaller than a predefined threshold. This number of arrays is also related with the redundancy parameter, which is described later.

Finally, it is important to emphasize that although we have focused the descriptions above on the DOP, the same conclusions and definitions are completely valid for PDOP, HDOP and VDOP. The only difference lays in the mathematical formulation used to calculate each of these. However, these formulations commonly include the calculation of the three parameters at the same time. The election of the DOP component to be analyzed directly depends on the scenario to be designed. For example, if it is only airport surface, the most appropriate is to analyze only the HDOP, whereas for wide area scenarios (e.g., en-route phase) both HDOP and VDOP should be analyzed. Furthermore, it is also possible to directly analyze the PDOP, but for a clearer understanding is better to separately analyze it as the HDOP and VDOP.

\section{Measurement Error Balance}

In the design of a MLAT system is very important to perform a balance of the possible errors that can affect each station. This balance can be performed in several ways, each of these basically differing in the close they are to the real/operational case. The most basic way is to assume an equally and fixed amount of error for all the stations and for all the surveillance volume. This form is the most inaccurate way although it can be valid for some cases, like a small airport surface scenario. Another form, more accurate than the previous one, is by assuming the amount of error is different for each station and spatially variant.

As we have commented in $\S 2.1 .2$, the measurement errors in MLAT systems are assumed to be Gaussian distributed of zero mean and standard deviation greater than zero. Therefore, the measurements error balance consists in calculating the standard deviation of this distribution. This overall standard deviation can be obtained, for each station, as in (2.6). 
It is evident that an important part of the design accuracy depends on this measurements error balance, particularly, on the accuracy of estimating the standard deviation of every error source. So, this part can make the difference between a system design which is useful in theory and also in the operational case or only in the theory. Some of these standard deviation values should be provided by the manufacturer and other ones should be estimated by means of some physical models.

\section{Theoretical System Accuracy}

The theoretical system accuracy is the parameter that shows the accuracy of calculating the target position, for a particular spatial point, within the surveillance volume. This parameter includes both the effects of the DOP and those of the measurement errors. This parameter is called theoretical because only reflects the best case (or lower bound) of the accuracy that can be obtained with a certain system geometry and an amount of measurements errors. For practical issues this is the parameter used to obtain the system design.

Equivalently to the DOP, this parameter is also analyzed for $3 \mathrm{D}, 2 \mathrm{D}$ and vertical localization as it was already described in $§ 2.1 .2$.

The method used to calculate the theoretical system accuracy through this work has been developed with the active participation of the author of this thesis and is reported in [20]. This method is based on the Cramèr-Rao Lower Bounds (CRLB) analysis, which is a well-known technique in statistics, which sets a lower bound on the variance of an unbiased estimator.

\section{Redundancy}

The redundancy is one of the factors that determine the system availability and continuity in the presence of any failure of its parts. In this concept, the receiving station is treated as a unity.

The redundancy in a MLAT system is expressed in terms of $N_{s}-N_{f}$, where $N_{s}$ is the total number of stations composing the system and $N_{f}$ is the maximum number of stations that can fail or being inactive without altering (reducing) the overall system performance. In other words, it means that even failing $N_{f}$ stations, the system will continue providing the surveillance service within the required performance levels. Such a failure or inactivity can be due to either scheduled or spontaneous causes. The distributed network nature of the MLAT system allows it to efficiently provide good redundancy levels. However, this aspect must be considered in the design process. The way to take into account this aspect is something simple. The system design can be obtained such that, for each point within the surveillance volume, the system must operates with the same 
performance levels for $N_{c}-N_{f}$ stations than for $N_{c}$, where we remember that $N_{c}$ refers to the number of stations that have LoS in a particular point.

\section{Total Coverage}

The total coverage is an auxiliary concept which merges the concepts of system accuracy (either operational or theoretical) and redundancy. It is a "yes or not" parameter (or numerically "1 or 0") that computes the number of spatial points that are covered with LoS, for more than a predefined number of stations, and within a system accuracy better or equal than a predefined threshold.

The usefulness of this concept appears when solving the MLAT system design as an optimization problem. Although it is not explicitly taken into account in the next subsection of this chapter, due to the fact that it is only a composed parameter, it must be defined because we will use it in a posterior chapter.

\subsubsection{Performance Parameters vs. Design Parameters}

As we have stated before, the system design must be performed by taking into account a set of design parameters that allow the optimization of the system functions, which accordingly allow the optimization of the system performance. A general scheme, which for the sake of clarity is focused only on the positioning function, is shown in Fig. 3.3.

The system functions can be divided into a subset that is equipment dependent and other one that depends on the whole system. To design each of these functions a set of system design parameters is taken into account. Particularly, for the positioning function, those parameters are the LoS, the DOP, the measurement errors balance, the theoretical system accuracy and the redundancy. The right configuration of these parameters allows, in a theoretic sense, the system to provide a suitable positioning function and

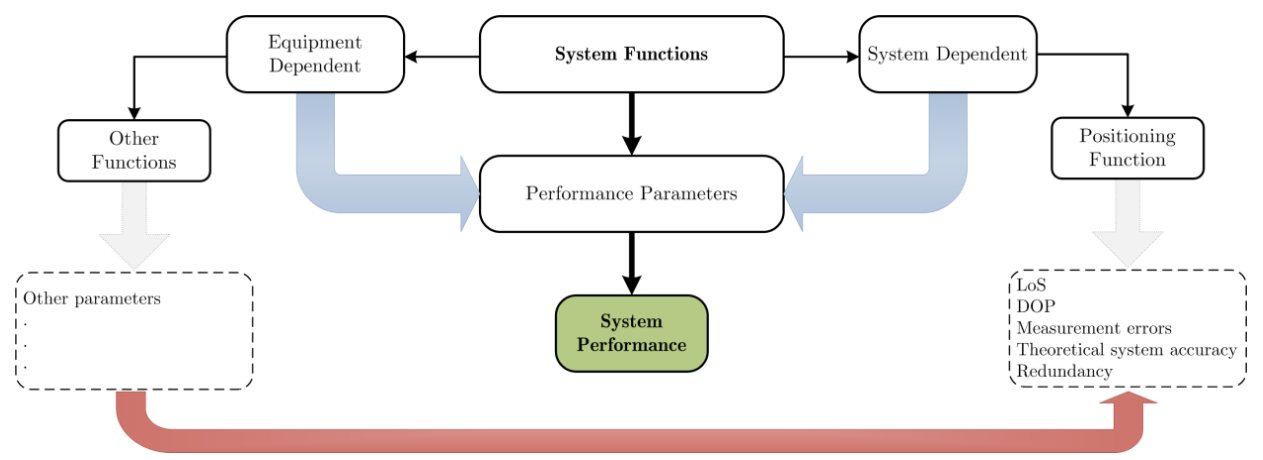

Fig. 3.3 Performance parameters vs. design parameters. 
this one, at the same time, allows to improve an important number of performance parameters. Among the performance parameters described in $\S 3.1 .1$, those strongly related with the positioning function are the SPoD, latency, operational system accuracy and availability.

The SPoD strongly depends on the positioning function because if the target position is calculated with accuracy out of the operational levels, the system neglects the corresponding report and therefore this parameter is reduced. In this sense, it is clear to see that the theoretical system accuracy must be within the predefined levels under any eventuality. Furthermore, also the redundancy parameter plays an important role on the SPoD parameter, because if the appropriate redundancy level is not configured, then, the suitable number of stations needed to allow the positioning function of providing the required operational accuracy cannot be available.

The latency dependency on the positioning function is more operative than theoretical. So, as it is shown in Chapter 7, the computational time directly depends on the way the position is calculated, it is, on the localization algorithm. In this sense, if the suitable localization algorithm or strategy is not used, this function will introduce a time delay that can exceed the maximum allowed latency. However, due to the computer processing advances, this dependency can be usually neglected. Furthermore, due to the fact that is more an operational relation, this aspect is not taken into account during the design process because, in a theoretical sense, no model can be applied to this dependency, even we now know that it exists.

The operational system accuracy dependency on the positioning function is direct and the most obvious. This dependency can be easily understood by remembering that the higher the theoretical accuracy, which includes the effect of the DOP and measurement errors, the higher can be the operational one.

The availability dependency on the positioning function is connected with the system geometry (i.e., on the number of stations and the corresponding spatial distribution), whose quality is reflected by the DOP parameter. So, the system geometry must allows the system providing the same accuracy levels, throughout time, even in the presence of any station failure or other situation that reduces their number. In this sense, the redundancy parameter must be set so that it allows preserving the DOP levels.

Finally, there exists another dependency between the design parameters of the different functions. In the case of positioning function this dependency is in one direction as depicted in Fig. 3.3. For example, the measurements errors depends also on the way the measurements devices (either in the receiving station or in the CPS) are designed and 
manufactured. Nevertheless, due to the nature of this dependency, henceforth we assume that this kind of dependency does not exist and we take it into account by introducing certain modelled uncertainty into the design process.

\subsubsection{Particularities for the MLAT Stations Deployment}

Deploying the stations in those theoretically ideal sites is not always possible, either due to site availability reasons, for restrictions or normative, for difficult access, for economical costs, because maybe this ideal site is in middle of a runway, etc. In this sense, the stations deployment is a specific process that depends on the scenario characteristics and on the particular system manufacturer. For example, for the same scenario, two different companies can provide different designs for the layout of the stations that, at least theoretically, meet all the requirements and restrictions imposed by the customer. Nevertheless, there are some general guidelines than can be taken into account when deploying the stations. These are commented below.

First of all, the possible sites where a priori is easier to deploy the stations must be identified. For example, those sites where already there are communication or surveillance infrastructures, mobile communication, TV or radio towers or electric power supply. Also, those sites which can be rent-free, or another characteristic that implies some economical or effort saves, should be identified. All of these sites should have certain priority in the design process.

For airport surface surveillance, special care must be taken for installing stations near to high density building areas because it can generate multipath problems. If the installation of a station in a site with these characteristics is required, some strategies should be contemplated, like to move the station within certain spatial range, for which the system performance parameters do not significantly change, or even to use sectorial antennas that limit the signal reception in some directions for which, obviously, the surveillance service is not necessary.

When dedicated interrogating stations must be implemented, it should be taken into account that, in order to reduce its number, the corresponding site (or sites) should be that which provides the highest LoS percentage over the entire surveillance volume.

Depending on the surveillance volume characteristics, it can be necessary the implementation of antennas with different radiation pattern, either horizontal or vertical. Because it affects the measurements accuracy, this radiation pattern should be also taken into account in the design process. 


\subsection{Solutions for MLAT Systems Design: State of the Art}

The main objective of MLAT systems is the hyperbolic localization of targets equipped with a transponder (or equivalently the non-transponder device for surface targets). Therefore, in general terms, the design of these systems is mainly focused on the optimization of that function, in such a way that a set of requirements and restrictions are satisfied as better as possible. More specifically, the design of a MLAT system consists in finding the best spatial distribution of the stations (i.e., the system geometry), which allows satisfying certain performance levels, which for this case are those described in $\S 3.1 .1$ and $\S 3.1 .2$. Likewise, this optimization is essentially supported on the basic theory about the accuracy of MLAT systems, particularly, on the knowledge about the system geometry influence on this accuracy. In this sense, the works which have been developed so far can be classified into three different groups. The first one comprises all the works describing the basic theory for analyzing the theoretical system accuracy. The second one comprises all the works that apply the basic theory (i.e., the works of the first group) in order to obtain some general guidelines for the station deployment; these works are commonly developed under some real/operational cases. The third group is composed of the works that provides some general frameworks which allows the "automatic" system design under some conditions. Furthermore, this last group normally makes also use of the first two groups (much more of the first one). In the following, the most relevant published works, within each of these groups, are briefly described in chronological order.

Before proceeding with the description of the works within each of the groups commented above, it is worth saying that a significant number of them have been developed for non hyperbolic localization systems, i.e., for the so called distributed sensor networks. For this reason, it is important to firstly describe the difference between these and the MLAT systems. In this sense, it is right to say that not all the distributed sensor networks based systems are geometric localization systems (as the MLAT is); so, the MLAT systems can be seen as a particular case of the first ones. The distributed sensor networks also comprise some systems that are only intended to individually collect certain kind of data, but not for a joint localization process. Therefore, in these cases, the spatial distribution of the stations (or sensors) is guided solely by the condition of LoS coverage. Within these systems can be mentioned the high precision seismic networks [21], where each station individually collects the corresponding data; in this system the objective is to deploy the stations in such a way that they cover the widest possible portion of the terrain. Nevertheless, the basis of these systems can be seen, from a common point of view, as far as all of them 
deploy a number of stations to meet some requirements and restrictions. In the remaining part of this section, unless it is clarified, we will refer always to target localization systems.

Within the first group, one of the first works developed for the analysis of the accuracy of hyperbolic localization systems was published in 1975 in [22]. In that paper a method for calculating the accuracy of a particular location estimator was derived. This method basically links the error covariance matrix to the moments and products of inertia of a mass configuration that is easily determined from the system geometry. Furthermore, the same author, in the same year, published in [23] a method that derives the boundaries for the first method in [22]. Three years later, in [24] some formulas were derived for estimating the length and cosines directions of the semiaxes of the error ellipsoids in multilateration, in terms of the R.M.S errors and direction cosines of the distance measurements. Then, in [25] a method for estimating the lower bounds on 2D localization errors in acoustic passive arrays was presented. The systems studied were intended for tracking the 2D target positions and velocities, and the method used for estimating the lower bounds of that tracking process was the Cramèr-Rao inequality. Furthermore, this works assumed ideal signal propagation, thus taking into account only instrumental errors (zero mean Gaussian distributed independent of the signal and of the remaining receivers). Thereafter, in [26] two approximate formulas, which relate the accuracy of the TDOA and differential Doppler measurements to the "onesigma width" of the constant lines characterized by those measured quantities on the surface of the Earth, were provided. The usefulness of these formulas was the easy computation of an approximate level of the localization accuracy. Then, in [3] was provided perhaps the most complete performance analysis until that time (1984) for passive localization systems, including also the hyperbolic ones. In this work, the concentration ellipse, the circular error probability, and the PDOP were defined for those systems. Many years later, in [4] some useful approximations for calculating the PDOP in 2D scenarios were presented. The derivations in this work were developed in the same sense than in [3]. Finally, the most recent work (up the time of writing this thesis) for analyzing the MLAT systems accuracy was published in [20]. In this work, the CRLB is used to calculate the lower bounds of accuracy for both standard (i.e., only TOA/TDOA measurements) and enhanced (i.e., RTD, AOA, time integration and the combination of them along with the standard version measurements) MLAT systems. Moreover, in this work also the signal propagation is modelled and a complete measurement error balance is taken into account.

Within the second group of works, in [27] a step by step manual process is presented for locating the sensors measuring the bearing of acoustic signals emitted from some targets. Such a process is based on the inspection 
of the Fisher Information Matrix (FIM) and it is composed of three guidelines for emplacing the sensors. Then, in [28-29] the authors presented a theoretical analysis of the CRLB for source localization based only on TDOA measurements and they provided some ideal geometries, which are called platonic (e.g., the tetrahedron, octahedron, hexahedron or cube, dodecahedron, and the icosahedron) and that minimizes the variance bounds for localization, assuming the same measurement errors in all the stations. In a posterior work [30], similar conclusions than in [28-29] were provided by one the authors of these previous works, but in this case a numerical deterministic method called spherical codes was used. Then, in [31] a particular analysis for the MLAT system of Boryspil airport (Kiev, Ukraine) was performed. In this work, some guidelines are obtained about the best system geometry configuration for that airport. In the same year, in [32] an analysis for source localization systems with decoupled range and bearing estimation, for the case of localizing only one target, was presented. Such analysis was developed for the case of stations measuring TOA, TDOA or AOA and the tool used for the accuracy analysis was the CRLB. Nevertheless, this analysis was restricted only to the case of the stations confined on or inside a circular area. The main conclusion of this work was that the optimum configuration for the system geometry is with the stations equally spaced on a ring-shape figure with some stations in the centre of it. Then, in [33] it is also concluded that the best configuration of the stations, for TDOA localization, is obtained by emplacing all the stations equally spaced on a ring-shaped figure (or concentric rings). This conclusion is obtained by means of mathematical analysis of the FIM, particularly by finding the configuration that minimizes the determinant of it, and by several simulations. Thereafter, in [34] the optimization of the number of the stations and their positions, under the layout of Boryspil airport (Kiev, Ukraine), by means of genetic algorithms is presented. The quality criterion used in this work is only the minimization of the volume of the error ellipsoid. However, the process to "estimate" the minimum number of stations is manual, and it consists basically in adding a pair of stations until some condition of DOP is satisfied. Moreover, in this work only the results are shown and no formal procedure is described; for this reason this work is classified into this group. Finally, in [35] a practical validation of the DOP parameter (for 2D localization) properties is performed by means of real measurements in the MLAT system of the Adolph Würth Airport (Schwäbisch Hall, Germany). The main conclusions of this work did not go beyond the qualitative validation of the theory about DOP parameter in that particular scenario. Additionally, it is important to emphasize that the works [34-35] have been published after the first publication [36] of this thesis concerning the optimization of the MLAT systems layout. 
From the description above of the second group works, it can be seen that all of them, under different conditions and methods, approximately arrive to the same conclusion, that is, the stations (or sensors) must be emplaced over a set of specific figures called platonic (the ring figure can be also considered as particular 2D case of a platonic). Nevertheless, it can be easily understood that for most of the real scenarios, e.g., an airport for the case of this thesis, it is strongly difficult to emplace the stations following those geometric figures because of the intrinsic limitations of them. Therefore, these works are only useful as a reference/ideal configurations that should be reached but not as a general procedure to design a real MLAT system.

Within the third group of works, in [37] an optimization procedure to emplace the sensors in a distributed sensor networks, based on simulate annealing, is proposed. In this work the sensors can be deployed only over a regular grid and the only parameters taken into account are the LoS coverage and the cost of emplacing a sensor in each particular point of the grid. Then, in [38] the authors use a metaheuristic method called diversified local search for emplacing the sensors of an indoor local positioning system. In this work, only the parameters of DOP and LoS coverage are used to evaluate the quality of the design. Furthermore, the size of the scenarios is very small (e.g., meeting rooms of $6 \mathrm{~m} \times 7 \mathrm{~m}$ ) and no signal propagation effects are considered. In the same year, in [21] a simple heuristic procedure is proposed to solve the same problem as described in [37]. Then, in [39] a deterministic numerical method that seeks to minimize only the condition number of the geometric matrices of two different TDOA localization estimators is proposed. Although the results shown in this work are useful, the proposed method is highly restrictive as the number of required stations increases. Furthermore, with the condition number optimization this method only optimizes the upper bound for the estimator error variances (i.e., the maximum variance) and does not guarantee whether the minimum variances are reached, which is a more realistic case. Most recently, in [40] an optimization procedure, based on random search and generalized pattern search, is proposed for emplacing the station of an AOA localization system. The problem optimized in this work is $2 \mathrm{D}$ and the parameter used for evaluate the system design is the theoretical accuracy as provided by a CRLB analysis. Furthermore, no signal propagation effects are taken into account. Finally, in [41] a simple application of the genetic algorithms for emplacing four MLAT stations, which measures only the TOA, in a wide area $(200 \mathrm{~km} \times 200 \mathrm{~km})$ is presented. In this work, the parameter to evaluate the system design quality is only the theoretical system accuracy at three different flight levels. Moreover, in this work no LoS coverage is taken into account and neither the signal propagation effects (assume stations equally affected by noise), thus leading to non-realistic designs. 
Also, the configuration of the genetic algorithm seems to be not computationally efficient, as a high number of individuals are used. Additionally, this work has been published after the first publication [36] of this thesis concerning the optimization of the MLAT systems layout.

From this last group description, several observations can be made. First, it can be observed that no many works have been developed for dealing with the problem of MLAT system design. Moreover, although some of the works cited above could be used for such a purpose, they do not contemplate several parameters that are highly important for MLAT systems, like the probability of detection, the signal propagation effects, a complete measurements error balance, real constraints due the scenario layout, and the possibility to also design the enhanced version of MLAT systems, whose implementation is in some sense growing. Furthermore, the works described above neither present any general framework for allowing the possible system expansions. All of these have motivated us to develop the part of the thesis concerning to the solution of these issues. 



\section{Optimization Theory: A Short Review}

As we have previously described in Chapter 3, one of the general objectives of this thesis is the development of general design strategies for the automatic design of MLAT systems. These general strategies are based on the metaheuristic optimization methods, particularly on the well known Genetic Algorithms (GA). Therefore, in this chapter we describe the basic concepts for this kind of optimization methods. To do this, we start with describing the concept of Combinatorial Optimization (CO) problems, which basically are the numerical mean to introduce a real world problem into a computer aided process. Then, we briefly describe the concept of metaheuristic optimization methods, making emphasis on the GA. For the latter, we make the description focused on the specific GA implemented in this thesis. The purpose of this chapter is to describe the entire optimization context, which supports the general design strategies proposed in Chapter 4. It is important to note that we have selected the GA, instead other kind of metaheuristic optimization method, because they are the most stablished methods in the literature and because the aim of this part is to develop the design strategies and not to develop a new optimization method.

\subsection{Combinatorial Optimization Problems}

In engineering, mathematics or computer sciences, many real-world problems (or theoretical ones) can be stated as optimization problems. In this kind of problems, the main objective is to find the "best" configuration of a set of variables which satisfy some goals. In a general sense, optimization problems can be grouped into two families: those where the solution can be represented as a set of real-valued variables and those where the solution can be represented as a set of discrete variables [42-43]. Particularly, among those whose solutions are represented by discrete variables there is a class called Combinatorial Optimization $(\mathrm{CO})$ problems. 
There exist in the literature many definitions for $\mathrm{CO}$ problems. According to [42], in a CO problem we are looking for an object from a finite, or possibly countable infinite, set. This object is typically an integer number, a subset, a permutation, or a graph structure. It should be pointed out that for optimization problems with real-valued solutions, which are commonly known as continuous optimization problems [42], we look for a set of real numbers or even for a function. More formal, the definition of a CO problem is given as follows [43]:

$$
\begin{aligned}
& \text { A CO problem } P=(\mathcal{S}, f) \text { can be defined by a set of } \\
& \text { variables } X=\left\{x_{1}, \ldots, x_{n}\right\} \text {; variable domains }\left\{D_{1}, \ldots, D_{n}\right\} \\
& \text { some constraints among variables (which can be or not } \\
& \text { the same for each of these), and an objective (also called } \\
& \text { fitness or cost function) function } f \text { to be minimized, } \\
& \text { where } f: D_{1} \times \ldots \times D_{n} \rightarrow \mathbb{R}^{+}, \text {and a set of feasible } \\
& \text { assignments } \mathcal{S}=\left\{s=\left\{\left(x_{1}, v_{1}\right), \ldots,\left(x_{n}, v_{n}\right)\right\} \mid v_{i} \in D_{i}\right\} \text {. }
\end{aligned}
$$

In the definition above, $\mathcal{S}$ is commonly known as the search (or solution) space, as each element of this can be seen as a candidate solution. When solving a CO problem, the key idea is to find a solution $s^{*} \in \mathcal{S}$, whose values satisfy all the constraints, with minimum objective function value, that is, $f\left(s^{*}\right) \leq f(s) \forall s \in \mathcal{S}$. In this case, $s^{*}$ is called globally optimal solution of $(\mathcal{S}, f)$ and the corresponding set of these $\mathcal{S}^{*} \subseteq \mathcal{S}$ is called the set of globally optimal solutions.

When the variable domains, the constraints and objective function take some values and a particular form, this specific situation is known as the instance of an optimization problem and is commonly denoted as $I$. Therefore, it is clear that we really solve an instance of an optimization problem rather than a problem itself.

Finally, it is important to comment that three different versions of $\mathrm{CO}$ problems can be found, namely [42], [44]: optimization problems, evaluation problems and decision problems (or recognition problems). The first ones are defined as those problems which look for a feasible solution $s^{*}$ that minimize (or maximize) the objective function $f$, the second ones are those problems which look for the minimum value $f^{*}$ of the objective function, while the latter are those problems which look to know whether or not there is a feasible solution $s \in \mathcal{S}$ such that $f(s) \leq \vartheta$ (a given threshold value). Further, based on the assumption that the objective function is easily computed (that is a reasonable assumption for a large number of applications), the optimization problems are considered as the hardest problems among the three ones previously described, while the decision ones are recognized as the "easiest" [42]. Under this definition, the problems dealt with in this work belong to the optimization problems group and unless otherwise stated we always refer to $\mathrm{CO}$ problem as an optimization problem. 


\subsubsection{Complexity of a CO Problem}

Another important point in the basic study of $\mathrm{CO}$ problems is the analysis of the corresponding problem complexity. This analysis allows us to categorize $\mathrm{CO}$ problems in different groups depending on their difficulty. Two important points to be analyzed in this aspect are: the relation between the objective function and constraints with the solution variables and, maybe more important, that one related with the required time to solve the particular problem.

Regarding to the first point commented above, an optimization problem can be classified as a Linear Programming (LP) problem, Quadratic Programming (QP) problem or Nonlinear Programming (NP) problem. In LP problem both the objective function and the constraints are linear functions of the solution variables, in QP problems the objective function is quadratically related with the solution variables whilst for the constraints the relation is linear and, finally, in NP problems both the objective function and constraints can be nonlinear functions of the solution variables [45-46]. Solving NP problems is more difficult than solving LP or QP problems. The solution of a NP problem generally requires an iterative procedure to establish a direction of search for the major iterations.

The second point is that related with the time to solve the optimization problem, specifically the characterization of this time. Because there is a high density of information and definitions, and because it is not the aim of this work to go in depth about this topic, nothing more than using it to define the order of problem complexity, we are going to provide only the elementary and more used definitions in the literature. Before providing these definitions, it is important to emphasize that when we refer the word time, it is related more to the number of steps (or operations) required to solve a specific problem than to the absolute processor time.

A standard and basic working definition to the characterization of the required time is based on the term polynomial time. This term refers to the asymptotic time upper bound (the worst case) to solve a given problem with an input of size $n$. In this way, a polynomial time is that one which can be expressed as a polynomial function of the input size, i.e., the order of the upper bound is $\mathcal{O}\left(n^{k}\right)$ for some constant $k$, e.g., a polynomial of $\mathcal{O}\left(n^{3}\right)$, $\mathcal{O}\left(n^{2}\right), \quad \mathcal{O}(1), \quad \mathcal{O}(n \log n)$. Likewise, a non-polynomial time (sometimes referred also as exponential time) is that one who cannot be expressed as a polynomial function of the input size, e.g., $\mathcal{O}\left(2^{n}\right), \mathcal{O}\left(n^{n}\right), \mathcal{O}(n !)$. Although the complexity theory, based on the time characterization, was originally designed for decision problems [42], [44], [47-48], it can be directly used for optimization problems with or without the same notation (see [49] and its references for other kind of notation). In this work, we use the same 
notation than the decision problems because it is widely accepted for the classical works we have used to support this part of the thesis.

Based on the definitions given above, optimization problems can be basically classified into two main complexity classes: class $P$ or class NP problems, where the word $P$ stands for deterministic Polynomial and the words NP stands for Nondeterministic Polynomial [42], [44], [47]. Standard definitions are [47-48]: the class $P$ problems are those that have a polynomial-time deterministic algorithm, i.e., these kind of problems can be solved in a time of $\mathcal{O}(p(n))$, where $p(n)$ is a polynomial on $n$, and a correct solution is always obtained. The class NP problems are those that can be solved in a polynomial time but by a non-deterministic algorithm.

Commonly, all problems that are in the class $P$ also are in class $N P$, thus $P \subseteq N P$. However, so far the question about whether class $P$ is a proper subset of class $N P$ (i.e., $P \subset N P$ ) or whether class $N P$ is equal to class $P$ (i.e., $P=N P$ ) has been not answered and, for now, the researchers assume $P \neq N P$ and that there are some problems that are in class $N P$, but not in class $P$. These last problems are difficult as no polynomial-time algorithms exist for them. This asseveration leads to another useful class, which is strongly connected with some optimization problems: the class NPhard [43], [47]. Specifically, a problem is classified as class NP-hard if an algorithm for solving this problem is polynomial-time reducible to an algorithm that is able to solve any problem in class NP [42], [44], [47]. Therefore, class NP-hard problems are at least as hard as any other problem in class NP, although they might be harder [44]. Furthermore, class NP-hard problems are not necessarily in class NP.

Another important concept in $\mathrm{CO}$ problems related with the complexity classes, which was introduced in [50], is the concept of class NP-complete problems as a subset of class NP. In this sense, a given problem is classified as class NP-complete if this is in class NP and is also in class NP-hard. In this sense, class NP-complete problems can be considered as the most difficult problems that are in class NP [44].

\subsubsection{On the Solution of CO problems}

The solutions for $\mathrm{CO}$ problems can be obtained by different methods that are based on a wide range of mathematical principles. In a general sense, these algorithms solve problems instances by exploring the search space efficiently. Moreover, normally these algorithms are related with the class NP-hard problems.

The algorithms for solving $\mathrm{CO}$ problems can be classified as complete or approximate algorithms [42-43], [51]. The complete algorithms ensure, for finite size instances of a $\mathrm{CO}$ problem, an optimal solution in bounded time. However, for $\mathrm{CO}$ problems that are class NP-hard no polynomial time 
algorithm exists (assuming that $P \neq N P$ ) and, therefore, complete methods need exponential computation time in the worst-case [43], which often leads to computational times too high for practical applications. This fact generates the necessity of using approximate algorithms, where the warranty of finding an optimal solution is sacrificed in order to obtain a good (or acceptable) solution in a significantly reduced amount of time.

Roughly speaking, among the approximate methods two kinds of these can be found: the constructive methods and the local search methods. Constructive algorithms start from "zero" and build solutions by cleverly adding components until a solution is complete, whilst local search algorithms start from an initial point (in some cases obtained by previous problem knowledge or by some kind of constructive algorithm) and iteratively try to replace the current solution for a better one by exploring only a reduced search space, which is normally user-defined. Constructive algorithms are faster and, in some sense, provide inferior quality solutions than the local search ones. However, this speed and quality of solutions should be measured by taking into account the amount of computational resources needed in respect of the particularities of the $\mathrm{CO}$ problem or desired solution.

Among the constructive algorithms, in the last three decades, a new kind of approximate algorithms have been developed which basically tries to combine basic heuristic methods in higher and well-structured level frameworks. This kind of algorithms is usually known as metaheuristic optimization methods and the introduction of these is the objective of the next subsection.

\subsection{Introduction to Metaheuristic Optimization Methods}

The term metaheuristic was introduced in [52] and is a compound word of two Greek words. The first one is meta, that means "beyond, in an upper level" and the second one is heuristic, which is a term derived from the verb heuriskein that means "to find" [43]. As it is commented in [43], up to now there is no a universal definition for the term metaheuristic. In the following we will try to state one of those ones within the current literature and which fits better the application addressed in this thesis.

According to [53], a metaheuristic can be defined as "an iterative generation process which guides a subordinate heuristic by combining intelligently different concepts derived from classical heuristics, artificial intelligence, biological evolution, natural and physical sciences, for exploring and exploiting the search space, using learning strategies that structure information in order to find efficiently near-optimal solutions". Moreover, as 


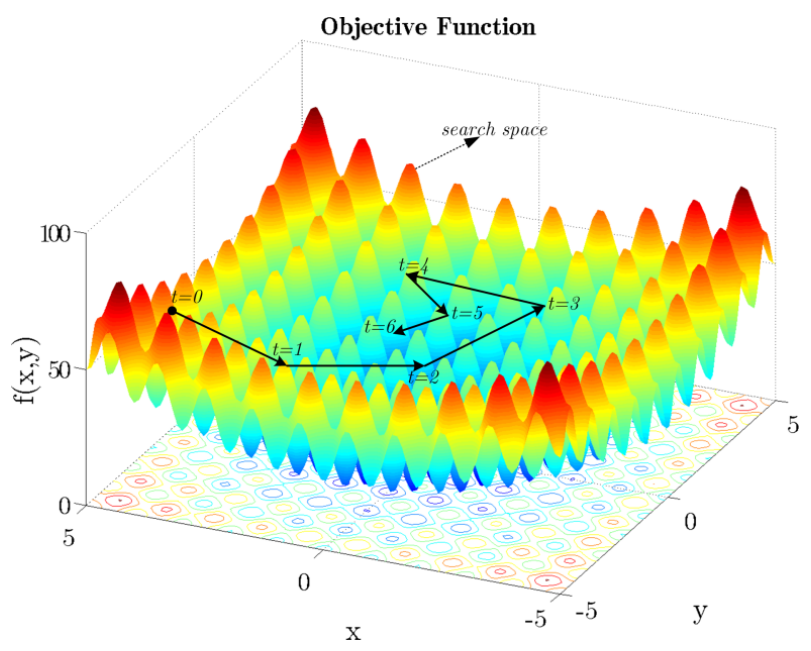

(a) Trajectory-based methods.

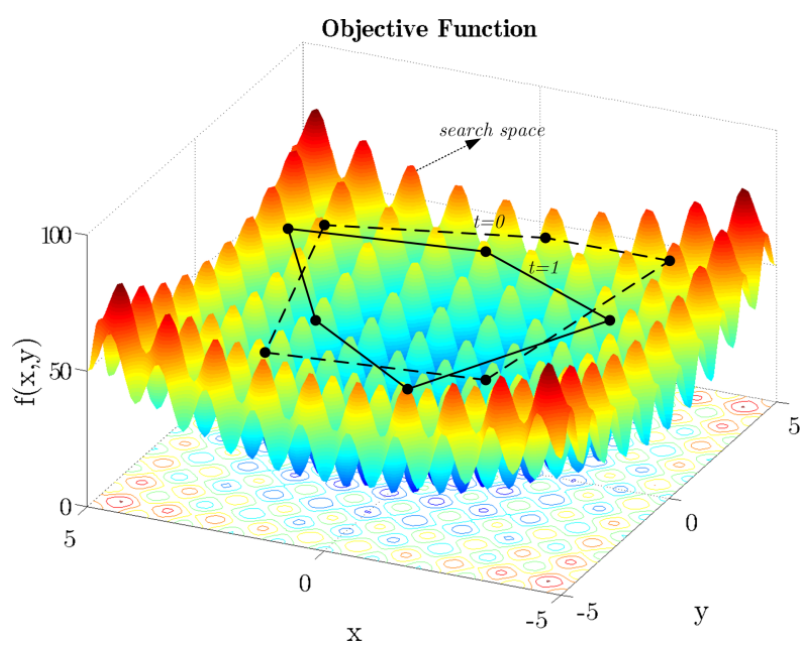

(b) Population-based methods.

Fig. 4.1 Trajectory-based methods vs. Population-based methods: Illustration of the search space exploration.

described in [43], [54], "the main goal of a metaheuristic is to avoid the disadvantages of iterative improvement and, in particular, multiple descent, by allowing the local search to escape from local optima" and "the main difference to pure random search is that in metaheuristic algorithms randomness is not used blindly but in an intelligent, biased form".

In a general sense, metaheuristic optimization algorithms include, but are not restricted to, Ant Colony Optimization (ACO), Evolutionary Computation (EC), including Genetic Algorithms (GA), Iterated Local 
Search (ILS), Simulated Annealing (SA), Particle Swarm Optimization (PSO), and Tabu Search (TS). However, a useful classification (one of those described in [43]) divides these methods into two groups, namely, population-based and trajectory-based (or single point search) algorithms. This classification is based on the number of solutions used by the method at any time. Trajectory-based methods work on single solutions at the same time and describe a trajectory in the search space during the search process. Among them are, for example, Iterated Local Search or Tabu search. On the other hand, regarding to population-based methods, they work with several solutions at the same time, i.e., they perform search processes which describe the evolution of a set of points in the search space. Examples of these are Genetic Algorithms or Ant Colony Optimization. Fig. 4.1 shows an illustration of this concept.

Most metaheuristic optimization methods, belonging to the classification presented above, share several concepts and principles. However, particularly more for population-based algorithms, because they deal with population of solutions that consequently provides an intrinsic way for the exploration and exploitation of the search space, the final behaviour and performance, of each particular method, are strongly connected with the way the population is manipulated. Therefore, due to the fact that in this thesis only the particular population-based method of genetic algorithms is used, it is better to directly focus the attention only on the introduction of this method. The latter is the aim of the next subsection.

\subsection{Review on Genetic Algorithms}

The Genetic Algorithms (GA) are nature-inspired metaheuristic optimization methods, which belong to the broader family of Evolutionary Computation (EC), and that solve both constrained and unconstrained optimization problems based on the process that drives the biological evolution. Due to the fact that these algorithms belong to the broader family of EC, before go into insight of GA a brief introduction of EC is provided below.

\subsubsection{Evolutionary Computation}

The EC is a collective term for the set of all optimization methods that are inspired by the Darwinian evolution [55]. In this family of algorithms, a solution for a particular optimization problem is called individual and the corresponding set of solutions, at a specific time (or iteration), is called population. Each iteration of the algorithm corresponds to a generation, where a specific group of operators is applied to individuals of the current 


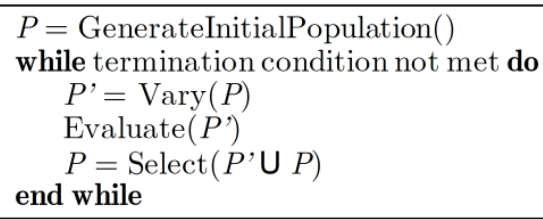

Fig. 4.2 General algorithm for EC.

population, in order to generate the individuals of the population for the next generation. This process, in a general sense, is described by the pseudo-code in Fig. 4.2. First, an initial population is generated (e.g., randomly) and then, while a particular condition is not satisfied, the algorithm apply the group of operators (denoted by the function Vary), evaluate the new population (function Evaluate) and select (function Select) a new population among the set individuals resulting from the union of the last population and the current one.

In the current literature (see [55] for a complete survey) the EC is divided in three main groups: evolutionary programming [56], evolutionary strategies [57] and genetic algorithms [58]. Furthermore, as it is stated in [55], algorithms belonging to the evolutionary programming and evolutionary strategies are more applicable to continuous optimization problems, while GA are more specific for combinatorial optimization problems. This is one of the reasons to select GA among the different EC methods.

\subsubsection{Genetic Algorithms}

The basic principles of GA were introduced in [58] and a more extensive and complete description of these can be found in [59-62]. In this thesis, we follow the EC structure to describe the general GA highlighting only those particular aspects which are implemented in this work. The steps to explain the GA are as follows: fundamental theory, problem adaptation, and GA operators or functions.

\section{Fundamental Theory}

The GA are adaptive optimization algorithms based on the genetic processes of the living organisms postulated by Darwin [63], which basically state that throughout generations they evolve following the principles of natural selection and the survival of the fittest. These natural principles also stipulate that those organisms which are more able to survive and attract mates have high probability of generate offspring and vice versa. This means that the good characteristics, commonly called genes, of the fittest individuals are preserved for successive generations, and probably 
increasing their presence in the population. Moreover, the combination of well-adapted individuals can generate other ones even with better genes than their predecessors. These basic principles help the organisms to evolve with their environmental changes and thus survive.

Genetic algorithms are capable of creating solutions to real-world problems by imitation of the genetic processes described above. To do this, GA use, because they are a kind of EC algorithm, a population of individuals where each of these represents a feasible solution (normally numerical) to the problem. Every individual is scored with a value that provides information about the quality of the corresponding solution. The higher is this value, the better is the adaptation to the problem of this individual and, therefore, the higher is the probability of survive and reproduce itself by combining his genes with other individual similarly selected. In such a way, a new population (set of solutions), which replace the last one, is generated. Furthermore, it is expected that good features of the previous population are preserved and the poor ones are improved, thus producing a mean quality of this new population better than that of the previous one. If the GA is well designed and implemented, convergence to a near-optimal solution can be ensured.

In order to computationally implement the ideas described above, a data structure and some procedures must be developed. The data structure refers to the adaptation of the problem, i.e., the numerical representation of the individuals, commonly called codification, and the real-valued space to score them, which is called fitness function. On the other hand, the procedures in GA are commonly known as operators and they are basically three: selection, crossover and mutation. Additionally, other specific functions can be implemented to improve the convergence of the algorithm.

There has been a variety of data structures and procedures proposed over the years [64]. Each set of these characterizes a particular GA. In this thesis we use a kind of canonical GA [60], whose general pseudo-code is shown in Fig. 4.3. In general sense, for a canonical GA, first, a population of individuals is generated by some means (e.g., randomly); then, the

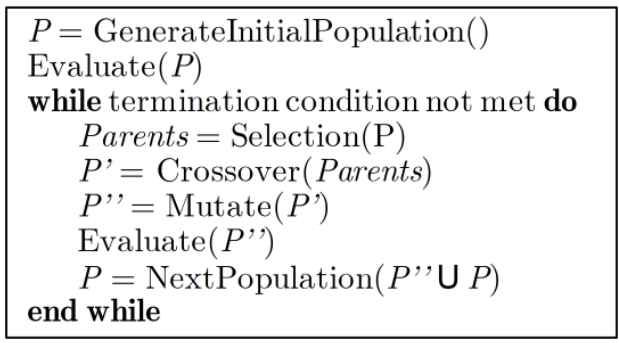

Fig. 4.3 Canonical algorithm for GA. 
quality of each individual is evaluated by means of a fitness function, which assigns a real value to the particular solution. These compose the initialization of the algorithm. After this initialization, the GA repeatedly modify the population of individuals until a stipulated condition is satisfied. At each step, the GA select the individuals, by means of a selection operator, among the current population to be parents and uses them to produce the individuals (called children) of the new population. For this, GA apply the operators of crossover and mutation. Once a new population is generated, the quality of each individual from it is evaluated. Finally, the next population is obtained by choosing a set of individuals among the union between the current population and the new one. In the GA terminology each of these iterations is called generation.

Due to the wide options present in the current literature, in the following we only describe the specific ones implemented in this work.

\section{Problem adaptation}

The first point in the problem adaptation is the numerical representation of an individual. As stated before, for GA this concept is referred as codification. There are several ways to represent an individual in GA and it often strongly depends on the problem characteristics and the corresponding search space. The most usual codification of an individual is as a set of parameters, where each of these can be binary values (called codification with a binary alphabet $\{1,0\}$ ), or integer numbers (also called codification with a real-integer valued alphabet $\left\{\mathbb{N}^{+}\right\}$). A general structure of an individual is shown in the following equation:

$$
y=\left[\begin{array}{llll}
p_{1} & p_{2} & \ldots & p_{I L}
\end{array}\right]_{1 \times I L}
$$

where each parameter $p_{i}$ can take a value either from the binary alphabet of from the real-integer valued alphabet. Commonly, for a given problem only one kind of structure and alphabet is used, i.e., all the individuals in the problem take the form of (4.1) and all their parameters take values from the same alphabet. In the GA terminology, each parameter $p_{i}$ is known as gene and the corresponding set of these (vector $y$ ) as chromosome. Moreover, when a particular chromosome takes values for every gene, this is referred as a genotype and the particular solution this genotype encode (e.g., the numerical value) is called phenotype [60]. These two last concepts are useful to make emphasis in the difference between the presentation of a solution and the solution itself.

The length $I L$ of an individual can be set as fixed or variable, depending on the particular problem. For the application dealt with in this thesis, that length can be both, fixed, in which case it will be the same for all individuals, and variable, in which case it become as another parameter to 
be optimized. Moreover, the values that an individual can take depend also on the problem constraints and parameter bounds, as naturally should be defined for any $\mathrm{CO}$ problem. To provide a more applied viewpoint, we refer the constraints as design requirements and restrictions, and the parameter bounds are simply the available points in the search space, which is a discrete set.

Regarding to the second point in problem adaptation is the concept of fitness function. The fitness function is a numerical function which assigns to each individual a real value that scores his quality regarding to the desired solution, i.e., $f: y \rightarrow \mathbb{R}^{+}$. This function is commonly designed for every specific problem and its inputs are mainly the genotypes, problem constraints and parameter (genes) bounds. This function should be carefully designed in such a way that it proportionally represents the actual quality of all individuals. Otherwise, a wrong design of this function can quickly lead the algorithm to poor-quality areas of the search space. In other words, the fitness function is the function to be minimized by the optimization algorithm.

\section{GA Operators}

As shown in Fig. 4.3, the main operators for GA are selection, crossover and mutation. These operators use individuals to produce a new population at each generation. Basically, first, the selection operator selects some individuals to be the parents, then, the crossover operator combines pair of parents to produce children for the new population, after that, the mutation operator changes some genes of individual parents to create other children. Finally, a next generation is obtained by selecting a certain number of individuals among the union set between the current population and the new population (i.e., the children). This final selection of the next population depends on a particular strategy which can be composed by other additional process. Among these processes we particularly use the socalled elitist strategy [60], [64], which is described later.

The selection operator selects a group of individuals in the current population to be parents and who contribute their genes to the children of the new population. Usually, the individuals with high fitness function values have high probability to be chosen as parents. However, different selection operators can be implemented, among those are [60], [64] stochastic uniform, remainder, uniform, roulette, tournament or even a combination of these. For the application dealt with in this thesis we use the stochastic uniform selection operator [60], [64], which is described below.

The stochastic uniform operator selects individuals proportionally to their fitness values but in a stochastic sense. To easily describe this operator, it is useful to make use of a circumference. Assume a 


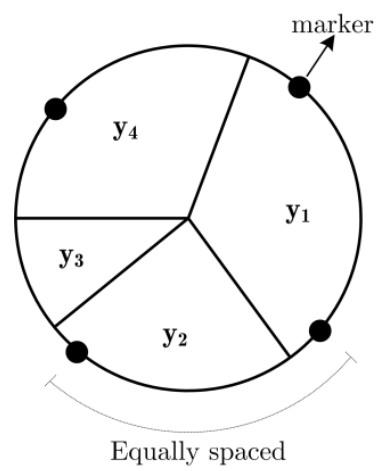

Fig. 4.4 Stochastic uniform selection operator.

circumference is divided into as many portions as individuals compose the population, where each of these portions represent an individual and its size is proportional to the inverse (the inverse because we are dealing with minimization problems) of the fitness value of the individual it represents. This operator moves a marker along the circumference with steps of equal size and for each movement the operator selects, as parent, the individual corresponding to the portion of the circumference where the marker lies. This movement is performed once per parent. The first point where the marker starts is a number, less than the step size, coming from a uniform random distribution. The number of steps (i.e., the number of parents) depends on the predefined probability of crossover. An illustration of this operator for a population of four individuals and a number of four parents is shown in Fig. 4.4. In this figure can be observed that individuals $y_{1}$ (twice), $y_{2}$ and $y_{4}$ are the ones selected as parents.

The crossover operator combines pair of parents, previously selected, to create children for the new population. This operator basically combines the genes of the parents and the procedure for performing this defines the kind of crossover operator used. Additionally, this operator is not applied to all the selected parents but it depends on a certain predefined probability (probability of crossover). Among different crossover operators are [60], [64] scattered, single point, two points, intermediate, heuristic or arithmetic. For the application dealt with in this thesis we use the intermediate crossover operator [60], [64], which is described below.

The intermediate crossover operator creates children from a weighted sum of the corresponding pair of parents. It can be expressed as follows:

$$
\text { child }=\operatorname{round}\left(y_{1}+a \times \text { ratio } \times\left(y_{2}-y_{1}\right)\right)
$$

where $a$ is a random number coming from a standard uniform distribution, ratio is a predefined control variable lying in the interval $[0,1]$, round is 
the round operator (it approximates the value to the nearest integer value) and, $y_{1}$ and $y_{2}$ are the corresponding parents.

The mutation operator changes some genes of some individual parents to create other children. This operator is not applied to all parents but it depends on a certain predefined probability. Among different mutation operators are [60], [64] Gaussian, uniform or adaptive feasible. The use of a mutation operator helps to avoid the problem of premature convergence, providing to all the points in the search space to be explored a probability higher than zero. For the application dealt with in this thesis we use the uniform mutation operator, which is described below.

The uniform mutation operator is a two step operator. First, the operator selects probabilistically a fraction from the individual for mutation, where each of these has the same probability of being mutated and which is previously defined. Then, the fraction is changed by a random number uniformly selected from the corresponding range for individual parameters (i.e., the parameter bounds).

For obtaining the next generation, a steady-state scheme is used [43], [64-65]. It transfers individuals of the current population to the next one. In this sense, the next population is formed by a certain number of individuals from the current population and the remaining ones from the children. From the current population are taken the $E_{c}$ individuals with the best fitness values and the remaining ones are taken as the $I L-E_{c}$ children with the best fitness values. The particular strategy of preserving the best $E_{c}$ individuals from the current population is known as the elitist strategy. The particular values of these variables are described and discussed later.

Finally, another important aspect when applying GA, or any metaheuristic optimization method, is the way to deal with infeasible solutions. This kind of solutions can appear due to the application of crossover or mutation operators and they can be, basically, of two forms. The first one is related to those solutions with components (parameters of individuals) lying out of the parameter bounds, whilst the second one appears when the solutions do not meet the constraints. The first kind of infeasible solution does not appear in this application because of the discrete-closed nature of the search space. However, it is possible to obtain infeasible solutions of the second kind. There are mainly three ways to deal with this kind of solutions [65]. The simplest action is to reject the infeasible solutions, but obtaining feasible solutions is difficult and it can lead the algorithm to very long-term convergence (i.e., divergence in practical terms). Another option is to penalize this solutions when computing the fitness function, which is a more appropriate option, even in some cases unavoidable [43]. The last option is try to repair the solution. However, this solution can be computationally expensive. In this thesis we 
use the option of penalizing the solutions in the fitness function. The way to penalize these solutions is described later. 


\section{General Procedures for Optimizing the Multilateration Layout}

In this chapter we describe the proposed general procedures for designing MLAT systems. Herein, we merge the concepts described in Chapter 3 and Chapter 4 to set up the general procedures. We first convert and express the MLAT system design problem into a combinatorial problem, performing also the corresponding complexity analysis of solving this problem in a computer. In this part, we also demonstrate that there is no deterministic algorithm (or procedure) for solving the MLAT design problem in polynomial time and, hence, that the most near optimal solution is by implementing a metaheuristic optimization algorithm. Then, the proposed general design procedure is fully described in $\S 5.3$, whilst the corresponding proposed design strategies are in $\S 5.4$. Finally, each of the developed strategies is tested over the Barcelona - El Prat (Barcelona, Spain) airport layout. Three different simulations and their convergence analysis are also provided. The contributions described in this chapter have been published in [20], [36], [66-68].

\subsection{The MLAT System Design as a Combinatorial Optimization Problem}

We have seen in a previous chapter what must be done and taken into account to perform the design of a MLAT system. Moreover, the different procedures or solutions which have been proposed in the literature, along with the models used to simulate the system performance, have been also briefly discussed. Within these chapters, we discussed the design of a MLAT system as guided by three main elements, namely, the system requirements, the restrictions and the resources. Likewise, the main goal of any design was stated as achieving the maximum compliance with the requirements, by using the fewest amount of resources, and satisfying all the restrictions as depicted in Fig. 5.1. 


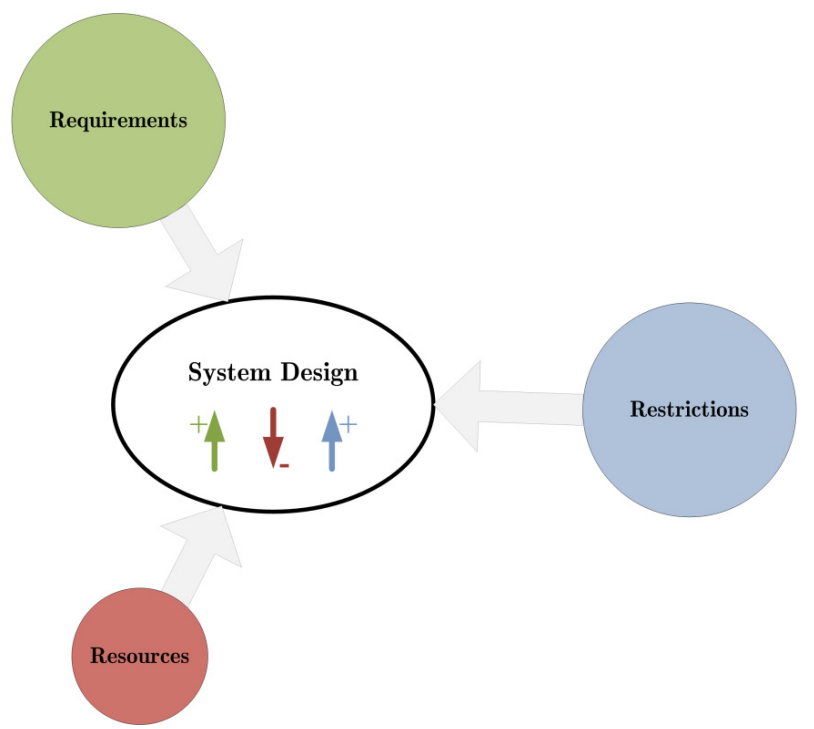

Fig. 5.1 System design elements.

As we have also stated before, the requirements are mainly based on the system performance parameters (see §3.1.1), the restrictions can be those imposed by regulatory bodies, by the nature of the scenario, etc., whereas the resources, which can be economic, logistics or time, are all directly related with the number of the stations, i.e., the smaller the number of these the fewer the amount of resources that must be spent. Therefore, in this way, it is clear to understand that the part of the design goal related with the use of the possible fewest amount of resources is directly connected with the use of the minimum possible number of stations. Similarly, the requirements and restrictions compliance is directly related with the stations position, i.e., the system geometry (see $§ 3.1 .2$ and §3.1.3). Nevertheless, in general, choosing the number of stations and their locations, to fully meet the design goal, is not an obvious task and the system designers have to make several attempts, by trial and error, before obtaining a satisfactory spatial distribution of the stations. As a matter of fact, we should remember that to perform this design, multiple factors (i.e., the design parameters) must be taken into account, and besides they are not one-to-one related with the requirements (i.e., the performance parameters) neither with the restrictions, but through several nonlinear mathematical models.

With the optimization theory, provided in Chapter 4, in hands, it can be seen that the whole design process can be automatically and accurately performed if all the aspects listed above are comprised into an optimization problem, whose general objective is to find the minimum number of stations 
and their locations, which satisfy all the requirements and restrictions. Such an optimization problem, as it is described in $\S 4.1$, can be of two forms, namely, a continuous optimization problem or a $\mathrm{CO}$ problem. The difference between these kinds of problems basically lies on the characteristics of the search space. In this work, we propose stating the MLAT system design as a CO problem. Moreover, once the MLAT system design is stated as a $\mathrm{CO}$ problem, it must be mapped to the context of some optimization algorithm in order to obtain a solution. In the next subsection, the full adaption of the MLAT design to a CO problem is described; essentially, each of the particularities of the MLAT design problem is fitted to the corresponding components of a CO problem.

\subsubsection{Mapping the MLAT System Design to a CO Problem}

As described in $\S 4.1$, a CO problem is composed of a set of variables, of variable domains, of constraints among variables, a goal function and a search space. Thus, the key idea when solving a CO problem consists in assigning to each problem variable a value, which lies within the corresponding variables domain and which satisfies the constraints among them, in such a way that the goal function, applied to the set of these assigned values (i.e., the problem solution), is minimized.

It is important to remember that, as we discussed in $\S 3.1$, the requirements for the design of MLAT systems, which are basically the system performance parameters, must be entered into the design process by means of the design parameters, because the last can be theoretically modelled. Therefore, the design parameters are those taken into account for fitting the MLAT design into a CO problem. The relation among them has already been described in $\S 3.1 .3$.

In the following, the description of every $\mathrm{CO}$ problem component, focused on the MLAT system design, is provided. In other words, and more specific to the optimization context, the instance $I$, as defined in $\S 4.1$, is set for the MLAT system design problems.

\section{Problem Variables}

The problem variables are defined, in a general sense, as the number of stations and their positions, and they can be represented by $X=$ $\left\{N_{s}, x_{1}, \ldots, x_{N_{S}}\right\}_{N_{s}+1}$, where $N_{s}$ is the estimated number of stations and $x_{i}$ the estimated position for the $i$ th station. The particular structure that takes the stations position depends on the characteristics of the search space; this aspect is discussed later.

This representation for the problem variables is the most general because, as it will be shown later, we propose several kinds of problem designs where the number of stations $N_{S}$ can be a fixed input parameter or 
being part of the set of variables $X$. Moreover, it can be also seen that for the problems dealt with in this thesis, the size of set $X$ can be fixed or changeable. These particularities are analyzed later on.

\section{Search Space}

The search space is composed of all possible sites where a receiving station can be emplaced, i.e., the search space contains all the possible values that can be assigned to the problem variables.

The definition of this space can be of two forms, continuous or discrete, and depending on this feature we deal with different optimization problems (see §4.1). For a given scenario, we can define the search space as the whole surveillance volume referenced in any geographical reference system. In this case, and assuming for clarity a Cartesian reference system, the values that can be assigned to the problem variables (regardless of the number of stations $N_{S}$ ) is any subset of coordinates $(x, y, z)$ lying within the predefined volume. So, it is clear to see for this case that, every $x_{i}$ is really a vector of three real-valued components and, therefore, the search space is defined in a continuous form. On the other hand, if we define the search space as a finite set of numbered sites, we are dealing with a discrete search space.

Throughout the development of this thesis, we have proposed the definition of the search space with both forms, continuous and discrete. We have found better results (in terms of convergence and in terms of closeness to the real/operational case) by implementing a discrete search space. For this reason, this is the only one presented in this thesis. However, for the interested reader, the results for the continuous search space can be found in $[36]$.

The discrete search space that we have defined is denoted by $\mathcal{S}$, whose size (i.e., the number of predefined sites) is $\ell_{\mathcal{S}}$, where $\ell_{\mathcal{S}}$ is such that $N_{s}<\ell_{\mathcal{S}}$. In this search space every site is numbered, regardless of any priority, from 1 to $\ell_{\mathcal{S}}$. Moreover, the use of this discrete search space allows us to implicitly take into account some practical limitations in the scenario as power supply or sites availability, and enabling also to obtain more realistic design as only the actually available sites are used. On the contrary, with the continuous search space some additional routines have to be implemented to avoid emplacing the stations in non-realistic sites.

Finally, a second search space must be used in case of implementing the optimization of the number of stations. This search space is, by definition, discrete and is denoted as $\mathcal{R}_{S}$. It is composed by integer values, lying within certain range denoted by $\mathcal{R}_{s}=\left[N_{s_{1}}, N_{s_{2}}\right]$, where $N_{s_{2}}>N_{s_{1}}$. The selection of both values is commonly intuitively guided by the experience of the designer, although the first value $N_{s_{1}}$ directly depends on the localization scheme and must be $N_{s_{1}} \geq 3$ (see $\S 2.1 .2$ ). 


\section{Variable Domains}

The variable domains define the range of values that can be assigned to each of the problem variables in $X$. In this work, all the values belonging to the search space can be assigned to every problem variable, so that the set of domains as defined in $\S 4.1$ is, for this case, only one and is denoted by $D_{\mathcal{S}}$.

From the theoretical fundamentals of MLAT systems, it can be understood that a station should not be emplaced in the same site than another one. Therefore, it can be raise the question about why do not set different domains for the problem variables to avoid that situation. This is, of course, a correct solution but maybe not the most efficient as a manual pre-processing should be performed to define the individual domains, and precisely it is the kind of steps which we are trying to avoid in this thesis part. Nevertheless, we propose to control that situation, and other ones which will be commented later, by means of the constraints imposed to the problem variables.

\section{Variables Constraints}

The variable constraints introduce the design restrictions into the $\mathrm{CO}$ problem and thus take them into account during the optimized process design. Really, we refer as restrictions in a more practical context and as constraints in a more numerical/optimization context. There are mainly three kinds of constraints, namely, those due to the scenario layout, those that are conceptual, and those that are due to the regulatory bodies.

Within the constraints due to the scenario layout we can find some forbidden areas where no stations can be emplaced, e.g., a runway or apron areas. Due to the nature of our proposed search space this kind of constraints are incorporated from the definition of it.

The conceptual constraints come from the knowledge of the MLAT systems theory, particularly of the DOP and its influence on the theoretical system accuracy. Among these are: a minimum spatial separation between any pair of receiving stations, and that one due to the fact that no station can be emplaced in the same site than another one. Although the second constraint can be seen as a particular case of the first one, we treat them separately due to the different influence level they have on the DOP. These constraints are implemented on the fitness function, which is defined later.

The last class of constraints are those imposed by the regulatory bodies, which particularly for the design and deployment of MLAT systems are described in [1-2]. Specifically, the most important restriction coming from these documents is the one related with the requirements of SPoD and availability, and the design parameter of redundancy, which is the minimum number of stations that must cover with LoS every point of the 
surveillance volume (see $§ 3.1 .2$ and $§ 3.1 .3$ ). This constraint is defined as LoS redundancy.

\section{Fitness Function}

The fitness function assigns a numerical and suitable score to the system design, thus quantifying it in respect of the requirements, restrictions (or constraints) and the amount of used resources. In other words, the fitness function is the numerical way to introduce into the optimization problem the requirements (which in this step are the design parameters as stated before), the restrictions (which are the constraints), and the amount of resources used in the design (which, as we have stated, it corresponds to the number of stations). The definition of every component of the $\mathrm{CO}$ problem is crucial to obtain a suitable performance and to ensure convergence when implementing an optimization algorithm to solve it. Therefore, the definition of the fitness function is considered as the most critical point in this process, because it is the only problem component that inform to the optimization algorithm about the quality of the solutions that it is finding. So, it is clear that the score provided by this function must truly reflect the closeness of the solution to the compliance of the requirements and restrictions.

The fitness function is specific to each kind of design but, in a general sense, the function that we propose in this work is a weighted sum that takes the following form:

$$
f(s)=1-\sum_{i=1}^{\text {cond }} \delta w_{i} c_{i} ; \quad \delta=\left\{\begin{aligned}
1, & c_{i}: \text { requirement } \\
-1, & c_{i}: \text { constraint }
\end{aligned}\right.
$$

where cond is the total number of requirements and constraint, $s=$ $\left\{\left(x_{1}, v_{1}\right), \ldots,\left(x_{n}, v_{n}\right) \mid v_{i} \in D_{i}\right\}$ is the particular solution to the CO problem, $c_{i}$ is the normalized cost of satisfying the $i$ th requirement or constraint, and $w_{i}$ is a normalized weight factor that controls the relevance of $c_{i}$ on the design problem. The corresponding values of $w_{i}$ and the functions to obtain the different $c_{i}$, for each application considered in this thesis, are described later on.

\subsection{Complexity Analysis of the MLAT Design Problem}

Once mapped the MLAT system design into a $\mathrm{CO}$ problem and before providing the description of the general procedure for solving it, an important aspect that must be analyzed is the complexity of the underlying optimization problem. As we have described in §4.1.1, two important 
aspects should be analyzed for understanding the complexity of the problem, namely: the relation between the fitness function and constraints (the latter being included in the first one) with the problem variables, and the aspect related with the required processing resources (i.e., the number of operations) for solving the problem. These two points are briefly analyzed in the following.

The relation between the fitness function and the problem variables can be understood by analyzing the expression in (5.1) and each of its components. From (5.1) we can see that the fitness function is not directly related with the problem variables (i.e., the number of stations and their locations) but through the different cost functions $c_{i}$ that are used. There are several cost functions (see $\$ 5.4$ ) that are used in this thesis for solving the different system design strategies. However, to define this kind of complexity, it is enough to analyze those functions which have the highest order of relation with the problem variables. This cost functions are those related with the system accuracy and with the SPoD. For the system accuracy, we have seen in $\S 2.1 .2$ that the relation between this and the stations position is highly non linear and also spatial-variant, whereas the $\mathrm{SPoD}$ is only related with the number of stations but also with a highly non linear relation (cf. (5.8) later on). In this sense, it is clear to conclude that the MLAT system design as a $\mathrm{CO}$ problem belongs to the nonlinear programming problems.

Regarding the amount of processing resources that must be used for solving the MLAT design problem, it is advisable to previously analyze the essential of this as a CO problem. For the sake of simplicity, we first analyze the case of optimizing only the stations position. So, this problem consists of selecting a subset of $N_{S}$ values (stations position) from a set of $\ell_{\mathcal{S}}$, regardless of the order of this and without the possibility to have two or more identical values. In this sense, the total number of possibilities, which we have to form the subset of $N_{s}$ values, is given by the following combinatorial expression:

$$
C_{1}\left(\ell_{S}, N_{s}\right) \equiv\left(\begin{array}{c}
\ell_{S} \\
N_{S}
\end{array}\right)=\frac{\ell_{S} !}{\left(\ell_{S}-N_{S}\right) ! N_{s} !}
$$

where, from the definition of the factorial, it can be understood that $N_{s}$ must be such that $0 \leq N_{s} \leq \ell_{\mathcal{S}}$. It is also in agreement with the real situation because it makes no sense to have more stations to emplace than available sites.

In other words, (5.2) provides the number of possible combinations (i.e., the number of possible solutions) that can be formed from the discrete search space $\mathcal{S}$, given its size $\ell_{\mathcal{S}}$ and the number of stations to be deployed $N_{s}$. 
So far, no deterministic method, which finds the best solution, has been proposed for solving the MLAT design problem under all the requirements and restrictions. Then, in this case, such a deterministic algorithm capable of finding the best solution is the "brute-force" algorithm; i.e., by testing all the possibilities and saving the best one. Therefore, it means that a total of $C_{1}$ operations must be performed to obtain the best subset from $\mathcal{S}$, which satisfies all the requirements and restrictions. In this sense, it can be seen that the complexity of the problem, in terms of number of operations, depends on the behaviour of $C_{1}$, which at the same time depends on two different inputs: $\ell_{\mathcal{S}}$ and $N_{s}$. The latter is an important difference between this optimization problem and other ones well studied and presented in the literature, because these last commonly depend only on one input parameter. Thus, we have to analyze the behaviour of (5.2) in respect of each of these inputs, in order to characterize the problem complexity.

A first idea about the behaviour of $C_{1}$ can be obtained by plotting it in respect of one of its parameters, setting the other one at a fixed value, and vice versa. This analysis is shown in Fig. 5.2 (the vertical axes are in logarithmic scale). In part (a) of this figure, $C_{1}$ is plotted as a function of $N_{S}$ for several fixed values of $\ell_{\mathcal{S}}$, whereas in part (b), $C_{1}$ is plotted as a function of $\ell_{S}$ for several fixed values of $N_{S}$. In part (a), it can be observed that, for every fixed value of $\ell_{\mathcal{S}}, C_{1}$ is symmetric in respect of $N_{S}$, and it increases non-linearly in the left part, then it reaches a maximum value and, after that, it decreases also non-linearly. This analysis suggests that the problem complexity, in respect of $N_{s}$, increases for some values of $N_{s}$ (i.e., for $N_{s}<\ell_{s} / 2$ ) and, then, it decreases for the remaining values that this parameter can take (i.e., for $\ell_{\mathcal{S}} / 2<N_{s}<\ell_{\mathcal{S}}$ ). However, some remarks about this initial conclusion should be done before proceeding with this analysis. The first one is related with the fact that this graphical analysis only shows the dependency in respect of one input parameter and not in respect of both, which is most general. The second one is related with the real, operative situation and is that it is not common to have approximately the same values of $N_{s}$ than $\ell_{\mathcal{S}}$, therefore, it is more common working within the left side of each of the curves in part (a) of Fig. 5.2. On the other hand, in part (b) of Fig. 5.2, it can be observed that $C_{1}$, as a function of $\ell_{\mathcal{S}}$, increases non-linearly for the entire domain and for all values of $N_{s}$. Then, from this first analysis, we can conclude that the MLAT design as a $\mathrm{CO}$ problem require an amount of computational resources that vary non-linearly with the size of the input parameters, i.e., either $N_{\mathcal{S}}$ or $\ell_{\mathcal{S}}$.

The graphical analysis performed above is useful to obtain a first idea about the problem complexity and the number of fitness function evaluations that must be run in case of solving the problem by means of the "brute-force" algorithm. However, it is not enough to fully characterize the 


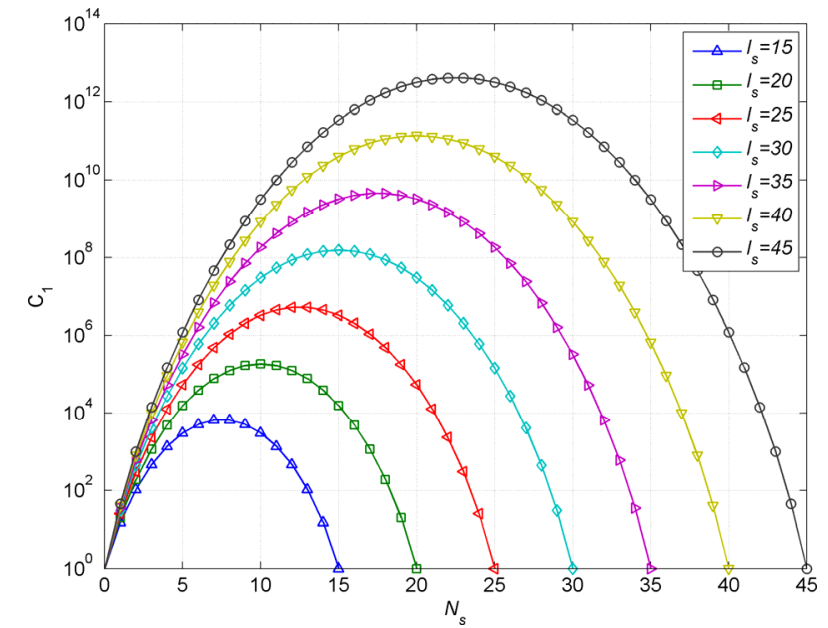

(a) Number of solutions in respect of the number of required stations.

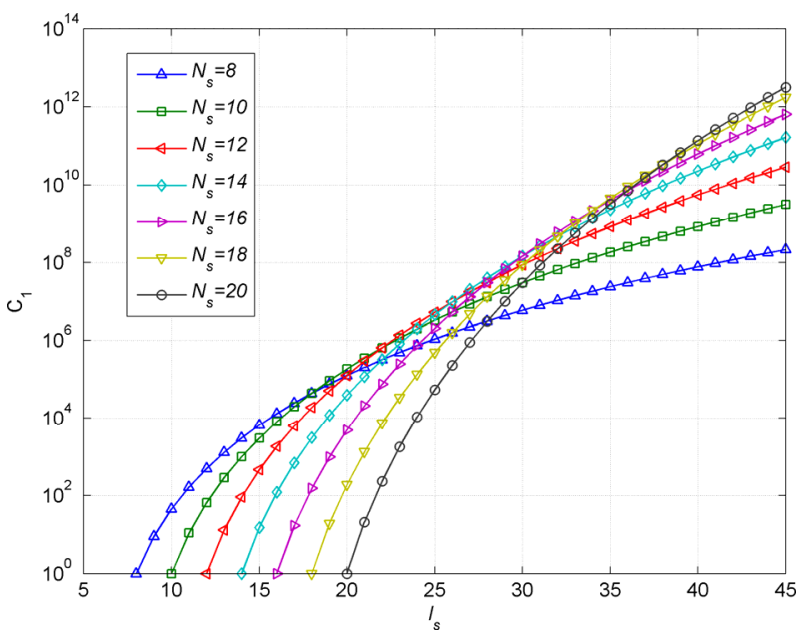

(b) Number of solutions in respect of the search space size.

Fig. 5.2 Number of possible solutions in respect of the search space size and of the number of required stations. The vertical scales are logarithmic.

problem complexity. Then, in the following, a more rigorous analysis of (5.2) is described. It consists in expressing $C_{1}$ as a function of only one parameter that comprises both $\ell_{s}$ and $N_{s}$, and then obtain an expression that explicitly shows the relation between $C_{1}$ and the input variables size.

Due to the fact that $N_{s}<\ell_{\mathcal{S}}$, we can assume that both input parameters, for the most of real, operative situations are related by a proportional constant such that $\ell_{\mathcal{S}}=a N_{s}$, where $a>1$, or equivalently 
$N_{s}=b \ell_{\mathcal{S}}$, where $b=1 / a$ and it is $0<b<1$. In this sense, $C_{1}$ can be expressed as a function of only one input parameter as follows:

$$
C_{1}\left(\ell_{\delta}\right)=\frac{\ell_{\delta} !}{\left(\ell_{\delta}-b \ell_{\delta}\right) !\left(b \ell_{\delta}\right) !}
$$

or

$$
C_{1}\left(N_{s}\right)=\frac{\left(a N_{s}\right) !}{\left(a N_{s}-N_{s}\right) ! N_{s} !}
$$

To easily manipulate the equations above, it is more practical to express them by using the Stirling's approximation [69] for factorials, which for convenience is written herein as:

$$
n ! \approx \sqrt{2 \pi n}\left(\frac{n}{e}\right)^{n}
$$

where $e$ is the Euler number.

Then, it can be demonstrated (see Annex A for the full demonstration) that, after few manipulations, (5.3) and (5.4) can be expressed, respectively, as follows:

$$
C_{1}\left(\ell_{\mathcal{S}}\right) \approx \sqrt{\frac{1}{2 \pi(1-b) b \ell_{\mathcal{S}}}}\left(\frac{1}{1-b}\right)^{(1-b) \ell_{\mathcal{S}}}\left(\frac{1}{b}\right)^{b \ell_{\mathcal{S}}} ; \quad 0<b<1
$$

and

$$
C_{1}\left(N_{s}\right) \approx \sqrt{\frac{a}{2 \pi(a-1) N_{s}}}\left(\frac{1}{a-1}\right)^{(a-1) N_{s}}(a)^{a N_{s}} ; \quad a>1
$$

From these expressions, it is explicit that, for both cases (5.5) and (5.6), the amount of operations that must be performed, by the "brute-force" algorithm, exponentially grows with the size of the input parameters, either $\ell_{s}$ or $N_{s}$. For a clearer interpretation, (5.5) is plotted in Fig. 5.3, for several values of the proportional constant $b$. It can be observed that the problem complexity posses a symmetric behaviour with the constant $b$, whose minimum values are obtained for $b=0.1$ and $b=0.9$, and the maximum is at $b=0.5$. However, in any case, the required number of operations is significantly large, and much more if we consider that these operations are matrix multiplications and inversions for a number of points belonging to spatial grid. It is worth saying that (5.5) and (5.6) are equivalent because every input parameter comprises the other one by means of the relations described above. Therefore, the complexity analysis can be considered complete by inspecting only some of these two expressions. 


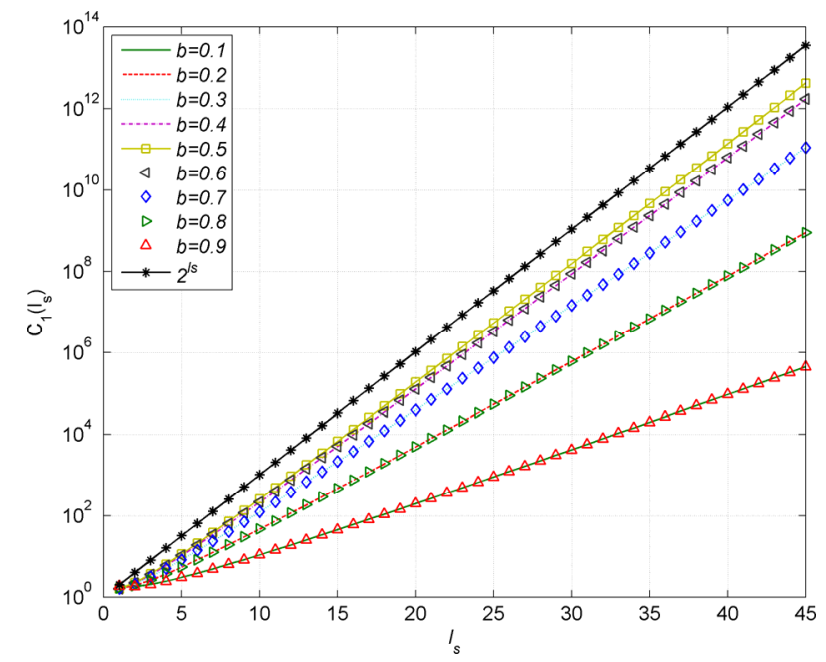

Fig. 5.3 Joint analysis of the problem complexity.

In (5.5) and (5.6), it can be also seen that $C_{1}$ is damped by a variable of $\mathcal{O}\left(\ell_{S}{ }^{-\frac{1}{2}}\right)$ and $\mathcal{O}\left(N_{s}{ }^{-\frac{1}{2}}\right)$, respectively. However, it can be proved that the effect of this damped variable is negligible in practical terms. For example, it can be easily demonstrated that it reaches its minimum value at $b=0.5$, in which case (5.5) takes the following form:

$$
C_{1}\left(\ell_{\mathcal{S}}\right)=\sqrt{\frac{2}{\pi \ell_{\mathcal{S}}}} 2^{\ell_{\mathcal{S}}}
$$

where the damped variable is clearly identified as the factor $\sqrt{2} / \pi \ell_{s}$. For a quick and practical comparison, this particular case is also plotted in Fig. 5.3 (black asterisks) with the damped factor suppressed. It is clear that, regardless of the absolute values, the damped version of $C_{1}\left(\ell_{\delta}\right)$ follows the same tendency of the undamped version.

Finally, remembering the concepts set in $\S 4.1 .1$, now we can easily conclude that the MLAT design CO problem belongs to the class NP problems. On the other hand, the classification of the problem within one of the classes derived from class NP requires a wider research that is out of the scope of this thesis, and it does not provide of significant improvement regarding the objectives of this thesis.

Regarding to the MLAT design problem consisting in the optimization of both the number of stations and their locations, the total of possibilities we have to form the solution is given by the following expression: 


$$
C_{2}\left(\ell_{\mathcal{S}}, N_{S}\right)=\sum_{i=N_{S_{1}}}^{N_{S_{2}}} \frac{\ell_{\mathcal{S}} !}{\left(\ell_{\mathcal{S}}-i\right) ! i !}
$$

where $N_{s_{1}}$ and $N_{s_{2}}$ are the boundaries of the second search space $\mathcal{R}_{s}$, previously defined.

It is easy to conclude, without any more analysis, that the problem $C_{2}$ is as least as hard as the first one $C_{1}$.

\subsection{Description of the Overall Design Procedure}

The general design procedure we have developed in this thesis is aimed at the designing of standard MLAT systems (e.g., with only TOA/TDOA receiving stations) or of its improved version (e.g., with the combination of TDOA/RTD or TDOA/AOA receiving stations). In this sense, the system design is obtained by calculating the minimum number of stations and their locations (stations sites) that maximize the LoS coverage, the system redundancy and the theoretical system accuracy. As the system redundancy is directly related with the LoS coverage, henceforth, unless otherwise stated, we will merge these two concepts into the LoS coverage one.

The calculations in this procedure are performed on the $\mathrm{CO}$ problem previously defined. Nevertheless, as we have commented before, the CO problem must be mapped into the context of some optimization algorithm, which for this case is the GA, in order to obtain the final solution. Through this section, we treat the optimization algorithm as a black box that receive some information and provide a modified version of it, then, in the next section, we fully describe every proposed design strategy along with the corresponding mapping to the GA context.

\subsubsection{General Framework}

The general idea used for this procedure is based on the integration, into an iterative routine, of different information and several numerical tools, in order to obtain a suitable MLAT system design (i.e., the number of stations and their locations), which efficiently satisfy the imposed requirements and restrictions. This general idea is illustrated in Fig. 5.4. In the following lines, this general idea is described.

As we can observe from Fig. 5.4, the general framework proposed in this thesis is composed by a core (i.e., the optimization algorithm), some inputs of different kinds, some numerical tools required to perform additional and necessaries calculations, and some outputs. In this figure, there are some black and white boxes and other ones that are coloured. The coloured ones 


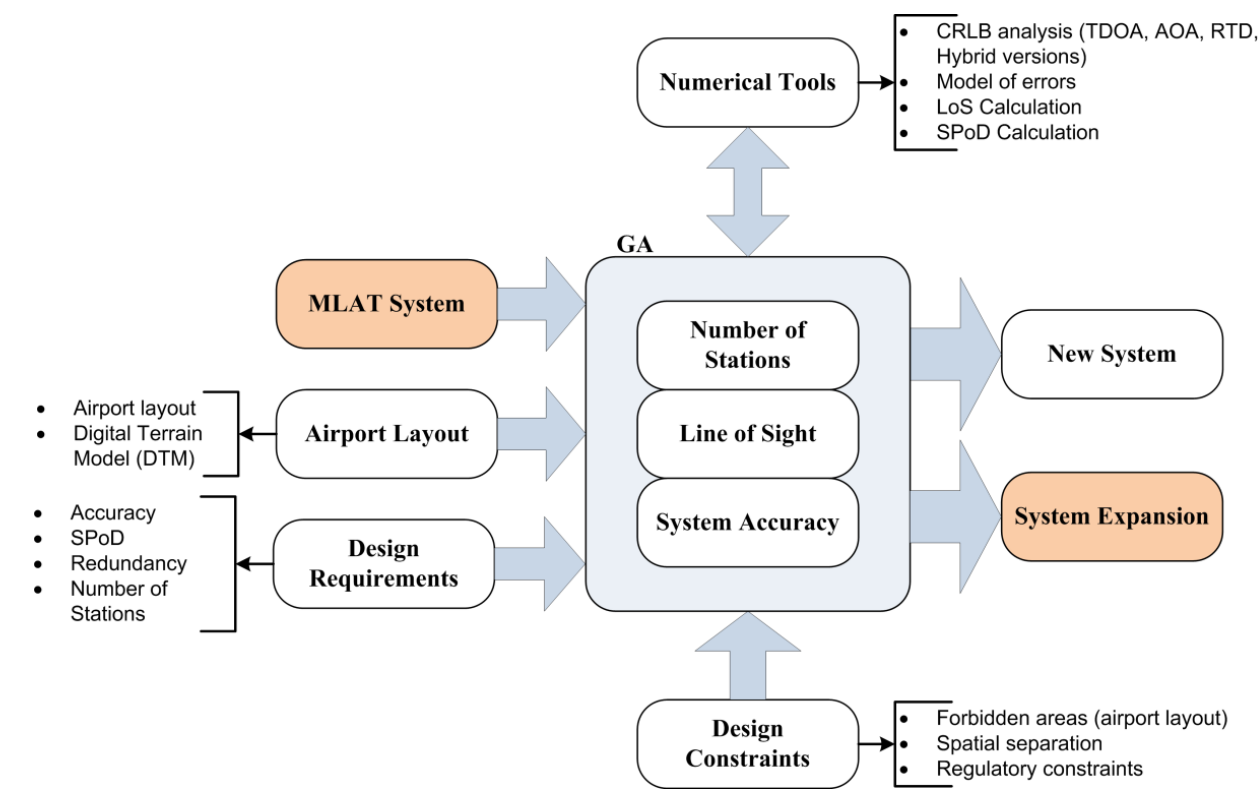

Fig. 5.4 General framework for designing MLAT systems.

are additional parts which are used to obtain possible systems validation or expansions. Firstly, we will explain the general framework based on the black and white boxes and then, the corresponding functionality that makes use of the coloured ones.

\section{Inputs}

The inputs are composed of the information about the scenario characteristics, specifically the airport layout, the design requirements and the design constraints.

The airport layout is basically composed by any numerical data that describes the airport buildings, the runways, stand and apron areas, etc. In this thesis, we use a general Data Exchange Format (DXF) file from Autodesk $^{\odot}$, which contains this information in form of polylines. Moreover, it is required information about the terrain model, i.e., basically the information about the terrain elevation or heights. For this thesis we use the standard Digital Terrain Model (DTM), which provides numerical information for the height of the terrain over a local reference frame, organized in matrix form, where each matrix position represents the height (commonly in meters) for a spatial grid with certain resolution.

The requirements, as we have commented before, are introduced into the framework by translating them into the system design parameters. This information is provided numerically and has been fully described in Chapter 
3. Within this information are the lower bound(s) for the theoretical system accuracy, the SPoD and redundancy, and the number of stations to be emplaced or the range of them, as defined in \$5.1.1, depending on the particular design goal.

The design constraints also have to be provided numerically, and as it was stated in $\S 5.1 .1$, they are mainly the coordinates, over a local reference frame, of the forbidden areas, the minimum spatial separation among stations and the regulatory ones, as the redundancy for the LoS coverage (i.e., LoS redundancy).

\section{Numerical Tools}

Within the necessaries numerical tools are those for performing the calculations of the theoretical system accuracy, the error models or error balance, the system probability of detection and the LoS.

The numerical tools for performing the theoretical system accuracy and error balance calculations is that described in [20]. The LoS calculations are performed by a proprietary routine made by the research group where this thesis is developed. Basically, this tool makes use of the DTM and performs a ray trace analysis to detect if between two given points there are or not LoS. Finally, the SPoD calculation is described later on (see §5.3.2).

\section{Outputs}

The outputs of this procedure are the assigned values to the $\mathrm{CO}$ problem variables, i.e., the number of required receiving stations and their locations. The format of each of these outputs is as described in $\S 5.1 .1$.

\section{Framework Core}

As we have commented before, the core of this procedure is the optimization algorithm of GA. This algorithm takes all the inputs and iteratively seeks to minimize the number of stations, to maximize the LoS coverage and to maximize the theoretical system accuracy; the latter is equivalent to find the optimal spatial distribution of the stations. The particular GA used and its operators, adapted to this work, have been previously described in $\S 4.3 .2$.

\section{Additional Functionality}

The design procedure proposed in this thesis is not only useful to obtain MLAT designs from zero, but also to analyze and validate if any previous design is the most suitable option to satisfy the set of requirements and restrictions, with a given amount of resources, whether it could be improved by some feasible, but not obvious, position changes of the stations or, even, to satisfy the requirements and restrictions with a smaller number of them. 
Likewise, if the surveillance volume increases, this procedure is also useful to obtain the new system design based on the already deployed one. In both cases, this additional functionality requires an additional input that is basically the existing system design, which is denoted as $\boldsymbol{M L A T}$ system. On the other hand, the corresponding additional output to this additional functionality, which we denote as system expansion, can contain either the information about the validation of the system design, about the new system changes (e.g., the new positions of some stations), or even a complete new design that satisfies all the requirements and limitations more effectively than the one at the input.

\subsubsection{Iterative Numerical Procedure}

The iterative, numerical, procedure developed in this thesis to perform the optimization process of the MLAT systems design is shown in Fig. 5.5. This procedure consists of three steps, namely, Initialization (A), System Design Evaluation (B), and GA (C). In other words, this iterative procedure is the framework core described in the last subsection. In the following, these steps are fully described.

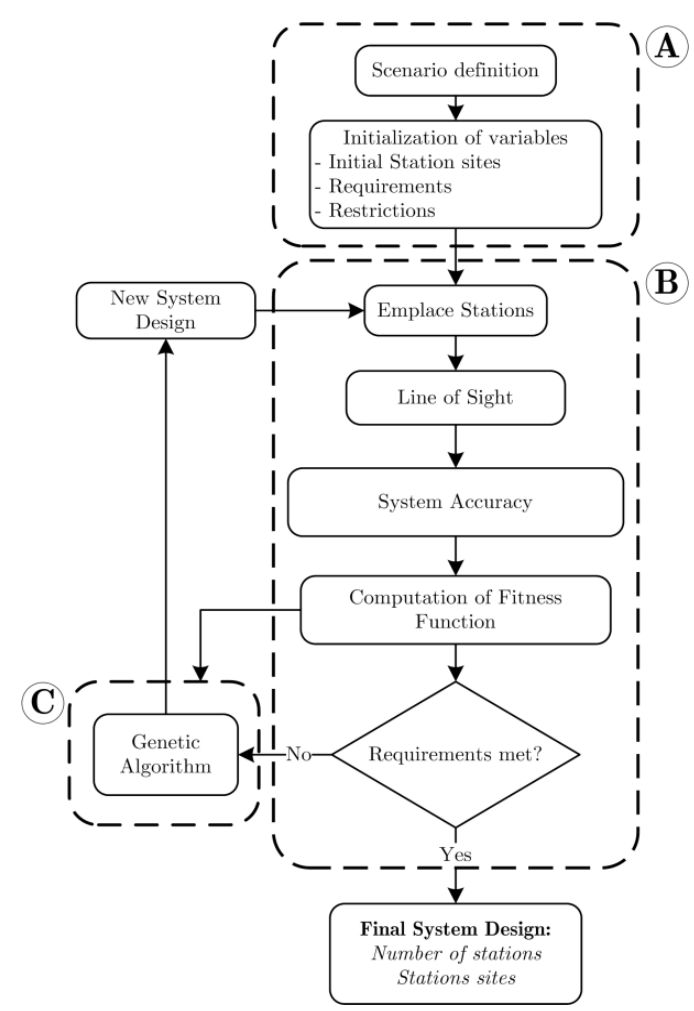

Fig. 5.5 Flowchart for the iterative procedure (the framework core). 


\section{Initialization}

In this step, all the problem characteristics are defined. In the scenario definition, the search spaces $\left(\mathcal{S}\right.$ and $\left.\mathcal{R}_{S}\right)$ of possible sites to emplace the stations and the one for the possible number of them are selected, and some areas of interest (areas to calculate the system parameters -basically LoS and the theoretical system accuracy) are defined. Then, the initial station sites (commonly by a random selection) and all the variables are initialized. The variables can be classified either as requirements or as restrictions. The requirements mainly are the number of stations to emplace (or the corresponding range defined by $\mathcal{R}_{s}$ ), the required system accuracy and the $\mathrm{SPoD}$. Of course, all these are input data to the problem. On the other hand, the restrictions mainly are the LoS redundancy, which is the minimum number of stations that must cover a point within the coverage area, in order to satisfy the requirements of $\mathrm{SPoD}$, and the minimum spatial separation between the $i$ th and $j$ th station, which is denoted as $\Delta_{i, j}$.

In this work, the restriction of LoS redundancy is calculated based on the manufacturer data about the probability of detection of each station. The $\mathrm{SPoD}$, for a given point $j$, can be calculated as follows:

$$
S P o D_{N_{s j}}=\sum_{k=0}^{N_{s j}-N_{s, \min }}\left(\frac{N_{s j} !}{\left(N_{s j}-k\right) ! k !}\right) \operatorname{PoD}_{\text {station }}^{N_{s j}-k}(1-P o D)^{k}
$$

where $P o D_{\text {station }}$ is the probability of detection of a single station (that should be provided by the manufacturer), $N_{s j}$ is the number of stations that cover the $j$ th point, and $N_{s, \min }$ is the minimum number of stations needed to calculate the position, which is commonly assumed to be $N_{s, \min }=4$ and depends on the localization algorithm implemented in the system. In this sense, the minimum number of stations, that make the $S P o D_{N_{S j}}$ equal or greater than the corresponding requirement for the $\mathrm{SPoD}$, can be estimated by imposing this condition and solving (5.8) for $N_{s j}$. This value is taken as the LoS redundancy restriction. Moreover, this value also depends on the performance of the localization algorithm used and in any case it can be modified (normally increased); in the remaining of this part, and unless otherwise stated, we assume that the LoS redundancy as calculated by solving (5.8) also satisfies the localization algorithm performance. Finally, it is worth saying that, due to the easy computation of (5.8), no explicit expression has to be found for $N_{s j}$, but only by a simple evaluation of it, in a given domain, is enough to find the solution.

\section{System Design Evaluation}

In this step, the quality of the partial design is evaluated (i.e., the partial spatial distribution of the MLAT stations). For this, the LoS and the 
theoretical system accuracy are calculated (only in the areas of interest previously defined) and these values are introduced into the fitness function, which follows a particular form of (5.1), to obtain the score that quantifies the quality of the system design, regarding the requirements and restrictions as defined in the first step.

The LoS calculation is performed only at the points within the areas of interest and the theoretical system accuracy is obtained only at the points that satisfy the requirement of LoS redundancy. Moreover, this accuracy calculation is performed by using all the stations within the LoS for each point, i.e., according to Fig. 3.2 we use a full calculation scheme. We have also tested the design optimization by using a calculation by arrays but, better results (in terms of convergence and realistic final designs) have been found by using the full calculation form. However, the interested reader can also find the corresponding results, of using the calculation by arrays form, in [36].

Finally, in this step, a decision line is implemented to decide if the stop condition (basically the compliance with the requirements and restrictions, or a fixed number of iterations) is reached. If "yes", the partial solution is taken as the final design, whereas in the case of "no" the procedure continues refining the partial solution, obviously until the stop condition is reached.

\section{Genetic Algorithm}

In this step, a GA is used to iterate and modify the partial solution that will be evaluated by the second step of this procedure. The GA repeatedly modifies a population of individual solutions. At each step, it randomly selects individuals among the current population to be parents and uses them to produce the children for the next generation. After successive generations, and if the algorithm is well calibrated, the population "evolves" toward an optimal solution [59-60], i.e., a system design that efficiently satisfies all the requirements and restrictions. The full description of the GA implemented in this thesis is provided in $\S 4.3 .2$, therefore, only the specification about the parents structure and the adaptation of the MLAT design CO problem (i.e., of the problem variables) to that algorithm is described in this section.

The adaption of the problem variables to the parent structure is referred as the codification of the algorithm and, as we described in $\S 4.3 .2$, it can be a set of parameters (cf. (4.1)) that can take values from a binary or realvalued alphabet. In this thesis we have used a real-valued alphabet due to the direct association that can be done between the problem variables and the individual built from this alphabet. In this sense, an individual as described by (4.1) takes the same form than the set $X$ of problem variables 
described in this chapter. It is shown, for the most general case, in the following equation:

$$
y=X=\left[N_{S}, x_{1}, \ldots, x_{N_{S}}\right]_{N_{S}+1}^{T}
$$

or equivalently, for the case of optimizing only the spatial distribution of the station and not the number of them, it takes the following form:

$$
y=X=\left[x_{1}, \ldots, x_{N_{S}}\right]_{N_{S}}^{T}
$$

where we have to remember that for the case of (5.9), the length of the individual is changeable and depends on its first element, whereas for the case of (5.10) that length is fixed and user-defined.

On the other hand, for the GA parameters choice, there is no any deterministic methodology for carrying out this process because it is strongly problem-dependent. Consequently, an accepted methodology to select these parameters is based on an empirical selection conducted through an exhaustive number of simulations. Thus, the latter is the methodology used in this thesis for performing the GA parameters choice. Then, after this selection process, the set of selected parameters, which has been found to be optimal in a wide sense, is described in Table 5.1.

Table 5.1 Parameters for GA.

\begin{tabular}{|l|l|}
\hline \hline \multicolumn{1}{|c|}{ Parameter } & \multicolumn{1}{c|}{ Value/Option } \\
\hline Population size & 10 \\
\hline Selection & Stochastic uniform \\
\hline Elite count & 2 \\
\hline Crossover fraction & 0.01 \\
\hline Type of crossover & Intermediate \\
\hline Probability of mutation & $10 \%$ \\
\hline \hline
\end{tabular}

Finally, it is worth saying that the GA has been chosen for this work because this is one of the most well-established and proved metaheuristic optimization methods, which can be found in the literature. Nevertheless, due to the modularity of the iterative procedure proposed herein, its extension to any other optimization method, such as Particle Swarm Optimization (PSO) [70] or Ant Colony Optimization (ACO) [71], is straightforward.

\subsection{Proposed Design Strategies}

In this thesis, we propose three different strategies to be used along with the procedure developed herein. These strategies, from our point of view, 
cover almost all the possible system designs either with standard MLAT systems (only with TOA/TDOA receiving stations) or with its enhanced versions (i.e., with TOA/TDOA and AOA or RTD stations). All these share the same design objective, which essentially is to obtain the system design that satisfies all the requirements and restrictions with the fewer amount of resources. The difference among them lies in the variables to be optimized and in the kind of system to be implemented (i.e., standard or enhanced MLAT). In this sense, the first proposed strategy is basically intended to design a MLAT system with a fixed number of TDOA stations, the second one is intended to design a MLAT system with a variable number of stations, whilst the third one is intended to design a enhanced MLAT system, either with the combination of TDOA/AOA or TDOA/RTD stations.

On the other hand, referring to an optimization context, the differences among these strategies, besides the conceptual one, basically lies in the definition of the GA individuals, in the fitness functions and in some configuration of the numerical tools used, particularly that one to obtain the theoretical system accuracy. In the following, each of these strategies is fully described, making emphasis in these aspects.

\subsubsection{Standard MLAT System with a Fixed Number of Stations}

This first strategy is the standard one proposed herein to be used along with the general procedure described in Fig. 5.5. It consists of the design of a standard MLAT system with a fixed and user-defined number of TDOA stations.

For this strategy, an individual is an array in the form of (5.10) with $N_{s} \times 1$ size, where the $i$ th position represents the index (from the search space $\mathcal{S}$ ) of the possible position for the $i$ th station.

The fitness function, which takes a particular form of (5.1), is defined as follows:

$$
f\left(\boldsymbol{y}_{t}\right)=1-\left(w_{1} f_{T C}\left(\boldsymbol{y}_{t}\right)-w_{2} f_{R o S}\left(\boldsymbol{y}_{t}\right)\right)
$$

where $f_{T C}$ is a function that quantifies the requirement of total coverage for a partial solution $y_{t}$ at time $t$, i.e., the percentage of points that are covered for more than LoS redundancy stations within a theoretical accuracy better than the corresponding value defined in the requirements and, $f_{R o S}$ is a function that quantifies the restriction of minimum spatial separation between any pair of stations of a partial solution $y_{t}$ at time $t$. These two functions can be calculated as follows: 


$$
f_{T C}\left(\boldsymbol{y}_{t}\right)=\frac{\text { Points with total coverage }}{\text { Total points evaluated }}
$$

and

$$
f_{\text {RoS }}\left(\boldsymbol{y}_{t}\right)=\frac{\text { Total of } \Delta_{i, j} \text { with } \Delta_{i, j}<\Delta_{\text {min }}}{\text { Total of } \Delta_{i, j}}
$$

The value of the weight factors depends on the importance given to each requirement or restriction on the design; they can be chosen by the designer, taking into account that the weight factor associated with the total coverage, e.g., $w_{1}$ for this case, must be much greater than the other ones; normally greater than 0.8 . In this thesis, and based on our simulations (reported and non reported in this thesis), we propose the use of $w_{1}=0.95$ and $w_{2}=0.05$, and in the remaining of this part the same reasoning is used to define the corresponding weight factors. The only condition that must be satisfied is that the sum of these must be equal to 1 .

The function in (5.13) penalizes those solutions with stations close to each other at a distance smaller than a predefined spatial threshold, which we denote as $\Delta_{\min }$. However, there exists the possibility, as we have commented before, of obtaining solutions with two (or more than two) stations in the same site. These particular situations are penalized directly in (5.11) instead than in (5.13). It is because the influence of having two stations in the same site, on the theoretical system accuracy, is negatively much stronger than having them close to each other a distance small than $\Delta_{\min }$ but greater than zero. Therefore, this particularity must be also stronger penalized. Then, the final expression for the fitness function takes the following form:

$$
f\left(x_{t}\right)=\left\{\begin{aligned}
1-F_{R_{1}}\left(x_{t}\right), & \text { if all } \Delta_{i, j}>0 \\
1, & \text { if at least one } \Delta_{i, j}=0
\end{aligned}\right.
$$

being

$$
F_{R_{1}}\left(\boldsymbol{x}_{t}\right)=w_{1} f_{T C}\left(\boldsymbol{x}_{t}\right)-w_{2} f_{R o S}\left(\boldsymbol{x}_{t}\right)
$$

Importantly, this procedure to penalize this kind of restriction is also used in the remaining strategies.

Finally, the last element that must be defined is the kind of numerical tool to be used for calculating the theoretical system accuracy. For this strategy, it is only necessary to implement the calculation of the TOA/TDOA error balance and the lower bounds for the standard MLAT systems [20]. 


\subsubsection{Standard MLAT System with a Variable Number of Stations}

The second strategy proposed in this thesis consists of the design of a standard MLAT system with a variable number of stations. In this case, the objective is not only to define the stations sites but also to calculate a relative minimum number of stations that satisfies all the assumed requirements and restrictions. In other words, to estimate the minimum number of stations, and their locations, that provides the maximum coverage with the maximum accuracy levels.

For this strategy, it is necessary not only to define the search space $\mathcal{S}$ as in the first one, but also that one to stipulate the range of the number of stations, i.e., the second search space $\mathcal{R}_{S}$.

An individual for this case is an array of variable length in the form of (5.9), where the first position sets this variable length. In this individual the $i$ th position represents the index (from the search space $\mathcal{S}$ ) of the possible position for the $i$ th- 1 station.

The fitness function, which takes a particular form of (5.1), is defined as follows:

$$
f\left(\boldsymbol{y}_{t}\right)=\left\{\begin{aligned}
1-F_{R_{2}}\left(\boldsymbol{y}_{t}\right), & \text { if all } \Delta_{i, j}>0 \\
1, & \text { if at least one } \Delta_{i, j}=0
\end{aligned}\right.
$$

being

$$
F_{R_{2}}\left(\boldsymbol{y}_{t}\right)=w_{1} f_{T C}\left(\boldsymbol{y}_{t}\right)-w_{2} f_{R o S}\left(\boldsymbol{y}_{t}\right)-w_{3} f_{R o N S}\left(\boldsymbol{y}_{t}\right)
$$

and $f_{\text {RoNS }}$ is a function that quantifies the cost of using the estimated number of stations, i.e., the greater this number is, the stronger the solution must be penalized as the objective is the use of the minimum number of stations. Thus, this function is expressed as follows:

$$
f_{\text {RoNS }}\left(\boldsymbol{y}_{t}\right)=\frac{\boldsymbol{y}_{t}(1)-\min \left(\mathcal{R}_{s}\right)}{\max \left(\mathcal{R}_{S}\right)-\min \left(\mathcal{R}_{s}\right)}
$$

The rest of functions used in (5.15) are the same as defined for the first strategy.

The value of the weight factors are chosen in the same sense as the first strategy and are $w_{1}=0.85, w_{2}=0.05$, and $w_{3}=0.1$.

Finally, the numerical tool for calculating the theoretical system accuracy is exactly the same as in the first strategy. 


\subsubsection{Enhanced MLAT System with a Fixed Number of Stations}

The third strategy proposed in this thesis consists of the design of an enhanced MLAT system with a fixed and user-defined number of stations, either TDOA/AOA or TDOA/RTD.

For this strategy, an individual is an array in the form of (5.10) with $N_{S} \times 1$ size, where the $i$ th position represents the index (from the search space $\mathcal{S}$ ) of the possible position for the $i$ th station. Moreover, the first $N_{s e}$ array elements of this individual represent the position of the enhanced stations. Likewise, another difference of this in respect of the individual defined in the first strategy, for the standard version of MLAT systems, is that, for this case, the individual positions that represent the enhanced stations are used by an additional function that takes into account the accuracy improvement due to these additional measurements. It is shown in the description of the corresponding fitness function.

The fitness function, which takes a particular form of (5.1), is defined as follows:

$$
f\left(\boldsymbol{y}_{t}\right)=\left\{\begin{aligned}
1-F_{R_{3}}\left(\boldsymbol{y}_{t}\right), & \text { if all } \Delta_{i, j}>0 \\
1, & \text { if at least one } \Delta_{i, j}=0
\end{aligned}\right.
$$

being

$$
F_{R_{3}}\left(\boldsymbol{y}_{t}\right)=w_{1} f_{T C}\left(\boldsymbol{y}_{t}\right)+w_{2} f_{L o s}\left(\boldsymbol{y}_{t}\left(1: N_{\mathrm{se}}\right)\right)-w_{3} f_{R o S}\left(\boldsymbol{y}_{t}\right)
$$

where $y_{t}\left(1: N_{s e}\right)$ refers to the first $N_{s e}$ elements of the particular individual, and $f_{\text {LoS }}$ is a function that quantifies the relative LoS coverage of the $N_{s e}$ enhanced stations and can be calculated as follows:

$$
f_{\text {LoS }}=\frac{\sum_{i=1}^{N_{s e}} \text { Number of points covered by } \boldsymbol{y}_{t}(i)}{\text { Total points evaluated } \times N_{\text {se }}}
$$

The rest of functions used in (5.17) are the same as defined for the first strategy.

The value of the weight factors are chosen in the same sense as the first strategy and are $w_{1}=0.9, w_{2}=0.05$, and $w_{3}=0.05$.

The usefulness of this strategy is justified because it is not enough to add the enhanced measurements only to those stations with the highest percentage of LoS coverage, which is equivalent of using the first strategy and simply assign the enhanced measurements to those particular stations. Moreover, it is also important to optimize the location of the enhanced stations because they significantly influence on the overall system accuracy, as it has been demonstrated for MLAT systems in [20].

Finally, the numerical tool for calculating the theoretical system accuracy is different from those used for the first and the second strategies. 
In this case, a different model must be used as the enhanced measurements must be introduced in this calculation. This particular form is called as CRLB for enhanced MLAT systems in [20].

\subsection{Simulation and Results}

To validate the procedure and the design strategies proposed in this thesis, three different simulations (one for every proposed strategy) have been carried out over the layout of Barcelona - El Prat (Spain) airport. The common objective for all the simulations is to obtain an MLAT system that covers the three runways, the taxiways, and the apron centrelines, given a set of requirements and restrictions. The first simulation is based on the use of only a fixed set of TDOA stations, the second one is based on the use a variable set of TDOA stations, whereas the third on is based on the use of TDOA stations along with one TDOA/AOA. Fig. 5.6 shows the Barcelona - El Prat airport layout and all the possible sites where the stations can be emplaced, i.e., the search space $\mathcal{S}$. For these simulations, the size $\ell_{\mathcal{S}}$ of the search space $\mathcal{S}$ is $\ell_{\mathcal{S}}=41$.

For all the simulations, the antenna station height (i.e., of the mast length) has been assumed to be equal to $2 \mathrm{~m}$ and the calculations for LoS and the theoretical system accuracy are performed for a spatial grid of $5 \mathrm{~m}$ $\times 5 \mathrm{~m}$. This spatial grid is also in agreement with the DTM used to calculate the LoS. The GA parameters are those ones described in Table 5.1 .

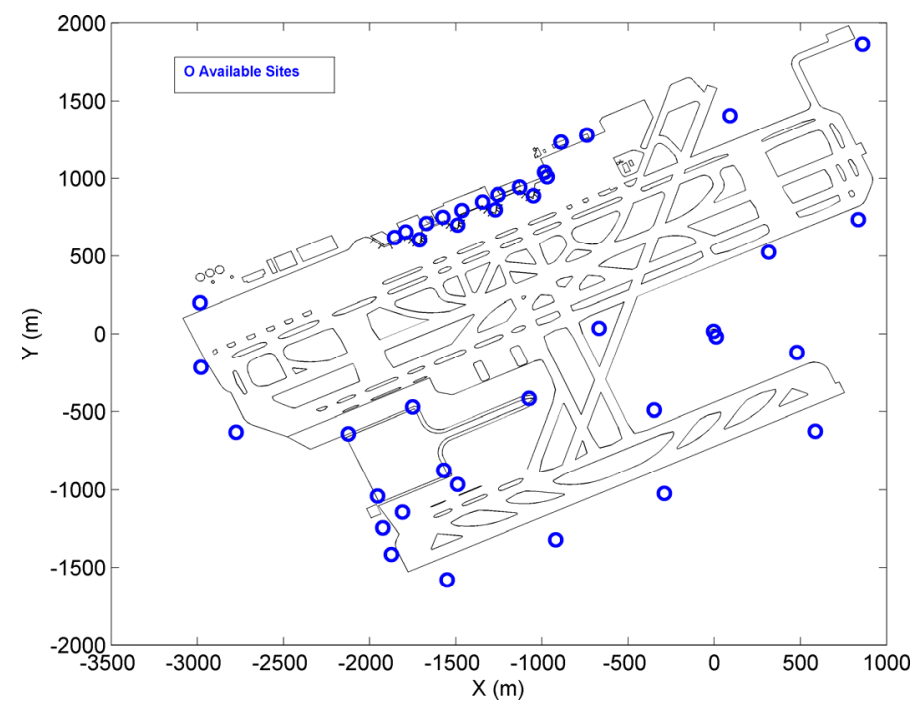

Fig. 5.6 Barcelona - El Prat airport layout with the possible sites to emplace the stations. 


\subsubsection{MLAT System with a Fixed Number of TDOA Stations}

This first simulation shows an application of the first strategy proposed in this thesis. The main objective is to design a MLAT system with a fixed number of TDOA stations, i.e., to find a spatial distribution of the stations which provides the better performance levels, fulfilling the given restrictions. Due to the fact that these simulations are performed over an airport surface scenario, the system requirements are those described in [1], which are basically: horizontal accuracy must be within $3.75 \mathrm{~m}(\sigma)$ and the SPoD must be better than $99.9 \%$. The number of stations to use in this design is $N_{s}=12$ and they measure only the TOA. The restriction of LoS redundancy, using a station probability of detection of $P o D_{\text {station }}=97 \%$, provided by a quick evaluation of (5.8), is 7 and the minimum spatial separation is set to $\Delta_{\min }=400 \mathrm{~m}$. The results for this scenario are shown in Fig. 5.7 - Fig. 5.9 .

Fig. 5.7 shows the horizontal accuracy for this scenario and how the corresponding areas of interest are covered with the assumed accuracy requirements. From the theory [3-4], it is well known that a convenient system geometry, to obtain high-accuracy levels, is to set the stations in a polygon enclosing the area(s) of interest (see also Fig. 2.8). In Fig. 5.7, it can be observed that the proposed procedure provides a solution that agrees with this theoretical aspect.

Another important aspect, when applying optimization methods, is the convergence rate, which is shown in Fig. 5.8. In this scenario, the number of possible combinations, as provided by $(5.2)$, is $7.8987 \times 10^{9}$ and a relative good solution is obtained within 50 iterations, which means only 500 problem evaluations. At this number of iterations, it can be considered that the procedure has converged. However, it is advisable to expend more iterations (up to 200) because the random component of the GA allows the procedure to explore new values in the search space. It is also important to emphasize that, sometimes, this random component can move the mean fitness value (line with circles in Fig. 5.8) to a worst value, but because this procedure always save the best solution (best fitness value), it does not represent a problem in the algorithm convergence. In any case, the total number of problem evaluations is significantly much smaller than that value provided by (5.2). The latter justifies the use of this procedure instead of the full evaluation of all possible combinations of the problem.

Finally, Fig. 5.9 shows the SPoD for the whole airport and we can see that, for every point, this value is over the required one. 


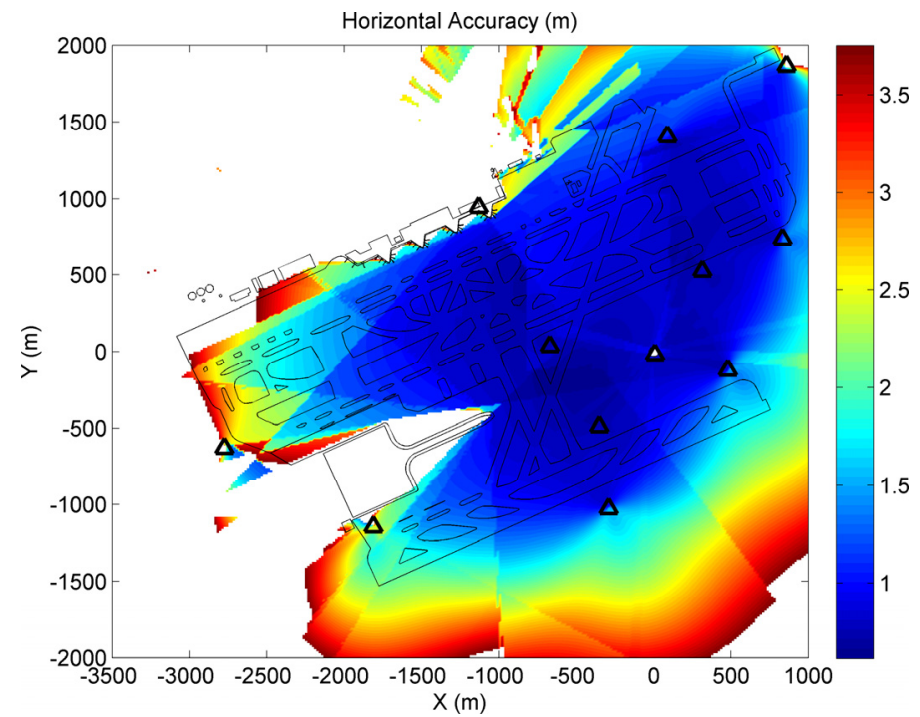

Fig. 5.7 Horizontal accuracy for the design with a fixed number of TDOA stations.

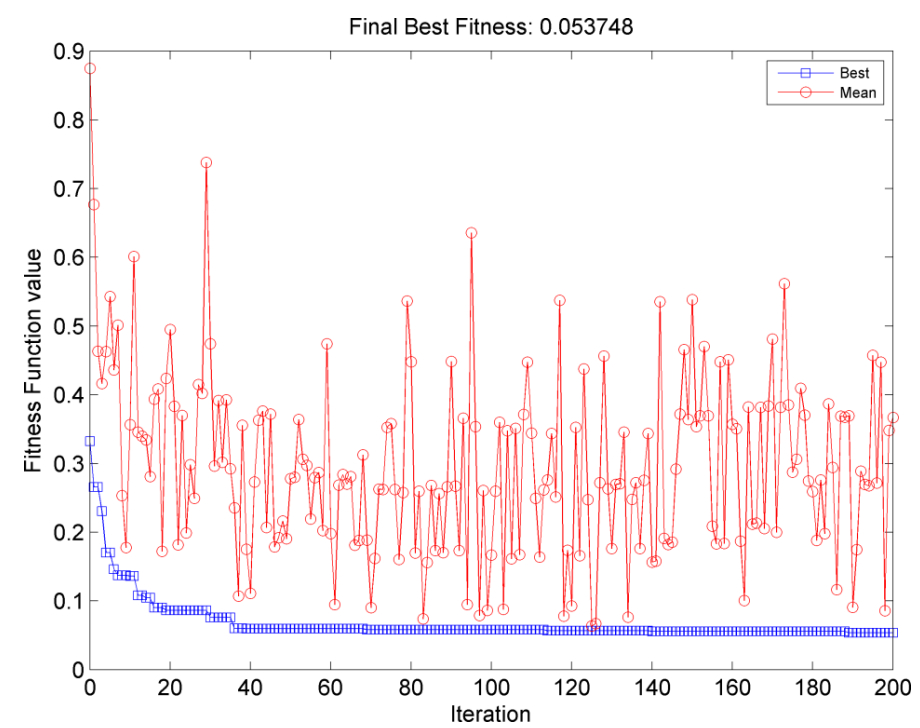

Fig. 5.8 GA convergence for the design with a fixed number of TDOA stations. 


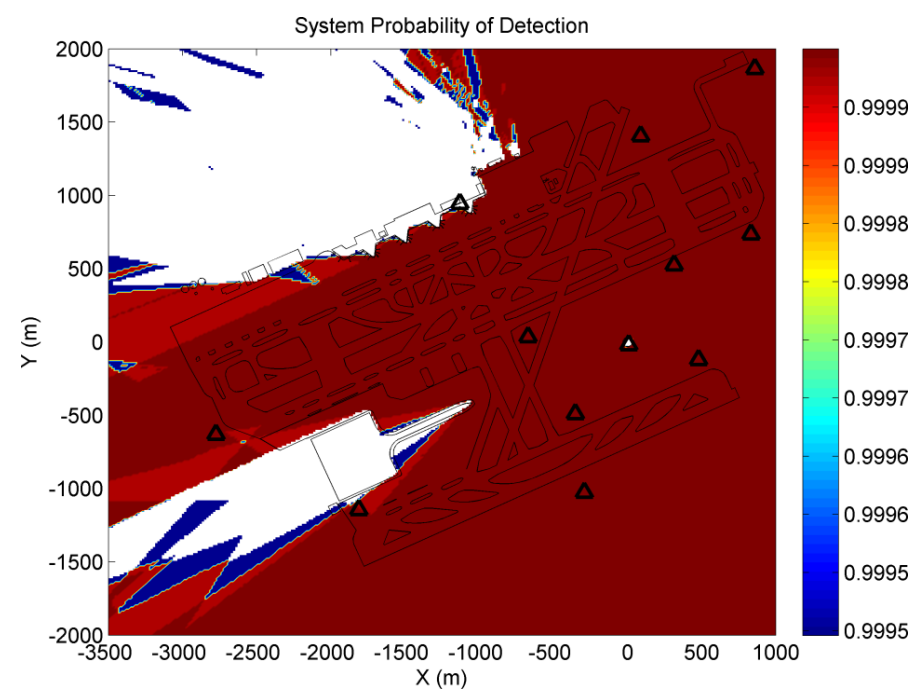

Fig. 5.9 SPoD for the design with a fixed number of TDOA stations.

\subsubsection{MLAT System with a Variable Number of TDOA Stations}

This second simulation shows an application of the second strategy proposed in this thesis. The main objective in this scenario is to design a MLAT system with a variable number of TDOA stations, i.e., to find the minimum number of stations, and their locations (system geometry), which provides the better performance levels, fulfilling the given restrictions. All the requirements and restrictions for this problem are those described for the first problem in §5.5.1. Moreover, for this problem, it is necessary to stipulate a range for the number of stations, i.e., the additional search space $\mathcal{R}_{s}$. For this particular, we have set this search space as $\mathcal{R}_{s}=[7,15]$. The results for this scenario are shown in Fig. 5.10 - Fig. 5.12.

Fig. 5.10 shows the results for the horizontal accuracy. Also, in this scenario, all the areas of interest are covered satisfying all the requirements and restrictions. The important aspect in this scenario is that the minimum number of stations calculated is 11, it is one less station than in the first scenario, which shows that it is possible to reach all the requirements and restrictions by using a fewer amount of resources. This kind of simulation is useful to know an approximate minimum number of stations that meets the requirements and restrictions or to validate whether a given design is the most optimal or not. Nevertheless, due to the random component of the GA, it is advisable to run the procedure, for this kind of designs, once or twice more, just to validate the calculated minimum number of stations. 


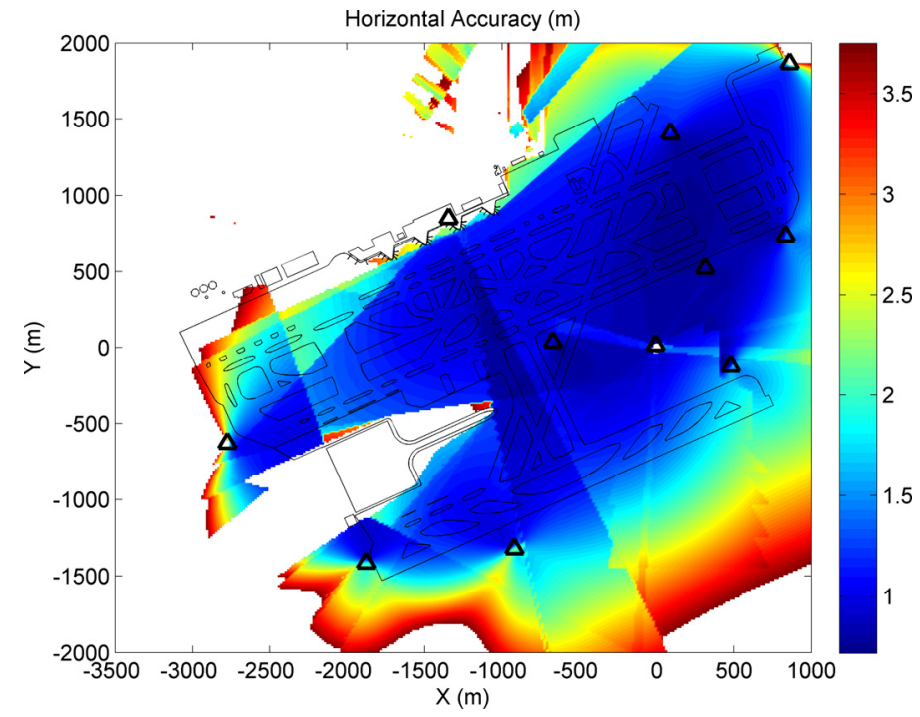

Fig. 5.10 Horizontal accuracy for the design with a variable number of TDOA stations.

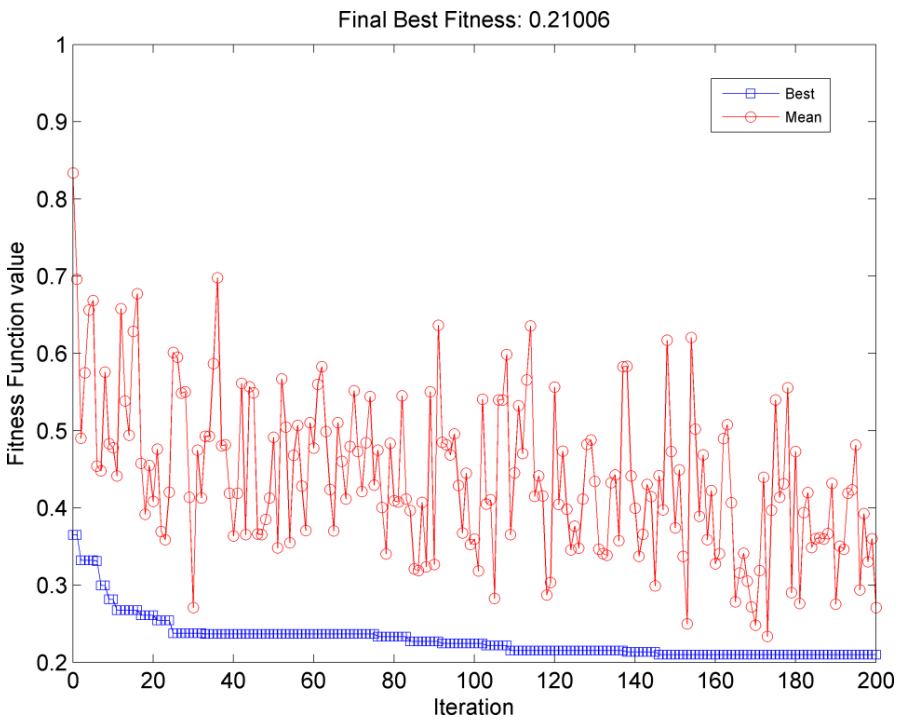

Fig. 5.11 GA convergence for the design with a variable number of TDOA stations.

Regarding the procedure convergence, which is shown in Fig. 5.11, it can be observed that a good solution is found after 150 iterations. This greater number of iterations, in respect of that for the first scenario, can be understood because the complexity of this problem (number of possible combinations, cf. (5.7)) is much greater $\left(1.2894 \times 10^{11}\right)$ than that of the first one. 


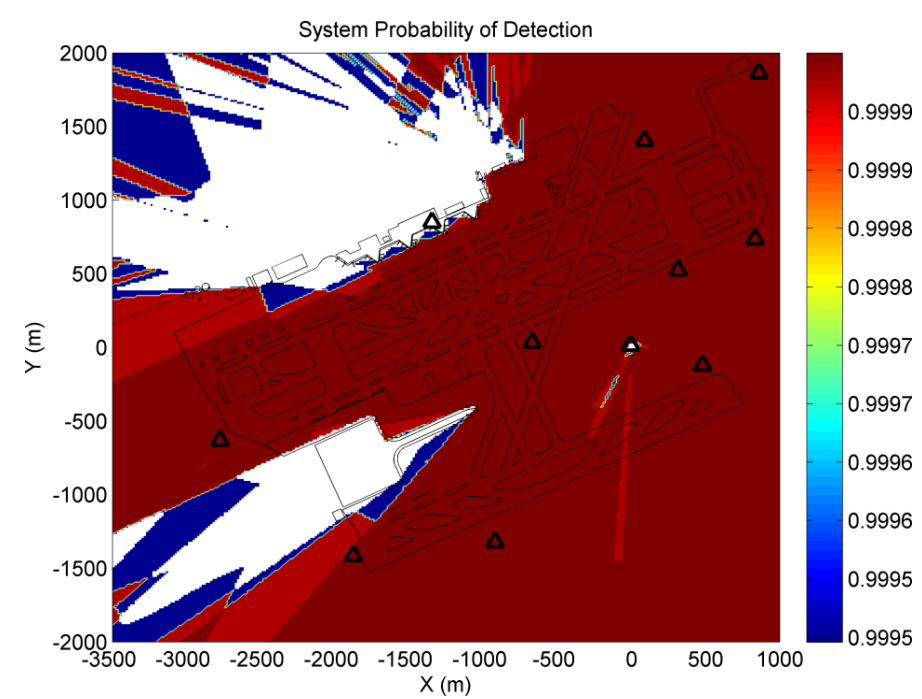

Fig. 5.12 SPoD for the design with a variable number of TDOA stations.

Finally, Fig. 5.12 shows the SPoD for the whole airport and we can see that, for every point, this value is over the required one.

\subsubsection{MLAT System with a Fixed Number of TDOA/AOA Stations}

This last application shows the application of the third strategy proposed in this thesis. The main objective is to design an enhanced MLAT system with a fixed number of TDOA/AOA stations. In this problem, the TDOA/AOA station measures the elevation (vertical) AOA. Normally, this kind of measurement capability is added to improve the horizontal accuracy in surface movement applications [20]. Likewise, as demonstrated in [20], only by adding one AOA station is enough to obtain significant accuracy improvements, of course, assuming that this particular station covers with LoS all areas where the improvement is required. Therefore, for this simulation we use only one (i.e., $N_{s e}=1$ ) TDOA/AOA station and it is designated to be the number one in the individual solutions of (5.10). All the requirements and restrictions for this problem are those described for the first problem in $\S 5.5 .1$. Moreover, the additional AOA measurement error is assumed to be $10^{-3} \mathrm{rad}[20]$. The results for this scenario are shown in Fig. 5.13 - Fig. 5.15.

Fig. 5.13 shows the horizontal accuracy for this scenario and how the corresponding areas of interest are covered with the assumed accuracy requirements. The complexity of this problem is basically of the same order than that of the first one but, here the theoretical system accuracy calculation has been carried out by taking into account the accuracy 


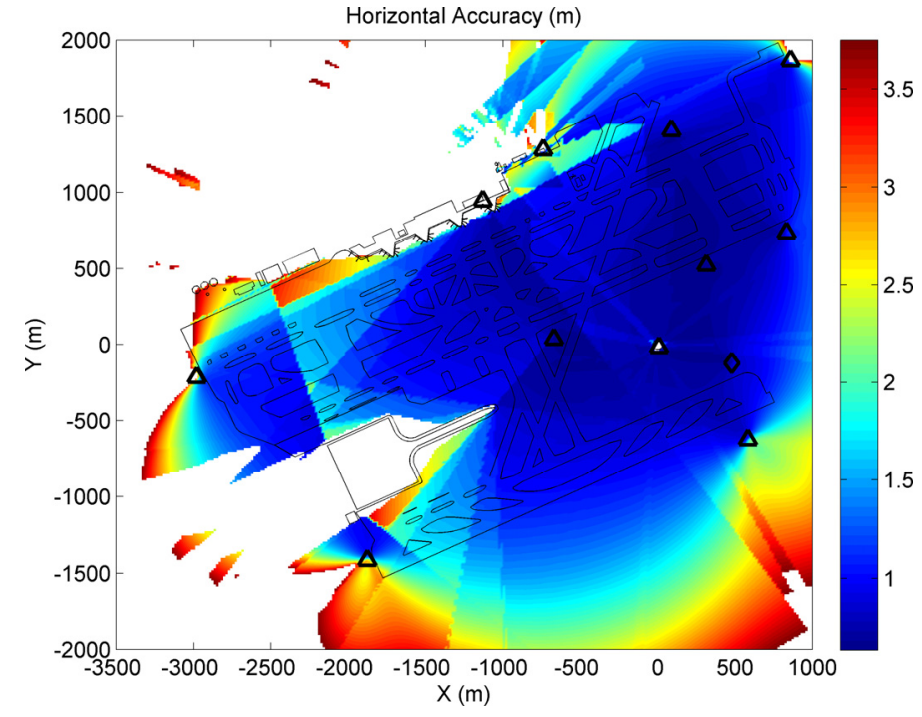

Fig. 5.13 Horizontal accuracy for the design with a fixed number of TDOA/AOA stations.

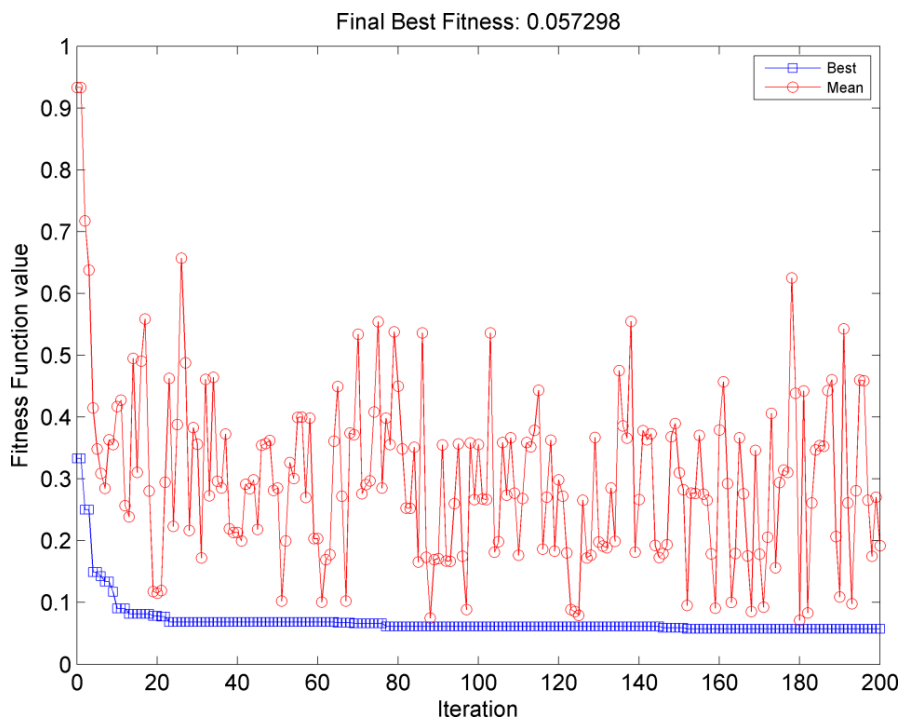

Fig. 5.14 GA convergence for the design with a fixed number of TDOA/AOA stations.

improvement provided by the TDOA/AOA station. The final site for this station is shown in Fig. 5.13 as a diamond. Also, for this kind of scenario, it is advisable to run the procedure once or twice more.

Regarding to the procedure convergence, which is shown in Fig. 5.14, a good solution is found after 50 iterations and also at this number of iterations it can be considered that the procedure has converged. Moreover, 


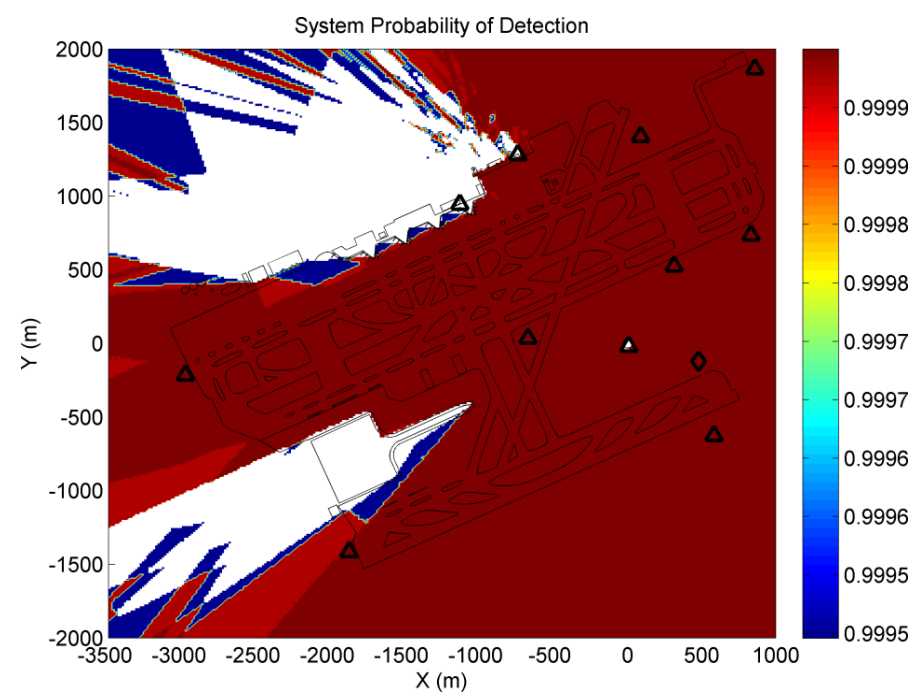

Fig. 5.15 SPoD for the design with a fixed number of TDOA/AOA stations.

also for this case, due to the additional iterations, it can be observed that the mean fitness value (line with circles in Fig. 5.14) is moved to slightly higher values for iterations between 60 and 80, but, after that, it decreases and approximately maintains a constant value after 100 iterations.

Finally, Fig. 5.15 shows the SPoD for the whole airport and we can also see that, for every point, this value is over the required one. 
Part III

Multilateration Algorithms and Their Improvements 



\section{Setting the Stage}

In this chapter, we set the stage for the third part of this thesis. We describe the basic foundations for understanding the MLAT localization problem as a numerical inverse one. First, the localization problem is fully described by means of the well known Time Difference of Arrival (TDOA) technique. The concept of hyperbolic system of equations as an inverse problem is also provided and placed into a general scheme, which divides the localization problem into two main components that can be individually analyzed: the data model and the numerical algorithm. Then, a short review for the mathematics of inverse problem is provided in $\S 6.2$, including the description of the ill-conditioned inverse problems. The concept of Singular Value Decomposition (SVD), as a tool for analyzing inverse problems, is introduced in $\S 6.3$ along with other useful numerical tools, which are based on that one. Finally, a short description of the classical numerical solution of inverse problems, in the sense of Least Squares (LS), and the well known pseudoinverse matrix is provided in $\S 6.4$. The purpose of this chapter is to provide the reader of the basic concepts for constructing, analyzing, and solving inverse localization problems in MLAT systems. It supports the developments of Chapter 7 and Chapter 8.

\subsection{Localization Problem in Mode S Multilateration}

In Mode S Multilateration systems, a number of ground stations (at least three for 2D localization or four for 3D localization) are placed in some strategic locations around the airport or the area to be covered. The system uses the Mode A/C and Mode S based transmissions; i.e., the spontaneous Mode S squitter, the asynchronous transponder replies as well as the responses to interrogations elicited by the system itself (see $\S 2.1 .3$ for a detailed description of the signals). Then, the signal is sent to the Central Processing Subsystem (CPS) where the transponder position is calculated [1-2]. This calculation is based on the TDOA principle, where the intersections of multiple hyperbolas (or hyperboloids), which have been 


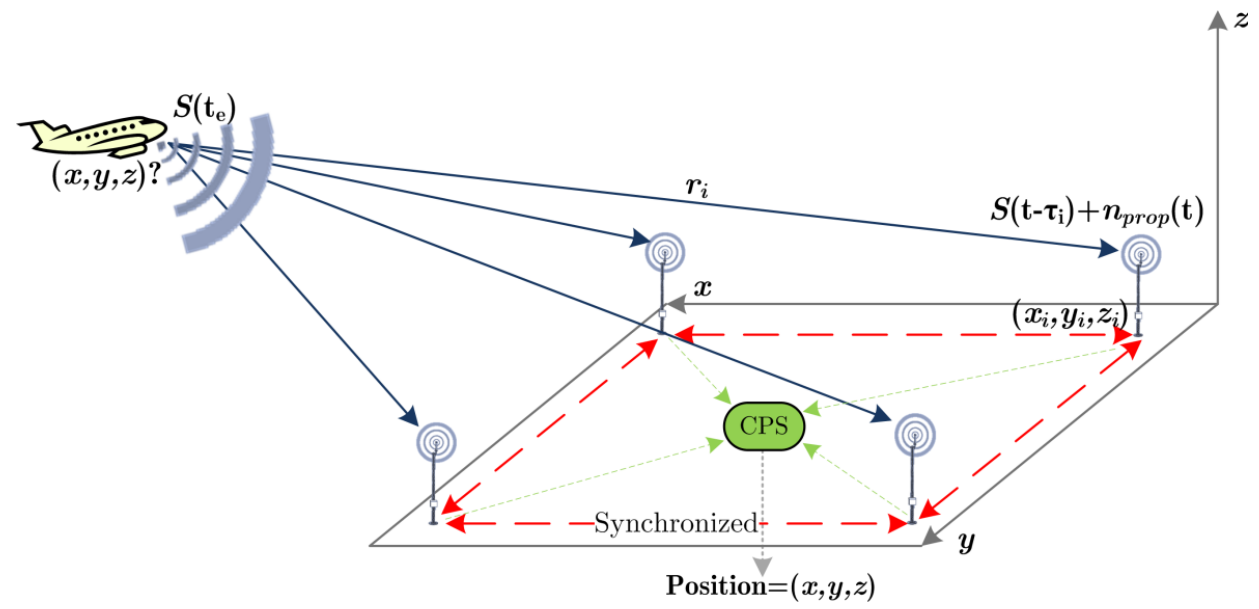

Fig. 6.1 Localization problem in Mode S Multilateration.

created with the pertaining Time of Arrival (TOA) differences, are computed. A standard example of these systems is shown in Fig. 6.1.

In the following subsection, the mathematical bases of this problem are described. The objective of this subsection is to set up a standard mathematical framework that describes the problem to be solved with the methods developed in this thesis.

\subsubsection{General Problem Description}

The standard localization problem for passive localization systems, like Mode S MLAT, is based on the configuration of a system of equations with the available information (e.g., system geometry and TOA/TDOA measurements) in order to calculate the unknown target (aircraft or vehicle) position in an assigned geographical reference system. There exists several methods to construct this system of equations and all of them are based on the same principle. The aim of this subsection is to describe such a principle.

In Fig. 6.1, the variable $r_{i}$ is the 3D distance between the target and $i$ th station. This distance, for each station, can be expressed as a nonlinear function $f_{i}(x, y, z)$ of the target position, as follows:

$$
r_{i} \equiv f_{i}(x, y, z)=\sqrt{\left(x-x_{i}\right)^{2}+\left(y-y_{i}\right)^{2}+\left(z-z_{i}\right)^{2}}, \quad i=1, \ldots, N_{s}
$$

being $N_{s}$ the number of MLAT ground (or receiving) stations, $\left(x_{i}, y_{i}, z_{i}\right)$ are the known coordinates of the ith station and $c$ is the speed of light. This function can be expressed in terms of the parameter that each station measures, i.e., the TOA, as shown below: 


$$
f_{i}(x, y, z)=c\left(T O A_{i}-t_{e}\right)
$$

where $t_{e}$ is the emission time of the signal (i.e., the time at which the signal is transmitted by the transponder), as depicted in Fig. 6.1. Importantly to emphasize that both $t_{e}$ and $T O A_{i}$ are clock times rather than absolute propagation times. Then, from (6.2) it is clear that:

$$
T O A_{i}=\frac{f_{i}(x, y, z)}{c}+t_{e}
$$

Now, because what we really know is a measured TOA quantity instead an exact value, it is more convenient expressing (6.3) as follows:

$$
\widehat{T O A}_{i}=\left(\frac{1}{c} f_{i}(x, y, z)+t_{e}\right)+n_{i}, \quad i=1, \ldots, N_{s}
$$

where the superscript " $\wedge$ " denotes a measured quantity and the term $n_{i}$ represents the measurement noise affecting the $i$ th station.

Finally, the TDOA between the $i$ th station and the reference one (without loss of generally, the station number 1 can be assumed as the reference one) is:

$$
\begin{gathered}
\widehat{T D O}_{i, 1}=\left(\frac{f_{i}(x, y, z)}{c}+t_{e}\right)-\left(\frac{f_{1}(x, y, z)}{c}+t_{e}\right)+n_{i, 1}, \\
i=2,3, \ldots, N_{s}
\end{gathered}
$$

where $n_{i, 1}$ is the equivalent measurement noise for the TDOA quantity. Finally, the TDOA measurement can be expressed as follows:

$$
\begin{aligned}
& \widehat{T D O A}_{i, 1}=\frac{1}{c} \sqrt{\left(x-x_{i}\right)^{2}+\left(y-y_{i}\right)^{2}+\left(z-z_{i}\right)^{2}} \\
&-\frac{1}{c} \sqrt{\left(x-x_{1}\right)^{2}+\left(y-y_{1}\right)^{2}+\left(z-z_{1}\right)^{2}}+n_{i, 1}
\end{aligned}
$$

Note from (6.6) that the term of the emission time $t_{e}$ has disappeared because the subtraction of the two TOA quantities in (6.5). This fact is one of the advantages of the TDOA principle as $t_{e}$ is an unknown quantity and thus the estimation of it is avoided.

On the other hand, theoretically for $N_{s}$ stations it is possible to obtain a total of $\left(\begin{array}{c}N_{s} \\ 2\end{array}\right)$ equations in the form of (6.6). Nevertheless, it can be demonstrated that all of these equations can be completely determined from a linear combination of only $N_{s}-1$ equations in the noiseless case, e.g., $\widehat{T D O A}_{i, 1}, i=2, \ldots, N_{s}$. Then, for simplicity and without loss of generality, from now on, we assume that with $N_{S}$ stations only a system of $N_{S}-1$ hyperbolic equations in the form of (6.6) can be set. 


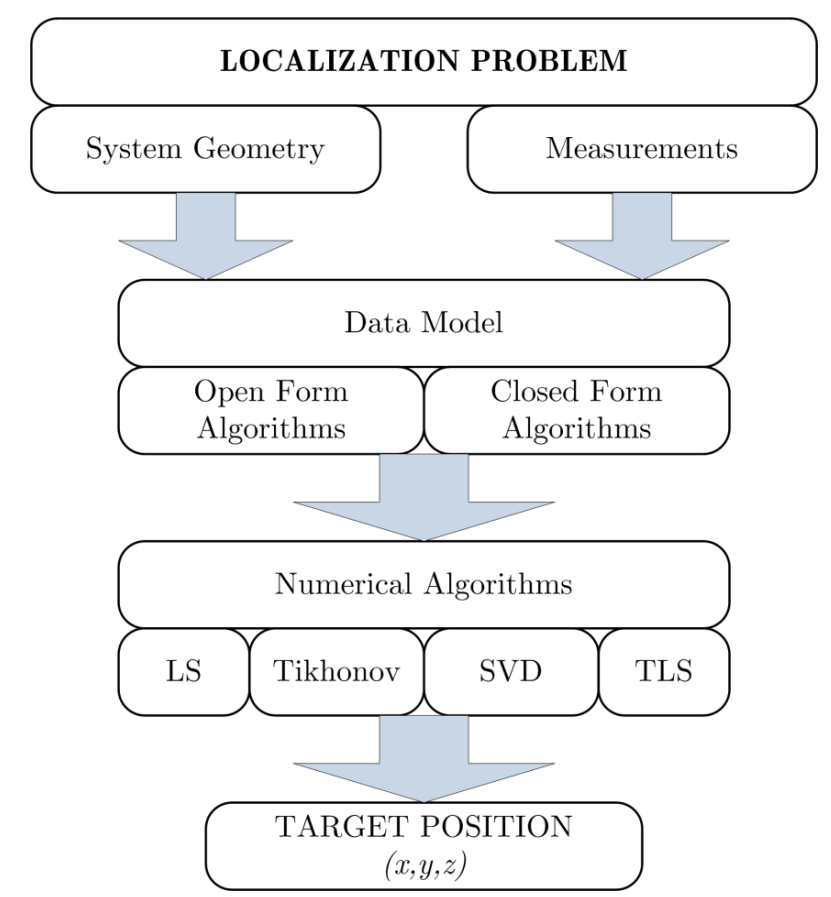

Fig. 6.2 General scheme of a localization problem in MLAT.

The localization problem consists of finding or estimating the target position given the set of $N_{s}-1$ hyperbolic equations. As we have stated in $\S 2.1 .2$, this localization problem can be expressed as a mathematical operator $\mathcal{M}$ (cf. (2.5)) which converts a set of measurements into a numerical target position by solving an inverse problem. Moreover, we also stated in $\S 2.1 .2$ that the particular form this operator can take depends on the kind of measurements and on the procedure to deal and to solve the set of hyperbolic equations, which is known as localization algorithm. For the standard version of MLAT, the kind of measurements is the set of $N_{s}-1$ TDOAs, whilst for the localization algorithms there is an extensive literature describing a vast number of methods. However, as a common factor, all localization algorithms are basically composed of two elements: a data model that allows the construction of the inverse problem (commonly a linear one) and a numerical algorithm for solving it and obtain a numerical data for the target position as depicted in Fig. 6.2.

To construct the inverse problem the algorithms can be summarized into two families: open form algorithms and closed form algorithms. Each of these kinds of algorithms has some advantages and disadvantages, depending on the application or the scenario to be used. In Chapter 7 we provide a short review of the most relevant localization algorithms for MLAT systems and perform a rigorous comparison among them. 
For the numerical algorithms, the most common one used in the literature is the Least-Squares (LS) method which leads to a minimum 2norm solution [72]. However, as it will be explained later on $\S 6.2$ and analyzed on $\S 7.5$, under some conditions this algorithm does not reach accurate results. On the other hand, there is a set of algorithms, called regularization algorithms, like the Tikhonov regularization [73-74], the Singular Value Decomposition (SVD) based regularization [75-76] and the Total Least Squares (TLS) [75], [77-78], which have been used for different scientific applications but not widely applied (even not used so far) for the MLAT localization problem. The complete description of these methods and the corresponding application to the MLAT localization problem is one of the main objectives of this thesis, and the remaining part of it is dedicated to this topic.

The remaining subsections of this chapter are dedicated to the review of the mathematical bases of inverse problems, the SVD and other numerical tools.

\subsection{An Inverse Problem Description}

Inverse problems arise from the necessity of knowing the source (or sources) that generates some physical phenomena [72], [79-80]. Normally, in a classical direct problem we know some sources and a mathematical model that describes the behaviour of the physical phenomena in terms of the sources. In this way, at any time it is possible to obtain the answer of the physical phenomena (or system) for any specific source. On the other hand, when the source is unknown (e.g., the target position from which a signal is transmitted) but the answer is known (e.g., a TOA/TDOA corresponding to the signal emitted at the unknown target position), often by measurements of some natural variables, it is necessary to formulate a model that allows knowing the source of these answers. This model is called inverse problem and a classical mathematical representation for describing it can be as follows:

$$
\int_{\Omega} \text { source } \times \text { system } d \Omega=\text { answer }
$$

where $\Omega$ is a domain, depending on the particular problem and $\times$ denotes the particular interaction.

In the model of (6.7) the objective is to find the source (source), given a mathematical model describing the system (system) and a set of data (inevitably noisy) containing the answer (answer). For solving (6.7) by a numerical method, which is the most common practice, it is necessary to 
convert the continuous model in (6.7) into a discrete one [79-80], resulting in the following:

$$
\boldsymbol{A \theta}=\widehat{\boldsymbol{m}}
$$

where $\boldsymbol{A}$ is a matrix describing the behaviour of the system (or physical phenomena); it is often called coefficient matrix [79-80], $\boldsymbol{\theta}$ is a column vector representing the unknown source and $\widehat{\boldsymbol{m}}$ is a column vector containing the answer (e.g., measurements by means of some instrument).

Achieving and solving a system of equations like (6.8) is the main objective of any localization algorithm in MLAT systems. The data model in Fig. 6.2 provides a procedure to manipulate the set of equations in the form of (6.6) in order to explicitly obtain a coefficient matrix $\boldsymbol{A}$ and a measurement vector $\widehat{\boldsymbol{m}}$, and the numerical algorithm solves that resulting system of equations. It is, the particular form each inverse localization problem can take depends on the pair composed of data model and numerical algorithm.

\subsubsection{Ill-Conditioned Problems}

There are two possible options when the inverse problem in (6.8) is solved, i.e., the problem can be well-conditioned or ill-conditioned. These concepts go back to Jacques Hadamard [8]. He postulated three conditions to define when an inverse problem is well-conditioned or ill-conditioned. These conditions are:

1. The solution exists.

2. The solution is unique.

3. The solution continuously depends on the problem data.

If the three above conditions are satisfied, the problem is defined as wellconditioned but, if at least one of them is not satisfied, the problem is defined as ill-conditioned [73], [76], [79-80]. This ill-conditioning may occurs due to the use of an incorrect mathematical model, in which case it should be modified, or because this ill-conditioning is an intrinsic part of the formulation. In the MLAT localization problem the data model is basically subject to the kind of measurements and to the system geometry. As we will show through Chapter 7, the ill-conditioning in MLAT localization problem mainly appears when only TOA/TDOA measurements are used; in this case the ill-conditioning can be considered as an intrinsic part of the formulation and the target position must be calculated under these conditions. However, the TOA/TDOA data model can be modified and improved by combining them with other kind of measurements (e.g., RTD or AOA), and the resulting data model will posses better numerical characteristics [20]. Besides the strategy of combining different 
measurements, there are other options to improve the TOA/TDOA data model. Nevertheless, all of these have some advantages and disadvantages and their use is not always possible. The particular aspects commented in this paragraph, along with the analysis of the ill-conditioned localization problem, are studied later in Chapter 7.

Solving well-conditioned problems may be easy, but when solving the illconditioned problem we can find several difficulties. This latter kind of problems are common in many applications in science and engineering, like acoustics [81], astrometry [82], computerized tomography [83], continuation problems [84], early vision [85], electromagnetic scattering [86], geophysics [87], inverse geo- and helioseismology [88], mathematical biology [89], image restoration [90], remote sensing [91], inverse scattering theory [92], signal processing [93] and statistics [94]. Moreover, as we have analyzed in this thesis, the ill-conditioned problems also appears in some typical scenarios in the localization process for MLAT systems (see $\S 7.5$ later).

In ill-conditioned problems, it is normal to deal with a coefficient matrix $\boldsymbol{A}$ which is characterized by a very high condition number, i.e., by a large ratio between the largest and the smallest singular values of the coefficient matrix (see $\S 6.3$ for more details). It means that in the system of equations shown in (6.8) some (or all) of the equations are numerically linearly dependent. It is, the coefficient matrix is unstable [72], [76].

\subsubsection{Solving Ill-Conditioned Problems}

When solving ill-conditioned problems it is necessary to apply strategies that satisfy the three Hadamard's condition. It is, to convert an illconditioned problem into a well-conditioned one. These strategies are commonly known as regularization methods. The regularization methods add additional information, about the desired solution, to the problem in order to achieve a stable coefficient matrix $\boldsymbol{A}$ [74], [76], [79-80], and thus obtain and stable and useful solution. The general idea of this aspect is shown in Fig. 6.3.

Mathematically, the basic idea supporting the regularization methods consists of allowing certain residual [95] associated with the regularized solution, whose norm, which is called residual norm, can be defined as

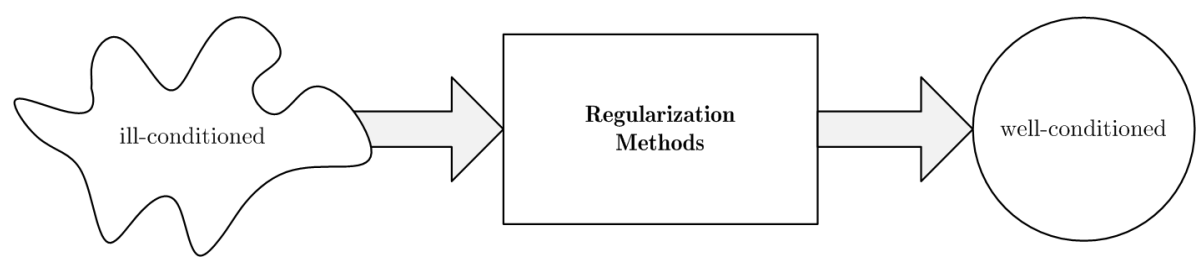

Fig. 6.3 Regularization strategy. 
follows:

$$
\rho(\boldsymbol{\theta})=\|\boldsymbol{A} \boldsymbol{\theta}-\widehat{\boldsymbol{m}}\|_{2}
$$

where \|\|$_{2}$ denotes the 2-norm operator [72], and then they can (in general terms) use one of the following schemes [76] to find a solution:

1. Minimize $\rho(\boldsymbol{\theta})$ subject to the constraint that $\boldsymbol{\theta}$ belongs to a specified subset, i.e., $\boldsymbol{\theta} \in S_{\boldsymbol{\theta}}$.

2. Minimize $\rho(\boldsymbol{\theta})$ subject to the constraint that some measurement $\Omega(\boldsymbol{\theta})$ of the size of $\boldsymbol{\theta}$ is less than some specified upper bound $\delta$, i.e., $\Omega(\boldsymbol{\theta}) \leq \delta$.

3. Minimize $\Omega(\boldsymbol{\theta})$ subject to the constraint $\rho(\boldsymbol{\theta}) \leq \alpha$.

4. Minimize a linear combination of $\rho(\boldsymbol{\theta})^{2}$ and $\Omega(\boldsymbol{\theta})^{2}: \min \left\{\rho(\boldsymbol{\theta})^{2}+\right.$ $\left.\lambda^{2} \Omega(\boldsymbol{\theta})^{2}\right\}$, where $\lambda$ is a specified weighting factor.

The main hypothesis in all the above schemes is that a regularized solution which have a permissible residual norm and which satisfies the additional constraint will be not too far from the exact, ideal solution. On the other hand, as it was described in [96], statistically the regularization process decreases the size of the covariance matrix at the expense of adding some amount of bias to the solution. The specific analysis of this situation for the localization process in MLAT system is discussed in $§ 8.1 .3$

Before solving an ill-conditioned problem it is important to check some important aspects about the problem. In [76] the author suggests some important points, which are summarized below:

- What kind of ill-conditioning does the problem have?

- Is it possible to regularize?

- Which additional information is available?

- Is this additional information suitable for stabilization purposes?

- Which method is more adequate to efficiently and reliably solve the problem on a computer?

- How much stabilization should be added?

Now, before continue with the study and description of some regularization methods, it is important to give an overview of the most common tools to analyze ill-conditioned problems, which basically are the SVD, the condition number and the numerical rank. This is the purpose of the next sections. 


\subsection{Singular Value Decomposition (SVD) and Other Numerical Tools}

Before we go into insight of the regularization methods, it is convenient to understand the superior numerical "tool" [72], [76] to analyze the illconditioned problems, i.e., the (ordinary) SVD. This tool is the basis of all methods and procedures discussed in this thesis, and it is also useful to classify the ill-conditioned problems into the two possible classes: ill-posed or rank-deficient.

The source of the ill-conditioned problems is that in matrix $\boldsymbol{A}$ to be inverted, there are, at least, two or more equations which are linearly dependent [72], [76], [79]. The SVD can identify these linear dependencies.

Also in this part, the concepts of condition number, rank and numerical rank of the matrix are reviewed. These parameters along with the SVD are very useful to identify the class of a given inverse problem and also to estimate the right parameters used by the regularization methods.

\subsubsection{The Ordinary SVD}

Let assume a rectangular or square matrix $\boldsymbol{A} \in \mathbb{R}^{m \times n}$, where without loss of generality $m \geq n$. The SVD of $\boldsymbol{A}$ is defined as the decomposition of the matrix into three independent matrices [72], [76], [97] as follows:

$$
\boldsymbol{A}=\boldsymbol{U} \boldsymbol{\Sigma} \boldsymbol{V}^{T}=\sum_{i=1}^{n} \boldsymbol{u}_{i} \sigma_{i} \boldsymbol{v}_{i}^{T}
$$

where $\boldsymbol{U}=\left[\boldsymbol{u}_{1}, \ldots, \boldsymbol{u}_{n}\right] \in \mathbb{R}^{m \times n}$ and $\boldsymbol{V}=\left[\boldsymbol{v}_{1}, \ldots, \boldsymbol{v}_{n}\right] \in \mathbb{R}^{n \times n}$ are matrices with orthonormal columns $\boldsymbol{u}_{i}$ and $\boldsymbol{v}_{i}$ respectively. Therefore, these two matrices satisfy the following identity:

$$
\boldsymbol{U}^{T} \boldsymbol{U}=\boldsymbol{V}^{T} \boldsymbol{V}=\boldsymbol{I}_{n}
$$

where $\boldsymbol{I}_{n}$ is the $n \times n$ identity matrix.

Matrix $\boldsymbol{\Sigma}$ is $n \times n$ diagonal with nonnegative elements $\left(\sigma_{1}, \ldots, \sigma_{n}\right)$ appearing in nonincreasing order such that $\sigma_{1} \geq \sigma_{2} \geq \cdots \geq \sigma_{n} \geq 0$. The elements of matrix $\boldsymbol{\Sigma}$ are called singular values of $\boldsymbol{A}$ and the columns vector $\boldsymbol{u}_{i}$ and $\boldsymbol{v}_{i}$ are the left and right singular vectors of $\boldsymbol{A}$, respectively. The matrices $\boldsymbol{U}, \boldsymbol{\Sigma}$ and $\boldsymbol{V}$ can be obtained by using some numerical routines. However, it is not the goal of this thesis to describe such procedures.

The SVD of $\boldsymbol{A}$ provides two sets of orthonormal basis vectors, i.e., the columns of matrix $\boldsymbol{U}$ and the columns of matrix $\boldsymbol{V}$. Both sets allow to transform matrix $\boldsymbol{A}$ into a diagonal matrix by projecting it on these two bases, as follows: 


$$
\boldsymbol{U}^{T} \boldsymbol{A} \boldsymbol{V}=\boldsymbol{\Sigma}=\left[\begin{array}{cccc}
\sigma_{1} & 0 & \cdots & 0 \\
0 & \sigma_{2} & \cdots & 0 \\
\vdots & \vdots & \ddots & \vdots \\
0 & 0 & \cdots & \sigma_{n}
\end{array}\right]_{n \times n}
$$

With matrix $\boldsymbol{\Sigma}$ in hand, is easier to check some numerical properties of the matrix $\boldsymbol{A}$ [72], [76]. For example, if the last singular value $\sigma_{n}$ is zero or very close to zero, it gives an indication that matrix $\boldsymbol{A}$ is ill-conditioned. Therefore, a small singular (or a group of these) value indicates some linear dependencies in the matrix $\boldsymbol{A}$.

\subsubsection{Null Space and Range of a Matrix}

Before continue with the description of the SVD based numerical tools, it is important to define the concepts of null space and range of a matrix.

The null space [72] (also known as kernel) of a $m \times n$ matrix $\boldsymbol{A}$ is the set of all vectors $\boldsymbol{\theta}_{\mathcal{N}}$ satisfying that $\boldsymbol{A \theta}_{\mathcal{N}}=\mathbf{0}$. Generally the null space is denoted by the operator $\mathcal{N}(\cdot)$. The mathematical definition of null space is expressed as follows:

$$
\mathcal{N}(\boldsymbol{A})=\left\{\boldsymbol{\theta}_{\mathcal{N}} \in K^{n}: \boldsymbol{A \theta}_{\mathcal{N}}=\mathbf{0}\right\}
$$

where $K$ is a field, which in the context of this thesis is the field of real numbers, and $\mathbf{0}$ is the zero vector with $m$ components.

The range [72] (also known as the column space) of a $m \times n$ matrix $\boldsymbol{A}$ is the set of all possible linear combinations of its column vectors. Mathematically, it means that if matrix $\boldsymbol{A}$ has columns vectors denoted as $\boldsymbol{a}_{i}$, where $i=1, \ldots, n$, the range of $\boldsymbol{A}$ will be the set of vectors resulting from the following linear combination:

$$
\sum_{i=1}^{n} c_{i} \boldsymbol{a}_{i}
$$

where $c_{i}$ is scalar. In other words, the range of a matrix can be also seen as the span of all of its columns $\boldsymbol{a}_{i}$. In the context of the linear system of equations, the range of $\boldsymbol{A}$ is the set of vectors $\widehat{\boldsymbol{m}}$ for which the system $\boldsymbol{A} \boldsymbol{\theta}=\widehat{\boldsymbol{m}}$ has a solution.

\subsubsection{The Generalized SVD (GSVD)}

The Generalized SVD (GSVD) is calculated for a pair of matrices $\boldsymbol{A}$ and $\boldsymbol{L}$. Assuming $\boldsymbol{A} \in \mathbb{R}^{m \times n}, \boldsymbol{L} \in \mathbb{R}^{p \times n}$ and that $m \geq n \geq p$. Also it is assumed that 
$\mathcal{N}(\boldsymbol{A}) \cap \mathcal{N}(\boldsymbol{L})=\{\mathbf{0}\}$ and that $\boldsymbol{L}$ is a full rank matrix. Then the GSVD is a decomposition of the matrix pair $(\boldsymbol{A}, \boldsymbol{L})$ in the following form [72], [76]:

$$
\begin{gathered}
\boldsymbol{A}=\boldsymbol{U}\left(\begin{array}{cc}
\boldsymbol{\Sigma} & 0 \\
\mathbf{0} & \boldsymbol{I}_{\boldsymbol{n}-\boldsymbol{p}}
\end{array}\right) \boldsymbol{X}^{-1} \\
\boldsymbol{L}=\boldsymbol{V}\left(\begin{array}{ll}
\boldsymbol{M} & \mathbf{0}
\end{array}\right) \boldsymbol{X}^{-1}
\end{gathered}
$$

where $\boldsymbol{U} \in \mathbb{R}^{m \times n}$ and $\boldsymbol{V} \in \mathbb{R}^{p \times p}$ are orthonormal, i.e., $\boldsymbol{U}^{T} \boldsymbol{U}=\boldsymbol{I}_{n}$ and $\boldsymbol{V}^{T} \boldsymbol{V}=\boldsymbol{I}_{p} . \boldsymbol{X} \in \mathbb{R}^{n \times n}$ is a square matrix and it is non-singular with columns that are $\left(\boldsymbol{A}^{T} \boldsymbol{A}\right)$-orthogonal, it is:

$$
\boldsymbol{X}^{T} \boldsymbol{A}^{T} \boldsymbol{A} \boldsymbol{X}=\left[\begin{array}{cc}
\boldsymbol{\Sigma}^{2} & \mathbf{0} \\
\mathbf{0} & \boldsymbol{I}_{n-p}
\end{array}\right]
$$

and the matrices $\boldsymbol{\Sigma}$ and $\boldsymbol{M}$ are $p \times p$ diagonal matrices with elements $\left(\sigma_{1}, \ldots, \sigma_{p}\right)$ and $\left(\mu_{1}, \ldots, \mu_{p}\right)$, respectively. These elements are non-negative, they are ordered as:

$$
\begin{aligned}
& 0 \leq \sigma_{1} \leq \cdots \leq \sigma_{p} \leq 1 \\
& 1 \geq \mu_{1} \geq \cdots \geq \mu_{p}>0
\end{aligned}
$$

and they are normalized such that:

$$
\sigma_{i}^{2}+\mu_{i}^{2}=1, \quad i=1, \ldots, p
$$

Moreover, for the GSVD, the generalized singular values $\gamma_{i}$ of the pair $(\boldsymbol{A}, \boldsymbol{L})$ are also defined. Each of these takes the following form:

$$
\gamma_{i}=\frac{\sigma_{i}}{\mu_{i}}, \quad i=1, \ldots, p
$$

and they obviously appear in non-decreasing order.

\subsubsection{Classification of the Ill-Conditioned Problems}

Once defined the SVD of a given matrix, it is important to define the different classes of problems that can appear in many applications. In [76] two classes of ill-conditioned problems are suggested, namely, the rankdeficient problems and the discrete ill-posed problems.

In rank-deficient problems the matrix $\boldsymbol{A}$ has a cluster of small singular values and also there is a well-determined gap between the large and small singular values. This means that one or more rows and columns of $\boldsymbol{A}$ are linearly dependent.

In discrete ill-posed problems there is no gap between the large and small singular values, instead, all of these gradually decay to zero. This fact 
makes the notion of numerical rank to be not useful to analyze this class of ill-conditioned problems.

\subsubsection{Basic Parameters to Analyze Ill-Conditioned Problems}

There are some basic parameters which are useful to quickly check if a given matrix $\boldsymbol{A}$ is ill-conditioned or not. The use of them depends on the class of problem but, normally, the condition number and rank of a matrix give a quick idea about the "amount" of ill-conditioning of the matrix [72], [76]. On the other hand, there is another parameter that is only useful when the problem is identified as a rank-deficient problem, which is called the numerical rank of a matrix [72], [76]. In this subsection all of them are reviewed.

\section{The Condition Number}

The condition number of a given matrix $\boldsymbol{A}$ is defined as the relation between the maximum and minimum singular values of that matrix [72], [76]. In other words, the condition number is the relation between the first and the last singular values. It is numerically defined as follows:

$$
\text { cond }=\frac{\sigma_{1}}{\sigma_{n}}
$$

A high condition number is found if the last singular value is close to zero. In this case the matrix $\boldsymbol{A}$ can be considered as an ill-conditioned matrix.

\section{The Rank of a Matrix}

The rank of a matrix $\boldsymbol{A}$ is defined as the number of linearly independent columns of $\boldsymbol{A}$ [72], [76]. In terms of the SVD of matrix $\boldsymbol{A}$, the rank is equal to the number of strictly positive singular values contained in matrix $\boldsymbol{\Sigma}$ (cf. (6.12)) [76]. When matrix $\boldsymbol{A}$ has some errors, like measurements errors, approximation and discretization errors, as well as rounding errors, this definition is not useful, because some columns that are found to be mathematically linearly independent under those noisy conditions, for a practical point of view should be considered as linearly dependent. Hence, in those cases is more useful to use the concept of numerical rank. This parameter is described below.

\section{Numerical Rank}

The numerical rank of a matrix $\boldsymbol{A}$ is the number of columns of this matrix that, in the presence of some error level $\epsilon$, are practically linearly independent [72], [76]. Formally, it is defined as the real and integer number $r_{\epsilon}$ that satisfies the following expression: 


$$
r_{\epsilon}=r_{\epsilon}(\boldsymbol{A}, \epsilon)=\min _{\|\boldsymbol{E}\|_{2} \leq \epsilon} \operatorname{rank}(\boldsymbol{A}+\boldsymbol{E})
$$

where matrix $\boldsymbol{E}$ is the equivalent perturbation matrix containing the errors of matrix $\boldsymbol{A}$. Note in (6.20) the important condition for the norm of the perturbation matrix $\boldsymbol{E}$.

The numerical rank takes sense only when the problem is defined as rank-deficient because in this case there is a well determined gap between some singular values and the rest of these, such that $r_{\epsilon}$ satisfies the following inequality:

$$
\sigma_{r_{\epsilon}}>\epsilon \geq \sigma_{r_{\epsilon}+1}
$$

Furthermore, the numerical $\epsilon$-rank defined in (6.20) can be defined with respect to the Frobenius norm $\|\cdot\|_{F}$ and in this case the numerical rank is the smallest integer $k$ for which [98]:

$$
\sigma_{k+1}^{2}+\cdots+\sigma_{n}^{2} \leq \epsilon^{2}
$$

\subsection{Least Squares (LS) and the Pseudoinverse}

The method of Least Squares (LS) [72] is a classical numerical tool for solving overdetermined system of equations (as the most common case in MLAT localization), i.e., the case of having more equations (or measurements) than unknown parameters. In these cases no exact solution can be achieved. Therefore, the LS method proposes that the best solution that can be found in these cases is the one which minimizes the sum of the squared differences between the measurements and the modelled ones. In other words, LS proposes to find a solution that minimizes the squared 2norm of a residual function as that described in (6.9). That is, for a given linear system of equations $\boldsymbol{A} \boldsymbol{\theta}=\widehat{\boldsymbol{m}}$, where $\boldsymbol{A}$ is a $m \times n$ coefficient matrix being $m>n, \boldsymbol{\theta}$ is the $n \times 1$ unknown vector, and $\widehat{\boldsymbol{m}}$ is the $m \times 1$ known measurement vector, the LS looks for a solution $\boldsymbol{\theta}$ that produces de minimum value for the following function:

$$
\|\boldsymbol{A \theta}-\widehat{\boldsymbol{m}}\|_{2}^{2}
$$

that is the squared version of (6.9).

Now, if matrix $\boldsymbol{A}$ has linearly independent columns (i.e., it is of full rank) and if the matrix $\boldsymbol{A}^{T} \boldsymbol{A}$ is invertible, then the LS leads to the following solution:

$$
\widehat{\boldsymbol{\theta}}=\left(\boldsymbol{A}^{T} \boldsymbol{A}\right)^{-1} \boldsymbol{A}^{T} \widehat{\boldsymbol{m}}
$$


where we have used the super script ${ }^{\wedge}$ to denote an estimated (non exact) solution.

However, if matrix $\boldsymbol{A}$ has some linearly dependent columns, the solution of (6.24) should be avoided and instead that the following option should be used:

$$
\widehat{\boldsymbol{\theta}}=\boldsymbol{A}^{\dagger} \widehat{\boldsymbol{m}}
$$

where $\boldsymbol{A}^{\dagger}$ is defined as the pseudoinverse [72] matrix of $\boldsymbol{A}$.

In terms of the SVD of $\boldsymbol{A}$, the pseudoinverse matrix $\boldsymbol{A}^{\dagger}$ can be obtained as:

$$
\boldsymbol{A}^{\dagger} \equiv \sum_{i=1}^{\operatorname{rank}(\boldsymbol{A})} \boldsymbol{v}_{i} \sigma_{i}^{-1} \boldsymbol{u}_{i}^{T}
$$

where $\boldsymbol{u}_{i}$ and $\boldsymbol{v}_{i}$ are the $i$ th left and right singular vectors of $\boldsymbol{A}$, respectively, $\sigma_{i}$ is the $i$ th singular value $\boldsymbol{A}$ as defined in $\S 6.3 .1$, and the function $\operatorname{rank}()$ denotes the rank of a matrix. When the solution $\widehat{\boldsymbol{\theta}}$ is obtained by using the pseudoinverse matrix it is defined as the minimum 2norm solution.

In terms of the matrices $\boldsymbol{U}, \boldsymbol{\Sigma}$, and $\boldsymbol{V}$ (see $§ 6.3 .1$ ), the pseudoinverse of $\boldsymbol{A}$ can be also defined as:

$$
\boldsymbol{A}^{\dagger}=\boldsymbol{V}_{n \times \operatorname{rank}(\boldsymbol{A})} \boldsymbol{\Sigma}_{\operatorname{rank}(\boldsymbol{A}) \times \operatorname{rank}(\boldsymbol{A})}\left(\boldsymbol{U}_{m \times \operatorname{rank}(\boldsymbol{A})}\right)^{T}
$$

Note that if matrix $\boldsymbol{A}$ is of full $\operatorname{rank}$, then $\operatorname{rank}(\boldsymbol{A})=n$ and, hence, the matrix $\left(\boldsymbol{A}^{T} \boldsymbol{A}\right)^{-1} \boldsymbol{A}^{T}$ is equal to the pseudoinverse matrix $\boldsymbol{A}^{\dagger}$. Therefore, for simplicity it is common to use the following equivalence:

$$
\boldsymbol{A}^{\dagger}=\left(\boldsymbol{A}^{T} \boldsymbol{A}\right)^{-1} \boldsymbol{A}^{T}
$$

Through this thesis we always make use of the equivalence (6.28) and indistinctly refer both as the pseudoinverse matrix, understanding that if $\boldsymbol{A}$ is not full rank, $\boldsymbol{A}^{\dagger}$ is obtained by (6.26) or (6.27). 


\section{Localization Algorithms for Mode S Multilateration}

In this chapter, we propose and develop a general frame for the analysis and comparison of localization algorithms. Generally, the localization algorithms are classified into two families: open form algorithms and closed form algorithms. In this chapter, we propose an additional and wider, but fully compatible, classification. This classification is based on a general framework that defines a localization algorithm as the pair composed by a data model and a numerical algorithm. This general frame and classification, as well as the relation with the classical one, are fully described in $\S 7.1$. Then, we briefly describe the most representative localization algorithms in the literature, and fix them into our proposed general frame in $\S 7.2, \S 7.3$, and $\S 7.4$. Once analyzed the localization algorithms, we describe a general procedure for analyzing the MLAT localization problem, specifically, for identifying the sources of the illconditioning and to get a general idea about the amount of this illconditioning. Finally, simulation and results for the comparison of the localization algorithms are presented in $\S 7.6$ for simulated scenarios, and in $\S 7.7$ for real data ones. General conclusions about the performance of all localization algorithms are provided. Besides the description of the novel general frame and the classification of localization algorithms, the purpose of this chapter is to demonstrate that there is no general algorithm that provides, under all conditions, the most statistically (low dispersion and unbiased) and numerically (stable and with robust convergence) efficient solutions for the target location. It also composes the main motivation for the proposed general localization strategy in Chapter 8. The contributions of this chapter have been published in [99-101].

\subsection{A Prelude to Localization Algorithms}

As we have described in $\S 6.1$, the localization problem consists of estimating the target position under any geographical reference system, given a set of 
physical measurements of signals emitted by aircrafts or vehicles. In general terms, the target position is that numeric parameter that satisfies a set of equations relating it with each measurement, e.g., the hyperbolic ones (6.6) for the case of standard MLAT systems. To set the latter in a computationally implementable context is the objective of the localization algorithms. To do this, they are composed of a data model and of a numerical algorithm. The data model explicitly relates the unknown target position with the set of known parameters (e.g., measurements and receiving stations position) by constructing a numerical inverse problem; the resulting inverse problem can be linear or non linear but, for practical issues, they are normally linear. The numerical algorithm solves the resulting inverse problem and allows the calculation of a numerical data for the target position under a specific geographical reference system (see Fig. 6.2), e.g., the Cartesian coordinate system. In this section, we describe a general framework that organizes the whole structure of any localization algorithm. This general framework is useful for understanding any of the localization algorithms that are described in this thesis, to develop a general classification for them, to facilitate an equivalent comparative analysis between them, and to identify their advantages and disadvantages, as well as to better identify which novelties are provided by the localization strategies provided in Chapter 8. This general framework is the result of an exhaustive literature search and posterior processing of it for selecting the most relevant and representative localization algorithms.

\subsubsection{The Data Model}

Before describing the different kinds of data models, it is important to introduce the concept of characteristic equation, which is very useful to explain and understand that classification and the posterior description of the localization algorithms. Essentially, a characteristic equation is the mathematical scalar element that relates the unknowns with the measurements collected by a set of receiving stations and their positions. In this sense, when this scalar element is transformed into a vector-matrix form, for a set of $N_{s}$ stations, it is called data model. In other words, the data model is the generalized version of a characteristic equation.

The data models used by the different localization algorithms can be classified as statistical approach based models, numerical approach based models, and algebraic approach based models. In the following the main characteristics of each of these are described.

The statistical approach based models assume certain statistical hypothesis about the measurements and the target position and set a probabilistic model that relates to each other. Most of these models are based on the Maximum Likelihood (ML) principle [102] due to the proven 
asymptotic consistency and efficiency of the ML estimators (MLE). These models require additional information about the measurement error distributions; usually Gaussian distributions are assumed. Furthermore, the resulting models are highly nonlinear in the unknown target position. Then, to solve this kind of models, linear approximations and iterative numerical methods are required. Consequently, they also require a good previous estimation of the solution to avoid the local minima and the convergence is not always guaranteed; for this reason the algorithms based on them are commonly classified as open form algorithms. On the other hand, if the statistical hypotheses are satisfied by the measured data, these models provide the most optimum estimators, i.e., estimators that are in practice unbiased and with its covariance matrix very close to its CRLB.

The numerical approach based models set a mathematical function that jointly relates the unknown target position, the measurements and a parameter derived from the target position (e.g., the target range) that naturally is also unknown. The resulting models are linear in one unknown given the other one. Then, they assume certain numerical approximations between the target position and its derived parameter in order to simplify the solution. The most common assumed approximation is that of mutual numerical independence between them. These numerical approximations are independent of the statistical distributions of the measurement errors. Most of them are based on the Least Squares (LS) principle, i.e., they set an error function whose squared norm is minimized. These models can be solved by direct optimization and do not require any previous estimation of the solution; for this reason the algorithm based on them are commonly classified as closed form algorithms. Furthermore, normally the computational cost required to solve this kind of models is lower than that required for the statistical approach based ones. On the other hand, all of algorithms based on these models introduce quadratic noise terms in the resulting inverse problem, and the solutions provided by them are biased and are not optimum in the statistical sense.

The algebraic approach based models do not use any statistical assumptions nor numerical approximations. They algebraically manipulate the hyperbolic equations until directly set an inverse problem that linearly relates the unknown target position with the known parameters (i.e., the measurements and the station positions). These models are very simple as only geometric relations are used. On the contrary, they usually require more stations to form the characteristic equation and introduce quadratic and cubic noise terms in the inverse problem. As the numerical approach based models, these ones do not require any previous estimation of the solution and can be solved by direct optimization; hence, the algorithms that use these models are also classified as closed form algorithms. Moreover, the solutions provided by the algorithms based on these models 
are also biased and are not optimum in the statistical sense. On the other hand, most of them require the lowest computational resources for solving the resulting inverse problem.

\subsubsection{The Numerical Algorithm}

The resulting linear inverse problem, obtained from any data model, must be numerically solved to obtain a numerical data for the target position. As we have also commented in $\S 6.1 .1$, the most used numerical algorithm to solve the resulting linear inverse problem is the LS, i.e., by the pseudoinverse matrix (see §6.4). Moreover, for the statistical approach based models that require iterative procedures because the nonlinearity of the resulting model, the Gauss-Newton method [103] is the most commonly used. All the localization algorithms analyzed in this chapter are solved by these methods. On the other hand, there also exists the well known regularization methods but, due to the fact that the application of them is one of the contributions of this thesis, they are treated in more detail in Chapter 8.

\subsubsection{The General Framework for Localization Algorithms}

The formulation of any localization algorithm can be summarized as depicted in Fig. 7.1. A localization algorithm starts by establishing a characteristic equation that can relate the unknown target position $\boldsymbol{\theta}$, the measurements $\widehat{\boldsymbol{m}}$, the position $\boldsymbol{\vartheta}_{i}$ of a finite number of stations, and optionally a derived parameter of the target position $\boldsymbol{\theta}$, which is denoted in Fig. 7.1 as $g(\boldsymbol{\theta})$. Then, depending on the procedure used to develop the characteristic equation, it is expressed in the sense of its corresponding data model. Furthermore, in this point, the data model is processed, and the statistical assumptions or numerical approximations, if applicable, are introduced to set up an inverse problem. This inverse problem can be generally composed of a coefficient matrix $\boldsymbol{G}$, an unknown vector $\boldsymbol{\theta}$, and a known measurement vector $\widehat{\boldsymbol{m}}$. As we will describe in the following subsections, different pairs of coefficient matrix and measurement vector will result in different localization problems. In other words, it means that every localization algorithm is, in principle, characterized by its own coefficient matrix and measurement vector. Finally, the inverse problem can be solved by any of the available numerical algorithms, and thus the solution for the target position $\widehat{\boldsymbol{\theta}}$ is obtained.

It is clear to see that a complete localization strategy is composed of the triplet formed by a coefficient matrix, a measurement vector and a numerical algorithm that operates with them. In this sense, the best 


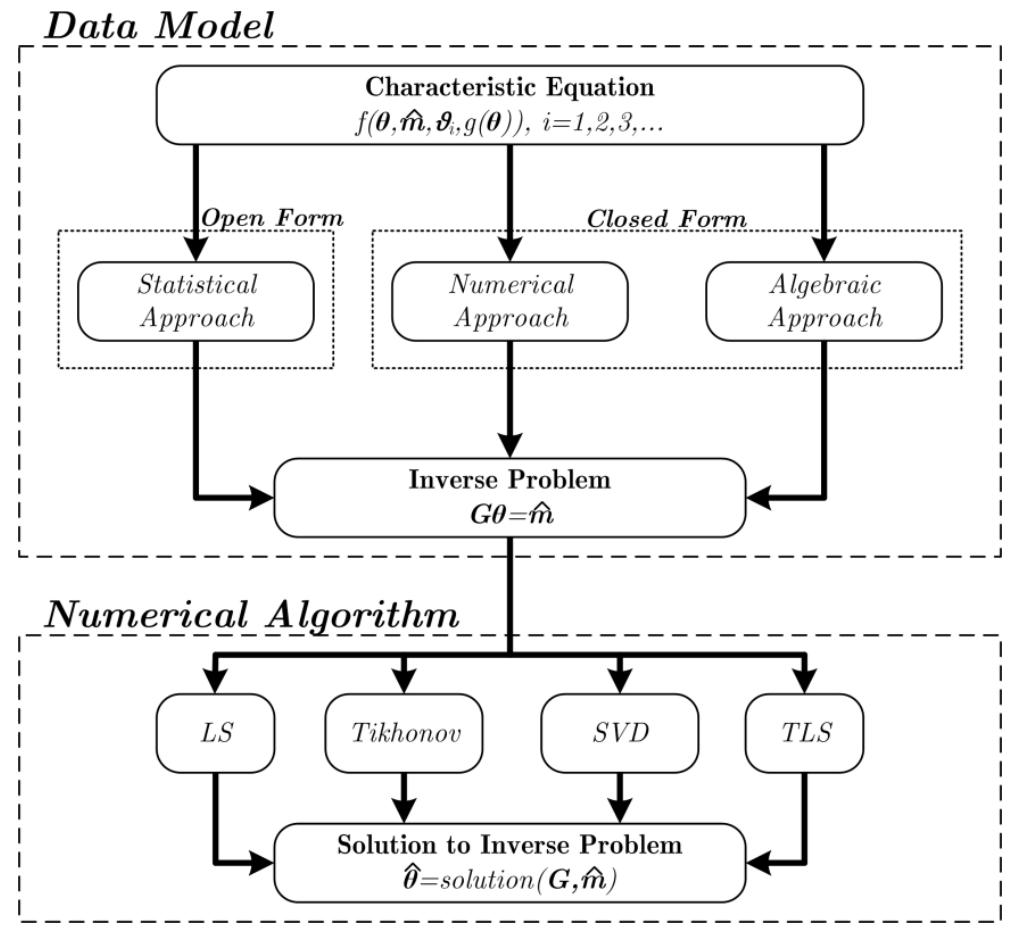

Fig. 7.1 General framework for localization algorithms.

localization algorithm, for a given scenario, is that whose triplet best fits to the particular conditions of such scenario.

\subsubsection{Setting a General Notation}

Because several localization algorithms, which have been developed in different works, will be described, analyzed and compared, it is advisable to set a general notation for the most used parameters and for those that are common to all algorithms analyzed herein. All the algorithms analyzed and developed in this thesis are referenced in a Cartesian coordinate system whose two dimensional case is always composed by the $(x, y)$ coordinates and the three dimensional one for $(x, y, z)$. It is shown in Fig. 7.2.

Generally, unless otherwise indicated, the origin of the coordinate system is set in an arbitrary spatial point. The vector containing the full set of target Cartesian coordinates is denoted as:

$$
\boldsymbol{\theta}=\left[\begin{array}{l}
x \\
y \\
z
\end{array}\right]_{3 \times 1}
$$

and the corresponding target range is denoted as $R_{S}$ and is defined as: 


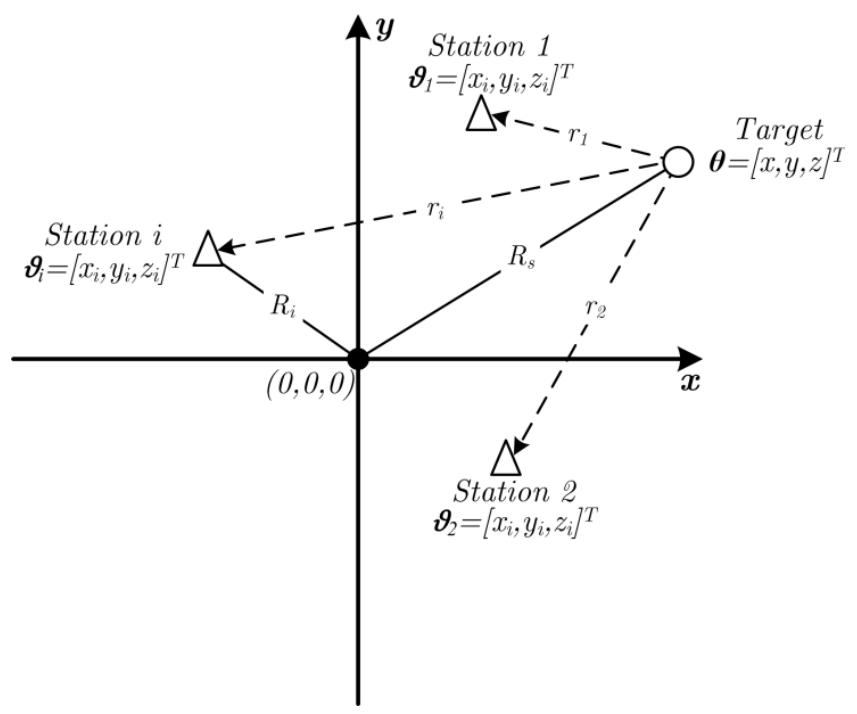

Fig. 7.2 General notation for localization algorithms.

$$
R_{s} \triangleq\|\boldsymbol{\theta}\|_{2}=\sqrt{x^{2}+y^{2}+z^{2}}
$$

The $i$ th station position is denoted as:

$$
\boldsymbol{\vartheta}_{i}=\left[\begin{array}{l}
x_{i} \\
y_{i} \\
z_{i}
\end{array}\right]_{3 \times 1}
$$

where the corresponding distance to the origin of the coordinate system is defined as:

$$
R_{i} \triangleq\left\|\boldsymbol{\vartheta}_{i}\right\|_{2}=\sqrt{x_{i}^{2}+y_{i}^{2}+z_{i}^{2}}
$$

The range from the target to the $i$ th receiving station is denoted as:

$$
r_{i} \triangleq\left\|\boldsymbol{\theta}-\boldsymbol{\vartheta}_{i}\right\|_{2}=\sqrt{\left(x-x_{i}\right)^{2}+\left(y-y_{i}\right)^{2}+\left(z-z_{i}\right)^{2}}
$$

Finally, we have defined the set of measured TDOA quantities in (6.6). However, it is more common, for simplicity, to use the set of range difference quantities, which simply can be obtained by multiplying the set of TDOA measurements by the speed of light as follows:

$$
m_{i, 1} \triangleq r_{i}-r_{1}=\operatorname{cTDOA}_{i, 1}, \quad i=2, \ldots, N_{s}
$$

and expressed in vector form they are: 


$$
\boldsymbol{m}=\left[\begin{array}{c}
m_{2,1} \\
\vdots \\
m_{N_{S}, 1}
\end{array}\right]_{\left(N_{S}-1\right) \times 1}
$$

where we have used the station 1 as the reference one. Moreover, in the case of noisy quantities, i.e., as obtained by measurements, they are denoted as $\widehat{m}_{i, 1}$ and $\widehat{\boldsymbol{m}}$, respectively.

\subsection{Open Form Algorithms}

The open form algorithms are based on the problem linearization, it is, this kind of algorithms linearizes (6.6) to obtain explicit linear relations between the range difference measurements $\widehat{\boldsymbol{m}}$ and the unknown target position $\boldsymbol{\theta}$. Normally, these algorithms use an iterative procedure and need for a suitable starting point (i.e., a priori information about $\boldsymbol{\theta}$ ). The most established open form algorithm, to solve the hyperbolic system of equations in Mode S Multilateration, is based on Taylor-series expansion $[3],[103]$.

\subsubsection{Taylor-Series Expansion Algorithm}

Initially, this method was presented in [103]. In this reference, the author used an algebraic procedure to describe the application of Taylor-series expansion to linearize (6.6) and obtain an explicit mathematical model to calculate the unknown position $\boldsymbol{\theta}$. Later, in [3] it was presented the accuracy analysis for some passive localization systems. In this reference the author shows an equivalent formulation to that presented in [103] but using a statistical approach. The statistical procedure allows the reader to get more insight of the real problem. Therefore, in this work the Taylor-series expansion method is summarized following the procedure shown in [3].

For real standard Mode S Multilateration systems (i.e., using only range difference measurements), it is more general to write (6.6) as follows

$$
\widehat{m}_{i, 1} \equiv c T D O A_{i, 1}=\left(f_{i}(x, y, z)-f_{1}(x, y, z)\right)+n_{i, 1}
$$

where $n_{i, 1}$ is the equivalent noise for the range difference measurement between the $i$ th station and the reference one. This noise basically depends on the capacity of each station to receive, time tag and sends to the CPS the corresponding TOA measurement. However, in a general sense, the noise is assumed to be zero-mean and Gaussian distributed (see $§ 2.1 .2$ for more details). 
The $N_{s}-1$ range difference equations in the form of (7.8) can be expressed in vector notation as follows:

$$
\widehat{\boldsymbol{m}}=\boldsymbol{m}(\boldsymbol{\theta})+\boldsymbol{n}
$$

where $\widehat{\boldsymbol{m}}$ is a $N_{s}-1$ column vector which contains all the range difference measurements, $\boldsymbol{m}(\boldsymbol{\theta})$ is also a $N_{s}-1$ column vector that takes the following form:

$$
\boldsymbol{m}(\boldsymbol{\theta})=\left[\begin{array}{c}
f_{i}(x, y, z)-f_{1}(x, y, z) \\
\vdots \\
f_{N_{S}}(x, y, z)-f_{1}(x, y, z)
\end{array}\right]_{\left(N_{s}-1\right) \times 1}, \quad i=1, \ldots, N_{s}
$$

Finally, $\boldsymbol{n}$ is the equivalent range difference noise measurement vector of $N_{s}-1$ size and it is assumed to be a multivariate zero mean and Gaussian distributed with covariance matrix given by:

$$
\boldsymbol{N}=E\left[(\boldsymbol{n}-E[\boldsymbol{n}])(\boldsymbol{n}-E[\boldsymbol{n}])^{T}\right]
$$

where $E[$ ] denotes the expectation operator. The form of this covariance matrix depends on the techniques to estimate the TOA/TDOA values.

In this way, the likelihood function for the unknown target position is given by [3]:

$$
\Lambda(\boldsymbol{\theta})=\frac{1}{(2 \pi)^{\frac{N_{S}-1}{2}} \operatorname{det}(\boldsymbol{N}(\boldsymbol{\theta}))^{\frac{1}{2}}} e^{-\frac{1}{2}\left\{(\hat{\boldsymbol{m}}-\boldsymbol{m}(\boldsymbol{\theta}))^{T} \boldsymbol{N}(\boldsymbol{\theta})^{-1}(\hat{\boldsymbol{m}}-\boldsymbol{m}(\boldsymbol{\theta}))\right\}}
$$

where $\operatorname{det}(\boldsymbol{N}(\boldsymbol{\theta}))$ denotes the determinant of the $\left(N_{S}-1\right) \times\left(N_{S}-1\right)$ measurement covariance matrix $\boldsymbol{N}(\boldsymbol{\theta})$. In this sense, the target position, for a given system and a set of measurements, is that $\boldsymbol{\theta}$ which maximizes the likelihood function (7.12) and therefore minimizes the following function:

$$
Q(\boldsymbol{\theta})=(\widehat{\boldsymbol{m}}-\boldsymbol{m}(\boldsymbol{\theta}))^{T} \boldsymbol{N}(\boldsymbol{\theta})^{-1}(\widehat{\boldsymbol{m}}-\boldsymbol{m}(\boldsymbol{\theta}))
$$

In order to find the $\boldsymbol{\theta}$ minimizing (7.13), the function $\boldsymbol{m}(\boldsymbol{\theta})$ is linearized by a Taylor-series expansion centred in a suitable staring point $\boldsymbol{\theta}_{0}=$ $\left[x_{0}, y_{0}, z_{0}\right]^{T}$ and it is only retained the terms of zero and first order, as follows:

$$
\boldsymbol{m}(\boldsymbol{\theta})=\boldsymbol{m}\left(\boldsymbol{\theta}_{0}\right)+\boldsymbol{G}\left(\boldsymbol{\theta}-\boldsymbol{\theta}_{0}\right)
$$

where $\boldsymbol{G}$ is the TDOA Jacobian matrix which takes the following form: 


$$
\boldsymbol{G}=\frac{1}{c}\left[\begin{array}{cccc}
\frac{x_{0}-x_{2}}{r_{2}}-\frac{x_{0}-x_{1}}{r_{1}} & \frac{y_{0}-y_{2}}{r_{2}}-\frac{y_{0}-y_{1}}{r_{1}} & \frac{z_{0}-z_{2}}{r_{2}}-\frac{z_{0}-z_{1}}{r_{1}} \\
\vdots & \vdots & \vdots \\
\frac{x_{0}-x_{N_{S}}}{r_{N_{S}}}-\frac{x_{0}-x_{1}}{r_{1}} & \frac{y_{0}-y_{N_{S}}}{r_{N_{S}}}-\frac{y_{0}-y_{1}}{r_{1}} & \frac{z_{0}-z_{N_{S}}}{r_{N_{S}}}-\frac{z_{0}-z_{1}}{r_{1}}
\end{array}\right]_{\left[N_{S}-1\right] \times 3}
$$

Now, defining $\widehat{\boldsymbol{m}}_{\Delta}=\widehat{\boldsymbol{m}}-\boldsymbol{m}\left(\boldsymbol{\theta}_{0}\right)$ and $\Delta \boldsymbol{\theta}=\boldsymbol{\theta}-\boldsymbol{\theta}_{0}$, then the function $Q$ in (7.13) can be rewritten as follows:

$$
Q(\Delta \boldsymbol{\theta})=\left(\widehat{\boldsymbol{m}}_{\Delta}-\boldsymbol{G} \Delta \boldsymbol{\theta}\right)^{T} \boldsymbol{N}(\boldsymbol{\theta})^{-1}\left(\widehat{\boldsymbol{m}}_{\Delta}-\boldsymbol{G} \Delta \boldsymbol{\theta}\right)
$$

Finally, the derivatives of (7.16) with respect to each component of $\Delta \boldsymbol{\theta}$ must be calculated and then set them to zero to obtain a direct expression for $\boldsymbol{\Delta} \boldsymbol{\theta}$. After several algebraic steps it can be demonstrated that:

$$
\widehat{\boldsymbol{\theta}}=\left(\boldsymbol{G}^{T} \boldsymbol{N}(\boldsymbol{\theta})^{-1} \boldsymbol{G}\right)^{-1} \boldsymbol{G}^{T} \boldsymbol{N}(\boldsymbol{\theta})^{-1} \widehat{\boldsymbol{m}}_{\Delta}+\boldsymbol{\theta}_{0}
$$

where the variable $\boldsymbol{\theta}$ has been changed for $\widehat{\boldsymbol{\theta}}$ just to emphasize that this is the variable estimated by the Maximum Likelihood Estimator (MLE) (7.12). Usually, due to the fact that the covariance matrix $\boldsymbol{N}(\boldsymbol{\theta})$ depends on the true target position $\boldsymbol{\theta}$ (which obviously is unknown), it is removed from (7.17), assuming an identity matrix. This assumption is only a practical mathematical simplification, because this matrix generally is not equal to the identity one. Physically, this assumption means that all the stations are equally affected by the noise; a fact that is not entirely true but it is a valid assumption to solve the problem. Moreover, (7.17) should be refined by several iterations until the method reaches a good accuracy. This iterative procedure can be expressed as follows:

$$
\begin{gathered}
\widehat{\boldsymbol{\theta}}^{k}=\left(\boldsymbol{G}\left(\widehat{\boldsymbol{\theta}}^{k-1}\right)^{T} \boldsymbol{G}\left(\widehat{\boldsymbol{\theta}}^{k-1}\right)\right)^{-1} \boldsymbol{G}\left(\widehat{\boldsymbol{\theta}}^{k-1}\right)^{T} \widehat{\boldsymbol{m}}_{\Delta}\left(\widehat{\boldsymbol{\theta}}^{k-1}\right)+\widehat{\boldsymbol{\theta}}^{k-1}, \\
k=1, \ldots, K
\end{gathered}
$$

where $\widehat{\boldsymbol{\theta}}^{0}=\boldsymbol{\theta}_{0}, \widehat{\boldsymbol{m}}_{\Delta}\left(\widehat{\boldsymbol{\theta}}^{k-1}\right)=\widehat{\boldsymbol{m}}-\boldsymbol{m}\left(\widehat{\boldsymbol{\theta}}^{k-1}\right)$ and $K$ is the maximum number of refinement iterations. It is worth to say that $\boldsymbol{\theta}_{0}$ is not necessary a previous estimation of the true target position $\boldsymbol{\theta}$. As it will be shown later, for some applications the starting point can be assumed as a fixed value for all the coverage area. The value of the starting point $\boldsymbol{\theta}_{0}$ normally has an important influence on the convergence of the method. This fact will be analyzed in later in this section.

Finally, the iterative procedure in (7.18) needs a stopping criterion and which is usually provided by the Euclidean difference between the solution at the $k$ th iteration and the $k$ th- 1 . When the difference between these two solutions is smaller than a predefined spatial threshold, the algorithm stops 
the iterative procedure, otherwise, it continues with the refinement process. This criterion can be expressed as follows:

$$
\left\|\widehat{\boldsymbol{\theta}}^{k}-\widehat{\boldsymbol{\theta}}^{k-1}\right\|_{2}= \begin{cases}\leq \Delta_{S}, & \text { stop } \\ >\Delta_{S}, & \text { continue }\end{cases}
$$

where $\Delta_{\mathrm{S}}$ is the predefined spatial threshold.

Equation (7.18) can be also seen as a Least Squares (LS) solution of the MLAT localization problem, which is the minimum 2-norm solution, it is, that solution which satisfy (cf. (6.24) and (6.25)):

$$
\widehat{\boldsymbol{\theta}}=\arg \min \left\{\|\boldsymbol{G} \boldsymbol{\theta}-\widehat{\boldsymbol{m}}\|_{2}^{2}\right\}
$$

The matrix $\left(\boldsymbol{G}^{T} \boldsymbol{G}\right)^{-1} \boldsymbol{G}^{T}$ in (7.28) is known in the literature [72] as the pseudoinverse matrix (see also $\S 6.4$ ) and it is denoted by $\boldsymbol{G}^{\dagger}$. Thus, the iterative estimator in (7.18) can be alternatively expressed as follows:

$$
\widehat{\boldsymbol{\theta}}^{k}=\boldsymbol{G}^{\dagger}\left(\widehat{\boldsymbol{\theta}}^{k-1}\right) \widehat{\boldsymbol{m}}_{\Delta}\left(\widehat{\boldsymbol{\theta}}^{k-1}\right)+\widehat{\boldsymbol{\theta}}^{k-1}, \quad k=1, \ldots, K
$$

As we have previously analyzed in [20], this strategy does not always provide acceptable accuracies because, in many operational conditions, the matrix $\boldsymbol{G}$ has some linearly dependent equations and in these cases the solution provided by (7.18) or (7.21) presents big errors. Therefore, we can conclude that solving the localization problem in the sense of LS has some disadvantages. Numerically, the most important one is related with the Hadamard's conditions [8] and is that the LS solution (i.e., that one provided by the pseudoinverse matrix) does not always satisfy the three Hadamard's conditions and therefore, for several cases, the solution provided by (7.18) may diverge to solutions that are very far from the exact one; that is, the localization problem becomes ill-conditioned. However, on the other hand, this algorithm has the important advantage that it is the only one that is linearly related to the noise, whilst the closed form algorithms are quadratically noise-dependent.

\section{About the Starting Point}

As we stated before, when using Taylor-series expansion method it is necessary to provide a starting point or a previous estimation about the position. Depending on the application scenario, this starting point can be defined as a fixed point in the scenario or it can be estimated by a previous execution of a closed form algorithm.

For airport surface surveillance, it has been found that defining the centre of the airport as the starting point, sometimes it is enough to obtain satisfactory results by using the LS solution. Nevertheless, it is worth to 
remember that the use of the LS solution does not always guarantee the convergence of the method.

For the surveillance out of the system perimeter (normally WAM) it is necessary to use a closed form algorithm to find the starting point. The application of a closed form algorithm to estimate the starting point is justified in the sense that, in many cases, this kind of algorithms are noisesensitive and the use of a Taylor-expansion method improves that previous solution.

Another important aspect is that the most ill-conditioned position component usually is the vertical one (i.e., the aircraft height) and all the numerical methods are affected by this problem. In this way, one feasible and reliable option is to take as the vertical component of the starting point the barometric altitude, which is present in all the downlink messages (Downlink Format -DF- 4, 5, 17, 20 and 21).

Although, as it will be shown in the results section, the most suitable source for the starting point depends on the system geometry, coverage range, number of available stations, etc., but, there is always a practical strategy to estimate it.

\subsection{Closed Form Algorithms}

On the other hand, as we have described in $\S 7.1$ the closed form algorithms can either define a quadratic relation between the range difference measurements $\widehat{\boldsymbol{m}}$ and the unknown target position $\boldsymbol{\theta}$ or directly define a linear inverse problem by algebraic manipulation of the hyperbolic equations. They can be one or two step algorithms, and they can provide one or two solutions of the target position. In the last case, it is necessary to choose one of the two possible solutions (i.e., in this cases they solve a quadratic unknown variable). Unlike the open form algorithms, the closed form ones do not need a suitable starting point but, as it will be shown later, the closed form algorithms introduce quadratic or cubic noise term in the corresponding mathematical model, that in some cases can lead to poorer accuracy levels. After our literature revision, we have found the most relevant and representative closed form algorithms, which can be used to solve the localization problem in Mode S MLAT, are those proposed by Schmidt [104], Smith and Abel [105-106], Friedlander [107], Schau and Robinson [108], Chan and Ho [109-110], the application of Bancroft algorithm [111] (initially developed for GPS), and an interesting algorithm reported in the open license website Wikipedia $^{\circledR}[112]$. 


\subsubsection{Schmidt Algorithm (Plane Intersection)}

This algorithm was proposed by Schmidt [104]. It is based on the plane intersection principle, which states that the range differences to three stations, of known positions, provide a straight line of position that geometrically represents the major axis of a general conic (e.g., ellipse, hyperbola, parabola), whose focus (in the absence of measurement noise) is the unknown target location. This algorithm is a one step algorithm that provides one solution for the target position. The characteristic equation of this algorithm is obtained by algebraic manipulation of the range difference equations (cf. (6.6)), i.e., by combining several equations in the form of (6.6) until obtaining a model whose only unknown variables are the set of target position coordinates. With this procedure, this algorithm directly leads to a linear inverse problem without any statistical or numerical assumption, i.e., this algorithm is composed of an algebraic approach based model. The formulation for this algorithm is summarized below.

In general terms, the main objective of the plane intersection principle is to set the unknown target location coordinates and the known parameters (e.g., stations positions and measurements) in a linear equation as follows:

$$
A x+B y+C z=D
$$

This equation represents a straight line in the two dimensional (i.e., without the $\mathrm{z}$ component) case or a plane in the three dimensional one.

Thus, after algebraic manipulation of the range difference equations (6.6), the characteristic equation of this algorithm is expressed as follows:

$$
\begin{aligned}
{\left[x_{1} \widehat{m}_{i, 2}+x_{2} \widehat{m}_{1, i}\right.} & \left.+x_{3}\left(-\widehat{m}_{i, 2}-\widehat{m}_{1, i}\right)\right] x \\
& +\left[y_{1} \widehat{m}_{i, 2}+y_{2} \widehat{m}_{1, i}+y_{3}\left(-\widehat{m}_{i, 2}-\widehat{m}_{1, i}\right)\right] y \\
& +\left[z_{1} \widehat{m}_{i, 2}+z_{2} \widehat{m}_{1, i}+z_{3}\left(-\widehat{m}_{i, 2}-\widehat{m}_{1, i}\right)\right] z \\
& =\frac{1}{2}\left[\widehat{m}_{2,1} \widehat{m}_{i, 2} \widehat{m}_{1, i}+R_{1}^{2} \widehat{m}_{i, 2}+R_{2}^{2} \widehat{m}_{1, i}\right. \\
& \left.+R_{i}^{2}\left(-\widehat{m}_{i, 2}-\widehat{m}_{1, i}\right)\right], \quad i=3, \ldots, N_{s}
\end{aligned}
$$

where the origin of the coordinates system is arbitrary. Note that this equation is composed by measurements provided by three stations. As it was stated in $\S 6.1 .1$, to preserve the independency among equations it is not advisable to use all the possible combinations. For this reason, it is assumed in (7.23) the index $i$ to be $i=3, \ldots, N_{s}$. In other words, to describe it in the same sense than $\S 6.1 .1$, this algorithm requires two reference stations to form one equation and, without loss of generality, we assume these two reference stations to be the stations 1 and 2. Therefore, for $N_{s}$ available 
stations, only a set of $N_{s}-2$ equations can be set of the inverse localization problem.

The coefficients of (7.22) can be easily identified from the characteristic equation (7.23) and they explicitly are:

$$
\begin{gathered}
A_{i}=x_{1} \widehat{m}_{i, 2}+x_{2} \widehat{m}_{1, i}+x_{3}\left(-\widehat{m}_{i, 2}-\widehat{m}_{1, i}\right) \\
B_{i}=y_{1} \widehat{m}_{i, 2}+y_{2} \widehat{m}_{1, i}+y_{3}\left(-\widehat{m}_{i, 2}-\widehat{m}_{1, i}\right) \\
C_{i}=z_{1} \widehat{m}_{i, 2}+z_{2} \widehat{m}_{1, i}+z_{3}\left(-\widehat{m}_{i, 2}-\widehat{m}_{1, i}\right) \\
D_{i}=\frac{1}{2}\left[\widehat{m}_{2,1} \widehat{m}_{i, 2} \widehat{m}_{1, i}+R_{1}^{2} \widehat{m}_{i, 2}+R_{2}^{2} \widehat{m}_{1, i}+R_{i}^{2}\left(-\widehat{m}_{i, 2}-\widehat{m}_{1, i}\right)\right]
\end{gathered}
$$

Finally, the localization inverse problem can be comprised in vectormatrix form as follows:

$$
\boldsymbol{G}_{\text {Schmidt }} \widehat{\boldsymbol{\theta}}_{\text {Schmidt }}=\widehat{\boldsymbol{m}}_{\text {Schmidt }}
$$

where

$$
\boldsymbol{G}_{\text {Schmidt }}=\left[\begin{array}{ccc}
A_{3} & B_{3} & C_{3} \\
\vdots & \vdots & \vdots \\
A_{N_{S}} & B_{N_{S}} & C_{N_{S}}
\end{array}\right]_{\left(N_{S}-2\right) \times 3}
$$

and

$$
\widehat{\boldsymbol{m}}_{\text {Schmidt }}=\left[\begin{array}{c}
D_{3} \\
\vdots \\
D_{N_{S}}
\end{array}\right]_{\left(N_{S}-2\right) \times 1}
$$

Several observations can be made to this algorithm. To form a characteristic equation a set of three stations are required. Then, to estimate a target location composed by a set of $n$ coordinates, it requires at least $n+2$ stations (i.e., four stations for $2 \mathrm{D}$ localization and 5 for $3 \mathrm{D}$ localization). From (7.24)-(7.27) it can be seen that this algorithm introduces a cubic noise term in the measurement vector, which is evident with the term $\widehat{m}_{2,1} \widehat{m}_{i, 2} \widehat{m}_{1, i}$, and also introduces noise terms in the coefficient matrix, aspects that can degrade the algorithm performance in the case of highly noisy measurements. The data model is set without any restriction; hence, also the inverse problem is solved without any restriction. On the other hand, the computational cost of using this algorithm is very low. 


\subsubsection{Smith and Abel Algorithm (Spherical Interpolation)}

This algorithm was proposed by Smith and Abel [105-106]. It is based on the spherical interpolation principle, which states that the range difference between two stations (e.g., the $i$ th station and the reference one), of known positions, geometrically represents (in the absence of noise) the distance from the $i$ th station to a sphere centred at the target position and passing through the reference one. This algorithm is a one step algorithm that provides one solution for the target position. The characteristic equation is obtained by mathematically manipulating only one equation in the form of (6.6); hence, this equation contains two unknown and mutually dependent parameters: the target position and the range (or distance) from this to a reference point. Thus, this algorithm solves the problem by using two error functions in the sense of LS and it is therefore called spherical LS criteria. The first criterion is used to obtain an expression that explicitly relates the target position $\boldsymbol{\theta}$ and its distance $R_{S}$ to a known reference point (henceforth called target range), then the second one is used to obtain a direct solution for $R_{S}$ and finally, this latter solution is introduced into the first LS criterion to obtain the solution for the target position. In this algorithm both the target range and the target position are assumed to be numerically independent, i.e., this algorithm is composed of a numerical approach based model. This algorithm is summarized below. It is important to take into account that the formulation below is expressed by assuming the origin of the coordinate system to be at the position of the reference station (station 1 ), i.e., $\boldsymbol{\vartheta}_{i}=\boldsymbol{\vartheta}_{i}-\boldsymbol{\vartheta}_{1}, R_{1}=0$ and $R_{s}=r_{1}$.

The characteristic equation, which is formed by two stations (the $i$ th station and the reference one) is expressed as follows:

$$
2 \boldsymbol{\vartheta}_{i}^{T} \boldsymbol{\theta}=R_{i}^{2}-\widehat{m}_{i, 1}^{2}-2 R_{s} \widehat{m}_{i, 1}, \quad i=2, \ldots, N_{s}
$$

Due to the fact that the measurements are not precisely performed, this algorithm proposes that the above equation has to be really expressed in terms of an error equation as follows:

$$
\epsilon_{i, 1}=R_{i}^{2}-\widehat{m}_{i, 1}^{2}-2 R_{s} \widehat{m}_{i, 1}-2 \boldsymbol{\vartheta}_{i}^{T} \boldsymbol{\theta}, \quad i=2, \ldots, N_{s}
$$

which expressed in vector-matrix form leads to the following data model:

$$
\boldsymbol{\epsilon}=\boldsymbol{\delta}-2 R_{S} \widehat{\boldsymbol{m}}-2 \boldsymbol{S} \boldsymbol{\theta}
$$

where

$$
\boldsymbol{S}=\left[\begin{array}{ccc}
x_{2} & y_{2} & z_{2} \\
\vdots & \vdots & \vdots \\
x_{N_{S}} & y_{N_{S}} & z_{N_{S}}
\end{array}\right]_{\left(N_{S}-1\right) \times 3}
$$




$$
\boldsymbol{\delta}=\left[\begin{array}{c}
R_{2}^{2}-\widehat{m}_{2,1}^{2} \\
\vdots \\
R_{N_{S}}^{2}-\widehat{m}_{N_{S}, 1}^{2}
\end{array}\right]_{\left(N_{S}-1\right) \times 1}
$$

The data model in (7.30) is linear in the target position $\boldsymbol{\theta}$ given the target range $R_{S}$ and it is linear in $R_{S}$ given $\boldsymbol{\theta}$. Then, when it happens (i.e., the error vector linear in the unknowns), a solution for the target position $\boldsymbol{\theta}$ given $R_{S}$ can be found by the minimization of the 2-norm of (7.30), which is also known as the LS solution and it is as follows:

$$
\widehat{\boldsymbol{\theta}}=\frac{1}{2} \boldsymbol{S}^{\dagger}\left(\boldsymbol{\delta}-2 R_{s} \widehat{\boldsymbol{m}}\right)
$$

where $\boldsymbol{S}^{\dagger}$ is the pseudoinverse matrix of $\boldsymbol{S}$. Moreover, this solution can be seen as the solution of the following inverse problem:

$$
\boldsymbol{S} \widehat{\boldsymbol{\theta}}=\frac{1}{2}\left(\boldsymbol{\delta}-2 R_{S} \widehat{\boldsymbol{m}}\right)
$$

Then, because $R_{S}$ is still unknown and no solution can be found by using (7.32), the authors propose to use a second error function by substituting (7.32) into (7.30) and thus obtain an explicit solution for $R_{S}$, as follows:

$$
\boldsymbol{\epsilon}^{\prime}=\boldsymbol{\delta}-2 R_{S} \widehat{\boldsymbol{m}}-\boldsymbol{S} \boldsymbol{S}^{\dagger}\left(\boldsymbol{\delta}-2 R_{S} \widehat{\boldsymbol{m}}\right)=\left(\boldsymbol{I}-\boldsymbol{S} \boldsymbol{S}^{\dagger}\right)\left(\boldsymbol{\delta}-2 R_{S} \widehat{\boldsymbol{m}}\right)
$$

or equivalently

$$
\boldsymbol{\epsilon}^{\prime}=\boldsymbol{P}_{S}^{\perp}\left(\boldsymbol{\delta}-2 R_{S} \widehat{\boldsymbol{m}}\right)
$$

where

$$
\boldsymbol{P}_{S}^{\perp}=\boldsymbol{I}-\boldsymbol{S} \boldsymbol{S}^{\dagger}
$$

Now, it is clear that the new equation error is linear in the unique unknown $R_{S}$. In this sense, it can be solved by minimizing also the 2-norm of $\boldsymbol{\epsilon}^{\prime}$ in (7.35). This solution is expressed as follows:

$$
\hat{R}_{S}=\frac{\widehat{\boldsymbol{m}}^{T} \boldsymbol{P}_{S}^{\perp} \boldsymbol{P}_{S}^{\perp} \boldsymbol{\delta}}{2 \widehat{\boldsymbol{m}}^{T} \boldsymbol{P}_{S}^{\perp} \boldsymbol{P}_{S}^{\perp} \widehat{\boldsymbol{m}}}
$$

Finally, a solution for the target position $\boldsymbol{\theta}$ can be obtained by substituting the value in (7.37) into (7.32), whose resulting expression is:

$$
\widehat{\boldsymbol{\theta}}_{\text {Smith\&Abel }}=\frac{1}{2} \boldsymbol{S}^{\dagger}\left(\boldsymbol{I}-\frac{\widehat{\boldsymbol{m}} \widehat{\boldsymbol{m}}^{T} \boldsymbol{P}_{S}^{\perp} \boldsymbol{P}_{S}^{\perp}}{\widehat{\boldsymbol{m}}^{T} \boldsymbol{P}_{S}^{\perp} \boldsymbol{P}_{S}^{\perp} \widehat{\boldsymbol{m}}}\right) \boldsymbol{\delta}
$$


This final solution can be seen as the solution of the following inverse problem:

$$
\boldsymbol{G}_{\text {Smith\&Abel }} \widehat{\boldsymbol{\theta}}_{\text {Smith\&Abel }}=\widehat{\boldsymbol{m}}_{\text {Smith\&Abel }}
$$

where

$$
\boldsymbol{G}_{\text {Smith\&Abel }}=\boldsymbol{S}
$$

and

$$
\widehat{\boldsymbol{m}}_{\text {Smith\&Abel }}=\frac{1}{2}\left(\boldsymbol{I}-\frac{\widehat{\boldsymbol{m}} \widehat{\boldsymbol{m}}^{T} \boldsymbol{P}_{S}^{\perp} \boldsymbol{P}_{S}^{\perp}}{\widehat{\boldsymbol{m}}^{T} \boldsymbol{P}_{S}^{\perp} \boldsymbol{P}_{S}^{\perp} \widehat{\boldsymbol{m}}}\right) \boldsymbol{\delta}
$$

To form a characteristic equation in this algorithm a set of two stations are required. Then, to estimate a target location composed by a set of $n$ coordinates, it requires at least $n+1$ stations (i.e., three for $2 \mathrm{D}$ localization and four for 3D localization). Statistically, the solution as provided by the estimator in (7.38) is biased and not optimal due to the mutual independence that is assumed between the target position and range, and because the inverse problem is solved without any restriction that compensates that assumption of independence. Moreover, for this method the coefficient matrix is constant for a given system as it only depends on the stations position. On the contrary, since the measurement vector (7.41) is a modified version of the pure range differences vector, it introduces quadratic noise terms and its existence directly depends on the stability of calculating the pseudoinverse of $\boldsymbol{S}$ (cf. (7.36)). On the other hand, the computational cost of using this algorithm is very low.

\subsubsection{Friedlander Algorithm (Spherical Interpolation)}

This algorithm was proposed by Friedlander [107]. It is also based on the spherical interpolation principle as Smith and Abel algorithm (see §7.3.2) and it was presented as an alternative solution to it. This algorithm is a one step algorithm that provides one solution for the target position. The characteristic equation is obtained equivalently to Smith and Abel and, hence, it also contains two unknown and mutually dependent parameters: the target position and the target range. The main difference between these two algorithms is that Friedlander solves the problem by eliminating one of the two unknown parameters in the data model; the target range to the reference station $r_{1}$ (also here the station 1 is assumed as the reference one). It is performed by premultiplying the corresponding data model by a matrix that contains the measurement vector in its null space. In this algorithm, due to the elimination of one of the two mutually dependent parameters, which is in some sense an strategy similar of assuming them to 
be mutually independent, the solution is provided with any restriction (i.e., that relating the two parameters), hence, this algorithm is composed of a numerical approach based model. Although the author states in his original work [107] that this algorithm is mathematically equivalent to that of Smith and Abel [105], we have observed in the results that they present different performance. This algorithm is summarized below. It is important to take into account that the formulation below does not set the origin of the coordinate system at any receiving station position but at an arbitrary point.

The characteristic equation, which is formed by two stations (the ith station and the reference one) is expressed as follows:

$$
2\left(\boldsymbol{\vartheta}_{i}-\boldsymbol{\vartheta}_{1}\right)^{T} \boldsymbol{\theta}=\left(R_{i}^{2}-R_{1}^{2}\right)-\widehat{m}_{i, 1}^{2}-2 r_{1} \widehat{m}_{i, 1}
$$

Note that this equation is equivalent to that of Smith and Abel in (7.28). The main difference is due to the fact that (7.28) is obtained by setting the origin of the coordinate system at the position of the reference station whilst in (7.42) this origin is set in an arbitrary point. Then, the data model expressed in vector-matrix form is as follows:

$$
\boldsymbol{S}_{\text {Fried }} \boldsymbol{\theta}=\boldsymbol{\mu}-r_{1} \widehat{\boldsymbol{m}}
$$

where

$$
\begin{gathered}
\boldsymbol{S}_{\text {Fried }}=\left[\begin{array}{ccc}
x_{2}-x_{1} & y_{2}-y_{1} & z_{2}-z_{1} \\
\vdots & \vdots & \vdots \\
x_{N_{S}}-x_{1} & y_{N_{S}}-y_{1} & z_{N_{S}}-z_{1}
\end{array}\right]_{\left(N_{S}-1\right) \times 3} \\
\boldsymbol{\mu}=\frac{1}{2}\left[\begin{array}{c}
R_{2}^{2}-R_{1}^{2}-\widehat{m}_{2,1}^{2} \\
\vdots \\
R_{N_{S}}^{2}-R_{1}^{2}-\widehat{m}_{N_{S}, 1}^{2}
\end{array}\right]_{\left(N_{S}-1\right) \times 1}
\end{gathered}
$$

Equivalently to Smith and Abel, the data model in (7.43) is linear in $\boldsymbol{\theta}$ given $r_{1}$ and it is linear in $r_{1}$ given $\boldsymbol{\theta}$. In this point, the author proposes to eliminate $r_{1}$ by premultiplying the data model in (7.43) by a matrix $\boldsymbol{M}$ containing the measurement vector $\widehat{\boldsymbol{m}}$ in its null space, such that $\boldsymbol{M} \widehat{\boldsymbol{m}}=\mathbf{0}$, as follows:

$$
\boldsymbol{M}=(\boldsymbol{I}-\boldsymbol{Z}) \boldsymbol{D}
$$

where $\boldsymbol{I}$ is a $\left(N_{s}-1\right) \times\left(N_{s}-1\right)$ identity matrix and

$$
\boldsymbol{D}=[\operatorname{diag}(\widehat{\boldsymbol{m}})]^{-1}=\left[\begin{array}{lll}
\hat{m}_{2,1} & & \\
& \ddots & \\
& & \widehat{m}_{N_{S}, 1}
\end{array}\right]_{\left(N_{s}-1\right) \times\left(N_{S}-1\right)}^{-1}
$$




$$
\boldsymbol{Z}=\left[\begin{array}{cccc}
0 & 1 & & 0 \\
& \ddots & \ddots & \\
& 0 & \ddots & 1 \\
1 & & & 0
\end{array}\right]_{\left(N_{S}-1\right) \times\left(N_{S}-1\right)}
$$

Matrix $\boldsymbol{Z}$ is really a circular shift matrix that can be obtained by circularly moving the columns of the identity matrix one step to the right.

After premultiplying (7.43) the inverse problem takes the following form:

$$
M \boldsymbol{S}_{\text {Fried }} \boldsymbol{\theta}=\boldsymbol{M} \boldsymbol{\mu}
$$

where the corresponding solution can be obtained as:

$$
\widehat{\boldsymbol{\theta}}_{\text {Fried }}=\left(\boldsymbol{S}_{\text {Fried }}^{T} \boldsymbol{M}^{T} \boldsymbol{M} \boldsymbol{S}_{\text {Fried }}\right)^{-1} \boldsymbol{S}_{\text {Fried }}^{T} \boldsymbol{M}^{T} \boldsymbol{M} \boldsymbol{\mu}
$$

This final solution can be seen as the solution of the following inverse problem:

$$
\boldsymbol{G}_{\text {Fried }} \widehat{\boldsymbol{\theta}}_{\text {Fried }}=\widehat{\boldsymbol{m}}_{\text {Fried }}
$$

where

$$
\boldsymbol{G}_{\text {Fried }}=\boldsymbol{M} \boldsymbol{S}_{\text {Fried }}^{T}
$$

and

$$
\widehat{\boldsymbol{m}}_{\text {Fried }}=\boldsymbol{M} \boldsymbol{\mu}
$$

To form a characteristic equation in this algorithm a set of two stations are required. Then, to estimate a target location composed by a set of $n$ coordinates, it requires at least $n+1$ stations (i.e., three for $2 \mathrm{D}$ localization and four for 3D localization). However, it can be demonstrated that matrix $\boldsymbol{M}$ is always a singular matrix (see $\S 6.3 .5$ ) with rank $N_{s}-2$. For this reason the author propose to use at least $n+2$ equations instead $n+1$. Based on our simulations, we can say that the non-fulfilment of this condition is the main reason of the bad performance of the algorithm under some situations but, in practical terms, this asseveration is not always true and the algorithm provides similar overall performance levels than the Smith and Abel algorithm. Moreover, also as Smith and Abel algorithm, this one is biased and not optimal, and the coefficient matrix of the inverse problem is constant for a given system. On the contrary, this method also uses a modified version of the pure range difference vector and it introduces quadratic noise terms. On the other hand, the computational cost of using this algorithm is also very low. 


\subsubsection{Schau and Robinson Algorithm (Spherical Intersection)}

This algorithm was proposed by Schau and Robinson [108]. It is based on the spherical intersection principle, which states that the range distance from the target to any station, of known position, geometrically represents (in the absence of noise) the radius of a sphere whose center is at the target position. This algorithm is a two step algorithm that provides two possible solutions for the target position; hence, a procedure to select one of them must be implemented. The characteristic equation is obtained by mathematically manipulating pairs of equations in the form of (6.1) to derive an expression equal to that of Smith and Abel (cf. (7.28)); hence, this equation also contains two unknown and mutually dependent parameters: the target position and the target range from this to a reference point. The difference in the procedure for developing the characteristic equation of this algorithm is that the authors use the unknown target ranges as function of the range differences to pairs of stations (e.g., the $i$ th station and the reference one), because the first ones are not measured in the standard version of MLAT systems. Furthermore, the proposed procedure for solving the problem is also different to those of Smith and Abel (see §7.3.2), and of Friedlander (see §7.3.3). In this algorithm, the main idea is to sequentially find the two unknown parameters by solving two separate problems. First, the target range to a reference point is obtained by solving a quadratic equation and then, an inverse problem is solved to find the target position. This inverse problem must to be solved twice; one for each solution of the quadratic equation for the target range. With this procedure, also the target position and range are assumed to be numerically independent; hence, this algorithm is composed of a numerical approach based model. This algorithm is summarized below. It is important to take into account that the formulation below is expressed by assuming the origin of the coordinate system to be at the position of the reference station (i.e., the station 1), i.e., $\boldsymbol{\vartheta}_{i}=\boldsymbol{\vartheta}_{i}-\boldsymbol{\vartheta}_{1}, R_{1}=0$ and $R_{s}=r_{1}$.

The characteristic equation, which is formed by two stations (the $i$ th station and the reference one), is expressed as follows:

$$
2 \boldsymbol{\vartheta}_{i}^{T} \boldsymbol{\theta}=R_{i}^{2}-\widehat{m}_{i, 1}^{2}-2 R_{s} \widehat{m}_{i, 1}, \quad i=2, \ldots, N_{s}
$$

Note that the above equation is equal to that of Smith and Abel (cf. (7.28)). Then, the data model of this algorithm, expressed in vector-matrix form, is as follows:

$$
\mathfrak{M} \boldsymbol{\theta}=\frac{1}{2}\left(\boldsymbol{\Delta}-2 R_{s} \widehat{\boldsymbol{m}}\right)
$$

where 


$$
\begin{aligned}
\mathfrak{M} & =\left[\begin{array}{ccc}
x_{2} & y_{2} & z_{2} \\
\vdots & \vdots & \vdots \\
x_{N_{S}} & y_{N_{S}} & z_{N_{S}}
\end{array}\right]_{\left(N_{S}-1\right) \times 3} \\
\boldsymbol{\Delta} & =\left[\begin{array}{c}
R_{2}^{2}-\widehat{m}_{2,1}^{2} \\
\vdots \\
R_{N_{S}}^{2}-\widehat{m}_{N_{S}, 1}^{2}
\end{array}\right]_{\left(N_{S}-1\right) \times 1}
\end{aligned}
$$

Note also that matrix $\mathfrak{M}$ and vector $\boldsymbol{\Delta}$ are equal to matrix $\boldsymbol{S}$ and vector $\boldsymbol{\delta}$ of Smith and Abel algorithm (cf. (7.31)). We have decided to do not use the same notation just to preserve it as presented in the original publications. Equivalently to Smith and Abel, in this algorithm the authors suggest using a spherical LS criterion for solving the inverse problem in (7.53), which leads to the following solution:

$$
\widehat{\boldsymbol{\theta}}=\frac{1}{2} \mathfrak{M}^{\dagger}\left(\boldsymbol{\Delta}-2 R_{s} \widehat{\boldsymbol{m}}\right)
$$

where $\mathfrak{M}^{\dagger}$ is the pseudoinverse matrix of $\mathfrak{M}$. As it was expected, this solution is also equivalent to that of Smith and Abel (cf. (7.32)). Indeed, at this point both algorithms are totally equivalent and their differences start with the way proposed for solving (7.55). When arriving to (7.31), Smith and Abel proposed to use a second spherical LS criterion, by substituting (7.31) into the first spherical LS criterion in (7.30), in order to explicitly obtain a unique solution for $R_{S}$ and, with this value, solve the linear inverse problem in (7.31). Herein, Schau proposed not to use a second spherical LS criterion but instead to introduce the LS solution (7.55) into the quadratic equation $R_{S}^{2}=\boldsymbol{\theta}^{T} \boldsymbol{\theta}$ and find its roots for $R_{S}$. After the appropriate mathematical operations, the final quadratic equation in $R_{S}$ is as follows:

$$
\begin{aligned}
R_{S}^{2}\left[4-4 \widehat{\boldsymbol{m}}^{T}\left(\mathfrak{M}^{\dagger}\right)^{T} \mathfrak{M}^{\dagger} \widehat{\boldsymbol{m}}\right] \\
+R_{S}\left[2 \widehat{\boldsymbol{m}}^{T}\left(\mathfrak{M}^{\dagger}\right)^{T} \mathfrak{M}^{\dagger} \boldsymbol{\Delta}+2 \Delta^{T}\left(\mathfrak{M}^{\dagger}\right)^{T} \mathfrak{M}^{\dagger} \widehat{\boldsymbol{m}}\right] \\
-\left[\Delta^{T}\left(\mathfrak{M}^{\dagger}\right)^{T} \mathfrak{M}^{\dagger} \Delta\right]=0
\end{aligned}
$$

The first step of this algorithm consists of finding the roots of (7.56). Once they are found, the second step consists of introducing the two values into the inverse problem (7.55) to obtain the corresponding solutions for the target position as follows:

$$
\widehat{\boldsymbol{\theta}}_{\text {Schau\&Robinson }}^{1,2}=\frac{1}{2} \mathfrak{M}^{\dagger}\left(\boldsymbol{\Delta}-2 \widehat{R}_{S}^{1,2} \widehat{\boldsymbol{m}}\right)
$$

For real applications the final solution must be chosen among the two provided by (7.57). For this, an intuitive and empirical procedure must be 
implemented. Finally, the final solution in (7.57) can be seen as the solution of the following inverse problem:

$$
\boldsymbol{G}_{\text {Schau\&Robinson }} \widehat{\boldsymbol{\theta}}_{\text {Schau\&Robinson }}^{1,2}=\widehat{\boldsymbol{m}}_{\text {Schau\&Ronbinson }}^{1,2}
$$

where

$$
\boldsymbol{G}_{\text {Schau\&Robinson }}=\mathfrak{M}
$$

and

$$
\widehat{\boldsymbol{m}}_{\text {Schau\&Ronbinson }}^{1,2}=\frac{1}{2}\left(\boldsymbol{\Delta}-2 \widehat{R}_{S}^{1,2} \widehat{\boldsymbol{m}}\right)
$$

To form a characteristic equation in this algorithm, a set of two stations are required. Then, to estimate a target location composed by a set of $n$ coordinates, it requires at least $n+1$ stations (i.e., three for $2 \mathrm{D}$ localization and four for 3D localization). Also, as the Smith and Abel algorithm, the solution (or solutions) provided by (7.57) is biased and not optimal due to the mutual independence that is assumed between the target position and range, and because the inverse problem is solved without any restriction that compensate that assumption of independence. Additionally, the quality of the solution is also subject to the quality of the procedure implemented to choose the final solution among the two possible. On the other hand, the coefficient matrix is constant for a given system as it only depends on the stations position. On the contrary, the measurement vector (7.60) is a modified version of the pure range difference vector, thus it also introduces quadratic noise terms and its existence directly depends on the quality of finding the roots of the quadratic equation (7.56), which could not exist. On the other hand, although the computational cost of using this algorithm is not as low as that of Smith and Abel or Friedlander algorithms, because the necessity of implementing the procedure for choosing the final solution, it can be considered still low.

\subsubsection{Chan and Ho Algorithm (Spherical Interpolation with Quadratic Correction)}

This algorithm was proposed by Chan and Ho [109-110]. It is also based on the spherical interpolation principle as Smith and Abel algorithm (§7.3.2) and it was presented as an improved, numerically corrected version of some closed form algorithms, specifically those of Smith and Abel, Friedlander (see §7.3.3) and Schau and Robinson (see §7.3.4), which are not optimal estimators in the statistical sense. This algorithm is a two step algorithm that provides two possible solutions for the target location; hence, a procedure to select one of them must be implemented. Nevertheless, the 
two steps proposed by Chan and Ho are two inverse problems, not as in the algorithms cited above. The first step, which we call initial estimation, consists of calculating an initial solution for both the target position $\boldsymbol{\theta}$ and the target range $r_{1}$ to the reference station, whilst the second one, which we call quadratic correction, consists of imposing a known constraint between $\boldsymbol{\theta}$ and $r_{1}$ in order to improve the accuracy of $\boldsymbol{\theta}$. The main objective of the second step is to compensate the mutual independence between target position and range that was assumed in the previous algorithms. The characteristic equation, which is only used for the first step, is obtained in a similar procedure as Smith and Abel and, hence, it also contains the two unknown and mutually dependent parameters of target position and range. The main difference with the previous algorithms is that they estimate the target range and then the target position (e.g., Smith and Abel or Schau and Robinson), or mathematically eliminate the target range from the data model (e.g., Friedlander), whilst the present one proposes to jointly estimate both parameters. However, this first step can be considered equivalent to the Smith and Abel algorithm as it also uses a spherical LS criterion and also assumes mutual independence between target position and range. For this reasons, Chan and Ho proposed the second step of quadratic correction that consists of obtaining a spatial increment that can be added to the initial estimation of the target position and thus force it to satisfy the constraint between it and the target range (cf. (6.1)). In other words, the second step is intended to make a better use of the information redundancy from the spherical interpolation based methods. The correction is performed on the squares of the target coordinates; from here the name of quadratic correction. On the other hand, due to the fact that the second step is just a correction inverse problem and not a localization one, we can classify the data model of this algorithm to the class of numerical approach based models. This algorithm is shown below. It is important to take into account that the formulation below does not set the origin of the coordinate system at any receiving station position but at an arbitrary point.

The characteristic equation, which is formed by two stations (the $i$ th station and the reference one) is expressed as follows:

$$
2\left(\boldsymbol{\vartheta}_{i}-\boldsymbol{\vartheta}_{1}\right)^{T} \boldsymbol{\theta}=\left(R_{i}^{2}-R_{1}^{2}\right)-\widehat{m}_{i, 1}^{2}-2 r_{1} \widehat{m}_{i, 1}, \quad i=2, \ldots, N_{s}
$$

Note that the above equation is equivalent to the ones of Smith and Abel (cf. (7.28)), Schau and Robinson (cf. (7.42)), and Friedlander (cf. (7.52)). Due to the fact that the measurements are not precisely performed, this algorithm, like Smith and Abel algorithm, also proposes to set the above equation in terms of an error equation as follows:

$$
\epsilon_{i, 1}=\frac{1}{2}\left(\widehat{m}_{i, 1}^{2}-R_{i}^{2}+R_{1}^{2}\right)+\left(\boldsymbol{\vartheta}_{i}-\boldsymbol{\vartheta}_{1}\right)^{T} \boldsymbol{\theta}+r_{1} \widehat{m}_{i, 1}, \quad i=2, \ldots, N_{s}
$$


which expressed in vector-matrix form leads to the following data model:

$$
\boldsymbol{\epsilon}=\boldsymbol{h}(\widehat{\boldsymbol{m}})-\boldsymbol{G}_{a}\left[\begin{array}{l}
\widehat{\boldsymbol{\theta}}_{a} \\
\hat{r}_{1}
\end{array}\right]
$$

where $\widehat{\boldsymbol{\theta}}_{a}$ is the first estimation of $\widehat{\boldsymbol{\theta}}, \hat{r}_{1}$ is the first estimation of the target range to the reference station, $\boldsymbol{h}(\widehat{\boldsymbol{m}})$ is expressed as a function of the range differences measurement vector to emphasize that it is a random vector instead a deterministic one, and

$$
\begin{gathered}
\boldsymbol{G}_{a}=-\left[\begin{array}{cccc}
x_{2}-x_{1} & y_{2}-y_{1} & z_{2}-z_{1} & \widehat{m}_{2,1} \\
\vdots & \vdots & \vdots & \vdots \\
x_{N_{S}}-x_{1} & y_{N_{S}}-y_{1} & z_{N_{S}}-z_{1} & \widehat{m}_{N_{s}, 1}
\end{array}\right]_{\left(N_{s}-1\right) \times 4} \\
\boldsymbol{h}(\widehat{\boldsymbol{m}})=\frac{1}{2}\left[\begin{array}{c}
\widehat{m}_{2,1}^{2}-\left(R_{2}^{2}-R_{1}^{2}\right) \\
\vdots \\
\widehat{m}_{N_{s}, 1}^{2}-\left(R_{N_{S}}^{2}-R_{1}^{2}\right)
\end{array}\right]_{\left(N_{S}-1\right) \times 1}
\end{gathered}
$$

The data model in (7.63) is linear in the target position and the target range (note the similarities with the previous algorithms). Then, in this case, assuming the target position and the target range mutually independent, a solution can be found as the minimization of the 2-norm of (7.63), which is also known as the LS solution and it is as follows:

$$
\left[\begin{array}{c}
\widehat{\boldsymbol{\theta}}_{a} \\
\hat{r}_{1}
\end{array}\right] \approx\left(\boldsymbol{G}_{a}^{T} \boldsymbol{G}_{a}\right)^{-1} \boldsymbol{G}_{a}^{T} \boldsymbol{h}(\widehat{\boldsymbol{m}})=\boldsymbol{G}_{a}^{\dagger} \boldsymbol{h}(\widehat{\boldsymbol{m}})
$$

where $\boldsymbol{G}_{a}^{\dagger}$ is the pseudoinverse matrix of $\boldsymbol{G}_{a}$.

This first step clearly shows the difference between this algorithm and the previous ones. As we commented in the introduction to this algorithm, Smith and Abel and Schau and Robinson arrive to the same data model (or equivalent) and then solve first for the target range and then for the target position, whilst Friedlander mathematically eliminates $r_{1}$ from the data model by premultiplying for a matrix that contains the measurement vector in its null space. On the contrary, this algorithm directly estimates both parameters in the same inverse problem. Moreover, the solution in (7.65) can be seen as the solution of the following inverse problem:

$$
\boldsymbol{G}_{a}\left[\begin{array}{c}
\widehat{\boldsymbol{\theta}}_{a} \\
\hat{r}_{1}
\end{array}\right]=\boldsymbol{h}(\widehat{\boldsymbol{m}})
$$

Then, the second step corrects the previous solutions $\widehat{\boldsymbol{\theta}}_{a}$ and $\hat{r}_{1}$ by calculating a spatial increment that force satisfying the following equality constraint: 


$$
\left(\widehat{\boldsymbol{\theta}}_{a, 1}-x_{1}\right)^{2}+\left(\widehat{\boldsymbol{\theta}}_{a, 2}-y_{1}\right)^{2}+\left(\widehat{\boldsymbol{\theta}}_{a, 3}-z_{1}\right)^{2}=\hat{r}_{1}^{2}
$$

Note that this equality constraint is imposed on the squares of the coordinates. Then, it is performed by solving a simple inverse problem given by:

$$
\widehat{\boldsymbol{\theta}}_{a}^{\prime}=\left(\boldsymbol{G}_{a}^{\prime T} \boldsymbol{G}_{a}^{\prime}\right)^{-1} \boldsymbol{G}_{a}^{\prime T} \boldsymbol{h}^{\prime}\left(\widehat{\boldsymbol{\theta}}_{a}\right)
$$

where

$$
\begin{aligned}
\boldsymbol{G}_{a}^{\prime} & =\left[\begin{array}{ccc}
1 & 0 & 0 \\
0 & 1 & 0 \\
0 & 0 & 1 \\
1 & 1 & 1
\end{array}\right]_{4 \times 3} \\
\boldsymbol{h}^{\prime}\left(\widehat{\boldsymbol{\theta}}_{a}\right) & =\left[\begin{array}{c}
\left(\widehat{\boldsymbol{\theta}}_{a, 1}-x_{1}\right)^{2} \\
\left(\widehat{\boldsymbol{\theta}}_{a, 2}-y_{1}\right)^{2} \\
\left(\widehat{\boldsymbol{\theta}}_{a, 3}-z_{1}\right)^{2} \\
\hat{r}_{1}^{2}
\end{array}\right]_{4 \times 1}
\end{aligned}
$$

Finally, the two solutions provided by this algorithm are:

$$
\widehat{\boldsymbol{\theta}}_{\text {Chan\&Ho }}^{1,2}= \pm \sqrt{\widehat{\boldsymbol{\theta}}_{a}^{\prime}}+\left[\begin{array}{l}
x_{1} \\
y_{1} \\
z_{1}
\end{array}\right]
$$

The first localization inverse problem (7.66) can be seen as the solution of the following general inverse problem:

$$
\boldsymbol{G}_{\text {Chan\&Ho,1 }} \widehat{\boldsymbol{\theta}}_{\text {Chan\&Ho,1}}=\widehat{\boldsymbol{m}}_{\text {Chan\&Ho,1}}
$$

where

$$
\begin{gathered}
\boldsymbol{G}_{\text {Chan\&Ho,1 }}=\boldsymbol{G}_{a} \\
\widehat{\boldsymbol{\theta}}_{\text {Chan\&Ho,1}}=\left[\begin{array}{c}
\widehat{\boldsymbol{\theta}}_{a} \\
\hat{r}_{1}
\end{array}\right] \\
\widehat{\boldsymbol{m}}_{\text {Chan\&Ho,1}}=\boldsymbol{h}(\widehat{\boldsymbol{m}})
\end{gathered}
$$

whilst the second one in (7.68) can be seen as the solution of the following general inverse problem:

$$
\boldsymbol{G}_{\text {Chan\&Ho }} \widehat{\boldsymbol{\theta}}_{\text {Chan\&Ho }}^{1,2}=\widehat{\boldsymbol{m}}_{\text {Chan\&Ho }}
$$

where 


$$
\begin{gathered}
\boldsymbol{G}_{\text {Chan\&Ho }}=\boldsymbol{G}_{a}^{\prime} \\
\widehat{\boldsymbol{m}}_{\text {Chan\&Ho }}=\boldsymbol{h}^{\prime}\left(\widehat{\boldsymbol{\theta}}_{a}\right)
\end{gathered}
$$

To form a characteristic equation in this algorithm a set of two stations are required. However, as the first inverse problem estimates, in the most general case, a total of $n+1$ unknowns ( $n$ coordinates and $r_{1}$ ), to estimate a target location composed by a set of $n$ coordinates, it requires at least $n+2$ stations (i.e., four for 2D localization and five for 3D localization). Furthermore, it is important to highlight that because the pseudoinverse matrix can also solve underdetermined inverse problems (i.e., in the case of having only $n+1$ stations for estimating $n$ coordinates), we have concluded from our simulations that the solutions provided for the target range $r_{1}$ are not useful (e.g., $\left.\hat{r}_{1}<0\right)$ and in these cases the second step significantly degrades the position accuracy provided by the first step. In the same sense, we have also proved that when only the solution for target position as provided by the first step (i.e., the parameter $\widehat{\boldsymbol{\theta}}_{a}$ ) is taken, it presents the same performance as the one provided by Smith and Abel algorithm in (7.38). On the other hand, the authors of this algorithm indicated in [109110] that this algorithm yields and unbiased solution with small standard deviation close to its CRLB when the noise level is moderate. However, these unbiased and small standard deviation have been not found in our simulations for both simulated and real scenarios. Furthermore, the authors of the algorithms also suggest that the inverse problem in (7.66) can be more accurately solved if the covariance matrix of the measurement errors is used, i.e., by using the weighted LS. Nevertheless, we have to say that this asseveration is naturally true when that covariance matrix can be accurately estimated, which is not the case of MLAT systems where highly different conditions for the stations of the same system can be found. In these cases the use of the covariance matrix of measurement errors can lead to performance degradations.

The most critical aspect for the performance of this method is the accuracy of the initial estimations $\widehat{\boldsymbol{\theta}}_{a}$ and $\hat{r}_{1}$, because the second step is a simple quadratic correction that directly depends on their quality. In that first step, the coefficient matrix (cf. (7.64) and (7.72)) is not constant and introduces noise terms because it contains the range difference measurements. Regarding to the measurement vector in (7.72), it is a modified version of the pure range difference measurements and it introduces quadratic noise terms as it contains the squares of the range difference measurements (cf. (7.64)). Although the authors of the algorithm state that it is optimal in the statistical sense and that it can be considered as an approximation of the MLE in the small error region, based on our set of simulations we have to say that, in practice, it is not true (at least for 
MLAT system applications). Finally, the computational cost of using this algorithm is greater than the previously analyzed ones.

\subsubsection{Bancroft Algorithm}

The Bancroft algorithm was initially developed by Bancroft [113] for GPS applications and the corresponding application for MLAT systems was developed by Geyer and Daskalakis [111]. However, we prefer to call it Bancroft algorithm because the second work is a direct application of the Bancroft work. It makes use of the TOA measurements rather than the TDOA or range difference one. This algorithm is a one step algorithm that provides two possible solutions for the target position; hence, an intuitive procedure to select one of them must be implemented. The characteristic equation of this algorithm is obtained by algebraic manipulation of the TOA equations (cf. (6.3)), directly leading to a data model that is then simplified by making use of the Lorenz inner product for time-space vectors [113]. This algorithm does not make any statistical or numerical assumption; hence, it is composed of an algebraic approach based model. This algorithm is summarized below. It is important to take into account that the formulation below does not set the origin of the coordinate system at any receiving station position but at an arbitrary point.

After algebraic manipulation of the TOA equation (6.3), the characteristic equation of this algorithm is expressed as follows:

$$
\begin{aligned}
& 2\left(x_{i} x+y_{i} y+z_{i} z-c^{2} \hat{t}_{i} t_{e}\right) \\
& \quad=x^{2}+y^{2}+z^{2}-c^{2} t_{e}^{2}+x_{i}^{2}+y_{i}^{2}+z_{i}^{2}-c^{2} \hat{t}_{i}^{2}
\end{aligned}
$$

where $\hat{t}_{i}=\widehat{T O A}_{i}$, i.e., the $i$ th TOA measurement and $t_{e}$ is the signal emission time (see Fig. 6.1). Then, expressing (7.75) in the vector-matrix form leas to the following data model:

$$
2 A_{l} s_{a}=\Lambda \mathbf{1}+\boldsymbol{v}
$$

where

$$
\begin{gathered}
\boldsymbol{A}_{l}=\left[\begin{array}{cccc}
x_{1} & y_{1} & z_{1} & -c \hat{t}_{1} \\
\vdots & \vdots & \vdots & \vdots \\
x_{N_{s}} & y_{N_{s}} & z_{N_{s}} & -c \hat{t}_{N_{s}}
\end{array}\right]_{N_{s} \times 4} \\
\boldsymbol{s}_{a}=\left[\begin{array}{c}
\boldsymbol{\theta} \\
c t_{e}
\end{array}\right]_{4 \times 1} \\
\Lambda=\left\langle\boldsymbol{s}_{a}, \boldsymbol{s}_{a}\right\rangle=x^{2}+y^{2}+z^{2}-c^{2} t_{e}^{2}
\end{gathered}
$$




$$
\begin{gathered}
\mathbf{1}=\left[\begin{array}{c}
1 \\
\vdots \\
1
\end{array}\right]_{N_{S} \times 1} \\
\boldsymbol{v}=\left[\begin{array}{c}
\left\langle\boldsymbol{s}_{1}, \boldsymbol{s}_{1}\right\rangle \\
\vdots \\
\left\langle\boldsymbol{s}_{N_{S}}, \boldsymbol{s}_{N_{S}}\right\rangle
\end{array}\right]_{N_{S} \times 1}=\left[\begin{array}{c}
x_{i}^{2}+y_{i}^{2}+z_{i}^{2}-c^{2} \hat{t}_{i}^{2} \\
\vdots \\
x_{N_{S}}^{2}+y_{N_{S}}^{2}+z_{N_{S}}^{2}-c^{2} \hat{t}_{N_{S}}^{2}
\end{array}\right]_{N_{S} \times 1}
\end{gathered}
$$

where the operator \langle\rangle denotes the Lorenz inner product of two vectors, and when these two vectors are the same one, the parameter $\Lambda$ is known as the Lorenzian norm. Then, assuming matrix $\boldsymbol{A}_{l}$ to be non-singular, the explicit solution to the data model in (7.76) can be expressed as follows:

$$
\begin{gathered}
\boldsymbol{s}_{a}=\frac{1}{2} \Lambda \boldsymbol{A}_{l}^{\dagger} \mathbf{1}+\frac{1}{2} \boldsymbol{A}_{l}^{\dagger} \boldsymbol{v} \\
\boldsymbol{s}_{a}=\Lambda \boldsymbol{d}+\boldsymbol{e}
\end{gathered}
$$

where

$$
\begin{gathered}
\boldsymbol{d}=\frac{1}{2} \Lambda \boldsymbol{A}_{l}^{\dagger} \mathbf{1} \\
\boldsymbol{e}=\frac{1}{2} \boldsymbol{A}_{l}^{\dagger} \boldsymbol{v}
\end{gathered}
$$

Then, as $\boldsymbol{d}$ and $\boldsymbol{e}$ are unknowns, this algorithm proposes to take the Lorenzian norm of both sides of (7.78), which results in the following quadratic equation:

$$
\alpha \Lambda^{2}+\beta \Lambda+\gamma=0
$$

where

$$
\begin{gathered}
\alpha=\langle\boldsymbol{d}, \boldsymbol{d}\rangle \\
\beta=2\langle\boldsymbol{d}, \boldsymbol{e}\rangle \\
\gamma=\langle\boldsymbol{e}, \boldsymbol{e}\rangle
\end{gathered}
$$

and whose solution can be easily determined by:

$$
\Lambda^{1,2}=\frac{-\beta \pm \sqrt{\beta^{2}-4 \alpha \gamma}}{2 \alpha}
$$

Finally, the two possible solutions provided by this algorithm can be obtaining by replacing the values of $\Lambda$ obtained with (7.81) in (7.78) as follows:

$$
\hat{\boldsymbol{s}}_{a}^{1,2}=\Lambda^{1,2} \boldsymbol{d}+\boldsymbol{e}
$$


To form a characteristic equation in this algorithm only one station is required as it is a TOA based algorithm. On the contrary, it has to estimate an additional parameter: the signal emission time $t_{e}$. Thus, to estimate a target location composed by a set of $n$ coordinates, it requires at least $n+1$ stations (i.e., three for 2D localization and four for 3D localization). For this method the matrix that must be inverted is not exact as it contains the TOA measurements (cf. (7.77)), which naturally introduce noise terms. In the same sense, the calculation of the inverse for matrix $\boldsymbol{A}_{l}$ could be not stable as the fourth column contains quantities that, normally, are several orders of magnitude greater than the remaining ones. Furthermore, this algorithm also introduces quadratic noise terms as the vector $\boldsymbol{v}$ contains the squares of the TOA measurements. Additionally, the roots provided by (7.81) sometimes can be complex numbers. In these cases, the real part of the complex number must be extracted. On the other hand, the computational cost of using this algorithm is very low.

\subsubsection{Wikipedia Algorithm (Plane Intersection)}

This algorithm is published in the open license website Wikipedia ${ }^{\circledR}$ [112]. This algorithm is totally based on the same algorithm of Schmidt (see $\S 7.3 .1$ ), i.e., it is also based on the plane intersection principle. It is a one step algorithm that provides one solution for the target position and the characteristic equation is also obtained by algebraic manipulation of the range difference equations (cf. (6.6)), directly leading to a linear inverse problem without making any statistical or numerical assumptions, hence, the data model of this algorithm has the same form as that of Schmidt (i.e., it belongs to the class of algebraic approach based models). The difference between the algorithm of Schmidt and this one is that it uses a different algebraic procedure to obtain the coefficients of the characteristic equation. Therefore, it is expected, as we have proved through our simulations, that both algorithms present different performance. On the other hand, it is important to say that this algorithm is not published (at the time of this thesis is written) in any scientific journal nor conference proceedings; for this reason we have directly cited the open license website. This algorithm is summarized below.

Equivalently to Schmidt algorithm, the present one also sets the unknown target location coordinates and the known parameters (e.g., the station positions and measurements) in a linear equation as follows:

$$
A x+B y+C z=D
$$

Thus, after the corresponding algebraic manipulation of the range difference equations (6.6), the characteristic equation of this algorithm is expressed as follows: 


$$
\begin{aligned}
\left(x_{i} \hat{m}_{2,1}-x_{2} \hat{m}_{i, 1}\right) x+\left(y_{i} \widehat{m}_{2,1}-y_{2} \widehat{m}_{i, 1}\right) y+\left(z_{i} \widehat{m}_{2,1}-z_{2} \widehat{m}_{i, 1}\right) z & \\
= & \frac{1}{2}\left[R_{i}^{2} \widehat{m}_{2,1}-R_{2}^{2} \widehat{m}_{i, 1}-\widehat{m}_{i, 1} \hat{m}_{2,1}\left(\widehat{m}_{i, 1}-\widehat{m}_{2,1}\right)\right], \quad i \\
& =3, \ldots, N_{s}
\end{aligned}
$$

Note that the coefficients are different from those of Schmidt (cf. (7.23)). Also for this algorithm it is assumed the index $i$ to be $i=3, \ldots, N_{s}$, i.e., this algorithm also requires two reference stations to form an equation and they are assumed to be the stations 1 and 2. Moreover, for $N_{s}$ available stations, only a set of $N_{s}-2$ equations can be set for the inverse localization problem. Then, the coefficients $A, B, C$, and $D$ of (7.83) can be easily identified from the characteristic equation (7.84) and they explicitly are:

$$
\begin{aligned}
& A_{i}=x_{i} \widehat{m}_{2,1}-x_{2} \widehat{m}_{i, 1} \\
& B_{i}=y_{i} \widehat{m}_{2,1}-y_{2} \widehat{m}_{i, 1} \\
& C_{i}=z_{i} \widehat{m}_{2,1}-z_{2} \widehat{m}_{i, 1} \\
& D_{i}=\frac{1}{2}\left[R_{i}^{2} \widehat{m}_{2,1}-R_{2}^{2} \widehat{m}_{i, 1}-\widehat{m}_{i, 1} \widehat{m}_{2,1}\left(\widehat{m}_{i, 1}-\widehat{m}_{2,1}\right)\right]
\end{aligned}
$$

Finally, the localization inverse problem can be computed in vectormatrix form as follows:

$$
\boldsymbol{G}_{\text {wikipedia }} \widehat{\boldsymbol{\theta}}=\widehat{\boldsymbol{m}}_{\text {wikipedia }}
$$

where

$$
\boldsymbol{G}_{\text {Wikipedia }}=\left[\begin{array}{ccc}
A_{3} & B_{3} & C_{3} \\
\vdots & \vdots & \vdots \\
A_{N_{S}} & B_{N_{S}} & C_{N_{S}}
\end{array}\right]_{\left(N_{S}-2\right) \times 3}
$$

and

$$
\widehat{\boldsymbol{m}}_{\text {Wikipedia }}=\left[\begin{array}{c}
D_{3} \\
\vdots \\
D_{N_{S}}
\end{array}\right]_{\left(N_{S}-2\right) \times 1}
$$

The same observations as discussed for Schmidt algorithm (see §7.3.1) also hold for this method.

\subsubsection{Summary of Localization Algorithms}

In the following table the most relevant characteristics of every of localization algorithm are summarized. 
Table 7.1 Summary of localization algorithms. A: Alegbraic, S: Statistical, N: Numerical.

\begin{tabular}{|l|c|c|c|c|c|c|c|}
\hline \hline Algorithm & $\begin{array}{c}\text { Data } \\
\text { Model }\end{array}$ & $\begin{array}{c}\text { Stations } \\
\text { for one } \\
\text { Eq. }\end{array}$ & $\begin{array}{c}\text { Min. } \\
\text { Num. of } \\
\text { Stats. }\end{array}$ & Steps & $\begin{array}{c}\text { Type of } \\
\text { Sols. }\end{array}$ & $\begin{array}{c}\text { Num. } \\
\text { of } \\
\text { Sol. }\end{array}$ & $\begin{array}{c}\text { Noise } \\
\text { Terms }\end{array}$ \\
\hline Schmidt & $\mathrm{A}$ & 3 & $n+2$ & 1 & Direct & 1 & $\begin{array}{c}\text { Linear } \\
\text { Cubic }\end{array}$ \\
\hline Taylor & $\mathrm{S}$ & 2 & $n+1$ & 1 & Iterative & 1 & Linear \\
\hline $\begin{array}{l}\text { Smith and } \\
\text { Abel }\end{array}$ & $\mathrm{N}$ & 2 & $n+1$ & 1 & Direct & 1 & Quadratic \\
\hline Friedlander & $\mathrm{N}$ & 2 & $n+1$ & 1 & Direct & 1 & Quadratic \\
\hline $\begin{array}{l}\text { Schau and } \\
\text { Robinson }\end{array}$ & $\mathrm{N}$ & 2 & $n+1$ & 2 & Direct & 2 & Quadratic \\
\hline $\begin{array}{l}\text { Chan and } \\
\text { Ho }\end{array}$ & $\mathrm{N}$ & 2 & $n+2$ & 2 & Direct & 2 & Quadratic \\
\hline Bancroft & $\mathrm{A}$ & 1 & $n+1$ & 1 & Direct & 2 & $\begin{array}{c}\text { Linear } \\
\text { Quadratic }\end{array}$ \\
\hline Wikipedia & $\mathrm{A}$ & 3 & $n+2$ & 1 & Direct & 1 & $\begin{array}{c}\text { Linear } \\
\text { Cubic }\end{array}$ \\
\hline \hline
\end{tabular}

It has to be remembered that $n$ is the number of target position coordinates to be estimated, i.e., $n=2$ for $2 \mathrm{D}$ localization and $n=3$ for $3 \mathrm{D}$ localization.

\subsection{Projected Algorithms}

Due to the fact that for LAM the target height is approximately of the same order of maginitude of that of the stations, the values of the third column of matrix $\boldsymbol{G}$ (cf. (7.15)) are close to zero and therefore, in many cases, the solution provided by (7.18) diverges with large errors. However, it is possible to make a practical approximation that avoids this problem. This approximation consists of solving (7.18) by using a sub matrix $\boldsymbol{G}_{2 D}$ of the full matrix $\boldsymbol{G}$ in (7.15). The matrix $\boldsymbol{G}_{2 D}$ takes the following form:

$$
\boldsymbol{G}_{2 D}=\frac{1}{c}\left[\begin{array}{cc}
\frac{x_{0}-x_{2}}{r_{i}}-\frac{x_{0}-x_{1}}{r_{1}} & \frac{y_{0}-y_{2}}{r_{i}}-\frac{y_{0}-y_{1}}{r_{1}} \\
\vdots & \vdots \\
\frac{x_{0}-x_{N_{S}}}{r_{N_{S}}}-\frac{x_{0}-x_{1}}{r_{1}} & \frac{y_{0}-y_{N_{S}}}{r_{N_{S}}}-\frac{y_{0}-y_{1}}{r_{1}}
\end{array}\right]_{\left(N_{S}-1\right) \times 2}
$$

This approximation has the problem that it introduces a spatial bias into the final solution. It is because the measurements vector $\widehat{\boldsymbol{m}}_{\Delta}$ in (7.18) is directly related with the time of signal propagation in a three- 


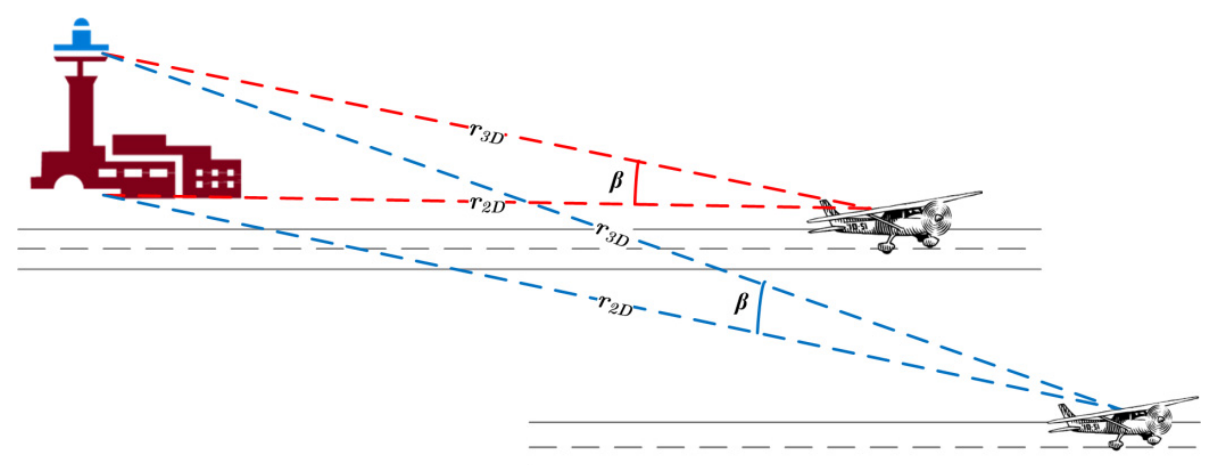

Fig. 7.3 Geometrical interpretation of projected algorithms.

dimensional space whilst the approximated matrix $\boldsymbol{G}_{2 D}$ assumes only a two dimensional space. The geometrical interpretation of this problem is shown in Fig. 7.3. In other words, the matrix $\boldsymbol{G}$ models the real physical phenomena, that is, the propagation of a signal through a path in a three dimensional space (i.e., $r_{3 D}$ in Fig. 7.3) and the measurements comprised in $\widehat{\boldsymbol{m}}_{\Delta}$ are related with the time of propagation along this path. On the other hand, the matrix $\boldsymbol{G}_{2 D}$ models the propagation of the signal along a path in a two dimensional projected space (i.e., $r_{2 D}$ in Fig. 7.3). It is evident that $r_{3 D} \geq r_{2 D}$ (generally it is $r_{3 D}>r_{2 D}$ ) and for this reason, this approximation introduces the spatial bias. This bias depends on the target position and sometimes, the error due to this reason takes values that can be neglected for LAM applications (e.g., smaller than 2 or $3 \mathrm{~m}$ ) but, sometimes it takes values that cannot be neglected.

The spatial bias for each measurement can be estimated as follows:

$$
\operatorname{bias}_{\widehat{m}}=r_{3 D}(1-\cos \beta)
$$

From (7.89) it can be seen more clearly that the measurement bias takes always a value greater than zero, with the exception of $\beta=0$. This bias is present at each station and the set of these generates the spatial bias in the final solution.

On the other hand, this approximation is also valid for the closed form algorithms. The main idea of this approximation is to remove all the terms related to the vertical coordinate, i.e., all terms containing information of the $z$ coordinate. For example, for Schau and Robinson algorithm (see $\S 7.3 .4)$ it is necessary to delete the $z$ coordinate from (7.54) and for Chan and Ho (see $\S 7.3 .5$ ), it is necessary to remove the $z$ coordinate from (7.64), (7.69), and (7.70). Furthermore, the spatial bias due to the 2D approximation also affects to these algorithms. 


\subsection{Analysis of Ill-Conditioned Problems in Mode S Multilateration}

There are several sources which generate the ill-conditioning of the inverse problem in Mode S MLAT. These sources can be classified into two groups; the first one is related to the system geometry and the second one is related to the measurement noise or the starting point for Taylor-series expansion method.

The sources of the ill-conditioning and their severity on the localization problem can be studied and identified by using several numerical tools like the Cramér-Rao Lower Bound (CRLB) accuracy analysis [20], Monte-Carlo simulations of (7.18) and the singular value analysis of $\boldsymbol{G}$ (cf. (7.15)) by means of the SVD [72]. In this subsection we show the application of these numerical tools to analyze and characterize the ill-conditioning in the localization problem for MLAT systems. By means of the CRLB analysis and the Monte-Carlo simulation for the location estimator in (7.18), it is possible to identify the source of the ill-conditioning, and by means of the SVD analysis it is possible to get an idea about the "level" of the illconditioning.

The application of the CRLB analysis and the Monte-Carlo simulation (with 100 trials), to identify the source (or sources) of ill-conditioning in MLAT localization (3D), is shown in Fig. 7.4 for a particular scenario (see $\S$ C.1.2 for scenario details). In this figure, the vertical axis shows the theoretical accuracy (standard deviation) as provided by the CRLB analysis described in [20] and the Root Mean Square (R.M.S) position error obtained from the Monte-Carlo trials. As it is described in [20], the CRLB mainly depends on the system geometry (i.e., on the matrix $\boldsymbol{G}$ ); in this case, we can identify that in those points where the CRLB analysis predicts poor accuracy values (e.g., R.M.S errors greater than $300 \mathrm{~m}$ ) the ill-conditioned problem is mainly due to the system geometry (e.g., the peaks before $5 \mathrm{~km}$ and around $18 \mathrm{~km}$ in Fig. 7.4). On the other hand, in those points where the CRLB analysis predicts good accuracy values (e.g., R.M.S errors smaller than $300 \mathrm{~m}$ ) but the R.M.S error of the location estimator does not reach the lower bounds, the ill-conditioned problem is mainly due to the measurement noise or to the quality of the starting point used in Taylor linearization (e.g., those points within $5-10 \mathrm{~km}$ in Fig. 7.4). Finally, in those points where R.M.S error and CRLB analysis reach about the same values, the problem is well-conditioned (e.g., those points beyond $20 \mathrm{~km}$ in Fig. 7.4).

The first step to understand if the localization problem in MLAT systems is rank-deficient or ill-posed, and to analyze the "level" of illconditioning, is to inspect the singular values of the matrix $\boldsymbol{G}$ for some specific points. This analysis is performed by using the ordinary SVD [72] 


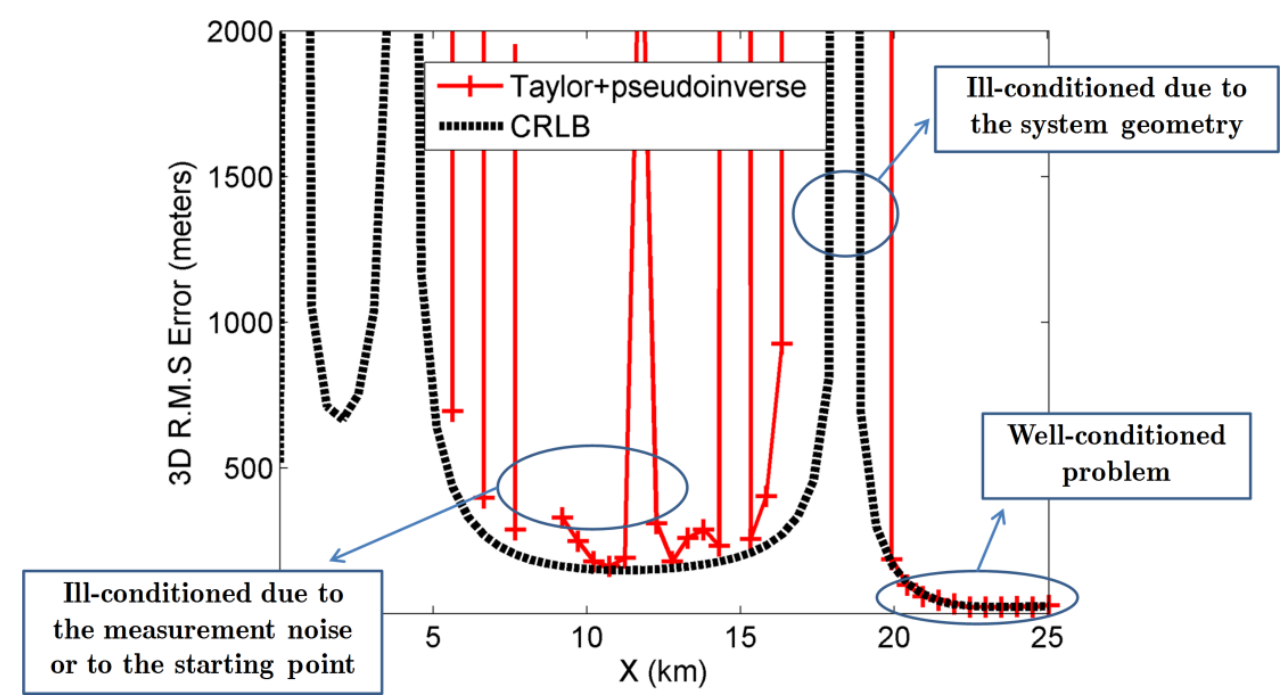

Fig. 7.4 Ill-conditioned problem in MLAT systems: an example.

which decomposes the matrix $\boldsymbol{G}$ into three independent matrices such that $\boldsymbol{G}=\boldsymbol{U} \boldsymbol{\Sigma} \boldsymbol{V}^{T}$ (cf. (6.10)), where $\boldsymbol{U}$ and $\boldsymbol{V}$ are matrices with orthonormal columns and $\boldsymbol{\Sigma}$, which is called matrix of singular values, is a $n \times n$ diagonal matrix with nonnegative elements (the singular values) appearing in nonincreasing order (cf. (6.12)). In the general case for MLAT localization problem, the variable $n$, which represents the number of coordinates for locating a target, is equal to 3 (i.e., there are three spatial coordinates composing the unknown target position $\boldsymbol{\theta}$ ). Furthermore, taking into account that the location estimator in (7.18) is iterative, the matrix of singular values for MLAT localization by means of that estimator is an hyper-matrix of the form:

$$
\boldsymbol{\Sigma}^{k}=\left[\begin{array}{ccc}
\sigma_{1}^{k} & 0 & 0 \\
0 & \sigma_{2}^{k} & 0 \\
0 & 0 & \sigma_{3}^{k}
\end{array}\right]_{3 \times 3 \times K} ; \quad k=1, \ldots, K
$$

being $K$ the total number of Taylor iterations.

The matrix of singular values contains the information about the severity of the ill-conditioned localization problem. The first aspect that can be analyzed is the condition number (see §6.3.5), defined for this case as cond $^{k}=\sigma_{1}^{k} / \sigma_{3}^{k} \quad$ (cf. (6.19)), which provides a quick and preliminary information about the "level" of ill-conditioning of the MLAT localization problem. The other important parameter to analyze from this matrix is the decay rate between two consecutive singular values (i.e., $\sigma_{2}^{k} / \sigma_{1}^{k}$ and $\left.\sigma_{3}^{k} / \sigma_{2}^{k}\right)$. However, because the Taylor based localization algorithm is 

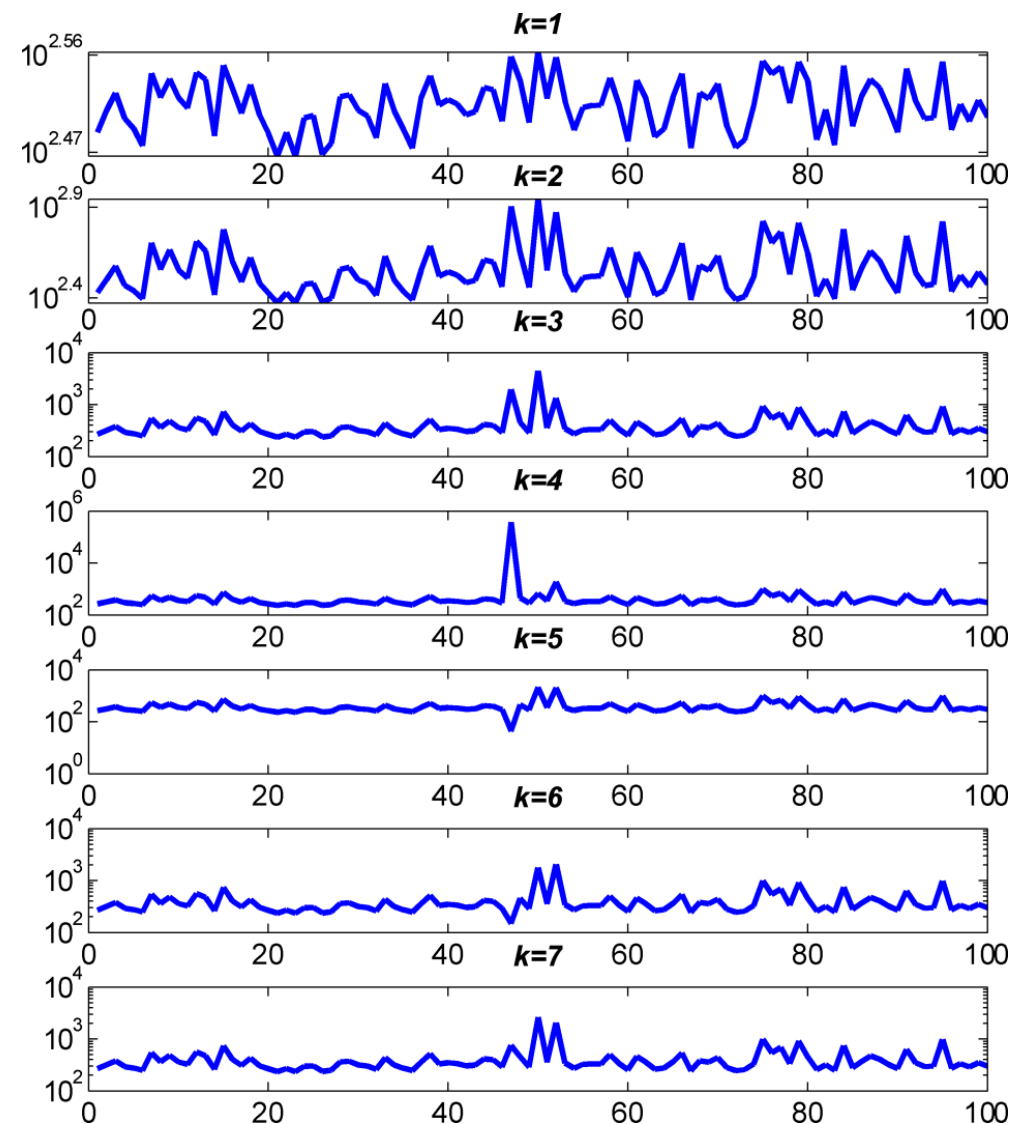

Fig. 7.5 Condition number for the first seven Taylor iterations. Vertical axis represents the condition number and horizontal axis the Monte-Carlo trials.

iterative, in this thesis we propose to analyze not only the decay rate between two consecutive values but also between the same singular values at two consecutive Taylor iterations. All of these results are shown below.

The condition number for the first seven Taylor iterations, for a particular point (around $17 \mathrm{~km}$ ) of the same scenario analyzed in Fig. 7.4, is shown in Fig. 7.5. The vertical axis represents the condition number and the horizontal axis the different Monte-Carlo trials for that point. This figure shows how the ill-conditioned problem is present in most of Taylor iterations. Although it is not a general rule, we have observed for long number of simulations that the "level" of ill-conditioning increases with the first Taylor iterations ( $k=1$ through $k=4$ in Fig. 7.4).

The next figures show the three singular values, in the Taylor iterations, for three different points (for the same scenario than in Fig. 7.4) where the localization problem is ill-conditioned due to the reasons commented above and also where it is well-conditioned. In the classical localization problem, 
there are three unknown variables (i.e., the three Cartesian coordinates) and the coefficient matrix is of $\left(N_{s}-1\right) \times 3$ size; therefore, this matrix has three singular values as we described before.

Part (a) of Fig. 7.6 shows the three singular values, for the Taylor iterations, of an ill-conditioned solution (given by the Pseudoinverse) produced by the measurement noise (or starting point). The three singular values are in non-increasing order. From this figure, it can be observed that the first two singular values $\left(\sigma_{1}\right.$ and $\left.\sigma_{2}\right)$ are around 1 but they decrease with the Taylor iterations and the third one oscillates on values very close to zero. For the first Taylor iterations there is a well determined gap between the first two singular values and the third one but, after those iterations this gap is slightly reduced.

Part (b) of Fig. 7.6 shows the corresponding singular values for a point where the ill-conditioned solution is produced by the system geometry. For this point, it is also observed that the third singular value is very close to zero (more than in the first situation), and that the two first ones start around 1 but they rapidly decrease with the Taylor iterations. Moreover, their decay rate is greater than that of the part (a) of Fig. 7.6, even the two first singular values are of the same order of the third one for the last Taylor iterations. The latter means the loss of a lot of information and therefore significant instabilities in the problem solution. Additionally, this observation also suggests that the ill-conditioning due to the system geometry could be stronger than that due to the measurement noise.

From both part (a) and part (b) of Fig. 7.6 it can be observed that for the first Taylor iterations there is a well determined gap between the two first singular values and the third one. In those iterations the problem could be a rank-deficient problem and, for the last Taylor iterations, this gap is reduced and all the singular values seem, on average, to decay gradually to zero. The latter suggests that in those points the problem is an ill-posed problem. However, this classification is only theoretical and in Chapter 8 the most relevant regularization methods are tested to solve this problem.

For a well-conditioned problem, at least the first and the second singular values approximately remain around the same value (in this case they are around 1 or greater) from the first Taylor iteration to the last one or increase with respect to it (see part (c) of Fig. 7.6). We have observed that, while the first two singular values maintain the same behaviour as described before, the problem remains well-conditioned independently of the third singular value.

Finally, it is important to emphasize that, besides the analysis shown in this section, all the information shown above is also useful to estimate some regularization parameters needed for the regularization methods applied in this thesis. This aspect will be shown in $\S 8.1 .4$ and $\S 8.2 .2$. 


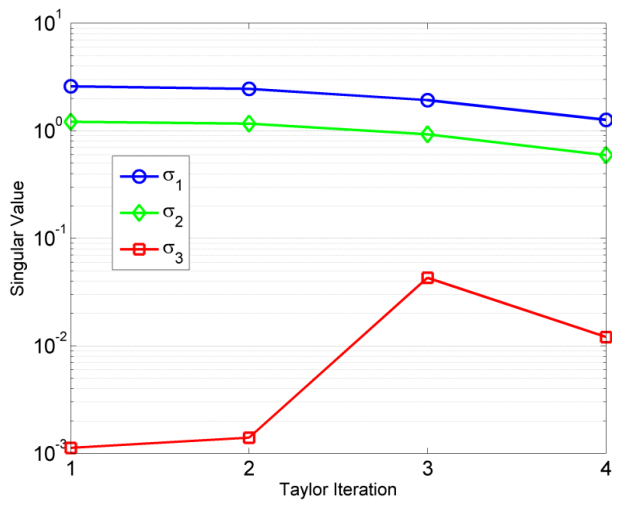

(a) Ill-conditioned due to the measurements noise.

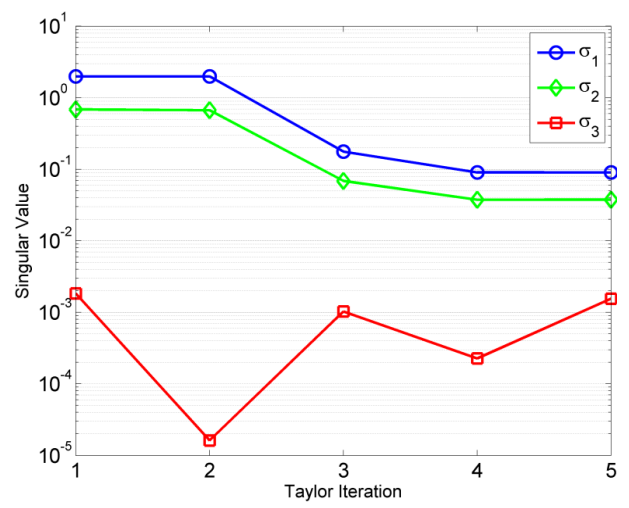

(b) Ill-conditioned due to the system geometry.

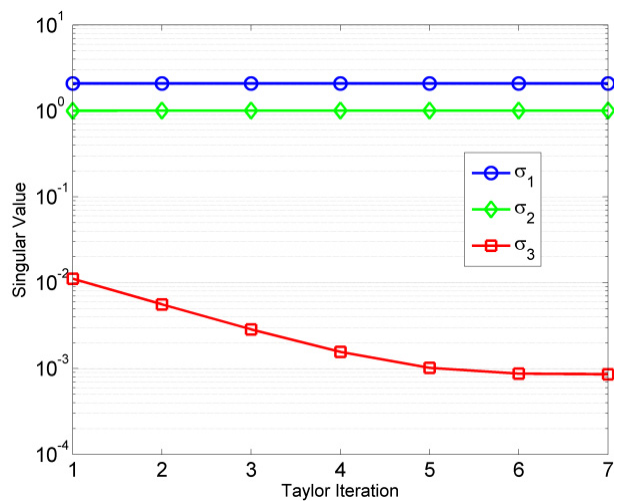

(c) Well-conditioned.

Fig. 7.6 Characterization of the singular value spectrum for the MLAT localization problem (vertical axes in log scale). 


\subsection{Simulation and Results: Comparison of Localization Algorithms}

In this part, the general performance of all the localization algorithms studied before is analyzed. To do this, we use three different scenarios of real MLAT systems. These systems are the WAM system of Innsbruck (Innsbruck, Austria) airport, the WAM system of Malpensa (Milan, Italy) airport, and the LAM system of Linate (Milan, Italy) airport. Each of these scenarios is fully described in Annex C. For these three scenarios we know the real system geometry (i.e., the stations position) and simulate the TOA measurements for a defined, realistic path. For this reason, in Annex C they are classified as "systems with real geometry and simulated measurements". In $\S$ C.1 we explain how these measurements are simulated. In the next section, we perform the same analysis but with real data.

To analyze the general performance of each localization algorithm, we estimate the Root Mean Square (R.M.S) error by performing a Monte-Carlo simulation with 100 trials. We separately analyze the horizontal (or 2D, $x, y$ - components) and the vertical (when apply) components of the R.M.S error for every localization algorithm. Moreover, in order to validate also the efficiency (in a statistical sense) of each algorithm, we also analyze the horizontal and vertical (when apply) bias. The R.M.S error tell us about how accurate is every localization algorithm, and the bias how exact they are. The $2 \mathrm{D}$ and vertical R.M.S error, for the ith simulated point, are respectively calculated as follows:

$$
\begin{gathered}
\text { R.M. } S_{2 D}(i)=\sqrt{\frac{\sum_{j=1}^{100}\left[\left(x_{j}^{\prime}(i)-x_{\text {exact }}(i)\right)^{2}+\left(y_{j}^{\prime}(i)-y_{\text {exact }}(i)\right)^{2}\right]}{100}} \\
\text { R.M. } S_{\text {vertical }}(i)=\sqrt{\frac{\sum_{j=1}^{100}\left(z_{j}^{\prime}(i)-z_{\text {exact }}(i)\right)^{2}}{100}}
\end{gathered}
$$

where $\left(x_{j}^{\prime}(i), y_{j}^{\prime}(i), z_{j}^{\prime}(i)\right)$ represents the target position as estimated by a localization algorithm for the $i$ th simulated point and the $j$ th Monte-Carlo trial, and $\left(x_{\text {exact }}(i), y_{\text {exact }}(i), z_{\text {exact }}(i)\right)$ represents the $i$ th exact target position, which for these simulations is known.

The $2 \mathrm{D}$ and vertical bias, for every algorithm, are respectively calculated for the ith simulated point as follows: 
$=\sqrt{\left(x_{\text {exact }}(i)-\frac{1}{100} \sum_{j=1}^{100} x_{j}^{\prime}(i)\right)^{2}+\left(y_{\text {exact }}(i)-\frac{1}{100} \sum_{j=1}^{100} y_{j}^{\prime}(i)\right)^{2}}$

$$
\text { bias }_{\text {vertical }}(i)=\sqrt{\left(z_{\text {exact }}(i)-\frac{1}{100} \sum_{j=1}^{100} z_{j}^{\prime}(i)\right)^{2}}
$$

For the Taylor algorithm that needs for a starting point, we use the corresponding position as provided by the Schau and Robinson algorithm. Particularly, for the Linate (surface movement) scenario we change the vertical component for a fixed value of $10 \mathrm{~m}$ (see $\S 8.6$ for a detailed analysis of the starting point for Taylor based algorithms). For these cases, because Schau and Robinson algorithm provides two possible solutions and, hence, one of them must be chosen, we implement an intuitive validation procedure to select the final solution. The latter is used only when it provides the starting point for Taylor algorithm. Furthermore, in order to know the best possible performance of every algorithm, when calculating the target position with the closed form algorithms that provide two possible solutions (i.e., Schau and Robinson, Chan and Ho, and Bancroft algorithms), we choose, as final solution, the one that is closer to the exact one. It is possible in this kind of simulations because we know the exact position value. However, for $\$ 7.7$, where we perform the experiment with real data and, therefore, the exact target position is not known, we implement the intuitive validation procedure for the three closed form algorithms that requires it. We have proved that this procedure is near to be fully optimal and, the most of time, it selects the best possible solution.

Taking into account the above paragraph, particularly for Taylor algorithm, one of the criterion to know whether it provides good performance levels or not, is to validate the ability of it to improve the position accuracy (i.e., R.M.S and bias) as provided by the closed form algorithm, which in this case is Schau and Robinson algorithm.

\subsubsection{Innsbruck WAM System}

The Innsbruck WAM system is composed of eight receiving stations, which are deployed around the mountains surrounding de Inn Valley (Tirol state at Austria). This system is intended for the Terminal Manoeuvring Area (TMA) surveillance. Therefore, for this system we simulate a takeoff line of 
approximately $80 \mathrm{~km}$ of length. The system layout and the takeoff line are depicted in Fig. C.1. The 2D and vertical R.M.S error for non-projected version of the localization algorithms are shown in Fig. 7.7 and Fig. 7.8, respectively, whilst the corresponding bias are shown in Fig. 7.9 and Fig. 7.10. We refer to every non-projected localization algorithm as the $3 \mathrm{D}$ version, whilst to the projected one as the $2 \mathrm{D}$ version. The corresponding 2D R.M.S error and bias for the projected version of the localization algorithms are respectively shown in Fig. 7.11 and Fig. 7.12. Note that for the projected version of localization algorithms the vertical component is not analyzed, as they do not calculate it.

In Fig. 7.7, for the 2D R.M.S error of non-projected localization algorithms, we observe that all the localization algorithms approximately present the same values until $30 \mathrm{~km}$ and thereafter, they present different performance. From that distance, the geometry quality rapidly changes (decreases the quality) and, therefore, not all the algorithms are equally efficient in recovering the target position information. However, these differences are just numerical, as all the algorithms (except the Bancroft one) provide 2D R.M.S values that are within the requirements for WAM [2]. In this situation, the Smith and Abel, and Chan and Ho algorithms, provide the best 2D R.M.S values and present the same values. Really, for this particular scenario, the 2D R.M.S error provided by these two algorithms are exactly the same, with the particular exception that Chan and Ho algorithm presents a particular peak at $X=0 \mathrm{~km}$ that is not shown in Fig. 7.7. However, the effect of this particular peak can be shown in Fig. 7.15 for the corresponding 2D bias analysis and in the first column of Table 7.2, §7.6.4. We later analyze more this particular. After these two algorithms, the Taylor algorithm is the one that provide better performance levels, thus improving the position accuracy as provided by the starting point (Schau and Robinson). Then, all the remaining algorithms, in practical terms, provide the same performance. The Bancroft algorithm, after $35 \mathrm{~km}$, is not capable to provide useful target position data, even when we are taking the best solution between the two ones provided by it.

The vertical R.M.S error is an important parameter for WAM surveillance as it informs about the quality, of each localization algorithm, for recovering the vertical target position component. For the non-projected version of localization algorithms at this scenario, this parameter is shown in Fig. 7.8. Similarly to the 2D R.M.S error, all the algorithms provide the same performance levels until $35 \mathrm{~km}$ and thereafter, they change and the general ranking performance in respect of the horizontal case also changes. The Taylor algorithm is the one that provides the best vertical performance (and with much difference regarding to the remaining ones), significantly improving the accuracy provided by its starting point. Moreover, in this case, the improvement added by the quadratic correction of Chan and Ho 


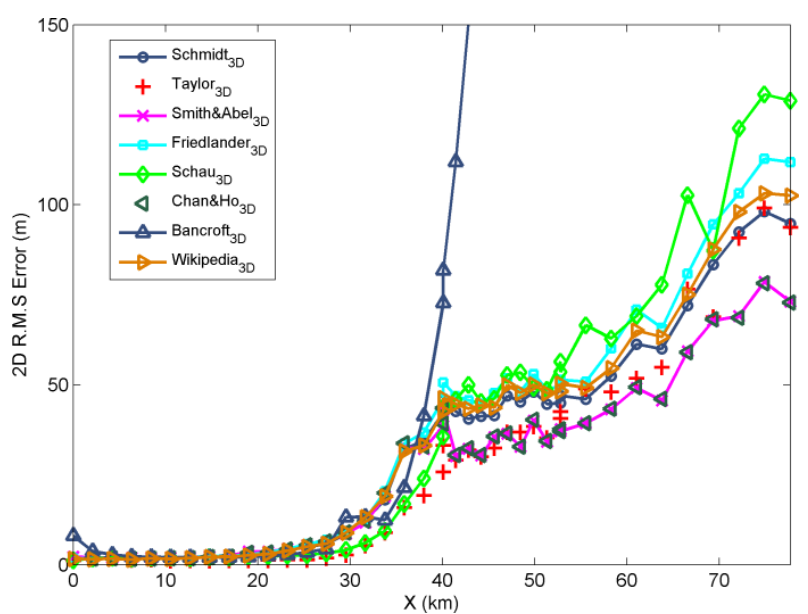

Fig. 7.7 2D R.M.S error for Innsbruck system: comparison of localization algorithms.

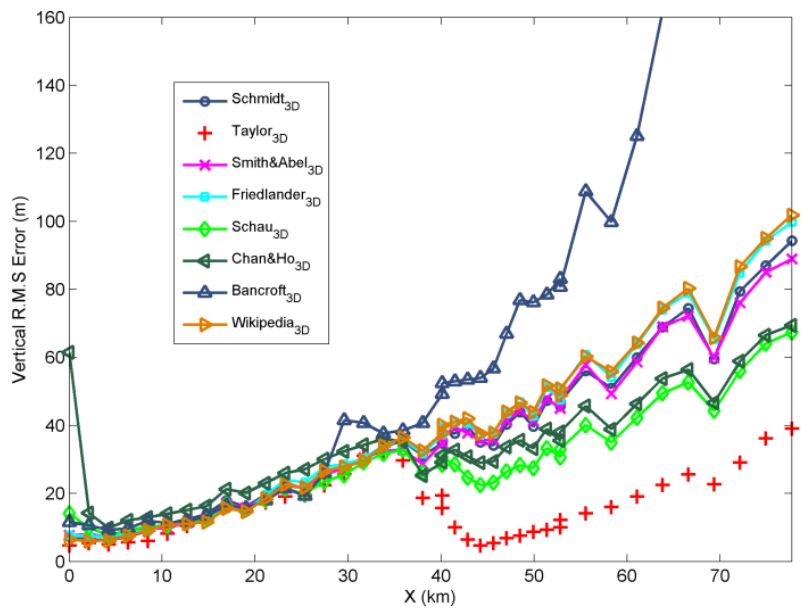

Fig. 7.8 Vertical R.M.S error for Innsbruck system: comparison of localization algorithms.

algorithm, in respect of Smith and Abel, can be clearly appreciated. However, we also observe the Schau and Robinson vertical performance is better than the one of Chan and Ho. The remaining algorithms approximately present the same performance levels, and again, the Bancroft one does not provide useful vertical position data.

The bias analysis is important for the operational systems because this effect cannot be generally corrected. In the case of a high R.M.S error, due to a high standard deviation, the results can be improved by implementing a set of tracking algorithms [114], which are implemented in all the ATC infrastructures. However, they are not capable to improve the possible amount of bias that each algorithm can introduce; the tracking algorithms start from the hypothesis that the estimated position data is unbiased. For 


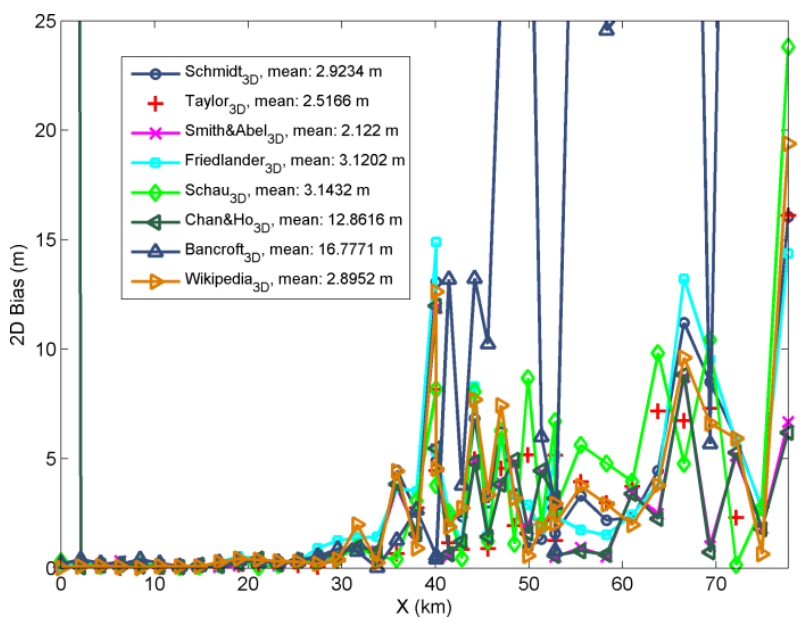

Fig. 7.9 2D bias for Innsbruck system: comparison of localization algorithms.

this reason, we separately analyze the amount of bias (both 2D and vertical) that each algorithm introduces. The $2 \mathrm{D}$ and vertical bias are respectively shown in Fig. 7.9 and Fig. 7.10. Additionally, in the legend of these figures, the mean value, for each algorithm, over the entire simulated takeoff line is shown. For the 2D bias, all the analyzed algorithms present small values of bias. Particularly, although Chan and Ho, and Smith and Abel algorithms are graphically the same, they present different numerical values due the particular peak that Chan and Ho presents at $X=0$. For the Bancroft algorithm, which introduces an average bias greater than 16 $\mathrm{m}$, it is due to its own characteristics. Finally, regarding to the vertical bias, the minimum biased solution is provided by the Taylor algorithm (an average value of $1.4 \mathrm{~m}$ ), and the remaining ones provide approximately the same values (around $3 \mathrm{~m}$ ). However, in practical terms, all of these algorithms present good general performance as $10 \mathrm{~m}$ of bias, at $60-70 \mathrm{~km}$ from the runway, is practically negligible due to the horizontal and vertical separations that are stipulated for the targets at these distances.

The R.M.S and bias analysis for the projected version of localization algorithms is shown in Fig. 7.11 and Fig. 7.12, respectively. In general, we observe that all algorithms present large 2D R.M.S error and bias values, being the Schau and Robinson algorithm the one that presents the "best" general performance. In any case, for the most of parts of the takeoff line, the performance provided by all the algorithms is considered poor. If we compare the bias (Fig. 7.12) with the R.M.S error (Fig. 7.11), we can observe the corresponding plots of bias and R.M.S error, for every algorithm, are significantly similar. It means that the R.M.S error is dominated for the bias introduced for these algorithms; it is also clear by analyzing the first column of Table 7.4, §7.6.4, where we can see the 
average bias values are approximately equal to the R.M.S error ones, for every algorithm. This large amount of bias appears because the errors due to the projection of this kind of algorithms (see $\S 7.4$ ), which for this case are significantly large, cannot be neglected. This additional bias does not allow the algorithm to provide suitable performance levels; all of these values are very far from those stipulated for WAM surveillance [2]. For this reason, the use of projected algorithms is not recommended for this kind of scenarios.

Finally, the average 2D and vertical R.M.S errors, and 2D and vertical bias, are shown in Table 7.2 and Table 7.3, $§ 7.6 .4$. Later in $\$ 7.6 .4$, based on these tables, we provide some general conclusion for the comparison of

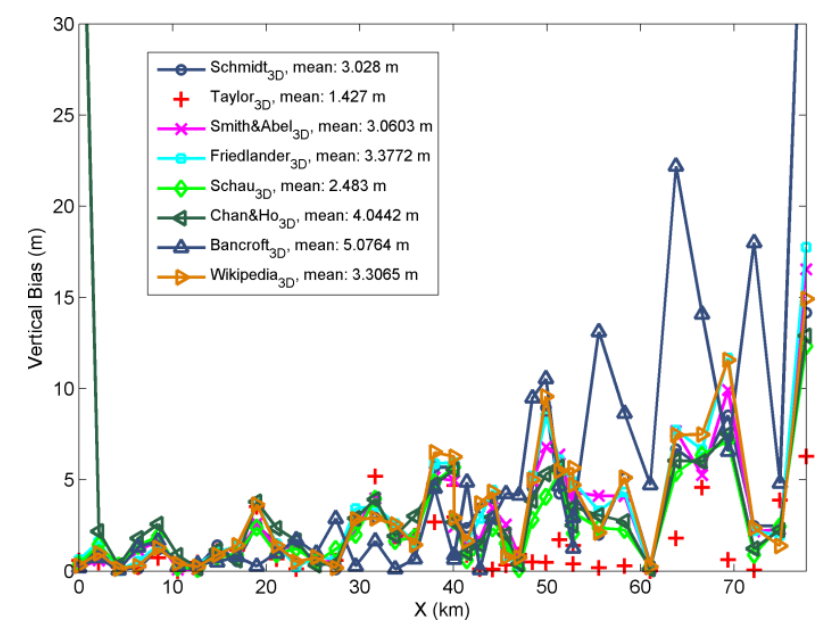

Fig. 7.10 Vertical bias for Innsbruck system: comparison of localization algorithms.

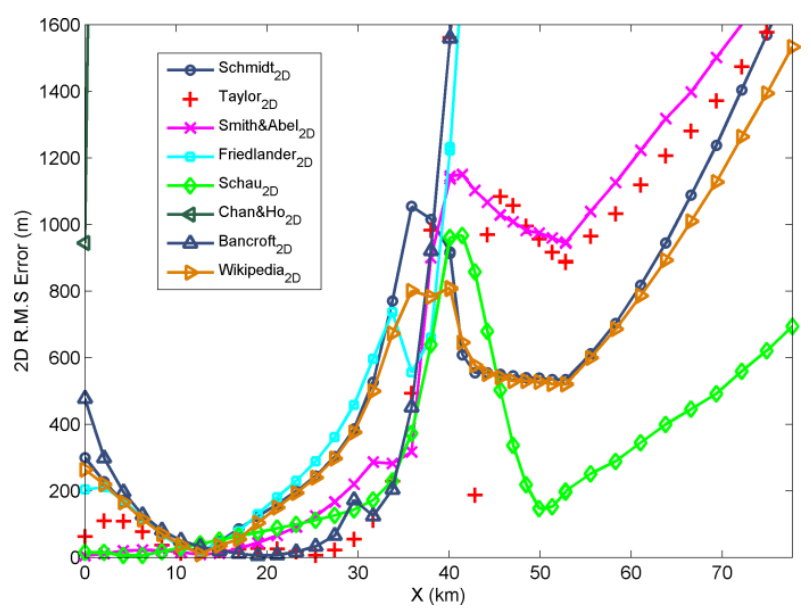

Fig. 7.11 2D R.M.S error for Innsbruck system: comparison of projected localization algorithms. 


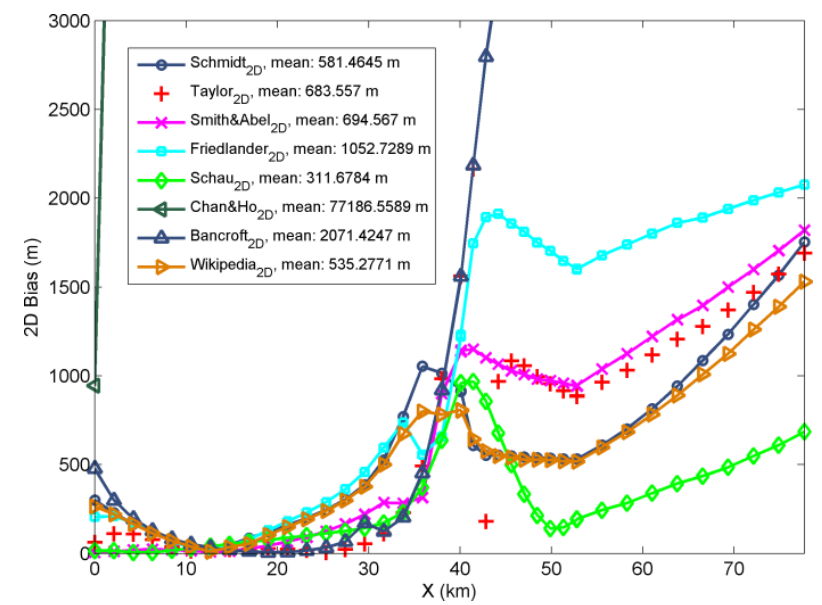

Fig. 7.12 2D bias for Innsbruck system: comparison of projected localization algorithms.

localization algorithms.

\subsubsection{Malpensa System}

The Malpensa WAM system (Milano, Italy) analyzed in this thesis is a proposal solution for the approach surveillance (up to $20-25 \mathrm{~km}$ from the runway threshold) that was firstly published in [7]. This system is composed of four receiving station and it is a well case study to analyze the performance of the localization algorithms under unfavourable system geometries. For this case, we simulate a typical $3^{\circ}$ ILS (Instrumental Landing System) takeoff line of $25 \mathrm{~km}$ of length. The system layout and the takeoff line are depicted in Fig. C.2. The 2D and vertical R.M.S error, for non-projected version of the localization algorithms are shown in Fig. 7.13 and Fig. 7.14, respectively, whilst the corresponding bias are shown in Fig. 7.15 and Fig. 7.16. The corresponding 2D R.M.S error and bias for the projected version of the localization algorithms are respectively shown in Fig. 7.17 and Fig. 7.18. Note that for the projected version of localization algorithms the vertical component is not analyzed, as they do not calculate it.

In Fig. 7.13, for the 2D R.M.S error, we observe Taylor algorithm does not provide useful position data as it diverges due the ill-conditioning of the localization problem in this scenario. Moreover, also the Smith and Abel, and Chan and Ho algorithms do not provide useful position data. Regarding to the Chan and Ho algorithm, it is because it requires at least $n+2$ stations to solve the 3D localization problem, as it has to find also a first estimation of the target range (cf. (7.65)). Under this situation of unfavourable geometry, basically this algorithm estimates a negative target 


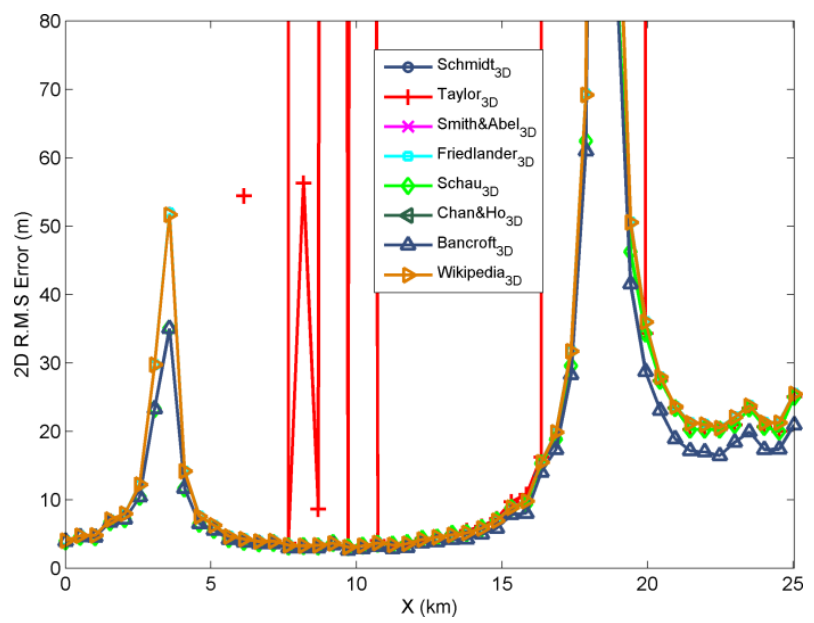

Fig. 7.13 2D R.M.S error for Malpensa system: comparison of localization algorithms.

range and then, when it applies the quadratic correction, the final solution diverges to inconsistence values. One option for this algorithm is to avoid this quadratic correction and take, as final solution, only the first solution for target position in (7.65). However, for this option, the target position is equivalent (or worse at it solves the problem with more unknowns) to that one of Smith and Abel algorithm, which also diverges in this scenario. The remaining algorithms approximately provide acceptable performance, presenting also the characteristic peaks around $4 \mathrm{~km}$ and $18 \mathrm{~km}$, due the illconditioning of this scenario.

For the case of vertical R.M.S error (see Fig. 7.14), only Taylor and Schau and Robinson algorithms are capable of recovering some useful information, but only beyond $20 \mathrm{~km}$ from the runway threshold. Before this point, any algorithm provides any useful data for the vertical target position component.

Regarding to the 2D bias analysis, with the exception of Taylor, Smith and Abel, and Chan and Ho, all the simulated algorithms introduce some amount of bias (in average smaller than $8 \mathrm{~m}$ ), which can be neglected in practical terms. However, they also present some large peaks around $4 \mathrm{~km}$ and $18 \mathrm{~km}$, as in the 2D R.M.S case. On the other hand, for the vertical bias analysis, all the algorithms introduces very large amount of biases and, hence, it shows that for this scenario, any algorithm is capable of recovering a useful vertical position component. It is because the system geometry is more unfavourable for this vertical component, i.e., the VDOP is very large. 


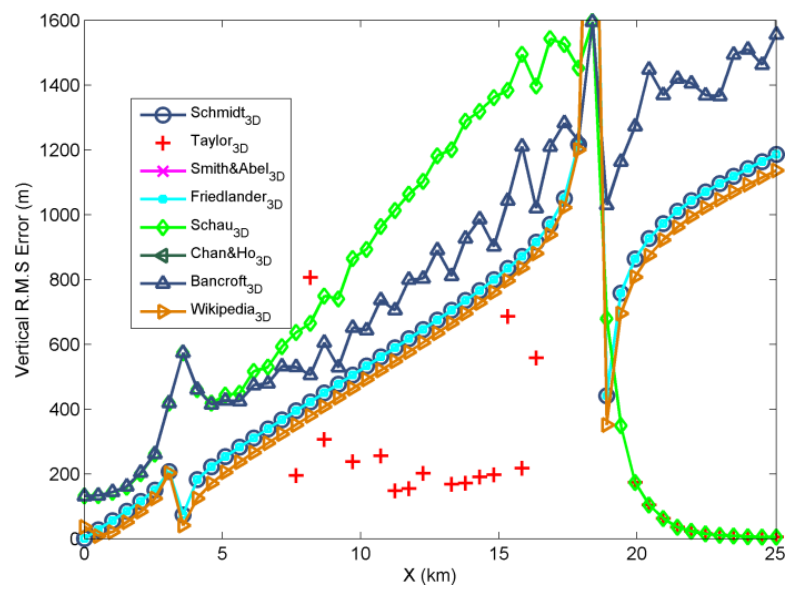

Fig. 7.14 Vertical R.M.S for Malpensa system: comparison of localization algorithms.

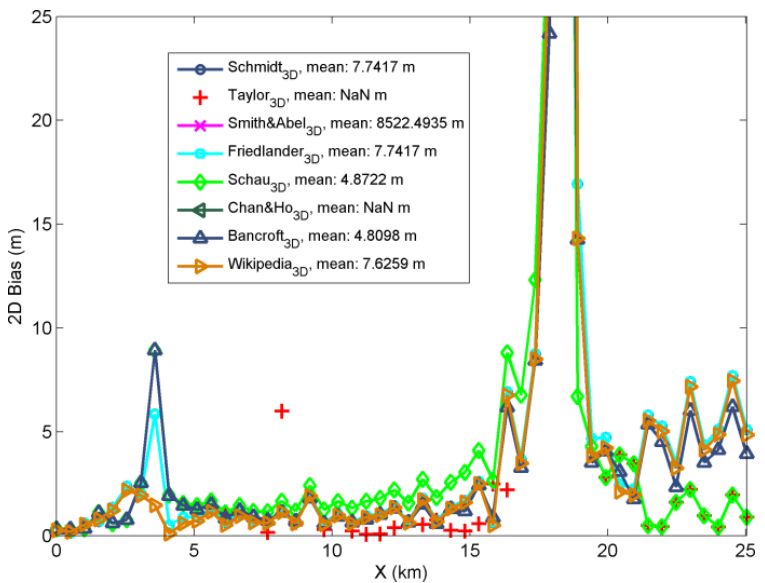

Fig. 7.15 2D bias for Malpensa system: comparison of localization algorithms.

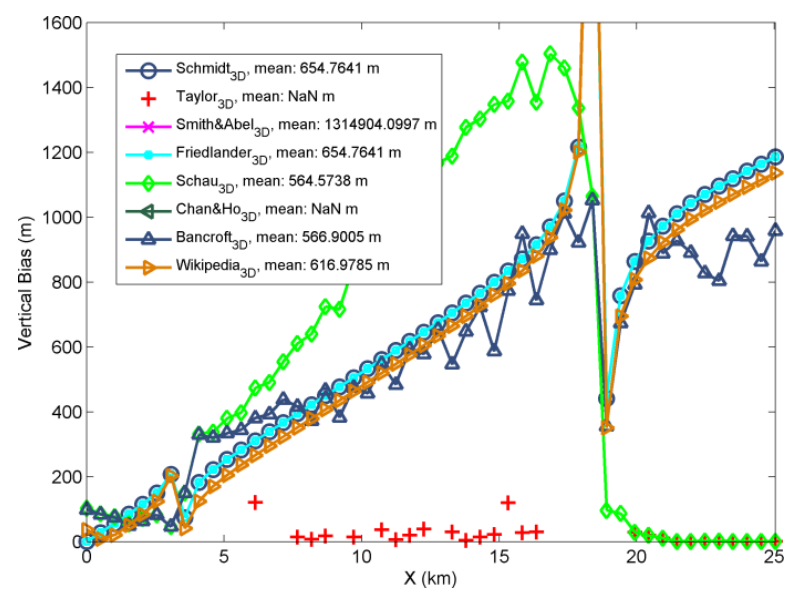

Fig. 7.16 Vertical bias for Malpensa system: comparison of localization algorithms. 
The 2D R.M.S and bias analysis for the projected version of localization algorithms is shown in Fig. 7.17 and Fig. 7.18, respectively. For this case, all the algorithms, including Taylor, and Smith and Abel, but with the exception of Chan and Ho, are capable of providing good performance levels. The reason for the projected version of the Chan and Ho algorithm is exactly the same as in its non-projected version. For the case of Smith and Abel algorithm that now provides very good accuracy, it is because, in its projected version, it has to calculate one less unknown (only two, i.e., $-x, y$-) with the same number of stations, which is translated in a improvement of the problem geometry conditioning for this particular algorithm. Moreover, the Schmidt, Smith and Abel, Friedlander, and Wikipedia algorithms provide exactly the same 2D R.M.S and bias (see Fig. 7.18 and the second column of Table 7.4, §7.6.4) for the entire takeoff line. In this scenario, the bias due to the projection is negligible for this scenarios and this fact allows them to improve the accuracy regarding to their non-projected versions. The main reason is because, in this scenario, the target flies to small flight levels than in Innsbruck scenario (see Fig. C.1 and Fig. C.2).

On the other hand, we can observe in Fig. 7.17 and Fig. 7.18 that Taylor, Schau and Robison, and Bancroft algorithms, although they provide good R.M.S and bias values for the most of takeoff line, they are stronger affected for the bias due to the projection. In this case, for these three algorithms, the bias and the R.M.S error are approximately the same for each of these (see the second column of Table 7.4, §7.6.4). It also means that the R.M.S is mainly dominated by the bias rather than by the standard deviation of every algorithm. Moreover, it also shows that the particular performance of every algorithm depends on the way to manage the hyperbolic equations and, therefore, not all the algorithms provide the same results under the same situations.

Finally, the average 2D and vertical R.M.S errors, and 2D and vertical bias, are shown in Table 7.2 and Table 7.3, §7.6.4. Later in $\S 7.6 .4$, based on these tables, we provide some general conclusion for the comparison of localization algorithms.

\subsubsection{Linate System (Surface Movement)}

The Linate LAM system is composed of eight receiving stations, which are deployed around the Linate airport (Milan, Italy). This system is intended for surface surveillance at Linate airport. Therefore, for this scenario we simulate a surface movement path that includes the runways, taxiways, and apron areas. The system layout and the surface movement path are depicted in Fig. C.3. For this scenario, only the 2D performance (i.e., the R.M.S and bias) for both non-projected and projected version of the localization algorithms is analyzed. The 2D R.M.S error and bias for the 


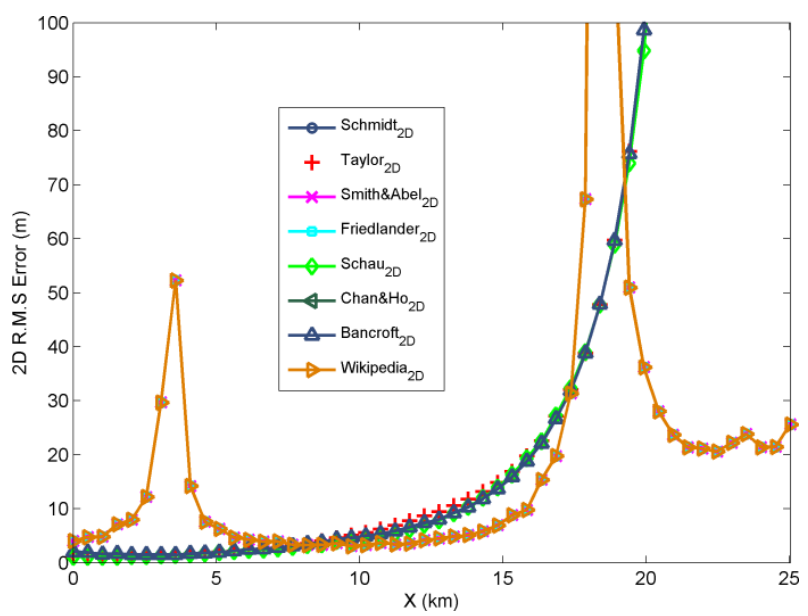

Fig. 7.17 2D R.M.S error for Malpensa system: comparison of projected localization algorithms.

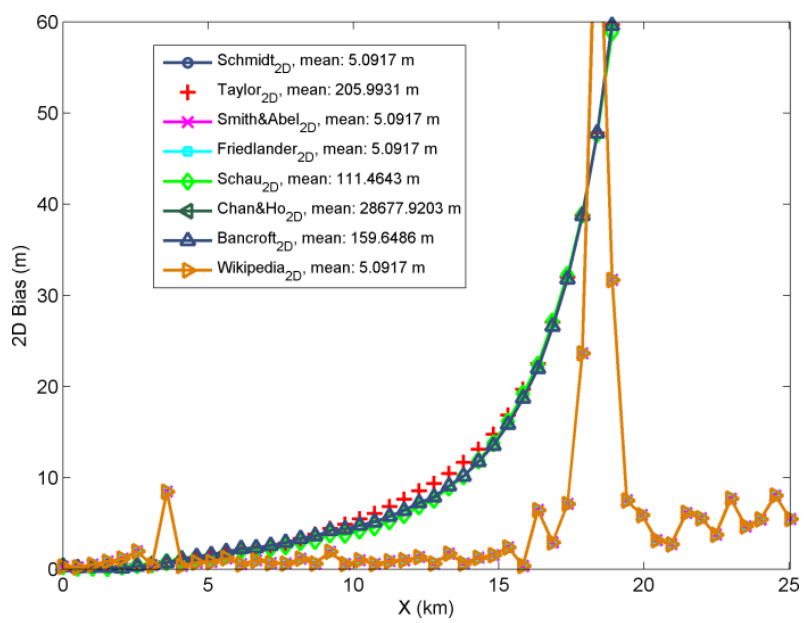

Fig. 7.18 2D bias for Malpensa system: comparison of projected localization algorithms.

non-projected localization algorithms are respectively shown in Fig. 7.19 and Fig. 7.20, whilst the corresponding ones for the projected versions are respectively shown in Fig. 7.21 and Fig. 7.22. Moreover, for this scenario, the possible failure of the station number 1 is simulated. The corresponding results are shown in Fig. 7.23 - Fig. 7.26.

For this scenario, all the non-projected localization algorithms (with the particular exception of Chan and Ho) provide a good average performance with the most of R.M.S errors being below $10 \mathrm{~m}$ and bias below $2.5 \mathrm{~m}$. Then, more specific, we observe all the algorithms present some large peaks of R.M.S (see Fig. 7.19) and bias (see Fig. 7.20) between the point $30-60$, around point 80 , and around point 130, being the Schau and Robinson, 


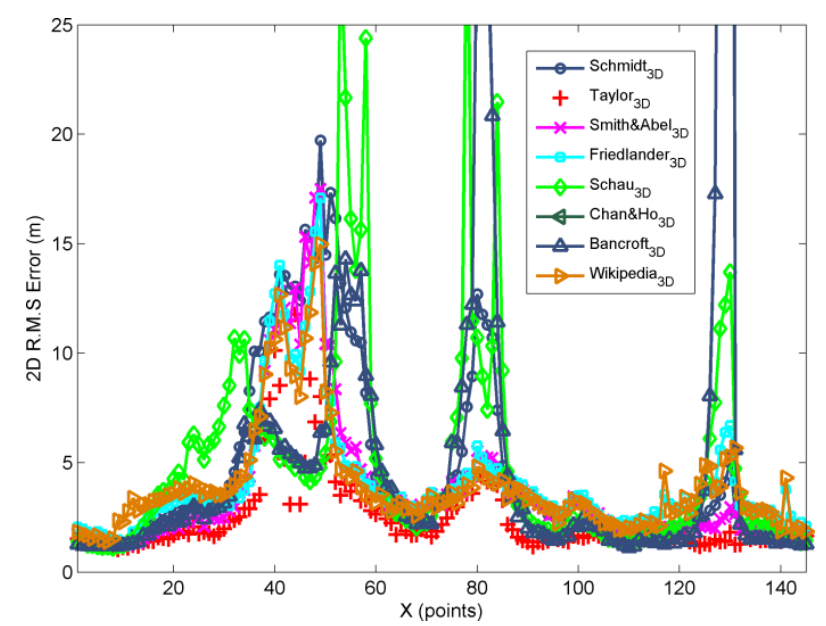

Fig. 7.19 2D R.M.S error for Linate system: comparison of localization algorithms.

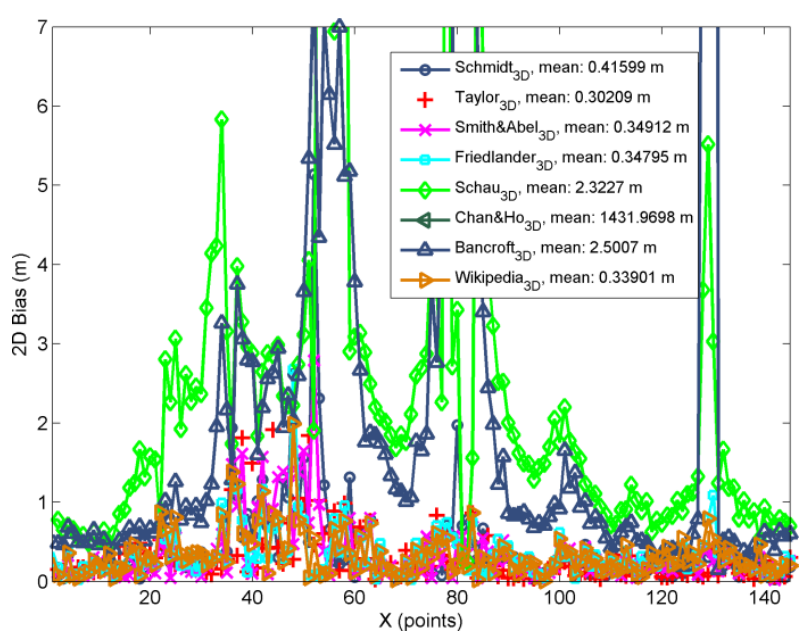

Fig. 7.20 2D bias for Linate system: comparison of localization algorithms.

Bancroft, and Schmidt, the algorithms that present the largest values. It is more clearly shown in the third column of Table 7.2, §7.6.4. Regarding to the bias analysis, all the algorithms (except Chan and Ho) can be considered numerically unbiased as they introduce only an average amount of bias smaller than $0.5 \mathrm{~m}$. Particularly, the Bancroft algorithm introduces an average bias of $2.5 \mathrm{~m}$, but it still can be considered small. Moreover, numerically, the Taylor algorithm is, again, the one that provides the minimum amount of bias. 


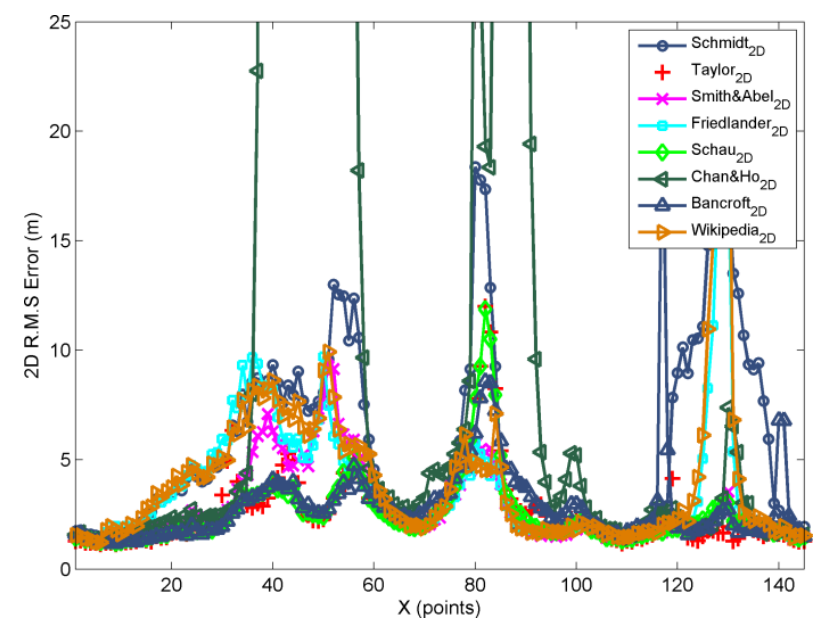

Fig. 7.21 2D R.M.S error for Linate system: comparison of projected localization algorithms.

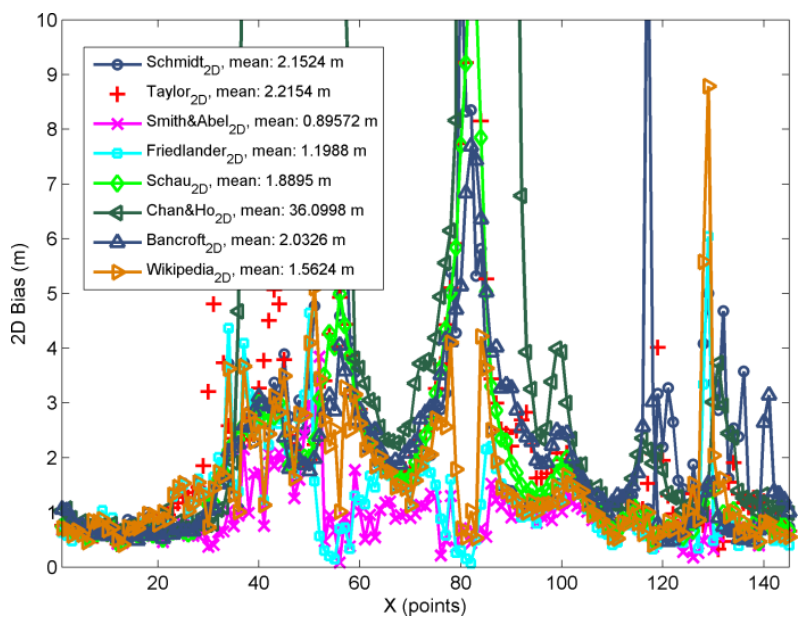

Fig. 7.22 2D bias for Linate system: comparison of projected localization algorithms.

On the other hand, regarding to the projected version of the localization algorithms, all of these provide also good performance levels. For this case, the projected Chan and Ho algorithm also provides good values for R.M.S error and bias. In general terms, the performance of the projected version of the algorithms is very similar to that of the non-projected versions (Fig. 7.21 and Fig. 7.22). More specific, the projected Schau and Robinson algorithm does not present the large peaks of R.M.S error and bias that appears in its non-projected form. In the same sense, the projected versions of Schmidt and Bancroft algorithms still keep some of the large peaks of R.M.S error. Also, for this case, the projected version Wikipedia and Friedlander algorithm present some large R.M.S error peaks around the 
point 130 (see Fig. 7.21). Moreover, we observe that some of projected algorithms (the Schmidt, Taylor, Smith and Abel, Friedlander, and Wikipedia) increase the amount of introduced bias. It is because the system geometry particularly affects the projection of those algorithms. Likewise, in the case of Schau and Robinson, and Bancroft algorithms, they reduce the amount of introduced bias regarding to their non-projected versions. However, these increments or reductions, for the amount of bias, in practical terms can be neglected and, hence, the performance of the nonprojected and projected algorithms for Linate scenario can be considered the same.

For the simulation of failure of one station (the station number 1 in Fig. C.3), the corresponding results are shown in Fig. 7.23 and Fig. 7.24 for the non-projected algorithms, whilst for the projected ones they are shown in Fig. 7.25 and Fig. 7.26. Under the failure of one station all the localization algorithms (non-projected and projected) degrades their overall performance. It is more clearly shown in Table 7.5, $\S 7.6 .4$ for the numerical analysis of this situation. It is expected because, generally, the system geometry quality decreases with a smaller number of stations, i.e., the HDOP increases. In Fig. 7.23 and Fig. 7.25 for the 2D R.M.S errors, we can see that the non-projected and projected versions of Smith and Abel, Friedlander, and Wikipedia algorithms, and the projected ones of Schau and Robinson, and Bancroft algorithms provide some acceptable performance levels. However, all of them present several large peaks of errors that are not suitable for surface surveillance [1]. Nowadays, this large peaks of errors are something smoothed by implementing tracking algorithms. Nevertheless, the tracking algorithms performance directly depends on the localization algorithms performance. Therefore, it is always advisable to provide localization strategies as accurate as possible.

Other important observation for the case of projected algorithms is that, for this case, the average amount of bias, of all them, is reduced regarding to the case of non station failure. It is because the new system geometry (i.e., without the station number 1 ) is not as negatively affected by the bias due to the projection as the first one. It is clearly understood by analyzing the system layout in Fig. C.3. In this system, the station number 1 is the highest station and it is very close to an area with a high density of points. Thus, the equivalent angle of projection (cf. (7.89)) is closer to $90^{\circ}$ and, hence, the amount of bias introduced by this station is not negligible. In this sense, when this station is not used, the bias due to the projection is reduced over that area. By contrary, with one less station, the overall position accuracy for all the algorithms decreases, as the R.M.S increment is dominated by the data dispersion rather than bias. 


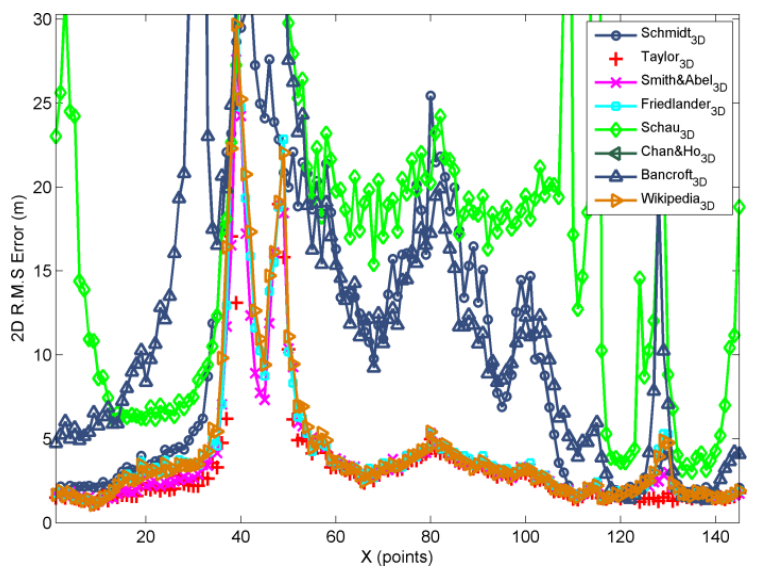

Fig. 7.23 2D R.M.S error for Linate system in the presence of the failure of station 1: comparison of localization algorithms.

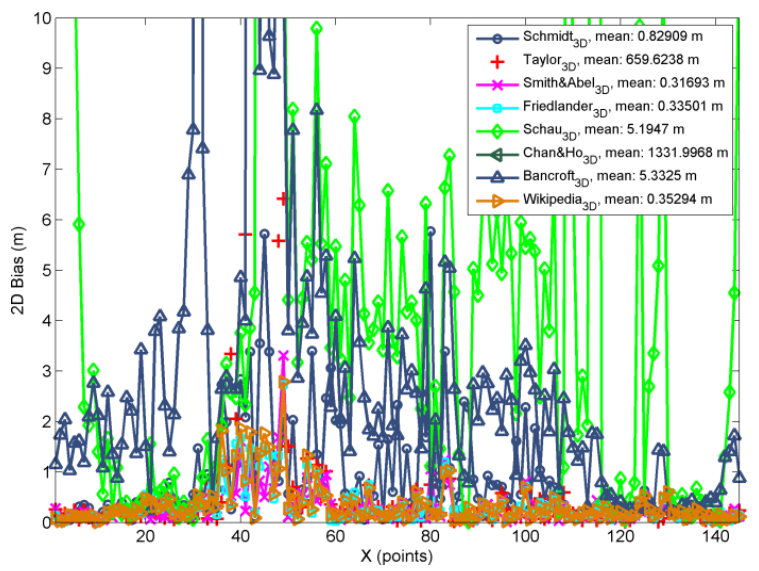

Fig. 7.24 2D bias for Linate system in the presence of the failure of station 1: comparison of localization algorithms.

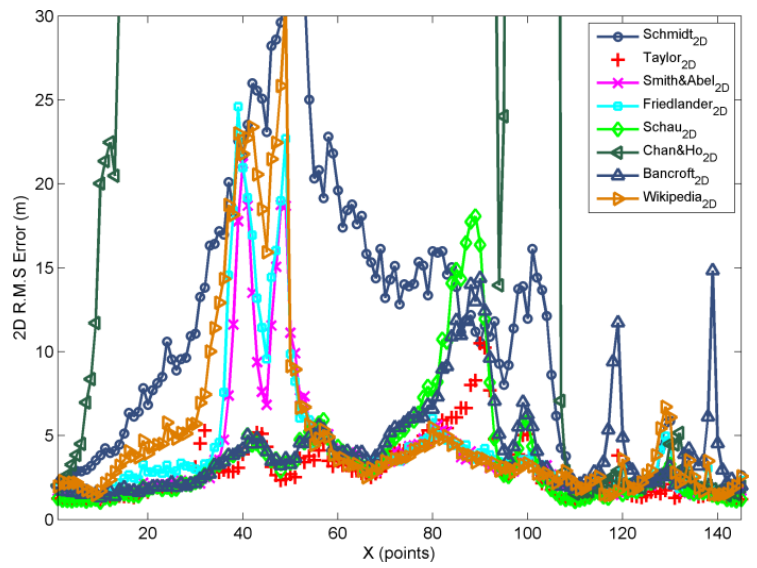

Fig. 7.25 2D R.M.S error for Linate system in the presence of the failure of station 1: comparison of projected localization algorithms. 


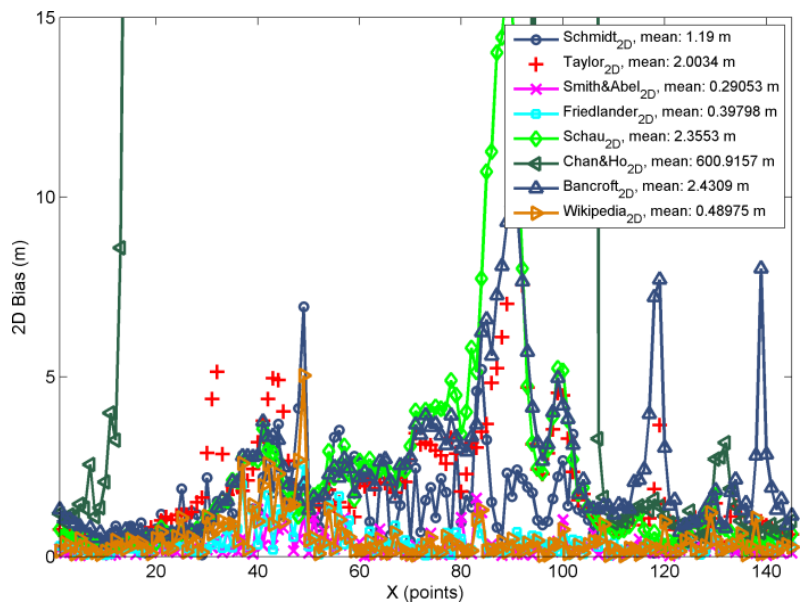

Fig. 7.26 2D bias for Linate system in the presence of the failure of station 1: comparison of projected localization algorithms.

\subsubsection{Numerical Comparison and General Conclusions for Simulated Scenarios}

In this section we provide some general conclusions about the comparison of localization algorithms. To do this, we support our analysis on the average values of R.M.S and bias, for every localization algorithms, and for the entire simulated paths. The values for the 2D R.M.S error and bias for the non-projected algorithms are shown in Table 7.2 , while the corresponding for the vertical position component analysis are shown in Table 7.3. The 2D R.M.S error and bias for projected algorithms are shown in Table 7.4.

For Innsbruck scenario, we have found the non-projected algorithms are practically unbiased through the entire takeoff line. It means that the R.M.S error is dominated by the standard deviation component, which is clear by comparing the amount of bias with the R.M.S error in the first column of Table 7.2. It also shows that the Innsbruck scenario presents a well-determined localization problem. Moreover, when it happens, the R.M.S error can be more reduced by implementing tracking algorithms, as it is due more to the standard deviation than for the bias. Exactly the same performance is appreciated for the vertical position component. In this sense, we conclude that all the analyzed non-projected localization algorithms are very statistically efficient, where the best performance is achieved by the Taylor algorithm, as it is capable of improving the accuracy of the starting point. Specially, this algorithm is highly efficient for recovering the vertical position component information, which is of vital importance for WAM surveillance applications. Thus, we recommend in 
these cases the use of a combined strategy composed of a closed form algorithm and the Taylor one. All commented above in this paragraph also show the theory described before in this chapter; that is, under suitable system geometries, all the localization algorithms are highly efficient, whilst under unfavourable system geometries this efficiency is reduced and some performance analysis must be carried out, in order to select the best option. In the same sense, we have observed that in the way that the system geometry is unfavourable (i.e., for high HDOP or VDOP), the R.M.S increments are also due to a bias increment, which is translated in an algorithm efficiency loss. Then, the best algorithm (or strategy) is the one that better exploit the statistical efficiency with high values of the DOP (either HDOP or VDOP), i.e., under an unfavourable system geometry.

Regarding to the projected localization algorithms in Innsbruck WAM system, all of these are significantly affected by the bias due to the projection. It is clear by analyzing the first column of Table 7.4, as the amount of bias, for every algorithm, is approximately equal to the R.M.S value. In this sense, we can conclude that the algorithms performance is mainly dominated by the bias due to the projection. Moreover, also the standard deviation of the algorithm, in these cases, is much smaller than the corresponding bias. Therefore, for this kind of scenario (WAM) is not advisable to use the projected version of the localization algorithms. In the same line, the opposite happens for the non-projected algorithms, that is: the R.M.S is mainly dominated by the standard deviation (the bias is smaller than that). It is because as the non-projected localization algorithms include the vertical position component, they are "worse" conditioned than the projected ones and, therefore, the data dispersion (i.e., the standard deviation) increases but, as they do not used projections, they are nearly unbiased. By contrary, when such a bias due to the projections can be neglected, the improvements in the R.M.S, due to the use of the projected algorithms, can be clearly appreciated. Likewise, when the problem is highly ill-conditioned, as in Malpensa case, the dispersion significantly increases and, neither the non-projected algorithms nor the projected ones can provide useful position data.

For Malpensa scenario, the best performance of the non-projected algorithms are provided by Schau and Robinson, and Bancroft algorithms, although also Schmidt, Friedlander and Wikipedia ones, provide similar values. However, all of these algorithms are also affected by the illconditioning and, hence, they present some large peaks of R.M.S errors. In turn, the Smith and Abel, and Chan and Ho algorithms do not provide any useful position data through the entire simulated takeoff line. The Taylor algorithm only provides useful position data in those points that are wellconditioned. Nevertheless, in practical terms, this algorithm does not work for this scenario. On the other hand, regarding to estimation of the vertical 
position component, any algorithm is capable to provide any useful data. It is also because this problem is more ill-conditioning in its vertical component; hence, any algorithm, no matter how robust it is, is capable of estimating an accurate vertical target position.

Regarding to the projected algorithms, the Schmidt, Friedlander, and Wikipedia ones, practically provide the same performance than in their non-projected versions (compare the second columns of Table 7.2 and Table 7.3). It means that, for this scenario, the projection does not significantly affect these algorithms. Likewise, the projected version of the Smith and Abel provides the same performance of these three algorithms (Schmidt, Friedlander, and Wikipedia). It is because now it is estimating one less unknown (i.e., the vertical one) with the same number of stations. It also shows one of the disadvantages of the non-projected version of this algorithm, that is, in the presence of small number of stations is not capable to provide accurate position data. Finally, for this scenario, the best performance is provided by the non-projected Schau and Robinson algorithm (see Table 7.2). Then, also the projected Schmidt, Wikipedia, and Friedlander algorithms provide good performance.

As a general conclusion for WAM scenarios, we find that the algorithms using algebraic approach based models (i.e., the Schmidt and Wikipedia ones) provide good and stable performance for all the scenarios, and in both non-projected and projected versions. Moreover, when these algorithms are solved by the pseudoinverse matrix, the condition of $n+2$ minimum stations (see Table 7.1, §7.3.8) can be reduced to $n+1$, in which case the localization problem becomes underdetermined, and evidently sacrificing the estimation of the vertical component. This robust performance of these algorithms, under the situation of small number of stations, is because they are the only ones that do not make numerical assumption in their data model. It allows the pseudoinverse matrix to accurately solve the underdetermined problem.

For Linate scenario, in general terms, with the exception of Chan and Ho algorithm, all the algorithms (non-projected and projected ones) provide good performance levels for surface surveillance (see the third column of Table 7.2 and Table 7.4). Likewise, under the failure of one station, the accuracy of all algorithms is reduced and, they provide different performance levels for different areas (see Table 7.5). Particularly, for this scenario we find the Smith and Abel, Friedlander, and Wikipedia algorithms approximately provide the best general performance.

On the other hand, more specific, under standard conditions (i.e., without the failure of stations), the Taylor algorithm is the one that provides the minimum amount of bias. It is in agreement with the previously described theory in this chapter, as the Taylor is the most (at least theoretically) efficient algorithm (minimum bias and variance) as long as the convergence can be guaranteed. The problem of this algorithm is 
that it does not always converge, as in the case of failure of one station. Regarding the projected Taylor algorithm, it keeps a smaller R.M.S error but the amount of introduced bias slightly increases. It is expected because it is something affected by the projection.

Table 7.2 Mean 2D R.M.S error and 2D bias values for localization algorithms. Values given in meters.

\begin{tabular}{|c|c|c|c|c|c|c|}
\hline \hline \multirow{2}{*}{ Algorithm Scenario } & \multicolumn{2}{|c|}{ Innsbruck } & \multicolumn{2}{c|}{ Malpensa } & \multicolumn{2}{c|}{ Linate } \\
\cline { 3 - 8 } & R.M.S & Bias & R.M.S & Bias & R.M.S & Bias \\
\hline Schmidt & 33.38 & 2.92 & 24.05 & 7.74 & 4.70 & 0.41 \\
\hline Taylor & 27.88 & 2.51 & NaN & NaN & 2.42 & 0.30 \\
\hline Smith\&Abel & 27.46 & 2.12 & 8523 & 8522 & 3.76 & 0.34 \\
\hline Friedlander & 37.21 & 3.12 & 24.05 & 7.74 & 4.08 & 0.34 \\
\hline Schau\&Robinson & 37.94 & 3.14 & 19.78 & 4.87 & 4.96 & 2.32 \\
\hline Chan\&Ho & 43.57 & 12.86 & NaN & NaN & 1175 & 1431 \\
\hline Bancroft & 180.60 & 16.77 & 18.50 & 4.80 & 5.91 & 2.50 \\
\hline Wikipedia & 34.98 & 2.89 & 24.11 & 7.62 & 3.96 & 0.33 \\
\hline \hline
\end{tabular}

Table 7.3 Mean vertical R.M.S error and vertical bias values for localization algorithms. Values given in meters.

\begin{tabular}{|c|c|c|c|c|}
\hline \hline \multirow{2}{*}{ Algorithm } & \multirow{2}{*}{ Scenario } & \multicolumn{2}{|c|}{ Innsbruck } & \multicolumn{2}{c|}{ Malpensa } \\
\cline { 2 - 5 } & R.M.S & Bias & R.M.S & Bias \\
\hline Schmidt & 36.74 & 3.02 & 654.8 & 654.7 \\
\hline Taylor & 16.13 & 1.42 & NaN & NaN \\
\hline Smith\&Abel & 36.3 & 3.06 & $1.315 \mathrm{E}+06$ & $1.314 \mathrm{E}+06$ \\
\hline Friedlander & 38.77 & 3.37 & 654.8 & 654.7 \\
\hline Schau\&Robinson & 28.35 & 2.48 & 646.8 & 564.57 \\
\hline Chan\&Ho & 33.67 & 4.04 & NaN & NaN \\
\hline Bancroft & 65.91 & 5.07 & 854.2 & 566.9 \\
\hline Wikipedia & 38.88 & 3.3 & 617 & 616 \\
\hline \hline
\end{tabular}


Table 7.4 Mean 2D R.M.S error and 2D bias values for projected localization algorithms. Values given in meters.

\begin{tabular}{|c|c|c|c|c|c|c|}
\hline \hline \multirow{2}{*}{ Algorithm } & \multirow{2}{*}{ Scenario } & \multicolumn{2}{|c|}{ Innsbruck } & \multicolumn{2}{c|}{ Malpensa } & \multicolumn{2}{c|}{ Linate } \\
\cline { 3 - 8 } & R.M.S & Bias & R.M.S & Bias & R.M.S & Bias \\
\hline Schmidt & 582 & 581 & 23.07 & 5.09 & 5.46 & 2.15 \\
\hline Taylor & 684 & 683 & 206.2 & 205.9 & 2.6 & 2.21 \\
\hline Smith\&Abel & 695 & 694 & 23.07 & 5.09 & 2.87 & 0.89 \\
\hline Friedlander & 1054 & 1052 & 23.07 & 5.09 & 3.75 & 1.19 \\
\hline Schau\&Robinson & 314 & 311 & 111.8 & 111.46 & 2.56 & 1.88 \\
\hline Chan\&Ho & 77190 & 77186 & 28680 & 28677 & 37.29 & 36 \\
\hline Bancroft & 2072 & 2071 & 160 & 159 & 2.89 & 2.03 \\
\hline Wikipedia & 536.5 & 535 & 23.07 & 5.09 & 4.1 & 1.56 \\
\hline \hline
\end{tabular}

Table 7.5 Mean 2D R.M.S error and 2D bias values for the localization algorithms, at Linate scenario, in the presence of a failure of station 1. Values given in meters.

\begin{tabular}{|c|c|c|c|c|}
\hline \hline \multirow{2}{*}{ Algorithm } & \multirow{2}{*}{ Scenario } & \multicolumn{2}{|c|}{ Linate } & \multicolumn{2}{c|}{ Linate (projected algorithms) } \\
\cline { 3 - 6 } & R.M.S & Bias & R.M.S & Bias \\
\hline Schmidt & 9.84 & 0.82 & 11.07 & 1.19 \\
\hline Taylor & 6601 & 659 & 2.85 & 2.00 \\
\hline Smith\&Abel & 3.97 & 0.31 & 3.88 & 0.29 \\
\hline Friedlander & 4.38 & 0.33 & 4.44 & 0.39 \\
\hline Schau\&Robinson & 22.65 & 5.19 & 3.69 & 2.35 \\
\hline Chan\&Ho & 1485 & 1331 & 602 & 600 \\
\hline Bancroft & 21.97 & 5.33 & 4.2 & 2.43 \\
\hline Wikipedia & 4.44 & 0.35 & 5.35 & 0.48 \\
\hline \hline
\end{tabular}

As a general conclusion, the Schmidt, Wikipedia, Schau and Robinson, and Friedlander algorithms roughly provide the best performance levels. Likewise, when its convergence can be guaranteed, the Taylor algorithm always significantly improves the accuracy provided by the previous commented algorithm by using their position data as its starting point. It is observed in Innsbruck scenario for both horizontal and vertical performance, and for Linate. Moreover, also it holds for Malpensa for those points where the DOP allows its convergence. Then, there is no a "superior" algorithm that provides the best performance under any scenario or situation. For this reason, a previous analysis of the algorithms performance is always advisable.

The above conclusion has motivated us to look for strategies that allow ensuring the Taylor algorithm convergence. Also, we consider important to emphasize that the computational cost of using a closed form algorithm, as the starting point of Taylor one, is very low due to the characteristics of them: they are not iterative and the formulation is very simple. 


\subsection{Experimental With Real Data}

To perform the experimental with real data, the company ERA A.S. has provided us of a record of TOA measurements of one of its operational system, the LAM system installed at Tallinn airport (Tallinn, Estonia). This system is intended for surface surveillance and is composed of fourteen receiving stations. The record of TOA measurements was taken through the entire airport surface following the requested procedures by the European regulatory bodies [1]. The record contains more than 4000 register (with an average period of $1 \mathrm{~s}$ ), where each register contains set of TOA measurements. From the information provided by the company, we know that these measurements are perturbed by multipath noise in some areas. Moreover, also the company above mentioned has provided us of highly accurate position reference data, which was simultaneously recorded with a GPS receiver with differential correction capabilities (DGPS). This data is used to evaluate the solutions accuracy of the localization algorithms analyzed and proposed in this thesis. The corresponding evaluation procedure is explained below. The system layout and the reference position data are depicted in Fig. C.4. Additionally, Fig. C.5 shows the LoS profile for this system, that is, this profile shows for every register the number of stations that measure the TOA of the emitted Mode S signal.

The procedure to evaluate the accuracy of the solutions, for this scenario, is something different that the one used for the simulated ones. In the previous scenarios $(\$ 7.6)$ we characterized every spatial point of the simulated path. It was possible as we performed Monte-Carlo simulations. In this case, the system accuracy is evaluated by calculating the errors (e.g., the Euclidean one) between the positions obtained by any localization algorithm and the corresponding ones provided by the DGPS. In other words, in this case the DGPS position data is assumed to be the exact one. Thus, an error distribution is obtained and its statistical characterization provides a reliable idea about the system accuracy. It is an established procedure that is requested by all the ANSP (Air Navigation Service Provider) in Europe and it is described by the EUROCAE MOPS (Minimum Operational Performance Specification) ED-117 [1]. The most used parameters to characterize the errors distribution, for any localization algorithm or strategy, are the standard deviation and the mean of that, and the Probability of Localization (PoL), also called probability of detection, under certain accuracy threshold $\delta$. The latter basically provides the percentage of estimated points that has an error smaller than the predefined accuracy threshold $\delta$. In this thesis, we use the Euclidean (or 2D) error, which is defined as follows: 


$$
\operatorname{error}_{2 D}(t)=\sqrt{\left(\hat{x}(t)-x_{D G P S}(t)\right)^{2}+\left(\hat{y}(t)-y_{D G P S}(t)\right)^{2}}
$$

where $(\hat{x}(t), \hat{y}(t))$ is the position calculated by a localization algorithm at a time $t$, and $\left(x_{D G P S}(t), y_{D G P S}(t)\right)$ is the reference position provided by the DGPS receiver at the same time $t$. The above 2D error can be also seen as:

$$
\operatorname{error}_{2 D}(t)=\sqrt{\operatorname{error}_{x}(t)^{2}+\operatorname{error}_{y}(t)^{2}}
$$

where $\operatorname{error}_{x}(t)=\hat{x}(t)-x_{D G P S}(t)$ and $\operatorname{error}_{y}(t)=\hat{y}(t)-y_{D G P S}(t)$, which compose the corresponding error distributions for the $x$ and $y$ coordinates, respectively. Now, the PoL is defined as the probability of calculate a target position with an error smaller than the predefined accuracy threshold $\delta$ and it can be expressed, for any localization method, as follows:

$$
P o L=\left(\frac{1}{L_{R}} \sum_{i=1}^{L_{R}} \operatorname{error}_{2 D}(i) \leq \delta\right) \times 100(\%)
$$

where $L_{R}$ is the total number of estimated positions, and the index $i$ represents the discrete number for every calculated error at every time $t$.

In this part, we show, for every localization algorithm, the plots for every estimated position coordinate (i.e., $x$ and $y$ ) in respect of the time of registers, and along with the corresponding coordinate as provided by the DGPS receiver. The time of registers is a UNIX time, given in format of seconds. Moreover, for each plot, in its legend, we also show the standard deviation and mean for the corresponding error distribution. The $x$ coordinate calculated for the non-projected algorithms are shown in Fig. 7.27 and Fig. 7.28, whilst the $y$ coordinate is shown in Fig. 7.29 and Fig. 7.30. The same results for the projected algorithms are shown in Fig. 7.31 Fig. 7.34. Finally, the standard deviation and mean values for the $2 \mathrm{D}$ errors distributions, and the $\mathrm{PoL}$ with $\delta=7.5 \mathrm{~m}$ and $\delta=15 \mathrm{~m}$ are shown in Table 7.6 for the non-projected algorithms, and in Table 7.7 for the projected ones.

From Fig. 7.27 to Fig. 7.34, we can see all the localization algorithms (non-projected and projected) provides position data with certain dispersion; it is clear as every plot is deviated from the corresponding DGPS data. Some algorithms provide higher dispersion and bias than others. However, from this figures it is not clear the difference among all algorithms. For this reason, we summarize the most important statistics in Table 7.6 and Table 7.7, and base our analysis on the values contained in them. 
Let us to firstly focus on the standard deviation and mean columns of Table 7.6, for the non-projected algorithms. These algorithms provide very large values of standard deviation and mean, significantly much greater than the requested one for surface surveillance [1]. In this sense, the Schmidt, Friedlander, and Wikipedia algorithms provide the smallest values (385 $\mathrm{m}$ and $37 \mathrm{~m}$ respectively). Then, the Smith and Abel, Schau and Robinson, Bancroft, algorithms provide greater values for standard deviation and mean. The Taylor algorithm is the one with the greatest values. The Chan and Ho algorithm deserves a special comment that is given later. The main reasons of these large values are mainly two: the first one is the conditioning of the localization problem for this scenario, which presents an equivalent characteristic to the one of Linate (see \$7.6.3), and the second one, the most relevant in this case, is the multipath noise that is contained in the TOA measurements. These two aspects generate the most deviated positions. In Fig. 7.27 to Fig. 7.34, for all the localization algorithms, the most of estimated coordinates are very close to the DGPS ones, and only for some specific time windows, they are highly deviated. In these time windows, which are equivalent to some airport areas, the largest errors are obtained. In this point, the parameter of PoL takes significant importance, as it tells us the percentage of point that has an error below the accuracy threshold. The values of PoL are shown in the third column of Table 7.6 for the non-projected algorithms, and the corresponding analysis is provided below.

For the case of PoL analysis in Table 7.6, the ranking of best algorithms something changes, and the Taylor algorithm is the one with the highest PoL for both accuracy thresholds $(7.5 \mathrm{~m}$ and $15 \mathrm{~m})$, i.e., Taylor algorithm is the one with the large amount of points with smaller errors. The reason of this contrast is quite simple, and is based on the fact that when Taylor does not converge, the results in those cases are very large but, when it converges, it is capable of providing the most smoothed and unbiased position data. By contrary, the closed form algorithms, although do not present those very large peak of errors, they are not statistically efficient. Regarding to the PoL of them, they provide similar values and much smaller than the ones of Taylor. Again, we conclude that the most efficient localization strategy is by combining a closed form algorithm and the Taylor one.

The Chan and Ho algorithm only provides $\mathrm{PoL}$ values smaller than $6.1 \%$. It shows the main disadvantage of this algorithm that is the low capability of obtained the first solution (cf. (7.65)), particularly the target range. In this case, when this algorithm applies the quadratic correction, as the target range is highly inaccurate (in some cases negative), this correction also leads to a highly inaccurate positions. We have found that if only the first solution, for target position, of this algorithm is taken as the 
final one, it presents an equivalent performance as Smith and Abel algorithm, as we have also described from the theoretical point of view. We do not show these additional results because the objective is to analyze the complete algorithm, and its main improvement that basically is the quadratic correction of the first target position estimation.

On the other hand, we consider important to comment that, in the CPS of the MLAT system, there is always implemented a set of tracking algorithms, which significantly reduce the standard deviation, of any of the analyzed localization algorithms, to values that are within the required standards. However, there is no the objective of this thesis to carry out the performance analysis of those tracking algorithms. The objective of this thesis part is to analyze the performance of the localization algorithms, because the more accurate the data of these algorithms, the more accurate the results of the tracking algorithms. Therefore, this analysis is intended to identify which localization algorithms provide the best statistical characteristics.

Regarding to the projected version of localization algorithms, we observe the same general tendency as in the case of non-projected algorithms. The most important difference is that, for the projected algorithms, the standard deviation and mean values of each of these are smaller than their corresponding values in the non-projected versions (compare Table 7.6 and Table 7.7). The reason of these smaller values is because the two dimensional projection does not significantly affect these algorithms in this scenario and, therefore, the possible introduced measurement bias is negligible regarding to the improvement in the standard deviation; improvement that is obtained for the better conditioned of the projected localization problem.

With this experiment, we obtain the same general conclusions than in the previous analysis for the simulated scenarios in $§ 7.6$. Equally than for Innsbruck, Malpensa, and Linate scenarios, the Taylor algorithm presents the best statistical characteristics, but it is not always numerically stable as its convergence is not guaranteed. In the same sense, the Schmidt, Wikipedia, Schau and Robinson, and Friedlander algorithms also present good performance levels. Additionally, for this experiment, we have found that Smith and Abel algorithm presents good performance. It is because the Tallinn system is composed of a large number of stations (14, with minimum 5 stations having LoS to the most of airport areas) and, therefore, is geometrically well conditioned. However, due to the non general stable performance of this algorithm, we do not recommend it for using in a combined strategy with the Taylor one. 

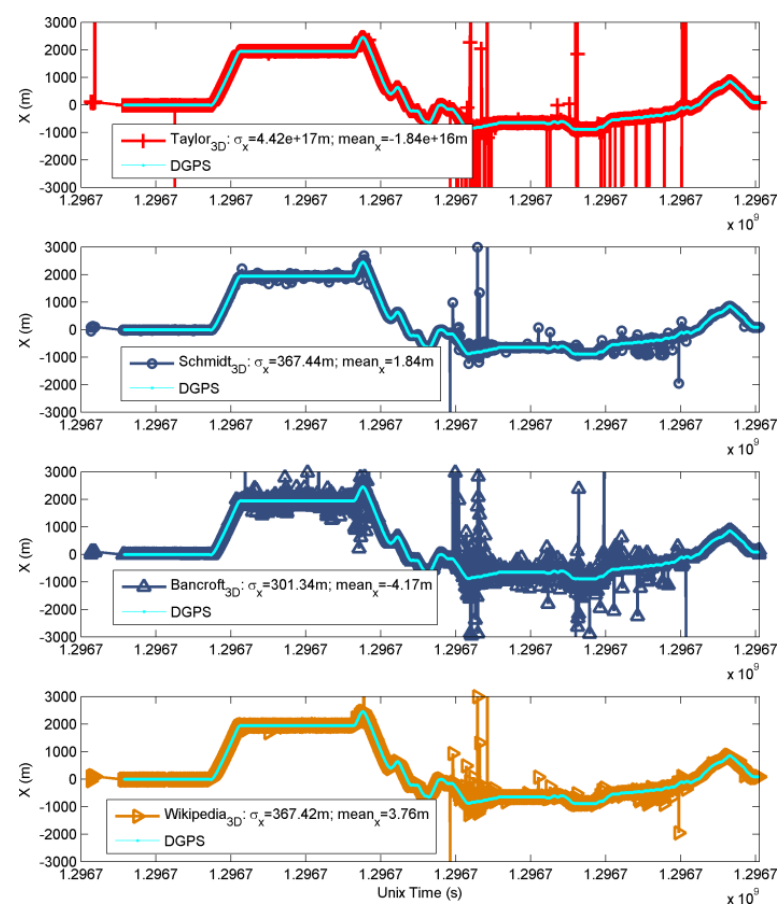

Fig. 7.27 $\mathrm{X}(\mathrm{t})$ for statistical and algebraic approach based models: comparison of localization algorithms.
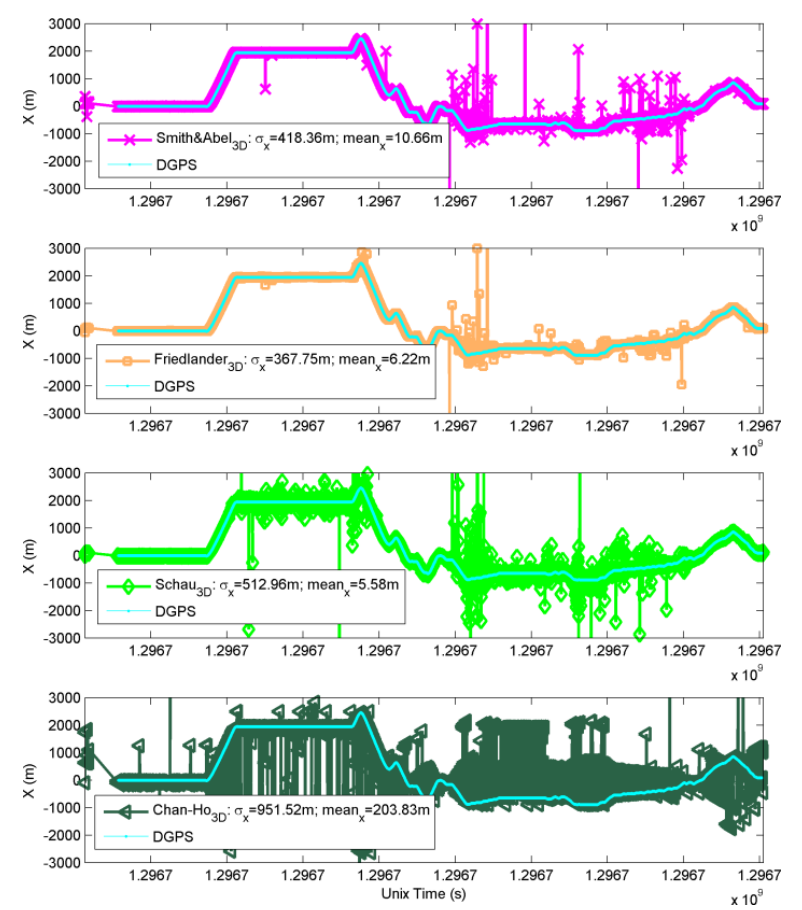

Fig. 7.28 X(t) for numerical approach based models: comparison of localization algorithms. 

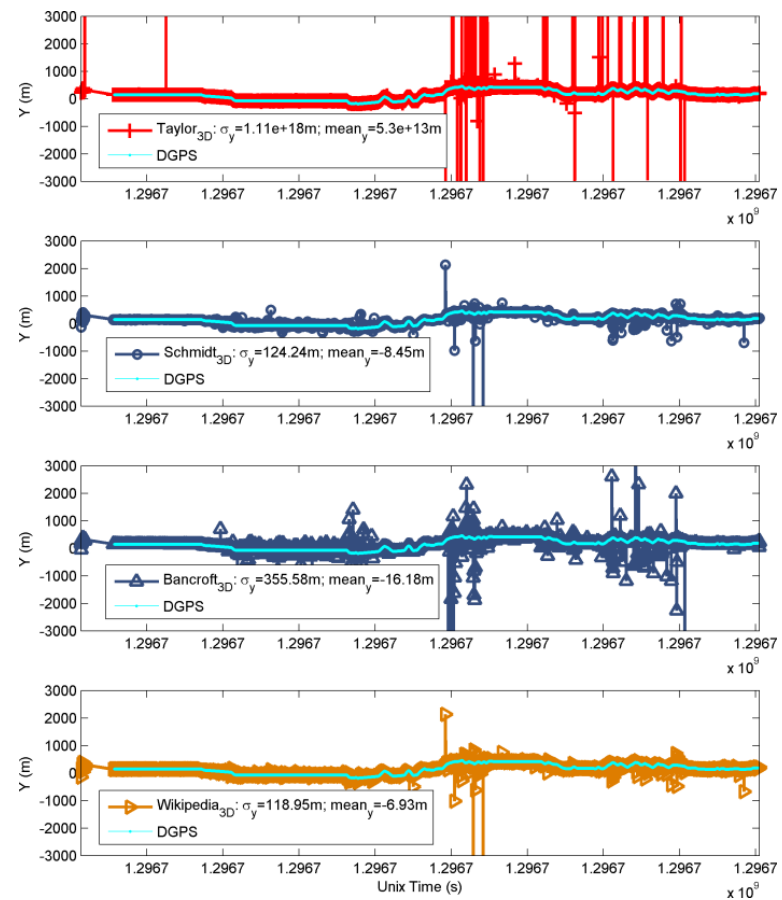

Fig. 7.29 $\mathrm{Y}(\mathrm{t})$ for statistical and algebraic approach based models: comparison of localization algorithms.
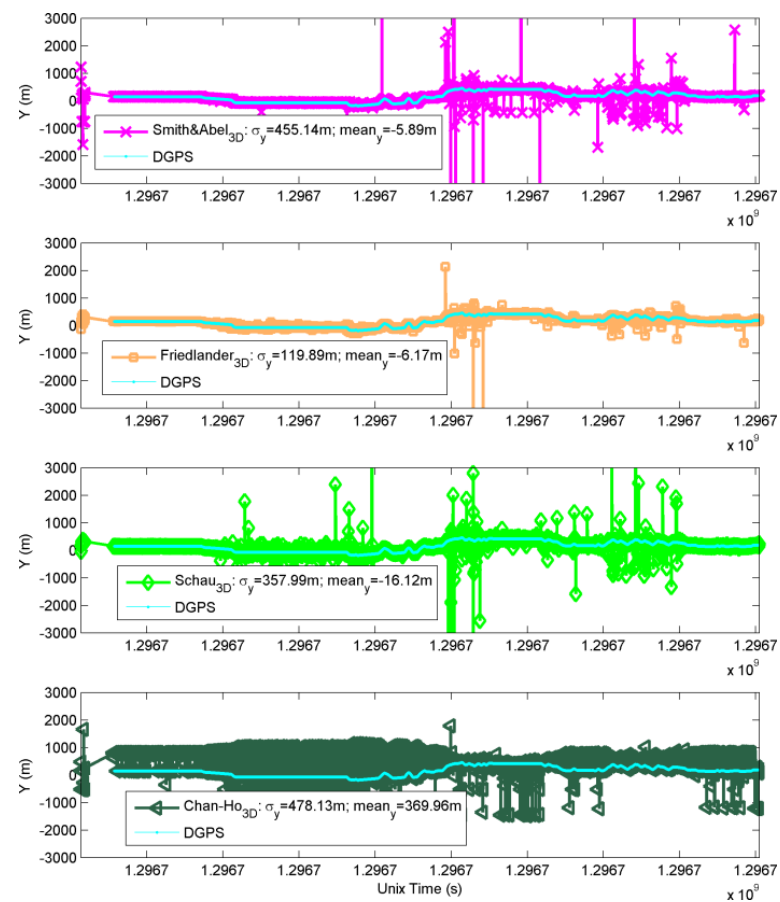

Fig. 7.30 Y(t) for numerical approach based models: comparison of localization algorithms. 

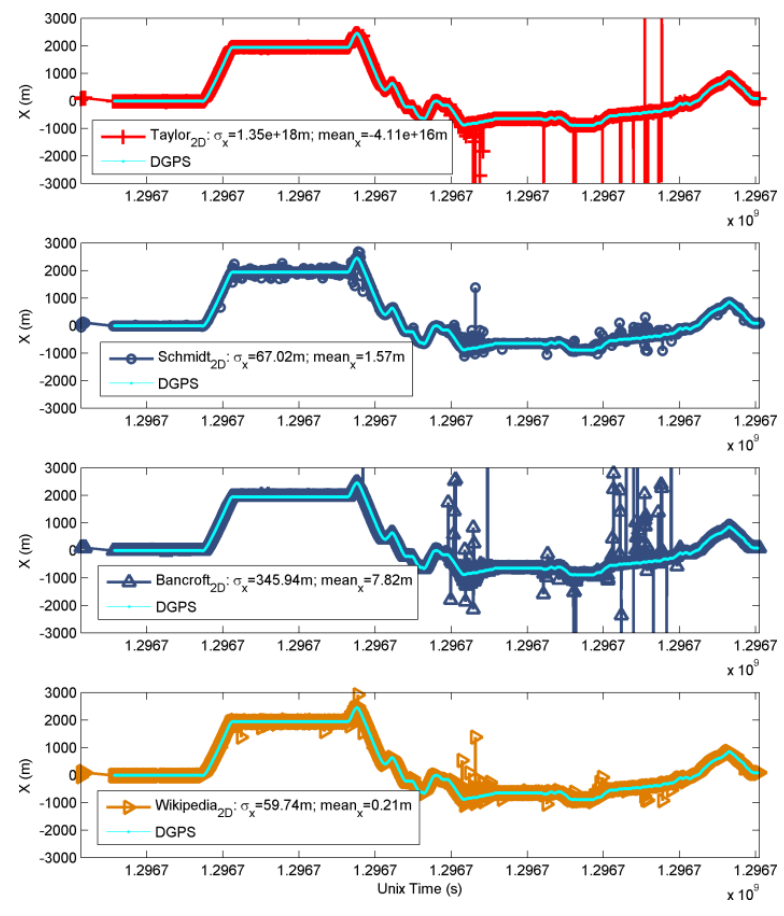

Fig. 7.31 X(t) for statistical and algebraic approach based models: comparison of projected localization algorithms.
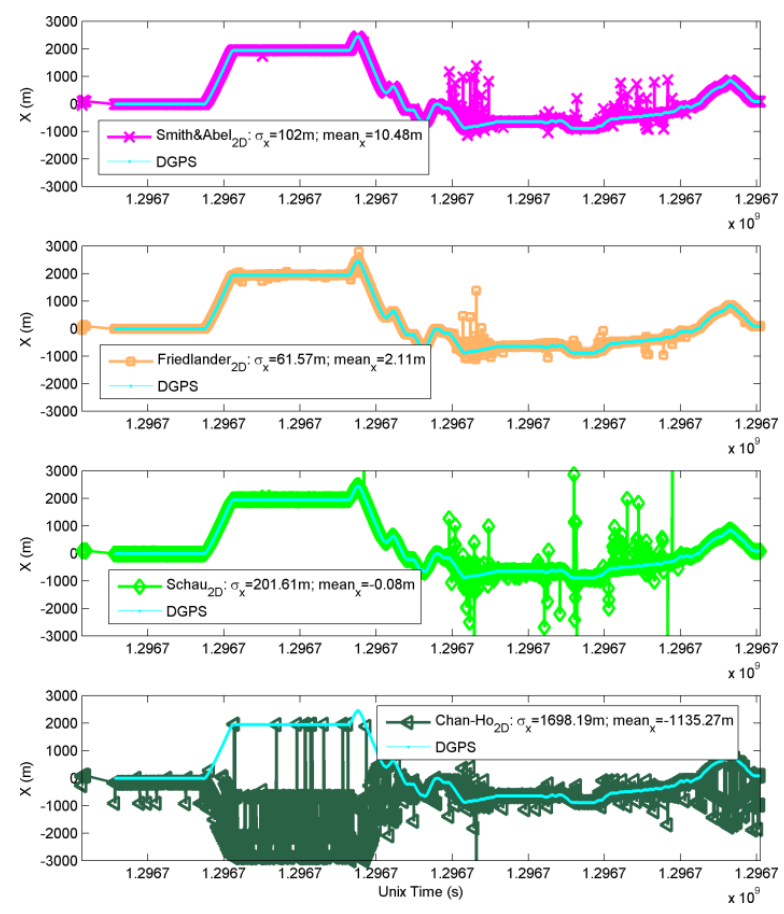

Fig. 7.32 $\mathrm{X}(\mathrm{t})$ for numerical approach based models: comparison of projected localization algorithms. 

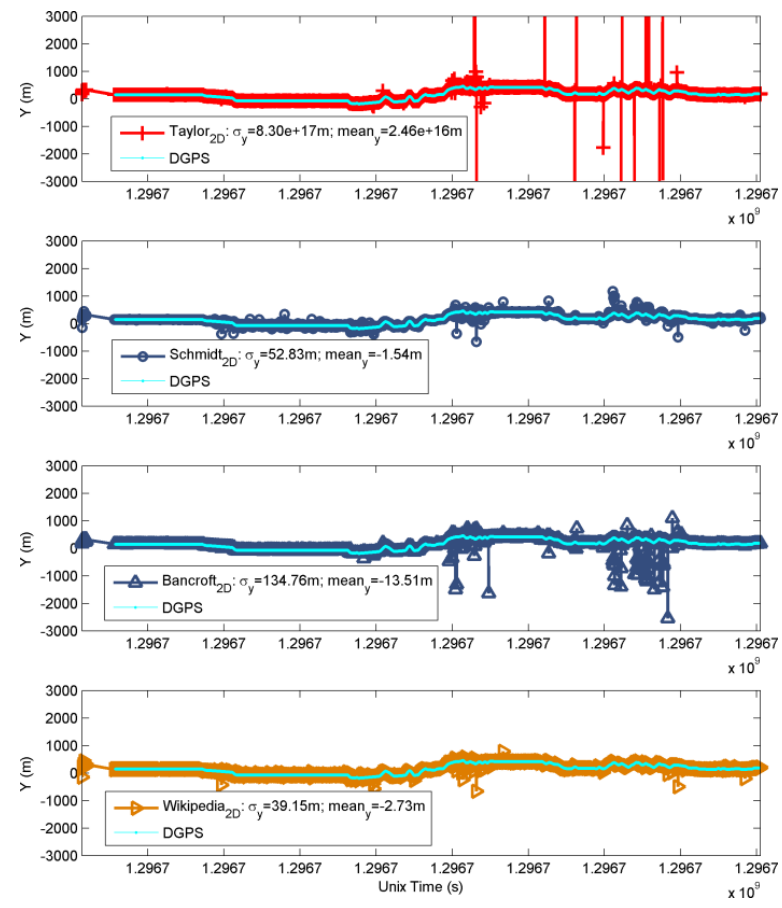

Fig. 7.33 $\mathrm{Y}(\mathrm{t})$ for statistical and algebraic approach based models: comparison of projected localization algorithms.
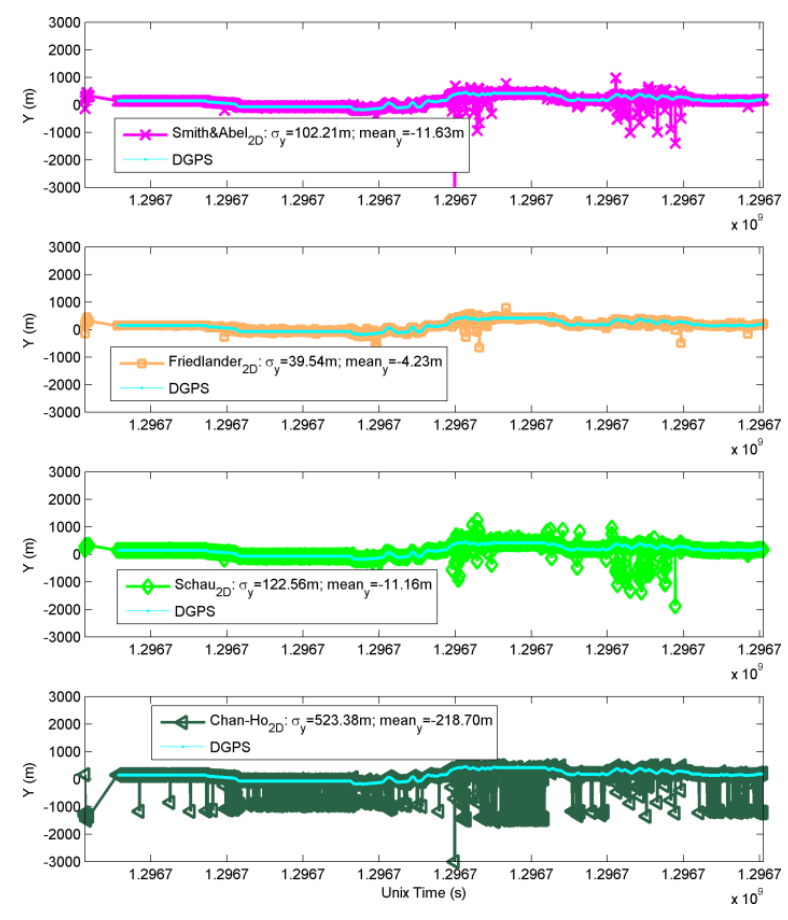

Fig. 7.34 Y(t) for numerical approach based models: comparison of projected localization algorithms. 
Table 7.6 Statistical parameters for the error distributions of localization algorithms. Values given in meters.

\begin{tabular}{|c|c|c|c|c|}
\hline \hline Algorithm & $\boldsymbol{\sigma}_{\mathbf{2 D}}(\mathbf{m})$ & $\mathbf{M e a n}_{\mathbf{2 D}}(\mathbf{m})$ & $\begin{array}{c}\text { PoL (\%) } \\
\boldsymbol{\delta}=\mathbf{7 . 5 m}\end{array}$ & $\begin{array}{c}\text { PoL (\%) } \\
\boldsymbol{\delta}=\mathbf{1 5 m}\end{array}$ \\
\hline Schmidt & 385.93 & 39.74 & 38.86 & 72.02 \\
\hline Taylor & $1.20 \mathrm{E}+18$ & $4.82 \mathrm{E}+16$ & 60.87 & 88.68 \\
\hline Smith\&Abel & 614.66 & 67.16 & 43.90 & 79.95 \\
\hline Friedlander & 385.15 & 36.67 & 41.24 & 75.24 \\
\hline Schau\&Robinson & 616.26 & 108.62 & 30.83 & 61.16 \\
\hline Chan\&Ho & 737.49 & 876.57 & 2.93 & 6.1 \\
\hline Bancroft & 456.06 & 97.61 & 31.74 & 59.45 \\
\hline Wikipedia & 384.66 & 35.38 & 45.49 & 77.36 \\
\hline \hline
\end{tabular}

Table 7.7 Statistical parameters for the error distributions of projected localization algorithms. Values given in meters.

\begin{tabular}{|c|c|c|c|c|}
\hline \hline Algorithm & $\boldsymbol{\sigma}_{\mathbf{2 D}}$ & Mean $_{\text {2D }}$ & $\begin{array}{c}\text { PoL (\%) } \\
\boldsymbol{\delta}=\mathbf{7 . 5 m}\end{array}$ & $\begin{array}{c}\text { PoL (\%) } \\
\boldsymbol{\delta}=\mathbf{1 5 m}\end{array}$ \\
\hline Schmidt & 80.14 & 29.40 & 37.56 & 70.99 \\
\hline Taylor & $1.59 \mathrm{E}+18$ & $5.81 \mathrm{E}+16$ & 68.54 & 94.25 \\
\hline Smith\&Abel & 141.70 & 31.92 & 46.47 & 79.50 \\
\hline Friedlander & 69.77 & 22.56 & 48.01 & 76.56 \\
\hline Schau\&Robinson & 231.55 & 46.67 & 38.28 & 76.13 \\
\hline Chan\&Ho & 1643.48 & 1339.15 & 8.79 & 19.46 \\
\hline Bancroft & 367.79 & 52.95 & 36.31 & 81.83 \\
\hline Wikipedia & 68.25 & 21.24 & 47.39 & 77.36 \\
\hline \hline
\end{tabular}





\section{Multilateration Localization by Using Regularization Algorithms}

We have studied and analyzed in Chapter 7 the different options for construct and solving the localization problem in MLAT systems. We have observed that for some scenarios, due to the system geometry, to the measurements noise and to the starting point quality (in the case of open form algorithms), the corresponding inverse problem is ill-conditioned and therefore the solution of the target position may be not correct or it may diverge with very large errors, i.e., in the case of ill-conditioning of the localization problem the estimated target position is not reliable.

Nowadays, the problem of ill-conditioning is solved by applying different hardware and software based solutions. Regarding to the hardware based solutions, one of these is by adding more stations, i.e., usually the problem of ill-conditioning is found for situations with a number of stations smaller than seven or eight [20]. Another one is by adding new measurement capabilities to the system, such as the AOA [20], [115] or RTD [20], [116], i.e., in a numerical sense, adding new measurements means to improve the corresponding data model and, therefore, to change the ill-conditioned coefficient matrix into a well-conditioned one. However, all these solutions, although are efficient options, require in many cases significant money investments. On the other hand, regarding the software based solutions, two possible options can be implemented. One is by using a horizontal (2D) projected version (see $\$ 7.4$ ) of the localization algorithms and solving the resulting system of equations with the pseudoinverse matrix. This option, although the corresponding coefficient matrix is initially well-conditioned, has the disadvantage that it adds a spatial bias (cf. (7.89)) due to the projection from $3 \mathrm{D}$ to $2 \mathrm{D}$ in the coefficient matrix but of course not in the measurements. The second software based option is by implementing the family of closed form localization algorithms (see §7.3). These algorithms have the advantage of not needing a starting point (an ill-conditioning source) but on the contrary, as we have analyzed in $\S 7.3$, they introduce quadratic and cubic relations between the solution (target position) and the 
measurement noise as well as the necessity, in some cases, to choose one of the two possible solutions. Horizontal projection for this kind of algorithms is also possible but the problem of spatial bias is equally present.

Once described the bases about the inverse problems and the localization problem in MLAT systems as well as the different solutions for solving it, in this part, several regularization methods are studied and applied to solve the MLAT localization problem. The solutions presented in this chapter can be classified as a kind of software based strategy, but they are intended to overcome those disadvantages commented above for both software and hardware based solutions. The regularization methods can be classified into two families [76], [80] namely; direct regularization methods and iterative regularization methods. The direct regularization methods are based on some kind of "canonical decomposition", such as, the QR factorization or the Singular Value Decomposition (SVD). For these methods, it is necessary to explicitly know the coefficient matrix of the system of equations and the solution is achieved by calculating the inverse of a modified version of that matrix. On the other hand, the iterative regularization methods avoid any decomposition and, instead, they are based on iterative schemes that access to the coefficient matrix via matrixvector products. This kind of methods does not need to explicitly know the coefficient matrix.

Since the coefficient matrix is explicitly known for the Multilateration localization problem, this work is only focused on the study and application of direct regularization methods. The direct regularization methods studied here are:

- Tikhonov regularization

- Truncated Singular Value Decomposition (T-SVD)

- T-SVD with sub-set selection (T-SVD SS)

- Truncated Total Least Squares (T-TLS)

In this thesis the regularization methods are only implemented along with the Taylor-series expansion method. As we have described in $\S 7.5$ the main problem for ensuring the convergence in the solution of the MLAT localization problem is the ill-conditioning of the corresponding coefficient matrix. Thus, with the setting and solution of the localization problem in the sense of regularization methods, we seek to turn the weaknesses of this localization algorithm (i.e., the necessity of a good starting point and the low or null convergence) into strengths while preserving its essential advantages (i.e., the statistical optimality). The contributions of this chapter have been published in [100-101], [117-118]. 


\subsection{Solving Localization Problem by Tikhonov Regularization}

The Tikhonov regularization was originally and independently derived by Tikhonov [74] and Phillips [73], and it has been used to solve the illconditioned problems in an important number of applications to engineering and science. Most of them cite this method as Tikhonov regularization; from that is the reason to call it by Tikhonov in this thesis. The main idea of this method is to incorporate a priori information about the size and smoothness of the final solution. This a priori information is in the form of a semi-norm. Generally, Tikhonov regularization leads to minimize not only the residual 2-norm but a trade-off between this residual 2-norm and the 2norm of the final solution. This principle is expressed as the following function [74], [76], [80]:

$$
\arg \min \left\{\|\boldsymbol{G} \boldsymbol{\theta}-\widehat{\boldsymbol{m}}\|_{2}^{2}+\lambda^{2}\|\boldsymbol{L} \boldsymbol{\theta}\|_{2}^{2}\right\}
$$

where $\lambda$ is the regularization parameter which controls the "importance" given to the minimization of the regularization term $\left(\|\boldsymbol{L} \boldsymbol{\theta}\|_{2}^{2}\right)$. The term $\|\boldsymbol{L} \boldsymbol{\theta}\|_{2}$ is also known as discrete smoothing norm (see $\S 6.2 .2$ ), and it can be also found as $\Omega(\boldsymbol{\theta})$. The matrix $\boldsymbol{L} \in \mathbb{R}^{n \times n}$ ( $n=3$ for $(x, y, z)$ localization) is called regularization matrix. In order to solve the Taylor-series expansion method in the sense of Tikhonov regularization, it is necessary to rewrite the likelihood function (7.12) taking into account of the minimization of (8.1). In this way the likelihood function for the Tikhonov method can be expressed as follows:

$$
\Lambda(\boldsymbol{\theta})=\frac{1}{(2 \pi)^{\frac{N_{S}-1}{2}} \operatorname{det}(\boldsymbol{N}(\boldsymbol{\theta}))^{\frac{1}{2}}} e^{-\frac{1}{2}\left\{(\hat{\boldsymbol{m}}-\boldsymbol{m}(\boldsymbol{\theta}))^{T} \boldsymbol{N}(\boldsymbol{\theta})^{-1}(\hat{\boldsymbol{m}}-\boldsymbol{m}(\boldsymbol{\theta}))+\lambda^{2}(\boldsymbol{L} \boldsymbol{\theta})^{T}(\boldsymbol{L} \boldsymbol{\theta})\right\}}
$$

where $\boldsymbol{\theta}=[x, y, z]^{T}$ is the target position and $\widehat{\boldsymbol{m}}$ is the range difference measurements vector (cf. (7.9) and (7.10)). In this application, we have defined the Maximum Likelihood Estimator (MLE) as the regularized $\boldsymbol{M L E}$ and the solution of this is the value of $\boldsymbol{\theta}$ that maximizes (8.2). In this way, the regularized MLE minimizes the following function:

$$
Q(\boldsymbol{\theta})=\left\{(\widehat{\boldsymbol{m}}-\boldsymbol{m}(\boldsymbol{\theta}))^{T} \boldsymbol{N}(\boldsymbol{\theta})^{-1}(\widehat{\boldsymbol{m}}-\boldsymbol{m}(\boldsymbol{\theta}))+\lambda^{2}(\boldsymbol{L} \boldsymbol{\theta})^{T}(\boldsymbol{L} \boldsymbol{\theta})\right\}
$$

Like in the case of Taylor-series method solved in the LS sense, it is necessary to expand the non-linear function $\boldsymbol{m}(\boldsymbol{\theta})$ by Taylor series centred also in a suitable starting point $\boldsymbol{\theta}_{0}=\left[x_{0}, y_{0}, z_{0}\right]^{T}$. This procedure leads to the following minimization function:

$$
Q(\Delta \boldsymbol{\theta})=\left\{\left(\widehat{\boldsymbol{m}}_{\Delta}-\boldsymbol{G} \Delta \boldsymbol{\theta}\right)^{T} \boldsymbol{N}(\boldsymbol{\theta})^{-1}\left(\widehat{\boldsymbol{m}}_{\Delta}-\boldsymbol{G} \Delta \boldsymbol{\theta}\right)+\lambda^{2}(\boldsymbol{L} \Delta \boldsymbol{\theta})^{T}(\boldsymbol{L} \Delta \boldsymbol{\theta})\right\}
$$


To calculate the value of $\boldsymbol{\Delta} \boldsymbol{\theta}$ that minimizes the function $Q(\Delta \boldsymbol{\theta})$, the derivative of (8.4), in respect of each component of $\boldsymbol{\Delta} \boldsymbol{\theta}$, are calculated and then set to zero to obtain a direct expression for $\boldsymbol{\Delta} \boldsymbol{\theta}$. This procedure is shown in the following lines

$$
\left.\nabla_{\Delta \boldsymbol{\theta}} Q(\Delta \boldsymbol{\theta})\right|_{\Delta \boldsymbol{\theta}=\Delta \widehat{\boldsymbol{\theta}}}=-\boldsymbol{G}^{T} \boldsymbol{N}(\boldsymbol{\theta})^{-1} \widehat{\boldsymbol{m}}_{\Delta}+\boldsymbol{G}^{T} \boldsymbol{N}(\boldsymbol{\theta})^{-1} \boldsymbol{G} \Delta \widehat{\boldsymbol{\theta}}+\lambda^{2} \boldsymbol{L}^{T} \boldsymbol{L} \Delta \widehat{\boldsymbol{\theta}}=0
$$

where (8.5) has been evaluated at $\Delta \widehat{\boldsymbol{\theta}}=\widehat{\boldsymbol{\theta}}-\boldsymbol{\theta}_{0}$. Then,

$$
\left(\boldsymbol{G}^{T} \boldsymbol{N}(\boldsymbol{\theta})^{-1} \boldsymbol{G}+\lambda^{2} \boldsymbol{L}^{T} \boldsymbol{L}\right) \Delta \widehat{\boldsymbol{\theta}}=\boldsymbol{G}^{T} \boldsymbol{N}(\boldsymbol{\theta})^{-1} \widehat{\boldsymbol{m}}_{\Delta}
$$

and the unknown variable $\Delta \widehat{\boldsymbol{\theta}}$ can be obtained by,

$$
\Delta \widehat{\boldsymbol{\theta}}=\left(\boldsymbol{G}^{T} \boldsymbol{N}(\boldsymbol{\theta})^{-1} \boldsymbol{G}+\lambda^{2} \boldsymbol{L}^{T} \boldsymbol{L}\right)^{-1} \boldsymbol{G}^{T} \boldsymbol{N}(\boldsymbol{\theta})^{-1} \widehat{\boldsymbol{m}}_{\Delta}
$$

Equation (8.7) is therefore the regularized MLE solution to the Taylorseries expansion method. Also from (8.7) it can be identified the following expression:

$$
\boldsymbol{A}_{\lambda}^{-1}=\left(\boldsymbol{G}^{T} \boldsymbol{N}(\boldsymbol{\theta})^{-1} \boldsymbol{G}+\lambda^{2} \boldsymbol{L}^{T} \boldsymbol{L}\right)^{-1} \boldsymbol{G}^{T} \boldsymbol{N}(\boldsymbol{\theta})^{-1}
$$

where $\boldsymbol{A}_{\lambda}^{-1}$ is known in the literature as the regularized inverse matrix of Tikhonov [74], [76]. Finally, the estimated target parameter is calculated by:

$$
\widehat{\boldsymbol{\theta}}_{\lambda}=\boldsymbol{A}_{\lambda}^{-1} \widehat{\boldsymbol{m}}_{\Delta}+\boldsymbol{\theta}_{0}
$$

where $\widehat{\boldsymbol{\theta}}$ has been written as $\widehat{\boldsymbol{\theta}}_{\lambda}$ to emphasize that this is the Tikhonov solution.

Due to the fact that the covariance matrix $\boldsymbol{N}(\boldsymbol{\theta})$ depends on the true target position $\boldsymbol{\theta}$, also for this regularized estimator it is removed from (8.8) assuming an identity matrix as in \$7.2.1. Furthermore, as (7.18), the regularized estimator in (8.9) should be refined by several iterations until the method reaches a good accuracy. The resulting regularized iterative procedure is expressed in this case as follows:

$$
\widehat{\boldsymbol{\theta}}_{\lambda}^{k}=\boldsymbol{A}_{\lambda}^{-1}\left(\widehat{\boldsymbol{\theta}}_{\lambda}^{k-1}\right) \widehat{\boldsymbol{m}}_{\Delta}\left(\widehat{\boldsymbol{\theta}}_{\lambda}^{k-1}\right)+\widehat{\boldsymbol{\theta}}_{\lambda}^{k-1}, \quad k=1, \ldots, K
$$

where $\widehat{\boldsymbol{\theta}}_{\lambda}^{0}=\boldsymbol{\theta}_{0}, \widehat{\boldsymbol{m}}_{\Delta}\left(\widehat{\boldsymbol{\theta}}_{\lambda}^{k-1}\right)=\widehat{\boldsymbol{m}}-\boldsymbol{m}\left(\widehat{\boldsymbol{\theta}}_{\lambda}^{k-1}\right)$ and $K$ is the maximum number of refinement iterations. It is worth to say that $\boldsymbol{\theta}_{0}$ is not necessarily a previous estimation of the true target position $\boldsymbol{\theta}$. As it will be shown later, for some applications the starting point can be assumed as a fixed value for all the coverage area. The value of the starting point $\boldsymbol{\theta}_{0}$ normally has an important influence in the convergence of the method but, for this application has been found that the performance of this solution is not as 
sensible as the LS solution. This fact will be analyzed in a posterior subsection.

Now, in order to make a correct application of the regularized MLE, it is very important to use the correct regularization parameter $\lambda$ and the correct regularization matrix $\boldsymbol{L}$, in order to obtain a valid solution.

The choice of regularization matrix $\boldsymbol{L}$ is directly connected with the statistics of the target position vector $\boldsymbol{\theta}$, i.e., with the correlation of the target position coordinates $(x, y, z)$. If the components of $\boldsymbol{\theta}$ are assumed to be non-random and uncorrelated (which is a realistic assumption, see [3], [20] for details), a standard choice of the regularization matrix $\boldsymbol{L}$ is to assume it as the $n \times n$ identity matrix. Moreover, in [119] the author showed that the existence of a minimum of the expected value for the residual error in Tikhonov method can be only guaranteed when $\boldsymbol{L}$ is the identity matrix, otherwise the Tikhonov solution could not be close to the exact one. Therefore, in the remaining of this thesis, unless otherwise indicated, the regularization matrix is assumed to be $\boldsymbol{L}=\boldsymbol{I}_{3 \times 3}$.

The choice of regularization parameter $\lambda$ is not as straightforward as the choice of the regularization matrix. There exists in the literature a set of numerical methods and procedures to calculate/estimate an approximated regularization parameter value. A correct statement is that this choice is connected with the SVD spectrum of the matrix $\boldsymbol{G}$. In this way, it is clear that in the solution of (8.10), the SVD spectrum should be inspected to calculate the regularization parameter. Therefore, the objective of the next subsection is to introduce the solution of (8.10) by means of an SVD routine instead of using a numerical one to obtain directly the inverse matrix (8.8), and also the description of a procedure to estimate a right regularization parameter $\lambda$.

\subsubsection{Solving Regularized MLE by SVD}

Equation (8.10) can be solved by a numerical routine to directly calculate the matrix $\boldsymbol{A}_{\lambda}^{-1}$. However, as it was commented above, it is necessary to inspect the SVD spectrum to estimate the regularization parameter and also because this spectrum provides an overall idea of how much illconditioned is the problem. The expression to calculate the Tikhonov regularized MLE solution by means of the SVD is shown in the following $[72],[76]$ :

$$
\widehat{\boldsymbol{\theta}}_{\lambda}^{k}=\left(\sum_{i=1}^{n} f_{i}^{k-1} \frac{\left(\boldsymbol{u}_{i}^{k-1}\right)^{T} \widehat{\boldsymbol{m}}_{\Delta}\left(\widehat{\boldsymbol{\theta}}_{\lambda}^{k-1}\right)}{\sigma_{i}^{k-1}} \boldsymbol{v}_{i}^{k-1}\right)+\widehat{\boldsymbol{\theta}}_{\lambda}^{k-1}, \quad k=1, \ldots, K
$$

where $\boldsymbol{u}_{i}$ and $\boldsymbol{v}_{i}$ are the left and right singular vectors of $\boldsymbol{G}$ respectively, while $\sigma_{i}$ is the $i$ th singular value of $\boldsymbol{G}$ (see $\S 6.3$ ) and they are obtained by 
an SVD routine. If $\boldsymbol{G} \in \mathbb{R}^{m \times n}$ (for this application $m=$ number of stations excluding the reference one- and $n=3$ ), then the SVD of $\boldsymbol{G}$ is a decomposition of the form [72], [76]:

$$
\boldsymbol{G}=\boldsymbol{U} \boldsymbol{\Sigma} \boldsymbol{V}^{T}=\sum_{i=1}^{n} \boldsymbol{u}_{i} \sigma_{i} \boldsymbol{v}_{i}^{T}
$$

where $\boldsymbol{U}=\left(\boldsymbol{u}_{1}, \ldots, \boldsymbol{u}_{i}\right) \in \mathbb{R}^{\left(N_{s}-1\right) \times n}$ and $\boldsymbol{V}=\left(\boldsymbol{v}_{1}, \ldots, \boldsymbol{v}_{i}\right) \in \mathbb{R}^{n \times n}$ are matrices with orthonormal columns, and $\boldsymbol{\Sigma}$ is a diagonal matrix such that $\boldsymbol{\Sigma}=$ $\operatorname{diag}\left(\sigma_{1}, \ldots, \sigma_{i}\right)$. Finally, $f_{i}$ are kwon as the Tikhonov filter factors [74], [76] and for this application $\left(\boldsymbol{L}=\boldsymbol{I}_{n}\right)$ takes the following form:

$$
f_{i}=\frac{\sigma_{i}^{2}}{\sigma_{i}^{2}+\lambda^{2}}
$$

\subsubsection{Effect of Tikhonov Regularization on the Localization Problem}

To understand how the Tikhonov method works on the localization problem, we explicitly obtain the matrix $\boldsymbol{A}_{\lambda}$. In the remaining descriptions of the regularized MLE we suppress the super-index $k$ for simplicity. The matrix $\boldsymbol{A}_{\lambda}$ can be expressed as follows:

$$
\boldsymbol{A}_{\lambda}=\left(\boldsymbol{A}_{\lambda}^{-1}\right)^{-1}=\left(\left(\boldsymbol{G}^{T} \boldsymbol{N}(\boldsymbol{\theta})^{-1} \boldsymbol{G}+\lambda^{2} \boldsymbol{L}^{T} \boldsymbol{L}\right)^{-1} \boldsymbol{G}^{T} \boldsymbol{N}(\boldsymbol{\theta})^{-1}\right)^{-1}
$$

After a few operations (8.14) takes the following form:

$$
\boldsymbol{A}_{\lambda}=\boldsymbol{G}+\lambda^{2}\left(\boldsymbol{G}^{T} \boldsymbol{N}(\boldsymbol{\theta})^{-1}\right)^{-1} \boldsymbol{L}^{T} \boldsymbol{L}
$$

Now, by expressing (8.15) in terms of the SVD of matrix $\boldsymbol{G}$, i.e., $\boldsymbol{G}=\boldsymbol{U} \boldsymbol{\Sigma} \boldsymbol{V}^{T}$ (see $\S 6.3 .1$ ), and neglecting the covariance matrix $\boldsymbol{N}(\boldsymbol{\theta})$ (see $\S 6.3)$, matrix $\boldsymbol{A}_{\boldsymbol{\lambda}}$ can be expressed as follows:

$$
\begin{gathered}
\boldsymbol{A}_{\lambda}=\boldsymbol{U} \boldsymbol{\Sigma} \boldsymbol{V}^{T}+\lambda^{2} \boldsymbol{U} \boldsymbol{\Sigma}^{-1} \boldsymbol{V}^{T} \\
\boldsymbol{A}_{\lambda}=\boldsymbol{U}\left(\boldsymbol{\Sigma}+\lambda^{2} \boldsymbol{\Sigma}^{-1}\right) \boldsymbol{V}^{T} \\
\boldsymbol{A}_{\lambda}=\boldsymbol{U} \boldsymbol{\Sigma} \boldsymbol{\Gamma}_{\lambda} \boldsymbol{V}^{T} \\
\boldsymbol{A}_{\lambda}=\boldsymbol{U} \boldsymbol{\Sigma}_{\lambda} \boldsymbol{V}^{T}
\end{gathered}
$$

where $\boldsymbol{\Gamma}_{\lambda}=\operatorname{diag}\left(\frac{1}{f_{1}}, \ldots, \frac{1}{f_{n}}\right), f_{j}$ is the $j$ th Tikhonov filter factor (cf. (8.13)), and $\boldsymbol{\Sigma}_{\lambda}=\boldsymbol{\Sigma} \boldsymbol{\Gamma}_{\lambda}$ is the corresponding matrix of singular values of $\boldsymbol{A}_{\lambda}$. In this sense, the final expression in (8.16) can be seen as the SVD of matrix $\boldsymbol{A}_{\lambda}$, whose left and right singular vectors are equal to the ones of matrix $\boldsymbol{G}$ and its singular values are an amplified version of those of matrix $\boldsymbol{G}$. Then, 
geometrically speaking it is known that the matrices $\boldsymbol{U}$ and $\boldsymbol{V}$ provide two set of orthonormal basis vectors, i.e., the columns of each of these, and, under this assumption, the singular values can be seen as the spectrum components of a matrix, and they are the only distinguishing factor between two matrices that share the same orthonormal basis, as the case of $\boldsymbol{A}_{\boldsymbol{\lambda}}$ and $\boldsymbol{G}$. Therefore, if we remember that matrix $\boldsymbol{G}$ contains the information about the system geometry, then taking into account that the information in matrix $\boldsymbol{A}_{\lambda}$ (i.e., its singular value spectrum) is a modified version of that one of matrix $\boldsymbol{G}$, we can say that $\boldsymbol{A}_{\lambda}$ represents a modified geometry of the system. In other words, the application of the regularized estimator can be seen as a system geometry correction, which converts the ill-conditioned scenario into an equivalent well-conditioned.

On the other hand, due to the fact that the minimum residual norm solution can only be obtained by the pseudoinverse matrix and as the matrix $\boldsymbol{A}_{\lambda}$ is different from the matrix $\boldsymbol{G}$, then the regularized MLE solution always has a bias regarding to the exact one [76], [119]. However and as it will be shown later, in many cases this bias can be neglected if the correct regularization matrix and the correct regularization parameter are used.

\subsubsection{Regularization Error of Tikhonov Regularization}

The application of any regularization method introduces some amount of error due to the approximations that are taken to allow solving the illconditioned problem [76], [98].

Substituting (7.9), (7.14) and (8.8) into (8.9), and remembering that $\widehat{\boldsymbol{m}}_{\Delta}=\widehat{\boldsymbol{m}}-\boldsymbol{m}\left(\boldsymbol{\theta}_{0}\right)$ and $\Delta \widehat{\boldsymbol{\theta}}=\widehat{\boldsymbol{\theta}}-\boldsymbol{\theta}_{0}$, the expression for $\widehat{\boldsymbol{\theta}}_{\lambda}$ can be rewritten as follows

$$
\begin{gathered}
\widehat{\boldsymbol{\theta}}_{\lambda}=\boldsymbol{\theta}+\left(\boldsymbol{G}^{T} \boldsymbol{N}(\boldsymbol{\theta})^{-1} \boldsymbol{G}+\lambda^{2} \boldsymbol{L}^{T} \boldsymbol{L}\right)^{-1} G^{T} \boldsymbol{N}(\boldsymbol{\theta})^{-1}\left\{\boldsymbol{m}(\boldsymbol{\theta})-\boldsymbol{m}\left(\boldsymbol{\theta}_{0}\right)\right. \\
\left.-\boldsymbol{A}_{\lambda}\left(\boldsymbol{\theta}-\boldsymbol{\theta}_{0}\right)+\boldsymbol{n}\right\}
\end{gathered}
$$

which is an equivalent formulation to that shown in [3] for the accuracy analysis of some passive localization systems. Equation (8.17) shows that the estimated target position is affected by an error that depends on two components, one is the regularized linearization error and the other one is the error due to the measurements noise.

For the Tikhonov method, the error can be expressed as follows [76]:

$$
\begin{gathered}
\boldsymbol{\theta}-\widehat{\boldsymbol{\theta}}_{\lambda}=\boldsymbol{G}^{\dagger} \boldsymbol{m}_{\Delta}(\boldsymbol{\theta})-\boldsymbol{A}_{\lambda}^{-1} \widehat{\boldsymbol{m}}_{\Delta} \\
\boldsymbol{\theta}-\widehat{\boldsymbol{\theta}}_{\lambda}=\left(\boldsymbol{G}^{\dagger}-\boldsymbol{A}_{\lambda}^{-1}\right) \boldsymbol{m}_{\Delta}(\boldsymbol{\theta})-\boldsymbol{A}_{\lambda}^{-1} \boldsymbol{n}
\end{gathered}
$$

where $\boldsymbol{G}^{\dagger}$ is the pseudoinverse matrix of $\boldsymbol{G}, \widehat{\boldsymbol{m}}_{\Delta}=\boldsymbol{m}_{\Delta}(\boldsymbol{\theta})+\boldsymbol{n}$ and $\boldsymbol{m}_{\Delta}(\boldsymbol{\theta})=$ $\boldsymbol{m}(\boldsymbol{\theta})-\boldsymbol{m}\left(\boldsymbol{\theta}_{0}\right)$. The first term in (8.18) is called regularization error and 
the second one is the perturbation error. The first term gives information about the system geometry correction error and the second one gives information about the measurements noise (instrumental errors, propagation effects, etc.). When very little regularization is introduced $(0 \leq \lambda \ll 1)$ most Tikhonov filter factors are close to 1 , and the overall error is dominated by perturbation error $\boldsymbol{A}_{\lambda}^{-1} \boldsymbol{n}$. This case is called undersmoothing and the result is that $\widehat{\boldsymbol{\theta}}_{\lambda}$ tends to the classical nonregularized solution (LS solution). On the other hand, when a large amount of regularization is introduced $(\lambda \gg 0)$ most filter factors are considerably small $\left(f_{i} \ll 1\right)$ and the overall error is dominated by the regularization error. This case is called oversmoothing and the result for the localization problem is that $\widehat{\boldsymbol{\theta}}_{\lambda}$ tends to $\boldsymbol{\theta}_{0}$.

In terms of the SVD of matrix $\boldsymbol{G}$, the overall error can be calculated as follows [76], [98]:

$$
\boldsymbol{\theta}-\widehat{\boldsymbol{\theta}}_{\lambda}=\sum_{i=1}^{n}\left(1-f_{i}\right) \frac{u_{i}^{T} \boldsymbol{m}_{\Delta}(\boldsymbol{\theta})}{\sigma_{i}} v_{i}-\sum_{i=1}^{n} f_{i} \frac{u_{i}^{T} \boldsymbol{n}}{\sigma_{i}} v_{i}
$$

Finally, it is evident by analyzing (8.18) (or (8.19)) that a correct implementation of the Tikhonov method is intended to balance the two error components (i.e., regularization and perturbation errors).

\subsubsection{Estimation of the Regularization Parameter for Tikhonov}

The selection/estimation of regularization parameter is one of the most critical aspects for the application of any regularization method. There exist many methods or procedures to calculate an approximated regularization parameter value. In [76] the author classifies these methods into two classes, based on the assumption about the measurement error 2-norm $\|\boldsymbol{n}\|_{2}$ (cf. (7.9)). The first class comprises the methods that are based on some a priori knowledge, or a good estimation of $\|\boldsymbol{n}\|_{2}$, and the second class comprises those ones that do not require any a priori knowledge or estimation of $\|\boldsymbol{n}\|_{2}$ but instead, they extract this information from the given measurement vector $\widehat{\boldsymbol{m}}$.

Within the first class, it is worth mentioning the discrepancy principle [120] which takes into account the measurements error 2-norm or its generalized version [121] (generalized discrepancy principle) which also takes into account possible errors $\boldsymbol{E}$ in the coefficient matrix (differential Jacobian matrix for this application) $\boldsymbol{G}$. The main idea of this methods is that, if $\boldsymbol{G} \boldsymbol{\theta}=\boldsymbol{m}$ (where $\boldsymbol{\theta}$ and $\boldsymbol{m}$ are the exacts quantities vectors) holds exactly, then the correct regularization parameter is that $\lambda$ for which the residual error $\left\|\boldsymbol{G} \widehat{\boldsymbol{\theta}}_{\lambda}-\widehat{\boldsymbol{m}}\right\|_{2}$ is equal to a specific value provided by a relation 
between the a priori upper bounds $\delta_{e}$ and $\delta_{E}$ for $\|\boldsymbol{n}\|_{2}$ and for $\|\boldsymbol{E}\|_{2}$ respectively. It is, the $\lambda$ that allows satisfying:

$$
\left\|\boldsymbol{G} \widehat{\boldsymbol{\theta}}_{\lambda}-\widehat{\boldsymbol{m}}\right\|_{2}=\delta_{e}
$$

where

$$
\|\boldsymbol{n}\|_{2} \leq \delta_{e}
$$

or in the generalized version case:

$$
\left\|\boldsymbol{G} \widehat{\boldsymbol{\theta}}_{\lambda}-\widehat{\boldsymbol{m}}\right\|_{2}=\delta_{0}^{\text {exact }}+\delta_{e}+\delta_{E}\|\boldsymbol{\theta}\|_{2}
$$

where $\delta_{0}^{\text {exact }}$ is an incompatibility measure of the inverse problem.

On the other hand, in the second class of methods, the goal is to minimize a function which approximates the total error (cf. (8.18)) and thus obtain a regularization parameter that provides an optimal point for the balance of the regularization and perturbation errors in $\widehat{\boldsymbol{\theta}}_{\lambda}$. Within these methods can be mentioned, the method described in [122-123] and the quasi-optimality criterion [124].

Additionally, there exist other methods that do not require information about $\|\boldsymbol{n}\|_{2}$. These are the Generalized Cross Validation (GCV) [76], [125] and the L-curve criterion [76], [98]. These methods are based on the hypothesis that a good approximation for the regularization parameter is that one which minimizes some specific functions. In the case of GCV method, it uses a function called GCV function [125-126] and in the case of L-curve criterion it uses a function called L-curve [98] which is a curve that shows the relation between the 2-norm of the regularized solution and the corresponding residual 2-norm.

It is important to say that all methods mentioned above provide regularization parameters values which allow finding accurate numerical solutions for a variety of applications (e.g., image processing, remote sensing, electromagnetic scattering, etc.), which have as a common aspect that are executed in non-real time. Moreover, it can be observed that all of these methods introduce a significant computational load because they are based either on the solution of an optimization problem, i.e., to find a parameter that satisfy some equalities (first class of methods), or find a parameter that minimize some special functions (second class of methods, GCV method and L-curve criterion). Therefore, due to their nature, the computation time required for applying these methods might be not suitable for real-time localization in MLAT systems.

In other words, and taking into account the main requirements for Air Traffic Control (ATC) operations, that are based on the data update time (time between one calculated position and the chronologically posterior [1- 
2]) and the system capacity (number of aircraft/vehicles that are in the coverage area in a time instant [1-2]), all methods commented above are not suitable for calculating the regularization parameter for the localization problem solution in MLAT systems. Therefore, in this thesis we propose two strategies to estimate this parameter. One of this is based on the evaluation of the problem for several regularization parameters values (no more than three) and the other one is based on the analysis of the singular values spectrum.

\section{Estimating by Residual Error Evaluation}

The first option for this application is to solve the problem for one, two or three regularization parameters and then choose that solution that corresponds with the minimum residual error. This option is valid for this application because the typical size of the coefficient matrices is normally smaller than $10 \times 3$. Obviously to implement this strategy a relative rigorous analysis of the problem scenario must be carried out. In general, the residual error for an inverse problem is given by:

error $_{j}=\frac{\left\|\boldsymbol{G}\left(\widehat{\boldsymbol{\theta}}_{\lambda_{j}}\right) \widehat{\boldsymbol{\theta}}_{\lambda_{j}}-\widehat{\boldsymbol{m}}\right\|_{2}}{\|\widehat{\boldsymbol{m}}\|_{2}}, j=1, \ldots$, total of evaluated values

Remembering that for Taylor-series expansion method, the matrix $\boldsymbol{G}$ is an approximation of an exact coefficient matrix, then (8.22) could not be a correct value for the residual error with respect to the true target position. Therefore, in this thesis we propose to calculate the residual error by replacing the regularized solution $\widehat{\boldsymbol{\theta}}_{\lambda_{j}}$ in the non-linear TDOA function (6.6), instead in the matrix $\boldsymbol{G}$, as follows:

$$
\text { error }_{j}=\frac{\left\|\boldsymbol{h}_{\lambda_{j}}-\widehat{\boldsymbol{m}}\right\|_{2}}{\|\widehat{\boldsymbol{m}}\|_{2}}, \quad j=1, \ldots
$$

where the vector $\boldsymbol{h}_{\lambda_{j}}$ is given by:

$$
\boldsymbol{h}_{\lambda_{j}}=\left[\begin{array}{c}
T D O A_{2,1}\left(\widehat{\boldsymbol{\theta}}_{\lambda_{j}}\right) \\
\vdots \\
T D O A_{N_{s}, 1}\left(\widehat{\boldsymbol{\theta}}_{\lambda_{j}}\right)
\end{array}\right]_{\left(N_{s}-1\right) \times 1}
$$

In other words, in this strategy we evaluate the quality of the regularized solution by directly using the non-linear problem in (6.6) instead of using the classical expression of matrix-vector product. 


\section{Estimating by Singular Value Spectrum Inspection}

The second option is based on a real-time inspection of the singular values spectrum of the coefficient matrix $\boldsymbol{G}$. Before describing the procedure proposed here, it is necessary to make use of the concepts of resolution matrix and averaging kernels. The resolution matrix, for any regularization method, is defined as follows [76]:

$$
\Xi \equiv A_{\lambda}^{-1} G
$$

The resolution matrix quantifies the amount of smoothing introduced by any particular regularization method. In this sense, the regularized solution $\widehat{\boldsymbol{\theta}}_{\lambda}$ can be written as follows (cf. (8.9)),

$$
\begin{gathered}
\widehat{\boldsymbol{\theta}}_{\lambda}=\boldsymbol{A}_{\lambda}^{-1} \widehat{\boldsymbol{m}}_{\Delta}+\boldsymbol{\theta}_{0} \\
\widehat{\boldsymbol{\theta}}_{\lambda}=\boldsymbol{A}_{\lambda}^{-1}\left(\boldsymbol{m}_{\Delta}(\boldsymbol{\theta})+\boldsymbol{n}\right)+\boldsymbol{\theta}_{0} \\
\widehat{\boldsymbol{\theta}}_{\lambda}=\boldsymbol{\Xi} \Delta \boldsymbol{\theta}+\boldsymbol{A}_{\lambda}^{-1} \boldsymbol{n}+\boldsymbol{\theta}_{0}
\end{gathered}
$$

In the case of the LS solution, i.e., by using the pseudoinverse matrix (which is the minimum residual 2-norm solution), the resolution matrix is $\boldsymbol{\Xi}=\boldsymbol{I}_{3}$ and, therefore, in the case of well-conditioned problem, the expected value of $\widehat{\boldsymbol{\theta}}_{L S}$ is such that $E\left[\widehat{\boldsymbol{\theta}}_{L S}\right]=\boldsymbol{\theta}$. However, for any regularization method, the resolution matrix is different from $\boldsymbol{I}_{3}$ and the regularized solution $\widehat{\boldsymbol{\theta}}_{\lambda}$ is a smoothed version of $\boldsymbol{\theta}$. Specifically, each component of the first term in (8.26) is a weighted average version of all elements in $\boldsymbol{\theta}$. The second term of (8.26) is the measurements noise contribution and the third one is the starting point for Taylor-series expansion method.

For Tikhonov regularization, the amount of smoothing is directly related to the regularization parameter $\lambda$ and satisfies that, if $\lambda=0 \rightarrow \boldsymbol{\Xi}=\boldsymbol{I}_{3}$ and if $\lambda=\infty \rightarrow \boldsymbol{\Xi}=\mathbf{0}_{3}$. It is evident that, in the first situation, the regularized solution $\widehat{\boldsymbol{\theta}}_{\lambda}$ tends to $\widehat{\boldsymbol{\theta}}_{L S}$ (undersmoothing case) and, in the second situation, $\widehat{\boldsymbol{\theta}}_{\lambda}$ tends to $\boldsymbol{\theta}_{0}$ (oversmoothing case). Consequently, it can be understood that the resolution matrix allows knowing how precise the regularized solution can reach the exact one.

On the other hand, to numerically analyze how much is smoothed each component of the regularized solution, it is necessary to inspect every row of the resolution matrix $\boldsymbol{\Xi}$. The $i$ th smoothed component can be written as follows [76]:

$$
(\boldsymbol{\Xi} \boldsymbol{\theta})_{i}=\boldsymbol{\xi}_{i}^{T} \boldsymbol{\theta}, \quad i=1, \ldots, 3
$$

The set of rows $\xi_{i}^{T}$ are known in the literature as averaging kernels [76], [88] of the problem. Particularly, for $(x, y, z)$ localization problems, it can be demonstrated that (see Annex B for the full demonstration): 


$$
\begin{aligned}
& \xi_{1}^{T}=f_{1} \boldsymbol{v}_{1}^{1} \boldsymbol{v}_{1}^{T}+f_{2} \boldsymbol{v}_{2}^{1} \boldsymbol{v}_{2}^{T}+f_{3} \boldsymbol{v}_{3}^{1} \boldsymbol{v}_{3}^{T} \\
& \xi_{2}^{T}=f_{1} \boldsymbol{v}_{1}^{2} \boldsymbol{v}_{1}^{T}+f_{2} \boldsymbol{v}_{2}^{2} \boldsymbol{v}_{2}^{T}+f_{3} \boldsymbol{v}_{3}^{2} \boldsymbol{v}_{3}^{T} \\
& \xi_{3}^{T}=f_{1} \boldsymbol{v}_{1}^{3} \boldsymbol{v}_{1}^{T}+f_{2} \boldsymbol{v}_{2}^{3} \boldsymbol{v}_{2}^{T}+f_{3} \boldsymbol{v}_{3}^{3} \boldsymbol{v}_{3}^{T}
\end{aligned}
$$

where $f_{j}$ is the $j$ th Tikhonov filter factor, $\boldsymbol{v}_{j}$ is the $j$ th right singular vector of matrix $\boldsymbol{G}$ and $\boldsymbol{v}_{j}^{i}$ is the $i$ th component of the $j$ th right singular vector. It can be observed from (8.28) that if the filter factors decrease (i.e., $\lambda$ increases), then, the averaging kernels also decrease and, therefore, the amount of smoothing introduced increases.

When the ill-conditioned appears, it is necessary to introduce some amount of smoothing. Now, the critical point is to know how much amount of smoothing is needed to avoid the ill-conditioned problem but also to reach an accurate solution.

Although it is not a fully general statement, it is appropriate to comment that there is a direct relation between the $i$ th averaging kernel $\boldsymbol{\xi}_{i}^{T}$ and the $i$ th Tikhonov filter factor $f_{i}$, such that, if $f_{i}$ decreases also the components amplitudes of $\xi_{i}^{T}$ decreases and, therefore, the amount of smoothing for the $i$ th component of the regularized solution, $\widehat{\boldsymbol{\theta}}_{\lambda}$, increases. Also, if $f_{i}$ increases the components amplitudes of $\xi_{i}^{T}$ increases and, therefore, the amount of smoothing for the $i$ th component of the regularized solution, $\widehat{\boldsymbol{\theta}}_{\lambda}$, decreases.

Finally, and based on the hypothesis that for Mode S Multilateration systems, the more ill-conditioned space coordinate is the vertical component, $z$, of the target position, it is proposed a formulation that seeks for introduce more amount of smoothing only for that vertical component. This formulation takes the following form:

$$
\lambda_{k}=\sigma_{3}^{k}+w\left(\sigma_{2}^{k}-\sigma_{3}^{k}\right)
$$

where $\lambda_{k}, \sigma_{2}^{k}, \sigma_{3}^{k}$ are the regularization parameter value and the second and third singular value of $\boldsymbol{G}$, respectively, for the $k$ th refinement iteration of Taylor-series expansion method. The factor $w$ is a factor that controls the weight of the term $\left(\sigma_{2}^{k}-\sigma_{3}^{k}\right)$. It has been observed that the value of this weight factor depends on the number of stations and the operational scenario, i.e., LAM or WAM.

On the other hand, there exists several works where a considerable number of analysis and simulations have been done in order to fully describe and understand the accuracy performance of LAM and WAM systems [3-4], [20]. One important conclusion extracted from these works is that, for a coverage area inside the system perimeter (the LAM), the 2D problem is better conditioned than the vertical one and, for coverage areas 
outside the system perimeter (far from that), the $2 \mathrm{D}$ and the vertical problem are conditioned in a similar way.

Additionally to (8.29) a strategy to update the weight factor $w$ for LAM localization problems is proposed. In this strategy it is proposed to update the value of $w$ with the refinement iterations, based on the hypothesis that, if for LAM the $2 \mathrm{D}$ problem is better conditioned, then the $2 \mathrm{D}$ convergence is more stable and with the refinement iterations the amount of illconditioned is reduced and, therefore, also the value of $w$ should be reduced (the less ill-conditioned, the lower the $\lambda$ should be). In this way, for LAM applications (8.29) takes the following form:

$$
\lambda_{k}=\sigma_{3}^{k}+w^{k}\left(\sigma_{2}^{k}-\sigma_{3}^{k}\right)
$$

where

$$
w^{k}=\left\{\begin{array}{rl}
0.8 w^{k-1}, & \sigma_{2}^{k} \geq \sigma_{2}^{k-1} \\
w^{k-1}, & \text { otherwise }
\end{array} ; \quad k=3, \ldots, K\right.
$$

where $K$ is the total number of Taylor iterations and $w^{1}=w^{2}=0.3$ for LAM applications.

On other hand, for WAM applications for near targets with small number of stations (normally smaller than five) it is used only (8.29) with $w=0.3$ and for WAM but, for far targets and a number of stations greater than five, it is used $w=0.03$. The particularities of this aspect are clearly shown in the results section.

\subsection{Solution by SVD Based Methods}

The classical solution by SVD is in connection with the Least Squares (LS) problem. The classical SVD says that, if $\boldsymbol{G}$ is invertible, then its inverse is given by [72], [76]:

$$
\boldsymbol{G}^{-1}=\sum_{i=1}^{n} \frac{\boldsymbol{v}_{i} \boldsymbol{u}_{i}^{T}}{\sigma_{i}}
$$

and then the solution $\widehat{\boldsymbol{\theta}}$ is given by:

$$
\widehat{\boldsymbol{\theta}}=\left(\sum_{i=1}^{n} \frac{\left(\boldsymbol{u}_{i}^{T} \widehat{\boldsymbol{m}}_{\Delta}\right) \boldsymbol{v}_{i}}{\sigma_{i}}\right)+\boldsymbol{\theta}_{0}
$$

On the other hand, based on the SVD it is defined the pseudoinverse matrix [72] $\boldsymbol{G}^{\dagger}$ of $\boldsymbol{G}$ as: 


$$
\boldsymbol{G}^{\dagger} \equiv \sum_{i=1}^{\operatorname{rank}(\boldsymbol{G})} \frac{\boldsymbol{v}_{i} \boldsymbol{u}_{i}^{T}}{\sigma_{i}}
$$

and the LS solution to the problem $\|\boldsymbol{G} \boldsymbol{\theta}-\widehat{\boldsymbol{m}}\|_{2}$ is given by:

$$
\widehat{\boldsymbol{\theta}}_{L S}=\boldsymbol{G}^{\dagger} \widehat{\boldsymbol{m}}=\left(\sum_{i=1}^{\operatorname{rank}(\boldsymbol{G})} \frac{\boldsymbol{u}_{i}^{T} \widehat{\boldsymbol{m}}_{\Delta}}{\sigma_{i}} \boldsymbol{v}_{i}\right)+\boldsymbol{\theta}_{0}
$$

The solution obtained by the pseudoinverse ensures the first two Hadamard's conditions but not the third one. It will be shown in the results section that when the problem is very ill-conditioned the solution in (8.35) does not provide accurate results.

A common case, solving localization problems in Mode S Multilateration is that there are more measurements, coming from the stations, than those necessaries to solve de system of equations. In this case, the system of hyperbolic equations is called over-determined system and the explicit solution can be expressed as follows:

$$
\widehat{\boldsymbol{\theta}}_{L S}^{\text {over }}=\left(\boldsymbol{G}^{T} \boldsymbol{G}\right)^{-1} \boldsymbol{G}^{T} \widehat{\boldsymbol{m}}_{\Delta}+\boldsymbol{\theta}_{0}=\boldsymbol{G}^{\dagger} \widehat{\boldsymbol{m}}_{\Delta}+\boldsymbol{\theta}_{0}
$$

where $\boldsymbol{G}^{\dagger}=\left(\boldsymbol{G}^{T} \boldsymbol{G}\right)^{-1} \boldsymbol{G}^{T}$ (see $\left.\S 6.4\right)$.

As it can be seen, the solution in (8.36) is totally equivalent than that in (7.17). The only difference is that, here the LS solution has been expressed in the SVD domain. Finally, also the solution provided by (8.36) must be iterated like in (7.18).

\subsubsection{Solution by Truncated SVD (T-SVD)}

When the matrix $\boldsymbol{G}$ is not exactly rank deficient, but instead numerically rank deficient in the presence of an error $\epsilon$, the solution by classical SVD should be avoided. In this case, it is better to take the rank- $\boldsymbol{k}_{\epsilon}$ matrix $\boldsymbol{G}_{\boldsymbol{k}_{\epsilon}}$ which is defined by [72], [76]:

$$
\boldsymbol{G}_{k_{\epsilon}} \equiv \sum_{i=1}^{k_{\epsilon}} \boldsymbol{u}_{i} \sigma_{i} \boldsymbol{v}_{i}^{T}
$$

where $k_{\epsilon}$ is a discrete value normally known as discrete regularization parameter (or truncation parameter) for T-SVD and the most common practice is to take $k_{\epsilon}=r_{\epsilon}$, i.e., the numerical rank of matrix $\boldsymbol{G}$ in the presence of a level error $\epsilon$. 
In other words, the main idea of this method consists of taking the first $k_{\epsilon}$ components of the matrix of singular values of $\boldsymbol{G}$ (cf. (6.12) or (8.12)) to construct the new coefficient matrix $\boldsymbol{G}_{\boldsymbol{k}_{\epsilon}}$, which initially has better numerical characteristics than $\boldsymbol{G}$. In this sense, this method is intended to neglect those singular values that are close to zero. Then, by using the same notation as in (7.18), the iterative location estimator in the sense of T-SVD can be expressed as follows:

$$
\begin{gathered}
\widehat{\boldsymbol{\theta}}_{k_{\epsilon}}^{k}=\left(\boldsymbol{G}_{k_{\epsilon}}\left(\widehat{\boldsymbol{\theta}}_{k_{\epsilon}}^{k-1}\right)^{T} \boldsymbol{G}_{k_{\epsilon}}\left(\widehat{\boldsymbol{\theta}}_{k_{\epsilon}}^{k-1}\right)\right)^{-1} \boldsymbol{G}_{k_{\epsilon}}\left(\widehat{\boldsymbol{\theta}}_{k_{\epsilon}}^{k-1}\right)^{T} \widehat{\boldsymbol{m}}_{\Delta}\left(\widehat{\boldsymbol{\theta}}_{k_{\epsilon}}^{k-1}\right)+\widehat{\boldsymbol{\theta}}_{k_{\epsilon}}^{k-1}, \quad k \\
=1, \ldots, K
\end{gathered}
$$

being $\widehat{\boldsymbol{\theta}}_{k_{\epsilon}}^{k}$ the solution provided by the T-SVD and $\widehat{\boldsymbol{\theta}}_{\boldsymbol{k}_{\epsilon}}^{0}$ the starting point as required for Taylor based methods. Note here that we have directly assumed the covariance matrix of the measurement errors (cf. (7.18)) to be the identity one as in $\S 8.1$, and the difference between $k$, which is the index for the iterations, and $k_{\epsilon}$ that is the notation for the regularization parameter for T-SVD.

The numerical solution of (8.38) can be also expressed in terms of the SVD of matrix $\boldsymbol{G}$ by using (8.37) as follows [76], [127]:

$$
\begin{aligned}
\widehat{\boldsymbol{\theta}}_{k_{\epsilon}}^{k}=\boldsymbol{G}_{k_{\epsilon}}^{\dagger}\left(\widehat{\boldsymbol{\theta}}_{k_{\epsilon}}^{k-1}\right) \widehat{\boldsymbol{m}}_{\Delta}\left(\widehat{\boldsymbol{\theta}}_{k_{\epsilon}}^{k-1}\right)+\widehat{\boldsymbol{\theta}}_{k_{\epsilon}}^{k-1} \\
=\left(\sum_{i=1}^{k_{\epsilon}} \frac{\left(\boldsymbol{u}_{i}^{k-1}\right)^{T} \widehat{\boldsymbol{m}}_{\Delta}\left(\widehat{\boldsymbol{\theta}}_{k_{\epsilon}}^{k-1}\right)}{\sigma_{i}^{k-1}} \boldsymbol{v}_{i}^{k-1}\right)+\widehat{\boldsymbol{\theta}}_{k_{\epsilon}}^{k-1}
\end{aligned}
$$

where the above expression should be evaluated for $k=1, \ldots, K$.

For this regularization method also those stop criteria described in (7.19) can be used. Generally, the pertaining scenarios for the application of these criteria along with the T-SVD are the same than those ones for Tikhonov regularization.

The solution provided by (8.39) is a unique solution with minimum 2norm and stable. In this way, this solution guarantees the three Hadamard's conditions. On the other hand, one of the most important aspects when using (8.39) is to know how to choose the correct level error $\epsilon$ or the direct choice of the regularization parameter $k_{\epsilon}$. The level error $\epsilon$ comes from the hypothesis that the matrix $\boldsymbol{G}$ is really in the form $\boldsymbol{G}=$ $\boldsymbol{G}^{\text {exact }}+\boldsymbol{E}$, where the $\boldsymbol{G}^{\text {exact }}$ is exactly rank deficient and $\boldsymbol{E}$ is some perturbation of $\boldsymbol{G}^{\text {exact }}$. As it was commented in $\S 6.3 .5$ the perturbation can be due to different sources. The author in [76] suggests that the level error $\epsilon$ has to satisfy $\|\boldsymbol{E}\|_{2} \leq \epsilon$. However, the perturbation matrix and its 2-norm are not known and the strategy commented before, to estimate the matrix 
rank, is not so useful in this case. The estimation of this parameter is the aim of the following subsection.

\subsubsection{Estimation of the Regularization Parameter for T-SVD}

The selection/estimation of the regularization parameter is also a critical aspect for the application of the T-SVD regularization. Unlike the Tikhonov regularization which has a continuous regularization parameter, the T-SVD regularization has a discrete one and the estimation or selection of it has some specific differences. From (8.37) it can be concluded that the discrete regularization parameter can take values from zero to $n$, which is the number of target position coordinates to be estimated, which for the general case of MLAT localization is equal to 3. However, if the regularization parameter is $k_{\epsilon}=3$, then no regularization is applied and therefore we obtain the LS solution in (7.18). Moreover, if the regularization parameter is $k_{\epsilon}=0$, all the singular values of the Jacobian differential matrix $\boldsymbol{G}$ are neglected and (8.39) does not lead to any valid solution. In this sense, it seems that the regularization parameter $k_{\epsilon}$ can take only two values (i.e., 1 or 2) in the particular case of requiring regularization for the localization problem in MLAT systems, and when no regularization is required this parameter takes only one value (i.e., 3).

On the other hand, basically, several methods to estimate the regularization parameter, from those described in $\S 8.1 .4$, can be also used to estimate the pertaining regularization parameter for T-SVD. However, the same disadvantages as commented in $\S 8.1 .4$ also hold for this method.

In this way, we have concluded that a feasible strategy to choose the regularization parameter is to solve the problem in (8.39) for $k_{\epsilon}=2$ and $k_{\epsilon}=3$. The option of $k_{\epsilon}=1$ has been widely tested and no useful solution is obtained because the loose of much information. Therefore, this option is not further considered. The same procedure as proposed for Tikhonov regularization in (8.23) - (8.24) is used for T-SVD.

Furthermore, an equivalent strategy to that described by (8.31), to improve the performance for LAM applications, can be also used for the TSVD regularization. It is described as follows,

$$
k_{\epsilon}^{k}=\left\{\begin{array}{ll}
3, & \sigma_{2}^{k} \geq \sigma_{2}^{k-1} \\
2, & \text { otherwise }
\end{array} ; \quad k=3, \ldots, K\right.
$$

where $K$ is the total number of Taylor iterations and $k_{\epsilon}^{1}=k_{\epsilon}^{2}=2$ for LAM applications. 


\subsubsection{Solution by T-SVD with a Sub-Set Selection (T-SVD SS)}

This method is similar to the T-SVD but here the $k_{\epsilon}$ most linearly independent columns from $\boldsymbol{G}$ are extracted instead the first $k_{\epsilon}$ columns. Therefore, it is needed the permutation matrix $\boldsymbol{\Pi}$ that minimizes the condition number of the new sub-matrix $\widehat{\boldsymbol{G}}_{\boldsymbol{k}_{\epsilon}}$. This new matrix consists of the first $k_{\epsilon}$ columns of the matrix $\boldsymbol{G} \Pi$. The T-SVD solution with subset selection is given by [72], [76]:

$$
\widehat{\boldsymbol{\theta}}_{k_{\epsilon}}^{\text {basic }}=\boldsymbol{\Pi}\left(\begin{array}{c}
\widehat{\boldsymbol{G}}_{k_{\epsilon}}^{\dagger} \widehat{\boldsymbol{m}}_{\Delta} \\
\mathbf{0}
\end{array}\right)+\left(\begin{array}{c}
\boldsymbol{\theta}_{0} \\
\mathbf{0}
\end{array}\right)
$$

where $\widehat{\boldsymbol{\theta}}_{k}^{\text {basic }}$ has zeros in those positions that corresponds to the neglected columns of $\boldsymbol{G}$. The matrix $\boldsymbol{\Pi}$ can be obtained by any routine for calculating the QR factorization. It is not the aim of this thesis to describe such a routine and we refer the interest reader to check the algorithms in [72] and the references therein.

By contrary to the T-SVD where we can force a truncation parameter $k_{\epsilon}$ and thus some amount of regularization is added, for the subset selection approach the equivalent regularization parameters depends on the estimation of an error level $\epsilon$ in the QR factorization. Due to the difficulties in estimation that error level, the QR factorization in this application always provides a matrix $\widehat{\boldsymbol{G}}_{\boldsymbol{k}_{\epsilon}}$ equal to the matrix $\boldsymbol{G}$ and, therefore, no regularization is added. For this reason we do not analyze more this method and no results are presented for it.

\subsection{Solution by Total Least Squares (TLS) Based Methods}

All methods we have described so far only take into account the possible errors in the measurement vector $\widehat{\boldsymbol{m}}$, i.e., they assume the errors sources when solving the general inverse problem $\boldsymbol{G} \boldsymbol{\theta}=\widehat{\boldsymbol{m}}$ are only contained in the measurement vector $\widehat{\boldsymbol{m}}$. However, it is also common to find cases when the matrix $\boldsymbol{G}$ is not precisely known or it has some errors as it can be obtained by measurements or by approximation of the exact operator. Furthermore, also linearization or discretization errors can appear. For these cases, the Total Least Squares (TLS) method [77], [128] was developed for solving the inverse problem by taking into account both the possible errors in the coefficient matrix $\boldsymbol{G}$ and their size in respect to those in the measurement vector $\widehat{\boldsymbol{m}}$.

The main idea of TLS method is to allow a residual vector (see $\S 6.4$ ) and a residual matrix. In this sense, the solution of the inverse problem consists in finding a vector $\boldsymbol{\theta}$ that satisfies the following minimization problem: 


$$
\min \left\|(\boldsymbol{G}, \widehat{\boldsymbol{m}})-\left(\widetilde{\boldsymbol{G}}, \widehat{\boldsymbol{m}}^{\prime}\right)\right\|_{F} \quad \text { subject to } \quad \widehat{\boldsymbol{m}}^{\prime}=\widetilde{\boldsymbol{G}} \boldsymbol{\theta}
$$

where $\widetilde{\boldsymbol{G}}$ is the perturbed coefficient matrix, $\widehat{\boldsymbol{m}}^{\prime}$ is the equivalent perturbed version of the measurement vector, and \|\|$_{F}$ denotes de Frobenius norm, which for a general $m \times n$ matrix $\boldsymbol{A}$ is defined as [72]:

$$
\|\boldsymbol{A}\|_{F}=\sqrt{\sum_{i=1}^{\min \{m, n\}} \sigma_{i}^{2}}
$$

being $\sigma_{i}$ the $i$ th singular value of matrix $\boldsymbol{A}$.

Thus, the solution for the localization problem by using (8.42) can be written in terms of the SVD of the overall matrix $\left[\boldsymbol{G}, \widehat{\boldsymbol{m}}_{\Delta}\right]$ as follows:

$$
\left[\boldsymbol{G}, \widehat{\boldsymbol{m}}_{\Delta}\right]=\overline{\boldsymbol{U}} \overline{\boldsymbol{\Sigma}} \overline{\boldsymbol{V}}^{T}
$$

being $\overline{\boldsymbol{\Sigma}}$ the matrix of singular values of $\left[\boldsymbol{G}, \widehat{\boldsymbol{m}}_{\Delta}\right]$ and it takes the following form:

$$
\overline{\boldsymbol{\Sigma}}=\operatorname{diag}\left(\bar{\sigma}_{1}, \ldots, \bar{\sigma}_{n+1}\right)
$$

where the operator diag denotes a diagonal matrix with its arguments in the main diagonal. Finally, the iterative location estimator can be obtained in the sense of TLS by:

$$
\widehat{\boldsymbol{\theta}}_{T L S}^{k}=-\overline{\boldsymbol{v}}_{1: n, n+1}^{k-1} \frac{1}{\bar{v}_{n+1, n+1}^{k-1}}+\boldsymbol{\theta}_{0}^{k-1}, \quad k=1, \ldots, K
$$

where $\overline{\boldsymbol{v}}_{1: n, n+1}^{k-1}$ is the $(n+1)$ th right singular vector of (8.44), of size $n \times 1$, for the $(k-1)$ th Taylor iteration, i.e., (8.44) is calculated for $\left[\boldsymbol{G}\left(\widehat{\boldsymbol{\theta}}_{T L S}^{k-1}\right), \widehat{\boldsymbol{m}}_{\Delta}\left(\widehat{\boldsymbol{\theta}}_{T L S}^{k-1}\right)\right]$.

When the problem is ill-conditioned, particularly with a rank deficient coefficient matrix, the solution (8.45) by TLS should be avoided and, equivalent to the case of the T-SVD, the use of the truncated version of the TLS is more advisable. This truncated version is described below.

\subsubsection{Solution by Truncated TLS (T-TLS)}

Similarly to the SVD and T-SVD solutions, when the matrix $\boldsymbol{G}$ is rank deficient or numerically rank deficient, it is common to neglect all zeros or small but non zero singular values of the overall matrix $[\boldsymbol{G}, \widehat{\boldsymbol{m}}]$ and solve the problem with the new truncated matrix. This solution is called Truncated TLS (T-TLS) [76-77]. This method modifies the initial ill-conditioned 
problem to a nearby one but, unlike to the T-SVD method, the modification depends on both $\boldsymbol{G}$ and $\widehat{\boldsymbol{m}}$.

If we define $k_{T-T L S}$ as the T-TLS truncation parameter, i.e., the number of retained singular values of $\left[\boldsymbol{G}\left(\widehat{\boldsymbol{\theta}}_{T L S}^{k-1}\right), \widehat{\boldsymbol{m}}_{\Delta}\left(\widehat{\boldsymbol{\theta}}_{T L S}^{k-1}\right)\right]$, and the matrix $\overline{\boldsymbol{V}}$ is partitioned into a $(n+1) \times(n+1)$ matrix such that:

$$
\overline{\boldsymbol{V}}=\left(\begin{array}{ll}
\overline{\boldsymbol{V}}_{11} & \overline{\boldsymbol{V}}_{12} \\
\overline{\boldsymbol{V}}_{21} & \overline{\boldsymbol{V}}_{22}
\end{array}\right)
$$

where $\overline{\boldsymbol{V}}_{11} \in \mathbb{R}^{n \times k_{T-T L S}}$, then, the iterative localization estimator in the sense of T-TLS can be obtained as follows:

$$
\begin{aligned}
\widehat{\boldsymbol{\theta}}_{k_{T-T L S}}^{k}=-\overline{\boldsymbol{V}}_{12}^{k-1}\left(\overline{\boldsymbol{V}}_{22}^{\dagger}\right)^{k-1}+\widehat{\boldsymbol{\theta}}_{k_{T-T L S}}^{k-1} \\
=-\overline{\boldsymbol{V}}_{12}\left(\overline{\boldsymbol{V}}_{22}^{T}\right)^{k-1}\left\|\overline{\boldsymbol{V}}_{22}^{k-1}\right\|_{2}^{2}+\widehat{\boldsymbol{\theta}}_{k_{T-T L S}}^{k-1}
\end{aligned}
$$

subject to the necessary condition that $\overline{\boldsymbol{V}}_{22} \neq 0$.

Note that if $k_{T-T L S}=n$, then $\widehat{\boldsymbol{\theta}}_{k_{T-T L S}}=\widehat{\boldsymbol{\theta}}_{T L S}$. Finally, also the most important aspect for the correct implementation of this method is to choose the correct truncation parameter $k_{T-T L S}$. To do this, the same principle as proposed for T-SVD (see $\$ 8.2 .2$ ) is used with the difference that for T-TLS based estimator the values $k_{T-T L S}=1$ and $k_{T-T L S}=2$ should be used. However, due to the size of the coefficient matrix, this kind of methods do not allow many possibilities for this truncation parameter and a general, roughly correct selection for ill-conditioned situations is $k_{T-T L S}=2$. Moreover, a difference between this truncation parameter and the one of the T-SVD must be taken into account. We have seen for the T-SVD algorithm that, the greater the regularization parameter, the less is the introduced amount of regularization. For the case of T-TLS is different: the greater the regularization parameter, the greater is the introduced amount of regularization.

In the theoretical sense, the main difference between the T-SVD solution and the T-TLS lies in the fact that, in the first one, the modification depends only on the coefficient matrix $\boldsymbol{G}$, while for T-TLS it depends on both the coefficient matrix and the measurement vector.

\subsection{General Localization Strategy and Additional Improvements}

Through the theoretical analysis and simulations we have performed for this thesis, we have identified several points that must compose a general localization strategy for MLAT systems. The main idea is based on the fact that there is no a unique superior algorithm that computes the target 
position with the best performance levels under any situation or scenario. Therefore, we propose the combined use of a closed form algorithm and an open form algorithm in order to obtain a statistically optimal (or near to optimal) localization strategy. Moreover, another simple but efficient strategies that allow improving the localization performance are proposed. The basic, canonical procedure for localization in MLAT system is shown in Fig. 8.1 and subsequently described. Then, the additional improvements are also described.

The basic scheme for the general localization strategy is composed of two main steps: area detection and localization process. The first one, area detection, is intended to estimate the possible geographical area where the aircraft/vehicle can be. The objective of this step is to inform to the second step the set of localization algorithms to be used for calculating the target position. We propose two kind of areas, namely, surface movement (i.e., the target is basically on the airport surface) or on flight (i.e., the target is taking off, landing or flying). To estimate the target location area it is used (simultaneously when it is possible) two ways, the first one is by reading the squitter/reply messages which normally contain some navigation data like the barometric altitude, ADS-B position, etc., and the other one is by calculating a first and quick position by means of a specific closed form algorithm. This step has to be used only in the track initiation process, which is a confidential time interval to initialize, reliably, the corresponding target track [1-2].

The second step in Fig. 8.1 is the localization process. This process is used once the area detection step has finalized and it is executed with a certain update rate that depends on the scenario characteristics (i.e., LAM or WAM), the users, agreements, etc. [1-2]. This process is based on the combined use of a closed form algorithm (a 3D version or a projected one, depending on the detected area) and the Taylor based algorithm along with some of the analyzed and proposed regularized estimators. For this aspect, we propose the use of Tikhonov based regularization or T-SVD one. A previous analysis of the particular scenario is always advisable in order to choose the estimator that better satisfies the conditions. With the information given by the first step, the procedure decides, to calculate the starting point, the use of the projected version (for surface movements) of the closed form algorithm or of the 3D, non-projected one (for on flight targets). Then, it is used the selected regularized location estimator to refine the starting point and thus obtain a more accurate position data. For the vertical component of the starting point, we propose to use a fixed value (e.g., $10 \mathrm{~m}$ ) for LAM applications (i.e., surface movements) as all the targets heights (i.e., the transponder height) are in a closed range of meters, while for WAM applications we propose the use of barometric altitude when it is present or the one as provided by the $3 \mathrm{D}$ closed form algorithm. 


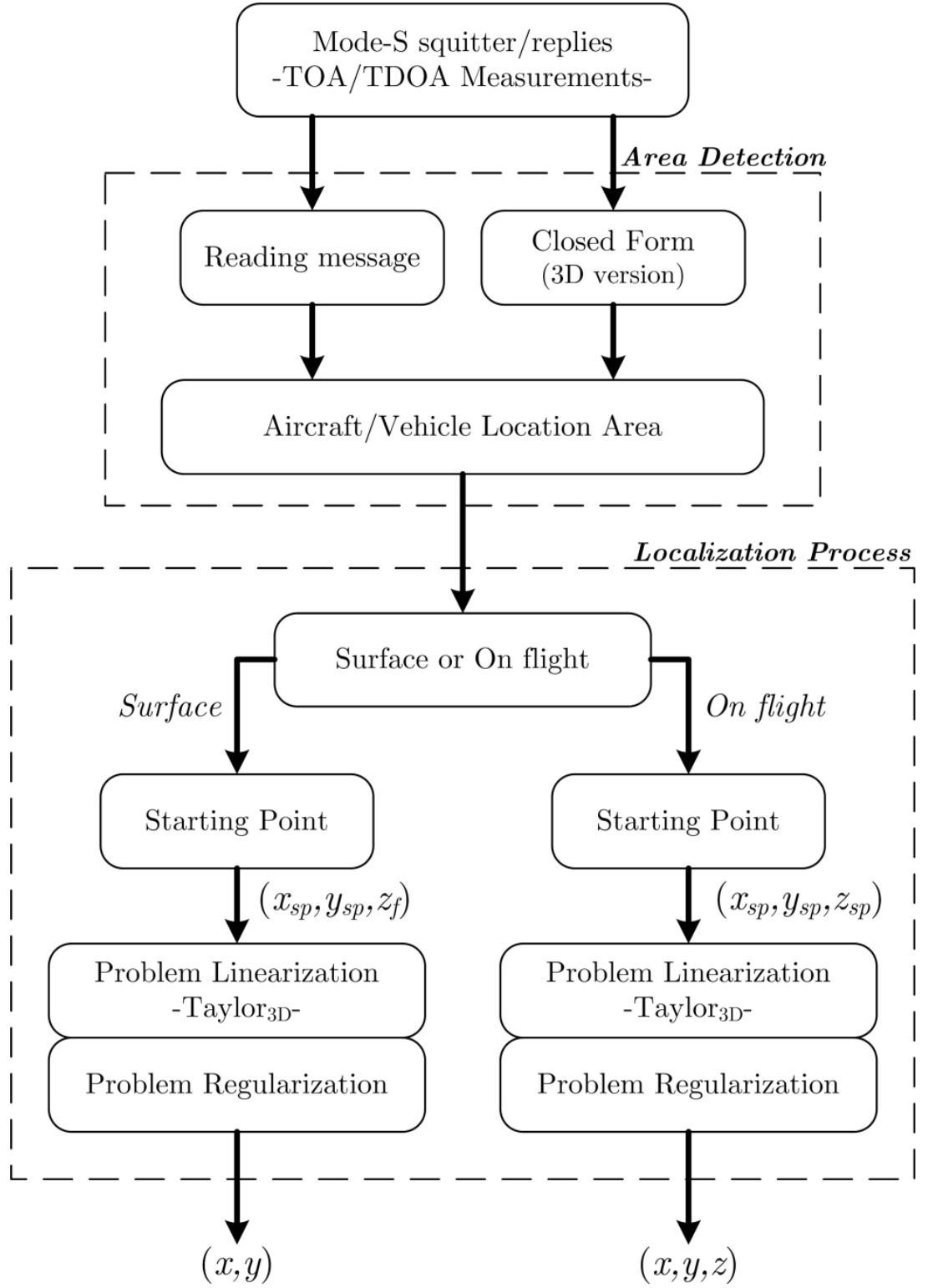

Fig. 8.1 Basic scheme for the general localization strategy.

In order to introduce a general notation, we call the strategy of solving the Taylor localization algorithm in the sense of any regularization method as the Regularized Location Estimator (RLE), and when we refer it, we are really referring to the regularized estimators proposed in this thesis. In the following subsection, some additional proposed improvements to the RLE are described. 


\subsubsection{Additional Improvements}

We propose two additional improvements for the general localization strategy described in Fig. 8.1. The first one is based on the previous selection of some measurements, i.e., in some scenarios, some measurements are highly perturbed for non random noises and, in these cases, any localization algorithm can obtain useful solutions, hence, it is advisable to remove these measurements from the localization inverse problem. The second one is based on the exploration of the set of $K$ partial solutions (see $\S 8.1$ ) provided by the iterative localization estimator, for each particular point. We have observed that, under some situations, the solution provided by this iterative procedure (i.e., the last partial one) is not always the closest one to exact target position. In this sense, we have seen that by an adequate exploration of the $K$ partial solutions, the accuracy of the iterative estimator can be improved. These additional improvements are described below. Then, a general flowchart, including them, is described.

\section{Measurements Selection (SM)}

There are situations for which some measurements are highly (or extremely highly) perturbed for non random noises, e.g., the ones due to non line of sight propagation (multipath). In such cases, it is advisable to remove these measurements from the localization inverse problem as no algorithm can reach a useful or reliable solution. To overcome this problem, we propose a simple strategy for selecting some measurements and to remove those possible highly perturbed ones. The main idea consists of sorting the TOA measurements from the smallest to the greatest, then the first four ones are retained, and finally the inverse problem is solved only with those four measurements.

The hypotheses for this is based on the fact that if the $1090 \mathrm{MHz}$ signal is not propagated through a straight line, to a receiving station, probably the TOA measured for such a station will be greater than those measured at the rest of the stations. Thereby, the reason to choose only four stations and no more, in the case of they are available, is to try to set a system of equations with unique solution as only three equations can be set with four stations. Moreover, as it is shown in the results section, the regularized location estimators allow ensuring the solution convergence even in the case of the minimum number of stations.

Although this strategy can, in some cases, make no use of the additional good measurements, we have found that when using any of our RLEs, the possible accuracy degradation is negligible, whilst the improvement on those cases of non random noises is much relevant. The advantages of this strategy are clearly shown when analyzing the real system data in the results part. 


\section{Solution Selection (SS)}

Due to the fact that the RLE is an iterative estimator (cf. (8.10)), which produces several partial solutions (i.e., one solution is produced for every iteration), we have observed that not always the last one (i.e., the final solution) is the best solution in respect of the exact target position. It is because the Taylor iterations, either for the non-regularized or for the regularized version, are intended for minimizing the 2-norm of a residual error function (see $\S 6.4$ and $\S 8.1$ ) but not in respect to the exact target position. In other words, the iterative localization algorithm estimates a target position $\boldsymbol{\theta}$ that better matches the measurement vector $\widehat{\boldsymbol{m}}$ in the system of equations $\boldsymbol{A} \boldsymbol{\theta}=\widehat{\boldsymbol{m}}$. Additionally, the regularized version also satisfies the minimization of the smoothed norm. However, any of these algorithms looks for a solution $\boldsymbol{\theta}$ that minimizes the distance to the exact target position. It is because it is not the aim of any localization algorithm and, the second one, because the exact target position is not known. To perform that minimization, we propose a criterion called "solution selection by nearest estimated neighbour". This criterion consists in choosing as final target position that partial solution with minimum distance to an estimated target position provided by a tracking algorithm. It is, the final solution for a time $t$ is taken as a value that satisfies the following expression:

$$
\widehat{\boldsymbol{\theta}}(t)=\arg \min \left\{\left\|\widehat{\boldsymbol{\theta}}^{k}(t)-\widehat{\boldsymbol{\theta}}_{\text {Track }}(t)\right\|_{2}\right\}
$$

where $\widehat{\boldsymbol{\theta}}_{\text {Track }}(t)$ is the position data provided by the tracking algorithm for time $t$ and $\widehat{\boldsymbol{\theta}}^{k}(t)$ is the $k$ th partial solution, provided by the RLE, for time $t$. This criterion is illustrated in Fig. 8.2.

In Fig. 8.2 the yellow star represent the starting point for the regularized estimator, the red circle is the final solution as provided by the regularized estimator, and the green squares are the partial solutions. The dash-dot line is the approximated trajectory that provides a tracking algorithm after processing the position data. In the illustration of Fig. 8.2, the red circle for

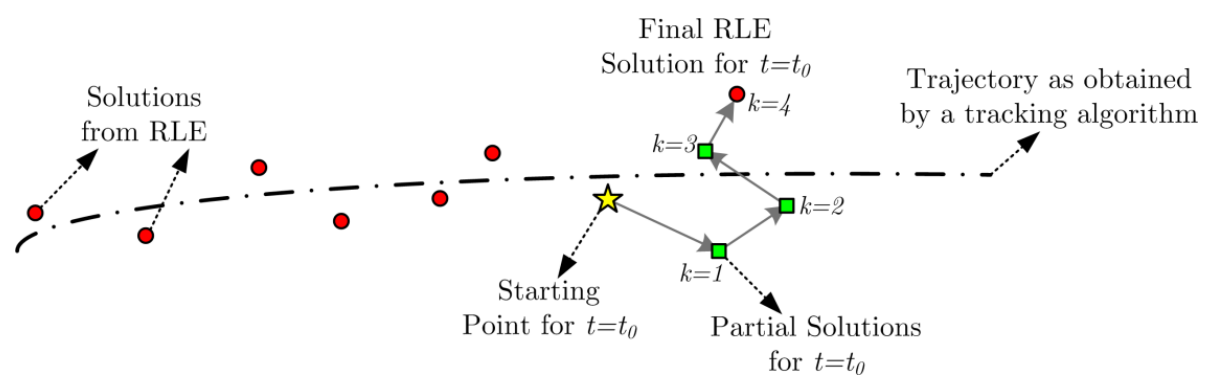

Fig. 8.2 Solution selection by nearest estimated neighbour. 
the iteration $k=4$ is the value that better matches the system of equation $\boldsymbol{A} \boldsymbol{\theta}=\widehat{\boldsymbol{m}}$, but the iteration for $k=3$ is the value that better satisfies the minimization problem in (8.48). Thus, the final solution, for the target position, as obtained by the solution selection by nearest estimated neighbour criterion is that of $k=3$. Moreover, in the cases of small measurement noises, generally the solution obtained by this criterion coincides with the last iteration of the regularized estimator. The reason to use the smoothed target position provided by a tracking algorithm is briefly described below.

It is known that the position data provided by implementing a tracking algorithm, under certain assumptions about the observation and process models used, is an estimation that minimizes the following mean-squared error [114]:

$$
E(t)=\int_{-\infty}^{\infty}\left(\boldsymbol{\theta}(t)-\widehat{\boldsymbol{\theta}}_{\text {Track }}(t)\right)^{T}\left(\boldsymbol{\theta}(t)-\widehat{\boldsymbol{\theta}}_{\text {Track }}(t)\right) P\left(\boldsymbol{\theta}(t) \mid \boldsymbol{Z}^{t}\right) \mathrm{d} \boldsymbol{\theta}
$$

where $P\left(\boldsymbol{\theta}(t) \mid \boldsymbol{Z}^{t}\right)$ is the condition probability function of the target position and $\boldsymbol{Z}^{t}$ represents the set of previous position data. Thus, if the tracking algorithm converges, in statistical sense the smoothed vector $\widehat{\boldsymbol{\theta}}_{\text {Track }}$ is the most nearest solution to the exact target position. Importantly to comment that the tracking algorithms are implemented in all the ATC infrastructures.

The improvement added by the two above strategies is significant when multipath problem appears, as it is the case of the real system evaluated in this thesis. This general localization strategy is analyzed in $\S 8.7$.

\section{Flowchart for the General Localization Strategy}

A general flowchart for implementing the general localization strategy and the additional improvements, proposed in this thesis, is shown in Fig. 8.3.

In the following, each step of the flowchart above is described.

1. In this point the TOA/TDOA measurements are processed to put them into a common clock frame. Moreover, some additional delays can be added. It depends on the particular architecture used to measure the TOAs or TDOAs. This processing is out of the scope of this thesis and, hence, we assume the TOA/TDOA quantities are suited to be used for the localization algorithms.

2. In the case that the received set of measurements are the first ones (understanding for first, the first one after area detection in Fig. 8.1) for a particular target (i.e., $t=1$ ), the starting position $\left(x_{s p}, y_{s p}\right)$ is calculated by means of a closed form algorithm. Then, this starting point is sent to the step number 5 to obtain 


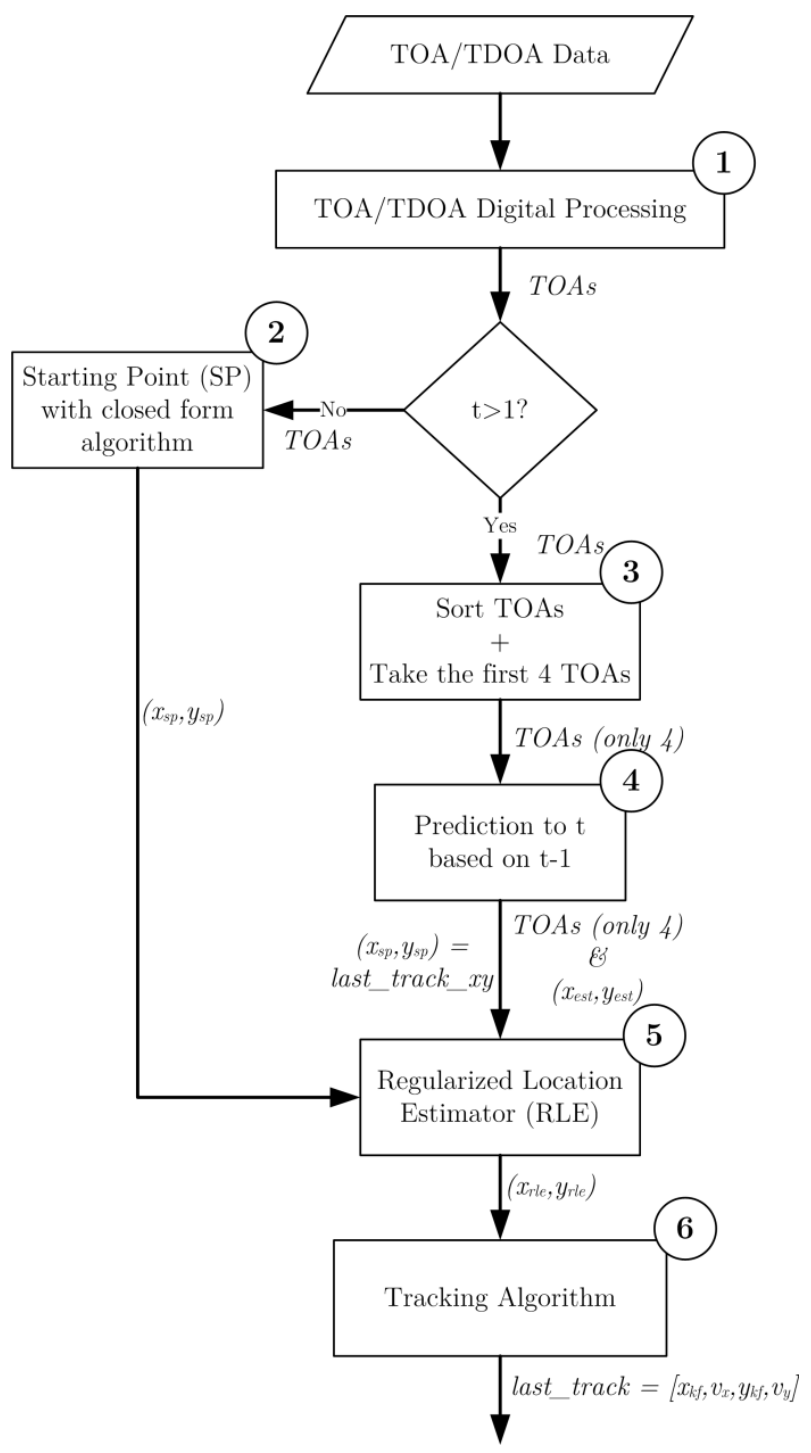

Fig. 8.3 Flowchart for the general strategy with the additional improvements.

an improved position with the Regularized Location Estimator (RLE).

3. In this point the selection of measurements is performed. This step is composed of two sub-steps as follows:

a. Sort TOAs: In this sub-step the TOA measurements are sorted from the smallest to the greatest. 
b. Take the first 4 TOAs: In this sub-step the first four TOA measurements, from the previous sub-step, are taken as described before.

4. In this point, a predicted position $\left(x_{e s t}, y_{e s t}\right)$ is estimated by a simple linear interpolation from the time of the last position to the current time. It is calculated by using the last valid track as provided by a tracking algorithm (e.g., a Kalman Filter -KF[114]) in the step number 6 . This predicted position is not used as a starting point for the RLE, it is used for an additional function within the RLE as described below.

5. In this point, the target position is calculated by means of the RLE. It is composed of two sub-steps.

a. In the first sub-step, the target position is calculated by means of some regularized localization algorithms described in this thesis. The starting point $\left(x_{s p}, y_{s p}\right)$ for $t=1$ is the one as provided by the closed form algorithm, whilst hereafter it is the last track as provided by the tracking algorithm.

b. The second sub-step is really the second additional improvement we propose in this thesis: "the solution selection by the nearest estimated neighbour". It has been described before.

6. In this step a tracking algorithm is used to smooth the solutions provided by the regularized location estimators.

\subsection{Simulation and Results: Analysis for Regularized Location Estimators}

In this part, we analyze the overall accuracy performance for the different Regularized Location Estimators (RLE) proposed in this thesis: the Tikhonov, the T-SVD and the T-TLS based RLEs. To do this we have used the scenarios of Innsbruck, Malpensa and Linate that are described in $\S$ C.1. For each scenario, we obtain the 2D and vertical (when apply) R.M.S errors and the spatial biases.

For these simulations, we only use the basic regularized estimators of Tikhonov (8.10), T-SVD (8.38), and T-TLS (8.47), i.e., without using the general localization strategy described in Fig. 8.3, because the main objective of this part is to validate the overall performance of the three RLE proposed in this thesis for both LAM and WAM operations. 


\subsubsection{Accuracy Analysis for Innsbruck WAM System}

For this scenario (see $\S$ C.1.1 for a complete description), the full starting point, i.e., $(x, y, z)$, is obtained by the closed form algorithm described in §7.3.4 (Schau \& Robinson algorithm). Moreover, the regularization parameter for Tikhonov method is estimated by $(8.29)$ with $w=0.03$; it is a WAM system with more than five stations. For the discrete regularization parameters for T-SVD and T-TLS based RLEs, we have used the procedures described in $\S 8.2 .2$ and $\S 8.3 .1$ respectively.

The Innsbruck system is a full operating system for the Terminal Manoeuvring Area (TMA) at Innsbruck airport. This system is fully operational and therefore it provides a well-defined accuracy. The horizontal accuracy for this system is shown in Fig. 8.4. This figure shows the 2D R.M.S error for the starting point (Starting point), for the classical

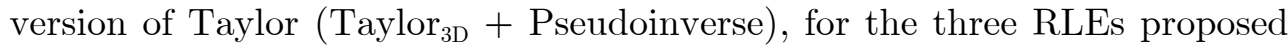
in this thesis, and the predicted performance as obtained by the CRLB analysis [20]. Firstly, it can be observed that the CRLB predicts a smoothed behaviour for the horizontal accuracy, which means that this system does not present ill-conditioning due to the system geometry. The starting point as provided by the closed form algorithm provides an acceptable accuracy and it is improved by the classical version of Taylor based algorithm. Moreover, the corresponding solutions for the Tikhonov and T-SVD RLEs also present a smoothed behaviour, which is equal to that of the classical version of Taylor. It is because the number of stations and their horizontal, and vertical separations, avoid the ill-conditioning of the problem. This fact also avoids the ill-conditioning due to the measurements noise. Regarding to the T-TLS based RLE, it also presents a smoothed behaviour for the 2D R.M.S error but it is slightly greater than those of Tikhonov and T-SVD.

The vertical accuracy is shown in Fig. 8.5 and it presents an equivalent behaviour, than that presented by the horizontal accuracy, for classical Taylor, Tikhonov and T-SVD. Again, the T-TLS presents a similar performance but with some particular degradations at the end of the take of line (beyond $60 \mathrm{~km}$ in Fig. 8.5).

Another important aspect, complementary to the accuracy analysis, when using regularization methods, is the possible amount of spatial bias that can be introduced by these in the solution. The horizontal and vertical biases, for the algorithms evaluated in this scenario, are shown in Fig. 8.6 and Fig. 8.7 respectively. In these figures can be observed that the Tikhonov and T-SVD RLEs reach the same amount of bias than the classical Taylor solution, which is always the minimum biased estimator. The T-TLS based RLE presents an average spatial bias performance similar 


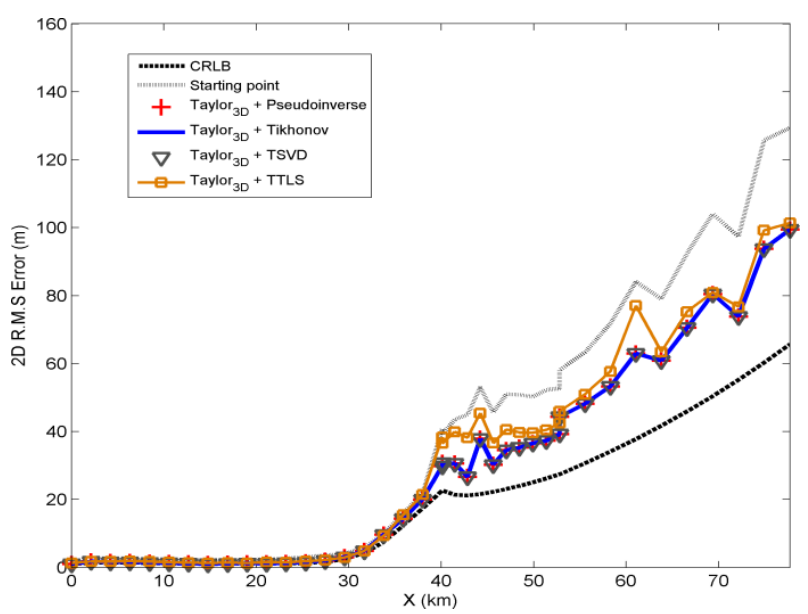

Fig. 8.4 2D R.M.S error for Innsbruck system.

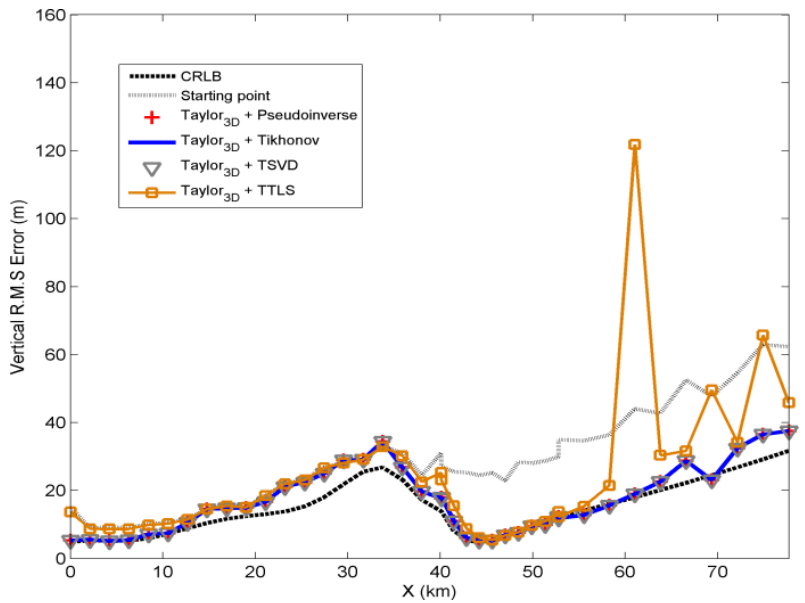

Fig. 8.5 Vertical R.M.S error for Innsbruck system.

to that of the classical Taylor solution, but with some degradation at the end of the takeoff line.

The bias analysis takes importance when tracking algorithms are used (as in all the ATC systems), because these algorithms improve the component of the R.M.S error, provided by the localization algorithm, that depends on the standard deviation but not on the bias. In this way, if the localization algorithm introduces a large amount of bias, also the smoothed solution after the tracking process will be far from the real one.

The objective of this simulation is to show that the application of the RLEs (more the Tikhonov and T-SVD versions), in the case of wellconditioned problem, provides the same results than the Pseudoinverse 


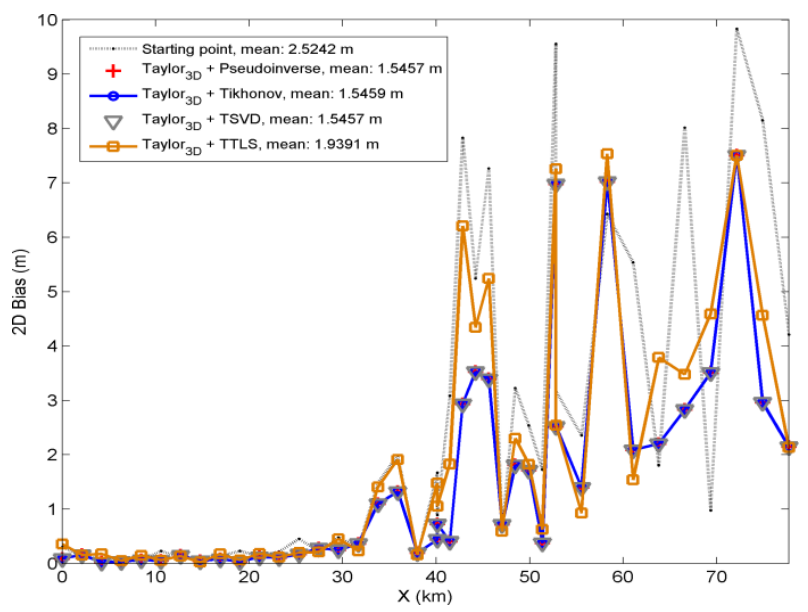

Fig. 8.6 2D bias for Innsbruck system.

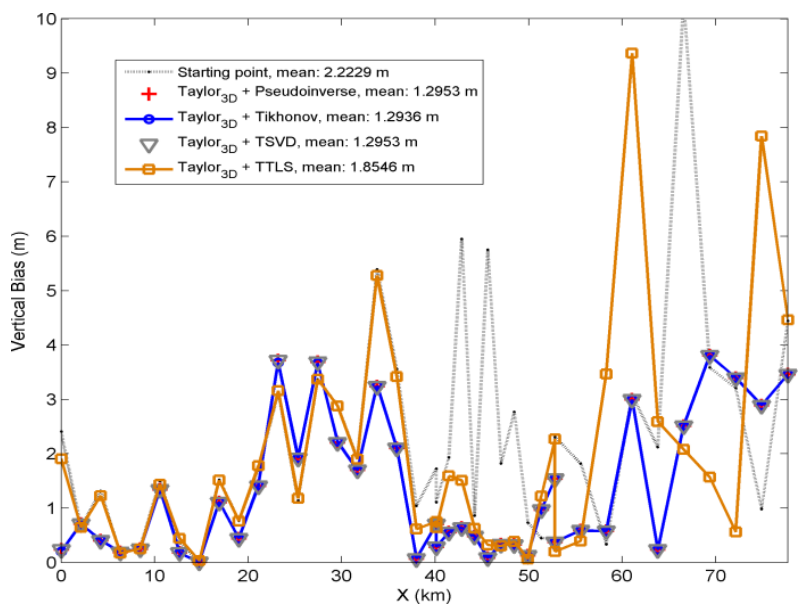

Fig. 8.7 Vertical bias for Innsbruck system.

solution (which is the minimum 2-norm solution) and therefore, it does not negatively affect the overall system accuracy.

\subsubsection{Accuracy Analysis for Malpensa WAM System}

For this scenario (see $\S$ C.1.2 for a complete description), the full starting point, i.e., $(x, y, z)$, is obtained by the closed form algorithm described in \$7.3.4 (Schau \& Robinson algorithm). Moreover, the regularization parameter for Tikhonov method is estimated by (8.29) with $w=0.3$; it is a WAM system but with a number of stations smaller than five. For the discrete regularization parameters for T-SVD and T-TLS based RLEs, we have used the procedures described in $\S 8.2 .2$ and $\S 8.3 .1$ respectively. 


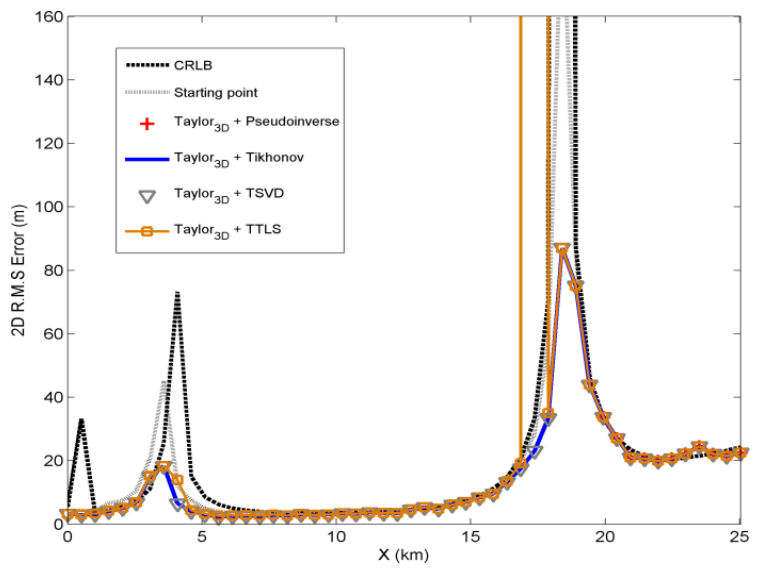

Fig. 8.8 2D R.M.S error for Malpensa system.

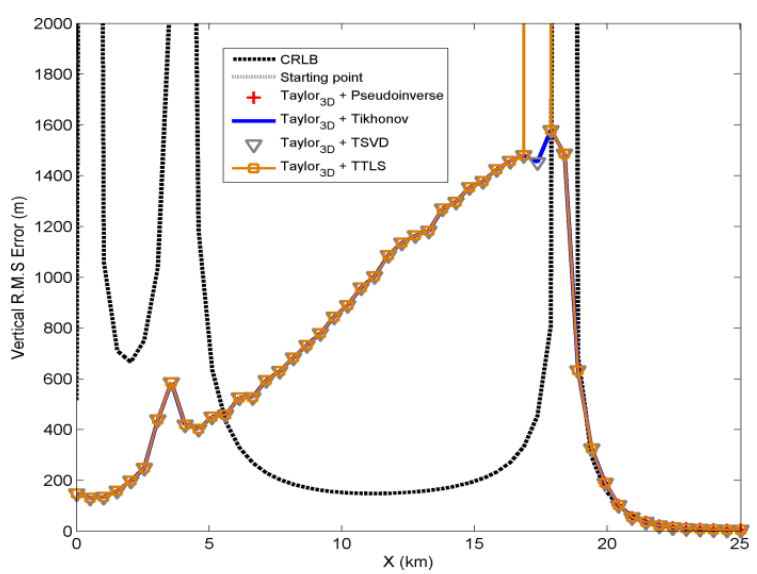

Fig. 8.9 Vertical R.M.S error for Malpensa system.

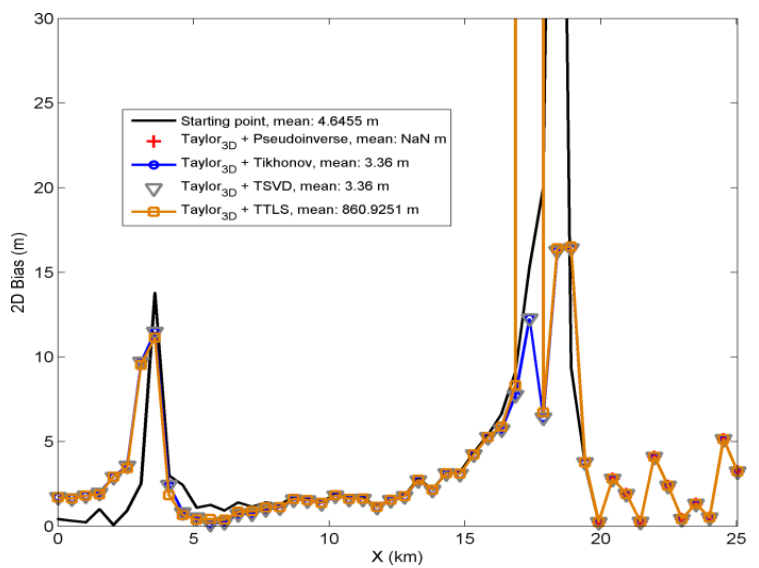

Fig. 8.10 2D bias for Malpensa system. 
The Malpensa system is a WAM system composed by four stations. In this scenario a typical $3^{\circ}$ ILS (Instrumental Landing System) takeoff line is simulated. As it is shown in [7], the location problem for this system is an ill-conditioned problem. Furthermore, the example shown in $\S 7.5$, Fig. 7.4, corresponds to this system. This ill-conditioning is due to the small number of stations and because they have relatively small vertical separations. The horizontal and vertical R.M.S errors for this scenario are shown in Fig. 8.8 and Fig. 8.9, respectively.

For the horizontal accuracy, it can be seen how the CRLB analysis predicts the ill-conditioning due to the system geometry and, therefore, it can be expected a bad accuracy for those points where the CRLB presents some peaks (within $0-5 \mathrm{~km}$ and $17-20 \mathrm{~km}$ ). The horizontal accuracy provided by the closed form algorithm approximately reaches the CRLB and it also presents bad accuracy levels for the same points than the CRLB. Then, the accuracy provided by the classical version Taylor method

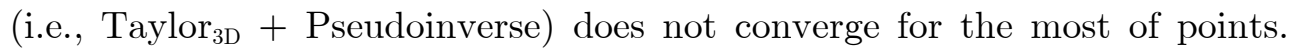
Specifically, it only converges for those points beyond $20 \mathrm{~km}$. In this way, it can be observed that this scenario presents an ill-conditioned problem due to both system geometry and measurements noise. Finally, it can be observed how the application of Tikhonov or T-SVD based RLEs significantly mitigates the ill-conditioning of the problem over the entire takeoff line. The T-TLS also presents the same behaviour but with a particular degradation near to $16 \mathrm{~km}$.

Regarding the vertical accuracy (see Fig. 8.9), the accuracy predicted by the CRLB analysis presents a greater amount of ill-conditioned than that for the horizontal case (the reason of this is the small vertical separation of the stations). In this case, the closed form algorithm provides a poor accuracy for the most of points, reaching acceptable values only beyond 20 $\mathrm{km}$. Furthermore, the classical version of Taylor method diverges for all the points before $20 \mathrm{~km}$ and it also reaches the CRLB beyond $20 \mathrm{~km}$. For this scenario, the application of all the RLEs does not improve the vertical accuracy and its vertical component tends to that of the starting point. The latter is a limitation of the application of regularization methods and it is because these methods filter out some components of the singular values spectrum, which leads to a loss of information and, therefore, they avoid the full and accurate recovery of all the position components.

On the other hand, the spatial horizontal bias for this scenario is shown in Fig. 8.10. For this analysis it is clear how the classical solution of Taylor algorithm presents an infinity average amount of bias (represented by the term NaN -Not a Number- in Fig. 8.10). By contrary, Tikhonov and T-SVD based RLEs present an acceptable average amount of bias (approximately of only $3 \mathrm{~m}$ ). For this case, also T-TLS based algorithm presents the same performance for the spatial bias but with a particular peak near to $16 \mathrm{~km}$. 


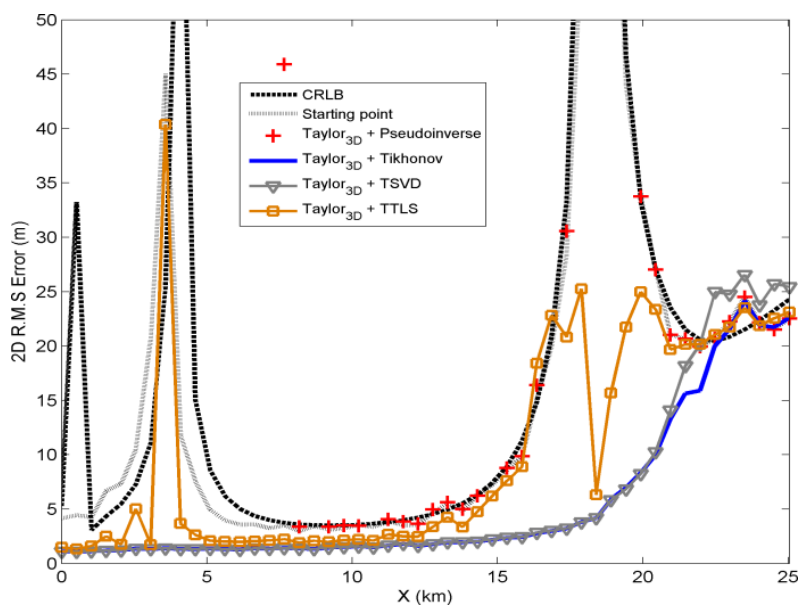

Fig. 8.11 2D R.M.S error for Malpensa system by using barometric altitude.

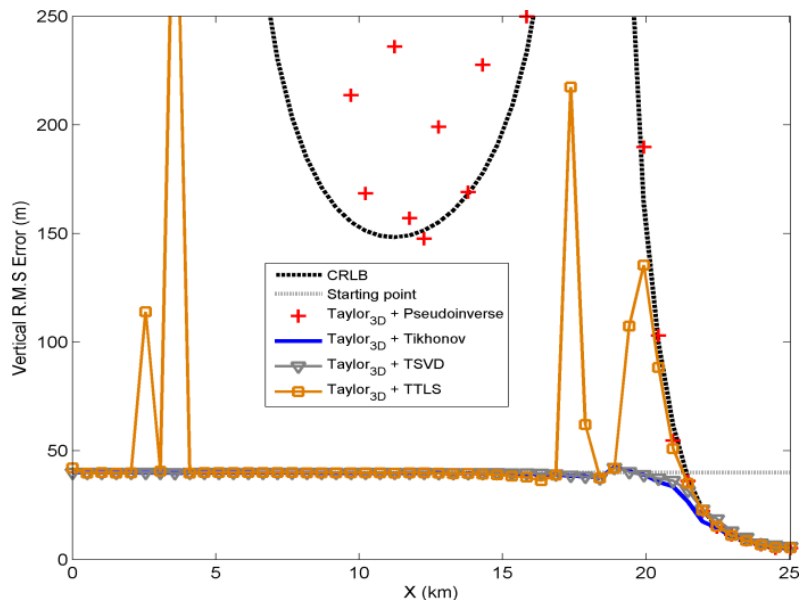

Fig. 8.12 Vertical R.M.S error for Malpensa system by using barometric altitude.

This is the reason for which the average bias of this estimator is very large (860 m).

In real applications, there exist other sources providing the vertical information, e.g., the barometer on board the aircraft which provides the barometric altitude. In standard conditions, this altitude information is reliable and accurate, and can be used by any localization system to improve the overall position accuracy. Based on this fact, if the vertical component, provided by the closed form algorithm, is modified by the barometric altitude contained in the reply/squitter messages (this data is present in the most of Downlink Formats -DF-, e.g., DF 4, 5, 17, 20 and 21 ), then it is possible to improve the overall accuracy provided by the RLEs. The results for this strategy, assuming the barometric altitude as the 


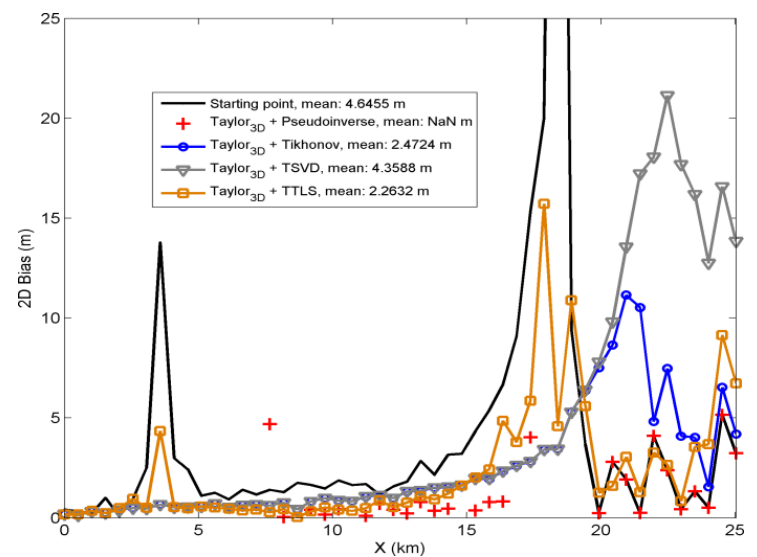

Fig. 8.13 2D bias for Malpensa system by using barometric altitude.

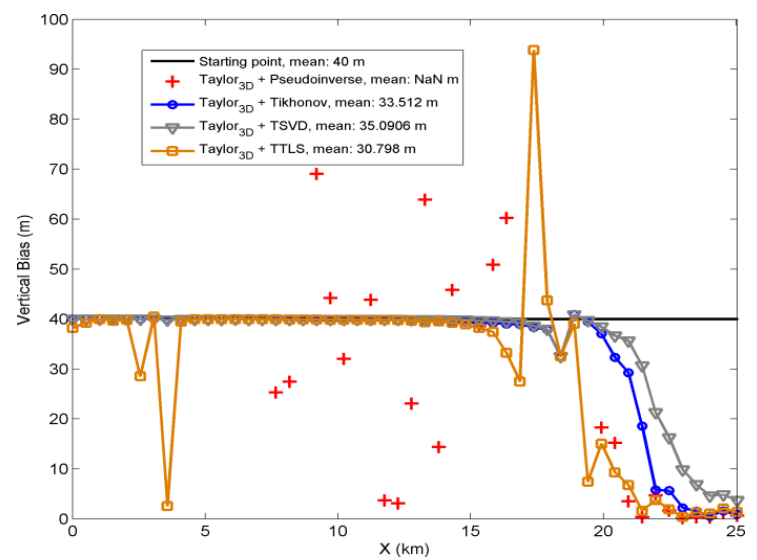

Fig. 8.14 Vertical bias for Malpensa system by using barometric altitude.

exact one plus a bias of $40 \mathrm{~m}$, are shown in Fig. 8.11 and Fig. 8.12 for the horizontal and vertical accuracies, respectively. Likewise, the corresponding horizontal and vertical biases are shown in Fig. 8.13 and Fig. 8.14, respectively.

For the horizontal accuracy, Fig. 8.11 shows how the addition of the barometric altitude information helps to improve the accuracy provided by the Taylor method solved by the Pseudoinverse matrix. However, this improvement is only for a small number of points. On the other hand, the use of this information along with the Tikhonov and T-SVD based RLEs improves significantly the horizontal accuracy over the entire takeoff line, providing accuracy levels below $25 \mathrm{~m}$ at $25 \mathrm{~km}$ from the runway, only with four stations (the EUROCAE requirements [1] ask for accuracy levels below $40 \mathrm{~m}$ at this distance). For this simulation it has been found the solution obtained by the application of Tikhonov regularization presents a slightly 
better accuracy for points beyond $22 \mathrm{~km}$. However, in practical terms this amount of improvement can be neglected. The horizontal accuracy of the T-TLS based algorithm is also improved but not as much as Tikhonov and T-SVD. Moreover, the accuracy of the closed form algorithm does not depend on this information and therefore it is the same with or without it.

Finally, for the vertical accuracy (see Fig. 8.12), the Taylor method solution obtained by the Pseudoinverse matrix does not converge for the most of points. In the case of the solution obtained by Tikhonov and TSVD based RLEs, they tend, in average, to the altitude provided by the barometer when the problem is strongly ill-conditioned, and tend to a better accuracy when the problem is well-conditioned (points beyond 20 $\mathrm{km}$ ). With some exceptions the same holds for the T-TLS based RLE. However, there are a set of points where the RLEs solutions improve the vertical accuracy provided by the barometer. These points are within $20-$ $22 \mathrm{~km}$ in Fig. 8.12. However, it is only a numerical result, because it is not a significant improvement, and the important aspect from this strategy is that the solutions obtained by the RLEs in any case avoid the divergence presented by the Pseudoinverse solution. In other words, the RLEs use the additional information when it is not capable to improve the accuracy; otherwise they improve it as shown in Fig. 8.12.

Regarding to the horizontal bias analysis, Fig. 8.13 shows the spatial bias for the starting point, for the Taylor method solved by the Pseudoinverse and by three RLEs. First, the classical version of Taylor method presents the minimum bias when it converges (e.g., some points within 10 and $15 \mathrm{~km}$ ). It is in concordance with the theory because, as it was commented in $\S 6.4$, it (LS solution) is the minimum 2-norm solution. However, in the rest of points the bias of this solution is infinitive (NaN). On the other hand, the solution provided by the application of Tikhonov based RLE introduces a bias but, as it is shown in Fig. 8.13, it is below $5 \mathrm{~m}$ for the most of points, and it is also smaller than that introduces by the starting point provided by the closed form algorithm. Only for a small number of points the bias introduced by Tikhonov regularization is greater than $5 \mathrm{~m}$ and below $10 \mathrm{~m}$ (around $20 \mathrm{~km}$ ). However, in average this bias is approximately $2.4 \mathrm{~m}$, which is a value that can be easily accepted for real applications. Moreover, the amount of bias introduced by the T-SVD based RLE is the same of that introduced by Tikhonov for points behind $20 \mathrm{~km}$ but, after that, the amount of bias introduced by T-SVD significantly increases up to the double of that introduced by Tikhonov based RLE. Finally, the T-TLS based RLE introduces greater amount of bias than TSVD based RLE for points around $16 \mathrm{~km}$, but smaller than that for points beyond $20 \mathrm{~km}$. Thus, in average the bias introduced by the T-TLS based algorithm is slightly smaller than that of the T-SVD based RLE but greater than the one of Tikhonov based RLE. The latter suggests that for this 
scenario the best overall accuracy performance is achieved by the application of Tikhonov regularization.

On the other hand, for the vertical bias analysis (see Fig. 8.14), all RLEs are biased to the vertical component as provided by the barometer, when the problem is strongly ill-conditioned (as the case previously shown in Fig. 8.12), and improve it when the problem is better conditioned. In this case, the T-TLS based RLE presents smaller vertical bias than Tikhonov and TSVD based one for some points around $4 \mathrm{~km}$ and beyond $20 \mathrm{~km}$. However, for other points it introduces greater bias than that of the barometric altitude. In the cases of Tikhonov and T-SVD, they reach the barometric altitude in the worst case. This result clearly shows one of the advantages of using the RLE proposed in this thesis (more the Tikhonov and T-SVD versions), that is, they improve when possible and, when not, they tend to the previous information.

\subsubsection{Accuracy Analysis for Linate (Surface) LAM System}

For this scenario (see $\S$ C.1.3 for a complete description), two kinds of simulations have been carried out. For the first kind, the starting point is set as the centre of the system perimeter as shown in Fig. C.3. The height of it is assumed to be $7 \mathrm{~m}$. For the second kind, the starting point is obtained by the closed form algorithm described in §7.3.4 (Schau \& Robinson algorithm) using also the corresponding approximation for LAM described in $\$ 7.4$ (i.e., the projected version of Schau \& Robinson algorithm). For this point the height of the starting point is also set to $7 \mathrm{~m}$, like in the first kind of simulations. Additionally, also the system performance in the presence of a possible failure of one station is analyzed. Because this system is only intended for surface surveillance, only the horizontal R.M.S error and bias are analyzed.

The regularization parameter for Tikhonov based RLE is estimated by (8.30), it is with the improvement for LAM systems. For T-SVD based RLE, the discrete regularization parameter is estimated for the procedure in $\S 8.2 .2$ with the corresponding LAM improvement described for (8.40). For T-TLS based RLE we have found that $k_{T-T L S}=2$ is the only option that provides acceptable results for LAM.

For the simulations of this scenario, we have used both version of the classical Taylor algorithm (the non-projected and projected versions), and the three proposed RLEs.

Due the number of stations composing this system (eight), it is initially geometrically well conditioned. However, due to the small vertical separation between them and the aircrafts/vehicles, it is expected some amount of ill-conditioning in this problem. 


\section{Accuracy Analysis by Using a Fixed Starting Point}

The horizontal R.M.S error and bias for this simulation are shown in Fig. 8.15 and Fig. 8.16, respectively. For this scenario, the CRLB predicts good horizontal accuracy levels over the entire simulated path, presenting only some peaks with no more than $14 \mathrm{~m}$ of R.M.S error (see Fig. 8.15). In this way, as we have previously commented, this problem is basically geometrically well-conditioned.

The non-projected version of Taylor algorithm, solved by the Pseudoinverse, practically does not converge for the entire path. This means that the problem solved by this method is ill-conditioned due to the measurements noise and also due the quality of the starting point. By contrary, the projected version of Taylor algorithm $\left(\right.$ Taylor $_{2 \mathrm{D}}+$ Pseudoinverse) converges for the entire path but, as we have previously analyzed in $\S 7.6 .3$, it introduces certain amount of bias. On the other hand, it is evident the significant accuracy improvement added by the application of Tikhonov and T-SVD based RLEs. In this problem, these RLEs significantly mitigate the ill-conditioning due to the measurements and to the quality of the starting point. Moreover, due to the fact that for this scenario a fixed starting point is used, the application of these regularization methods allows the utilization of Taylor based algorithm with the advantages of the open form algorithms (linearly related with the noise and the optimality in the statistical sense) and of the closed form algorithms (the non necessity of estimate a starting point). Furthermore, although Tikhonov and T-SVD based RLEs present similar 2D R.M.S errors, for some points within the 120 - 140 the accuracy provided by the T-SVD based RLE significantly degrades in respect of that provided by Tikhonov. In this simulation, the T-TLS based RLE provides acceptable accuracy but not as stable as that of Tikhonov and T-SVD. Practically, we can consider for this case that T-TLS based RLE does not converge.

Regarding to the bias analysis (see Fig. 8.16), the same general behaviour than that for the 2D R.M.S error is observed. That is, the nonprojected version of the classical Taylor algorithm does not converge and the average amount of bias is infinity $(\mathrm{NaN})$, adn the projected version of Taylor algorithm, although it converges, introduces certain amount of bias. Likewise, the amount of bias introduced by both Tikhonov and T-SVD based RLEs can be neglected in practical terms $(0.89 \mathrm{~m}$ for Tikhonov and $1.51 \mathrm{~m}$ for T-SVD) but, for those points within 120 - 140 the bias introduced by the T-SVD significantly increases regarding to that of the Tikhonov regularization. In any case, in average both estimators improve the overall performance of the classical solution provided by the projected version of Taylor solved by the Pseudoinverse. 


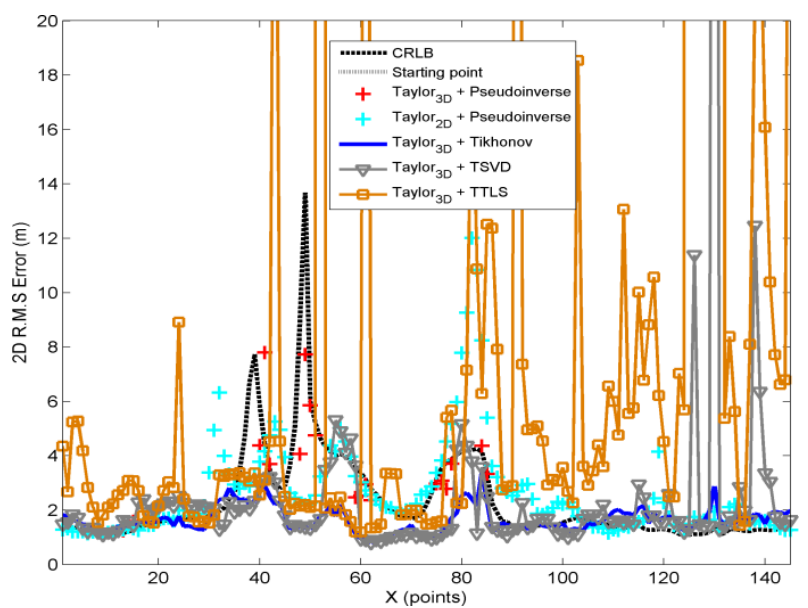

Fig. 8.15 2D R.M.S error for Linate system with a fixed starting point.

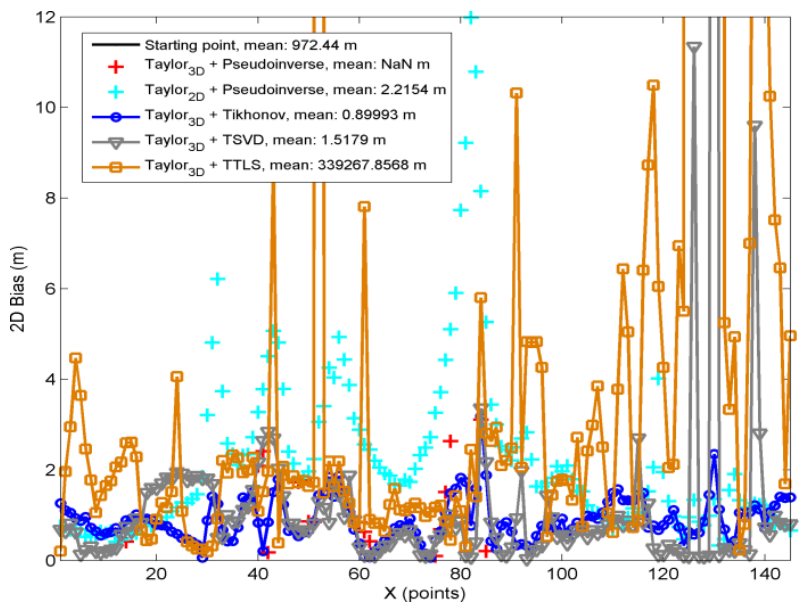

Fig. 8.16 2D bias for Linate system with a fixed starting point.

\section{Accuracy Analysis by Using the Starting Point Provided by Schau E Robinson Algorithm}

In this simulation we analyze the horizontal accuracy and bias of the estimators when the starting point is provided by the projected version of the Schau \& Robinson algorithm (see $\S 7.3 .4$ and $\S 7.4$ ). These results are shown in Fig. 8.17 and Fig. 8.18, respectively.

First, it should be noted that the quality (horizontal accuracy) of the starting point for this simulation (R.M.S error between $1 \mathrm{~m}$ and $12 \mathrm{~m}$ ) is significantly much better than that of the fixed point (R.M.S error between $260 \mathrm{~m}$ and $2650 \mathrm{~m}$, see Fig. 8.16). For this reason, the classical version of Taylor algorithm (Taylor $3 \mathrm{D}+$ Pseudoinverse) converges with stable 


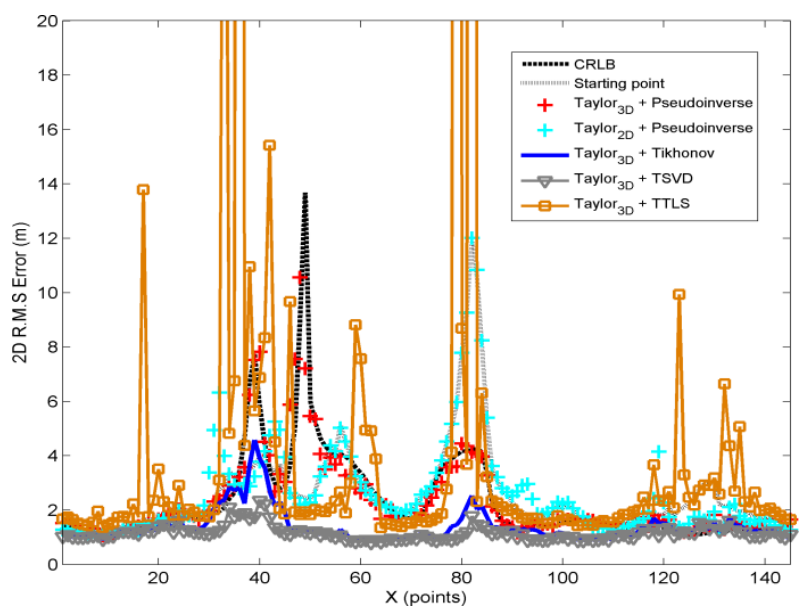

Fig. 8.17 2D R.M.S error for Linate system with starting point from projected version of Schau\&Robinson algorithm.

performance and reaches the CRLB. On the other hand, the horizontal accuracy provided by both Tikhonov and T-SVD based RLEs is below $5 \mathrm{~m}$ and for the most of points they improve the accuracy provided by the closed form algorithm, and by both version of Taylor (non-projected and projected). Additionally, for this simulation it is observed that the accuracy provided by T-SVD is better than that provided by the Tikhonov regularization, but not much. For the case of T-TLS based RLE, it converges and presents good accuracy levels, but not as well as Tikhonov and T-SVD. Furthermore, it also presents some peaks of R.M.S error that are greater than the corresponding values of the closed form algorithm and of the both version of Taylor. The same behaviour is observed for the horizontal bias analysis, and, in practical terms, the overall performance provided by both Tikhonov and T-SVD based RLEs can be considered the same $(0.22 \mathrm{~m}$ of bias for Tikhonov and $0.12 \mathrm{~m}$ for the T-SVD). In this case, also the T-TLS based RLE presents a small average amount of bias. However, it is greater than that of the non-projected Taylor algorithm and, therefore, its implementation is not justified.

The results shown in Fig. 8.15 - Fig. 8.18 suggest that Tikhonov and TSVD based RLEs are useful to solve the ill-conditioning of the location problem for LAM. In this sense, these results also suggest that Tikhonov regularization allows a more robust and reliable location strategy, as it is capable of reaching good accuracy values even in the presence of pooraccurate starting points. 


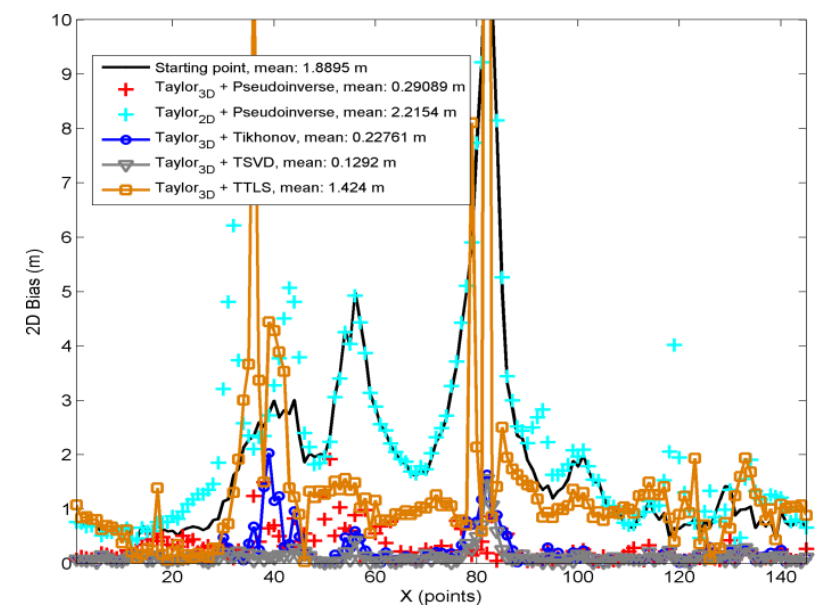

Fig. 8.18 2D bias for Linate system with starting point from projected version of Schau\&Robinson algorithm.

\section{Accuracy Analysis in the Presence of a Possible Failure of One Station (Fixed Starting Point)}

Another important aspect to be studied in the accuracy analysis is the behaviour of the overall system accuracy when one station fails. In this part, the possible failure of the station number 1 is simulated (see Fig. C.3), and the starting point is set to the centre of the system perimeter (see Fig. C.3). The failure is simulated simply by not using this station in the position estimation process. The 2D R.M.S error and bias analysis are shown in Fig. 8.19 and Fig. 8.20, respectively.

Under this situation, it is observed from Fig. 8.19 that with the failure of the station number 1, the accuracy provided by each algorithm decreases. Particularly, the non-projected version of Taylor does not converge for the most of points. The projected version of Taylor converges for the most of points but, it does not in those points within 117 - 140. It is because these points are in an area where the station number 1 significantly improves the HDOP of the system. In this sense, the localization problem for this scenario is ill-conditioned due to the starting point and geometry under the failure of that station. Moreover, also the T-SVD and T-TLS based RLEs does not converges for these points. Tikhonov based RLE is the only one that provides good accuracy values (below $6 \mathrm{~m}$ for the entire path) for the entire path under this situation. The same behaviour is present for the bias analysis, where Tikhonov provides an average amount of bias of only $2 \mathrm{~m}$. In other words, Tikhonov is the only method that allows obtaining the minimum bias under this situation: failure of one station and poor-accurate starting point. 


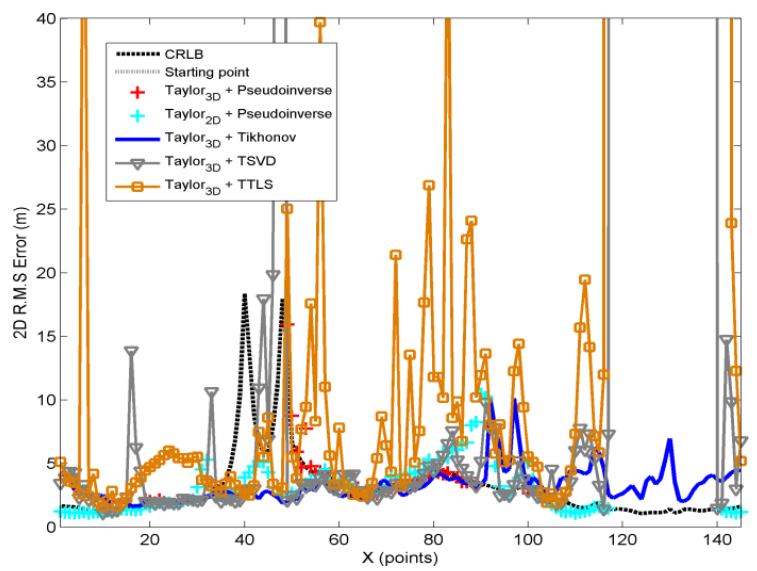

Fig. 8.19 2D R.M.S error for Linate system with a fixed starting point: failure of station 1.

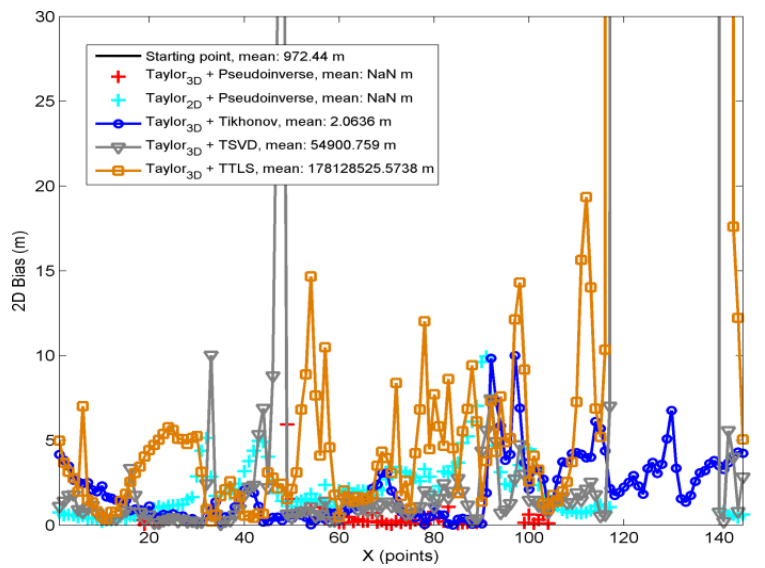

Fig. 8.20 2D bias for Linate system with a fixed starting point: failure of station 1

Accuracy Analysis in the Presence of a Possible Failure of One Station (Starting Point provided by Schau \& Robinson Algorithm)

In this part, we also perform the accuracy analysis under the failure of station number 1 but, in this case, the starting point is obtained by the projected version of Schau \& Robinson algorithm. The corresponding results for the 2D R.M.S error and bias are shown in Fig. 8.21 and Fig. 8.22 , respectively.

For this simulation, it is observed that although the classical nonprojected version of Taylor converges for an important number of points, it still does not provide accurate position data for other ones. In this case, the projected version of Taylor provides accurate results but, they are not as 


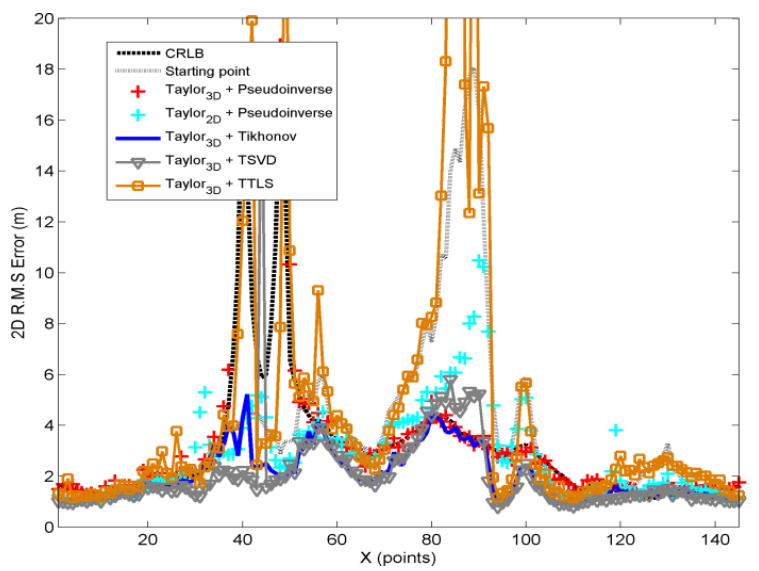

Fig. 8.21 2D R.M.S error for Linate system with starting point from projected version of Schau\&Robinson algorithm: failure of station 1.

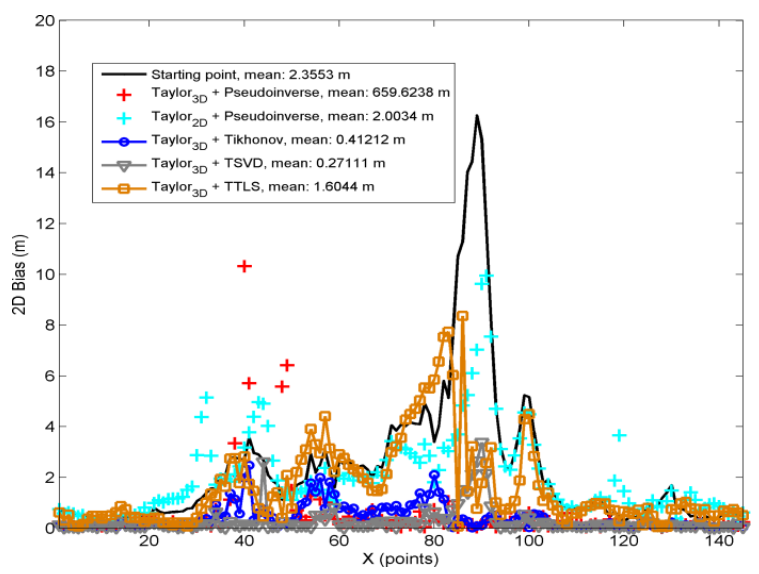

Fig. 8.22 2D bias for Linate system with starting point from projected version of Schau\&Robinson algorithm: failure of station 1.

well as those provided by Tikhonov and T-SVD based RLEs. Thus, it is also observed that Tikhonov and T-SVD based RLEs provide approximately the same overall accuracy performance. For the horizontal accuracy both algorithms improve, in the most of the simulated path, the accuracy provided by the starting point (i.e., the one of Schau \& Robinson algorithm). Moreover, regarding to the bias of the estimator, Tikhonov provides an average amount of bias of $0.41 \mathrm{~m}$ and T-SVD of $0.27 \mathrm{~m}$, which in practical terms are the same. Finally, the T-TLS based RLE provides accurate results for the most of points, and provides an average amount of bias of $1.6 \mathrm{~m}$. However, it also presents some peaks that make its implementation not viable in the situations of the failure of stations. 
From these results, we indentify some important conclusions for the application of RLEs for LAM operations. For those situations where a suitable number of stations are operational, both Tikhonov and T-SVD based RLEs provide good accuracy values and in all the cases improve the accuracy of the starting point. In this sense, when the quality of the starting point is relatively good (e.g., coming from the projected version of a closed form algorithm), the T-SVD provides (in a numerical sense) the best accuracy values (results in Fig. 8.17, Fig. 8.18, Fig. 8.21, and Fig. 8.22) but, if the quality of the starting point decreases, the best accuracy is achieved by the Tikhonov regularization (results in Fig. 8.15, Fig. 8.16, Fig. 8.19, and Fig. 8.20).

On the other hand, if some station (or stations) is not operational, Tikhonov regularization presents a more robust performance for the case of the fixed starting point (results in Fig. 8.19 and Fig. 8.20) and, for the case of the starting point coming from the projected version of a closed form algorithm, Tikhonov and T-SVD based RLEs provide the same performance. In the same sense, we conclude that the T-TLS based RLE, although it provides some good results, does not present significant improvements regarding to those of Tikhonov and T-SVD. Therefore, we do not include more this estimator in the remaining part of this thesis.

From the comments above, we also conclude that the application of both Tikhonov and T-SVD based RLEs provide significant accuracy improvements and that the Tikhonov regularization presents a more robust performance.

\subsection{Simulation and Results: Analysis of the Starting Point Quality}

In this part, the influence of the starting point quality, on the accuracy and convergence of the Taylor based algorithms (i.e., the non-projected version of Taylor solved by the Pseudoinverse matrix -classical solution-, the projected one, and the Tikhonov and T-SVD based RLEs) is studied. Also here, we only use the basic regularized estimators of Tikhonov (8.10) and T-SVD (8.38), i.e., without using the general localization strategy described in Fig. 8.3. As we have analyzed in $\S 8.5$, normally the Taylor based algorithms improve the accuracy of the starting point. Therefore, one of the objectives of this subsection is to know how robust is every regularized estimator proposed in this thesis, regarding to the quality (or accuracy) of the starting point. Likewise, the other objective of this analysis is to select the possible best option to provide a starting point for using along with our proposed general localization strategy in Fig. 8.3. Thus, first we shortly comment all the possible options to calculate/estimate a starting point and 
then, the most of them are tested for Linate (see §C.1.3) and Malpensa (see $\S$ C.1.2) scenarios.

The possible sources to calculate or estimate the starting point are summarized in Table 8.1.

Table 8.1 Possible sources for the starting point.

\begin{tabular}{|l|c|}
\hline \hline \multicolumn{1}{|c|}{ Source } & Notation \\
\hline Closed form algorithm & $\left(x_{\text {closed }}, y_{\text {closed }}, z_{\text {closed }}\right)$ \\
\hline Closed form + barometric altitude & $\left(x_{\text {closed }}, y_{\text {closed }}, z_{b}\right)$ \\
\hline Closed form + fixed altitude & $\left(x_{\text {closed }}, y_{\text {closed }}, z_{f}\right)$ \\
\hline Fixed point & $\left(x_{f}, y_{f}, z_{f}\right)$ \\
\hline Projected Closed form + barometric altitude & $\left(x_{\text {closed } D,}, y_{\text {closed } 2 D}, z_{b}\right)$ \\
\hline Projected Closed form + fixed altitude & $\left(x_{\text {closed } 2 D}, y_{\text {closed } D D}, z_{f}\right)$ \\
\hline Position data in DF $4,5,17,20,21$ & $\left(x_{D F}, y_{D F}, z_{D F}\right)$ \\
\hline Position after a tracking algorithm & $\left(x_{\text {Track }}, y_{\text {Track }}, z_{\text {Track }}\right)$ \\
\hline \hline
\end{tabular}

The use of the different sources commented in Table 8.1 depends on the application, i.e., LAM or WAM. For example, the fixed starting point is more useful for LAM applications, whilst the option of closed form algorithm + barometric altitude is more advisable for WAM applications or for an approach line (this part can be also considered as LAM). In this part, the two last options in Table 8.1 are not simulated. The option of the position data contained in the DF because we do not have any of this data, and the option corresponding to the tracking filter is tested with the real data experimentation in $\S 8.7$.

Another important aspect to use the sources for a starting point depends on the version of Taylor method used, i.e., the non-projected version or the projected one. For the projected version of Taylor it is necessary to set a starting point only with the horizontal coordinates, i.e., $(x, y)$.

\subsubsection{Feasible Options for LAM Operations}

The feasible options for LAM applications are shown in Fig. 8.23 and Fig. 8.24 for the non-projected version of Taylor method and for the projected one respectively.

The regularization methods have been not implemented for the projected version of Taylor because this problem is generally well-conditioned. Another reason to avoid the use of the regularization methods along with the Taylor ${ }_{2 \mathrm{D}}$ algorithm is the amount of bias introduced by this particular strategy can be large, i.e., the bias due to the projected plus the bias due the regularization method. 


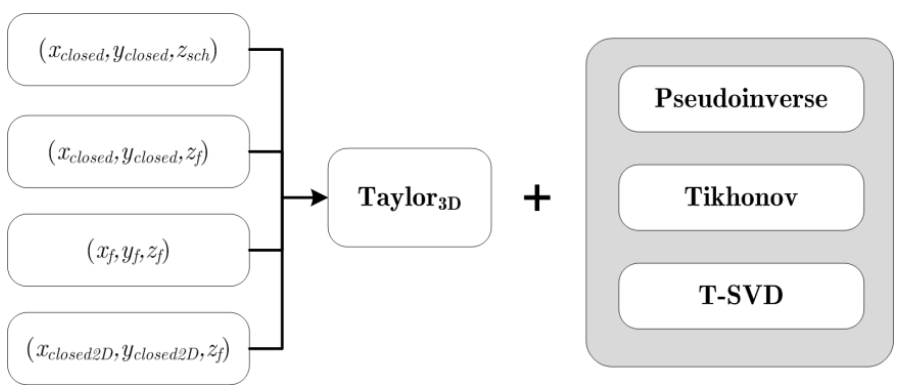

Fig. 8.23 Feasible options for the starting point in LAM operations for the non-projected version of Taylor based algorithms.

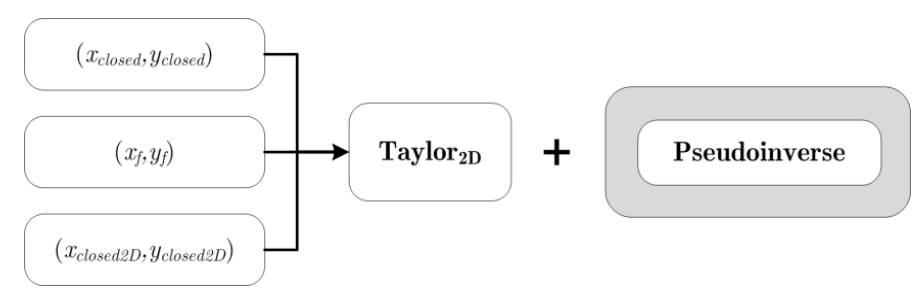

Fig. 8.24 Feasible options for the starting point in LAM operations for the projected version of Taylor based algorithms.

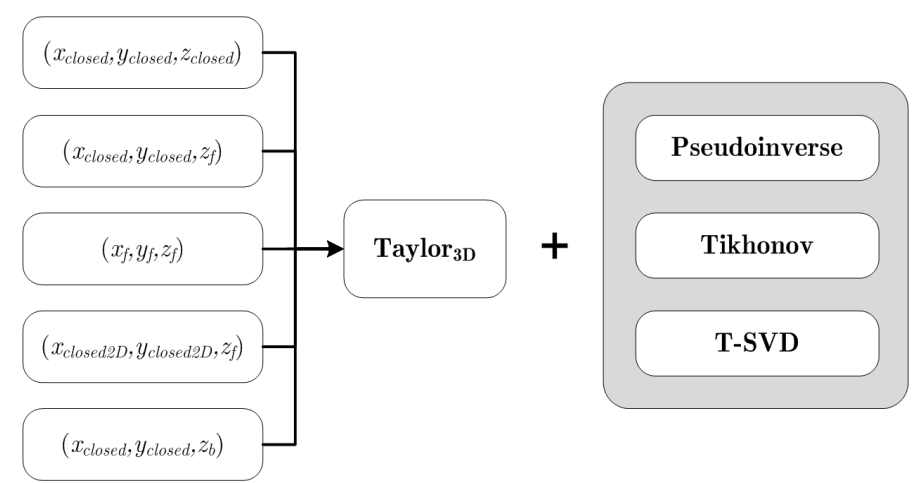

Fig. 8.25 Feasible options for the starting point in WAM operations for the non-projected version of Taylor based algorithms.

\subsubsection{Feasible Options for WAM Operations}

The feasible options to estimate the starting point for WAM operations are shown in Fig. 8.25. The use of the projected version of Taylor method is normally not advisable to be used for WAM, due to the amount of bias that it introduces. However, we also validate this option for providing the starting point. 


\subsubsection{Accuracy Analysis for LAM Operations}

For the analysis of the LAM accuracy -surface movements- regarding to the starting point it has been simulated all the possible combinations shown in Fig. 8.23 and Fig. 8.24 for the Linate scenario. For the options including a closed form algorithm we use the Schau \& Robinson (see $\$ 7.3 .4$ ) in the nonprojected and projected versions.

The regularization parameters used in these simulations are the same of the previous section for both Tikhonov and T-SVD based RLEs.

\section{Starting Point Provided by the Schau ${ }_{3 D}$ Algorihm}

The results for this simulation are shown in Fig. 8.26 and Fig. 8.27 for the 2D R.M.S error and 2D bias respectively. Then, Fig. 8.28 and Fig. 8.29 show the same parameters but for a simulation of the possible failure of the station number 1 .

For the first simulation (Fig. 8.26 and Fig. 8.27) we can observe that the starting point has a good accuracy and, in this way, it allows to the

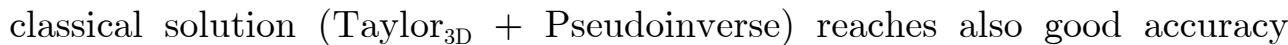
values. The Taylor $2 \mathrm{D}+$ Pseudoinverse solution also converges over the entire simulated path but, it adds an amount of bias that is of approximately $2 \mathrm{~m}$. Regarding to the RLEs solutions, Taylor $_{3 \mathrm{D}}+$ Tikhonov and Taylor $_{3 \mathrm{D}}+$ T-SVD provides good accuracy values. The Tikhonov solution reaches the same accuracy than the classical solution (i.e., the solution with minimum 2-norm or the minimum biased solution) and the TSVD solution introduces a bigger bias that is approximately of $2 \mathrm{~m}$.

On the other hand, for the simulation of a failure of station 1 (results in Fig. 8.28 and Fig. 8.29), the starting point has good accuracy values but the classical solution diverges with big errors in some parts of the simulated path. The Taylor ${ }_{2 \mathrm{D}}+$ Pseudoinverse solution provides good accuracy values and improves the accuracy of the starting point. Finally, the Tikhonov based RLE solution provides the best performance in this case with an average bias of $1.8 \mathrm{~m}$, and the T-SVD based one also provides good accuracy values but it does not improve the starting point accuracy, instead, this solution introduces bigger errors than that of the starting point. 


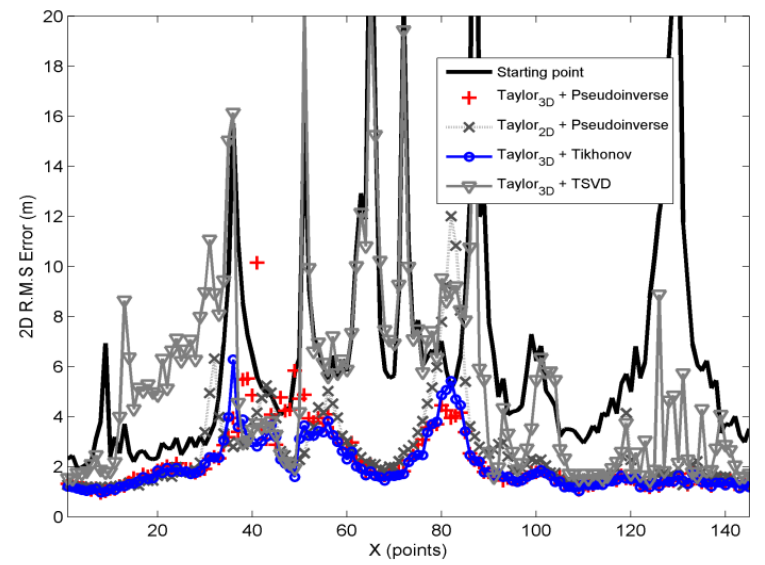

Fig. 8.26 2D R.M.S error with starting point provided by $\mathrm{Schau}_{3 \mathrm{D}}$.

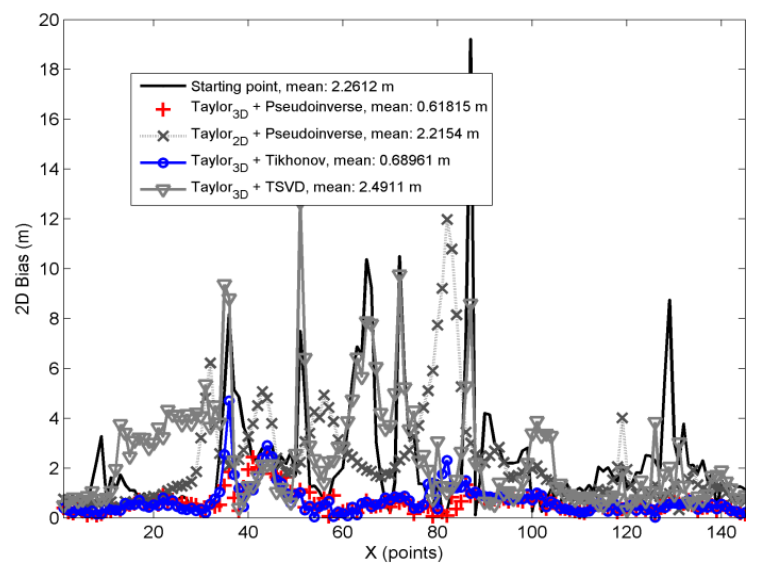

Fig. 8.27 2D bias with starting point provided by $\mathrm{Schau}_{3 \mathrm{D}}$.

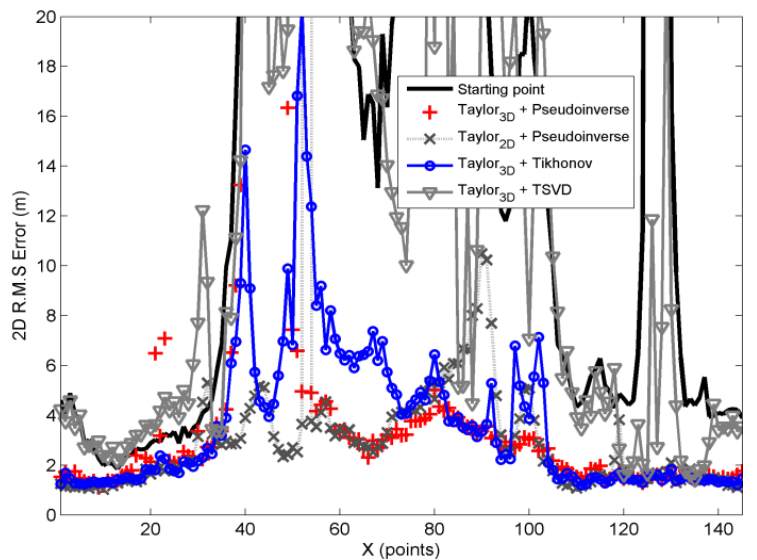

Fig. 8.28 2D R.M.S error with starting point provided by $\mathrm{Schau}_{3 \mathrm{D}}$ : failure of station 1. 


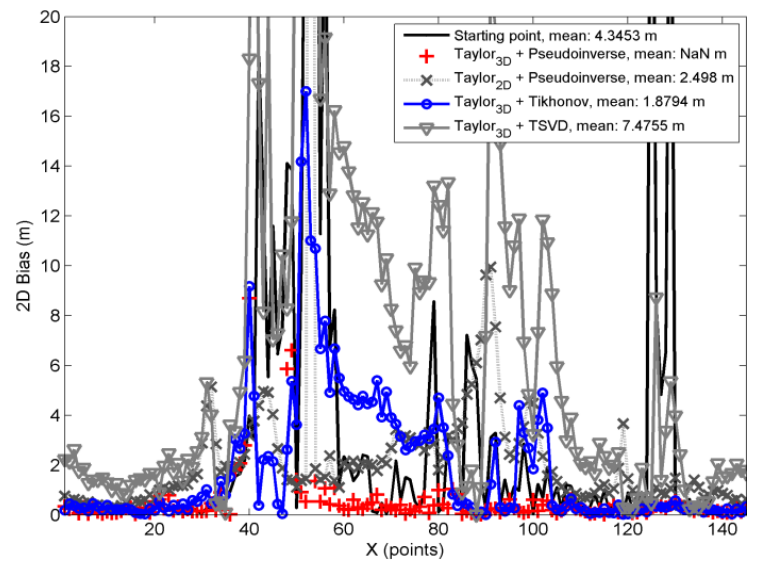

Fig. 8.29 2D bias with starting point provided by $\mathrm{Schau}_{3 \mathrm{D}}$ : failure of station 1 .

\section{Starting Point Provided by Schau ${ }_{3 D}$ Algorithm and a Fixed Altitude}

The results for this simulation are shown in Fig. 8.30 and Fig. 8.31 for the 2D R.M.S error and 2D bias respectively. Then, Fig. 8.32 and Fig. 8.33 show the same parameters but for a simulation of the possible failure of the station number 1. Furthermore, the fixed altitude is assumed equal to $7 \mathrm{~m}$.

For the first simulation (Fig. 8.30 and Fig. 8.31) we observe that the starting point has a good accuracy and it is improved by all the algorithms. The best performance in this case is achieved by the T-SVD based RLE solution but the classical and Tikhonov based one reach, in practical terms, the same performance. The solution provided by Taylor $_{2 \mathrm{D}}+$ Pseudoinverse introduces an amount of bias of approximately $2 \mathrm{~m}$. For this algorithm, the performance is the same as it does not use the height information. However, in the presence of a failure of station 1 (Fig. 8.32 and Fig. 8.33), the classical and T-SVD based solutions diverge with big errors. The Taylor ${ }_{2 \mathrm{D}}$ + Pseudoinverse also improves the accuracy of the starting point but the best performance is achieved by the Tikhonov based solution, which only introduces an amount of bias smaller than $1 \mathrm{~m}(0.6 \mathrm{~m})$. The latter again suggests that Tikhonov based RLE is a more fault-tolerant estimator than the remaining ones. 


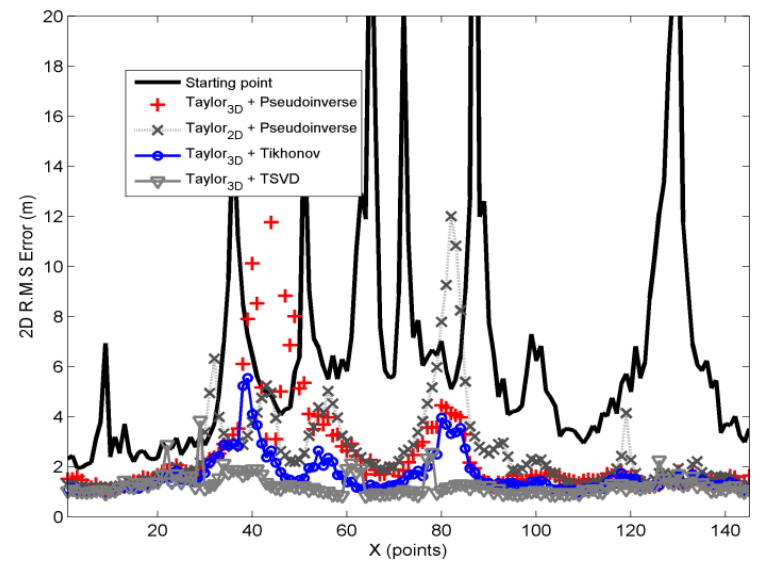

Fig. 8.30 2D R.M.S error with starting point provided by $S_{c h a u}+$ fixed altitude.

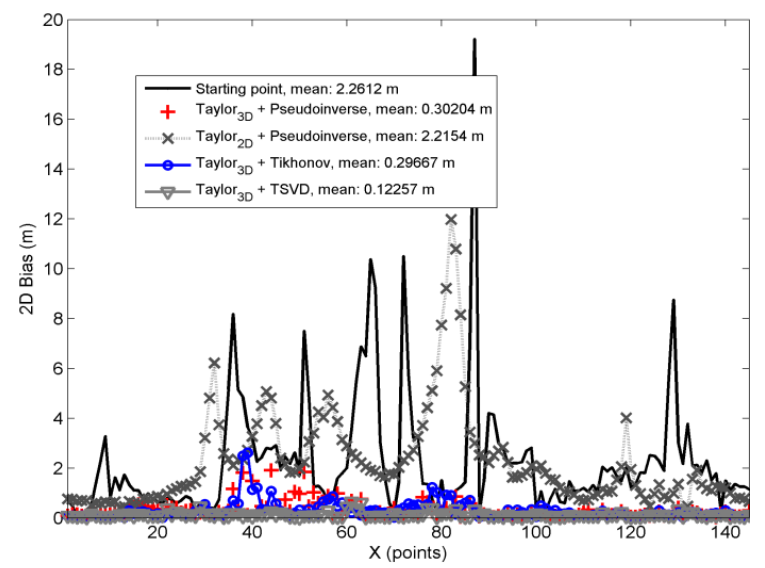

Fig. 8.31 2D bias with starting point provided by $\mathrm{Schau}_{3 \mathrm{D}}+$ fixed altitude.

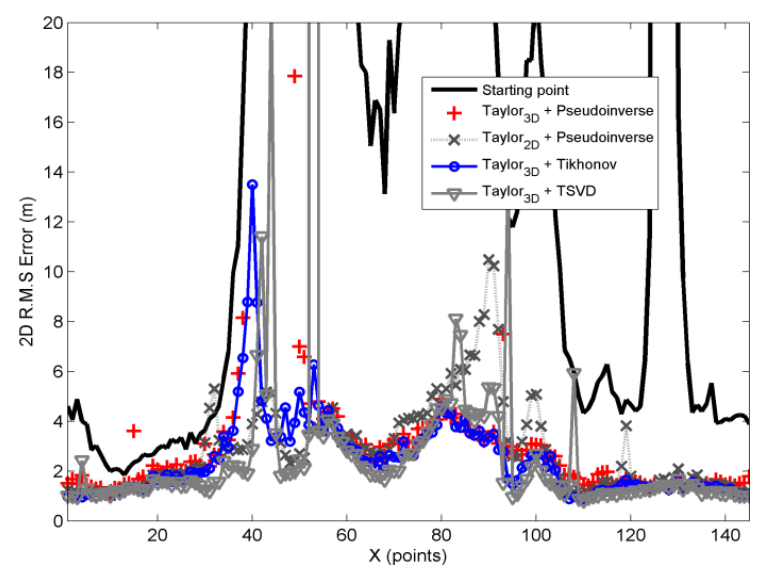

Fig. 8.32 2D R.M.S error with starting point provided by $S_{c h a u}$ D + fixed altitude: failure of station 1. 


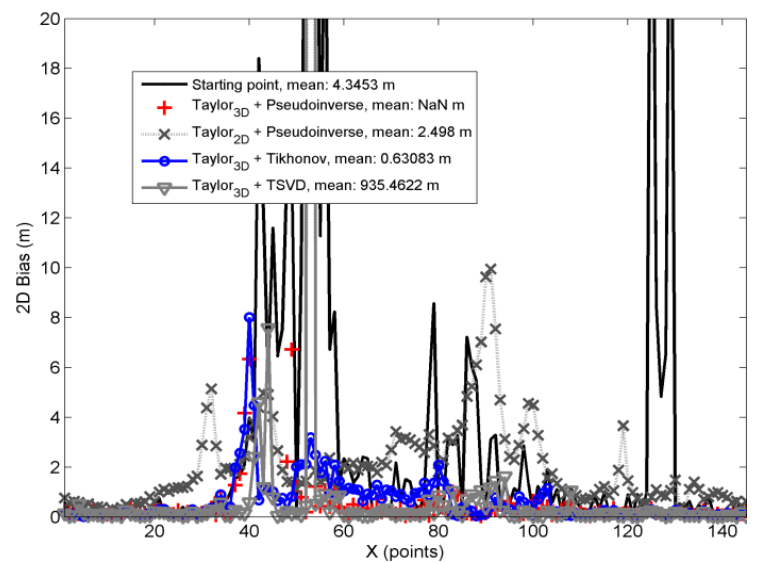

Fig. 8.33 2D bias with starting point provided by $\mathrm{Schau}_{3 \mathrm{D}}+$ fixed altitude: failure of station 1.

\section{Starting Point Provided by a Fixed Point}

The results for this simulation are shown in Fig. 8.34 and Fig. 8.35 for the 2D R.M.S error and 2D bias respectively. Then, Fig. 8.36 and Fig. 8.37 show the same parameters but for a simulation of the possible failure of the station number 1. Furthermore, the fixed point is that shown in Fig. C.3.

For the first simulation (Fig. 8.34 and Fig. 8.35) we observe that the starting point has, in average, a poor accuracy (an average bias of $972 \mathrm{~m}$ ). Firstly, for this simulation, the classical Taylor (non-projected) solution diverges with big errors for the most of simulated path. The Taylor ${ }_{2 \mathrm{D}}+$ Pseudoinverse solution provides good accuracy values and it introduces an amount of bias of approximately $2 \mathrm{~m}$. The Tikhonov based RLE solution provides the best performance (an average bias smaller than $0.5 \mathrm{~m}$ ) and the T-SVD based RLE one also provides good accuracy values but, for some parts of the simulated path, it introduces an amount of bias larger than 6 $\mathrm{m}$. Finally, in the presence of a possible failure of station 1 (Fig. 8.36 and Fig. 8.37), all the methods diverge to a large errors with the exception of the Tikhonov based RLE solution, which provides an average bias smaller than $1 \mathrm{~m}$ (see Fig. 8.37). 


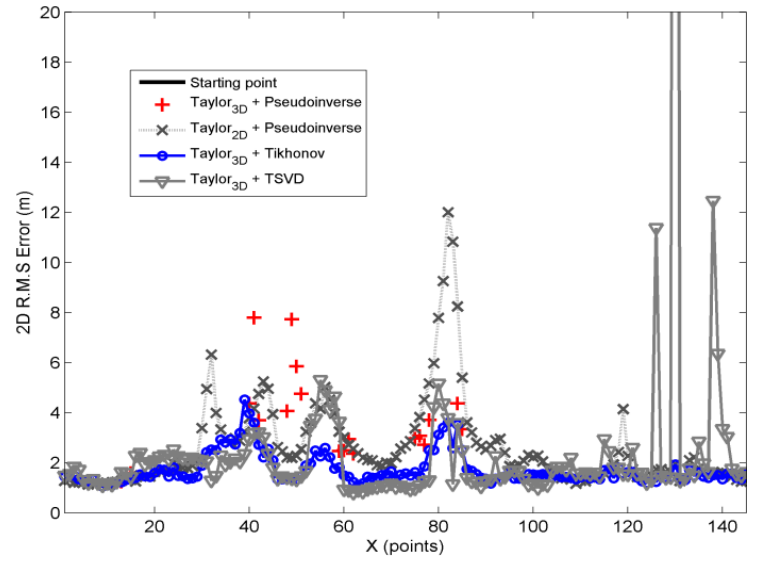

Fig. 8.34 2D R.M.S error with starting point set to a fixed coordinate.

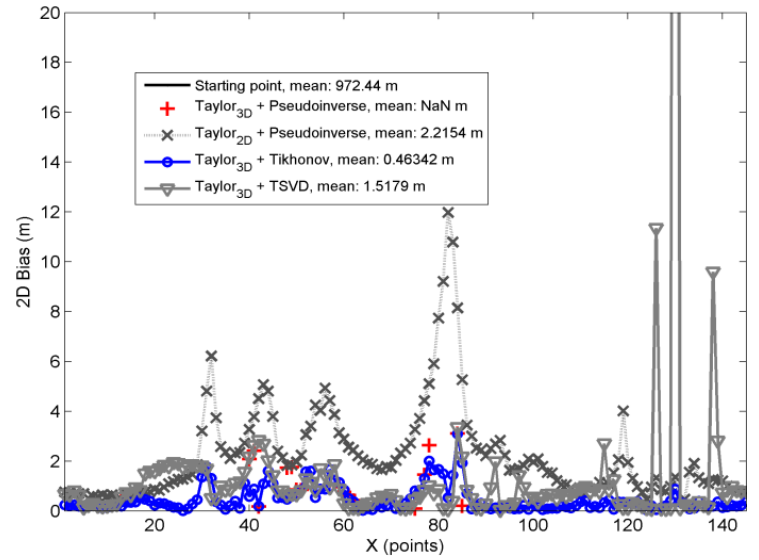

Fig. 8.35 2D bias with starting point set to a fixed coordinate.

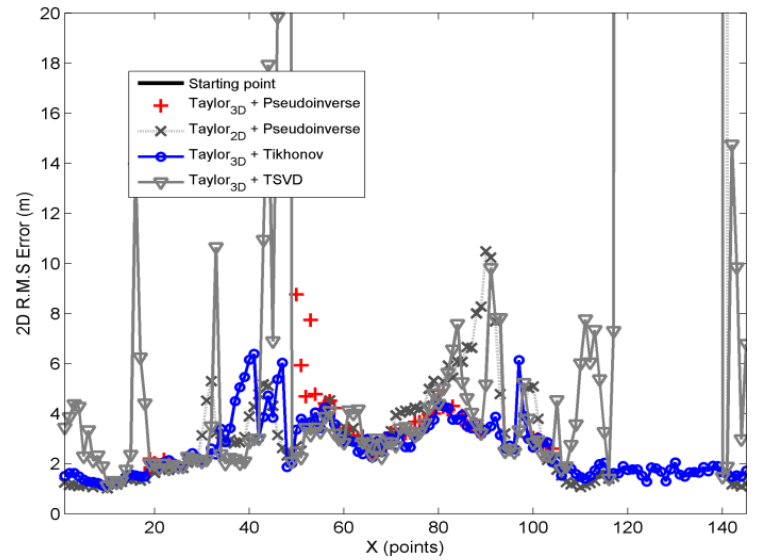

Fig. 8.36 2D R.M.S error with starting point set to a fixed coordinate: failure of station 1. 


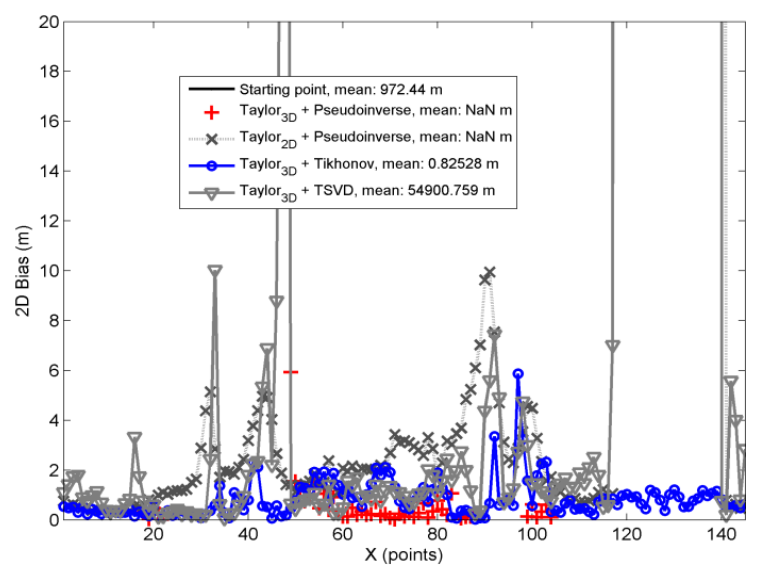

Fig. 8.37 2D bias with starting point set to a fixed coordinate: failure of station 1 .

\section{Starting Point Provided by Schau ${ }_{2 D}$ Algorithm and a Fixed Altitude}

The results for this simulation are shown in Fig. 8.38 and Fig. 8.39 for the 2D R.M.S error and 2D bias respectively. Then, Fig. 8.40 and Fig. 8.41 show the same parameters but for a simulation of the possible failure of the station number 1. Furthermore, the fixed altitude is assumed equal to $7 \mathrm{~m}$.

For the first simulation (Fig. 8.38 and Fig. 8.39) we observe that the starting point has a good accuracy and it is improved by all the algorithms. The best performance in this case is achieved by the T-SVD based RLE solution but, the classical Taylor and Tikhonov based one solutions reach, in practical terms, the same performance. The solution provided by Taylor $_{2 \mathrm{D}}+$ Pseudoinverse introduces an amount of bias of approximately 2 m. However, in the presence of a failure of station 1 (Fig. 8.40 and Fig. 8.41), the classical solution diverges with errors bigger than $100 \mathrm{~m}$. The Taylor $_{2 \mathrm{D}}+$ Pseudoinverse also improves the accuracy of the starting point under this situation but, the best performance is achieved by the Tikhonov and T-SVD based RLEs solutions, which only introduce an amount of bias smaller than $1 \mathrm{~m}$. 


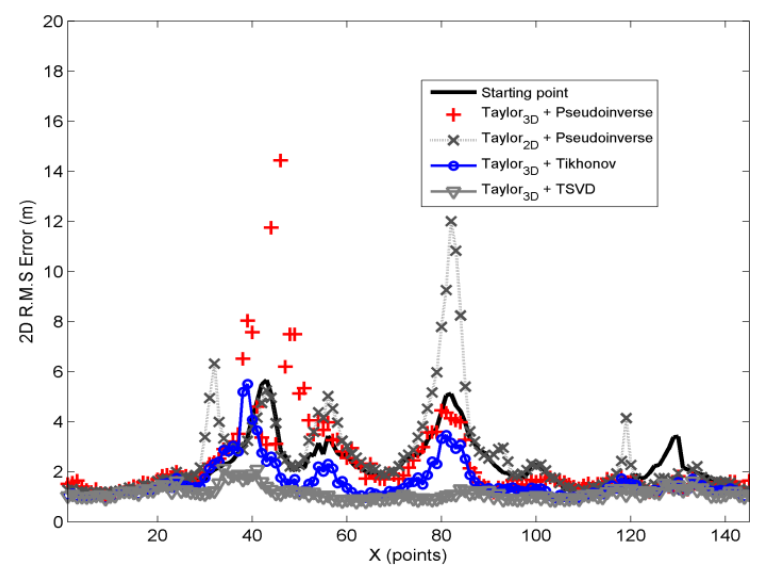

Fig. 8.38 2D R.M.S error with starting point provided by $\mathrm{Schau}_{2 \mathrm{D}}+$ fixed altitude.

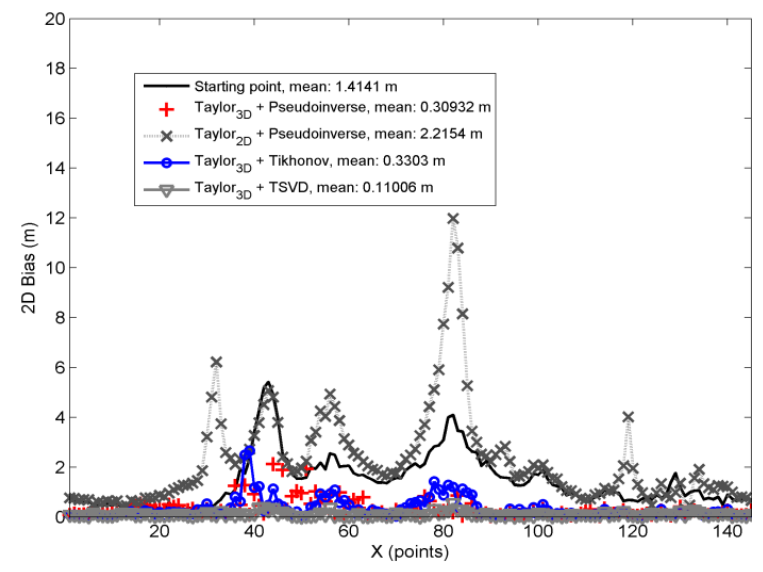

Fig. 8.39 2D bias with starting point provided by $\mathrm{Schau}_{2 \mathrm{D}}+$ fixed altitude.

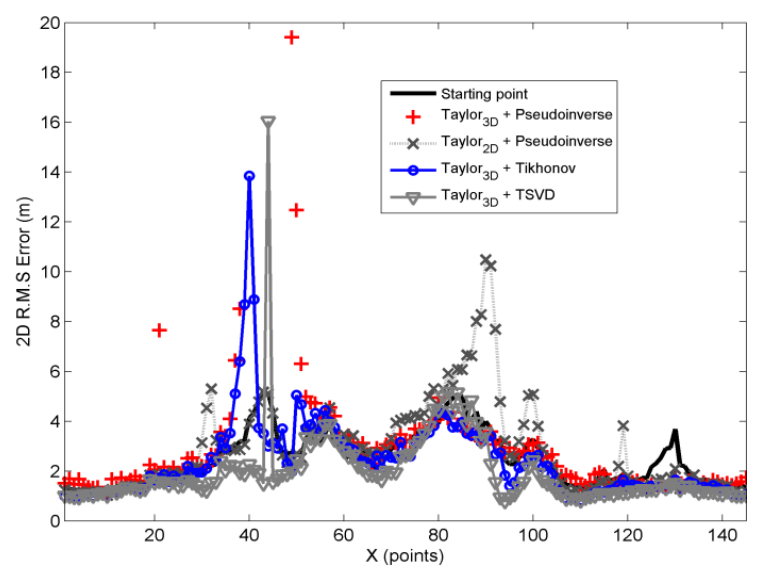

Fig. 8.40 2D R.M.S error with starting point provided by $\mathrm{Schau}_{2 \mathrm{D}}+$ fixed altitude: failure of station 1. 


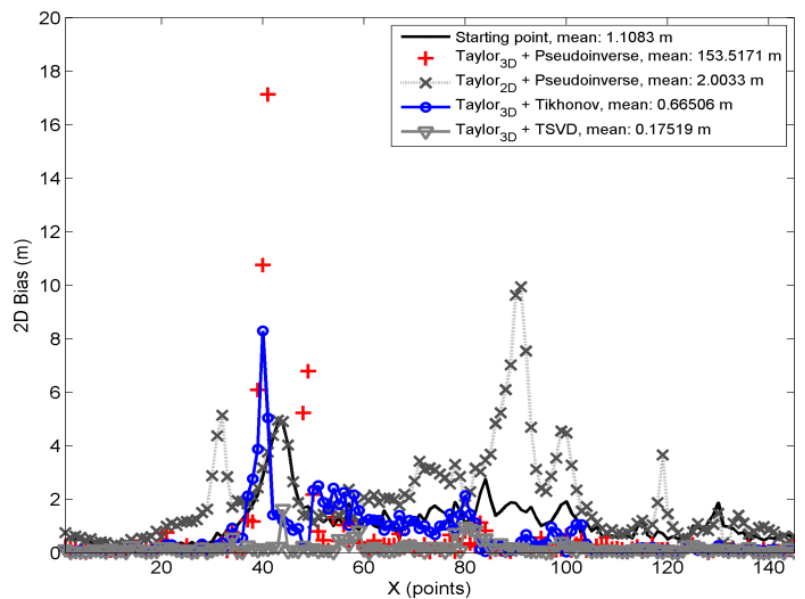

Fig. 8.41 2D bias with starting point provided by $\mathrm{Schau}_{2 \mathrm{D}}+$ fixed altitude: failure of station 1.

\section{Numerical Analysis}

In the following tables, we show the mean of the $2 \mathrm{D}$ bias and $2 \mathrm{D}$ R.M.S error for every algorithm and every starting point source, for the entire simulated path. Table 8.2 and Table 8.3 show the mean values for the simulations with all the stations operative and, Table 8.4 and Table 8.5 show the mean values for the simulations with the failure of station 1 . From these numerical results, we observe that the use of Taylor $_{3 \mathrm{D}}$ along with the Tikhonov regularization (i.e., the Tikhonov based RLE) provides good accuracy values for all the possibilities.

Table 8.2 Mean of the 2D bias over the simulated path of Linate system. Values given in meters.

\begin{tabular}{|c|c|c|c|c|c|}
\hline Algorithm & $\begin{array}{c}\text { Starting } \\
\text { Source }\end{array}$ & $\left(x_{s c h}, y_{s c h}, z_{s c h}\right)$ & $\left(x_{s c h}, y_{s c h}, z_{f}\right)$ & $\left(x_{f}, y_{f}, z_{f}\right)$ & $\left(x_{s c h 2 D}, y_{s c h 2 D}, z_{f}\right)$ \\
\hline \multicolumn{2}{|c|}{ Starting point } & 2.26 & 2.26 & 972.44 & 1.41 \\
\hline \multicolumn{2}{|c|}{$\begin{array}{c}\text { Taylor }_{3 \mathrm{D}}+ \\
\text { Pseudoinverse }\end{array}$} & 0.61 & 0.30 & $\mathrm{NaN}$ & 0.30 \\
\hline \multicolumn{2}{|c|}{$\begin{array}{c}\text { Taylor }_{2 \mathrm{D}}+ \\
\text { Pseudoinverse }\end{array}$} & 2.21 & 2.21 & 2.21 & 2.21 \\
\hline \multicolumn{2}{|c|}{ Talor $_{3 \mathrm{D}}+$ Tikhonov } & 0.68 & 0.29 & 0.46 & 0.33 \\
\hline \multicolumn{2}{|c|}{ Taylor $_{3 \mathrm{D}}+\mathrm{T}-\mathrm{SVD}$} & 2.49 & 0.12 & 1.51 & 0.11 \\
\hline
\end{tabular}


Table 8.3 Mean of the 2D R.M.S error over the simulated path of Linate system. Values given in meters.

\begin{tabular}{|c|c|c|c|c|c|}
\hline \hline Algorithm & $\begin{array}{c}\text { Starting } \\
\text { Source }\end{array}$ & $\left(\boldsymbol{x}_{\text {sch }}, \boldsymbol{y}_{\text {sch }}, \boldsymbol{z}_{\text {sch }}\right)$ & $\left(\boldsymbol{x}_{\boldsymbol{s c h},}, \boldsymbol{y}_{\text {sch }}, \boldsymbol{z}_{f}\right)$ & $\left(\boldsymbol{x}_{f}, \boldsymbol{y}_{f}, \boldsymbol{z}_{f}\right)$ & $\left(\boldsymbol{x}_{\text {sch } 2 \text { D }}, \boldsymbol{y}_{\text {sch } 2 \text { D }}, \boldsymbol{z}_{f}\right)$ \\
\hline Starting point & 6.54 & 6.54 & 972.4 & 2.21 \\
\hline $\begin{array}{c}\text { Taylor } \\
\text { Ps }\end{array}$ & 2.25 & 2.42 & NaN & 2.42 \\
\hline $\begin{array}{c}\text { Taylor } \\
\text { Pseudoinverse }\end{array}$ & 2.60 & 2.60 & 2.60 & 2.60 \\
\hline Talor $_{3 \mathrm{D}}+$ Tikhonov & 2.03 & 1.65 & 1.72 & 1.60 \\
\hline Taylor $_{3 \mathrm{D}}+$ T-SVD & 5.32 & 1.25 & 2.61 & 1.14 \\
\hline \hline
\end{tabular}

Table 8.4 Mean of the 2D bias over the simulated path of Linate system: failure of station 1. Values given in meters.

\begin{tabular}{|c|c|c|c|c|c|}
\hline \hline Algorithm & $\begin{array}{c}\text { Starting } \\
\text { Source }\end{array}$ & $\left(\boldsymbol{x}_{\boldsymbol{s c h}}, \boldsymbol{y}_{\boldsymbol{s c h}}, \boldsymbol{z}_{\text {sch }}\right)$ & $\left(\boldsymbol{x}_{\boldsymbol{s c h},}, \boldsymbol{y}_{\text {sch }}, \boldsymbol{z}_{f}\right)$ & $\left(\boldsymbol{x}_{f}, \boldsymbol{y}_{f}, \boldsymbol{z}_{f}\right)$ & $\left(\boldsymbol{x}_{\text {sch } 2 \boldsymbol{D}}, \boldsymbol{y}_{\text {sch } 2 \boldsymbol{D}}, \boldsymbol{z}_{f}\right)$ \\
\hline Starting point & 4.34 & 4.34 & 972.44 & 1.10 \\
\hline $\begin{array}{c}\text { Taylor } \\
\text { 3D }\end{array}$ & $\mathrm{NaN}$ & $\mathrm{NaN}$ & $\mathrm{NaN}$ & 153.51 \\
\hline $\begin{array}{c}\text { Pseudoinverse } \\
\text { Paylor }_{2 \mathrm{D}}+\end{array}$ & 2.49 & 2.49 & $\mathrm{NaN}$ & 2.00 \\
\hline Talor $_{\text {3D }}+$ Tikhonov & 1.87 & 0.63 & 0.82 & 0.66 \\
\hline Taylor $_{3 \mathrm{D}}+$ T-SVD & 7.47 & 935.46 & 54900.75 & 0.17 \\
\hline \hline
\end{tabular}

Table 8.5 Mean of the 2D R.M.S error over the simulated path of Linate system: failure of station 1. Values given in meters.

\begin{tabular}{|c|c|c|c|c|c|}
\hline Algorithm & $\begin{array}{l}\text { Starting } \\
\text { Source }\end{array}$ & $\left(x_{s c h}, y_{s c h}, z_{s c h}\right)$ & $\left(x_{s c h}, y_{s c h}, z_{f}\right)$ & $\left(x_{f}, y_{f}, z_{f}\right)$ & $\left(x_{s c h 2 D}, y_{s c h 2 D}, z_{f}\right)$ \\
\hline \multicolumn{2}{|c|}{ Starting point } & 23.22 & 23.22 & 972.4 & 2.47 \\
\hline \multicolumn{2}{|c|}{$\begin{array}{c}\text { Taylor }_{3 \mathrm{D}}+ \\
\text { Pseudoinverse }\end{array}$} & $\mathrm{NaN}$ & $\mathrm{NaN}$ & $\mathrm{NaN}$ & 1537 \\
\hline \multicolumn{2}{|c|}{$\begin{array}{c}\text { Taylor }_{2 \mathrm{D}}+ \\
\text { Pseudoinverse }\end{array}$} & 6.50 & 6.50 & $\mathrm{NaN}$ & 2.87 \\
\hline \multicolumn{2}{|c|}{ Talor $_{3 \mathrm{D}}+$ Tikhonov } & 3.75 & 2.47 & 2.59 & 2.40 \\
\hline \multicolumn{2}{|c|}{ Taylor $_{3 \mathrm{D}}+\mathrm{T}-\mathrm{SVD}$} & 15.83 & 11780 & 54910 & 1.98 \\
\hline
\end{tabular}

\subsubsection{Accuracy Analysis for WAM Operations}

For the analysis of the WAM accuracy, regarding to the starting point, we simulate all the possible combinations shown in Fig. 8.25 over a takeoff line for the Malpensa scenario (see Fig. C.2). Furthermore, the regularization parameters used in these simulations are the same of $\S 8.5 .2$ for both 
Tikhonov and T-SVD based RLEs. Because the reduced number of stations (only four) for this scenario, we do not simulate the possible failure of one of them.

\section{Starting Point Provided by Schau ${ }_{3 D}$ Algorithm}

The results for this simulation are shown in Fig. 8.42, Fig. 8.43, and Fig. 8.44 for the $2 \mathrm{D}$ and vertical R.M.S errors, and for the 2D bias, respectively. As we have previously analyzed in $§ 8.5 .2$ this problem is ill-conditioned. For the horizontal accuracy, the starting point provided by the full version of Schau algorithm provides poor accuracy values for those points within 2-5 $\mathrm{km}$ and $17-20 \mathrm{~km}$; in the remaining points it provides good accuracy values (with R.M.S errors below $30 \mathrm{~m}$ ). In this scenario, the Taylor $_{3 \mathrm{D}}+$ Pseudoinverse solution does not converge for those points before $20 \mathrm{~km}$, as it is clearly shown in Fig. 8.42. The Taylor $_{2 \mathrm{D}}+$ Pseudoinverse solution provides good accuracy values until a distance of $20 \mathrm{~km}$ from the runway; after that, the amount of bias due to the horizontal projection significantly affects the overall performance of this algorithm (see Fig. 8.44). Finally, Tikhonov and T-SVD based RLEs solutions improve, over the entire takeoff line, the accuracy of the starting point. In this case, both methods present the same performance.

On the other hand, no improvement is found for the vertical accuracy. It is because the pertaining accuracy of the starting point is significantly poor regarding to the small number of available stations. In this case, all the Taylor $_{3 \mathrm{D}}$ based RLE reach exactly the vertical component of the starting point.

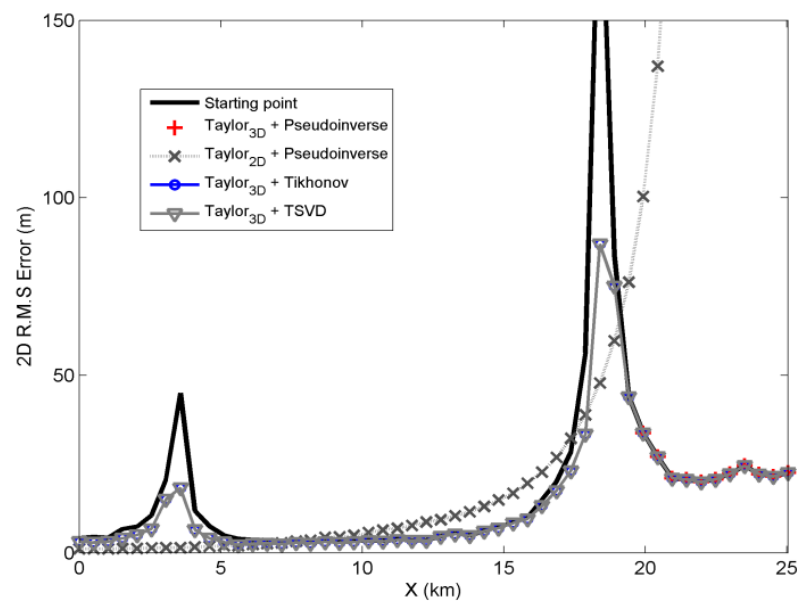

Fig. 8.42 2D R.M.S error with starting point provided by $\mathrm{Schau}_{3 \mathrm{D}}$. 


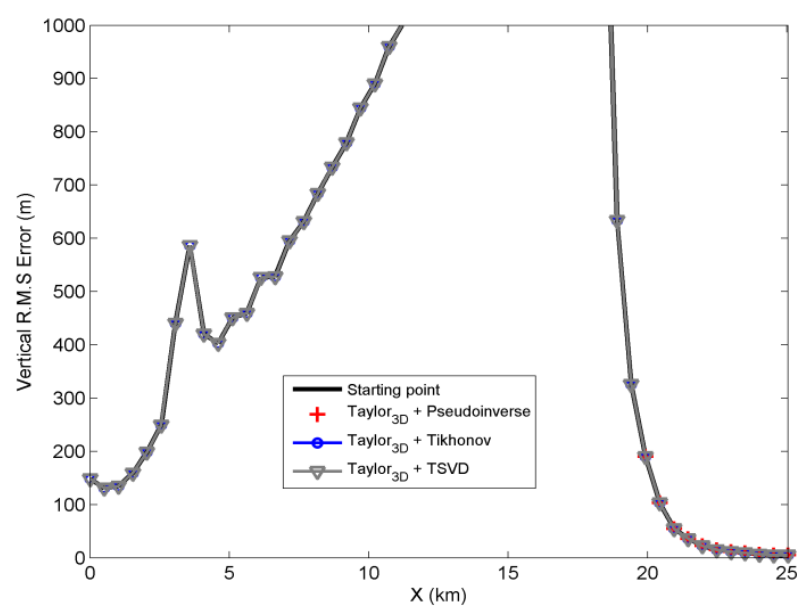

Fig. 8.43 Vertical R.M.S error with starting point provided by $\operatorname{Schau}_{3 \mathrm{D}}$.

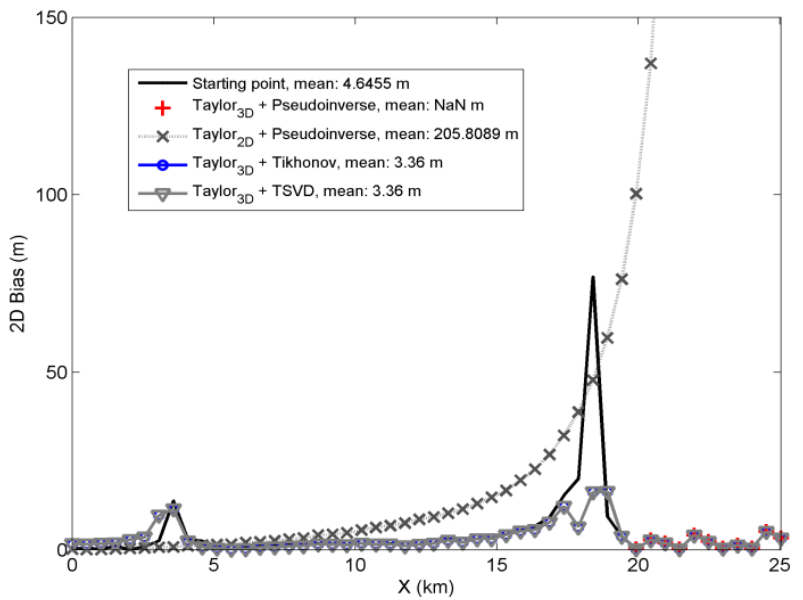

Fig. 8.44 2D bias with starting point provided by $\mathrm{Schau}_{3 \mathrm{D}}$.

\section{Starting Point Provided by Schau ${ }_{3 D}$ Algorithm and a Fixed Altitude}

The results for this simulation are shown in Fig. 8.45 and Fig. 8.46 for the $2 \mathrm{D}$ R.M.S error and 2D bias respectively. Because no improvement is achieved for the vertical position component, we do not show the corresponding R.M.S error for it. Furthermore, the fixed altitude is assumed equal to $25 \mathrm{~m}$.

In this case, we observe that the classical solution only converges for those points within 6-17 km. The Taylor $_{2 \mathrm{D}}+$ Pseudoinverse solution converges for all the points but it is affected by the large amount of bias for 


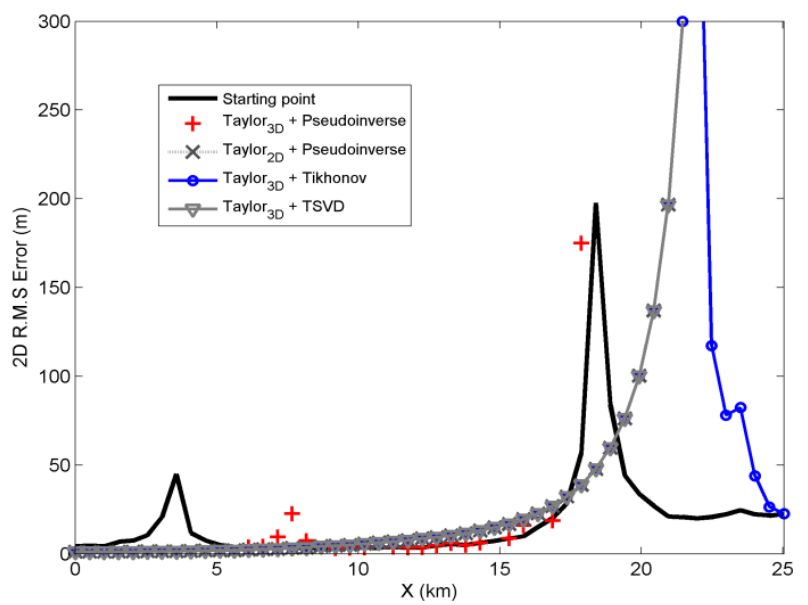

Fig. 8.45 2D R.M.S error with starting point provided by $\mathrm{Schau}_{3 \mathrm{D}}+$ fixed altitude.

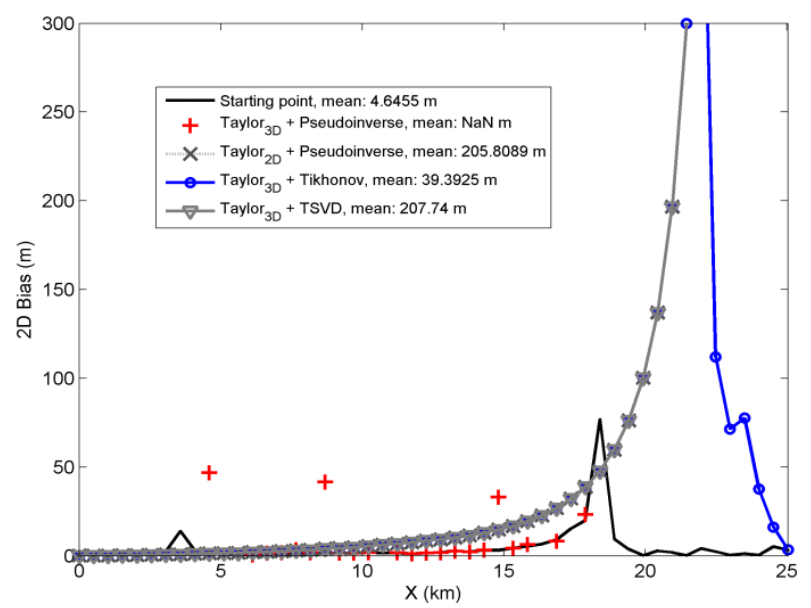

Fig. 8.46 2D bias with starting point provided by $\mathrm{Schau}_{3 \mathrm{D}}+$ fixed altitude.

points beyond $20 \mathrm{~km}$. The T-SVD based RLE solution provides the same performance than this. Finally, the Tikhonov based RLE solution provides better performance than the latter. However, after $20 \mathrm{~km}$ does not improve the accuracy of the starting point.

The results found in this simulation can be expected because also the regularization methods have a limit of improvement. In this way, the poor accuracy of the vertical component of the starting point and the small number of stations avoid a satisfactory convergence for those points beyond $20 \mathrm{~km}$, still using the regularization methods. 


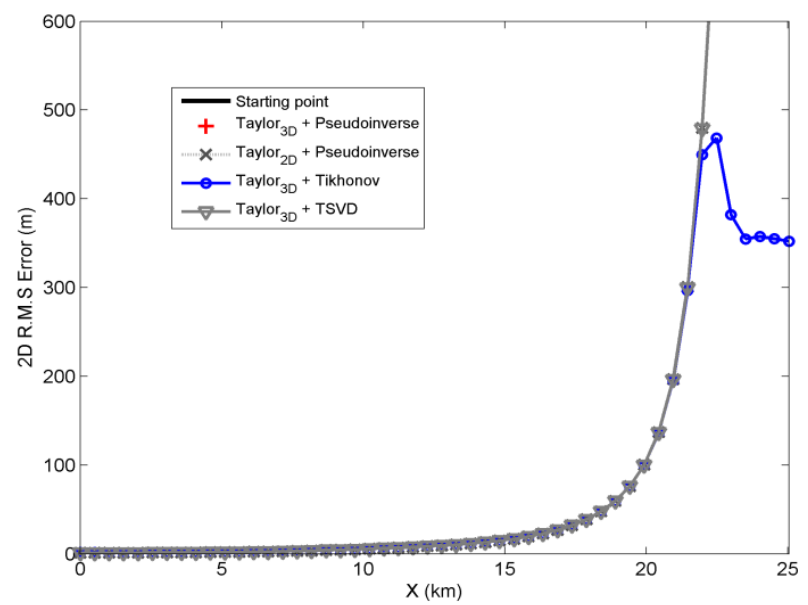

Fig. 8.47 2D R.M.S error with starting point set to a fixed coordinate.

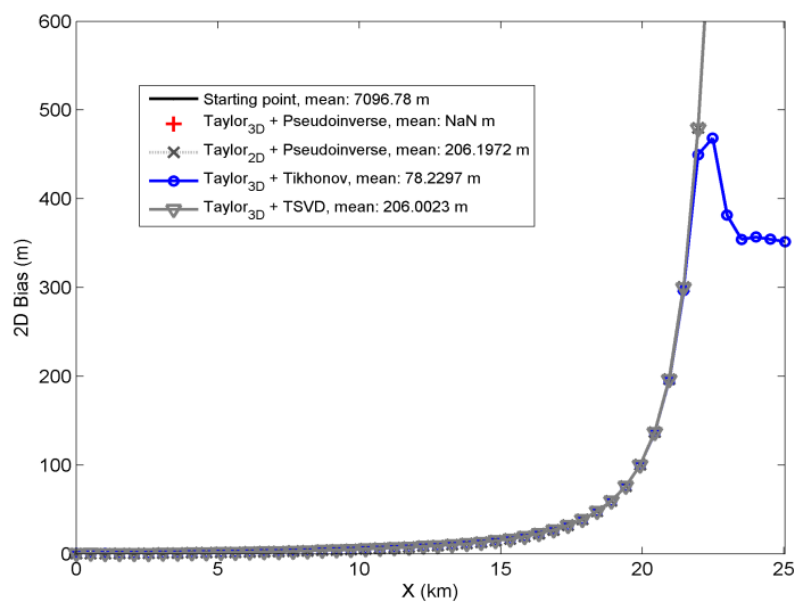

Fig. 8.48 2D bias with starting point set to a fixed coordinate.

\section{Starting Point Provided by a Fixed Point}

The results for this simulation are shown in Fig. 8.47 and Fig. 8.48 for the $2 \mathrm{D}$ R.M.S error and 2D bias respectively. Because no improvement is achieved for the vertical position component, we do not show the corresponding R.M.S error for it. Furthermore, the fixed altitude is assumed equal to $100 \mathrm{~m}$.

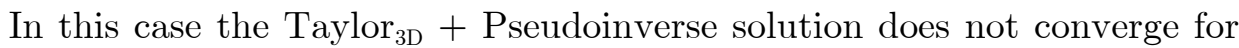
any point on the takeoff line. The remaining results and observations are significantly similar to those of the Fig. 8.45. For this reason, no more comments are provided for these results. 


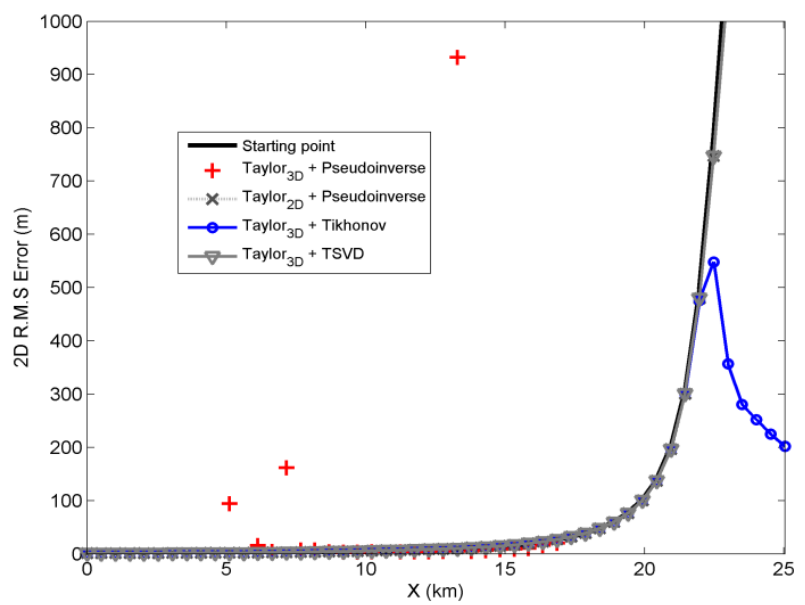

Fig. 8.49 2D R.M.S error with starting point provided by $\mathrm{Schau}_{2 \mathrm{D}}+$ fixed altitude.

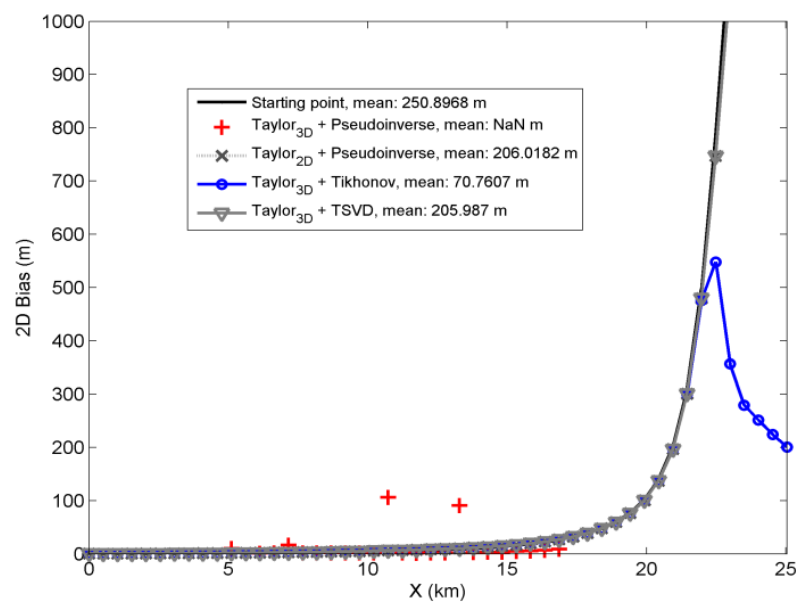

Fig. 8.50 2D bias with starting point provided by $\mathrm{Schau}_{2 \mathrm{D}}+$ fixed altitude.

\section{Starting Point Provided by Shcau ${ }_{2 D}$ Algorithm and a Fixed Altitude}

The results for this simulation are shown in Fig. 8.49 and Fig. 8.50 for the $2 \mathrm{D}$ R.M.S error and 2D bias respectively. Because no improvement is achieved for the vertical position component, we do not show the corresponding R.M.S error for it. Furthermore, the fixed altitude is assumed equal to $25 \mathrm{~m}$.

The results for this part are significantly similar to those in Fig. 8.45 and Fig. 8.47; for this reason no additional comments are done in this part. However, it is clear that under a geometrically ill-conditioned problem, the quality of the vertical component of the starting point plays an important 
role in the estimation of the corresponding horizontal ones. It is analyzed in the next simulation, where we use the barometer information.

\section{Starting Point Provided by Schau3D Algorithm and the Barometric Altitude}

The results for this simulation are shown in Fig. 8.51, Fig. 8.52, and Fig. 8.53 for the $2 \mathrm{D}$ and vertical R.M.S errors, and the $2 \mathrm{D}$ bias, respectively. Furthermore, the barometric altitude is simulated as $z_{b}=z_{\text {true }}+$ $\mathcal{N}(20 m, 5 m)$, where in this case $\mathcal{N}(20 m, 5 m)$ stands for a Gaussian distribution of $20 \mathrm{~m}$ of mean and standard deviation of $5 \mathrm{~m}$.

For this part, the altitude of the starting point is provided by the

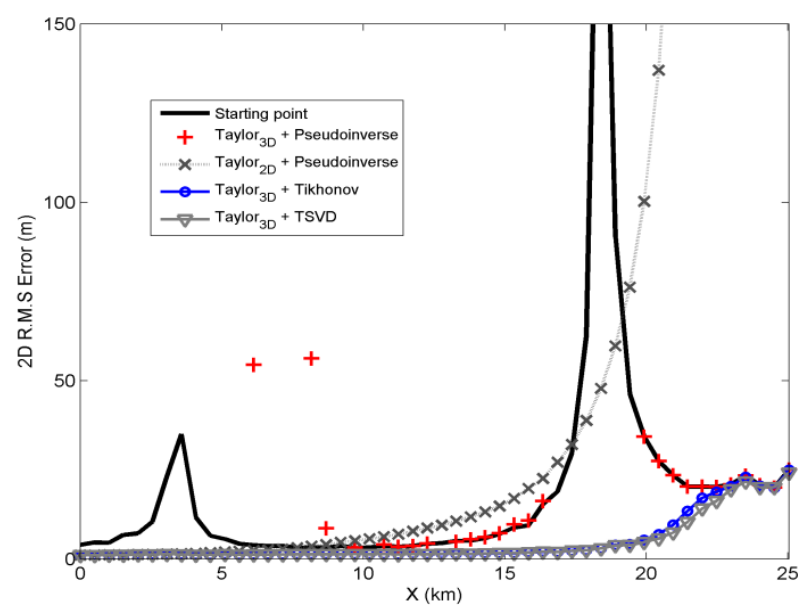

Fig. 8.51 2D R.M.S error with starting point provided by $\mathrm{Schau}_{3 \mathrm{D}}+$ barometric altitude.

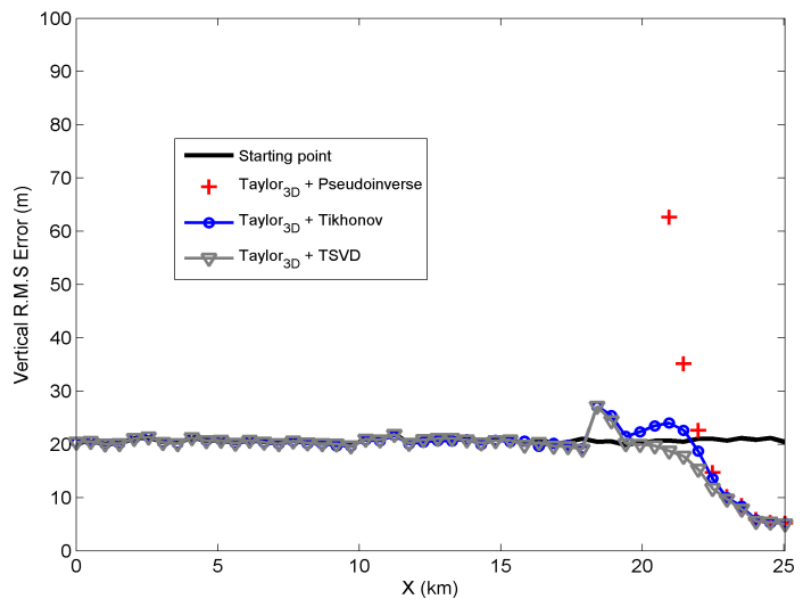

Fig. 8.52 Vertical R.M.S error with starting point provided by $\mathrm{Schau}_{3 \mathrm{D}}+$ barometric altitude. 


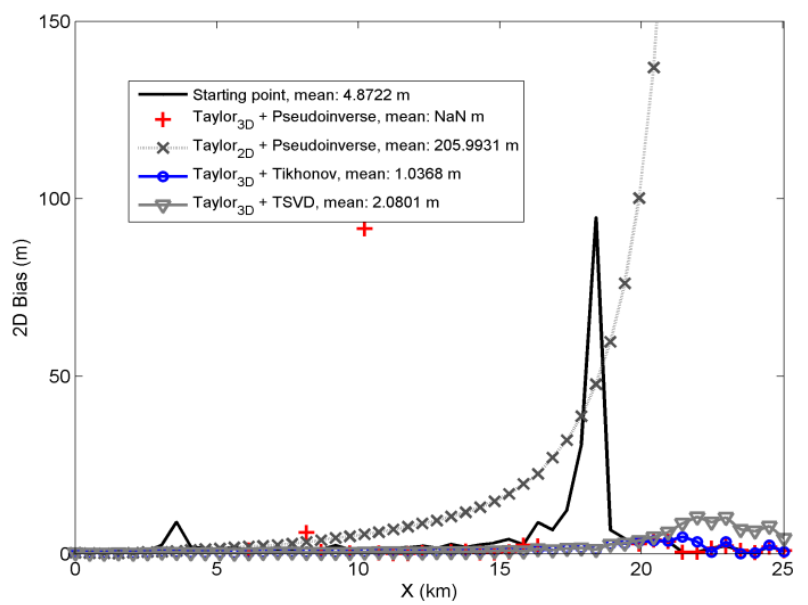

Fig. 8.53 2D bias with starting point provided by $\mathrm{Schau}_{3 \mathrm{D}}+$ barometric altitude.

barometric altitude. This fact allows the method to reach better accuracy values. In this case, the classical solution only converges for those points within $9-16 \mathrm{~km}$ and beyond $20 \mathrm{~km}$; in the remaining points it diverges to infinity errors due the geometrically ill-conditioning problem. The Taylor ${ }_{2 \mathrm{D}}$ + Pseudoinverse solution converges over the entire takeoff line but the amount of bias, due to the horizontal projection, is significantly large and avoid to this method improving the accuracy of the starting point, more for those points beyond $10 \mathrm{~km}$. Finally, the Tikhonov and T-SVD based RLEs solutions significantly improve the accuracy of the starting point over the entire takeoff line. In terms of the R.M.S error the Tikhonov and T-SVD based RLEs solutions present the same performance but, in the terms of the bias, the smallest one is obtained by the Tikhonov based solution (see Fig. $8.53)$.

Finally, regarding to the vertical accuracy, the classical solution provides good accuracy values only for those points beyond $20 \mathrm{~km}$ (where the problem is well-conditioned). The regularized solutions reach the same vertical component of the starting point until a distance of $20 \mathrm{~km}$ and, after that, they improve the accuracy of this component; i.e., the application of the regularization methods does not negatively affect the problem when it is well-conditioned.

\section{Numerical Analysis}

In the following tables, we show the mean of the $2 \mathrm{D}$ bias and 2D R.M.S error for every method and every source for the starting point, for the entire simulated path. Table 8.6 and Table 8.7 show the mean values for the simulations with all the stations operative. From these numerical results, we observe that the use of Tikhonov and T-SVD based RLEs allow 
providing good accuracy values for all the possibilities. Moreover, as we have previously analyzed, the Tikhonov based estimator is more faulttolerant and robust in the presence of starting point degradations.

From these results also we can conclude that the best option for providing a starting point for LAM operations is by using the $(x, y)$ components from a projected closed form algorithm along with a fixed height. We have particularly used the projected version of Schau \& Robinson algorithm, and useful results have been obtained. However, any of the remaining closed form algorithms, described and analyzed in $\S 7.3$ and $\$ 7.6$ respectively, could be used. On the other hand, for WAM operations, the best option for providing a starting point is by using the $(x, y)$ components from a non-projected closed form algorithm along with the information of altitude coming from the barometer. The use of barometric altitude is a realistic assumption as it is periodically sent by aircrafts in all the air spaces around the world [15]. Moreover, this altitude information is currently used for several ATC systems, like primary radars, secondary radars, and even MLAT systems, for directly inform to the air traffic controllers about the target height.

Table 8.6 Mean of the 2D bias over the entire takeoff line of Malpensa system. Values given in meters.

\begin{tabular}{|c|c|c|c|c|c|}
\hline \begin{tabular}{|l|l|} 
Algorithm & $\begin{array}{c}\text { Starting } \\
\text { Source }\end{array}$ \\
\end{tabular} & $\left(x_{s c h}, y_{s c h}, z_{s c h}\right)$ & $\left(x_{s c h}, y_{s c h}, z_{f}\right)$ & $\left(x_{f}, y_{f}, z_{f}\right)$ & $\left(x_{s \operatorname{ch} 2 D}, y_{s c h 2 D}, z_{f}\right)$ & $\left(x_{s c h}, y_{s c h}, z_{b}\right)$ \\
\hline Starting point & 4.64 & 4.64 & 7096.78 & 250.89 & 4.87 \\
\hline $\begin{array}{c}\text { Taylor }_{3 \mathrm{D}}+ \\
\text { Pseudoinverse }\end{array}$ & $\mathrm{NaN}$ & $\mathrm{NaN}$ & $\mathrm{NaN}$ & $\mathrm{NaN}$ & $\mathrm{NaN}$ \\
\hline $\begin{array}{c}\text { Taylor }_{2 \mathrm{D}}+ \\
\text { Pseudoinverse }\end{array}$ & 205.80 & 205.80 & 206.19 & 206.01 & 205.99 \\
\hline $\begin{array}{l}\text { Talor }_{3 \mathrm{D}}+ \\
\text { Tikhonov }\end{array}$ & 3.36 & 39.39 & 78.22 & 70.76 & 1.03 \\
\hline $\begin{array}{c}\text { Taylor }_{3 \mathrm{D}}+ \\
\text { T-SVD }\end{array}$ & 3.36 & 207.74 & 206.00 & 205.98 & 2.08 \\
\hline
\end{tabular}


Table 8.7 Mean of the 2D R.M.S error over the entire takeoff line of Malpensa system. Values given in meters.

\begin{tabular}{|c|c|c|c|c|c|c|}
\hline Algorithm & $\begin{array}{l}\text { Starting } \\
\text { Source }\end{array}$ & $\left(x_{s c h}, y_{s c h}, z_{s c h}\right)$ & $\left(x_{s c h}, y_{s c h}, z_{f}\right)$ & $\left(x_{f}, y_{f}, z_{f}\right)$ & $\left(x_{s c h 2 D}, y_{s c h 2 D}, z_{f}\right)$ & $\left(x_{s c h}, y_{s c h}, z_{b}\right)$ \\
\hline \multicolumn{2}{|c|}{ Starting point } & 18.49 & 18.49 & 7097 & 2512 & 19.78 \\
\hline \multicolumn{2}{|c|}{$\begin{array}{c}\text { Taylor }_{3 \mathrm{D}}+ \\
\text { Pseudoinverse }\end{array}$} & $\mathrm{NaN}$ & $\mathrm{NaN}$ & $\mathrm{NaN}$ & $\mathrm{NaN}$ & $\mathrm{NaN}$ \\
\hline \multicolumn{2}{|c|}{$\begin{array}{c}\text { Taylor }_{2 \mathrm{D}}+ \\
\text { Pseudoinverse }\end{array}$} & 206.10 & 206.10 & 206.4 & 206.3 & 206.2 \\
\hline \multicolumn{2}{|c|}{$\begin{array}{l}\text { Talor }_{3 \mathrm{D}}+ \\
\text { Tikhonov }\end{array}$} & 14.18 & 40.68 & 78.52 & 71.09 & 4.83 \\
\hline \multicolumn{2}{|c|}{$\begin{array}{c}\text { Taylor }_{3 \mathrm{D}}+ \\
\text { T-SVD }\end{array}$} & 14.18 & 208 & 206.3 & 206.2 & 4.51 \\
\hline
\end{tabular}

\subsection{Experimental With Real Data: General Localization Strategy}

To perform the experimental with real data, the company ERA A.S. has provided us of a record of TOA measurements of one of its operational system, the LAM system installed at Tallinn airport (Tallinn, Estonia). This system is intended for surface surveillance and is composed of fourteen receiving stations. The record of TOA measurements was taken through the entire airport surface following the requested procedures by the European regulatory bodies [1]. The record contains more than 4000 register (with an average period of $1 \mathrm{~s}$ ), where each register contains set of TOA measurements. From the information provided by the company, we know that these measurements are perturbed by multipath noise in some areas. Moreover, also the company above mentioned has provided us of highly accurate position reference data, which was simultaneously recorded with a GPS receiver with differential correction capabilities (DGPS). This data is used to evaluate the solutions accuracy of the RLEs and the general localization strategy proposed in this thesis. The system layout and the reference position data are depicted in Fig. C.4. Additionally, Fig. C.5 shows the LoS profile for this system, which shows for every register the number of stations that measure the TOA of the emitted Mode S signal.

The procedure to evaluate the accuracy of the solutions is exactly the same described in $\$ 7.7$. In this part we analyze the performance of the basic RLEs (i.e., the Tikhonov based estimator in (8.11) and the T-SVD based estimator in (8.39)) and the general localization strategy described in Fig. 8.3. Moreover, we also separately analyze the performance of the additional improvements proposed in $\$ 8.4 .1$, which allow us to clearly shown the significant accuracy increment that is obtained, also in the case of 
multipath noise. Regarding to the regularization parameters, we use the same as used for Linate scenario in $§ 8.5 .3$, as Tallinn system is obviously also a LAM system. The results for Tikhonov based RLE are shown in Fig. 8.54 and Fig. 8.55, for $x$ and $y$ coordinates, respectively, whilst for T-SVD based RLE are shown in Fig. 8.56 and Fig. 8.57. Each of these figures is equally divided in four subplots, which are organized in the same way from top to bottom: the first subplot shows the coordinate (either $x$ or $y$ ) as estimated only by the basic RLE (either Tikhonov in (8.11) or T-SVD in (8.39)), i.e., without the additional improvements proposed in $\S 8.4 .1$. The second subplot shows the estimated coordinate by using the RLE only with the additional improvement of measurement selection (SM). The third one is the estimated coordinate by using the RLE only with the additional improvement of solution selection (SS), and the last one shows the estimated coordinate by using the general localization strategy described in Fig. 8.3, i.e., by using the RLE along with the additional improvements of SM and SS. Also this last subplot shows the results of using a tracking algorithm (the basic Kalman Filter [114]) as described in the last step of Fig. 8.3. Finally, for all of these subplots the corresponding coordinate provided by the DGPS receiver is shown. The latter allow us to clearly observe the data dispersion. The numerical analysis is shown in Table 8.8 for the Tikhonov based RLE, and in Table 8.9 for the T-SVD one.

By looking the first subplot of each figure, we appreciate how the application of the basic RLE significantly improves the position data as provided by the classical solution of Taylor either in its non-projected version or in its projected one (compare with Fig. 7.27, Fig. 7.29, Fig. 7.31, and Fig. 7.33). Likewise, the successive subplots clearly show the accuracy improvement that is obtained in each of the general localization strategy steps, i.e., the accuracy improvement due to the SM, SS, and the combined use of both. In the last subplots, it is clear how the position data provided by the general localization strategy is very close to the reference position data (i.e., of the DGPS). The latter holds for both Tikhonov based strategy and T-SVD based one. Moreover, the small error peaks are corrected by the application of a simple tracking algorithm, as shown also in those last subplots.

Importantly to comment the significant improvement to the error peaks due to multipath that is obtained by the implementation of the SM and SS strategies. These improvements are evident by comparing the second and third subplots with the first one for each coordinate, and each RLE.

On the other hand, Table 8.8 and Table 8.9 show clearer the significant improvements provided by the RLEs proposed in this thesis, as the corresponding standard deviations and means are much smaller than those obtained by the classical localization algorithms in Table 7.6 and Table 7.7. Furthermore, the same holds for the PoL, as the values provided by the 
RLEs are greater than those ones for the classical localization algorithms. In this sense, we conclude the RLEs and the general localization strategy proposed in this thesis provide much better performance than the most established localization algorithms in the literature. Also, we consider important to comment that all the general conclusions obtained with the experimental with real data are fully in agreement with those ones obtained with the simulated scenarios in $\S 8.5$ and $\S 8.6$.

We have found through this experiment that the proposed general localization strategy, in practical terms, equivalently works with the Tikhonov based RLE or T-SVD based one. However, by comparing the fourth row of Table 8.8 for Tikhonov based RLE and the same one in Table 8.9, for T-SVD based one, we observe that Tikhonov provides performance parameters that are something better than the ones of T-SVD. Moreover, as we concluded in $\S 8.6$, Tikhonov based RLE is more robust under possible starting point accuracy degradations and possible failure of stations (the equivalent degradation of the quality of system geometry). Therefore, we recommend the preferable use of the general localization strategy with the Tikhonov based RLE rather than T-SVD based one, although obviously a previous analysis is always advisable.

The computational cost of implementing Tikhonov and T-SVD based RLE is exactly the same, as in both cases the SVD of the coefficient matrix must be calculated. As an example, the simulations presented in this thesis have been carried out in a personal computer of $1.3 \mathrm{GHz}$ of processor and 4 GB of RAM, and the average time of calculating one position with the general localization strategy of Fig. 8.3 was 8 ms (including the tracking algorithm), which is a time much smaller than the maximum delay of 250 ms, which is stipulated in EUROCAE MOPS ED-117 [1] for Mode S MLAT surface surveillance operations. 

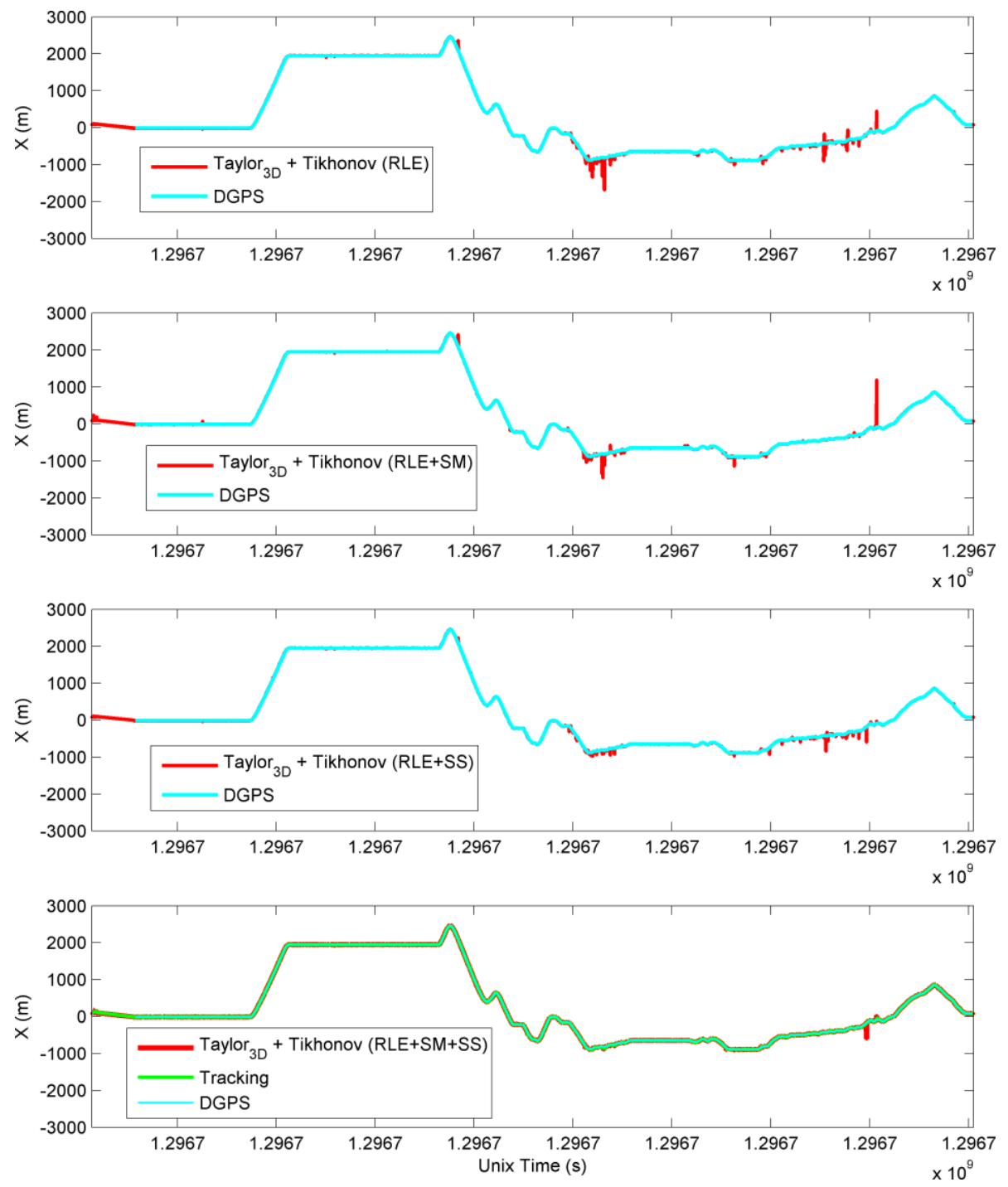

Fig. 8.54 X(t) for Tikhonov based RLE. 

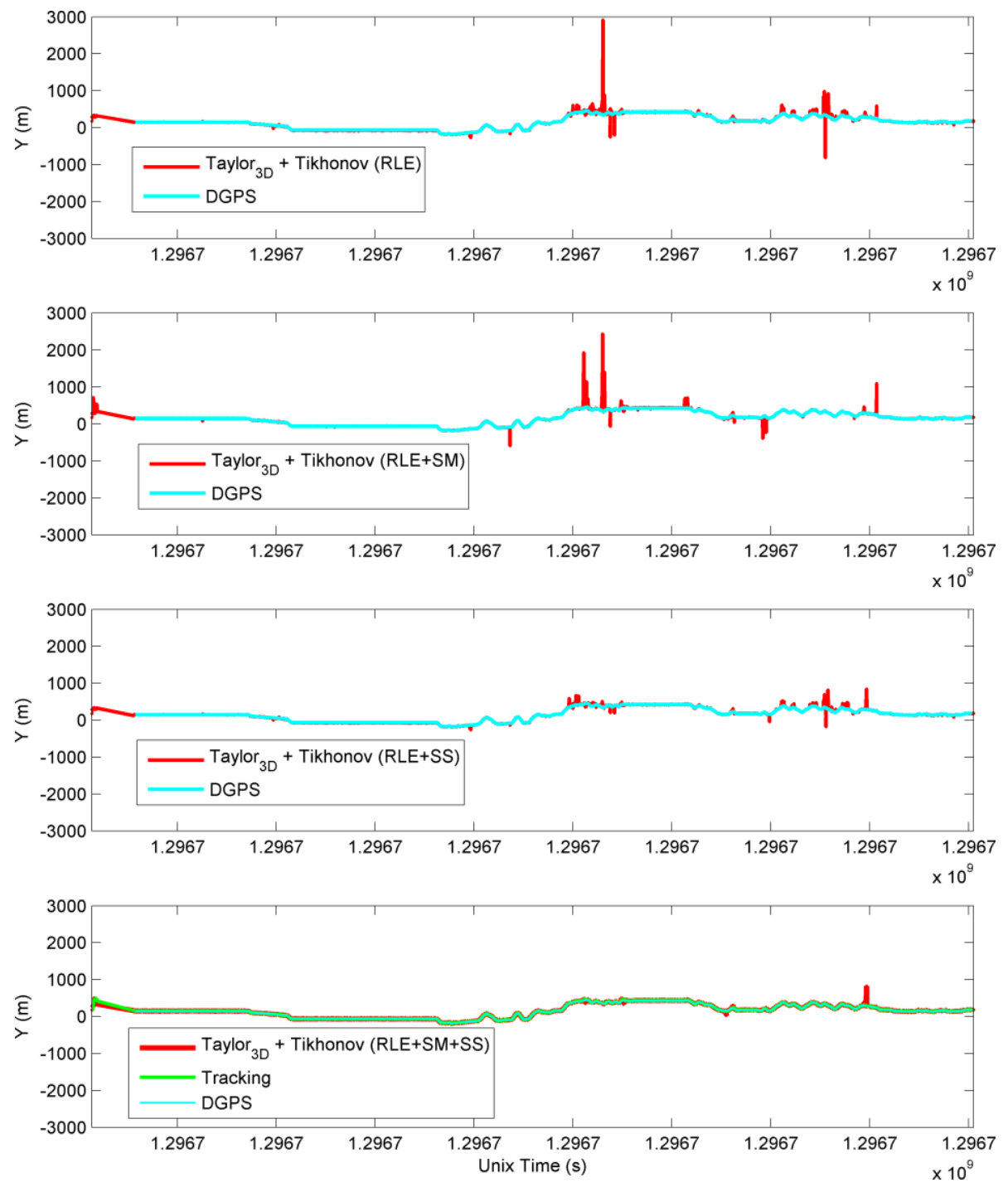

Fig. 8.55 Y(t) for Tikhonov based RLE. 

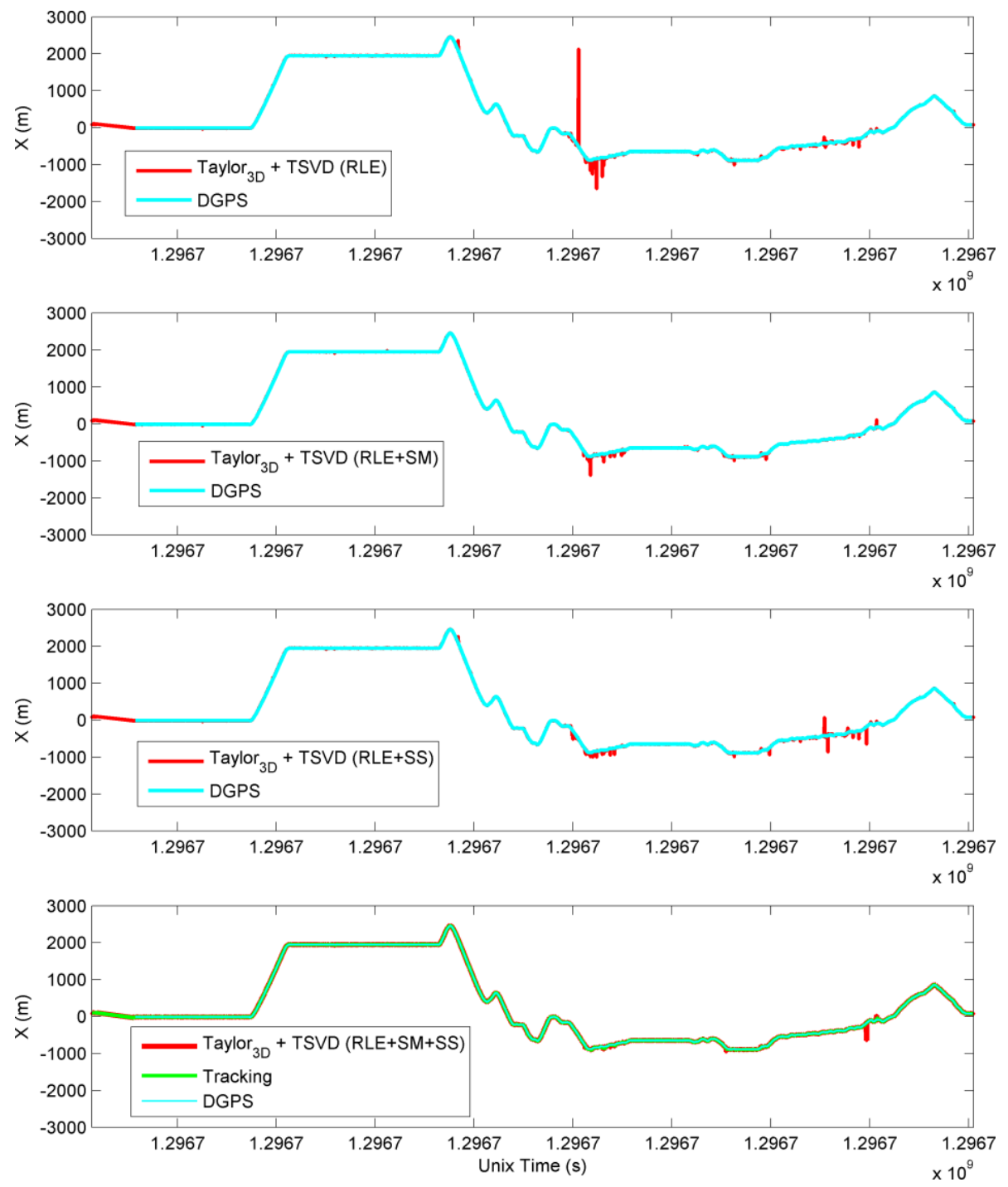

Fig. 8.56 X(t) for T-SVD based RLE. 

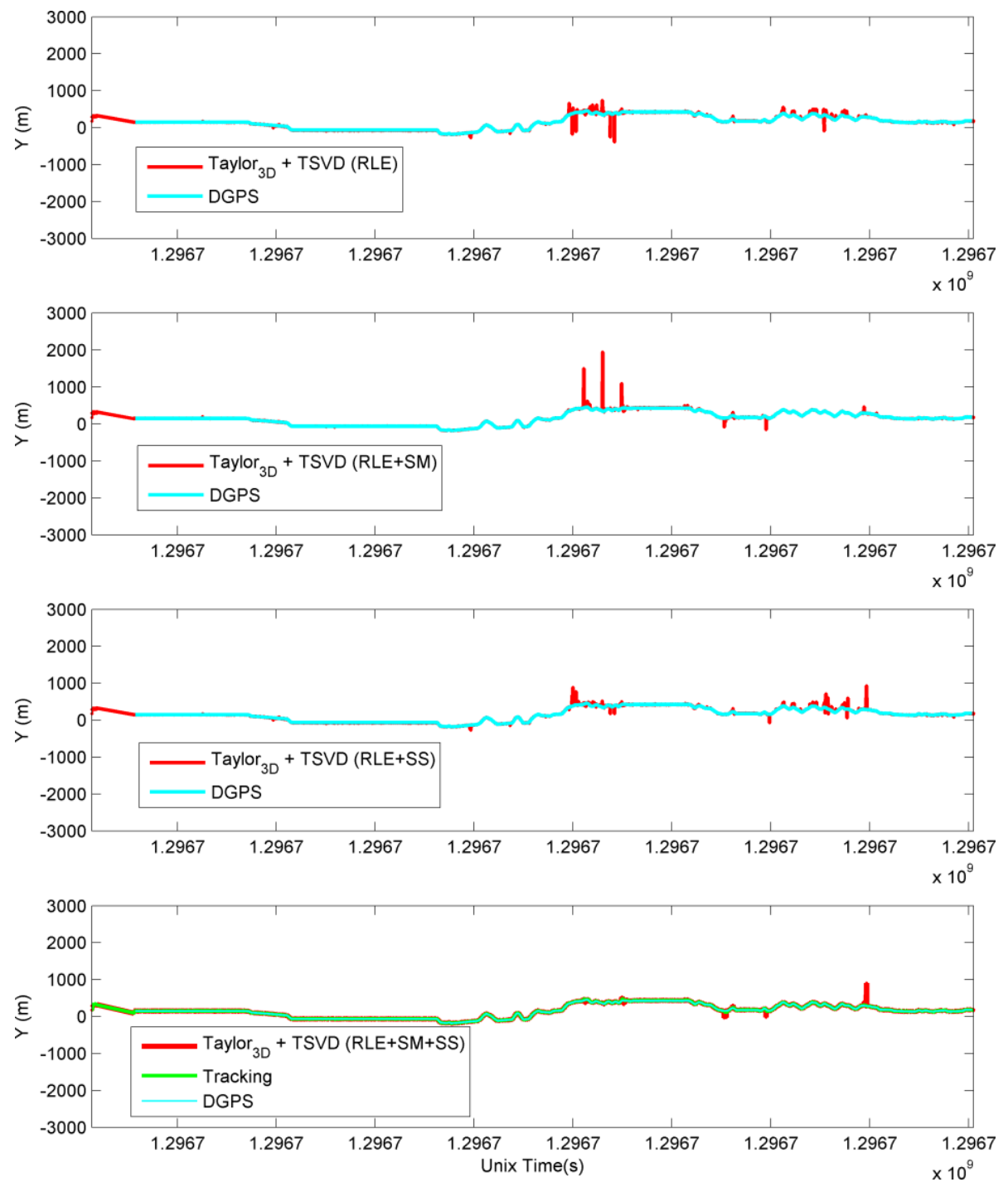

Fig. $8.57 \mathrm{Y}(\mathrm{t})$ for T-SVD based RLE. 
Table 8.8 Statistical parameters for the error distributions of the Tikhonov based RLE. Values given in meters.

\begin{tabular}{|c|c|c|c|c|}
\hline \hline Algorithm & $\boldsymbol{\sigma}_{\mathbf{2 D}}(\mathbf{m})$ & $\mathbf{M e a n}_{\mathbf{2 D}}(\mathbf{m})$ & $\begin{array}{c}\text { PoL (\%) } \\
\boldsymbol{\delta}=\mathbf{7 . 5 m}\end{array}$ & $\begin{array}{c}\text { PoL (\%) } \\
\boldsymbol{\delta}=\mathbf{1 5 m}\end{array}$ \\
\hline $\begin{array}{c}\text { Taylor } \\
\text { 3D } \\
\text { (RLE) Tikhonov }\end{array}$ & 60.32 & 13.08 & 67.96 & 89.73 \\
\hline $\begin{array}{c}\text { Taylor } \\
\text { 3D }+ \text { Tikhonov } \\
(\text { RLE+SM) }\end{array}$ & 59.15 & 10.15 & 71.73 & 93.41 \\
\hline $\begin{array}{c}\text { Taylor }_{3 \mathrm{D}}+\text { Tikhonov } \\
(\text { RLE+SS) }\end{array}$ & 27.20 & 10.30 & 68.78 & 90.45 \\
\hline $\begin{array}{c}\text { Taylor } \\
\text { 3D }+ \text { Tikhonov } \\
\text { RLE+SM+SS) }\end{array}$ & 13.14 & 6.30 & 74.02 & 95.14 \\
\hline Tracking & 6.07 & 5.68 & 76.55 & 95.40 \\
\hline \hline
\end{tabular}

Table 8.9 Statistical parameters for the error distributions of the T-SVD based RLE. Values given in meters.

\begin{tabular}{|c|c|c|c|c|}
\hline Algorithm & $\sigma_{2 D}(\mathrm{~m})$ & $\operatorname{Mean}_{2 \mathrm{D}}(\mathrm{m})$ & $\begin{array}{c}\text { PoL (\%) } \\
\delta=7.5 m\end{array}$ & $\begin{array}{l}\text { PoL (\%) } \\
\delta=15 m\end{array}$ \\
\hline $\begin{array}{c}\text { Taylor }_{3 \mathrm{D}}+\text { T-SVD } \\
(\mathrm{RLE})\end{array}$ & 53.09 & 11.41 & 68.44 & 90.29 \\
\hline $\begin{array}{c}\text { Taylor }_{3 \mathrm{D}}+\mathrm{T}-\mathrm{SVD} \\
(\mathrm{RLE}+\mathrm{SM})\end{array}$ & 35.23 & 7.82 & 72.69 & 94.25 \\
\hline $\begin{array}{c}\text { Taylor }_{3 \mathrm{D}}+\mathrm{T}-\mathrm{SVD} \\
(\mathrm{RLE}+\mathrm{SS})\end{array}$ & 27.76 & 10.15 & 68.32 & 90.26 \\
\hline $\begin{array}{c}\text { Taylor }_{3 \mathrm{D}}+\mathrm{T}-\mathrm{SVD} \\
(\mathrm{RLE}+\mathrm{SM}+\mathrm{SS}) \\
\end{array}$ & 15.45 & 6.65 & 73.05 & 94.73 \\
\hline Tracking & 6.67 & 5.84 & 76.60 & 95.04 \\
\hline
\end{tabular}




\section{Conclusions and Perspectives}

In this thesis we have proposed and developed a set of new strategies to improve the MLAT systems performance in the ATC operations. Such strategies are focused on the automatic, and optimized design of MLAT systems, and on the performance improvement of the localization function, which is basically the core function of these systems. This thesis has been divided into three parts. The first one, horizontal to the remaining two, provides all the theoretical foundations for MLAT systems and describes how they are framed into the general ATC infrastructure. We consider this part a small contribution to the knowledge as far as no public documents, describing these topics, were found before this thesis. In the following, the main conclusions and specific contributions to the knowledge of the second and third parts of this thesis are provided. Thereafter, we describe some open research lines from this work, and finalize by providing the merits of the author of this thesis.

\subsection{Conclusions for Design Strategies}

In this thesis part, we have developed the design strategies for the automatic, and optimized design of MLAT systems in both standard (i.e., by only using TOA/TDOA measurements) and enhanced (i.e., by the combining use of RTD/TDOA and AOA/TDOA) forms.

First, we have developed a framework for the general problem of MLAT system designs. This general framework describes the system performance parameters, which are usually set as the requirements for a system design, and the system design parameters, which are the ones that allow the computer modelling and optimization of the system functions. Moreover, this framework also describes how the system performance and design parameters are related. This framework composes the core of the general design strategy proposed in this thesis. 
We have performed an exhaustive state of the art review of the currently proposed solutions for MLAT systems design, and we have found that all of them can be classified into three groups. The first group comprises the works describing the basic theory for analyzing the theoretical accuracy of MLAT systems. The second one comprises all the works that apply the basic theory (i.e., the first group works) to obtain some general guidelines for the station deployment (i.e., the system geometry), and the third one comprises the works that provide some general frameworks, which allows the "automatic" system design under some conditions. We have also found that the majority of works in the literature belong to the first two groups, and no many works have been developed for dealing with the problem of automatic MLAT system design. Moreover, we have also found that all the revised works do not contemplate some parameters, which are very important for MLAT systems, like the probability of detection, the signal propagation effects, the measurement error balance, the real constraints due to the scenario layout, and the possibility to also design the enhanced version of MLAT systems, whose implementation is growing nowadays.

We have developed the mathematical adaptation of the MLAT system design problem into a combinatorial optimization one. In this adaptation adaption, we have mapped every part of the MLAT design problem into every part of a general combinatorial optimization problem (i.e., the problem variables, search space, variable domains, variable constraints, and fitness function). It allowed us to implement the particular metaheuristic optimization technique of genetic algorithms, which is the numerical mean that finds the best system design parameters that optimize the system performance ones.

We have rigorously analyzed and demonstrated the computer complexity of the MLAT system design as combinatorial optimization problem. We have demonstrated the MLAT system design problem belongs to the class of NP problems and, hence, that there is no a deterministic algorithm that could find an optimal solution in polynomial time.

Finally, in this thesis part, we have developed an efficient procedure to define the layout of standard and enhanced MLAT systems. This procedure is based on the use of genetic algorithms along with the integration of different information and several numerical tools such as the general CRLB analysis. Moreover, practical and useful strategies to apply the procedure have been also proposed and fully described. They are useful not only to design new standard and enhanced MLAT systems but also to validate whether a previous system design could be the optimum solution with regard to a set of available resources and to analyze possible system expansions. The procedure and strategies developed in this thesis are very useful because they avoid the full evaluation of all the possibilities. Instead of this, we have found that only the evaluation of the $6 \times 10^{-6} \%$ of all the 
possible options is enough to obtain satisfactory results, i.e., a system design that satisfies all the requirements and restrictions.

Three kinds of designs have been presented and evaluated. The first one is able to design new MLAT systems with a fixed number of TDOA stations but also to validate whether a final design (clearly before the implementation) can be improved by feasible, but not obvious, sites changes. The second one provides a strategy to obtain a minimum number of stations that satisfies all the stipulated requirements and restrictions. The third one is proposed to design enhanced MLAT systems, i.e., by using other type of measurements such as AOA or RTD. For the third design, an example with a MLAT system using TDOA/AOA stations has been presented, but the use with other measurements combinations is straightforward. Finally, it is worth to say that also these design strategies can be used together in order to obtain more reliable results, e.g., firstly the second design can be used to obtain a possible minimum number of stations that meets all the requirements and restrictions and then, by means of the first design (or the third one in the case of enhanced MLAT systems), obtain the optimum sites or just to validate the set obtained with the second one. Due to the modularity of our proposed design strategy, the use of new requirements or restrictions is also possible only by simply modifying the corresponding cost function and their weight factors.

\subsection{Conclusions for Localization Strategies}

In this thesis part, we have developed the strategies for localization in Mode $\mathrm{S}$ MLAT systems. These strategies have been only proposed for the standard version of MLAT systems (i.e., for only TOA/TDOA measurements).

First, we have described all the theoretical foundations of the localization in MLAT systems and its numerical solution. Particularly, we have described the general MLAT localization as an inverse problem, the current algorithms to solve it (i.e., the LS and pseudoinverse concepts), and its numerical characteristics, emphasizing on the ill-conditioning of it under some situations. It has allowed us to identify the techniques used in other scientific fields to solve the equivalent ill-conditioned inverse problems. Moreover, we have described the canonical tool for analyzing ill-conditioned problems (i.e., the SVD) and the basic parameters to analyze the illconditioned inverse problems (i.e., condition number, rank, numerical rank, and SVD spectrum). In this part, we have provided all the concepts which support all our developments.

We have performed an exhaustive revision of the state of the art for the localization algorithms, which can be used in MLAT systems. Based on this 
state of the art review, we have proposed a general, novel framework to understand, and compare the localization algorithms. In this framework, a localization algorithm is characterized by the pair formed by a data model and a numerical algorithm. For the data models, we propose three different approaches that encompass the most of the localization algorithms found in literature. We have also demonstrated that this classification is fully compatible with the current one, which only classifies the algorithms as open or closed form algorithms. The proposed data models are the statistical approach based models, which construct the localization problem by making some statistical assumptions, the numerical approach based ones, which make some numerical assumption among the unknown variables, and the algebraic approach based ones, which do not make any statistical or numerical assumption. On the other hand, for the numerical algorithms, we have found the most of them are based in the LS sense. Likewise, we have described all the localization algorithms that, from our point of view, represent the majority of them in the literature.

We have tested all the described localization algorithms for both simulated scenarios and scenarios with real data. For all the cases the general conclusions we have found are the same. We have found the most statistically optimal solutions are provided by the algorithms using a statistical approach based model, as long as the statistical hypotheses are met and the algorithm convergence is reached. For MLAT systems, we have also shown that the statistical hypotheses are always satisfied in real data scenarios but, the convergence of these algorithms when solved in the sense of LS is unstable and, hence, not always guaranteed. The algorithms that use a numerical approach based model are by definition no statistically optimal, but they provide better convergence characteristics and the computational cost of implementing them is low. However, they sometimes needs for suitable geometry conditions to obtain satisfactory results. Regarding the algorithms that use an algebraic approach based model, they also are not statistically optimal by definition as they set the localization problem without taking into account of any statistical characteristic of the measurements. However, this kind of algorithms, due to their nature, provides a stable performance, even in the case of small number of stations. By contrary, besides they are not statistically optimal, they also need one more stations than the remaining ones.

We have concluded that, in order to obtain the most efficient (statistically and numerically speaking) localization strategy, it is always advisable to use the combination of a statistical approach based model algorithm (that is an open form algorithm) along with either a numerical or an algebraic approach based model algorithm (that are closed form algorithms). 
We have fully analyzed the numerical characteristics of the localization problem for MLAT systems, when it is solved in the sense of Taylor series expansion algorithm. This analysis has been presented in the sense of the SVD spectrum of the coefficient matrix. In this way, we have found that the SVD spectrum of the coefficient matrix changes with the Taylor refinement iteration and, therefore, it can be classified as a rank-deficient or discrete ill-posed problem. Furthermore, we have identified three possible sources for the ill-conditioning of the localization problem, i.e., the system geometry, the measurements noise, and the quality starting point for Taylor method. When the ill-conditioning is due to the system geometry it was observed that the problem tends to be more rank-deficient and when it is due to the measurements noise, or to the starting point, it was observed that the problem tends to be more discrete ill-posed.

We have studied several regularization methods, in order to propose new localization algorithms for Mode S MLAT, and a new general localization strategy for all the scenarios, but particularly for those cases that present some amount of ill-conditioning. Several regularization methods have been studied and tested, namely, Tikhonov regularization, Truncated SVD (TSVD), T-SVD with sub-set selection (T-SVD SS), and Truncated Total Least Squares (T-TLS). Within these methods, it has been found that the best performances are obtained by the application of Tikhonov and T-SVD methods along with the Taylor series expansion algorithm. Thus, with these two methods we have proposed two Regularized Location Estimators (RLE).

For every RLE we have developed a novel and efficient strategy to estimate the corresponding regularization parameters. Moreover, a particular procedure to refine the estimation of the regularization parameter, in the case of LAM surveillance, has also been developed. Particularly, for Tikhonov based RLE, due to its continuous nature, we have also analyzed its influence on the localization problem, specifically, it has been described the geometrical correction and the effects of the regularization and perturbation errors.

We have found that the correct implementation of Tikhonov and T-SVD regularization methods always significantly mitigates the ill-conditioning of the localization problem. The application of any regularization method always introduces certain amount of bias on the results. However, in this thesis, we have demonstrated, by means of simulations of both simulated and real data scenarios, that with the use of the correct regularization parameter this amount of bias can be neglected in practical terms.

We have analyzed, by means of simulations, the different and possible sources to calculate the starting point for Taylor based algorithms (for the classical solution in the sense of LS and the RLEs proposed herein). Furthermore, it has also been analyzed the accuracy performance in the 
presence of a possible failure of one station. Regarding the results for Tikhonov and T-SVD based RLEs, we have found that, although both methods provide good accuracy performances, Tikhonov is more stable and reliable in the presence of possible degradations of the starting point and of the failure of some stations. For example, particularly for LAM (but also for WAM), when using the Tikhonov based RLE, it is enough to use only the centre of the airport as the starting point (a fixed value) without the need of a closed form algorithm.

We have developed a general regularized localization strategy along with a set of additional improvements. It is based on the combined use of a closed form algorithm and the RLE developed herein (either the Tikhonov or T-SVD based RLE). The general strategy is composed of two steps, the first step is called area detection and it is intended to detect the situation of the aircraft/vehicle (i.e., if the target is on the surface or on flight). The first step must be only carried out in the first time at the Mode $\mathrm{S}$ squitter/replies from an aircraft or vehicles are detected by the system. Then, the second step, called localization process, is a recursive process which is intended to calculate the position of the aircrafts/vehicles. Moreover, the additional improvements are the measurements selection and solution selections.

We have tested the proposed general localization strategy in both simulated and real data scenarios. The simulated scenarios are composed by real geometries and simulated measurements, whilst the real data scenario is composed by both real geometries and real measurements. We have found, for all the cases, significant improvements when using the proposed general localization strategy with regard to all the localization algorithms presented in the literature and also analyzed in this thesis. Moreover, we have also found the proposed additional improvements help to significantly improve the system performance in the case of highly noisy measurements, even for the real data scenario we have found these strategies allows to mitigate the errors due to multipath problem.

Finally, we consider important to emphasize that when the localization problem is well-conditioned (e.g., Innsbruck WAM system) the classical solution without the use of any of the proposed RLE (i.e., by the pseudoinverse matrix) provides good accuracy performance. However, the use of the proposed RLEs has the same accuracy performances. For these reason, we propose the general use of the proposed RLEs to solve the localization problem. 


\subsection{Future Work}

Once finalized this thesis, some research lines remain open and should be further investigated. These open lines are listed below:

- The design strategies proposed in this thesis take into account an important number of performance parameters, which are introduced into the resulting combinatorial optimization problem by means of the system design parameters. However, effects like those due to multipath propagation from buildings have been not introduced. Therefore, it should be interesting to introduce these effects for all the kind of measurements, i.e., TOA/TDOA, RTD and AOA, in order to obtain more realistic system designs.

- Although the metaheuristic of GA used in this thesis provides suitable convergence rates, it should be interested to validate novel techniques, like Ant Colony Optimization (ACO) or Particle Swarm Optimization (PSO), which have presented better performances in other scientific fields.

- For the regularized location estimators we have described some analytical expressions that show the existence of regularization and perturbation errors, which are affected by the amount of regularization that is introduced. Thus, it is interesting to obtain some analytical procedures that allow the previous analysis of the accuracy for the regularized location estimators.

- We have proposed some additional improvements that allow the mitigation of the errors due to the multipath effect. However, although they are efficient for the scenarios tested in this thesis, they should be further investigated to increase their robustness.

- We have proposed a general localization strategy for using with the standard MLAT systems, i.e., by only using TOA/TDOA measurements. Moreover, we have also shown that these regularized estimators improve the position accuracy also in the case of wellconditioned problems. Therefore, it should be advisable to investigate the usefulness of these algorithms for the enhanced version of these systems, i.e., by also using RTD or AOA measurements, which in principle are also well-conditioned problems.

- We consider that the validation of the general localization strategy proposed in this thesis is enough to demonstrate the real usefulness of it. However, it would be advisable to validate it with more real data, coming from other operational systems. 


\subsection{Author's Merits}

The scientific and academic contributions produced during the $\mathrm{PhD}$ course of the author are presented. None of these publications have previously formed part of other works. These publications are listed below:

\section{Peer-Reviewed (indexed) Journal Papers}

- I. A. Mantilla-Gaviria, M. Leonardi, J. V. Balbastre-Tejedor, and E. d. l. Reyes, "On the application of singular value decomposition and Tikhonov regularization to ill-posed problems in hyperbolic passive location," Mathematical and Computer Modelling, vol. 57, pp. 19992008, April 2013.

- I. A. Mantilla-Gaviria, M. Leonardi, G. Galati, J. V. BalbastreTejedor, and E. d. l. Reyes, "An effective procedure to design the layout of standard and enhanced mode-S multilateration systems for airport surveillance," International Journal of Microwave and Wireless Technologies, vol. 4, pp. 199-207, 2012.

- I. A. Mantilla-Gaviria, M. Leonardi, G. Galati, J. V. BalbastreTejedor, and E. d. 1. Reyes, "Efficient location strategy for airport surveillance using mode-s multilateration systems," International Journal of Microwave and Wireless Technologies, vol. 4, pp. 209216, 2012.

- G. Galati, M. Leonardi, I. A. Mantilla-Gaviria, and M. Tosti, "Lower bounds of accuracy for enhanced mode-s distributed sensor networks," IET Radar Sonar and Navigation, vol. 6, pp. 190-201, March 2012.

\section{Peer-Reviewed (non-indexed) Journal Papers}

- I. A. Mantilla-Gaviria, R. F. Ruiz, J. V. Balbastre-Tejedor, and E. d. l. Reyes, "ETP/GDOP Behaviour study for n-sensors arrays in a multilateration radar system," Iteckne: Innovación e Investigación en Ingeniería, vol. 6, pp. 29-34, June 2009.

\section{Papers in International Conferences}

- I. A. Mantilla-Gaviria, R. F. Ruiz, J. V. Balbastre-T, and E. d. 1. Reyes, "Application of Metaheuristic Optimization Techniques to Multilateration System Deployment," in Proceedings of Enhanced Solutions for Aircraft and Vehicle Surveillance Applications, ESA VS 2010, German Institute of Navigation (DGON), Berlin, Germany, March 16-17, 2010, p. Session 2B/3.

- I. A. Mantilla-Gaviria, M. Leonardi, J. V. Balbastre-T, and E. d. 1. Reyes, "Singular value decomposition and Tikhonov regularization in hyperbolic passive location," in Proceedings of Mathematical 
Modelling in Engineering 83 Human Behaviour, Valencia, Spain, September 6-9, 2011, pp. 183-188.

- I. A. Mantilla-Gaviria, M. Leonardi, G. Galati, J. V. Balbastre-T, and E. d. 1. Reyes, "Strategies to Design and Deploy Mode-S Multilateration Systems," in Proceedings of Tyrrhenian International Workshop on Digital Communications - Enhanced Surveillance of Aircraft and Vehicles (ESAV'11), Capri, Italy, September 12-14, 2011, pp. 167 - 172.

- I. A. Mantilla-Gaviria, M. Leonardi, G. Galati, J. V. Balbastre-T, and E. d. 1. Reyes, "Improvement of multilateration (MLAT) accuracy and convergence for airport surveillance," in Proceedings of Tyrrhenian International Workshop on Digital Communications Enhanced Surveillance of Aircraft and Vehicles (ESAV'11), Capri, Italy, September 12-14, 2011.

- I. A. Mantilla-Gaviria, M. Leonardi, G. Galati, and J. V. BalbastreTejedor, "Comparison of localization algorithms for Multilateration (MLAT) systems in airport surface surveillance," in Proceedings of International Symposium Enhanced Solutions for Aircraft and Vehicle Surveillance Applications, ESA VS 2013, German Institute of Navigation (DGON), Berlin, Germany, March 20-21, 2013, p. Session 4c.

\section{Papers in National Conferences}

- I. A. Mantilla-Gaviria, R. F. Ruiz, J. V.Balbastre-Tejedor, and E. d. 1. Reyes, "Evaluación de la mejora de la PTE/GDOP con relación al número de sensores en un sistema de multilateración radar," in Proceedings of the XXIV Simposium Nacional de la Unión Científica Internacional de Radio (URSI 2009), Santander, Cantabria, Spain, 16/18 de Septiembre de 2009.

- I. A. Mantilla-Gaviria, R. F. Ruiz, E. J. Gómez-Pérez, J. V. Balbastre-Tejedor, and E. d. l. Reyes, "Distribución de las estaciones receptoras de un sistema de multilateración en un entorno aeroportuario mediante un algoritmo de optimización," in Proceedings of the XXV Simposium Nacional de la Unión Científica Internacional de Radio (URSI 2010), Bilbao, País Vasco, Spain, 15/17 de Septiembre de 2010.

\section{Other Related Publications}

- R. F. Ruiz, I. A. Mantilla-Gaviria, E. J. Gómez-P, J. V. BalbastreT, and E. d. l. Reyes, "A genetic algorithm and local search for the automated calculation of dilution of precision of mode-s multilateration systems at airports," in Proceedings of Tyrrhenian International Workshop on Digital Communications - Enhanced 
Surveillance of Aircraft and Vehicles (ESAV'11), Capri, Italy, September 12-14, 2011, pp. 259 - 263.

- E. J. Gómez-Pérez, I. A. Mantilla-Gaviria, R. F. Ruiz, and J. V. Balbastre-Tejedor, "Tracking time domain to compensate temporary performance degradations of receivers stations in multilateration systems," in Proceedings of the International Conference on Radar Systems, Glasgow, UK, October 22-25, 2012.

- I. A. Mantilla-Gaviria, E. J. Pérez-Gómez, R. F. Ruiz, R. F. FloresAcedo, M. Muñoz-Martínez, and J. V. Balbastre-Tejedor, "A local data fusion implementation for Multilateration (MLAT) track update with ADS-B data," in Proceedings of International Symposium Enhanced Solutions for Aircraft and Vehicle Surveillance Applications, ESAVS 2013, German Institute of Navigation (DGON), Berlin, Germany, March 20-21, 2013, p. Session 1.

- E. J. Gómez-Pérez, I. A. Mantilla-Gaviria, R. F. Ruiz, A. LópezBailon, J. V. Balbastre-Tejedor, and E. d. l. Reyes, "Aplicación de filtros estimadores en la evaluación de sistemas de guiado y control de tráfico en superficie," in Proceedings of the XXV Simposium Nacional de la Unión Científica Internacional de Radio (URSI 2010), Bilbao, País Vasco, Spain, 15/17 de Septiembre de 2010.

- R. F. Ruiz, I. A. Mantilla-Gaviria, E. J. Gómez-Pérez, J. V. Balbastre-Tejedor, and E. d. 1. Reyes, "Algoritmo genético y búsqueda local para el cálculo de cobertura DOP/PTE con alto grado de precisión en el diseño de un sistema de multilateración de control aeroportuario," in Proceedings of the XXV Simposium Nacional de la Unión Científica Internacional de Radio (URSI 2010), Bilbao, País Vasco, Spain, 15/17 de Septiembre de 2010.

\section{Technical Disseminations}

- I. A. Mantilla-Gaviria, M. Leonardi, and G. Galati, "Lower bounds of accuracy for Mode S distributed sensors network," Università degli studi di Roma "Tor Vergata", Rome, Italy, June 2010

- I. A. Mantilla-Gaviria, M. Leonardi, and G. Galati, "Regularization methods for solving ill-conditioned location problems in Mode $\mathrm{S}$ Multilateration," Università degli studi di Roma "Tor Vergata", Rome, Italy, May 2011.

- I. A. Mantilla-Gaviria, J. V. Balbastre-Tejedor, "Sistemas de multilateración para el control de tráfico aéreo," Unidad Administrativa Especial de Aeronáutica Civil (UAEAC), Colombia, March 2012. 


\section{Academic Activities}

During his $\mathrm{PhD}$ years, the author has tutored some final degree projects and master thesis, as well as taught some courses in the field of air traffic control and surveillance systems. These academic activities are listed below:

- Final Degree Project: "Desarrollo y aplicación de filtros estimadores para la evaluación de sistemas avanzados de control y guiado de tráfico en superficie (A-SMGCS)," at Escuela Técnica Superior de Ingenieros de Telecomunicación, Universidad Politécnica de Valencia, September 2010; Author: E. J. Gómez-Pérez; Tutors: I. A. Mantilla-Gaviria and J. V. Balbastre-Tejedor.

- Final Degree Project: "Development and ant colony optimization algorithm for the design of multilateration (MLAT) systems," at Politecnico di Torino (Turin, Italy), July 2012; Author: C. Díaz; Tutors: I. A. Mantilla-Gaviria, J. V. Balbastre-Tejedor, and G. Vecchi.

- Master Thesis: "Tracking en el dominio del tiempo para compensar degradaciones temporales en el funcionamiento de estaciones receptoras en sistemas de multilateración," at Escuela Técnica Superior de Ingenieros de Telecomunicación, Universidad Politécnica de Valencia, July 2012; Author: E. J. Gómez-Pérez; Tutors: I. A. Mantilla-Gaviria and J. V. Balbastre-Tejedor.

- Training Course: "Sistemas de Multilateración (MLAT) para el control de tráfico aéreo," at Centro de Formación Permanente (CFP), Universidad Politécnica de Valencia, 2012, Duration: 24.5 hrs, Professors: J. V. Balbastre-Tejedor and I. A. Mantilla-Gaviria. 

A

\section{Development of Combinatorial by Means of Stirling's Approximation}

The full description of the development of combinatorial formula in (5.3) and (5.4) is provided in this annex. For convenience, the combinatorial formulas in (5.3) and (5.4) and the Stirling's approximation [69] are rewritten, respectively, in the following:

$$
\begin{gathered}
C_{1}\left(\ell_{S}\right)=\frac{\ell_{S} !}{\left(\ell_{S}-b \ell_{S}\right) !\left(b \ell_{S}\right) !} \\
C_{1}\left(N_{s}\right)=\frac{\left(a N_{s}\right) !}{\left(a N_{s}-N_{s}\right) ! N_{S} !} \\
n ! \approx \sqrt{2 \pi n}\left(\frac{n}{e}\right)^{n}
\end{gathered}
$$

Now, the Stirling's approximation is replaced in $C_{1}\left(\ell_{\delta}\right)$ as follows

$$
C_{1}\left(\ell_{\mathcal{S}}\right)=\frac{\sqrt{2 \pi \ell_{\mathcal{S}}}\left(\frac{\ell_{\mathcal{S}}}{e}\right)^{\ell_{\mathcal{S}}}}{\sqrt{2 \pi(1-b) \ell_{\mathcal{S}}}\left(\frac{(1-b) \ell_{\mathcal{S}}}{e}\right)^{(1-b) \ell_{\mathcal{S}}} \sqrt{2 \pi b \ell_{\mathcal{S}}}\left(\frac{b \ell_{\mathcal{S}}}{e}\right)^{b \ell_{\mathcal{S}}}}
$$

then, rearranging the common terms,

$$
\begin{gathered}
C_{1}\left(\ell_{\mathcal{S}}\right)=\sqrt{\frac{2 \pi \ell_{\mathcal{S}}}{2 \pi(1-b) \ell_{\mathcal{S}} 2 \pi b \ell_{\mathcal{S}}}}\left(\frac{\ell_{\mathcal{S}}}{e}\right)^{\ell_{\mathcal{S}}}\left(\frac{(1-b) \ell_{\mathcal{S}}}{e}\right)^{(b-1) \ell_{\mathcal{S}}}\left(\frac{b \ell_{\mathcal{S}}}{e}\right)^{-b \ell_{\mathcal{S}}} \\
C_{1}\left(\ell_{\mathcal{S}}\right)=\sqrt{\frac{1}{2 \pi(1-b) b \ell_{\mathcal{S}}}\left(\frac{\ell_{\mathcal{S}}}{e}\right)^{\ell_{\mathcal{S}}+b \ell_{\mathcal{S}}-\ell_{\mathcal{S}}-b \ell_{\mathcal{S}}}(1-b)^{(b-1) \ell_{\mathcal{S}}(b)^{-b \ell_{\mathcal{S}}}}}
\end{gathered}
$$




$$
C_{1}\left(\ell_{\mathcal{S}}\right)=\sqrt{\frac{1}{2 \pi(1-b) b \ell_{\mathcal{S}}}}\left(\frac{1}{1-b}\right)^{(1-b) \ell_{\mathcal{S}}}\left(\frac{1}{b}\right)^{b \ell_{\mathcal{S}}} ; \quad 0<b<1
$$

Finally, the explicit expression for $C_{1}\left(N_{S}\right)$ is straightforward by replacing the relation $\ell_{\mathcal{S}}=a N_{S}$ into $C_{1}\left(\ell_{\mathcal{S}}\right)$. 
B

\section{Development of Averaging Kernels for Tikhonov Regularization}

The full description of the formulas for the averaging kernels in (8.28) is provided in this annex. For convenience, let rewriting (8.26) only in terms on the smoothed version of the exact target position, i.e., by omitting the noise and starting point terms, as follows:

$$
\widehat{\boldsymbol{\theta}}_{\lambda}=\boldsymbol{\Xi} \boldsymbol{\theta}
$$

Then, the definition of the resolution matrix is expressed as (8.25):

$$
\Xi=A_{\lambda}^{-1} G
$$

and the inverse matrix of Tikhonov can be expressed in terms of the SVD of matrix $\boldsymbol{G}$ as follows:

$$
\boldsymbol{A}_{\lambda}^{-1}=\left(\boldsymbol{U} \boldsymbol{\Sigma} \boldsymbol{\Gamma}_{\lambda} \boldsymbol{V}^{T}\right)^{-1}=\boldsymbol{V} \boldsymbol{F}_{\lambda} \boldsymbol{\Sigma}^{-1} \boldsymbol{U}^{T}
$$

where matrix $\boldsymbol{\Gamma}_{\boldsymbol{\lambda}}$ has been defined in (8.16) as a diagonal matrix containing the inverse of the Tikhonov filter factors. Thus, matrix $\boldsymbol{F}_{\lambda}$ is a diagonal matrix containing the filter factors as follows:

$$
\boldsymbol{F}_{\lambda}=\boldsymbol{\Gamma}_{\lambda}^{-\mathbf{1}}=\left[\begin{array}{lll}
f_{1} & & \\
& \ddots & \\
& & f_{n}
\end{array}\right]_{n \times n}
$$

being $f_{j}$ the $j$ th Tikhonov filter factor. Then, the resolution matrix can be also expressed in terms of the SVD of matrix $\boldsymbol{G}$ as follows:

$$
\Xi=\left(\boldsymbol{V} \boldsymbol{F} \boldsymbol{\Sigma}^{-1} \boldsymbol{U}^{T}\right)\left(\boldsymbol{U} \boldsymbol{\Sigma} \boldsymbol{V}^{T}\right)=\boldsymbol{V} \boldsymbol{F} \boldsymbol{V}^{T}
$$

The above resolution matrix can be expressed in terms of the right singular vectors as: 


$$
\boldsymbol{\Xi}=\boldsymbol{V} \boldsymbol{F} \boldsymbol{V}^{T}=\sum_{j=1}^{n} f_{j} \boldsymbol{v}_{j} \boldsymbol{v}_{j}^{T}
$$

where $\boldsymbol{v}_{j}$ is the $j$ th right singular vector of matrix $\boldsymbol{V}$. Now, if we define the resolution matrix as:

$$
\mathbf{\Xi}=\left[\begin{array}{lll}
\xi_{1}^{1} & \xi_{1}^{2} & \xi_{1}^{3} \\
\xi_{2}^{1} & \xi_{2}^{2} & \xi_{2}^{3} \\
\xi_{3}^{1} & \xi_{3}^{2} & \xi_{3}^{3}
\end{array}\right]_{n \times n}
$$

It is straightforward that every averaging kernel can be obtained as:

$$
\boldsymbol{\xi}_{i}=\left[\begin{array}{l}
\xi_{i}^{1} \\
\xi_{i}^{2} \\
\xi_{i}^{3}
\end{array}\right]_{n \times 1}=\sum_{j=1}^{n} f_{j} \boldsymbol{v}_{j}^{i} \boldsymbol{v}_{j}
$$

where $\boldsymbol{v}_{j}^{i}$ is the $i$ th component of the $j$ th right singular vector. Finally, developing the above expression for the $i$ th averaging kernel it is obtained the expression in (8.28) as:

$$
\xi_{i}^{T}=f_{1} \boldsymbol{v}_{1}^{i} \boldsymbol{v}_{1}^{T}+f_{2} \boldsymbol{v}_{2}^{i} \boldsymbol{v}_{2}^{T}+f_{3} \boldsymbol{v}_{3}^{i} \boldsymbol{v}_{3}^{T}
$$




\section{Simulated Scenarios}

In this thesis we validate the proposed localization estimators and strategies by using two kinds of scenarios. The first ones are MLAT systems with real geometries and simulated TOA measurements. Within this kind of scenarios, we simulate three different systems: the WAM system of Innsbruck (Austria) Airport, the WAM system of Malpensa (Milan, Italy) airport (it was a particular proposal for that airport), and the LAM system of Linate (Milan, Italy) airport. The latter has been provided by Thales Italia S.p.A. On the other hand, the second kind of scenario is a MLAT system with real geometry and real measurement data. It is the LAM system of Tallinn (Tallinn, Estonia) airport, whose real data have been kindly provided by the company ERA A.S. (www.erabeyondradar.com). In the following we describe the system geometries and paths of each of system mentioned before.

\section{C.1 Scenarios with Real Geometry and Simulated Measurements}

In this kind of scenarios we use the real system geometry, a real flight or surface movement path, and the simulated TOA measurements. These simulated TOAs are generated by adding, to the exact values, a random error that follows a Gaussian distribution of zero mean and standard deviation $\sigma_{T O A}$. Moreover, for setting this standard deviation we have used the general simulation model as proposed in [20]. Basically this model takes into account of the errors due to propagation loss, to the quantisation effects of the receivers, to the stations synchronization, etc. (see also Table 2.1 and $(2.6))$. 


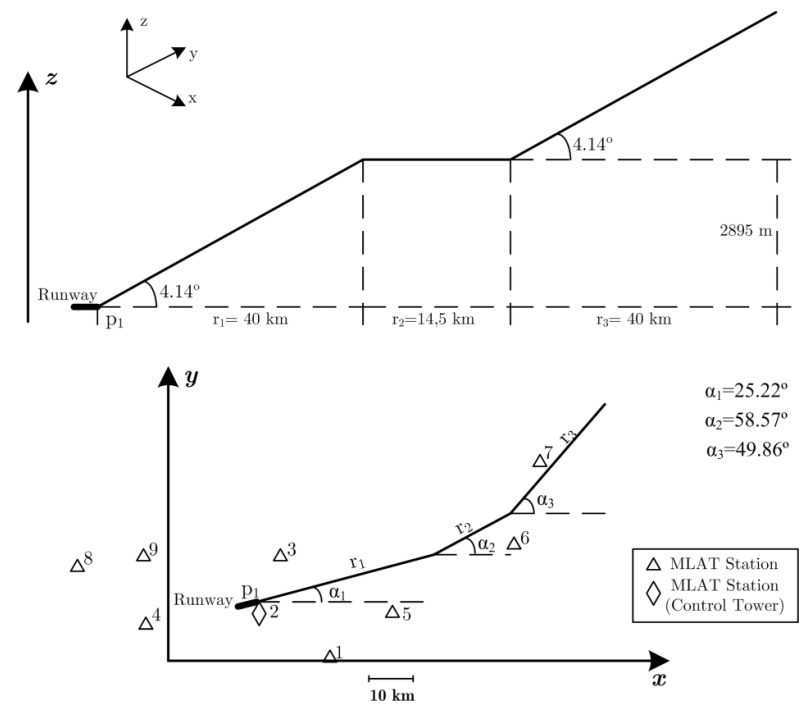

Fig. C.1 Innsbruck system layout (top: vertical profile, bottom: horizontal profile).

\section{C.1.1 Innsbruck System}

The Innsbruck airport (IATA code: INN) WAM system is composed of nine stations (three receiver/transmitter -R.T.-, five receivers only -R.O.-, and one reference transponder), and a redundant processing subsystem. The coordinates of the nine stations are shown in Table C.1 and the layout of the system and the flight path are shown in Fig. C.1.

In this scenario we assume all the stations have line of sight to all the simulated points.

Table C.1 Stations coordinates for the Innsbruck WAM system.

\begin{tabular}{|c|c|c|c|c|}
\hline \hline Num. & Type & Lat. & Lon. & Alt $^{9}$ ( $\left.\mathbf{m}\right)$ \\
\hline 1 & Ref. Trans. & $47^{\circ} 12^{\prime} 13.4^{\prime \prime}$ & $11^{\circ} 27^{\prime} 36.7^{\prime \prime}$ & 2245 \\
\hline 2 & R.T./Ref. Trans. & $47^{\circ} 15^{\prime} 28.2^{\prime \prime}$ & $11^{\circ} 21^{\prime} 9.8^{\prime \prime}$ & 616 \\
\hline 3 & R.O. & $47^{\circ} 18^{\prime} 46.4^{\prime \prime}$ & $11^{\circ} 23^{\prime} 10.3^{\prime \prime}$ & 2336 \\
\hline 4 & R.T. & $47^{\circ} 14^{\prime} 37.4^{\prime \prime}$ & $11^{\circ} 10^{\prime} 51.9^{\prime \prime}$ & 1910 \\
\hline 5 & R.O. & $47^{\circ} 15^{\prime} 11.6^{\prime \prime}$ & $11^{\circ} 33^{\prime} 20.9^{\prime \prime}$ & 1360 \\
\hline 6 & R.T. & $47^{\circ} 19^{\prime} 15.7^{\prime \prime}$ & $11^{\circ} 44^{\prime} 30.8^{\prime \prime}$ & 1895 \\
\hline 7 & R.O. & $47^{\circ} 24^{\prime} 34.6^{\prime \prime}$ & $11^{\circ} 47^{\prime} 15.1^{\prime \prime}$ & 1006 \\
\hline 8 & R.O. & $47^{\circ} 18^{\prime} 11.9^{\prime \prime}$ & $11^{\circ} 04^{\prime} 23.11^{\prime \prime}$ & 605 \\
\hline 9 & R.O. & $47^{\circ} 18^{\prime} 49.3^{\prime \prime}$ & $11^{\circ} 10^{\prime} 39.5^{\prime \prime}$ & 1459 \\
\hline \hline
\end{tabular}

${ }^{9}$ A.M.S.L.: Above Mean Sea Level. 


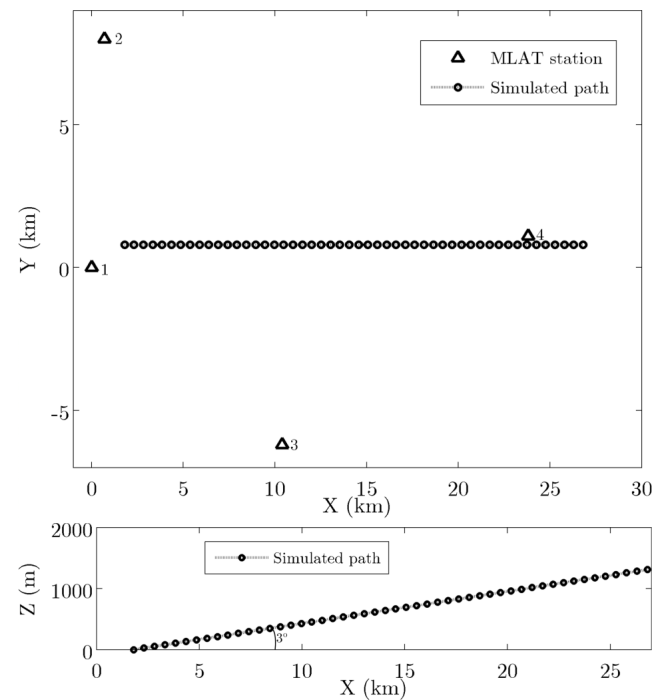

Fig. C.2 Malpensa system layout for the first takeoff line: Malpensa 1 (top: horizontal profile, bottom: vertical profile).

The level (A.M.S.L) of the runway is approximately $577 \mathrm{~m}$, the takeoff line is in north-east direction, and the coordinates (Lat., Lon.) of the initial point are $\left(47^{\circ} 15 ' 41.42^{\prime \prime}, 11^{\circ} 21^{\prime} 21.63^{\prime \prime}\right)$.

\section{C.1.2 Malpensa System}

The Malpensa airport (IATA code: MXP) WAM system is composed of four receiving stations. It was a proposal for solving the approach surveillance at Malpensa airport and was firstly described and analyzed in [7]. This scenario is a good case study for understanding the essentials of the ill-conditioning problems, and to clearly showing the improvements reached by using regularization strategies. For this particular system we simulate a takeoff line. The coordinates of the four stations are shown in Table C.2 and the layout with the takeoff line path is shown in Fig. C.2.

In this scenario we assume all the stations have line of sight to all the simulated points.

Table C.2 Stations coordinates for the Malpensa WAM system.

\begin{tabular}{|c|c|c|c|}
\hline \hline Num. & $\mathbf{X}(\mathbf{m})$ & $\mathbf{Y ~ ( m ) ~}$ & Alt. (m) \\
\hline 1 & -1800 & -800 & 40 \\
\hline 2 & -1100 & 7200 & 50 \\
\hline 3 & 8600 & -7000 & 45 \\
\hline 4 & 22000 & 300 & 47 \\
\hline \hline
\end{tabular}




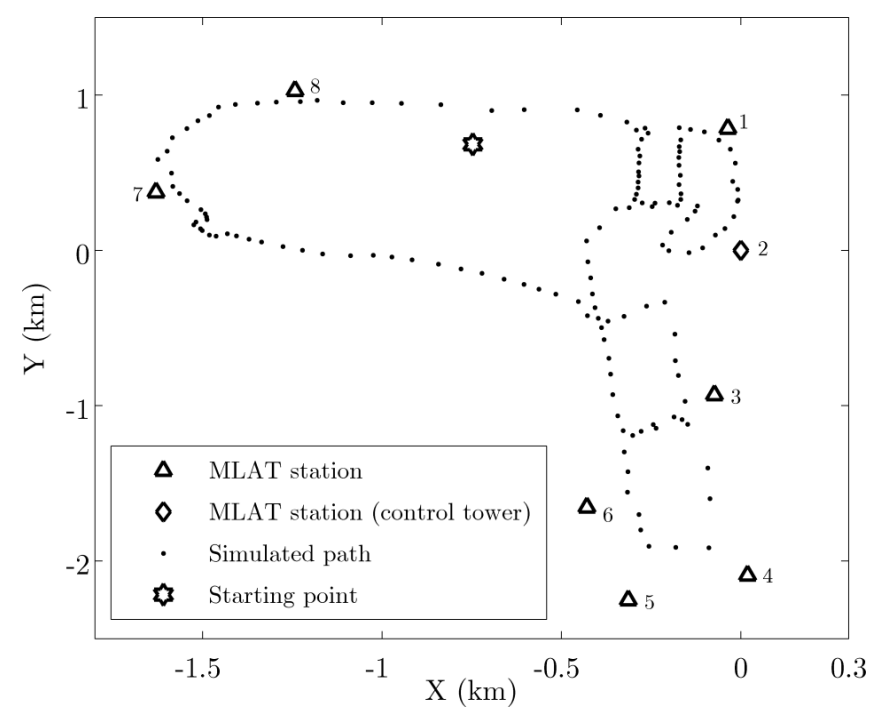

Fig. C.3 Linate system layout and the simulated surface movement.

\section{C.1.3 Linate System}

The Linate airport (IATA code: LIN) LAM system is composed of eight receiving stations and it is intended only for surface movement surveillance. The coordinates of the stations have been kindly supplied by Thales Italia S.p.A. (Dr. Ing. R. Scaroni) and are shown in Table C.3. For this system we simulate a surface movement path that is shown in Fig. C.3. In this figure the coordinate system is centred to the station emplaced in the control tower (the station number 2 depicted as the diamond in Fig. C.3).

In this scenario we assume all the stations have line of sight to all the simulated points.

Table C.3 Stations coordinates for the Linate LAM system.

\begin{tabular}{|c|c|c|c|}
\hline \hline Num. & Lat. & Lon. & Alt $^{10}$. (m) \\
\hline 1 & $45^{\circ} 27^{\prime} 32.2798^{\prime \prime}$ & $9^{\circ} 16^{\prime} 53.4155^{\prime \prime}$ & 168.199 \\
\hline 2 & $45^{\circ} 27^{\prime} 6.8428^{\prime \prime}$ & $9^{\circ} 16^{\prime} 54.9524^{\prime \prime}$ & 150.936 \\
\hline 3 & $45^{\circ} 26^{\prime} 36.6958$ & $9^{\circ} 16^{\prime} 51.4025^{\prime \prime}$ & 152.445 \\
\hline 4 & $45^{\circ} 25^{\prime} 59.038$ & $9^{\circ} 16^{\prime} 55.4563^{\prime \prime}$ & 151.821 \\
\hline 5 & $45^{\circ} 25^{\prime} 53.8948^{\prime \prime}$ & $9^{\circ} 16^{\prime} 40.1378^{\prime \prime}$ & 143.813 \\
\hline 6 & $45^{\circ} 26^{\prime} 13.2991^{\prime \prime}$ & $9^{\circ} 16^{\prime} 34.9441^{\prime \prime}$ & 145.4 \\
\hline 7 & $45^{\circ} 27^{\prime} 19.1738^{\prime \prime}$ & $9^{\circ} 15^{\circ} 39.9965^{\prime \prime}$ & 151.63 \\
\hline 8 & $45^{\circ} 27^{\prime} 40.3024^{\prime \prime}$ & $9^{\circ} 15^{\circ} 57.9054^{\prime \prime}$ & 150.34 \\
\hline \hline
\end{tabular}

${ }^{10}$ A.M.S.L.: Above Mean Sea Level. 


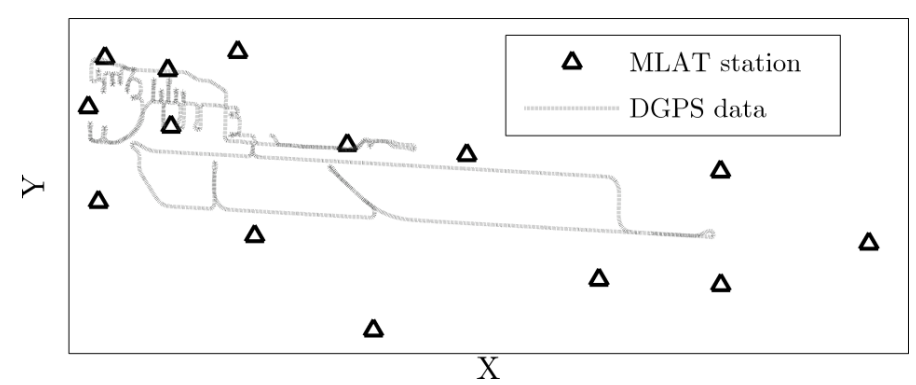

Fig. C.4 Tallinn system layout.

The level (A.M.S.L) of the runway is approximately $105 \mathrm{~m}$. Moreover, the six-peaks star in Fig. C.3 represents a fixed starting point (for Taylor based algorithms) for using in some simulations.

\section{C.2 Scenario with Real Data}

\section{C.2.1 Tallinn LAM System (ERA A.S.)}

The Tallinn airport (IATA code: TLL) LAM system is composed of fourteen receiving stations and it is intended only for surface movement surveillance. The information about this system has been provided by the company ERA A.S. (www.erabeyondradar.com). Due to a confidentiality agreement we cannot provide in this thesis of the numerical values for the station coordinates, therefore, only the system layout is shown in Fig. C.4. Moreover, to evaluate the algorithms accuracy, the company has provided us of reference position data as obtained with a DGPS receiver. The corresponding reference path is also shown in Fig. C.4.

On the other hand, Fig. C.5 shows the line of sight profile for the recorded TOA measurements in Tallinn airport, i.e., the number of stations that measure the TOAs for every position register. The total number of registers is 4230 . 


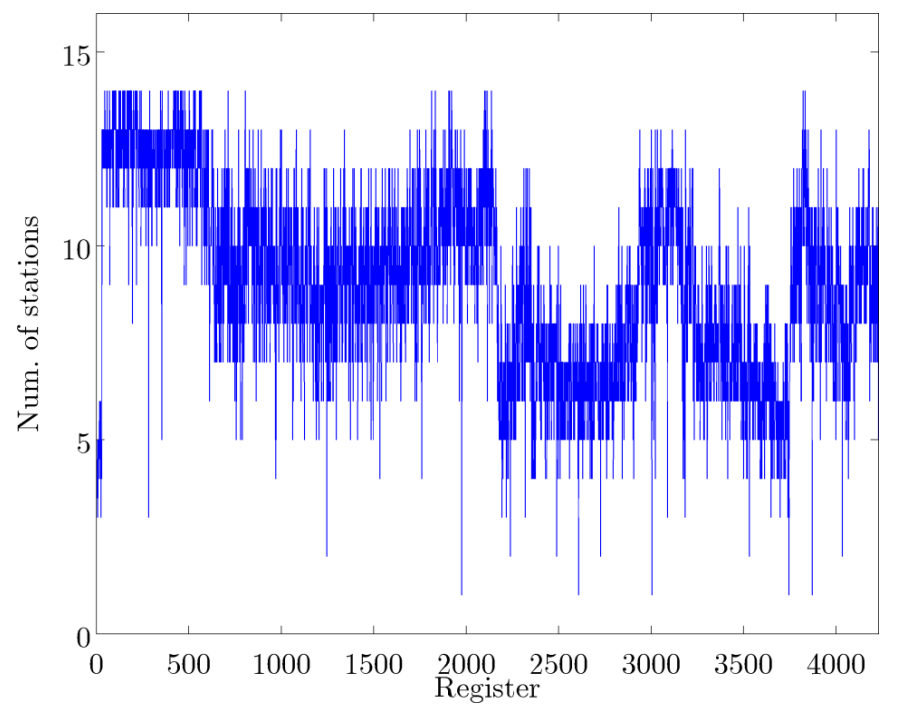

Fig. C.5 Line of sight profile for Tallinn system. 


\section{Bibliography}

[1] EUROCAE-WG-41, "ED-117, MOPS for Mode S Multilateration Systems for Use in Advanced Surface Movement Guidance and Control Systems (A-SMGCS)," ed: The European Organisation for Civil Aviation Equipment (EUROCAE), November 2003.

[2] EUROCAE-WG-70, "ED-142, Technical specification for Wide Area Multilateration (WAM) systems," ed: The European Organisation for Civil Aviation Equipment (EUROCAE), September 2010.

[3] D. J. Torrieri, "Statistical Theory of Passive Location Systems," IEEE Transactions on Aerospace and Electronic System, vol. AES20, pp. 183-198, March 1984.

[4] N. Levanon, "Lowest GDOP in 2-D scenarios," IEE Proc. Radar, Sonar Navig., vol. 147, pp. 149-155, June 2000.

[5] G. Galati, M. Leonardi, P. Magaró, and V. Paciucci, "Wide area surveillance using SSR mode-s multilateration: advantages and limitations," in European Radar Conference, EuRAD 2005, October 6-7, 2005, pp. $225-229$.

[6] G. Galati, M. Gasbarra, P. Magaró, P. D. Marco, L. Mene, and M. Pici, "New approaches to multilateration processing: analysis and field evaluation," presented at the European Radar Conference, EuRAD 2006, 2006.

[7] G. Galati, M. Leonardi, and M. Tosti, "Multilateration (Local and Wide area) as a distributed sensor system: Lower bounds of accuracy," in European Radar Conference, EuRAD, Amsterdam, October 30-31, 2008, pp. 196-199.

[8] J. Hadamard, Lectures on Cauchy's Problem in Linear Partial Differential Equations. New Haven: Yale University Press, 1923.

[9] Eurocontrol, "The ATM Surveillance Strategy for ECAC," Eurocontrol, 2008.

[10] "The LORAN-C System of Navigation," Jansky \& Bailey Engineers for U.S. Coast Guard, Washington D.C.1962.

[11] B. Forssell, Radionavigation Systems: Artech House, 2008. 
[12] A. D. McComas, "Brassboard Model ATCRBS Based Surface Trilateration Data Aquisition Subsystem," Departement of Transportation Systems Center No. 471-2513-999, August 1974.

[13] M. L. Wood, "Propagation of Mode S Beacon Signals on the Airport Surface," MIT Lincoln Laboratory Journal, vol. 2, 1989.

[14] M. I. Skolnik, Radar Handbook: McGraw-Hill, 1990.

[15] ICAO, "Annex 10, Aeronautical Communications, volume IV surveillance and collision avoidance systems," 4th ed: International Civil Aviation Organization, July, 2002.

[16] W. R. Hahn and S. A. Tretter, "Optimum processing for delayvector estimation in passive signal arrays," IEEE Transactions on Information Theory, vol. IT-19, pp. 608-614, September 1973.

[17] M. Stevens, Secondary Surveillance Radar: Artech House, 1988.

[18] G. Galati, M. Leonardi, P. D. Marco, L. Mené, P. Magaró, and M. Gasbarra, "New time of arrival estimation method for multilateration target location," presented at the Joint International Symposium on Sensors and Systems for Airport Surveillance JISSA 2005, Paris, France, June 20-21, 2005.

[19] "Surveillance Data Exchange, Part 7: Category 010, Transmission of Monosensor Surface Movement Data," European Organisation for the Safety of Air Navigation 2007.

[20] G. Galati, M. Leonardi, I. A. Mantilla-Gaviria, and M. Tosti, "Lower bounds of accuracy for enhanced mode-s distributed sensor networks," IET Radar Sonar and Navigation, vol. 6, pp. 190-201, March 2012.

[21] S. U. Khan, "Approximate optimal sensor placements in grid sensor fields," in IEEE 65th Vehicular Technology Conference, 2007, (VTC2007-Spring), Dublin, April 22-25, 2007, pp. 248-251.

[22] H. B. Lee, "A novel procedure for assesing the accuracy of hyperbolic multilateration system," IEEE Transactions on Aerospace and Electronic System, vol. AES-11, pp. 2-15, January 1975.

[23] H. B. Lee, "Accuracy Limitations of Hyperbolic Multilateration System," IEEE Transactions on Aerospace and Electronic System, vol. AES-11, pp. 16-29, January 1975.

[24] J. F. Cline, "Multilateration error ellipsoids," IEEE Transactions on Aerospace and Electronic System, vol. AES-4, pp. 665-667, July 1978.

[25] P. M. Schultheiss and E. Weinstein, "Lower bounds on the localization errors of a moving source observed by a passive array," IEEE Transactions on Acoustics, Speech, And Signal Processing, vol. ASSP-29, pp. 600-607, June 1981. 
[26] P. C. Chestnut, "Emitter location accuracy using TDOA and differential doppler," IEEE Transactions on Aerospace and Electronic System, vol. AES-18, pp. 214-218, March 1982.

[27] J. S. Abel, "Optimal sensor placement for passive source localization," in International Conference on Acoustics, Speech, and Signal Processing 1990, (ICASSP-90) Albuquerque, NM, April 3-6, 1990, pp. 2927-2930.

[28] B. Yang and J. Scheuing, "Cramer-Rao bound and optimum sensor array for source localization from time differences of arrival," in IEEE International Conference on Acoustics, Speech and Signal Processing 2005, (ICASSP 2005), Philadelphia, PA, USA, March 18-23, 2005, pp. IV-961 - IV-964.

[29] B. Yang and J. Scheuing, "A theoretical analysis of 2D sensor arrays for TDOA based localization," in IEEE International Conference on Acoustics, Speech and Signal Processing 2006, (ICASSP 2006), Toulouse, France, May 14-19, 2006, pp. IV-901 - IV-904.

[30] B. Yang, "Different sensor placement strategies for TDOA based localization," in IEEE International Conference on Acoustics, Speech and Signal Processing 2007, (ICASSP 2007), Honolulu, HI, April 15-20, 2007, pp. II-1093 - II-1096.

[31] I. Konchenko, "Availability analysis of the multilateration surveillance system in Kiev (Boryspil) airport," in Microwaves, Radar and Remote Sensing Symposium, 2008, (MRRS 2008), Kiev, Ukraine, September 22-14, 2008, pp. 83-85.

[32] K. C. Ho and L. M. Vicente, "Sensor allocation for source localization with decoupled range and bearing estimation," IEEE Transactions on Signal Processing, vol. 56, pp. 5773-5789, December 2008.

[33] J. T. Isaacs, D. J. Klein, and J. P. Hespanha, "Optimal sensor placement for time difference of arrival localization," in 48th IEEE Conference on Decision and Control 2009, (CDC 2009), Shanghai, China, December 15-18, 2009, pp. 7878-7884.

[34] I. Konchenko and F. Yanovsky, "Multilateration surveillance system arrangement at the airport area," in Photonics Applications in Astronomy, Communications, Industry, and High-Energy Physics Experiments 2010, Wilga, Poland May 24-29, 2010.

[35] A. Jasch, T. Feuerle, G. Scoor, and P. Hecker, "Geometrical siting considerations for wide area multilateration systems," in 2010 IEEE/ION Position Location and Navigation Symposium (PLANS), Indian Wells, CA, USA, May 4-6, 2010, pp. 1304-1308.

[36] I. A. Mantilla-Gaviria, R. F. Ruiz, J. V. Balbastre-T, and E. d. l. Reyes, "Application of Metaheuristic Optimization Techniques to Multilateration System Deployment," in Enhanced Solutions for Aircraft and Vehicle Surveillance Applications, ESAVS 2010, 
German Institute of Navigation (DGON), Berlin, Germany, March 16-17, 2010, p. Session 2B/3.

[37] F. Y. S. Lin and P. L. Chiu, "A near-optimal sensor placement algorithm to achieve complete coverage/discrimination in sensor networks," IEEE Communications Letters, vol. 9, pp. 43-45, January 2005.

[38] J. O. Roa, A. R. Jimémez, F. Seco, J. C. Prieto, and J. Ealo, "Optimal placement of sensors for trilateration: regular lattices vs meta-heuristic solutions," in 11th International Conference on Computer Aided Systems Theory (EUROCAST'07), Las Palmas de Gran Canaria, Canary Islands, Spain, February 12-16, 2007, pp. 780-787.

[39] J. Neering, C. Fischer, M. Bordier, and N. Maízi, "Optimal sensor configuration for passive position estimation," in 2008 IEEE/ION Position Location and Navigation Symposium, Monterey, CA, May 5-8, 2008, pp. 951-960.

[40] B. Omidali and S. A.-A. B. Shirazi, "Sensor placement to improve the positioning performance based on Angle of Arrival (AOA)," Wireless Engineering and Technology, vol. 1, pp. 41-45, July 2010.

[41] L. Bo, Z. Xuejun, and Z. Shuang, "Multilateration station location study based on genetic algorithm," in 2010 International Symposium on Computational Intelligence and Design (ISCID), Hangzhou, October 29-31, 2010, pp. 60-63.

[42] C. H. Papadimitriou and K. Steiglitz, Combinatorial optimization: algorithms and complexity. New York: Dover Publications, Inc., 1982.

[43] C. Blum and A. Roli, "Metaheuristics in combinatorial optimization: overview and conceptual comparison," ACM Computing Surveys, vol. 35, pp. 268-308, September 2003.

[44] F. Rothlauf, Design of modern heuristics: principles and application. New York, NY: Springer-Verlag Berlin and Heidelberg GmbH \& Co., 2011.

[45] A. L. Peressini, F. E. Sullivan, and J. J. U. Jr., The mathematics of nonlinear programming. New York, Inc.: Springer, 1988.

[46] D. P. Bertsekas, Nonlinear programming, 2nd ed.: Athenea Scientific, 1999.

[47] M. R. Garey and D. S. Johnson, Computer and intractability: a guide to the theory of NP-Completeness. New York, NY, USA: W. H. Freeman \& Co., 1990.

[48] A. Schrijver, Combinatorial optimization - polyhedra and efficiency. Berlin: Springer-Verlag, 2003.

[49] U. Flarup, "Optimization and evaluation problems over the real numbers," PhD. thesis, Department of Mathematics and Computer 
Science (IMADA), University of Southern Denmark, Odense, Denmark, April 2008.

[50] S. A. Cook, "The complexity of theorem-proving procedures," in Third ACM Symposium on Theory of Computing, New York, USA, 1971, pp. 151-158.

[51] G. L. Nemhauser and A. L. Wolsey, Integer and combinatorial optimization. New York: Wiley, 1988.

[52] F. Glover, "Future paths for integer programming and links to artificial intelligence," Comput. Oper. Res., vol. 13, pp. 533-549, 1986.

[53] I. H. Osman and G. Laporte, "Metaheuristics: a bibliography," Ann. Oper. Res., vol. 63, pp. 513-623, 1996.

[54] T. Stützle, "Local search algorithms for combinatorial problems analysis, algorithms and new applications," presented at the DISKIDissertationen zur Künstliken Intelligenz. infix, Sankt Augustin, Germany, 1999.

[55] L. Bianchi, M. Dorigo, L. M. Gambardella, and W. J. Gutjahr, "A survey on metaheuristics for stochastic combinatorial optimization," Natural Computing, vol. 8, pp. 239-287, 2009.

[56] L. J. Fogel, A. J. Owens, and M. J. Walsh, Artificial intelligence through simulated evolution. New York, NY, USA: Wiley, 1966.

[57] R. I. Rechenberg, Evolutionsstrategie:optimierung technischer systeme nach prinzipien der biologischen evolution. Stuttgart, Germany: Frommann-Holzboog, 1973.

[58] J. H. Holland, Adaptation in natural and artificial systems. Ann Harbor, MI, USA: The University of Michigan Press, 1975.

[59] D. E. Goldberg, Genetic Algorithms in Search, Optimization and Machine Learning. Boston: Addison-Wesley, 1989.

[60] L. Davis, Handbook of genetic algorithms. New Yor, NY, USA: Van Nostrand Reinhold, 1991.

[61] Z. Michalewicz, Genetic algorithms + data structures = evolution programs. Berlin Heidelberg: Springer Verlag, 1992.

[62] C. R. Reeves, Modern heuristic techniques for combinatorial problems. New York, NY, USA: John Wiley \& Sons Inc, 1993.

[63] C. Darwin, On the origin of species by means of natural selection. London, UK: Murray, 1859.

[64] S. N. Sivanandam and S. N. Deepa, Introduction to Genetic Algorithms. Berlin: Springer, 2007.

[65] A. Hertz and D. Kobler, "A framework for the description of evoluationary algorithms," European Journal of Operational Research, vol. 126, pp. 1-12, Oct. 2000. 
[66] I. A. Mantilla-Gaviria, M. Leonardi, and G. Galati, "Lower Bounds of accuracy for Mode S distributed sensors network," Università degli studi di Roma "Tor Vergata", Rome, June 2010.

[67] I. A. Mantilla-Gaviria, M. Leonardi, G. Galati, J. V. Balbastre-T, and E. d. 1. Reyes, "Strategies to Design and Deploy Mode-S Multilateration Systems," in Tyrrhenian International Workshop on Digital Communications - Enhanced Surveillance of Aircraft and Vehicles (ESA V'11), Capri, Italy, September 12-14, 2011, pp. 167 172 .

[68] I. A. Mantilla-Gaviria, M. Leonardi, G. Galati, J. V. BalbastreTejedor, and E. d. l. Reyes, "An effective procedure to design the layout of standard and enhanced mode-S multilateration systems for airport surveillance," International Journal of Microwave and Wireless Technologies, vol. 4, pp. 199-207, 2012.

[69] M. Abramowitz and I. A. Stegun, Handbook of mathematical functions: with formulas, graphs, and mathematical tables. Washington, USA: Dover Publications, June 1965.

[70] M. Clerc, Particle Swarm Optimization. London: ISTE, 2006.

[71] M. Dorigo and T. Stützle, Ant Colony Optimization. Cambridge, MA: MIT Press, 2004.

[72] G. H. Golub and C. F. V. Loan, Matrix Computations, Third ed. Baltimore: the Johns Hopkins University Press, 1996.

[73] D. L. Phillips, "A technique for the numerical solution of certain integral equations of the first kind," Journal of the ACM, vol. 9, pp. 84-97, 1962.

[74] A. N. Tikhonov, "Solution of incorrectly formulated problems and the regularization method," Sovieth Math. Dokl., vol. 4, pp. 10351038, 1963.

[75] P. C. Hansen, "Regularization, GSVD and truncated GSVD," BIT, vol. 29, pp. 491-504, September 1989.

[76] P. C. Hansen, Rank-Deficient and Discrete iII-Posed Problems, Numerical Aspects of Linear Inversion. Philadelphia: SIAM, 1998.

[77] S. V. Huffel and J. Vandewalle, The Total Least Squares Problem Computational Aspects and Analysis. Philadelphia: SIAM, 1991.

[78] R. D. Fierro and J. R. Bunch, "Collinearity and total least squares," SIAM Journal on Matrix Analysis and Applications, vol. 15, pp. 1167-1181, October 1994.

[79] S. Twomey, Introduction to the Mathematics of Inversion in Remote Sensing and Indirect Measurements. New York: Dove Publications, 1996.

[80] A. Ribés and F. Schmitt, "Linear Inverse Problem in Imaging," IEEE Signal Processing Magazine, vol. 25, pp. 84-99, July 2008. 
[81] F. Santosa, Y.-H. Pao, W. W. Symes, and C. Holland, Inverse problems of acoustic and elastic waves. Philadephia: SIAM, 1984.

[82] I. J. D. Craig and J. C. Brown, Inverse problems in astronomy. Bristol, UK: Adam Hilger, 1986.

[83] F. Natterer, The mathematics of computarized tomography. New York: Wiley, 1986.

[84] A. S. Carasso, "Overcoming Hölder discontinuity in ill-posed continuation problems," SIAM J. Numer. Anal., vol. 31, pp. 15351557, 1994.

[85] M. Bertero, T. A. Poggio, and V. Torre, "Ill-posed problems in early vision," in Proc. IEEE, 1988, pp. 869-889.

[86] R. F. Harrington, Field computations by moment methods. New York: Macmillan, 1993.

[87] W. Menke, Geophysical data analysis: discrete inverse theory. San Diego: Academic Press, 1989.

[88] J. Christensen-Dalsgaard, J. Schou, and M. Thompson, "A comparison of methods for inverting helioseismic data," Month. Not. R. Astr. Soc., vol. 242, pp. 353-369, 1990.

[89] J. J. M. Cuppen, "A numerical solution of the inverse problem of electrocardigraphy," PhD. Thesis, Department of Mathematics, University of Amsterdam, Amsterdam, 1983.

[90] H. C. Andrews and B. R. Hunt, Digital Image Restoration. Englewood Cliffs, NJ: Prentice-Hall, 1977.

[91] R. Barakat and G. Newsam, "Remote sensing of the refractive index structure parameter via inversion of Tatarski's integral equation for both spherical and plane wave situations," Radio Science, vol. 19, pp. 1041-1056, 1984.

[92] D. Colton and R. Kress, Integral equation methods for scattering theory. New York: Wiley, 1983.

[93] A. J. Thorpe and L. L. Scharf, "Data adaptive rank-shaping methods for solving least squares problems," IEEE Transactions on Signal Processing, vol. 43, pp. 1591-1601, 1995.

[94] D. M. Titterington, "Common structure of smoothing techniques in statistics," Int. Stat. Rev., vol. 53, pp. 141-170, 1985.

[95] C. D. Mol, "A critical survey of regularized inversion methods," in Inverse problems in scattering and imaging, M. Bertero and E. R. Pike, Eds., ed Bristol, UK: Adam Hilger, 1992, pp. 345-370.

[96] D. W. Marquardt, "Generalized inverses, ridge regression, biased linear estimation, and nonlinear estimation," Technometrics, vol. 12, pp. 591-612, August 1970.

[97] R. J. Hanson, "A numerical method for solving Fredholm integral equations of the first kind using singular values," SIAM Journal on Numerical Analysis, vol. 8, pp. 616-622, 1971. 
[98] P. C. Hansen, "Analysis of discrete ill-posed problems by meas of the L-curve," SIAM Review, vol. 34, pp. 561-580, 1990.

[99] I. A. Mantilla-Gaviria, M. Leonardi, G. Galati, and J. V. BalbastreTejedor, "Comparison of localization algorithms for Multilateration (MLAT) systems in airport surface surveillance," in International Symposium Enhanced Solutions for Aircraft and Vehicle Surveillance Applications, ESAVS 2013, German Institute of Navigation (DGON), Berlin, Germany, March 20-21, 2013, p. Session $4 \mathrm{c}$.

[100] I. A. Mantilla-Gaviria, M. Leonardi, J. V. Balbastre-T, and E. d. 1. Reyes, "Singular value decomposition and Tikhonov regularization in hyperbolic passive location," in Mathematical Modelling in Engineering \& Human Behaviour, Valencia, Spain, September 6-9, 2011, pp. 183-188.

[101] I. A. Mantilla-Gaviria, M. Leonardi, J. V. Balbastre-Tejedor, and E. d. l. Reyes, "On the application of singular value decomposition and Tikhonov regularization to ill-posed problems in hyperbolic passive location," Mathematical and Computer Modelling, vol. Available online from 21 March 2012, 2012.

[102] H. L. v. Trees, Detection, Estimation and Modulation Theory vol. Part I: Wiley-Interscience, 2001.

[103] W. H. Foy, "Position-Location Solution by Taylor-Series Estimation," IEEE Transactions on Aerospace and Electronic System, vol. AES-12, pp. 187-194, March 1976.

[104] R. O. Schmidt, "A new approach to geometry of range difference location," IEEE Transactions on Aerospace and Electronic System, vol. AES-8, pp. 821-835, Nov. 1972.

[105] J. O. Smith and J. S. Abel, "The spherical interpolation method of source localization," IEEE Journal of Oceanic Engineering, vol. OE12, pp. 246-252, January 1987.

[106] J. S. Abel and J. O. Smith, "The spherical interpolation method for closed-form passive source localization using range difference measurements," in IEEE International Conference on Acoustics, Speech, and Signal Processing, ICASSP '87, Dallas, Texas, USA, April 6-9, 1987, pp. 471-474.

[107] B. Friedlander, "A passive localization algorithm and its accuracy analysis," IEEE Journal of Oceanic Engineering, vol. OE-12, pp. 234-245, 1987.

[108] H. C. Schau and A. Z. Robinson, "Passive Source Localization Employing Intersecting Spherical Surfaces from Time-of-Arrival Differences," IEEE Transactions on Acoustics, Speech, And Signal Processing, vol. ASSP-35, pp. 1223-1225, August 1987.

[109] Y. T. Chan and K. C. Ho, "An efficient closed-form localization solution from time difference of arrival measurements," in IEEE 
International Conference on Acoustics, Speech, and Signal Processing (ICASSP) Adelaide, South Australia, Australia, April 19-22, 1994, pp. 393-396.

[110] Y. T. Chan and K. C. Ho, "A simple and efficient estimator for hyperbolic location," IEEE Transactions on Signal Processing, vol. 42, pp. 1905-1915, August 1994.

[111] M. Geyer and A. Daskalakis, "Solving passive multilateration equations using Bancroft's algorithm," in Digital Avionics Systems Conference, Bellevue, WA, USA, 1998, pp. F41/1 - F41/8.

[112] Wikipedia. http://en.wikipedia.org/wiki/Multilateration.

[113] S. Bancroft, "An algebraic solution of the GPS equations," IEEE Transactions on Aerospace and Electronic System, vol. AES-21, pp. 56-59, January 1985.

[114] Y. Bar-Shalom, X. R. Li, and T. Kirubarajan, Estimation with applications to tracking and navigation. New York, USA: WileyInterscience, 2001.

[115] C. Reck, U. Berold, and L. P. Schmidt, "High Precision DOA Estimation of SSR Transponder Signals," in IEEE International Conference on Wireless Information Technology and Systems, Honolulu, USA, Aug. 29 - Sept. 03, 2010, pp. 1 - 4.

[116] E. Perl and M. J. Gerry, "Target Localization Using TDOA Distributed Antenna," USA Patent US 2005/0035897 A1, Feb. 17 2005.

[117] I. A. Mantilla-Gaviria, M. Leonardi, G. Galati, J. V. Balbastre-T, and E. d. 1. Reyes, "Improvement of multilateration (MLAT) accuracy and convergence for airport surveillance," in Tyrrhenian International Workshop on Digital Communications - Enhanced Surveillance of Aircraft and Vehicles (ESAV'11), Capri, Italy, September 12-14, 2011.

[118] I. A. Mantilla-Gaviria, M. Leonardi, G. Galati, J. V. BalbastreTejedor, and E. d. 1. Reyes, "Efficient location strategy for airport surveillance using mode-s multilateration systems," International Journal of Microwave and Wireless Technologies, vol. 4, pp. 209$216,2012$.

[119] A. MacLeod, "Finite-dimensional regularization with nonidentity smoothing matrices," Linear Algebra Appl., vol. 111, pp. 191-207, 1988.

[120] V. A. Morozov, "On the solution of functional equations by method of regularization," Sovieth Math. Dokl., vol. 7, pp. 414-417, 1966.

[121] V. A. Morozov, Methods for solving incorrectly posed problems. New York: Springer-Verlag, 1984.

[122] H. Gfrerer, "An a posteriori parameter choice for ordinary and iterated Tikhonov regularization of ill-posed problems leading to 
optimal convergences rates," Math. Comp., vol. 49, pp. 507-522, 1987.

[123] T. Raus, "The principle of the residual in the solution of ill-posed problems with nonselfadjoint operator," Uchen. Zap. Tartu Gos. Univ., vol. 75, pp. 12-20 (in Russian), 1985.

[124] M. Hanke and T. Raus, "A general heuristic for choosing the regularization parameter in ill-posed problems," SIAM J. Sci. Comput., vol. 17, pp. 956-972, 1996.

[125] G. H. Golub, M. T. Heath, and G. Wahba, "Generalized crossvalidation as a method for choosing a good ridge parameter," Technometrics, vol. 21, pp. 215-223, 1979.

[126] G. Wahba, "Practical approximate solutions to linear operator equations when the data are noisy," SIAM J. Numer. Anal., vol. 14, pp. 651-667, 1977.

[127] J. M. Varah, "On the numerical solution of ill-conditioned linear systems with applications to ill-posed problems," SIAM Journal on Numerical Analysis, vol. 10, pp. 257-267, 1973.

[128] G. H. Golub and C. F. V. Loan, "An analysis of the total least squares problem," SIAM J. Numer. Anal., vol. 17, pp. 883-893, 1980. 